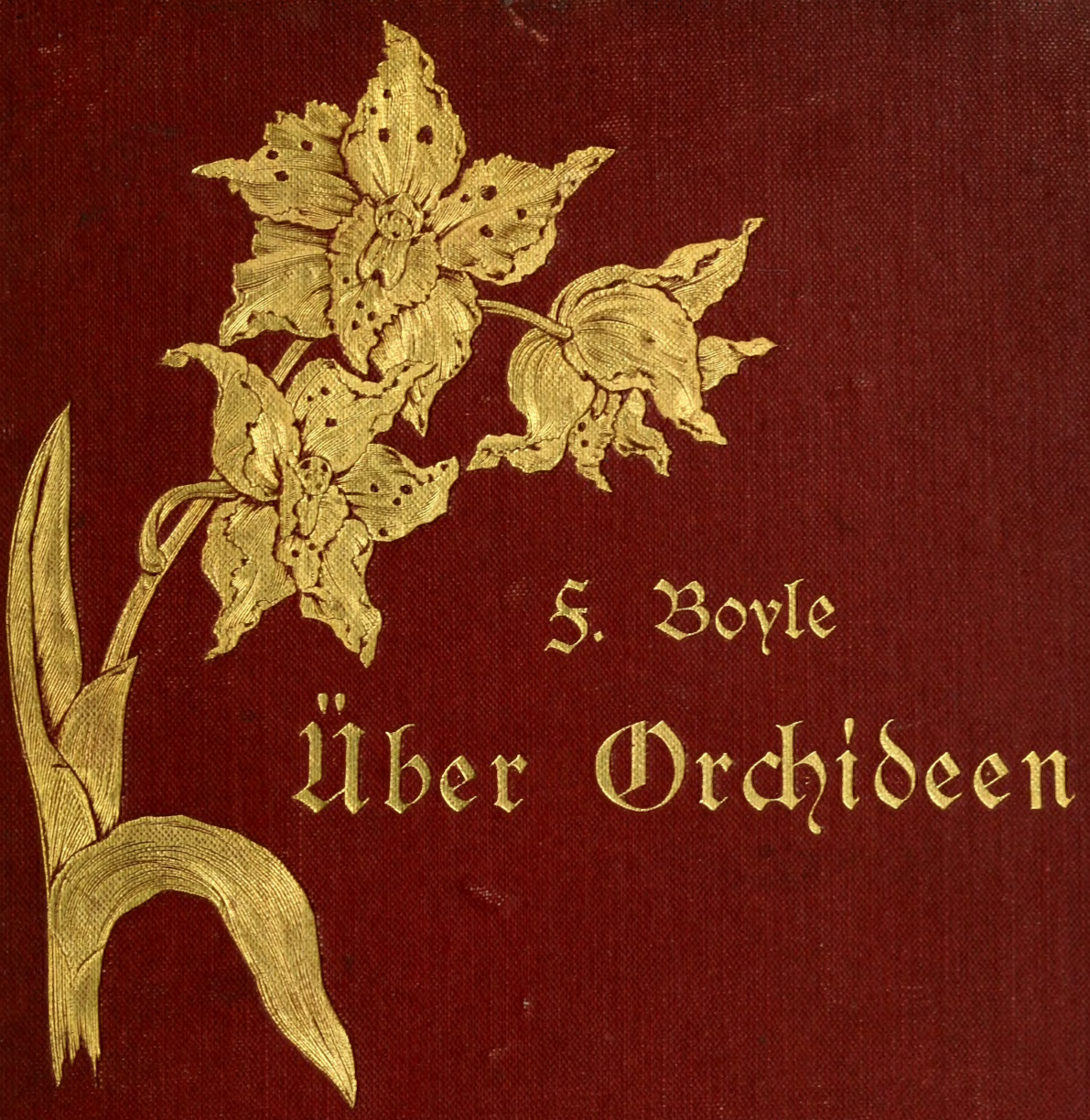




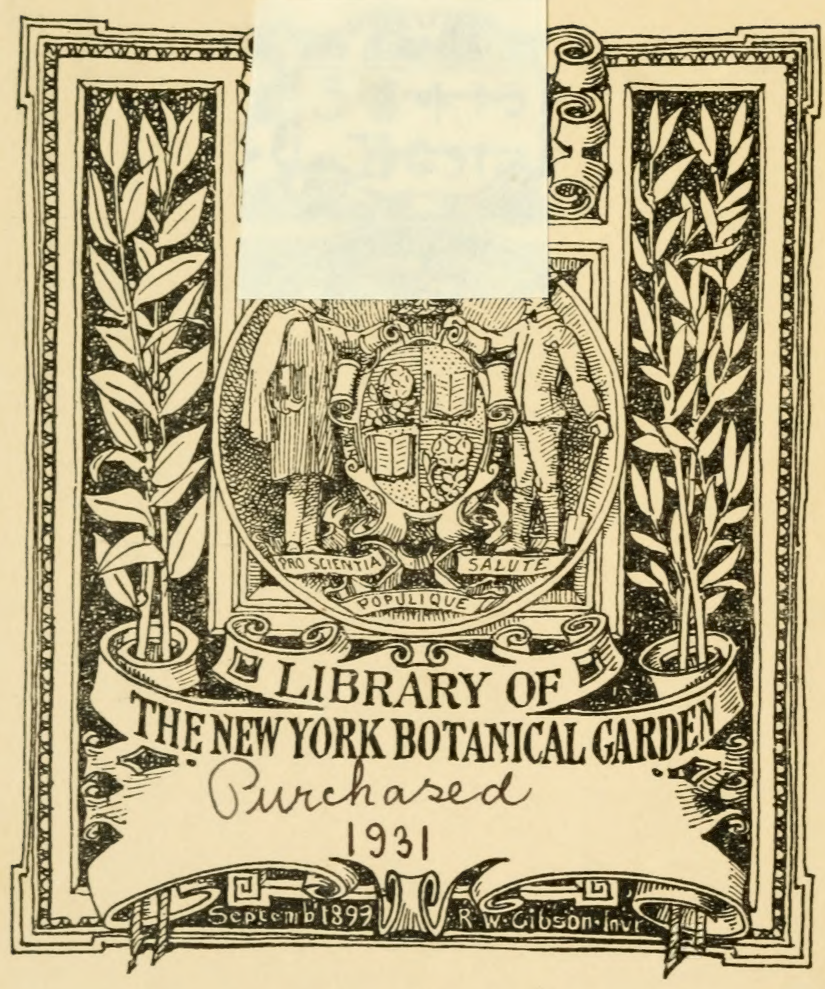



über Drdideen. 




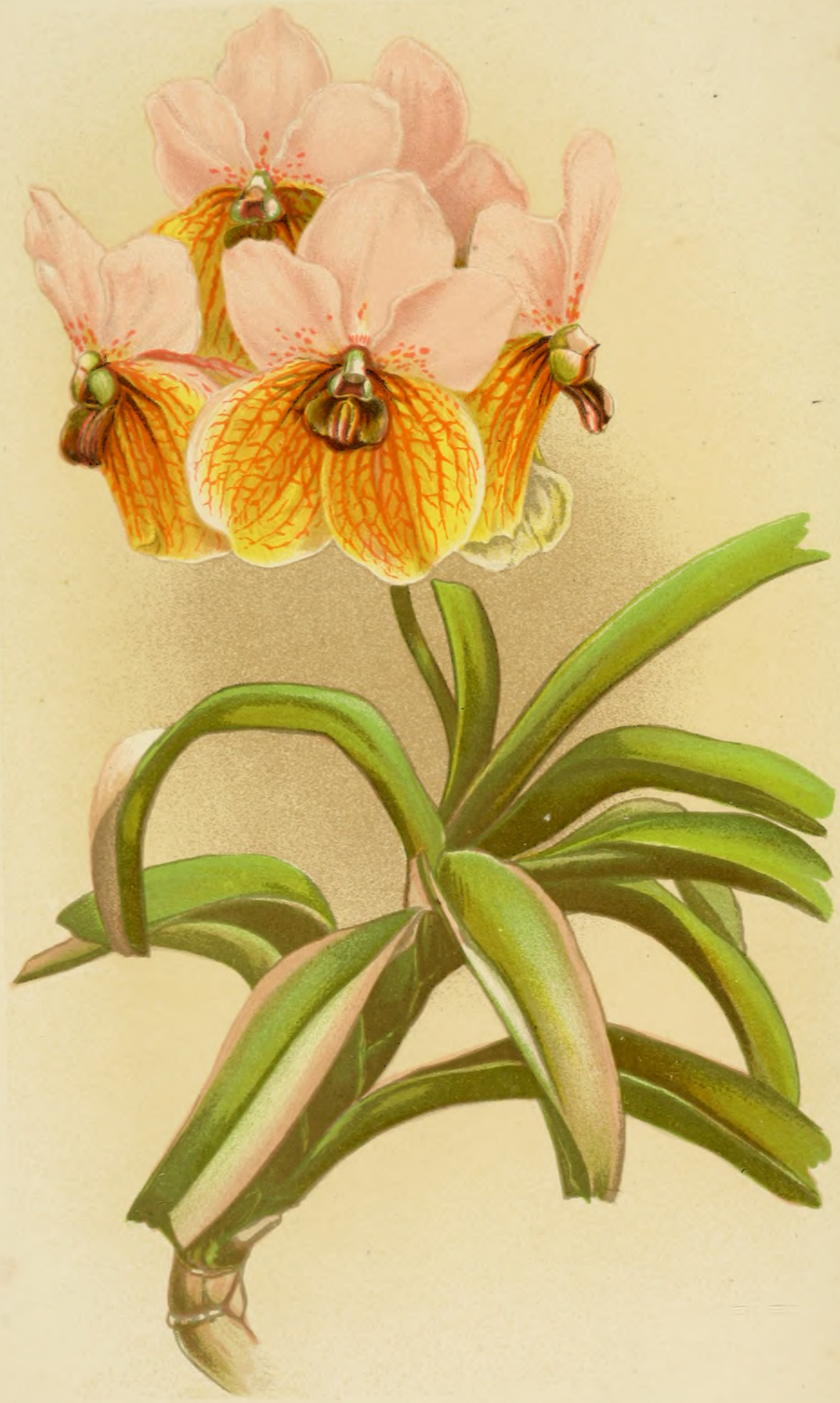




$$
\text { f. Boyle. }
$$

\section{ïber (Drabiden.}

\section{Dentiche Original=2lusgabe,}

herausgegeben vor

\section{Dr. $\mathfrak{f} \cdot \mathfrak{K} \mathfrak{x}$ änjlin,}

profeffor in Berlin.

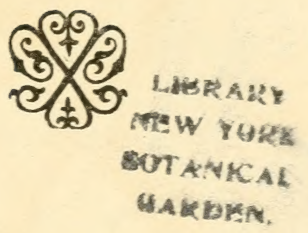

2rit 8 farbendrudtafeln.

Berliti.

Derlagsbudbandlung Paul Parev.

Berlag für Randivirtidaft, Bartenbau uto Foritweien.

SW., Eqebemannjtrafie 10.

1896. 
QL 66

Al

$B 655$

1896 


\section{Dorwort des herausgebers.}

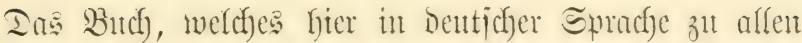
Denen reben joll, bie fït Surchidoen, iffre Sultur 1 mo alles, was

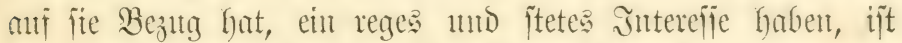

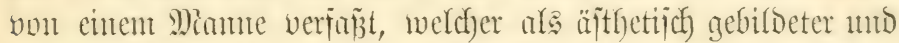
fïr jein Thenta Yeideniffraftlich) begeifterter Raie zu Rnien rebet. Er will begeiftern, wie er jelber begeiftert ift; er will den Srcfioeen Frembe emverfen bei allen benten, welche ciner

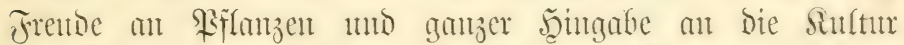

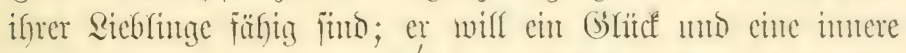

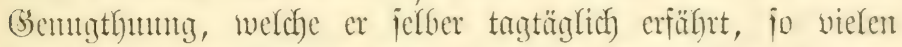

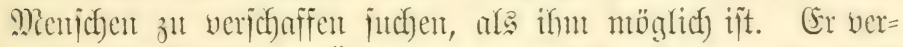
futcht alle Mittel ber Überredung, in allen Formen, bie nach

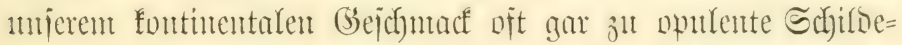

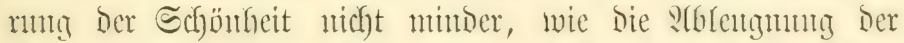

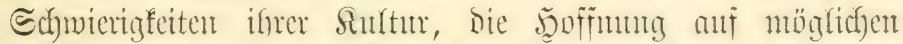

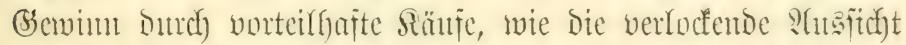

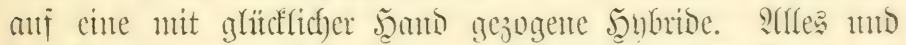

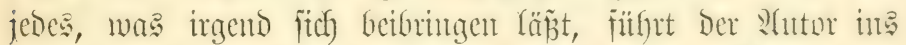

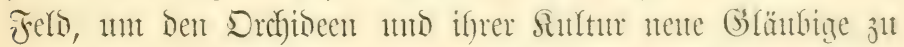

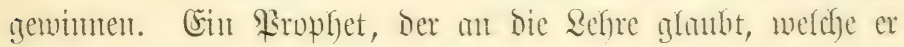
presigt, unt fie andareiten mill!

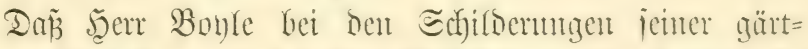
merijchen Berjuche unb bei ben Patichlä̈zen fïr Gegimtente

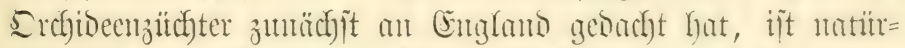

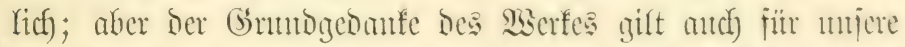

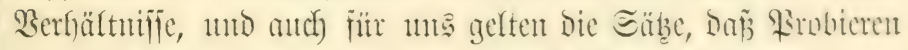

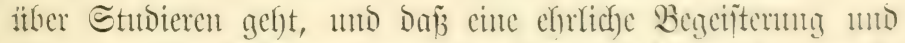
Siebe ju ben

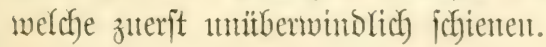




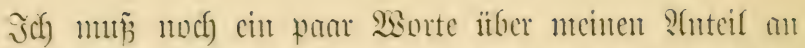

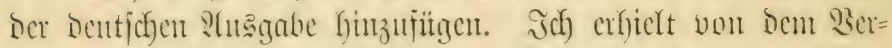
Yeger, Dem Dr. Finren, Das fertige Miantffript mit Der Bitte,

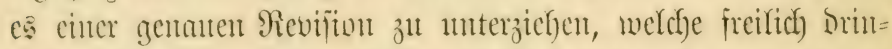
geno nötig war. Die ?tufangafapitel hatte ich) ganı nen ju itherjetzen; bei Den jpäteren Sapiteln, weldhe von vonnferein

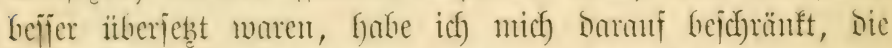

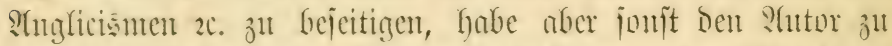

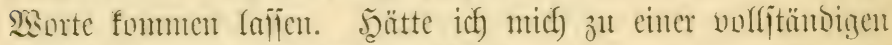

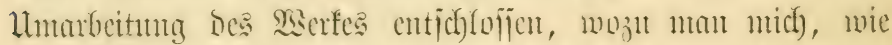
icf noch cinmal bemerfe, nicht aufgeforisert batte, fo witroc

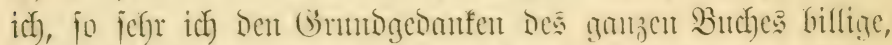

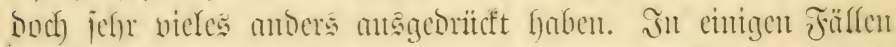

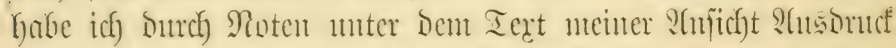

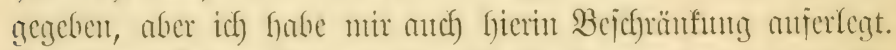
Eim Botanifer muñ ja notwenbigerweije manches anters be=

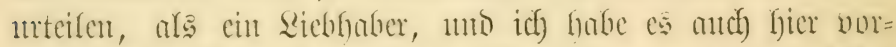

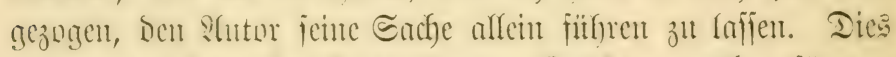
vormen zlt benterfen hiclt ich) Der Seritif gegentitber fïr $\mathrm{cr}=$

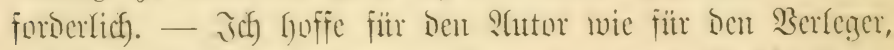

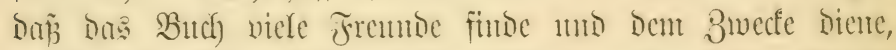

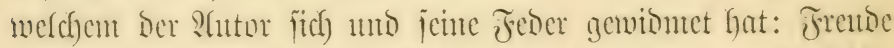
unto Begcifterung für Die Sultur Der Erchioneen jut erwecfent.

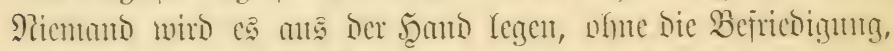

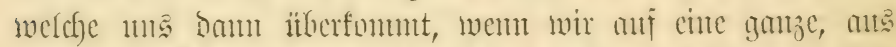

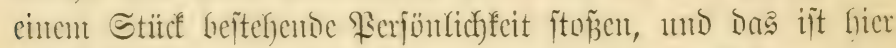

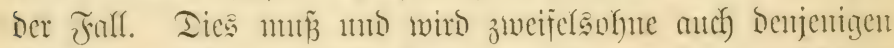

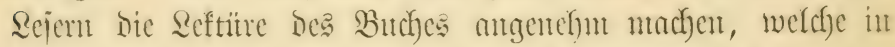

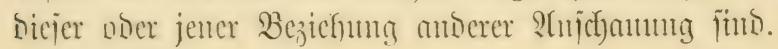

Berlin= Osr. Sidfterfelde, Friffling 1896.

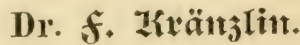




\section{Inthalt.}

Einteitung .................... Eeite 1

Frites fapitel: Wie iđ es anjing, Gartenbau ว̆l treiben . . 5

Breites Rapitel . . . . . . . . . . . . . . 11

Drittes fapitel . . . . . . . . . . . . . 17

Biertes Stapiter: Eine Srchibeen=2Uftion . . . . . . . 24

Jünjteß Sapitel: Srchibeen in allgemeinen . . . . . . 39

Sechites Stapitel: Stalthaus=Drdfideen . . . . . . 53

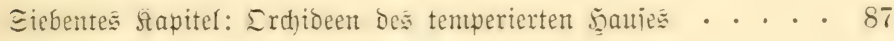

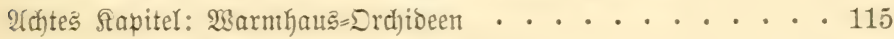

Reuntes Sapitel: Fine verjdyollene Drdibee. . . . . . . 143

Behntę FapiteY: Fine Drchideen=F̃arm ... . . . . 150

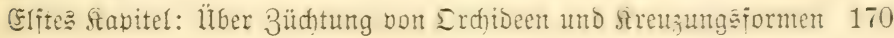





\section{Einleitun.}

Der Bwedf, welchen idf Durch bies Butch erreichen mollte, ijt an flariten in einem Brieje Dargelegt, melchen ich nor cinigen Whonaten an cine 3 eitung $=\Re$ Redaftion richtete und welchen ich) fier in Sortfaut mitteile:

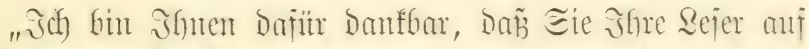

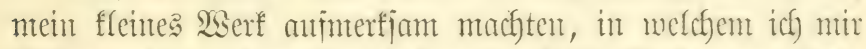

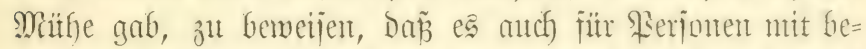

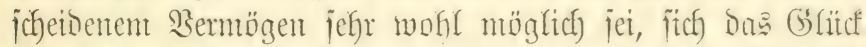

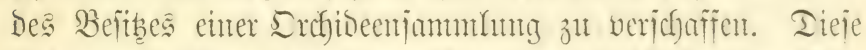
(Erfementmis allgemeiner zat mathen, erad te ich) fït Den Bei=

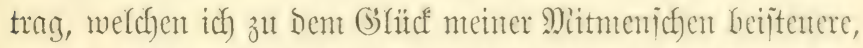
und ich Githe mir ein, Darjer ebenjoviel mert iit, als mantches

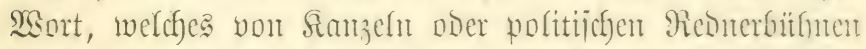

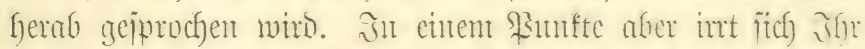
Berichteritatter, indont er mir zutgleid) cin Somm(iment mad)t,

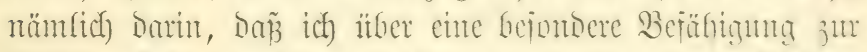

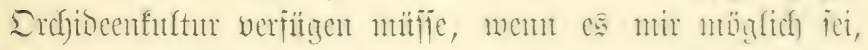

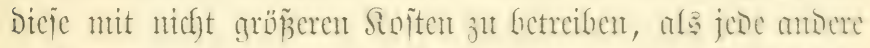

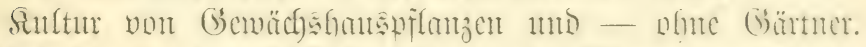

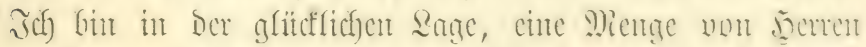

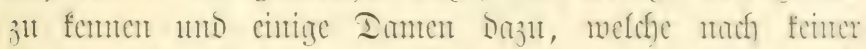

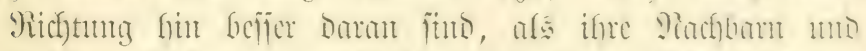
weld)e, gleid) mir, anth feine bcjontorent Echmierinfeiten

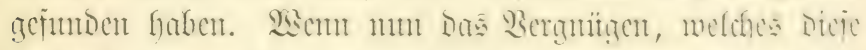

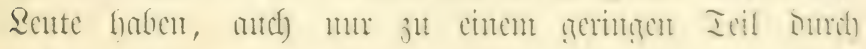
Drdideen. 


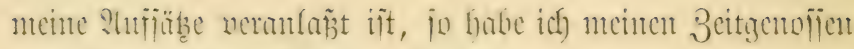
menigitens etwas (jittes gethan."

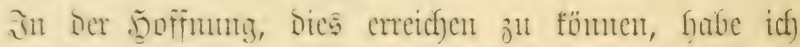

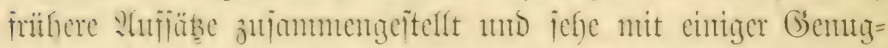

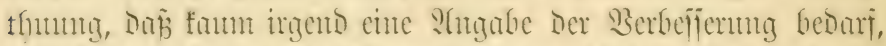

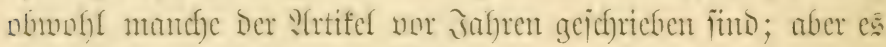

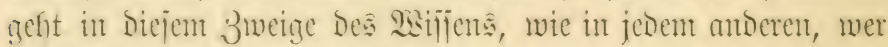

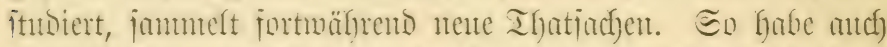

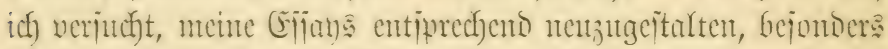

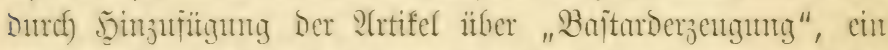

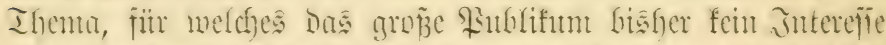
hatte, weil es abjolut nichts bavon erjufr); Denu tfatjächlich) hat f̈̈f) niemand Die Dithle gegeben, cinen Bericht itber bie

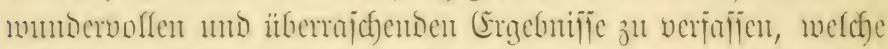

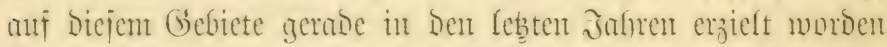

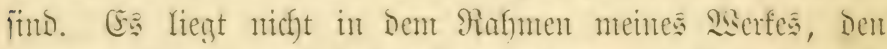
ganzen Ilmiang bicier 2(rbeiten Darjulegen, jedoch wird jeder,

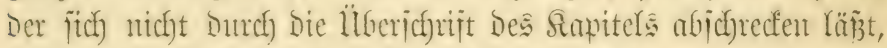

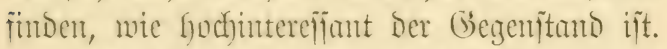

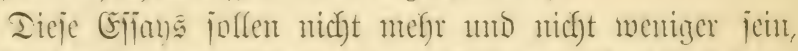

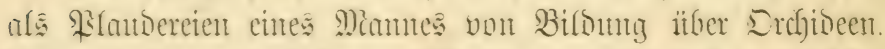
Eie enthalten matiurlidf eine Mienge von Thatjachen, weldhe,

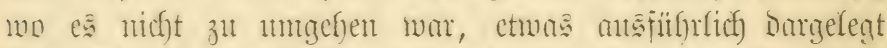

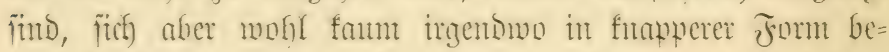
handelt fimben. Da mich alfes interefïtert, was Stchibeen

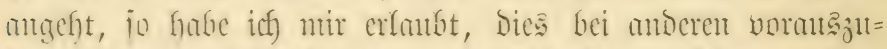

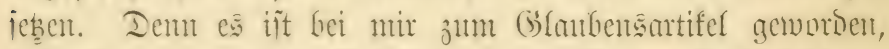

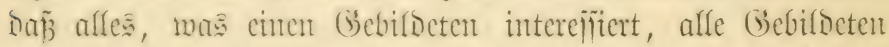
interefifteren mitije, jofern co nux in ciner flaren mo an= iprechenton Form worgetragen mirD. Bisher haben Gelehte

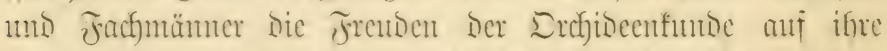

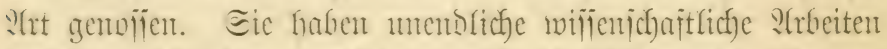

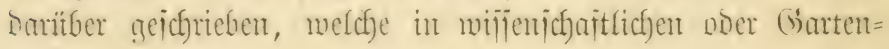




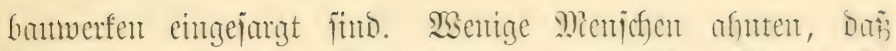

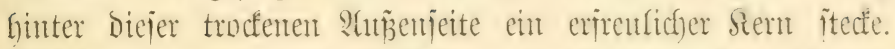

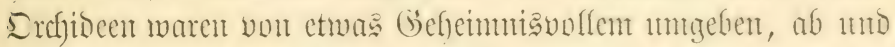

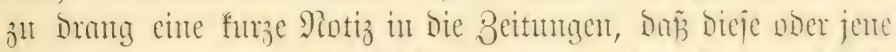
Siflanze mit einem unglaublichen Namen zu irgento cinem fahel=

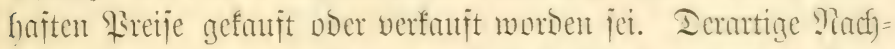

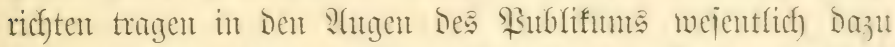
bei, bie ganze Frage unch umafhbarer ju machen, mo es it

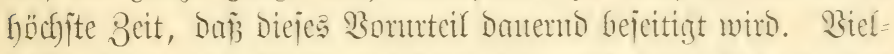

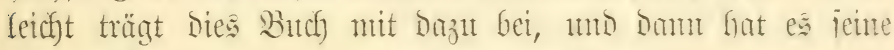
Schulloigfeit getthan - went es Rejer findet.

Die farbigen Iajeht füb verffeinerte Reprobuftionem der

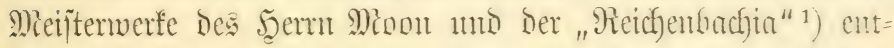

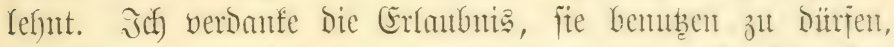

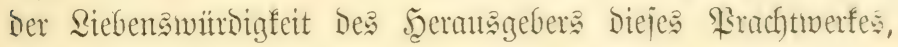
Şerm Friedr. Sander.

(Genane Sulturammeipungen habe idh nicht gegehent.

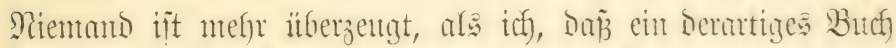

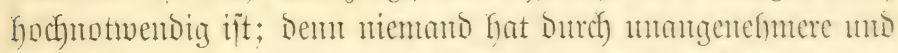

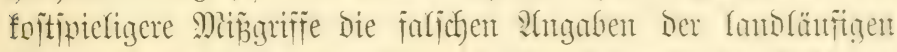

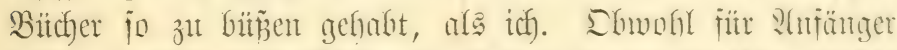

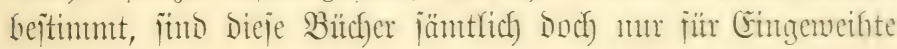

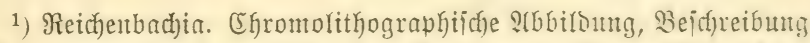
und Rufuranmeijung Der idönjten Drchibeen. Inter Mitwirfunty

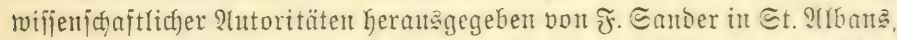

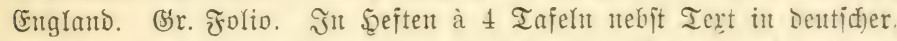

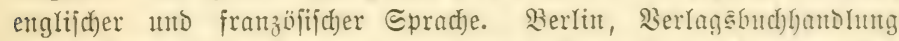
Baul Farey. 12 şefte bilben einen Banto. Subjfriptionaprets à ફ̧eft $10 \mathfrak{M}$.

Bereits erfduienten:

Erite Serie, Band 1, 48 Tafeln mit Tert, 1889. 220 9J.

2, 48 Iafeln mit $\mathfrak{T}$ ert, 1890.120 ,

3weite Serie, " 1, 48 Tafeln mit Tert, 1892. 120 "

2, 48 Tafeln mit Tert, 1894. 120 " 


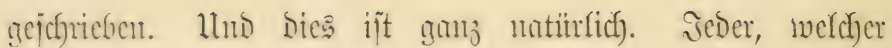
es, morin es inmer jein maty, ju eincr gemifjen Jertigfeit

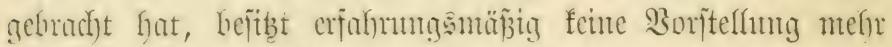

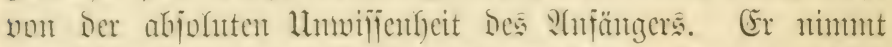

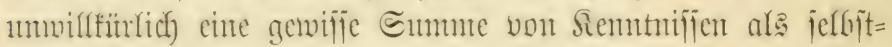

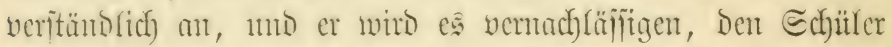
ither Die alfererjen Refrjäbe zu unterrichten, obwohl bieje Die

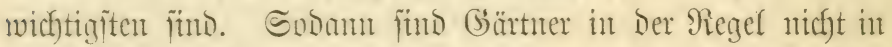
Der Sage nocr baran gemöhnt, bas, was fie jagen wollen,

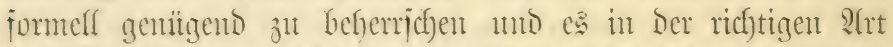

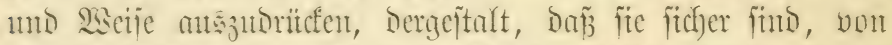

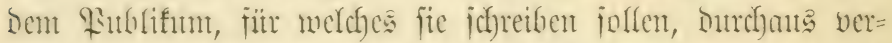

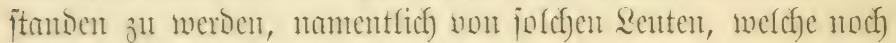
feine Affunng von Der Sache Gaben.

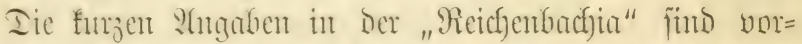
trefflid), aber wer citiert gern ?notijen, weld)e in Foliobänben zeritreut fino! Teitch's Manual of Orchidaceous Plants ift

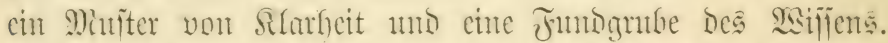

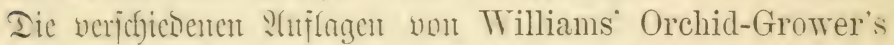

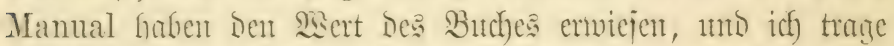

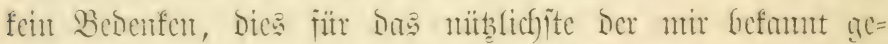

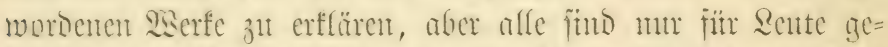

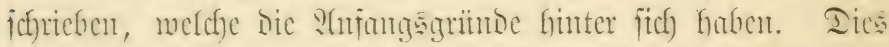

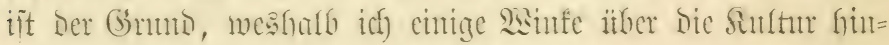

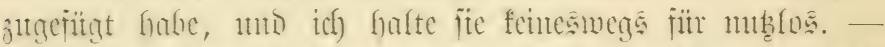

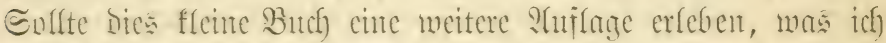

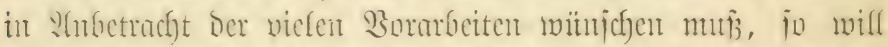

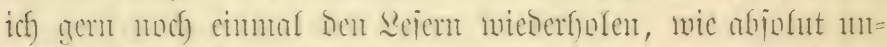

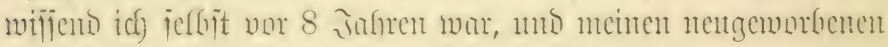

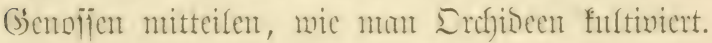




\section{(Er|tes Eapitel.}

\section{Wvic ich es antint Gartenbau sull treibent.}

Itm meine f́fente Biffa und alles, was p̈e emthält, hat fïf) bei meinen Befannten ein ganzer Surnus vou Eagen ge=

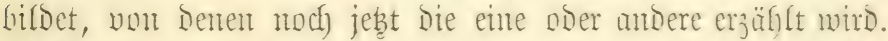

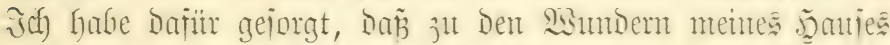

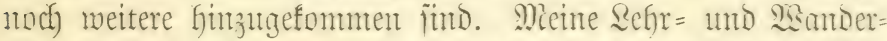
jofre fint vorither, aher indem ich Diejen gliteflichiten Ieil meine Reben jefy mìner meinen

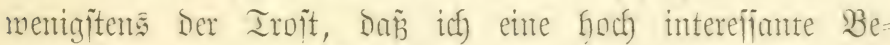

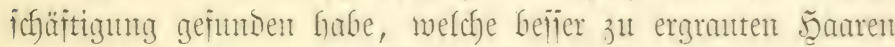

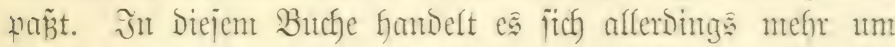

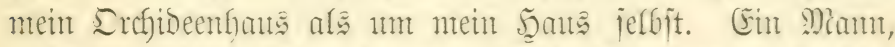
welcher in jeiner Jutgent wenig Botanif gelemt unt bies

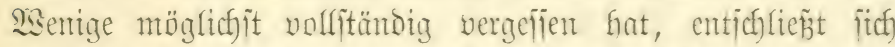
nicht leicht zul Diejem Gébiet Der höheren Gantenfunit, unt im Gefiugl jeiner abjoluten ltumifienheit wird er allen ben itber-

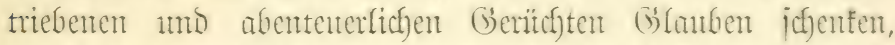
welche fitch an Erchidoen und ifre Sultur fnitpfen, und Da PGufflärung cine langiam arbeitento Miacht iit, wirb cin joldfer Peann jeinen gemacht hat, bevor er zum Biele gelangte.

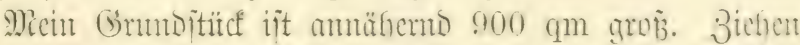

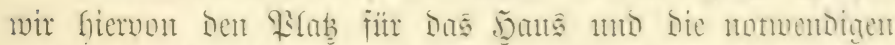

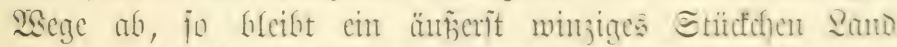

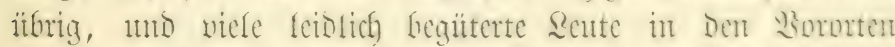




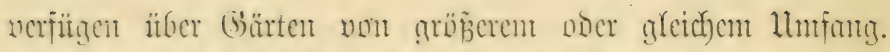

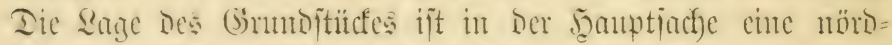

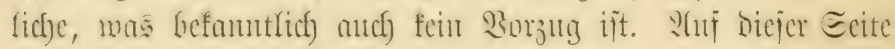

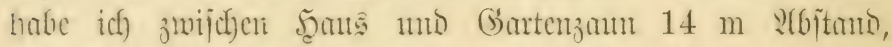
auf ber Sitjeite $16 \mathrm{~m}$, auf ber Sildjeite $19 \mathrm{~m}$ unb auf

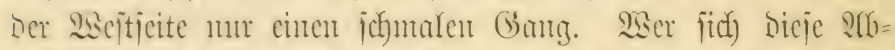

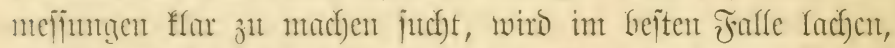

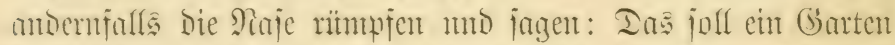

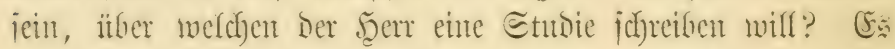

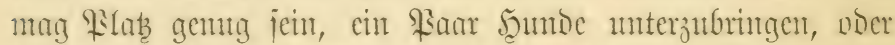
cine Partie Lawn-tennis jull ipeYen, ober einen Ieppicf) zu

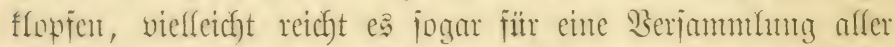

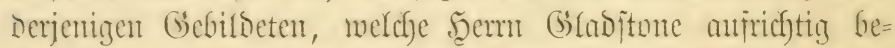
mumben, aber nimmerntef) fïtr einen Yeislichen Ganten mit

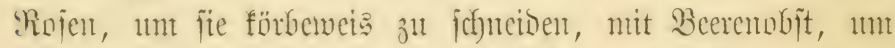
Frutchtgelee fït cine Fontilie jut fochen, mit Egampignonts, Iomaten, Sanjervien un Drchibeen. Dic indijchen Gautfer,

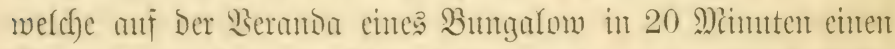
Mantgubaum hervormachjen lafien, mögen jo etwas fertig bringen, aher ein efrelicher (Effriftementich nicht. Hud Duch

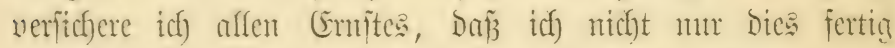

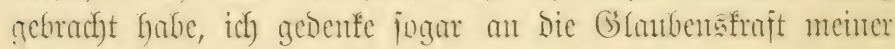

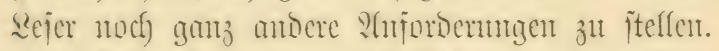

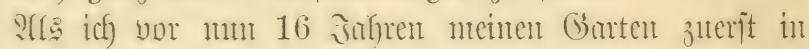

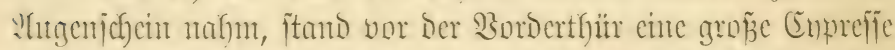

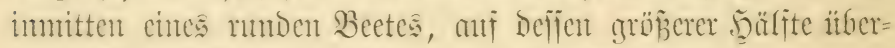
haupt feine Bhumen gebieffen, anf Der flemeren aber mur in

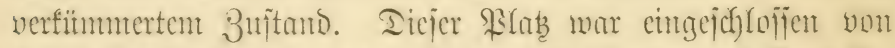

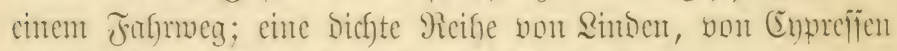
unterbrochen, itbermudberte bie Etadete mi affen હeiten, ein

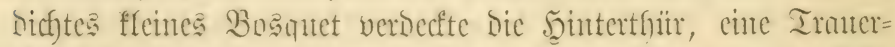

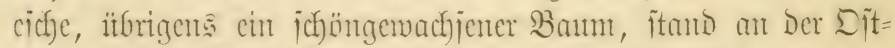

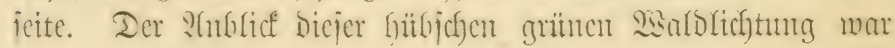




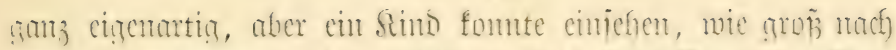

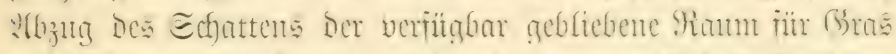

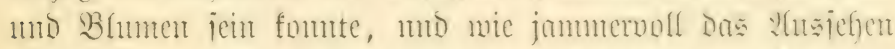

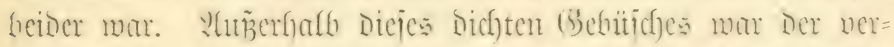

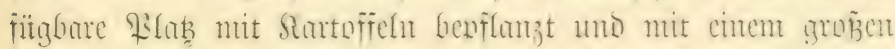
Befege fïr Şüfner bejebt.

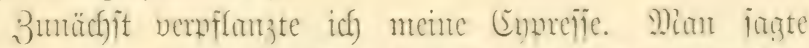

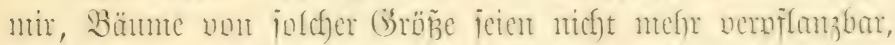

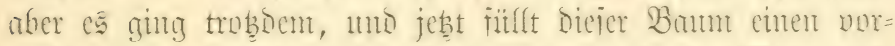

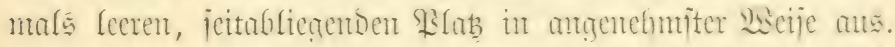

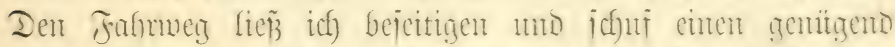

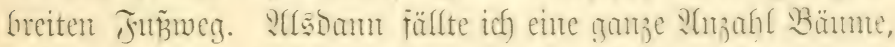

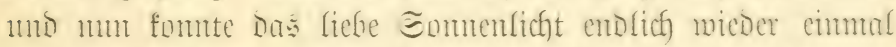

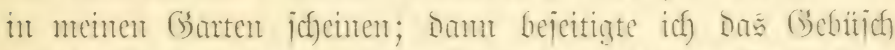

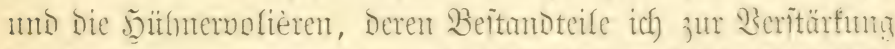

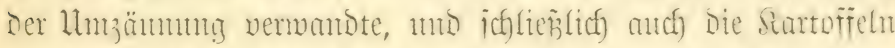
- fumz, icf muchte tabula rasa. Imm entfiés icf meine

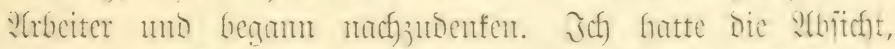

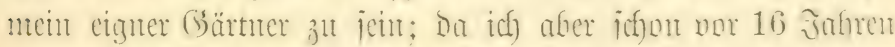

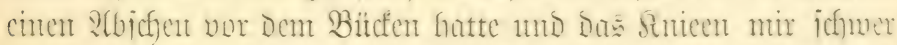

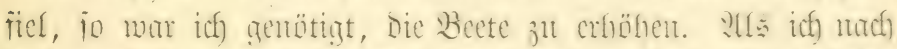

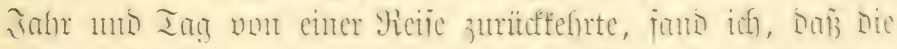

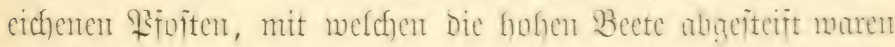

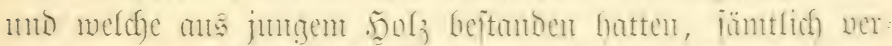
fanlt marent. Uln bicient lühelftano cin fiir allental burju bengen, wählte ich Ihombilnen als bifoîten, Die erite meiner

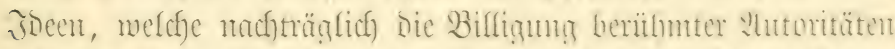

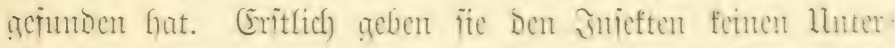

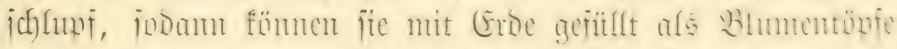

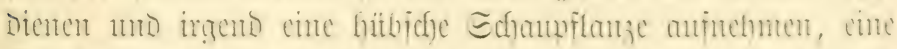

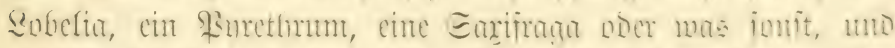

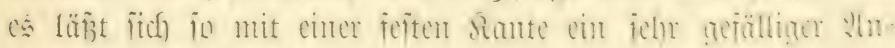

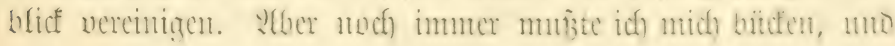




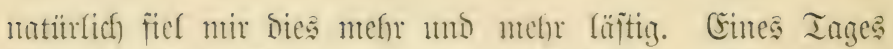

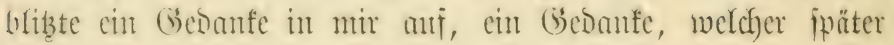
Das leitemise Primip mener Gartenfmit mumbe mo vielfeicht

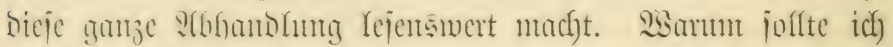

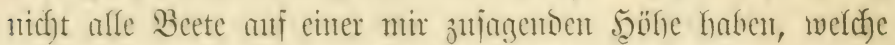
mir bas Bütfen erjparte? Da fein Gärtner frither anj emen äfnlichen Einfall geraten war, jo erjofien Der Sojtenpunft nächit als das cinjige Şundemis. Da ich gerabe bantals fïr

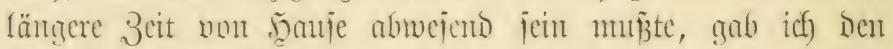

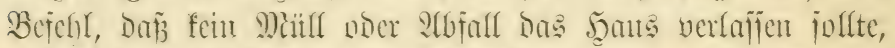

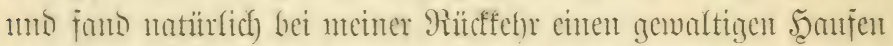

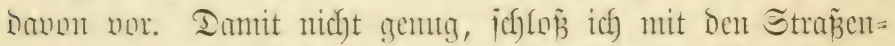
renigern einten Bertrag und erficlt vou ifunen Sefuricht zu

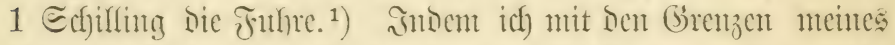

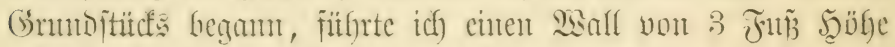

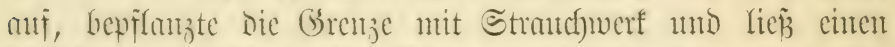
flitbich breiten Rand füt Bhtumen. Dies gelang ïber Ẽr= marten; Denn alfes, Bhtmen wie Strüudfer, trieben 1mL

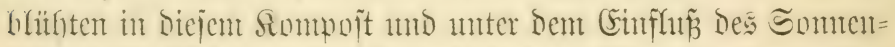

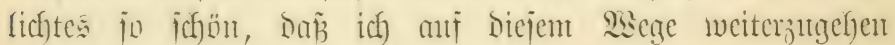
bejchlón.

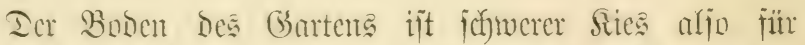

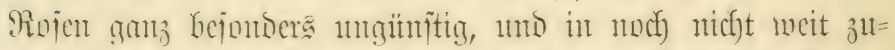
ritclfegentor Beit war ntein Garten ein Sumpf. Der fleine Rajen=

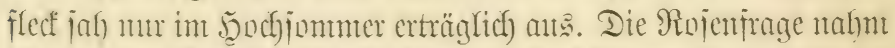

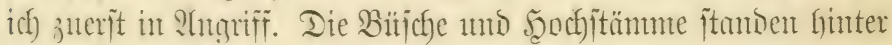

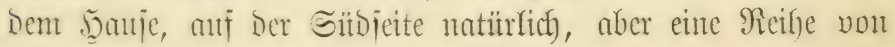

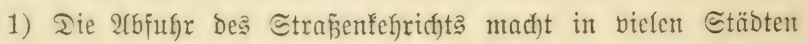
Englands viel größ̄ere folten, als bei uns in Dentic)land, Dasjelbe gilt von Der ausgeidad)teten Erde bei Renbauten, weldje gerabeznt cinc

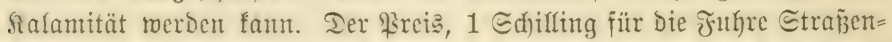

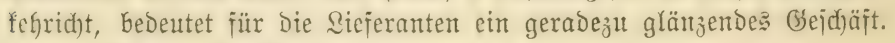
- Immerfung Des überiebers. 


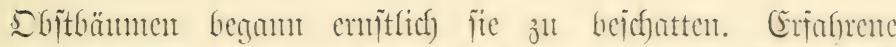

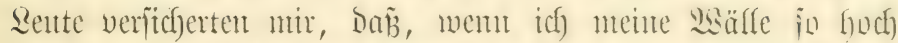

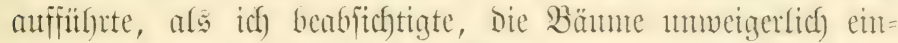

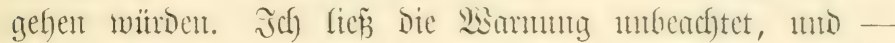

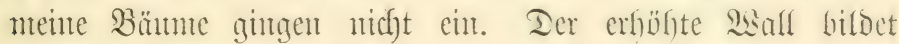

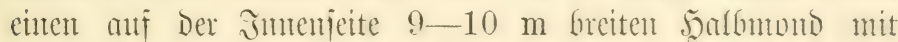

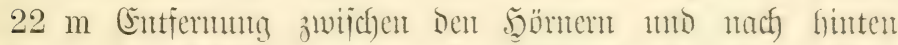

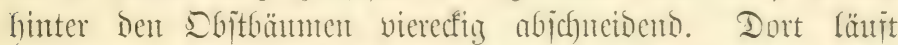

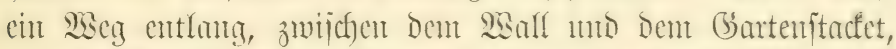

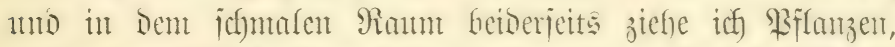
weldhe ntun nicht leicht jut fantfen befonmmt, wie Serbel, Echnitt=

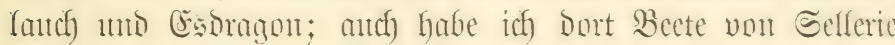

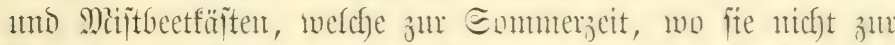

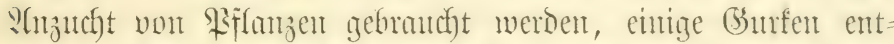

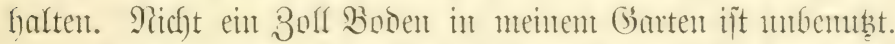

Der balfmento arjo ijt mit Bojen bejebt. Pachoent bor (Erbreich be erföhten

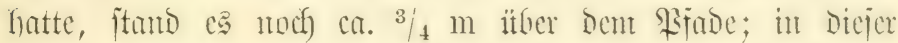

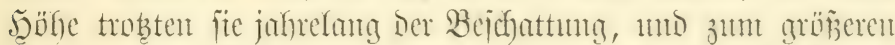
Teile merben fie bies femerhin thum, minteiten io longe,

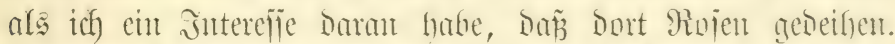

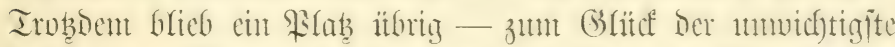

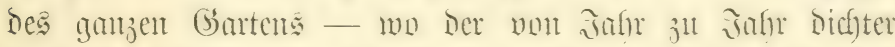

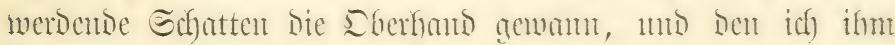
itbertajfen muñte. Dort pfflarzte ich) Saxifraga hỵmoides,

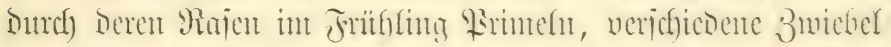

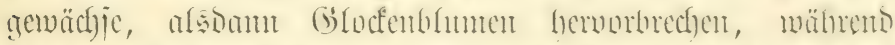

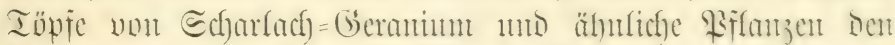

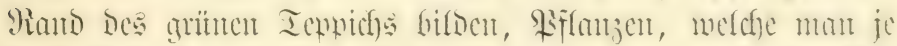

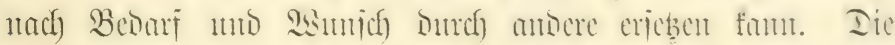
Bernentung Dicier Saxifraga it in ber Inat cine meiner

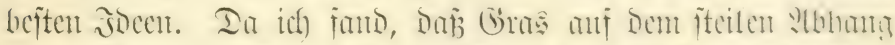

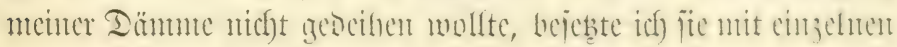




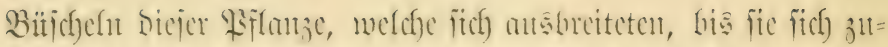

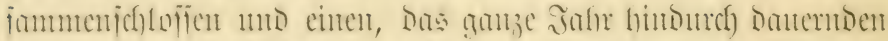

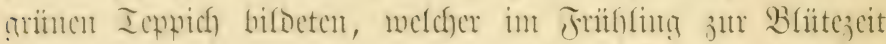

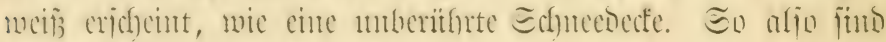

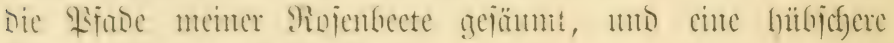
Siante ift jofwerlich Dentfor.

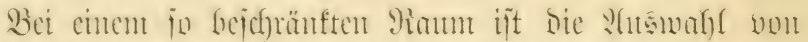

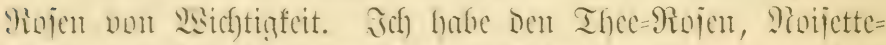

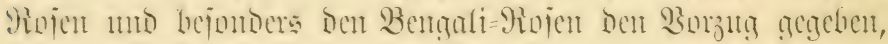

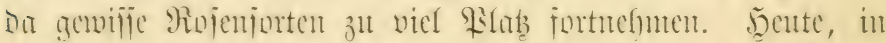

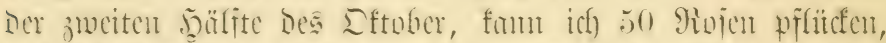

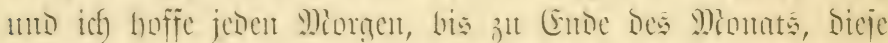

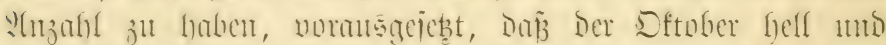

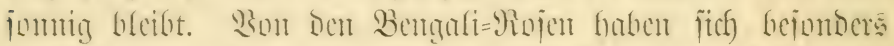

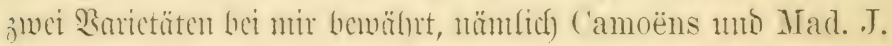
Messimy; ifne Fürbuntg ift vofflig anders, als bic Der anderen

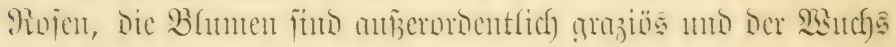
ift fräftig.

Ier winzig̣e, aber trotzom recht unumgenelume Rajen=

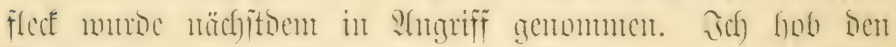

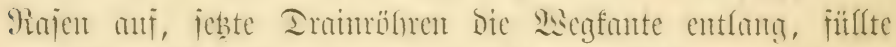

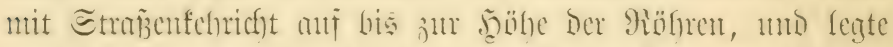

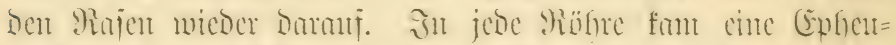

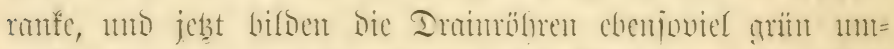

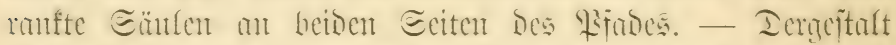

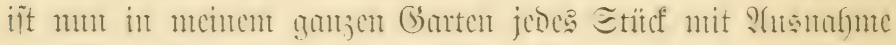

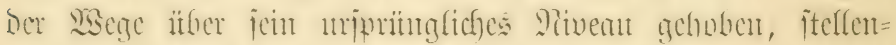

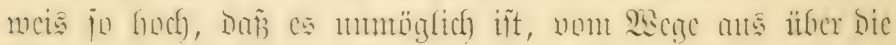

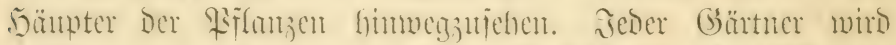

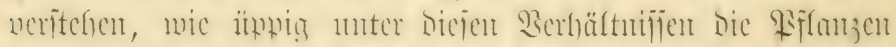

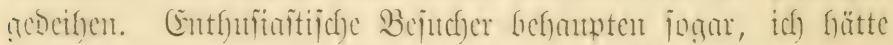

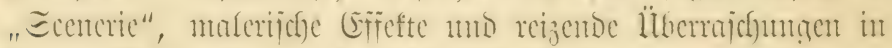

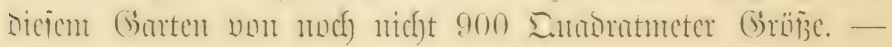




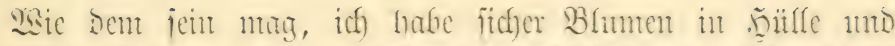

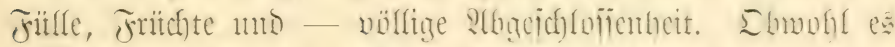

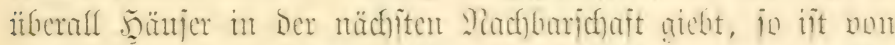

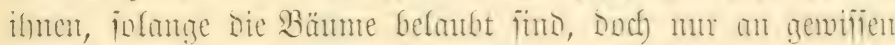
Stelfen ein fleines Stitdffen fichtbar.

\section{Bweites fapitel.}

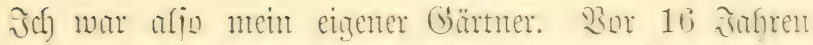

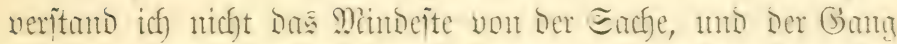

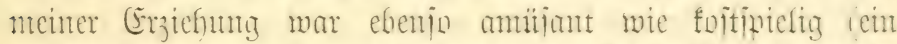

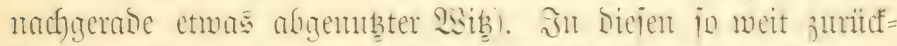
liegenten Tagen waren Die Beranten, Die harten, antantern=

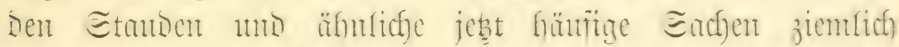
menbefunnt. Emitg itudierte ich Die Sataloge Der Büthter, ich)

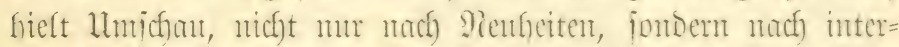

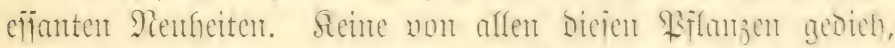

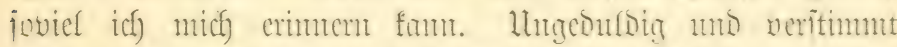

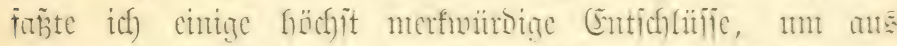

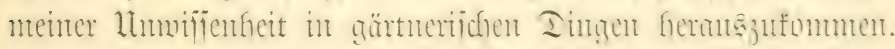
Einer Diejer (Entichliffile, Defïen ich mich noch erintere, wor Der,

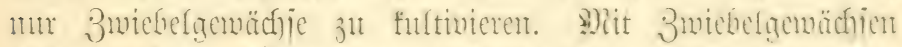

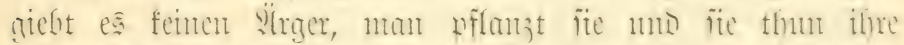

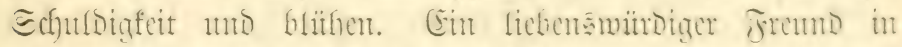

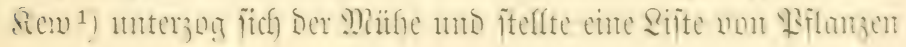

1) Sew, an Der Themje oberhalb Sonoons gelegen, ijt berifhmt

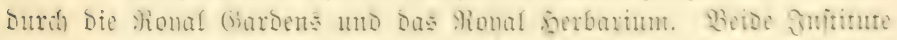

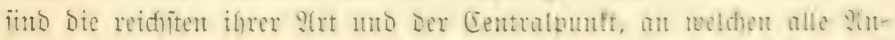




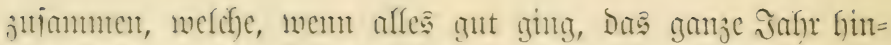

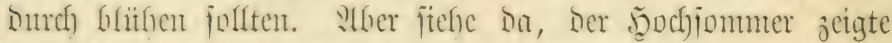

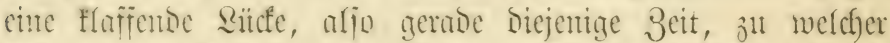

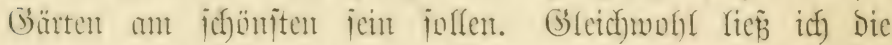

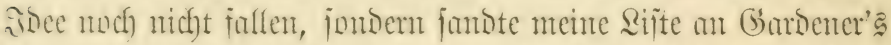

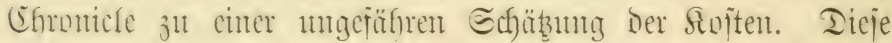

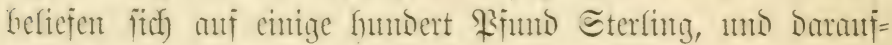

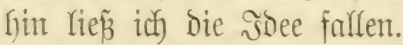

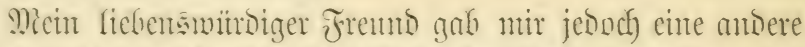

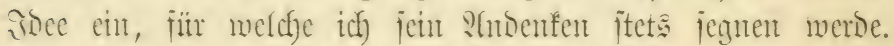

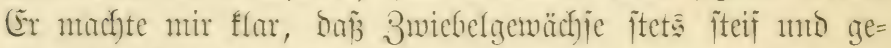

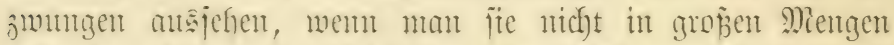
Lfflangt, wie mun êे leidyt mit Den filfigeren Eorten, wie

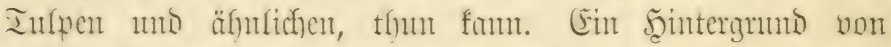

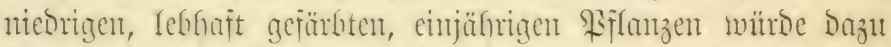

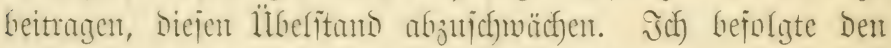
2amf mo befolge ifn his jum heutigen Iage, wo ich mefr. nou ber Eache veritefle. Eeit Diejer 3eit find Jrithlings=

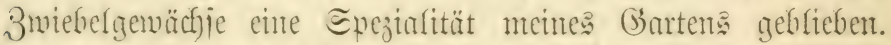

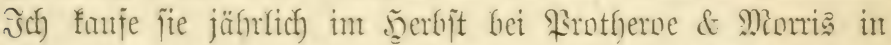
Efreanitide, aber nicht nach Den mir jugejanden Ratalogen, mo

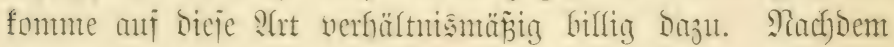

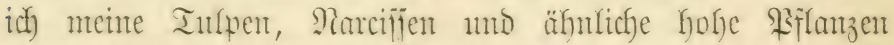
untergebracht habe, fïlle idf Den (S) numb ber Beete mit Myosotis noer Silene pendula noer beiben, welche wäfrento bes

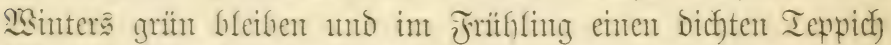

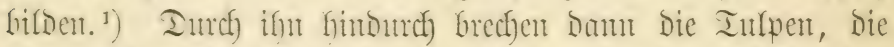

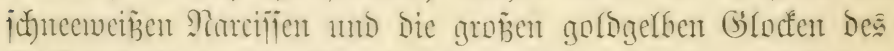
Iaffobiff (Nareissus Pseudo-Narcissus) und jefen anf Diejent

fragen gärtnerijân uno botantichen Jnfalts aus Englano uno allen britijchen Rolonien gerichtet werben.

1) כit in bent milden stlinta von @itidenglano möglid), bei uns

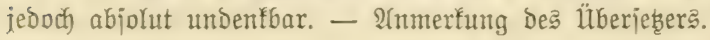




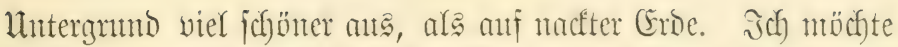

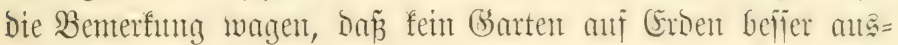
jeben fanm, als Der meinige, mem alle bicje Bmiebelgewächie

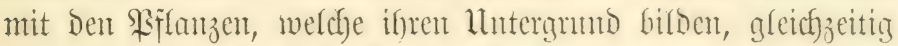

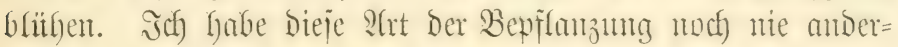
wärts gefutnden. ${ }^{1}$ )

Noch eines anderen $\mathfrak{B r o j e f t e s}$ erimmere ich mich.

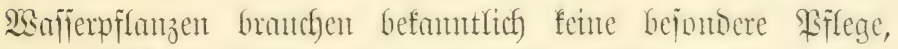
ber gejchicftejte Gbärtner fam fie nicht berbefjem mo ber um=

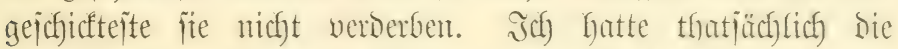

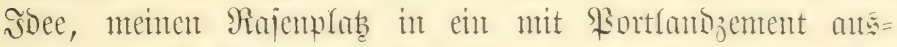

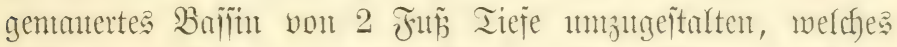
mit einem beizapparat veriefen merden follte, am barin

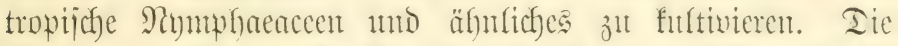

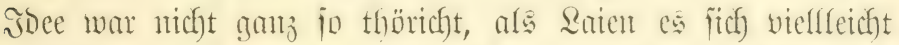
voritellen, Dem zmei meiner Befamten haben thatjüchlich Victoria regia in offenen (Sontenteichen fultiviert, aflerbintg

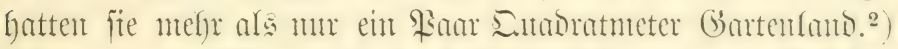

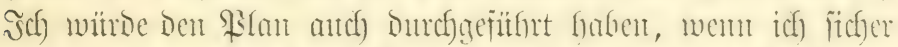

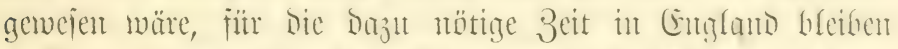

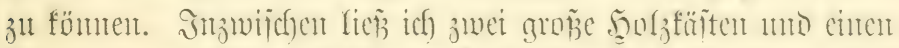

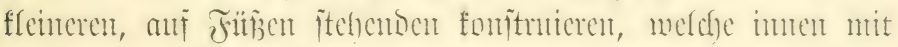

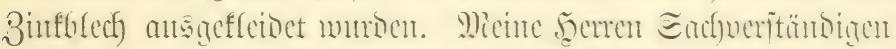

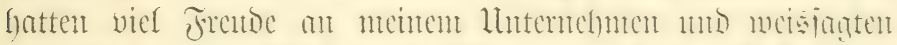

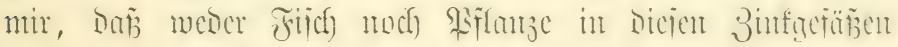

1) Iañ Dicie ?lat Der Bepflanzung ieltent nurfommt, iit ridftig:

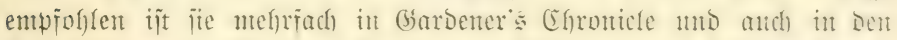

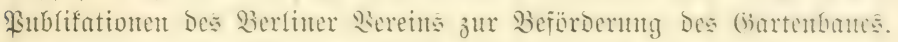
- 2rumerfung Des überīebers.

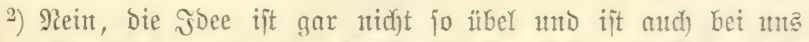

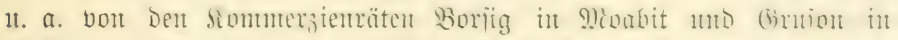

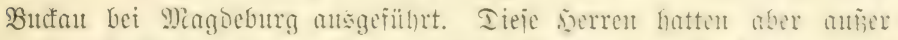

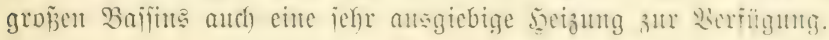




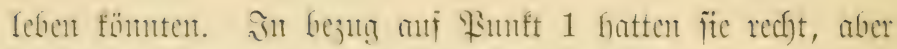

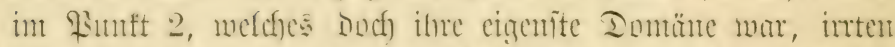

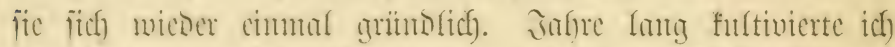

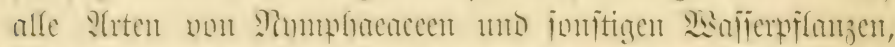

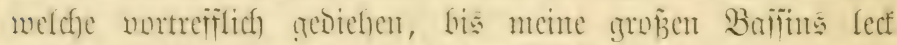

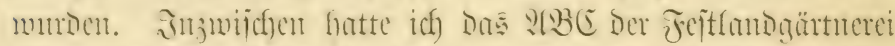

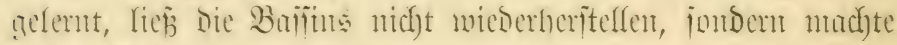

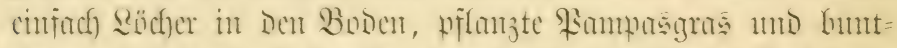

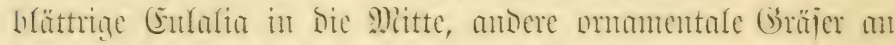

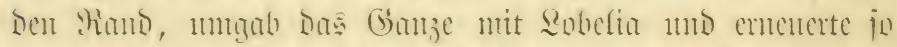

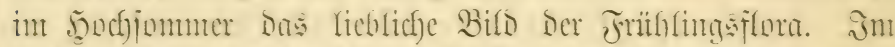
nüd)iten Jufure merne ich Dic Buifin mit Anomatheca cruenta bepflanzen, ben am idgünten biühenden Graje, wem

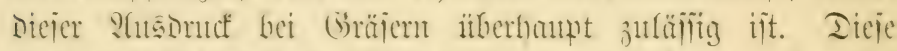

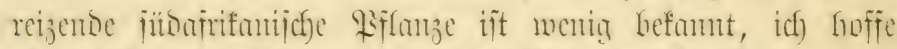

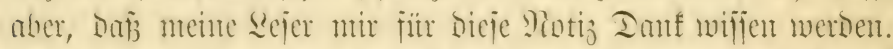

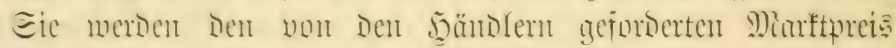

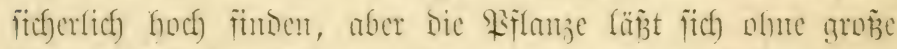
Echmierigfeit Dajut bringen, Eamen jut tragen mo Dam ver=

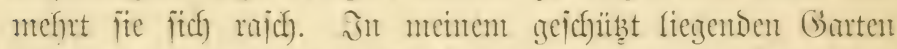

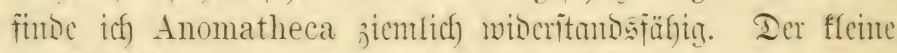
Binffefhälter exiititiert noch mo beherbergt Nymphaea odorata,

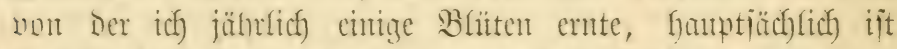
er aber Dem Aponogeton distachym itherfojien, ser fapt=

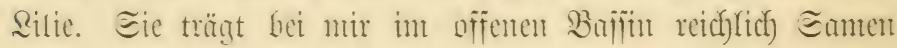
mo mem basifelfe tiejer läge, mitroen ifyre eigenartigen,

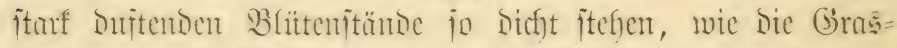

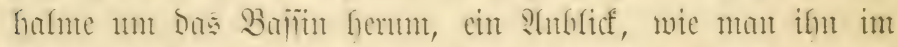

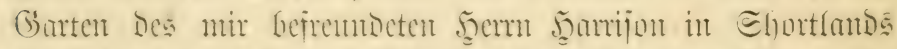

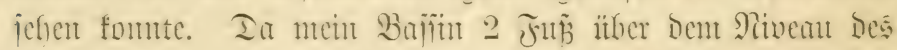

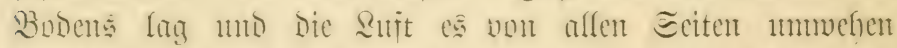

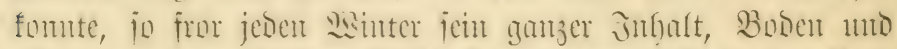

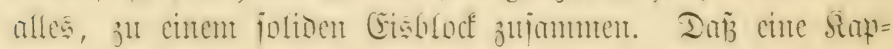




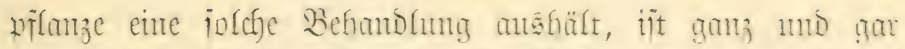
gegen Die Candläuffigen VIrngahen Der Bitcher, aber meine itarfen Aponogeton fieften bicie Ienmeraturen man, mu Die jungen

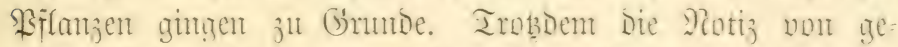

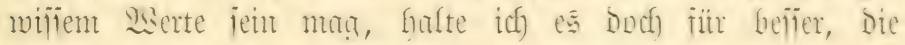
Snollen biejer SHFlanze frofftree zut itberwinternt.

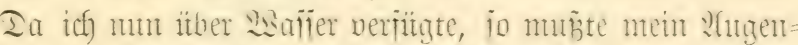

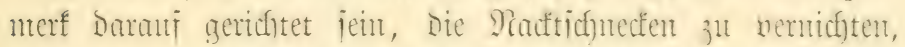

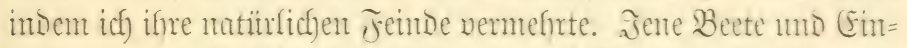
falintugen von Saxifraga hypnoides, weldye ich vorfin emvähnte,

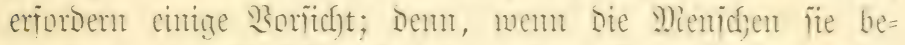

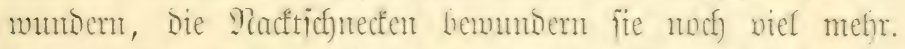

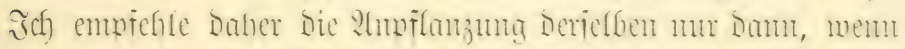

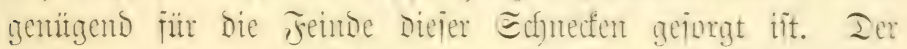

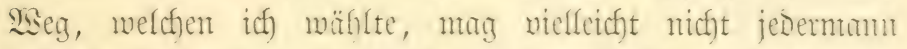

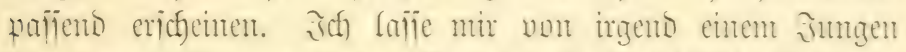
Fitr einten Eirpence cinen (Fimer volf Trofichlaidf beiorgent.

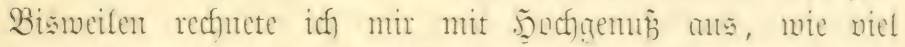
taulende fräftiger mo untemehmuntgäluitiger Batradjer ich erzichen uno zu meinem und meiner Plachban Beiten in Die

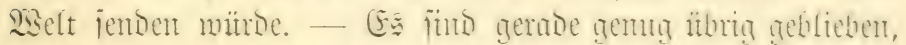
ltm mir ju Dienten; Dent icf) bemerfe mentị non Pladt=

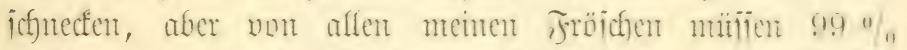

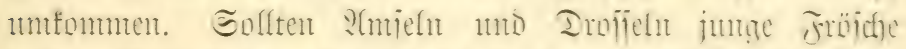

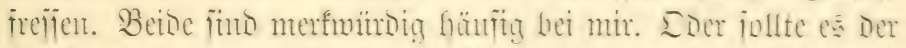
Getbrand (Dytiscus marginalis) iein, weldfer mir jumt ieinen

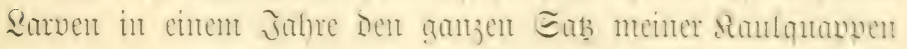

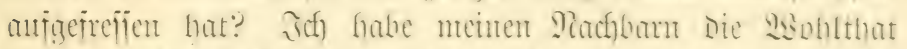

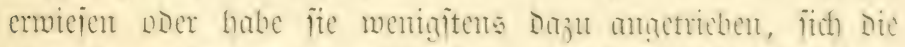

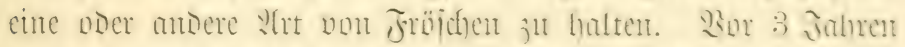

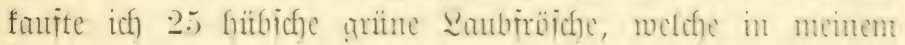

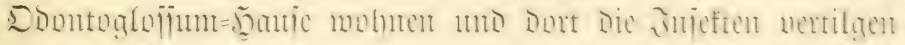

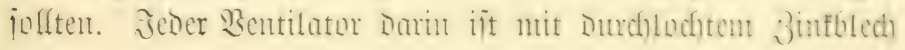




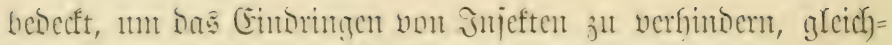

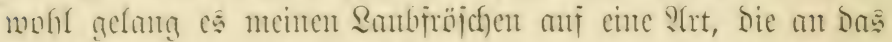

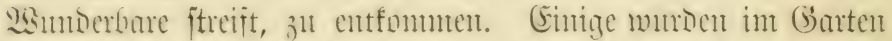
attrapiert uns zuritfigebrad)t, aber fie famben micherum ifren

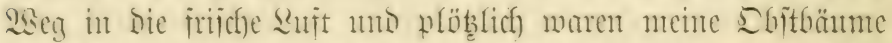

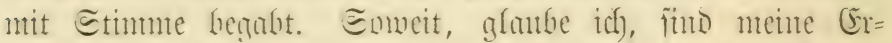
fafmmgen Diejelfen, wie Diejentigen aller Rente, weldye f̈td) mit

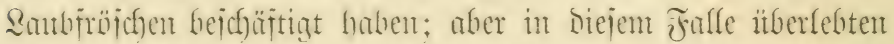
meine Fröjche 2 sinter, Damuter einen antergemöhn(ich) harten. Miente Fröiche inngen im nädjiten Eommer recht

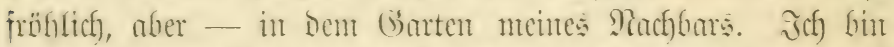

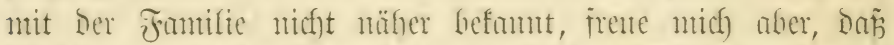

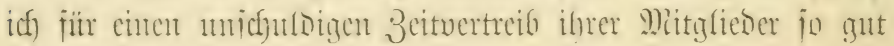
gejorgt habe.

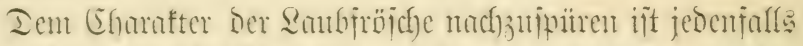

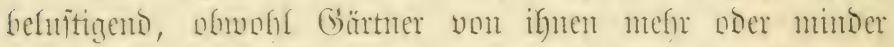

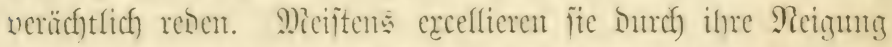

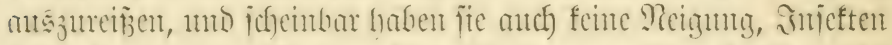

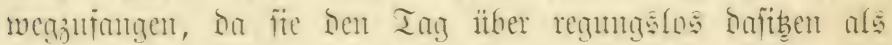

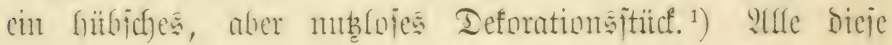

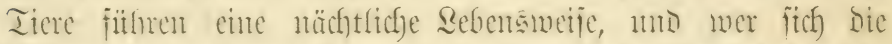

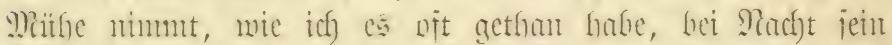

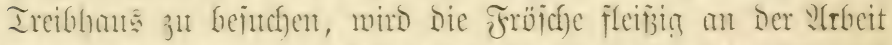
finten mo iefen, wic fie mit munderforer (belentigfeit smojichen

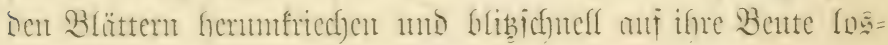

1) Bei Diejer ganjen ?tbfandung über Das Sebent und Ireiben

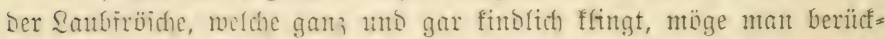

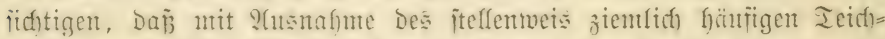
froides affe miere Surfhe in England fehlen. firöten tveroen, um

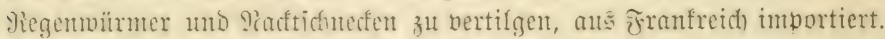

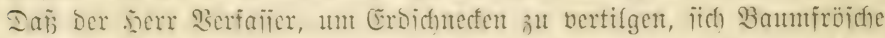

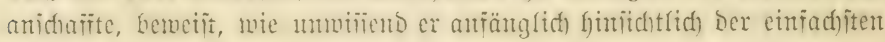
jragen mar. 



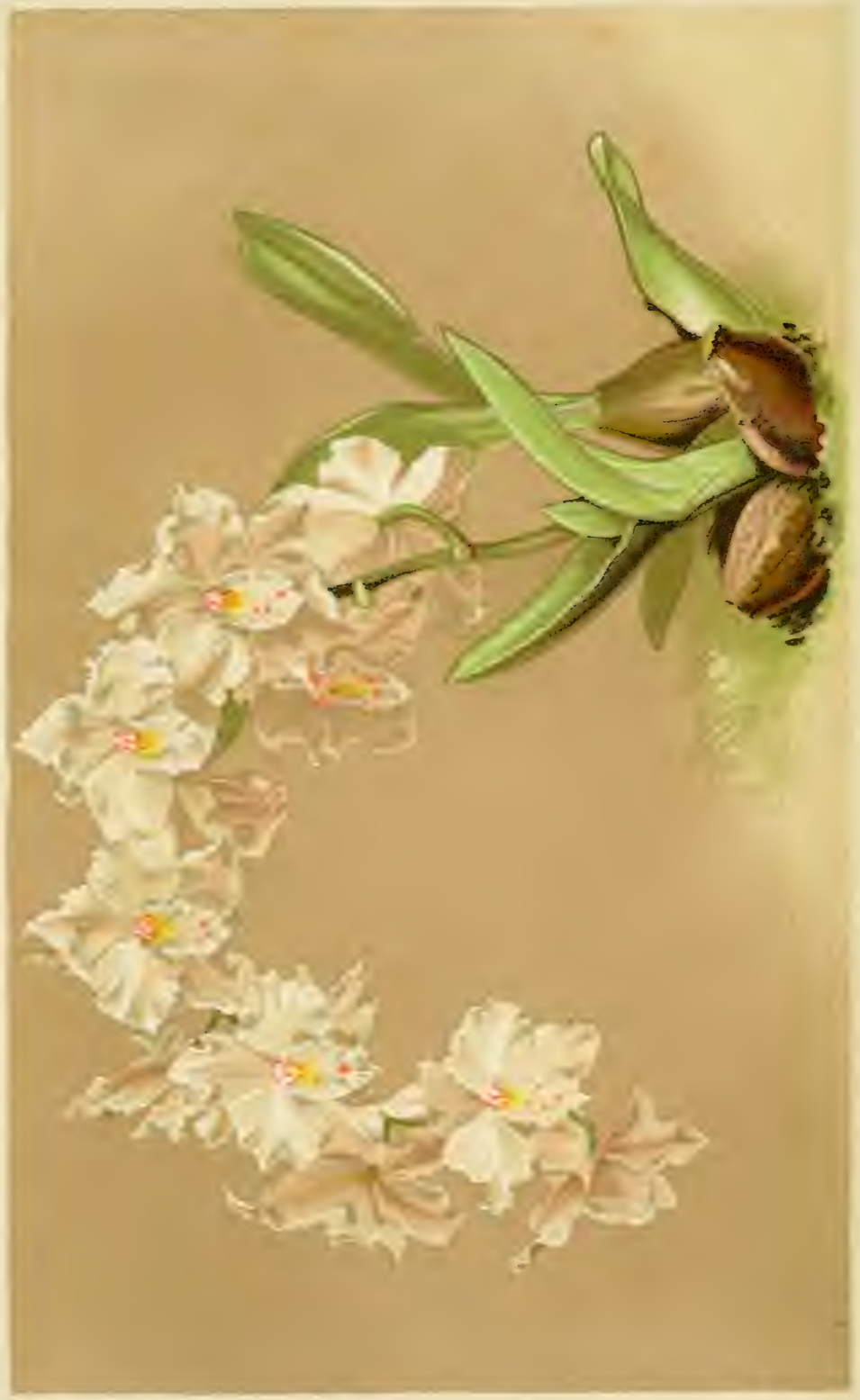




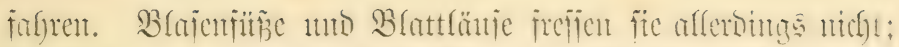

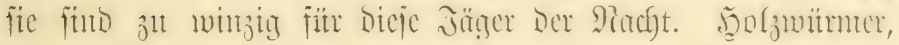
Impentiatio mo wor allen Dingen Echaben, bieje tötfidfen

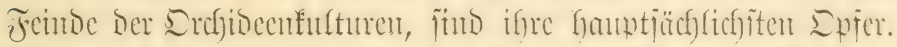

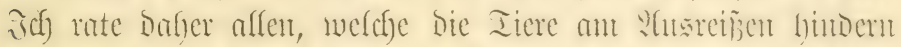

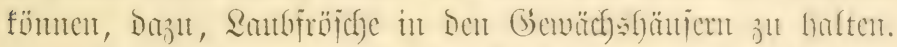

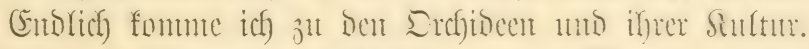

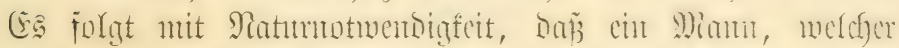

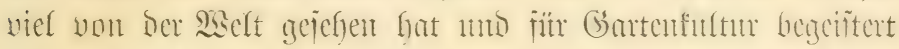

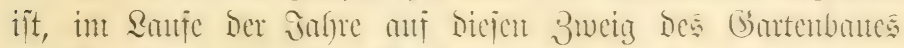

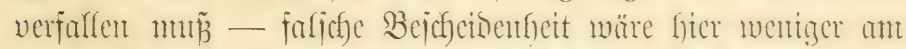

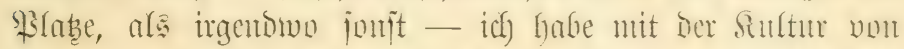

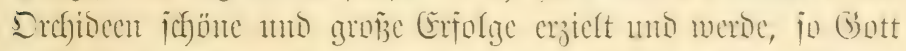

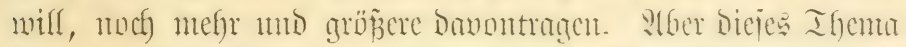

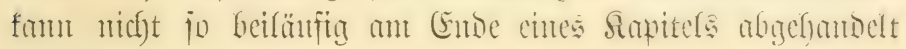
merion.

\section{Drittes finpitel.}

Sin Den Ingen menter Qefrering

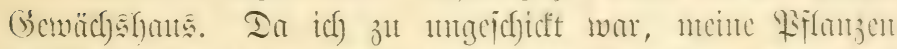

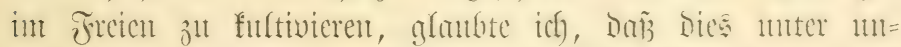

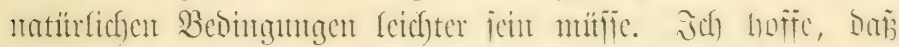

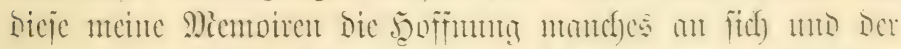

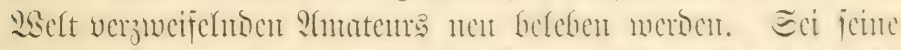

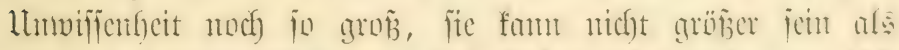

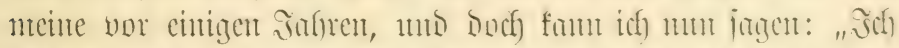

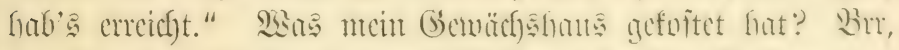

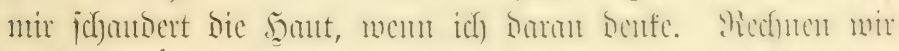

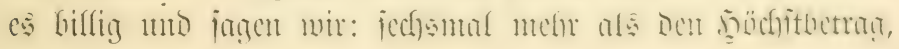




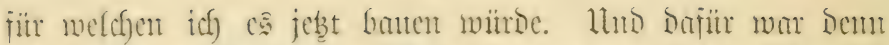

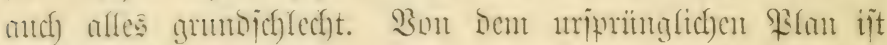

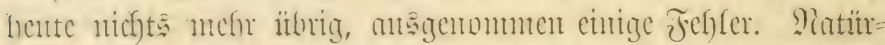

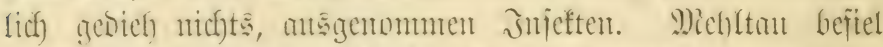

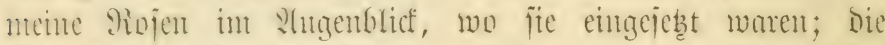

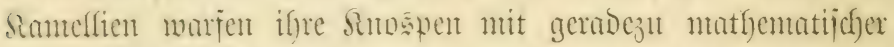

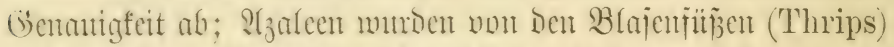

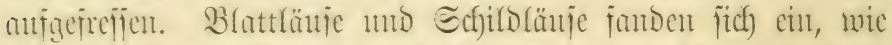

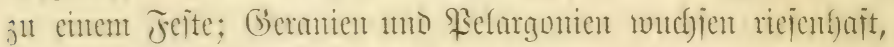

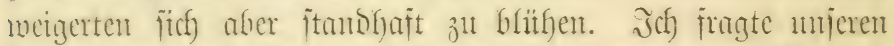

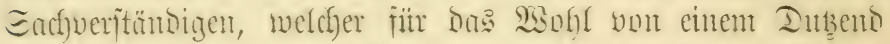
Biffengärten verantwortfich) war - cinen erfafrenten Miann,

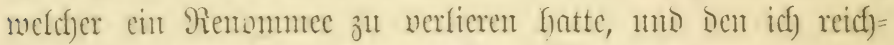
lich) Dafiir bezahlte. Der witroige Mianm jagte stad) ciner

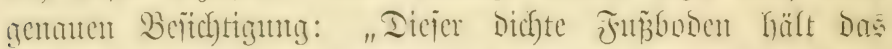

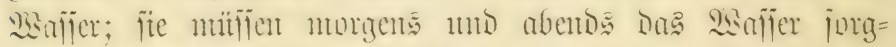
fältig arfwifichen Kaffen." Lho biejer gute Miam hatte

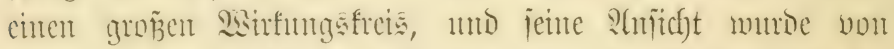

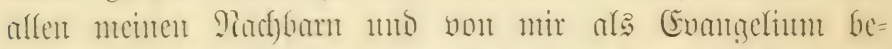

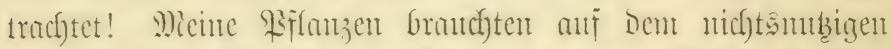

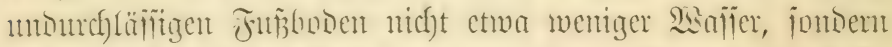

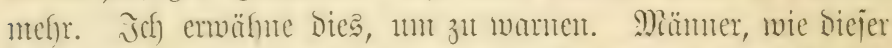
bifoen aber einten zientich) Gebententon Beitanoteil jeiner Silafife. $\mathfrak{A}($ S id $)$ mu an meiner Bartenfunt, Drimen und

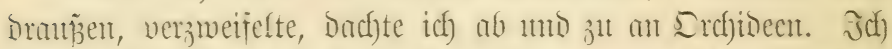

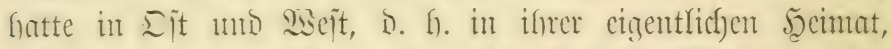

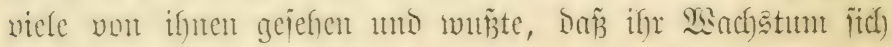
an ftrenge sejebe bindet.

Bhoere Biflanjen - Rojen z. B. - ipielen cincm fort= mäfrend Etreiche. Bafo verlangt iffre Befonndumg bies, bald jenes, Dinge, Deren Platur nicht cimmal jofar prëcififiert

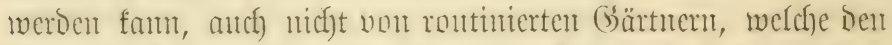

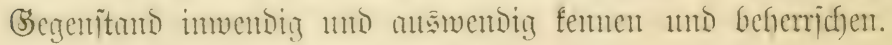




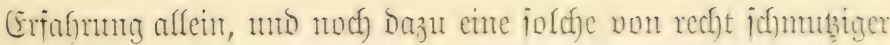

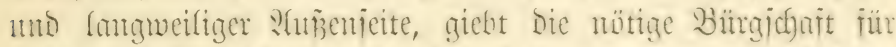

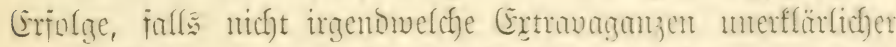

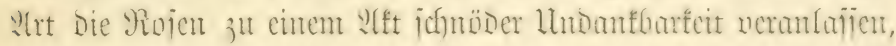

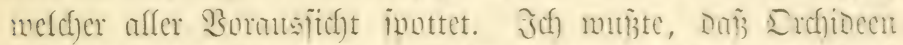

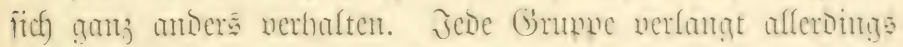
ihre eigenen Bedingumgen; fonm mon sicje midgt criüflen, fo

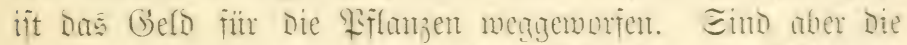

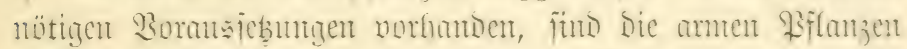

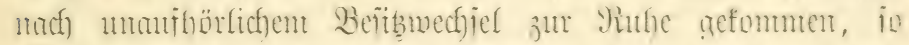

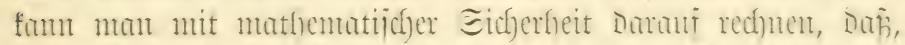
mem Die und Die Beflandung angemant miro, Dieje noer

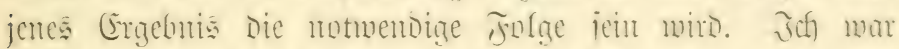

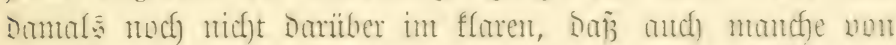

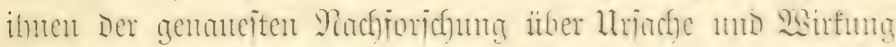

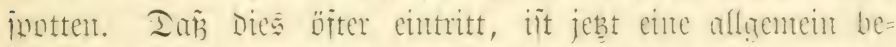
founte Ihatiache, fie itöbt aher Die Regel nich)t um; Dem Dicic

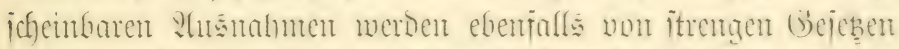

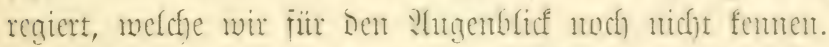

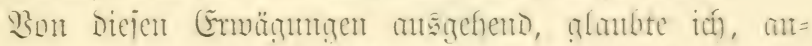

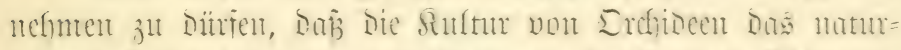

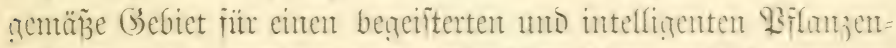

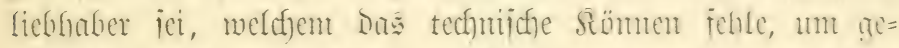
möhnliche Wiflanjen jiefen jul fömen; Dem — io nalm id) an - Erchiocenfultur it Stovfarbeit, Die anderen Sulturen Drandarbeit. P(ber idf) teilte Die aflgemein verbertete, jekt als

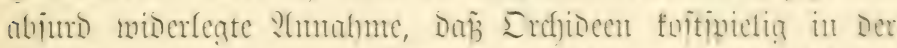

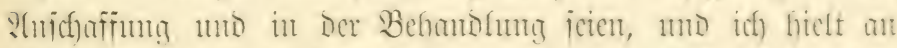

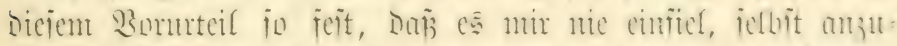

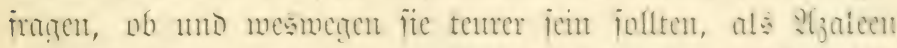

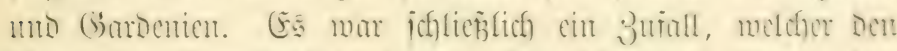

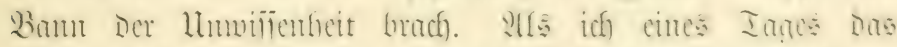

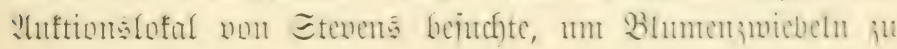




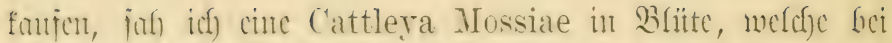

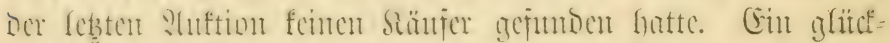

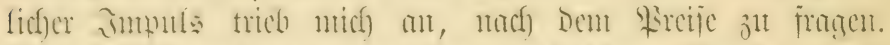

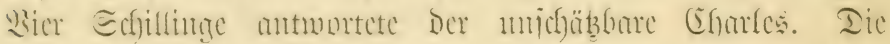

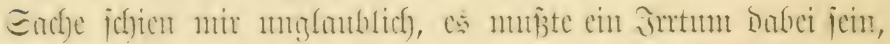

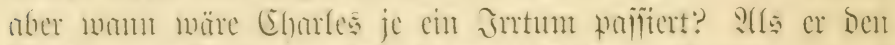

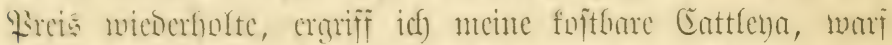

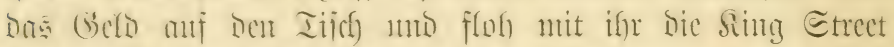

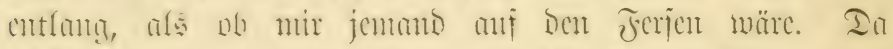

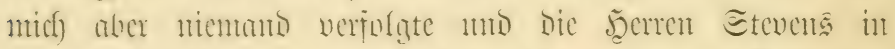

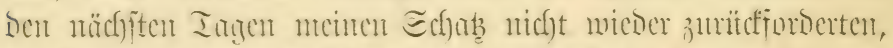

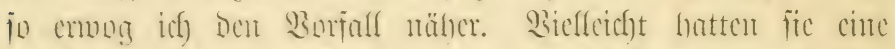

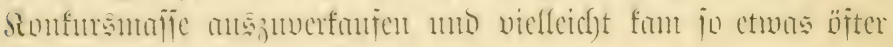

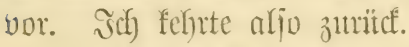

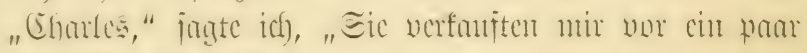
Iagen eine Cattleya Mossiae."

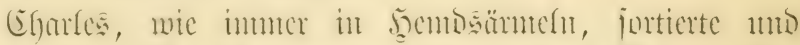

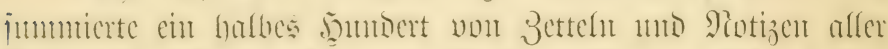

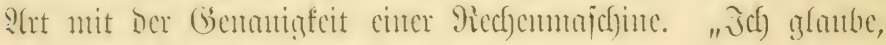
ja, Şerr", war jeine 2futwort.

"WSar fie nicht ziemlich) tenter?" fragte id ).

"Das ift ifree Sache, Şerr", antwortete er lachento.

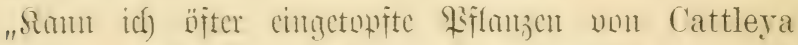

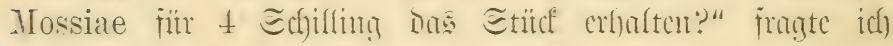
weiter.

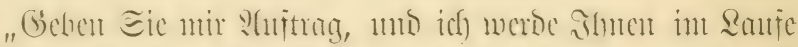

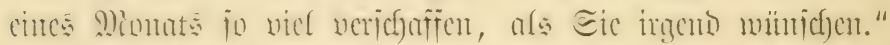

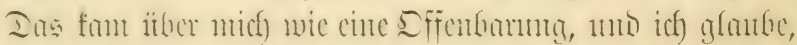

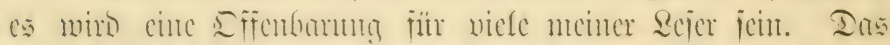

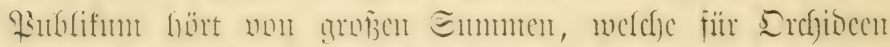

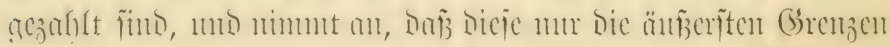

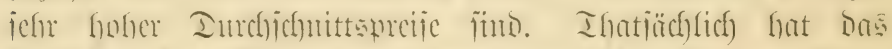

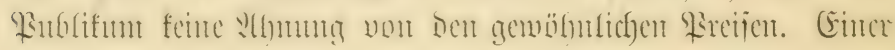




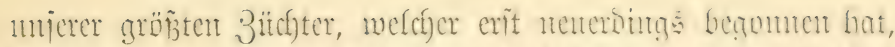

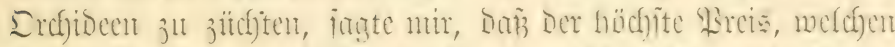

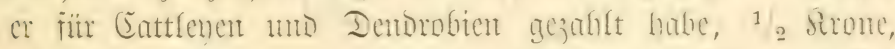

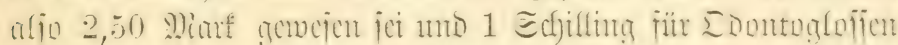

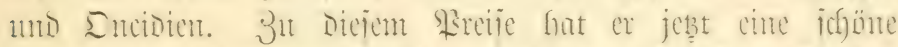

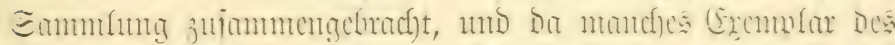

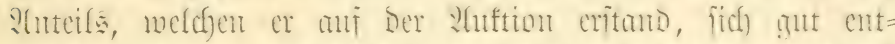

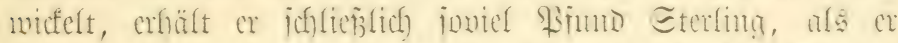

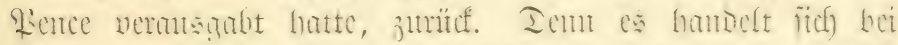

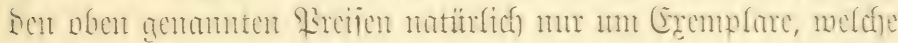

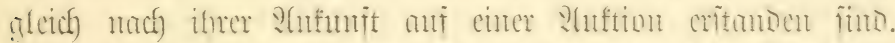

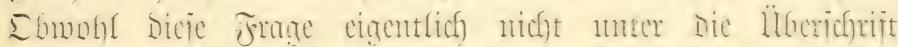

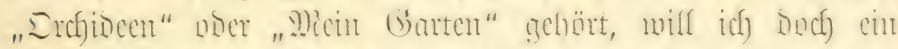

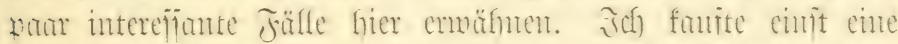

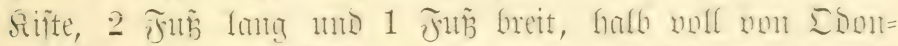

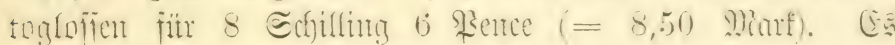
waren fleme (Exemtslare, aber tadelfow gejums, Dem wus Den

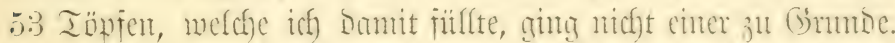

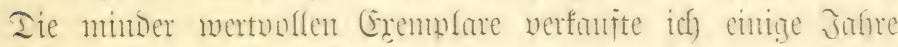

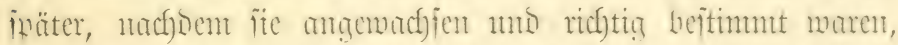

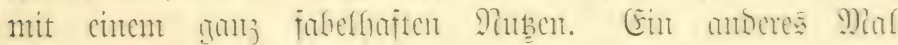
funte idf 3 Bimbel vun Odontoglossum Alexantrae rar.

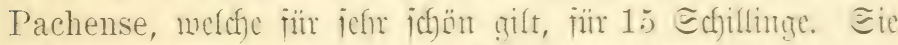

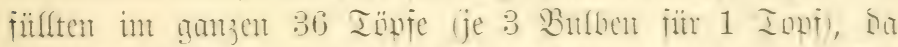

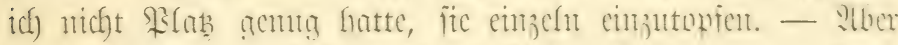

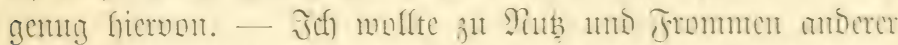

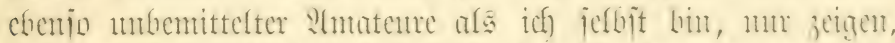

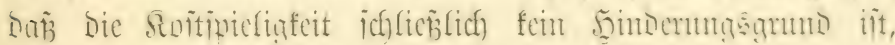

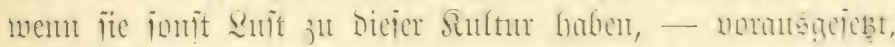

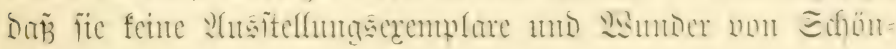
Geit verlangen.

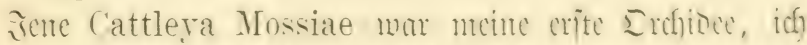

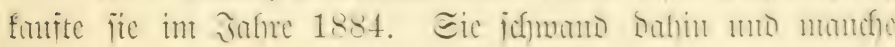




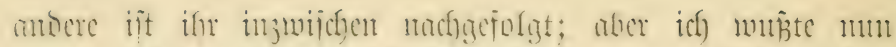

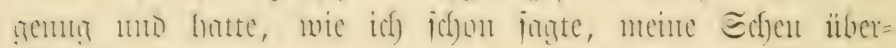

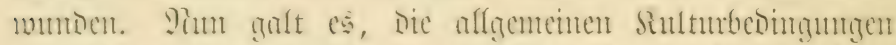

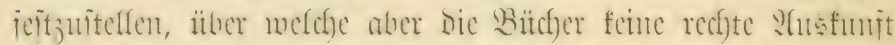

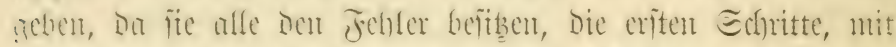

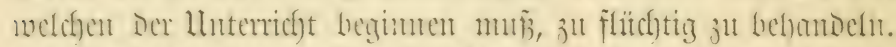

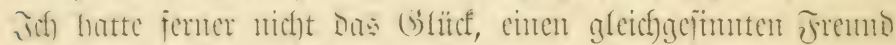

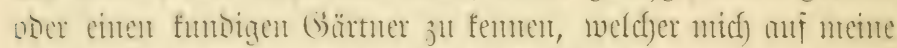

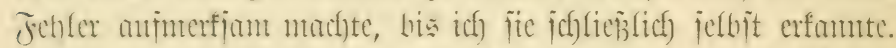

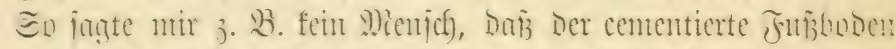

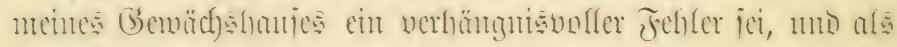
ich), hercits etmos entmuttigt, Den flemen, iu Der Einfeitume

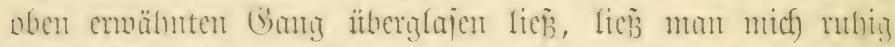

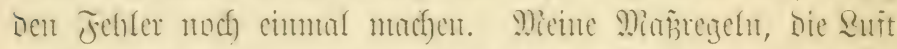
fentht ju crhalten, waren jum Ieif jefre fummetidf, aher fic ent=

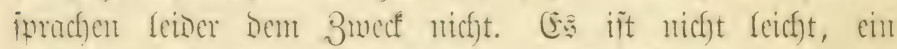
ianteres num flares Diaterial jum Benecfen Des Eement=

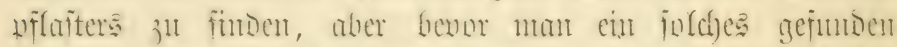

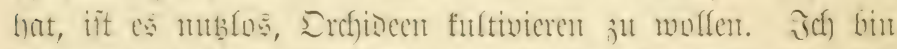

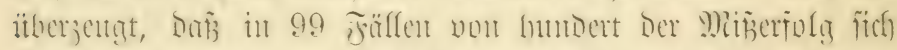

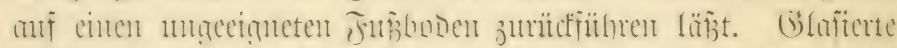

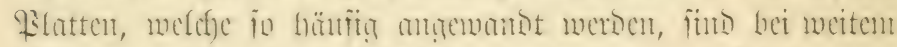

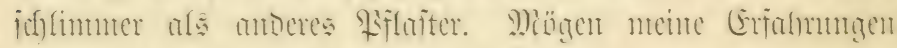
anderent Renten zur 23 anmung Dienten!

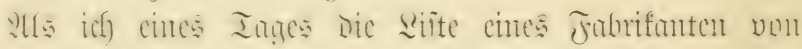

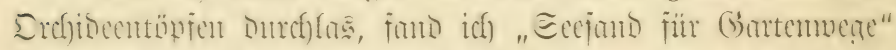

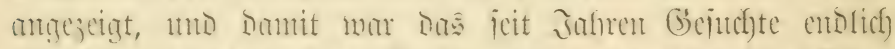

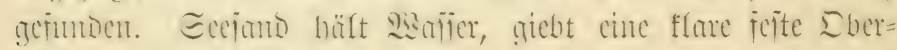

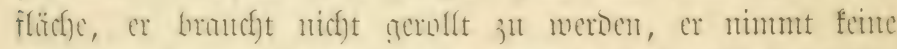

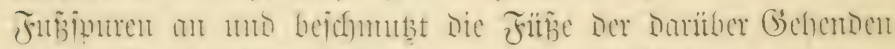

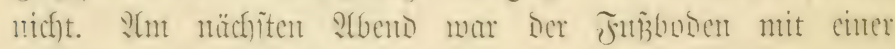

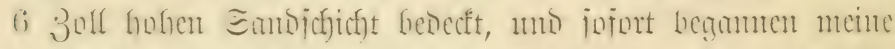

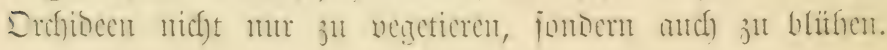




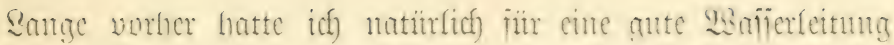

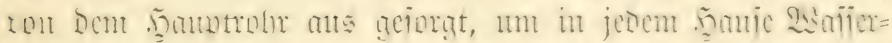

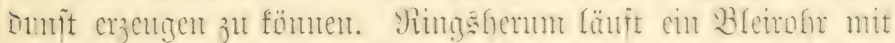

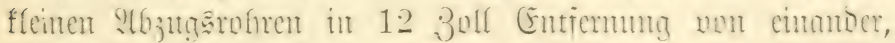

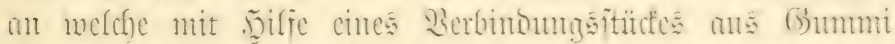

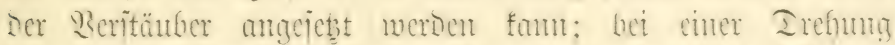

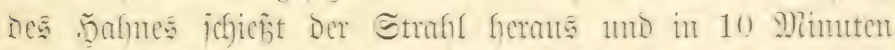

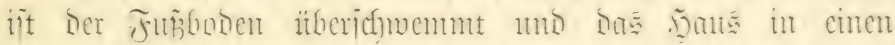

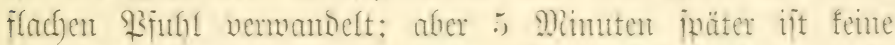

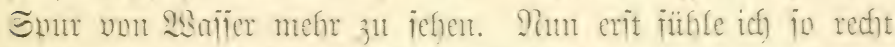

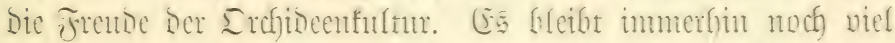
jul lemen, recht viel fogar. (5) gicht amäfiemo soono fultivierte

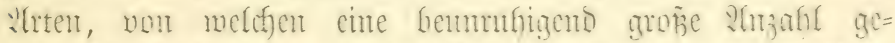

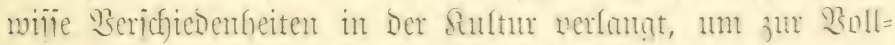

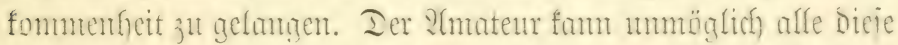

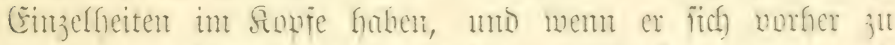
genau unterrichtet, wiro ex leidft in Echredfen geraten und

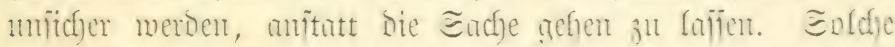

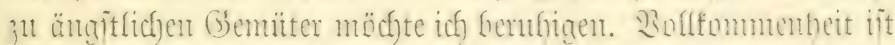

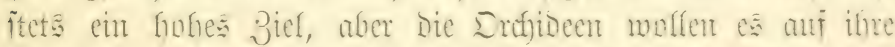

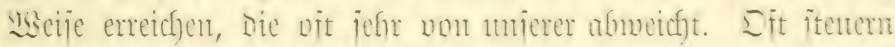

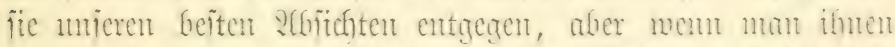

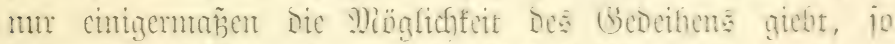

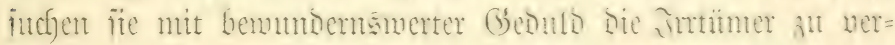

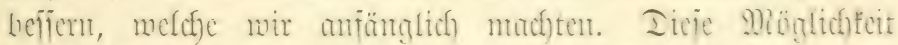
Des Gencifens fiegt, mie idh bereits ansgefübrt habe, in Den

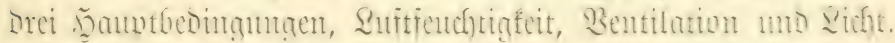
Sind Dicje Drei Fuftoren vorhanden, io ming man ju Den

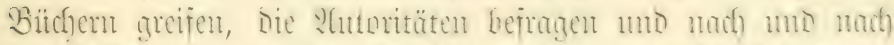

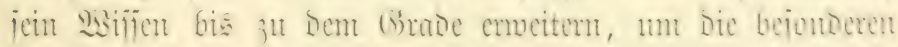

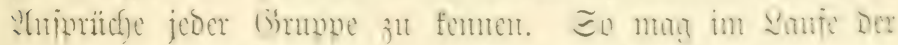

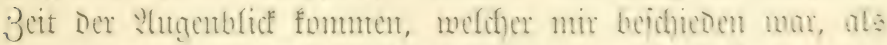

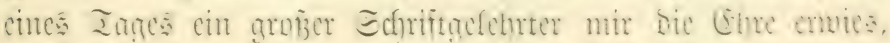




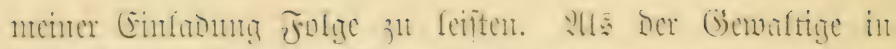
nein fleines EDontoglofium = Dam gemeten mar, mantorten

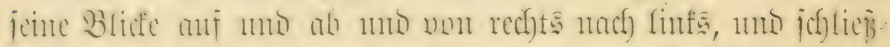

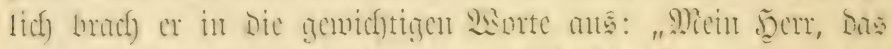

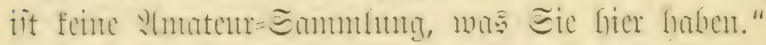

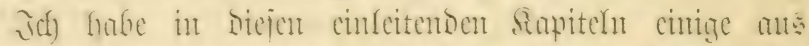

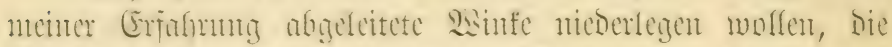

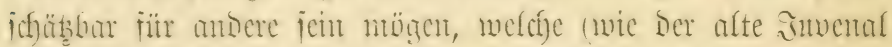

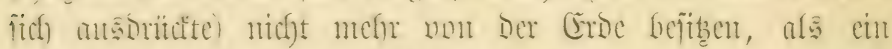

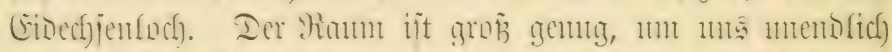

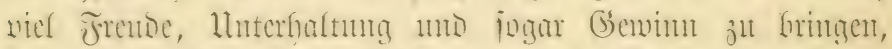

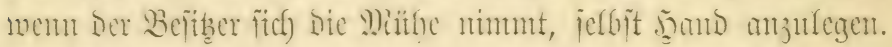

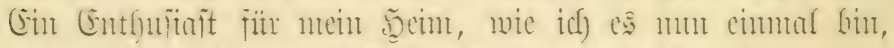

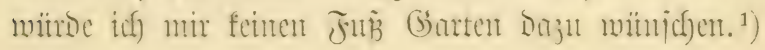

\section{Wirtes fanpitel.}

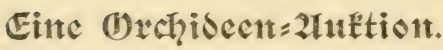

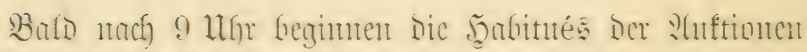

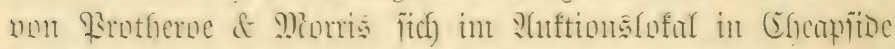

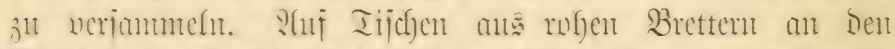

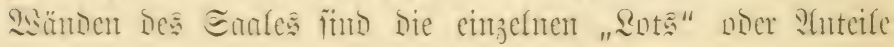

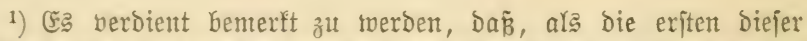

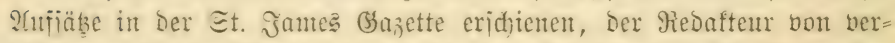

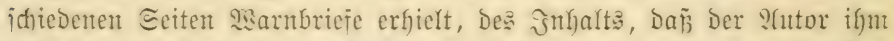

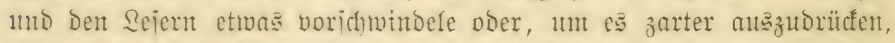

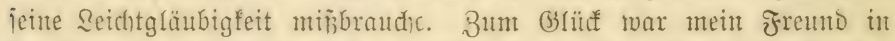
Der Rage, zu antworten, Dañ er periönlich jede (borantie für bie gìd)tig=

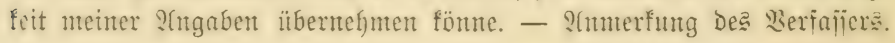




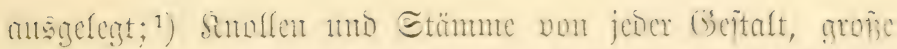

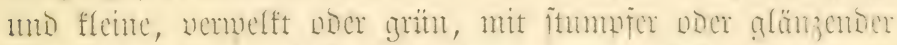

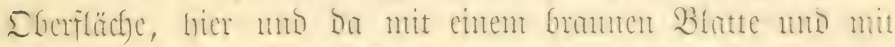

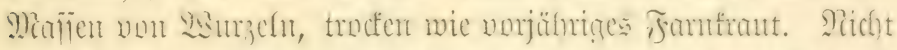

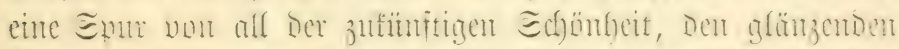

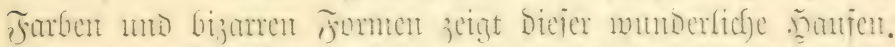

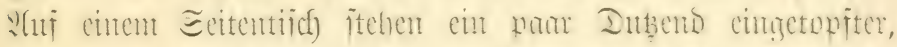

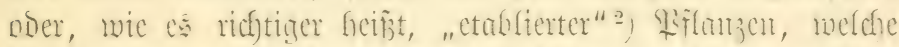

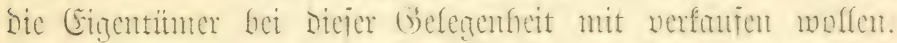

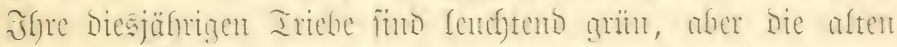
Buthen finto chenjo fafthos, abs sic ber nen angefonmenent.

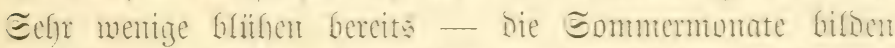

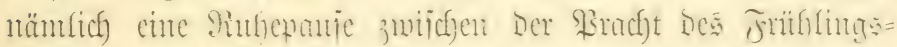

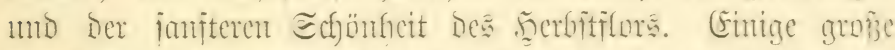
Iendrofien (Dendrob. Dahllonsianum) 3. B. 3cigen unzertige

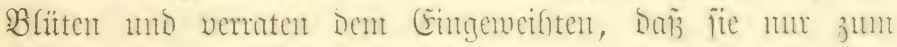

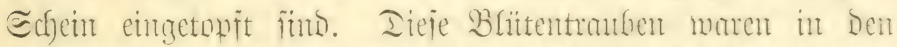

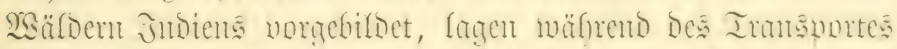

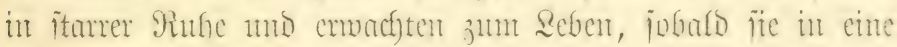

1. (E⿱ Drücfe. Jede ratiilenichaft und jedes Gemerbe fat ifr natimales oder internationales siotmälich, weldhes mir bie Cringetweiften veritehen uno

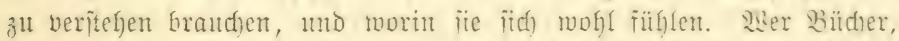

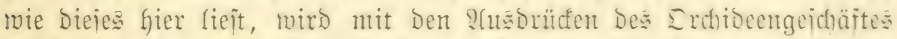

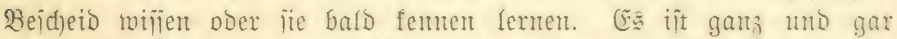

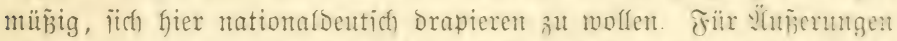
unieres liationalbemuñtieins itnd andere (belegentheitem geeignter, als

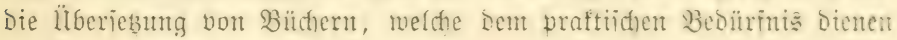
joflen. - Anmerfung des überiełers.

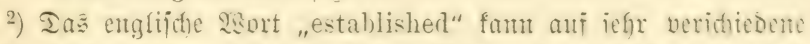

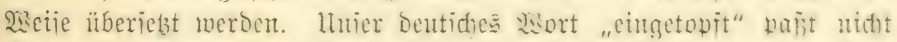
red)t für Erdhibeen, Da biele bon ifnen gar nidht in Tüpien fultiviert werden fömen, iondem in fïrben nder an Blöfen. "Bemur;elt" vaht

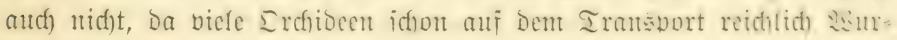
zeln birben. - Inmerfung des litberjebers. 


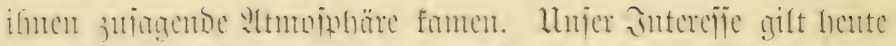

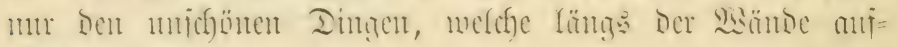
gechänft \iegen.

Die gemögntichen Bejutcher biejer :Luftionen Gilden cine

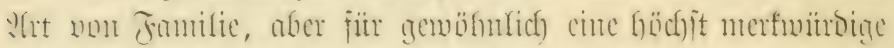

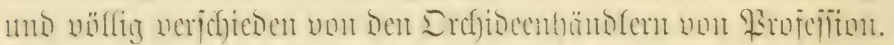

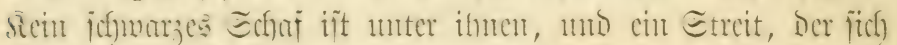

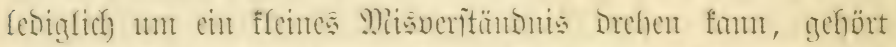

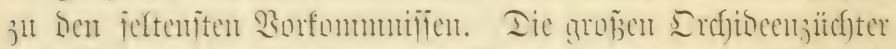

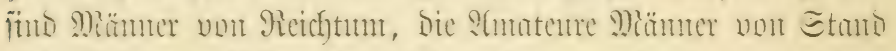

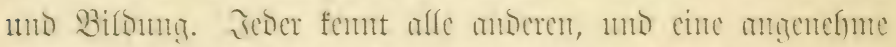

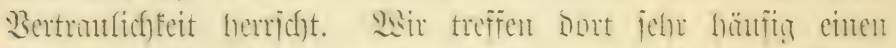

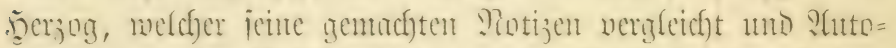

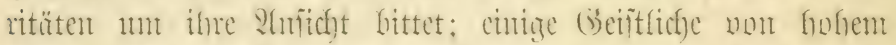

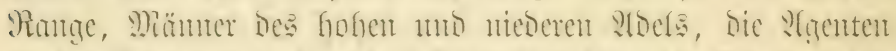
grofier S(mateure uns, wie jefloitueritännlich), Die Bertreter ber

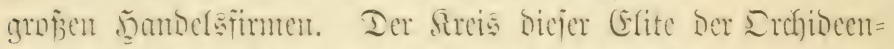

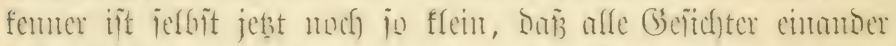

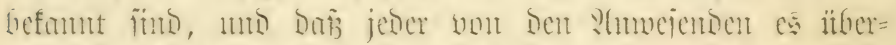

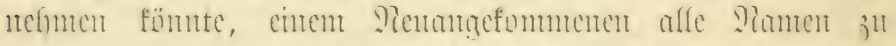

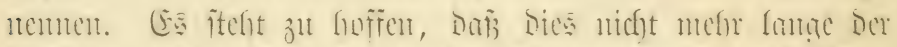

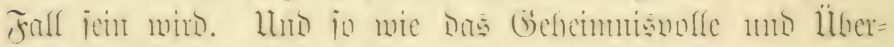

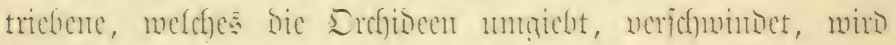

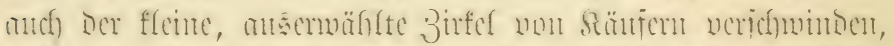

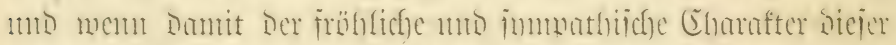

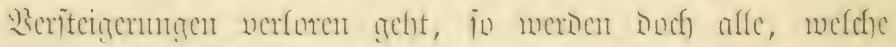

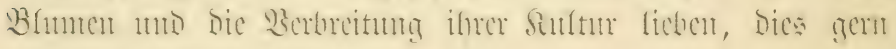
mit in Den Sinuf utchutent.

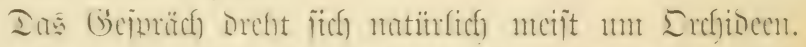

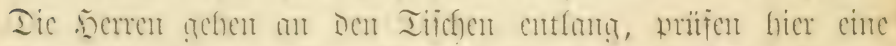

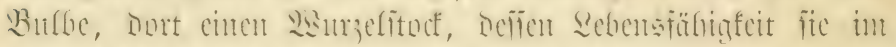

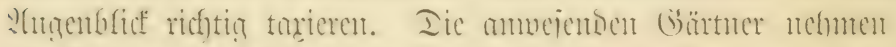

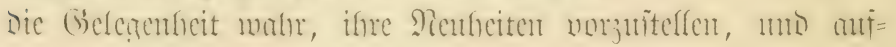




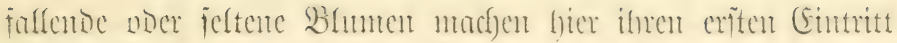

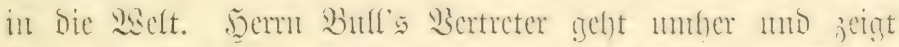
Dem einen oner anderen ber beren Den Jnhalt cince fleinen Echadftel, cinte Aristolnchia elegans, mit ifren ichöncu

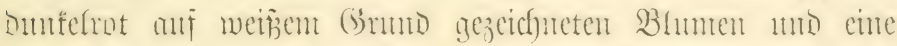
neuc vintetät vou Impatiens; er verteilt lesterc, mo bie

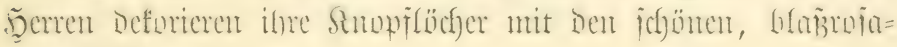
roten Błtuten.

Die Erregung fteigt jebet jelten noch fo hoch, wie in

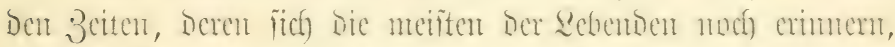

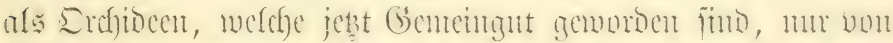

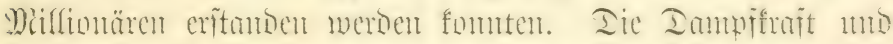

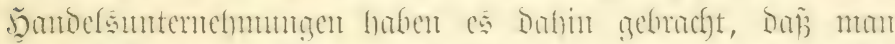

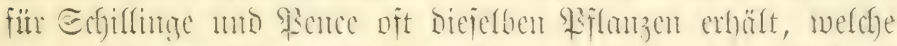

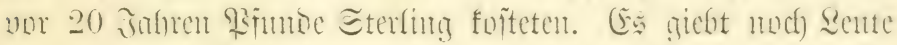

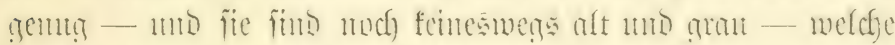
fidf ber Econe crimem, als Masdevallia Torarensis zuerit

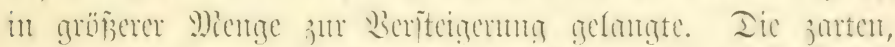

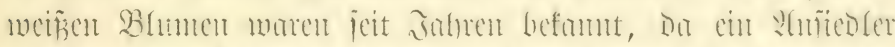

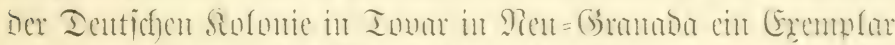

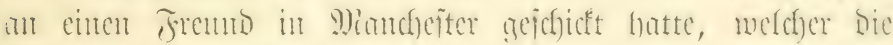

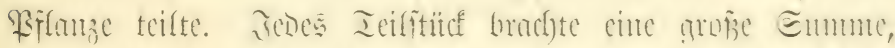

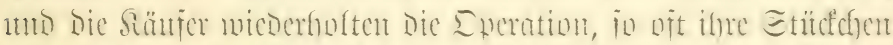

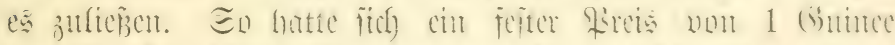

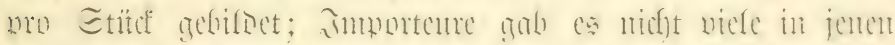

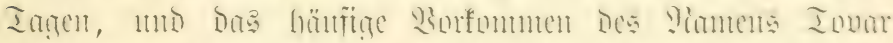

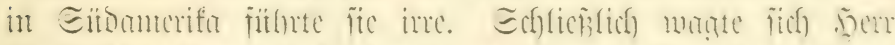

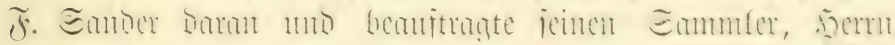

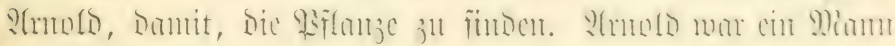

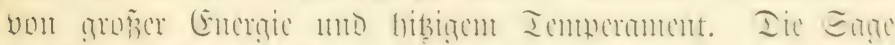

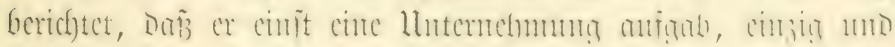

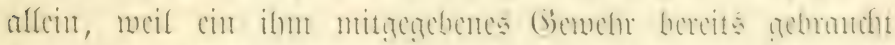

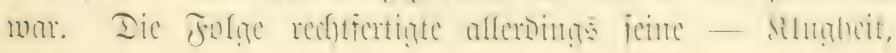




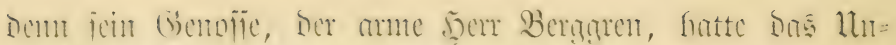

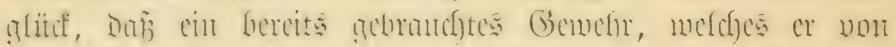

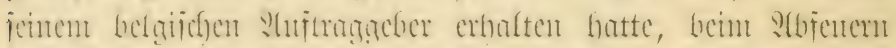

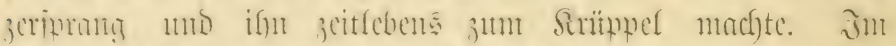

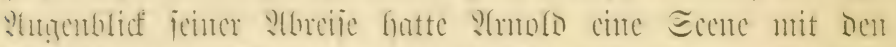

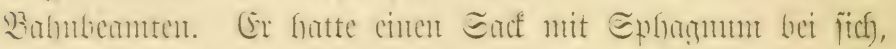
num icme foitharen Siflanjen ju verpacten, mo man mollte

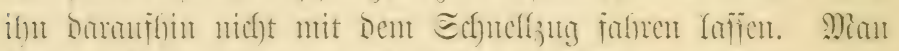

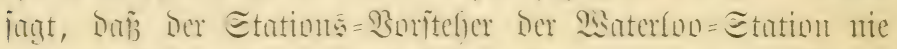

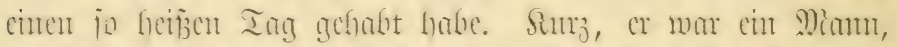
meldem ctmas jujttraten mar. Gin junger \$affagier Gemies

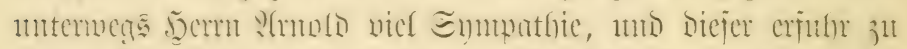

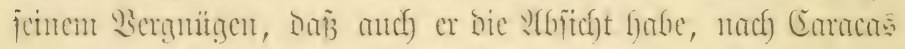

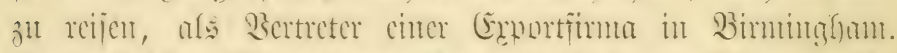

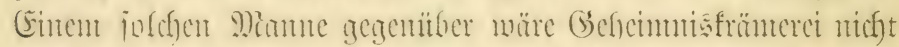
ant Rilabe gemejen, nut jo mentger, als bie Frngen biejes

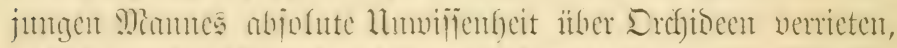

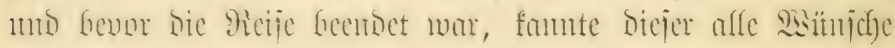

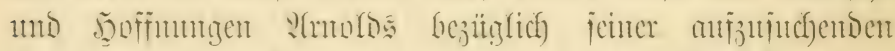

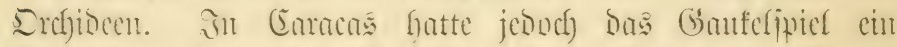

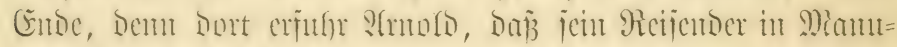

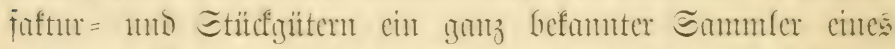

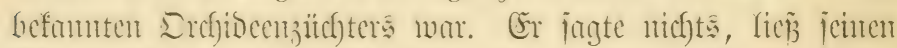

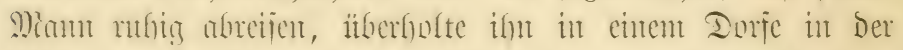

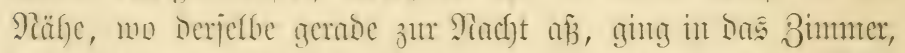

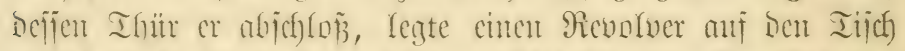

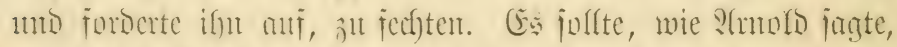

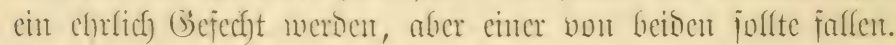
Ier Berräter man won ben Emit Der Rage jo itherjengt -

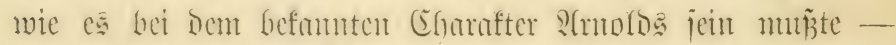

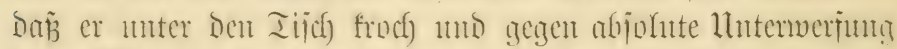

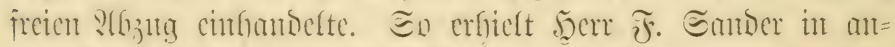
gentijener 3eit 40000 Etitćl Masdevallia Torarensis, meldfe 


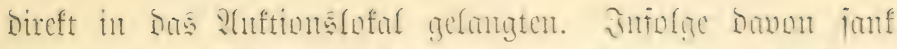

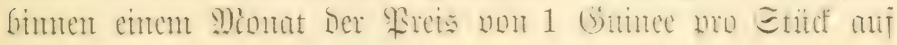
Den Bruchteil eines Exfjillings.

Zlnoere groise beriteigentugen maren bic, als Phalaenuis Sanderiana mo Vanda Sunderiana jum derfunif famen mo Eummen von ichier unglanhlidjer ñöhe angelegt untron. Eonam Dicjenige, als (rpripediun Spicerianum, Crpriped. Curtisii un Laelia anceps alba verfulft murden.

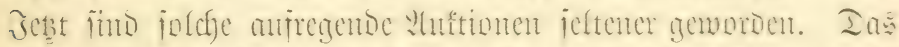

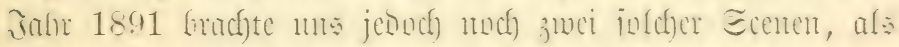
Cattleya labiata autumnalis nuo Dendrobium Phalaenopsis Schroederianum jutr Q Fiflange miro noch cimmal in einent bejomberen stapitel "ver=

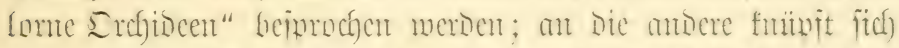

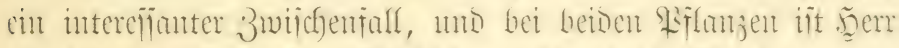
Eamber Der bुelo Der Gejchichte. Dendrol. Plaalaenopsis

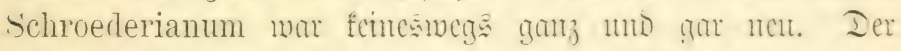

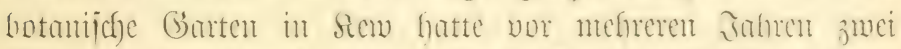

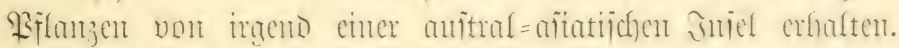

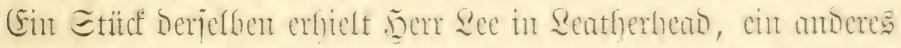

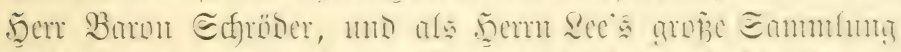

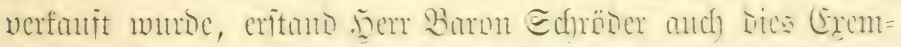

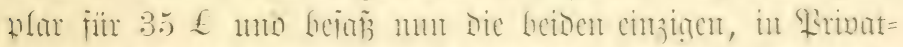

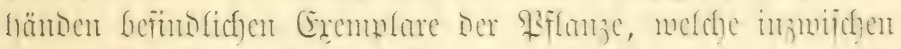
jeinen Ramen erfalten hatte.

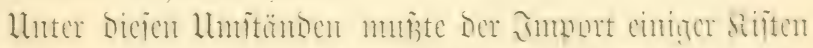

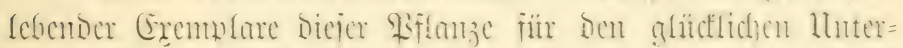

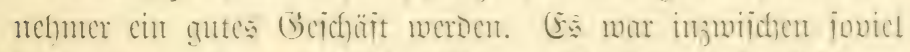

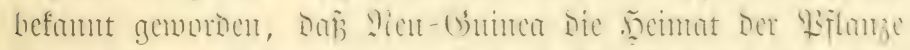

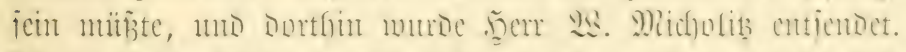

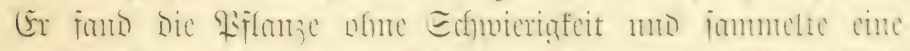

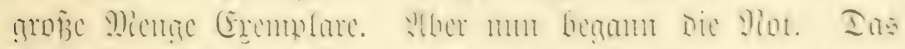

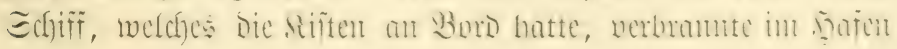




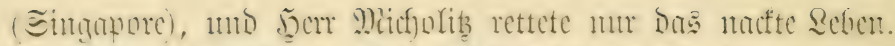

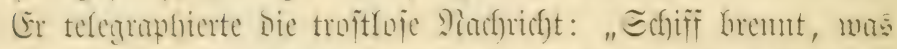

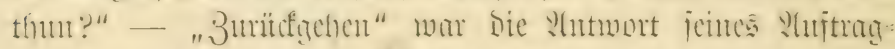

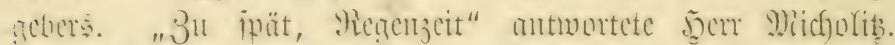

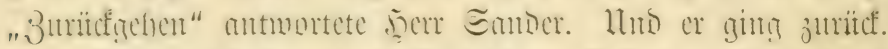

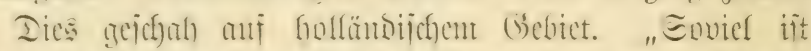

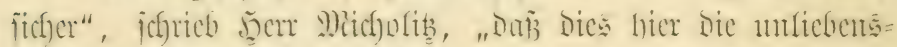

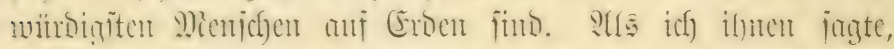

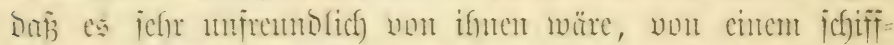

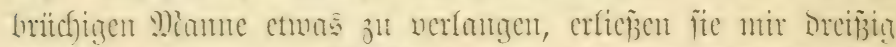

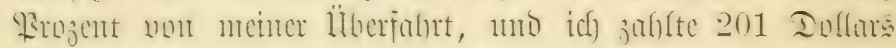

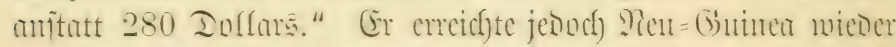

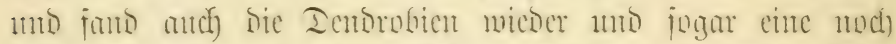

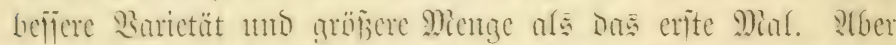

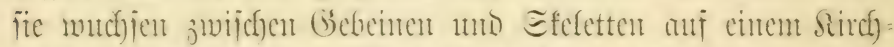

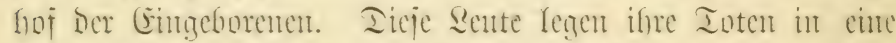

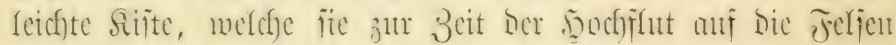

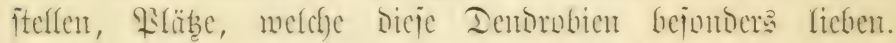

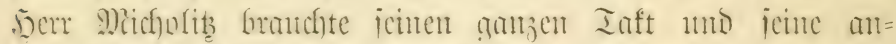

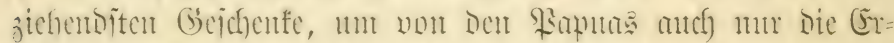

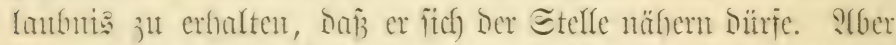

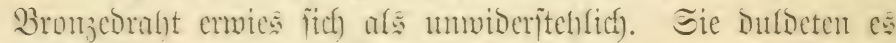

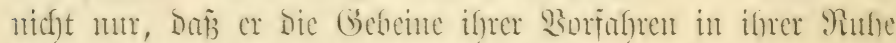

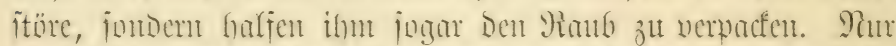

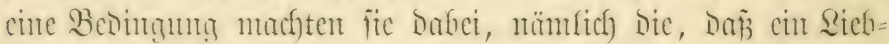

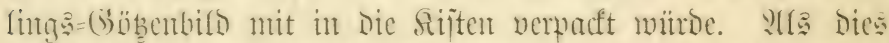
jugeitanten mar, fïfnrten fie cinen Ianz um bie Sijten anf mob halfen ite forttransortieren. Iicsmal ging affes gut,

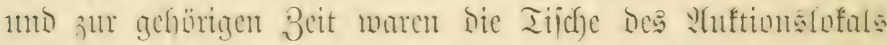
mit Imijenten ciner Fiflanze belanen, melde, Genor Di: Eenoung angefimbigt war, ju Den Pierfen einer Der reidjiteit Sanmlungen Der şelt gehört hatte.

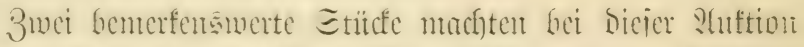

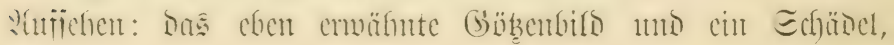




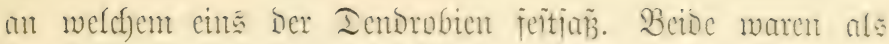

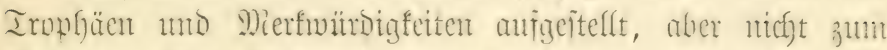

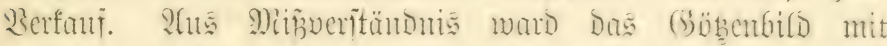
veriteigert. (5) frachte mux eine Selemigfeit ein - jenodh

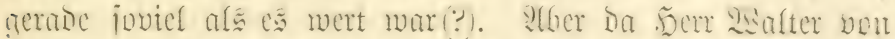

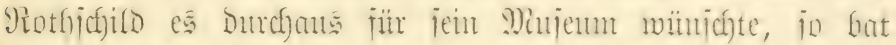

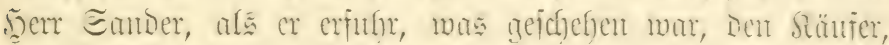
in Den Rëtdfali jut willigen; aber siejer weigerte fitch). $\left.{ }^{1}\right)$

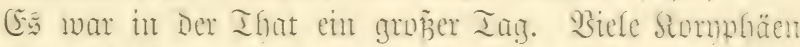

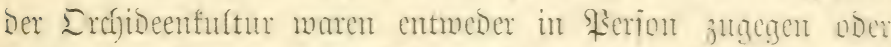

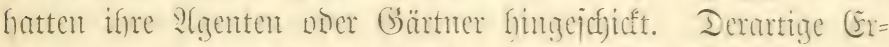

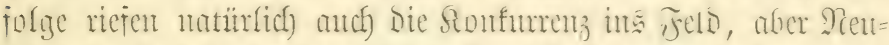

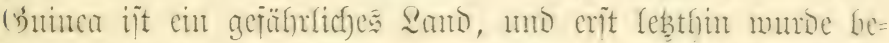

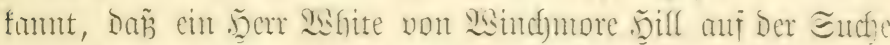
nach ebendemiclben Dendrobium Phalaenopsis Schroederianum jeinen Tod gefmben habe.

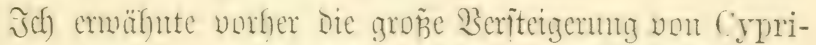

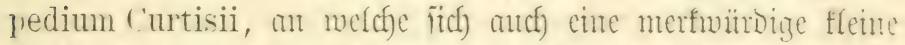

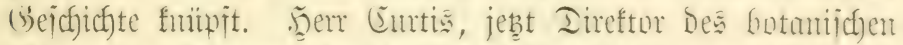
Gartens zu Renang, iande Dicje Pfflanze in Jafne 1852 ans

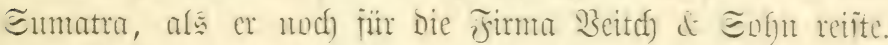
Iic Eemoung wax iefur flein und ba feine meitere folgte, io

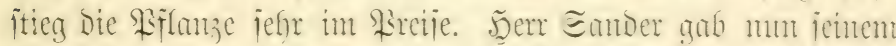

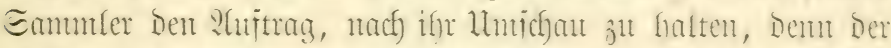

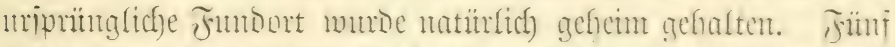

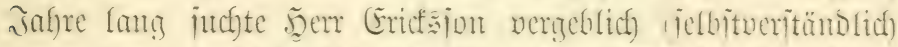
janmelte er in biejer Beit viclerlei amberes (bute) und anb ju=

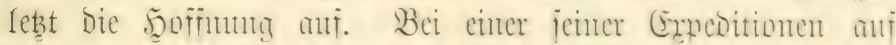

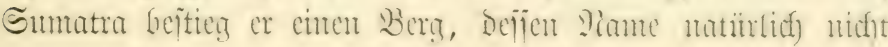

1) Die Stelfe lautet rörtlid): But Hon. Walt. de Rothschild fancier it for his musem, and on learning what hat hapenen Mr. Sander begged the purchaser to name his own price. That individual refused. 


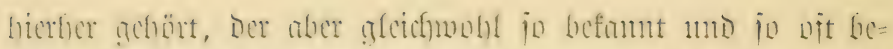

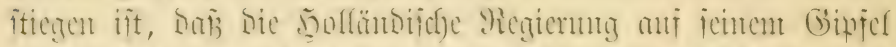

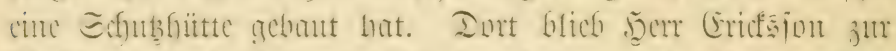

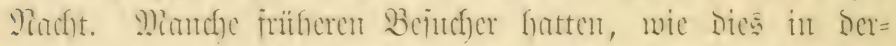

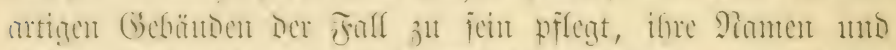

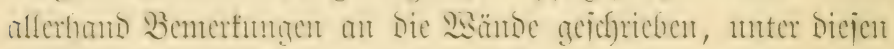

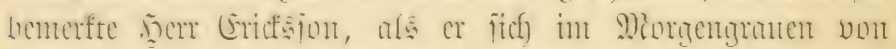
ichent Rager erfoh, Das Bifo ches Cypripedium mit grinter

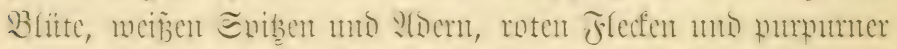

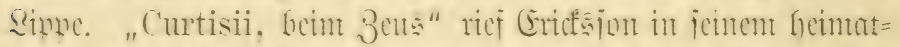

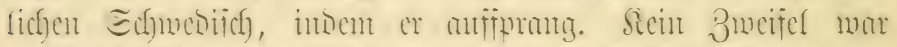

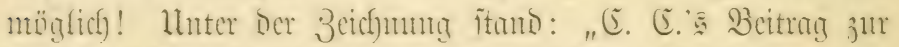

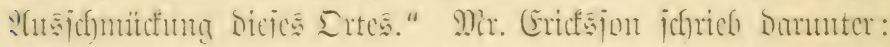

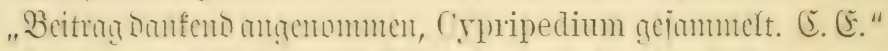

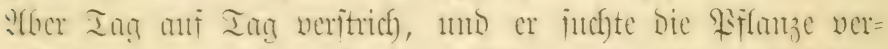

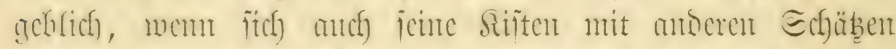

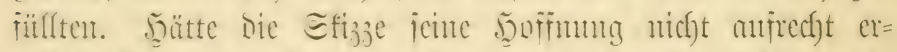

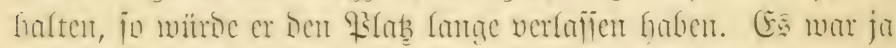

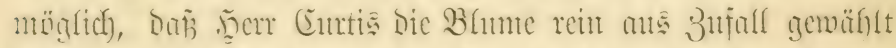

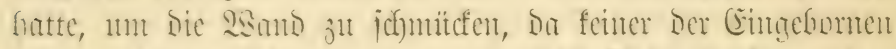

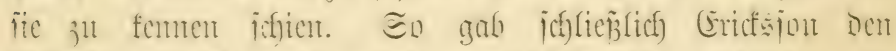

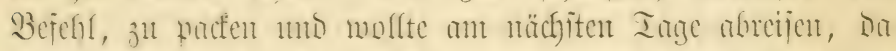

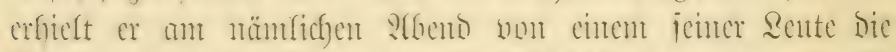

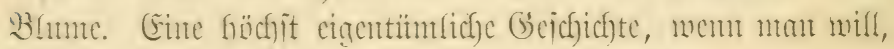
aber eine, Deren Genantigfeit ich verbitrgen fant.

Bu einer moneren Slaije vou Beriteigenungen, weldye

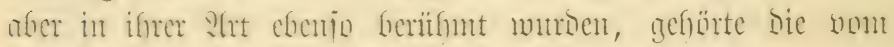

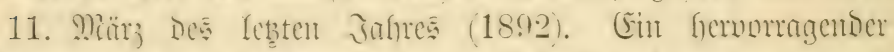

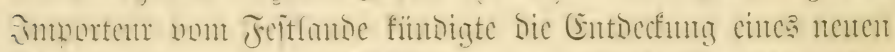

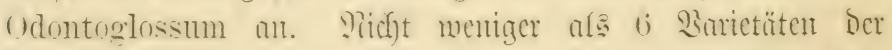

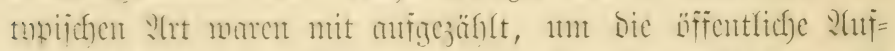

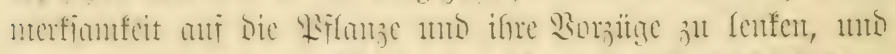

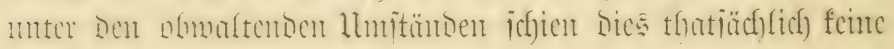




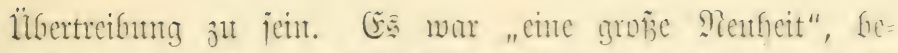

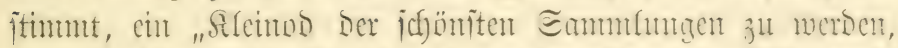
eine "Qiebling

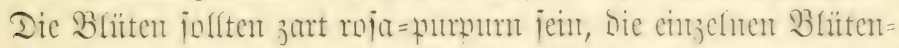

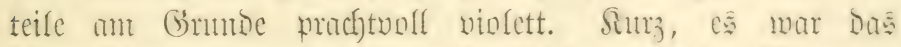
blante Odontoglossum uno verbiente jeflr wofil ben Beinamen "Coeleste". Llmo Der ganje Burrat von 200 Etituf follte

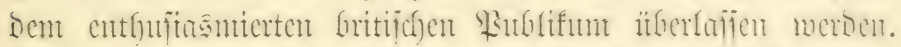

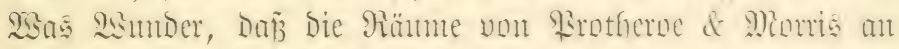
jenem Mergen bes 11. Miörz mit einer Dichten Menge gerüllt

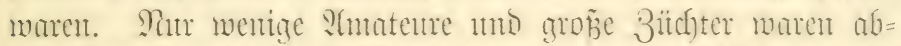

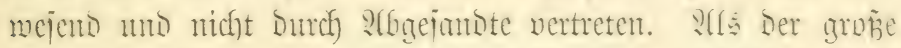

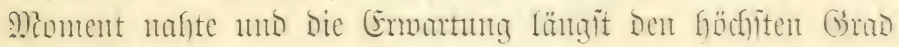

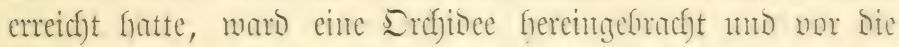

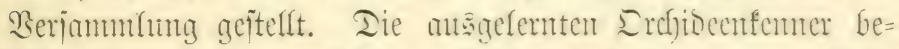
jaffen fie mit cinem flitchtigen Blict und ingtent Donn: "Echn nett, aher wir müchten ..Odontoglossum coeleste" jefen".

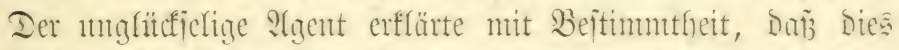

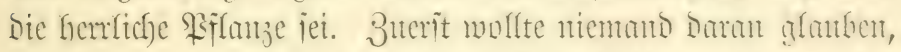
Da alle es für cinen gemanten Echerz fieltent; als ca aber

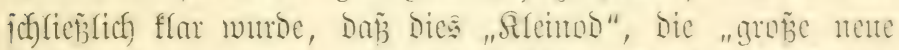

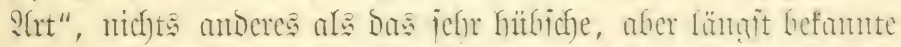
Odontoglossum ramosissimum ici, erfob fich cin folche (b)

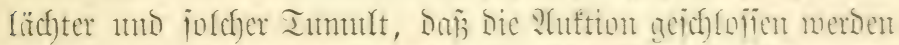

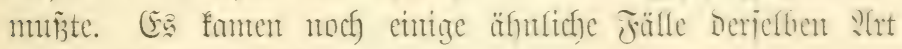
vor, welche aber nicht jo arg waren.

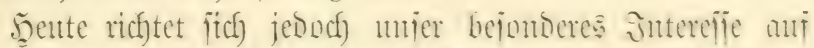

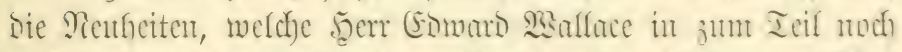
umbetretenten Gegenten gejammelt hat. Doer sistlace, melffer

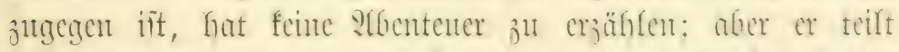

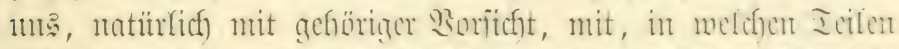

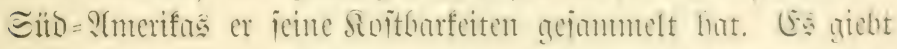

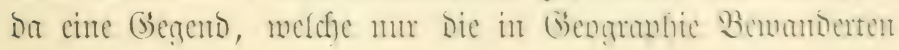




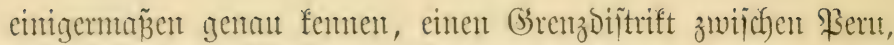
Étador, Eohtmbien, Benezutela umb Brafifien; cr wird vou

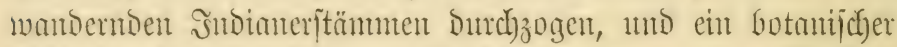

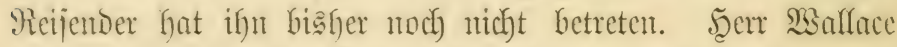
folgte Dem Butge ber Eentral=Corbifleren von Eolumbien aus

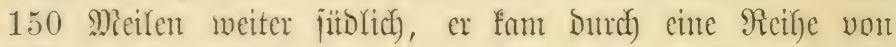
Ifyälen, die reichjten, weldye biejer trob feinter Jitgento weit gereīte Mann gejehen hatte, und weldhe Mitriaden von Şerben

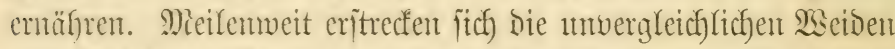
won Pajabena=Gras; aber bie wilden Serben, weldse nie in cinten Stall famen, fund ifye einzigen Bemofner. Şier wächjt an Bergabyängen bie jo jeltene weiz̉e Bletia Sherrattiana,

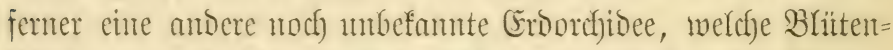

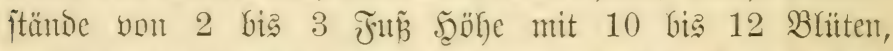
Dic Sobralia-äbnlich), purpumot und 3 bis 4 goll breit

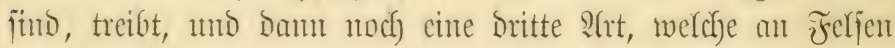
wächjt und wie Majjen von frijugejallentem Edynee ans fieft. Dieje Erjählumgen flingen anfregents, werben aber

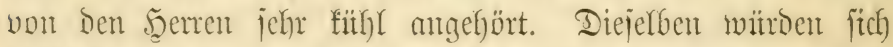
vielleicht fïr Den niebrigften \$ireis Derartiger Seltentyeiten, nod) mefre aber Dafïr interefifteren, welche etwaigen (Shancen fïr ein gritclfiches Gielingen bie Sultum Derjelben bietet. Şänoler funto

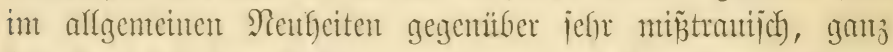
bejonders bei Orobrchideen, mo ans nafeliegenden Gritnden

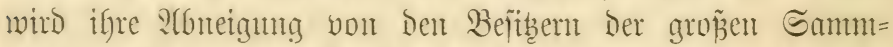

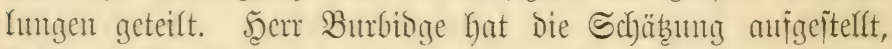

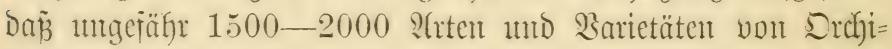
Deen in anjeren Eammtungen vorfanden fund, cine beun=

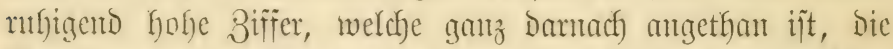

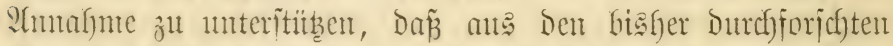

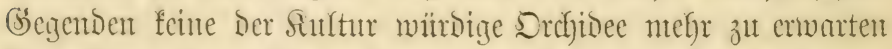

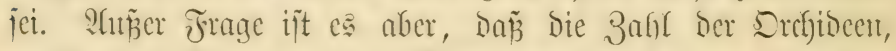
weldye bic Eammler bes Mitnefmens nicht mert geadhtet 


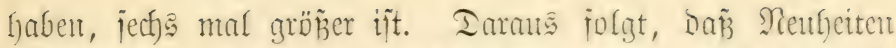

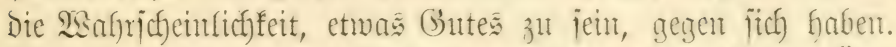
Biele : Prten you meiter Berfreitung zeigen je nadf Der D̈rt=

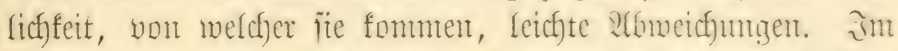

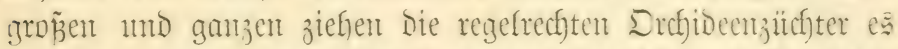

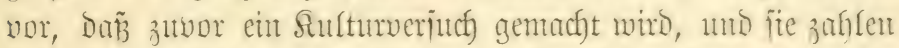

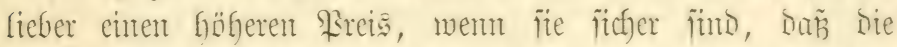

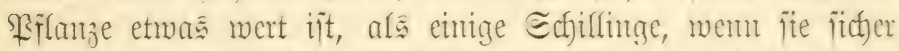
ermarten fönnen, von Der Siflanze mur Märfe uno bie itarte Mafricheinfichfeit cines Mitserjolges jn haben. - Ennit er=

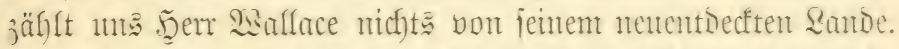

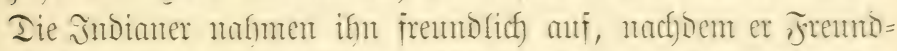
ichajt mit einer alten Fran geichlojien fatte, unis machten ifm mährend jeines viertägigen :Gufenthaltes Das Qefen nach ifreer P(rt angenefym.

Der Shtftionator hat inzmijgen mit jeinen Säufem ge= Wlanbert. Gr jüflt jelbit ein Jnterejie an iciner sisare, uno mie jollte es anders jein bei Gégenitänden von jolcher Ecchön= heit! (E⿱ Şlajīen ïbergehen, welche Der Figentitmer als "llnica" an= fieht, und von meldfen er bei Der Beriteigernuty unglatbliffe

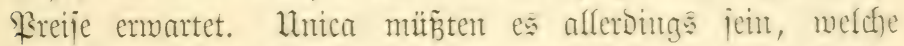
Die Fentermrobe Diejer icharfen uno geitbten ?angen unbefellingt paifieren föment. Plumeria alba 3. B. wiro vorgelegt, uno

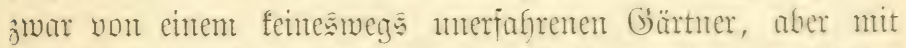
joviel Echen mo Boritcht, wie fie fird fïr cine Der exquijitejten

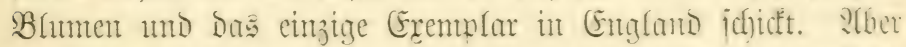

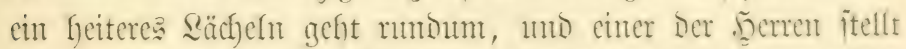

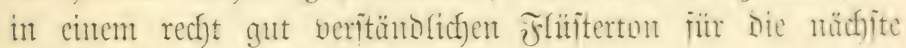
Beriteigerung ein Duthend Exemplare für nut cintu Bundfteil

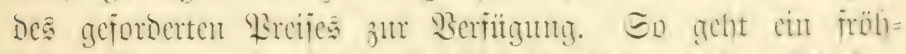

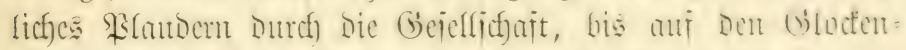

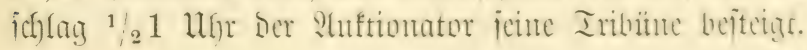


Giuffumbert Rot Odontoglossum Alexandrae be bejten Inpus mo von tabefloier Beichaffenfleit fonmen ifme zuerit

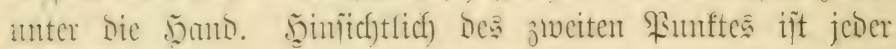

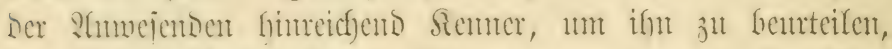
mo fiminichtlidf) Des eriten iit man gern geneigt, Den $\mathfrak{B} \mathrm{er}=$ fänfern Gitanten ju ichenfen. Die Buthen find prafl blanf mit Dem ffecinen, hervorbrechenten Triebe jwijchen ben

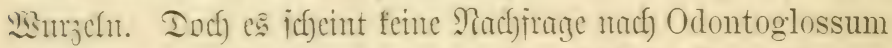

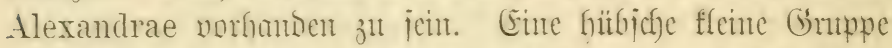
nou Bhuthen geht für 11 Edfilfinge fort, mo jede von ifnen miro int nüchjten Frtithling eine ober gar żwei Rijpen Der weiben, rothrmun geffecten Blitten tragen. Dam hört das

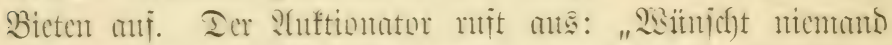

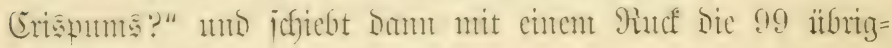

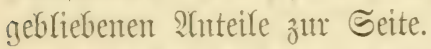

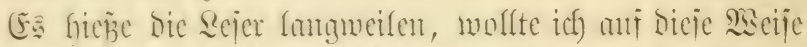

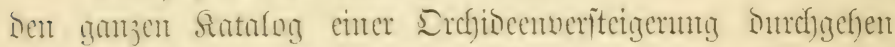

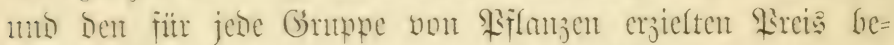

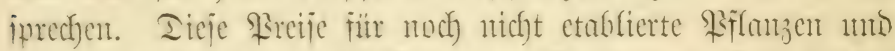

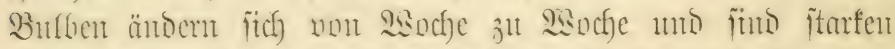

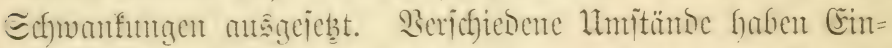

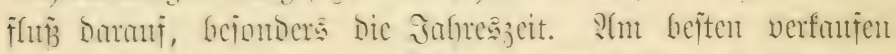
fie fidf) im Frithling, wo 9P(onate von Richt mo Eomten= ichein hevoritefen, um bie sirfinngen ber langen Reije ans

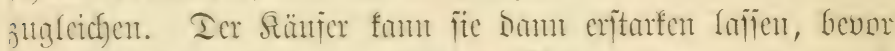

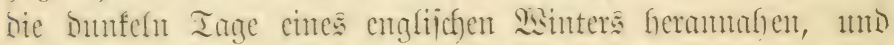

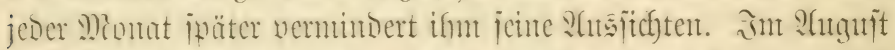

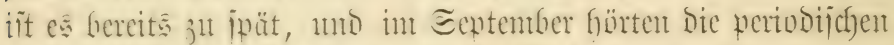

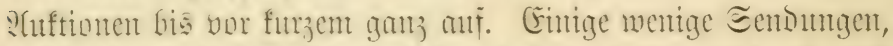

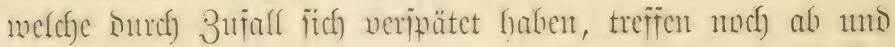

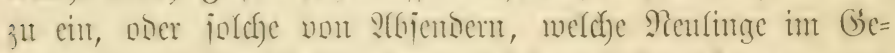

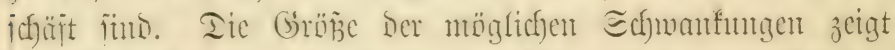

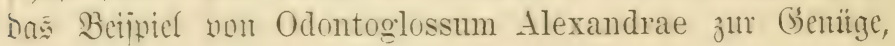




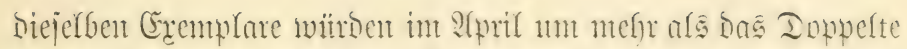
Des Sreijes unter hejtigem l̈beriteigern werfauft monden jein.

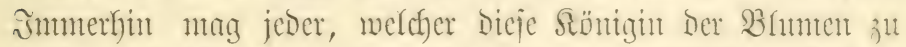

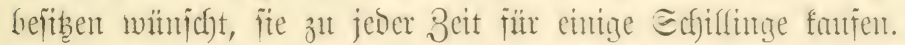

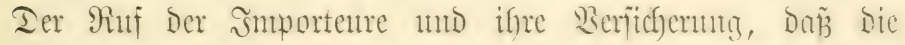

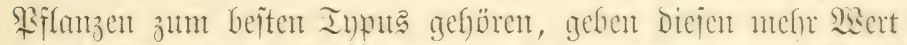

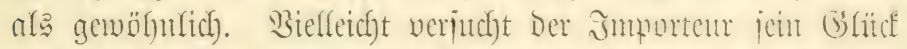

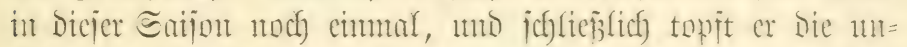
verfauften Burben ein und verfanft fie im nüchiten Frithlinụ als etabliert.

?hif bie Odontoglossum folgt vielfeicht cin Oncidium

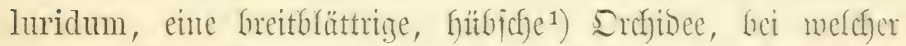

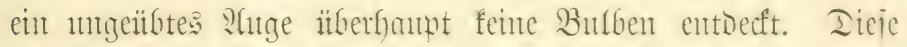
Piflanze beferricht immer(?) Die ?fuftion, wenn fie bitlfig an= geboten wird, uno 10 Ecfiffing mtag als cin guter Sireis für

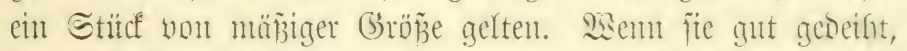

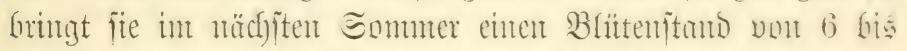

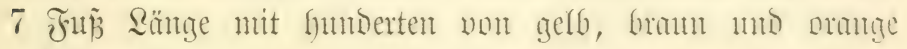
geflectten Błhment.

Oncidium juncifolium, Die nächìte an ber Rreifre, ijt

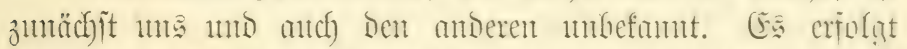

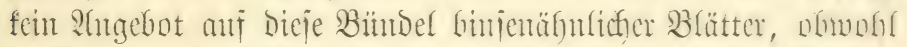

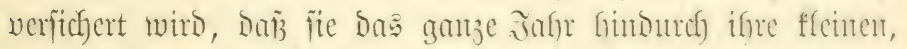
gelfen Bhumen hervorbringe. Epidendrum bicornutum anterer=

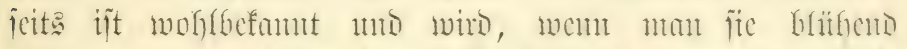
fiefht, was aber jelten ber Fall ijt, viel homumbert. Sic Beichreibung iffrer weiß̄en, rot geffecten Blïten flingt mehr

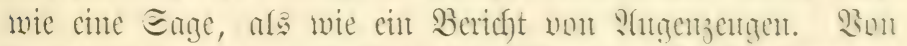

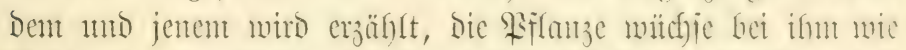

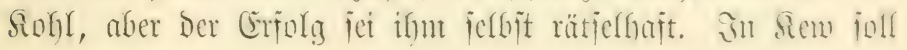

1) Ridyt inmer. Die Pflanze ift ungehener variabel unt hat

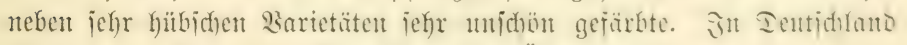
iît fie menig beliebt. - Stmerfung Des ltberjebers. 


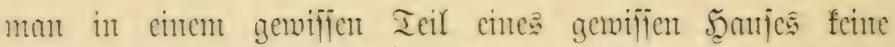
Edfmierigfeiten Bamit baben. Tie meiften Exemplare find gut

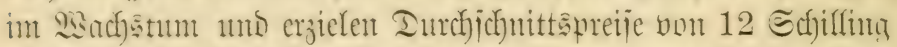

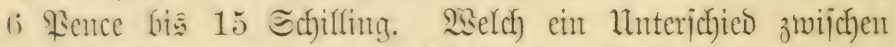

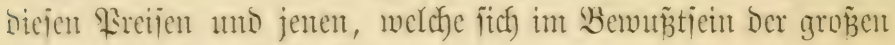
Menge feitgejebt haben!

Mir ipeziell fehlen alle jelbiterlebten Beipiele jolcher hohen Pireije, uns ich fam mur wiederfolen, baż hentzutage Echillinge ba gezafilt werocn, wo frither Rifunde Eterfing ge= gaflt muthen. ङo im Jafyre 1846. Damals brachte einte (1) Känfige Piflanze wie Barkeria elegans 5 bi $17 £$ mo ミtitc:; Epidendrum Stamfordianum 5 \&; Dendrobium formosum 1.) E; Aërides maculosum, crispum mo odoratum 20, 21 und 16 ?. Niemand Dari mu aber glantben,

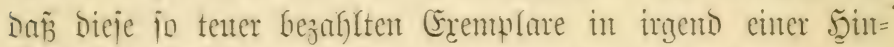
fithl befijer gewejen jeien, als bie, welche wir jebt erfolten, nein, gantz fither ît, baj̃ fite Dantals in ichlechterem Buitanto

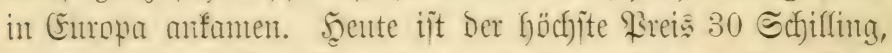

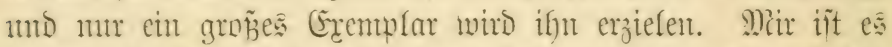
erftanticf), marum jo menig Reute Drefjideen fultivieren. Jenes

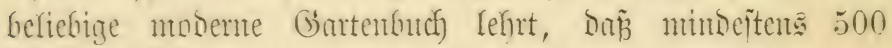

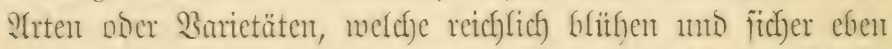

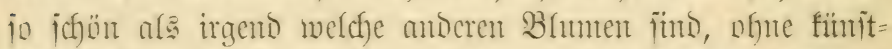

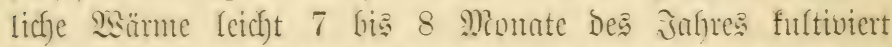

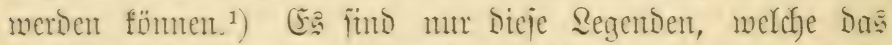

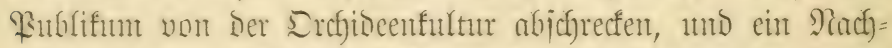
mittag in einent :Guftions Mittel, Diejelben zu vernichten.

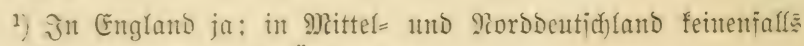

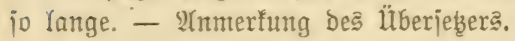




\section{finftes faptel.}

\section{(D) whidech int allachteinent.}

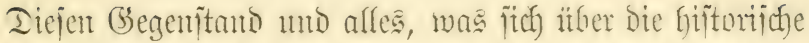

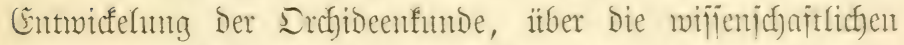
Fragen, weldye Gierbei in Betracht fonmen, nder gar itber bie praftijche Eeite Der Eache fagen lię̃e, in Dem furzen Rafment

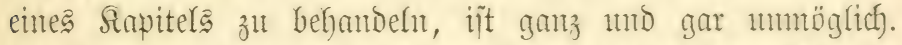

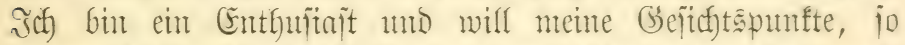
żmingento fie fït mich) jein mögen, fier nientantom anj=

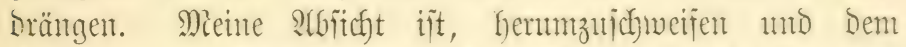
Flnge meiner Giedanfen zu folgen, wie fie entîtefen, biel= leicht nicht ganz ofune beitimmtes Biel, aber ofne mich an

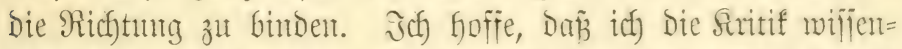
ichaftlich gebildeter Rejer nicht herantsiforbere mo jolche, melche Der Frage gleichgiltig gegenuiber Ftefen, unterfalte.

Siebensuntroige Rgilojophen, welche glantben, Dañ bie

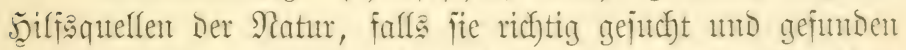
werden, bollanf gentitgen, jebes gejunde Beditrintis Der Nenticjent

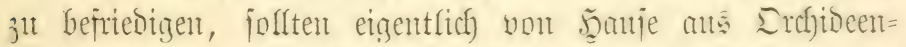

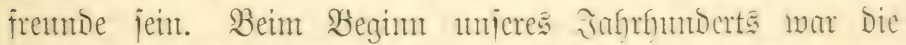
ISiffenjichaft Der Blumenzund zientich jo meit, wie wir hente

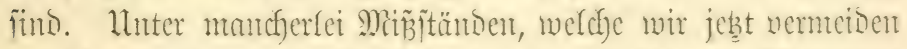

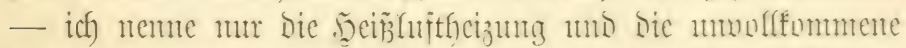

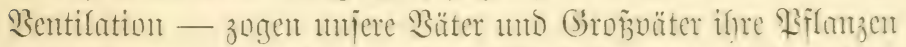
genau jo gut, wie wir; mancherlei Berfoeffenutgen - bejonters

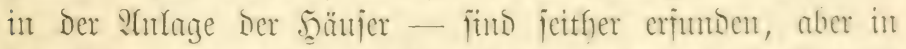

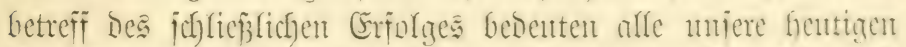

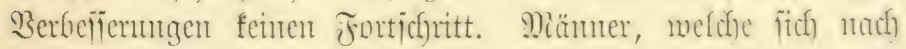

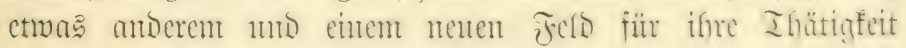

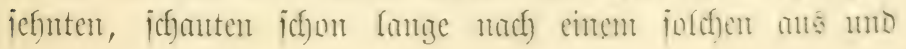




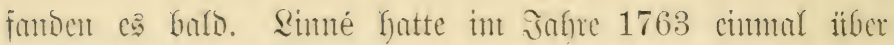

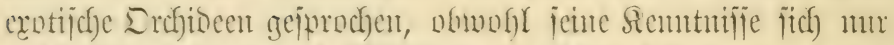

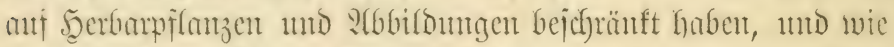

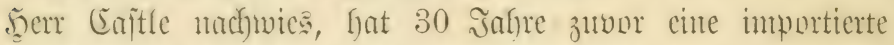

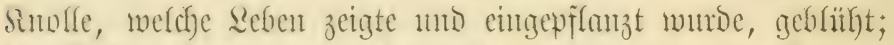
Den shament fement wir nicht meffr. So erfitheren bie (Sararten=

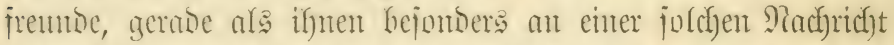

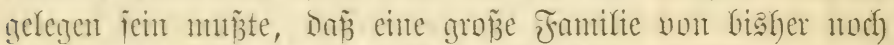
mulefumten

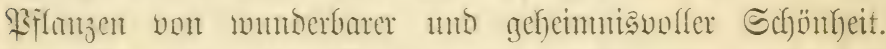

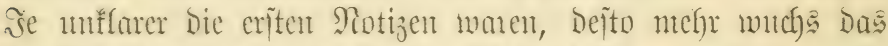

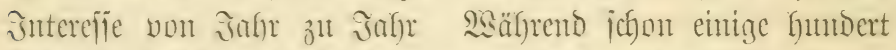

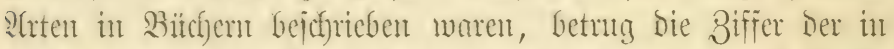

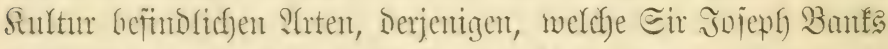

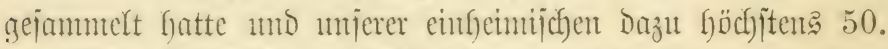

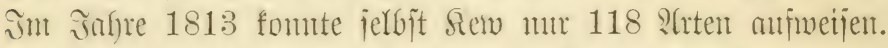

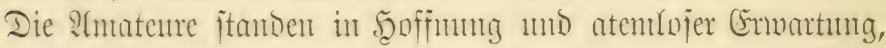
bis fie in den Befit biejer Schäbe fämen.

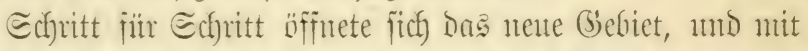

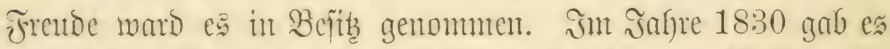
bereits che Pieife bur Eanmlungen, weldhe Damals fitr voll=

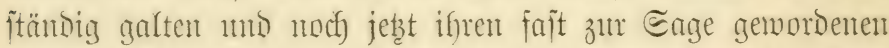

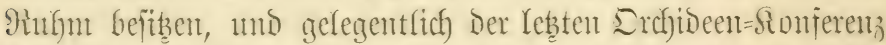

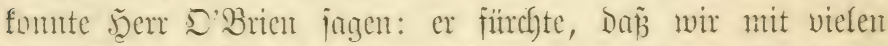
(5xemplaren nicht fonfurrieren fömuten, weld)e einitmals in

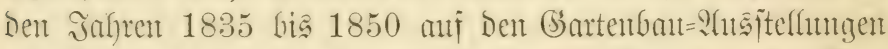

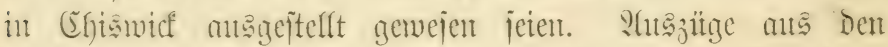
Berichten, weldhe er mitteilte, unterititben Dieje Samafyme. Mian fultivierte meniger P(rten, aber man fielt auj "๔chau=

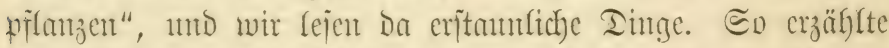
Mr. Sanvente, bie Mutter unjeres Sberhautes Eir Trevor

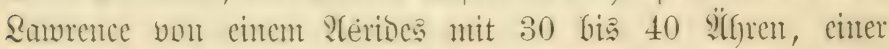
(Eattlena mit 20 Bliitenitänden, einen Epidendrum bicor- 


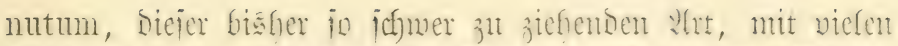
Blittentüunon uno einent Oncidium, weldes einen Blitten=

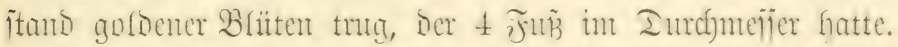

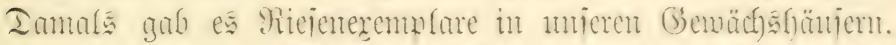

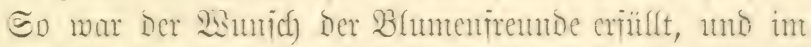

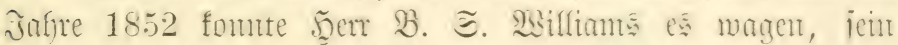
Budf) „Orchids for the Million“ zu Hublizieren, ein Buch), melches fpäter unter Den Iitel ,The Orchid Grower's Manual." cinen wohlverbienten Ruf erfielt. ${ }^{1}$ ) (Sine Bejolätigung wax

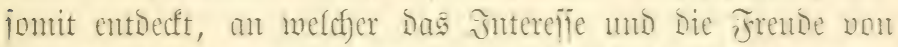

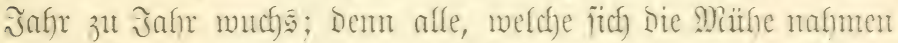

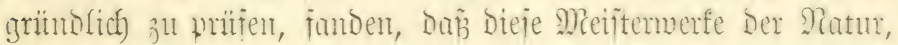

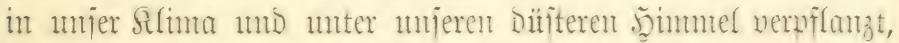
mit einer Eicherfeit und Regelmä̈̄igfeit jum B(ïhen gebrad)t werden fonnten, wie faum in ifrer Deimat. Die Edhwiertig= feiten Der Rultur ermicjen fict) im grofien und ganzen als

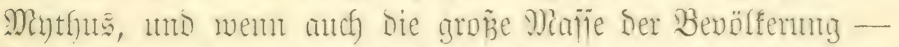

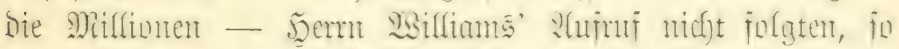
thaten es Die oberiten Befutanjend und Dieje mit Frenton. -

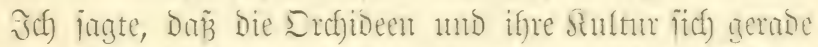

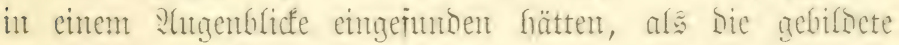

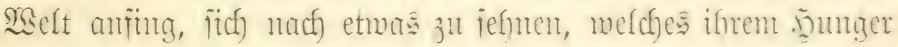
nach Beichäfitinng genitgte. Sente von Geichnad merden

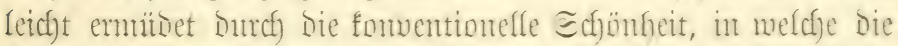
fimit aller irifheren Generationen ifn Echönfleitsiocal gefleioet

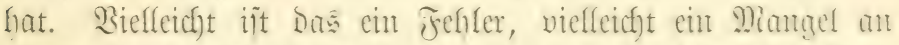

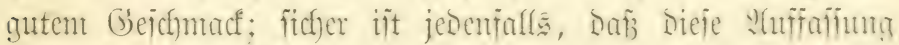

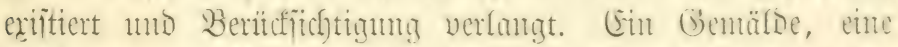
Bilojäule, ene Rorzellanvaje, furz irgend cin Benenfund Der

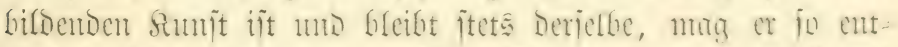
jücfend jein wie inmex. Ins Ënujigc, mas num Damit

1) Ias Bud iit in Der Iflat nortrefiflid) und hat bis iegt 7 iturfe 2uflagen exlebt. - 2anm. ๖. Überj. 


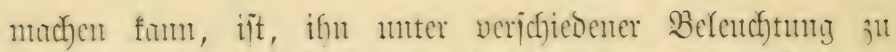
hetrachten. Théophile Gantier hat in einem Stugenflicf me bejomtenen Freimutes eimmal gejagt, mem Raphocls ", $3 e r=$

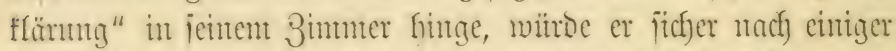

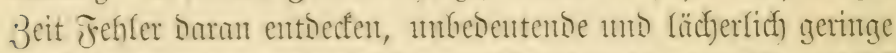

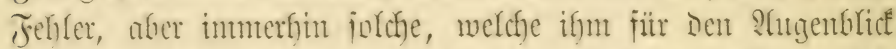

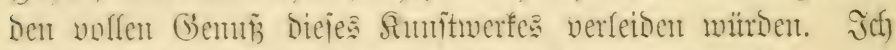
mmäfne Dicje Protiz fier, welche vielen meiner Rejer an=

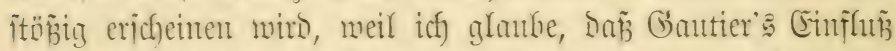

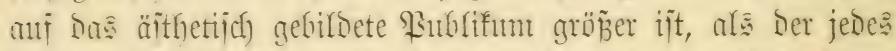
moeren Echriftitellers. Imilente, weldhe nie eine Beile jeiner

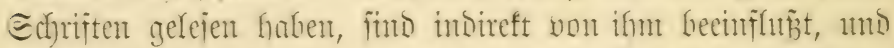

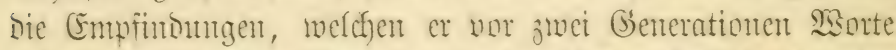

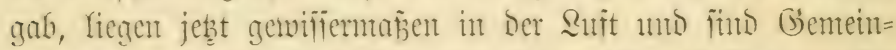

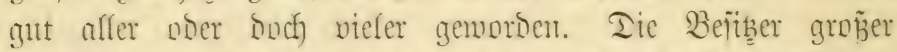

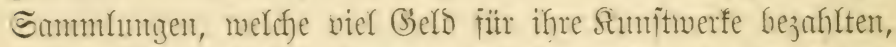
werDen fidc) natïrlidf) Dagegen itrüutben; aher in Der Reger

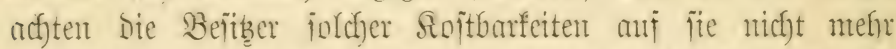
ars mif ifre mertvolfen Tijhte mis Etithle; berjenige jeboch,

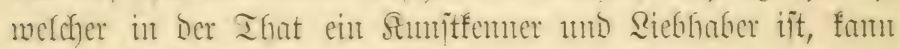

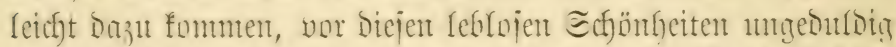
zut mernent, ment er fie itets vor fid hat. „D, Daßj bieje Rippen iprechen fömnten!" rief Eomper an. „D, Dä́ bieie herrfichen Geitaften fidc) nen gruppieren ober in cine andere

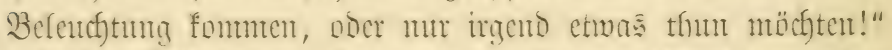

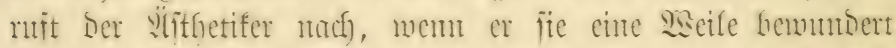

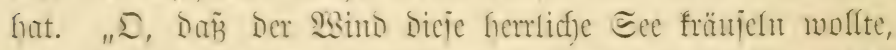

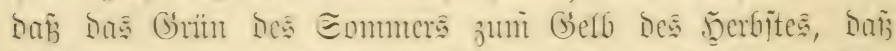

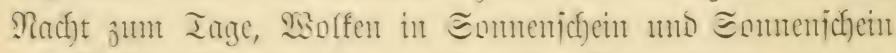

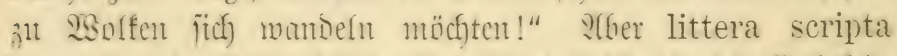

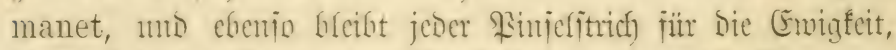

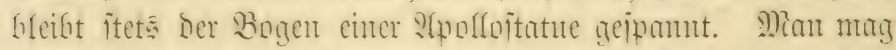

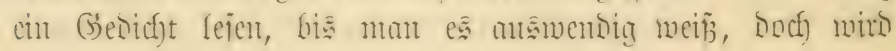


Ttets Das geiprochene Sart Die Berje mrierem Shr nahe Gringen, als wäre es Das erite Mial. Dialer mögen ifre Seimwand zur Seite ftellen mo nad) funzer Beit gleichjam

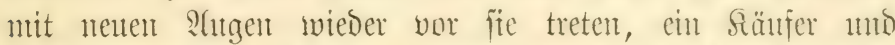
Siebgaber jebuch, welcher eimmal vou biejer Srantfyeit Der

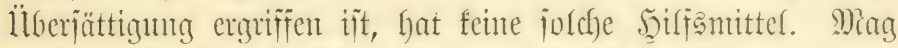

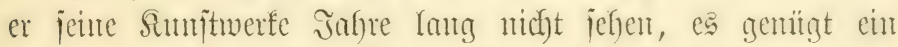

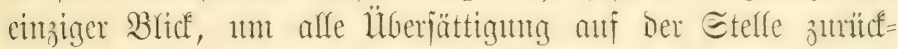
zumifen. Jid) habe jelbit einen Fall gentan beobachtet, wo ein

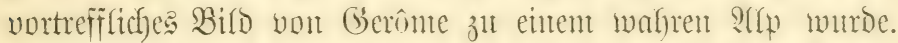

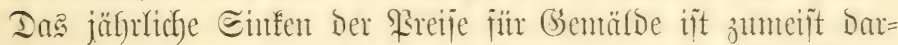

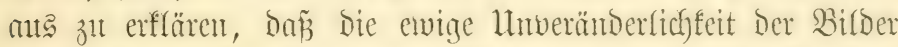
gerabezut eine Stwerfion gegen fie erzengt, umb bieje Stwerfiton macht fich gerabe unter ber befferen Slafje von Bejchitbern Der fumit bemerffor.

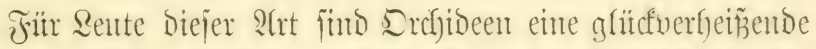

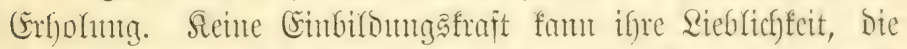

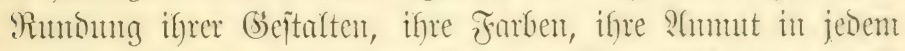

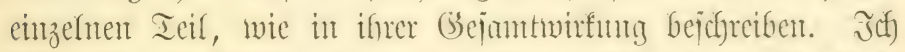
jah eimit in Jtalien (ober war es zut Sxford in Taylor's

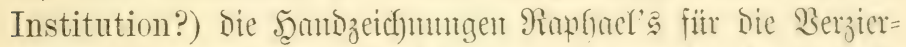

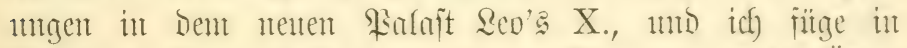

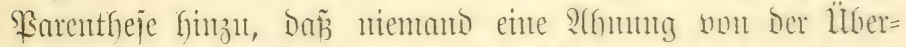

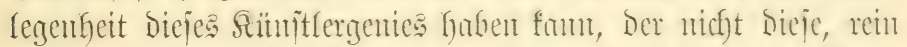
anf Den groben (Effeft bered)unten Etubien gejehen hat. Lluter

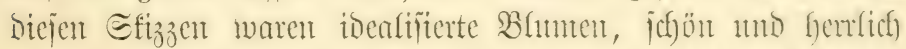

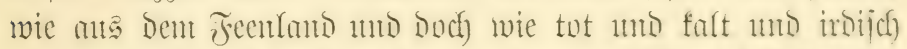

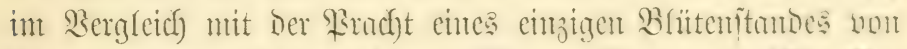

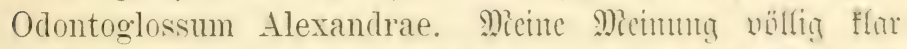

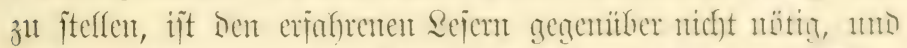
anderen Reuten merien s.

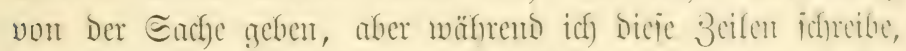
iteht bor mir che Ripe von Oncidium crispum. mo ither 


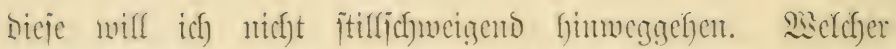

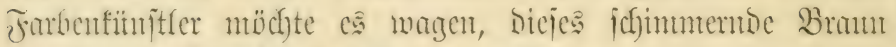
mit matter Golsfarbe zujanmenguftelfen; welcher ber form

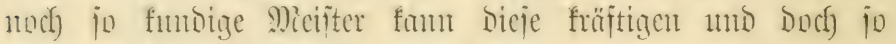

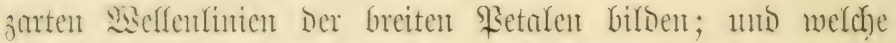

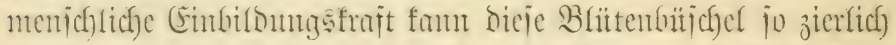

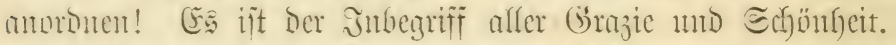

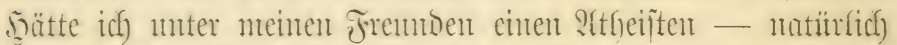

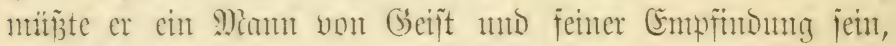

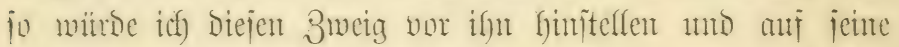

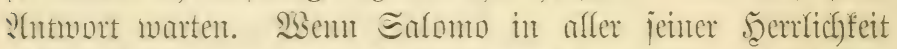
nitht geffeciset war, wie chne Silie anf Dent Felbe, jo haben

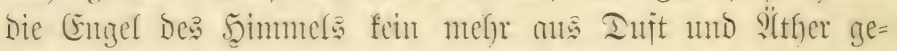
mobente Aleid als bie Blïten einer Drefibee. Dod) wieder zitr Sache. -

Biele Rente, Denen Der Gartenbant im allgemenen gleidf)=

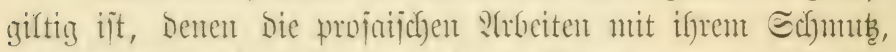

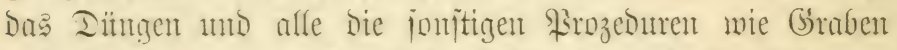

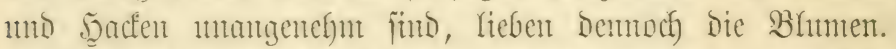

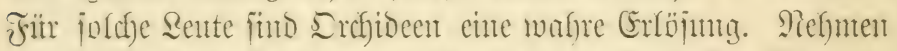

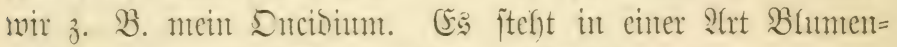

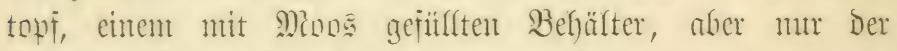
Bequemlichfeit harber; Denu Der ganze Bhlïtenjtanto mit jeinten grobien Bhunten entipringt ans einem Edheit Şorz. Sein

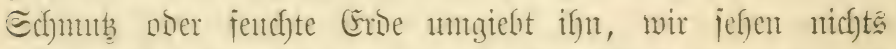

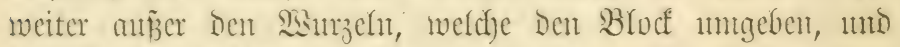

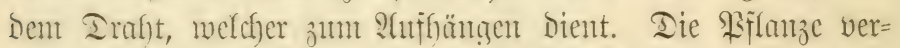
langt feine weitere sisartunty als iffr tägliches Bab. Ecit Dem

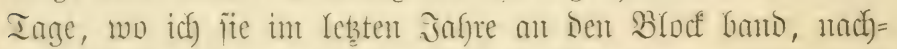
Sem fie iffrer somat mo all iffren Fremton entriffent mo

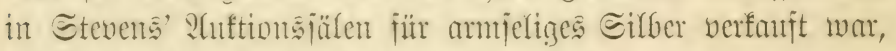

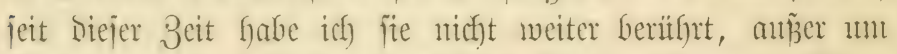

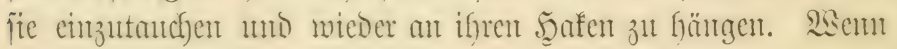




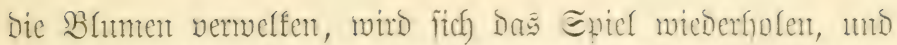
fie wird foffentfid) treiben nto meitemuadien, bis fie midf im

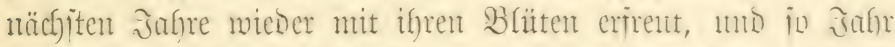

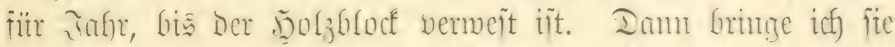

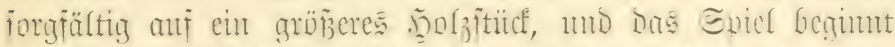

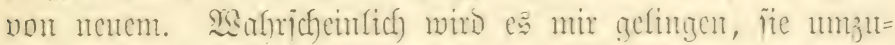

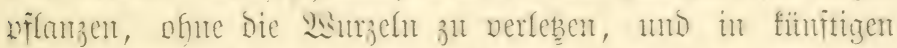

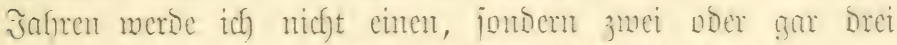

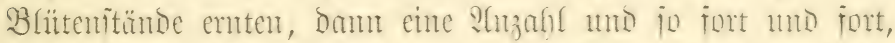

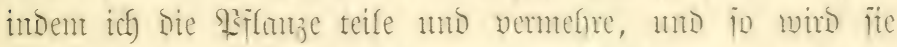

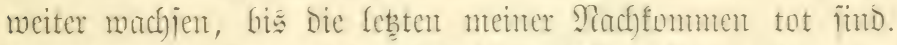

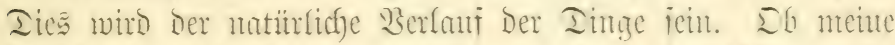

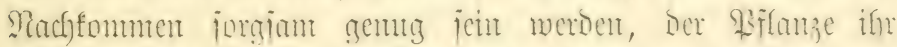

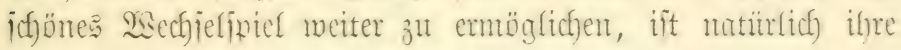
Sache unt wird meiner fontrolle entzogen jein.

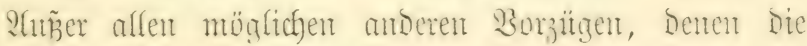

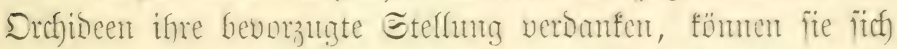

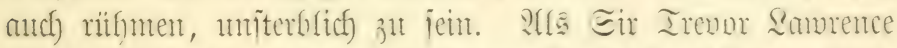

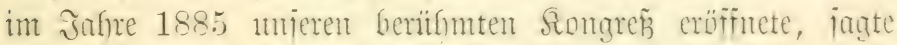

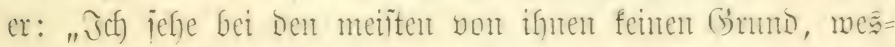

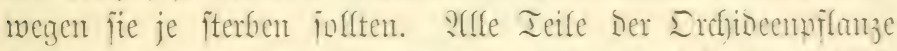

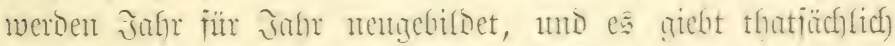

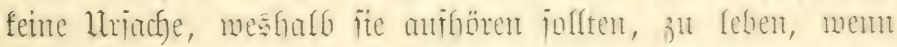
ïe nicht, wie bies in Der Gefongenjofaft borfonmen fant, Duref Fefler in Der Befandung getötet werden." Eir Trevor

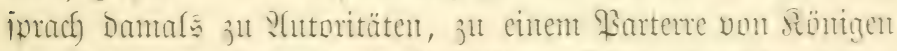
in Reiche Der Botmif, jonit flütte er fitch weiter ither Diejen

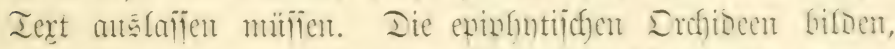

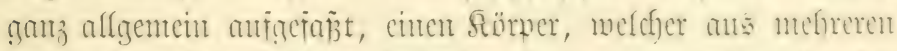

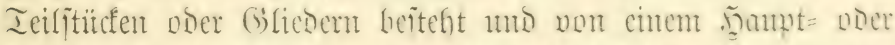
(bipfeltrieb abgeichlojien it. Die Cirfulation de Eafes

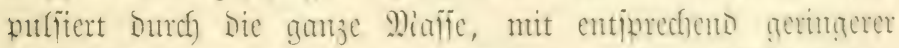
Sebhaftigfeit matïrlich) in ben Icilen, melde geblübt baben 


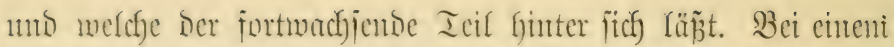

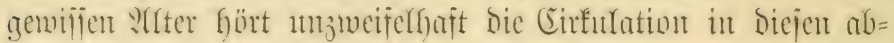

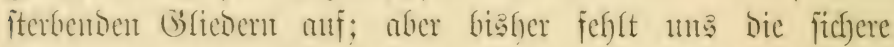
Senntuis, wie lange es Dautert, his die ansgeblithten Bulben

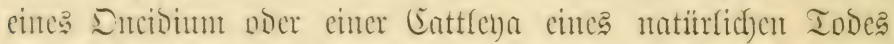

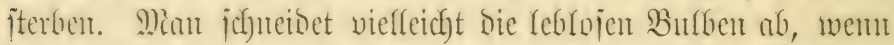
fie ju fanlen beginten, und fiehe Da, vielleidgt nach 12 Menaten

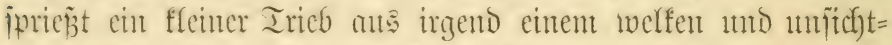

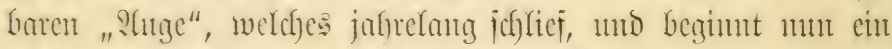

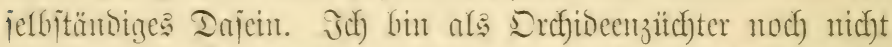

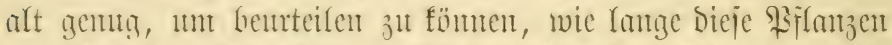

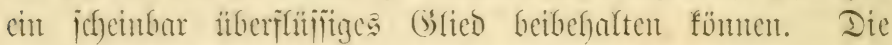
mundervolfe Srganijation biejer \$iflanzen ijt vor allen Dingen

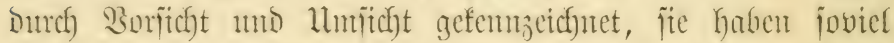

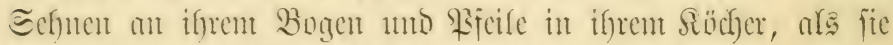
fïmen mo befralten fie jolange als möglich. Ier zarte,

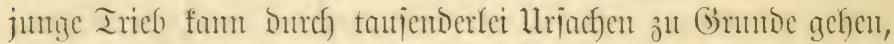
aber biejer Intrafl treibt bieje nubezäfmbaren Siflangen mu

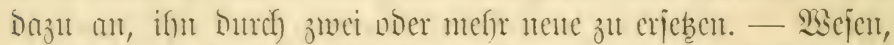

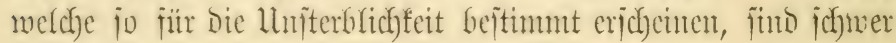
zll tötelt.

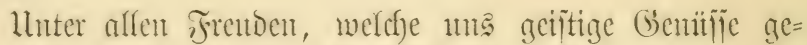
mähren, femte icf) feine, meldbe fich mit Der (bemugthunng ver=

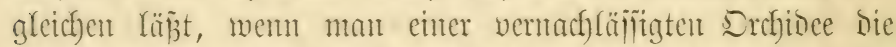

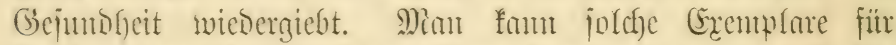

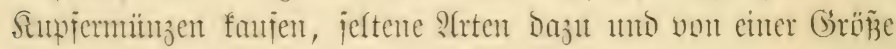

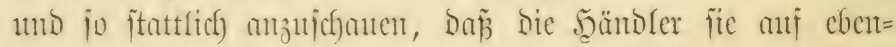

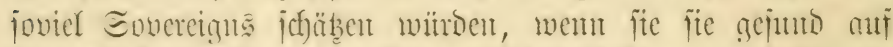

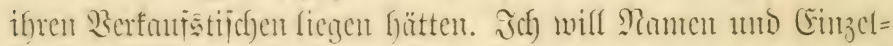

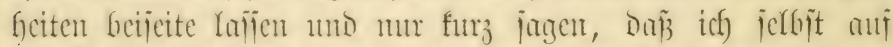

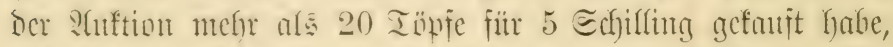

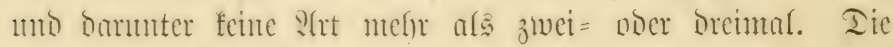

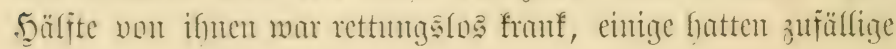


Beichäbigutgen erfitten, aber Der größ̃ere Teil war ein Epjer ungeichicfter Behandang gewejen, und auf Deren Sistederber=

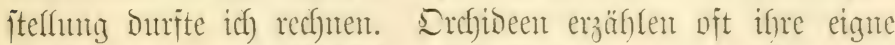

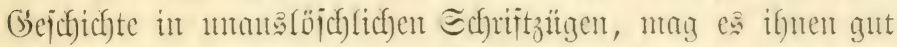

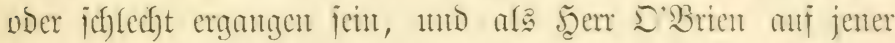
Sonferens thatjächlich wor Dem verjanmelten Rat Der er=

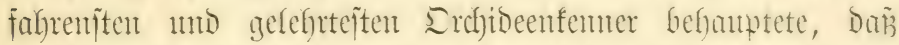

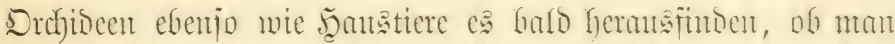

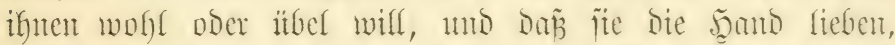

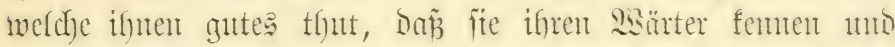

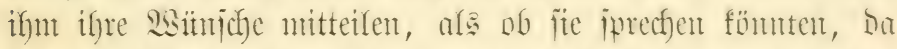

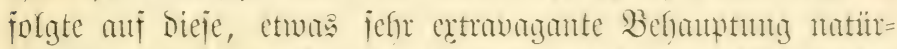
(ich) Şciterfeit, aber fein Epott, mo wer bie mentolich) vielen

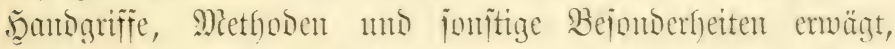
meldye bei mandyen Erchitieen angemanot werden mitījen, um ifnen gemtg zat thut, möchte fie fitr vermunthegabte Wejen Galtent.

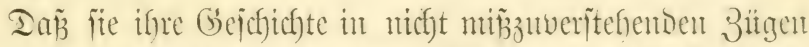
tragen, ift ohne weiteres jultugehen. Sich) habe ba eine Eattlena,

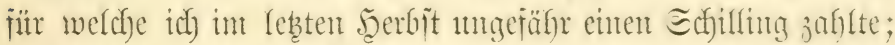
ich Kielt fie, obmogl fie feinen Pamen fatte, fïr wertwoll. Sie verriet mir, bar irgent ein granjanter Mienidg fie vor fïnf

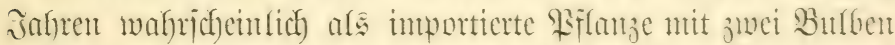

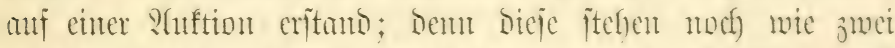

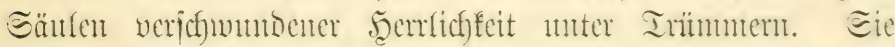

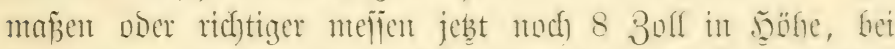

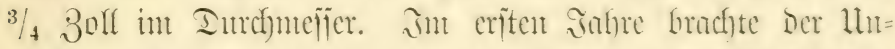

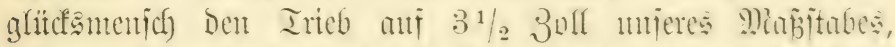

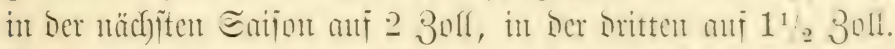

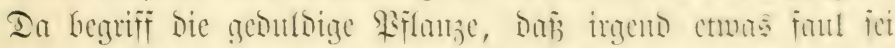

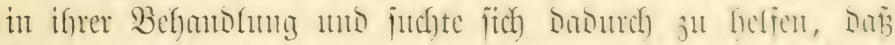

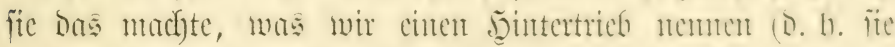

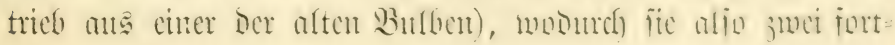




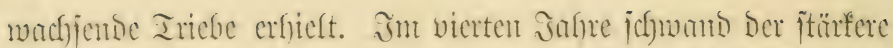

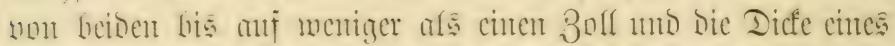

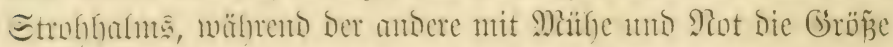

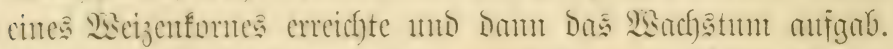

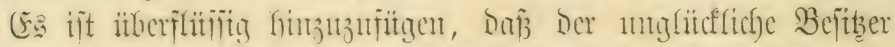
nie ente Epur ciner Bhitte zit jehen befonmen hat. Da eno=

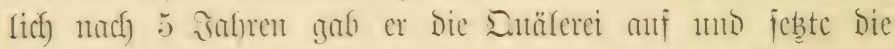

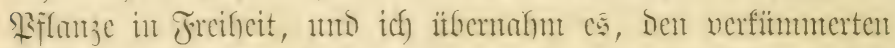

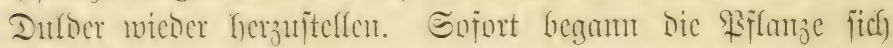

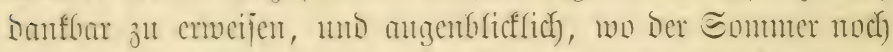

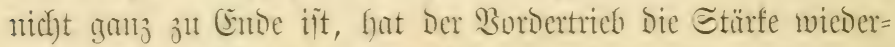
gemonnen, weldye er in brei Jafhen verloren hatte, wäfremo Der Fुintertrieb, welcher tot jut jein jofien, bic beite Butbe iťbertrumpft, welche mein Borgänger erzielt hat.

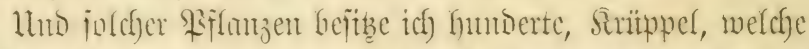

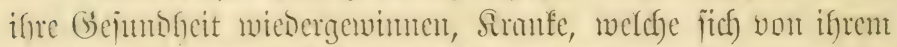
Sterfehett erfebert. Sb e w woht eine rufigere Frentio giefot,

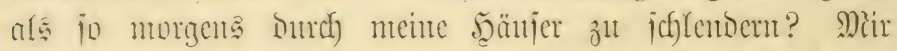

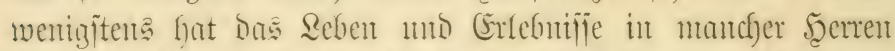

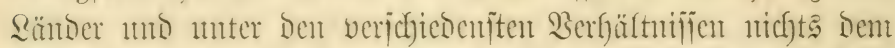

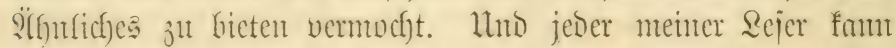
Dies erreichen; Dem idf) bin, aber nicht int gewölnnficfent

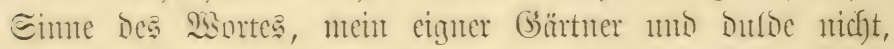
Dafis irgeno jemtano bie bant an meine Srefibeen legt. Hut

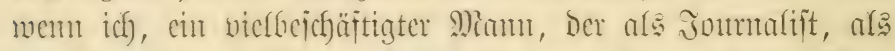

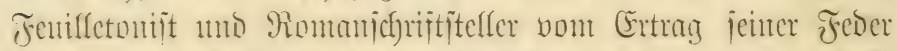

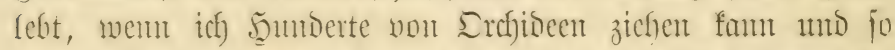

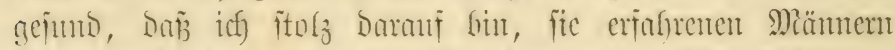

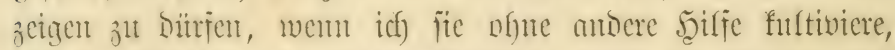

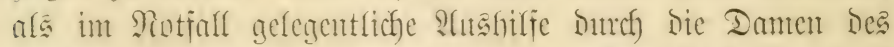

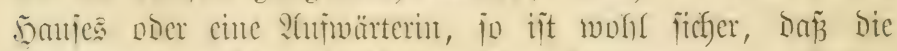

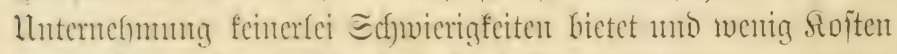

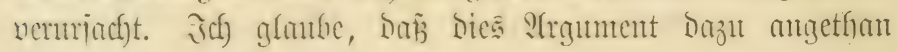




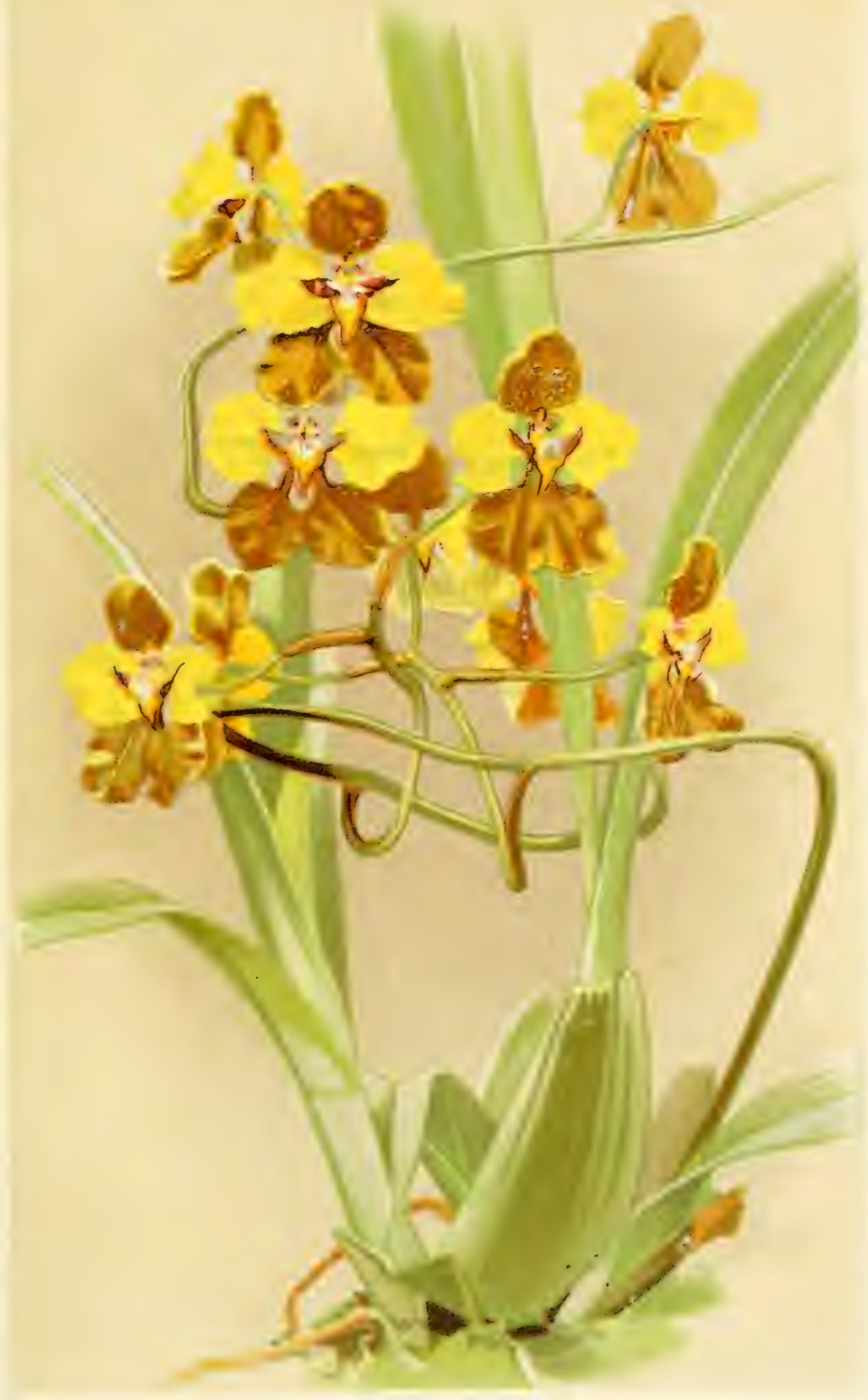





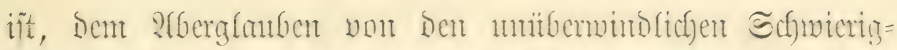

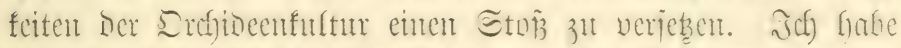

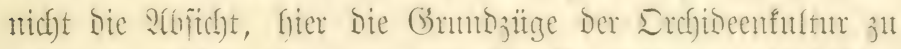

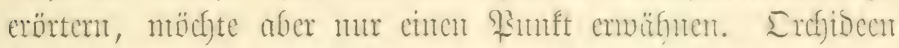

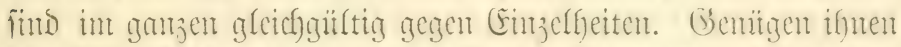
Die Sulturbebinguntgen in groß̌en und gantzen, fo werben

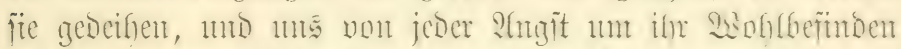

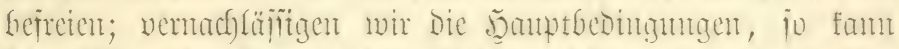

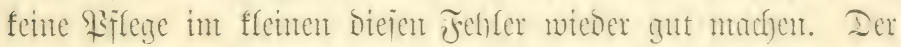

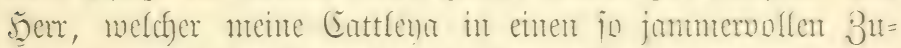

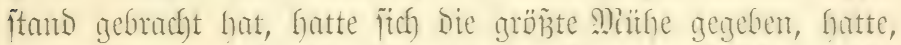
wie es iehr glanbhaft erichent, eine llmnenge Büther ntach)=

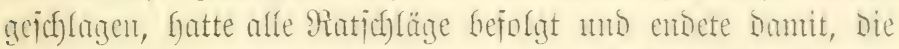

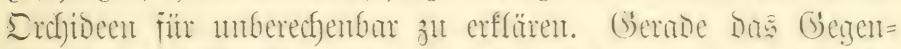

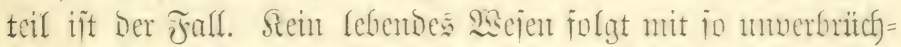

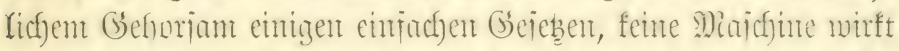

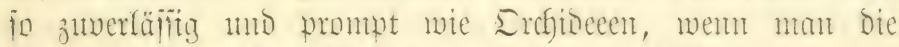
Gejejeb̧e, von meldyen ify Iajein affüngt, erijiflt.

STm beiten jefen wir bies an einigen, zut Beit nock nicht anfgeflärten Fö̈llen, melche ich) hier als Beipipiel an=

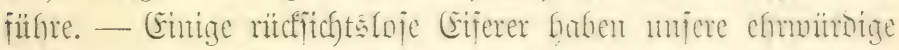

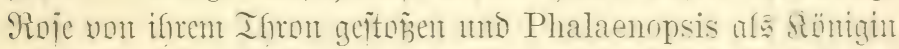
Der Bhtmen gehuldigt. Saljen mir Die Frage Der \$ictät

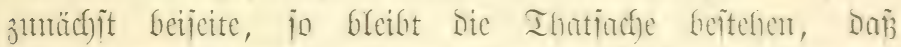

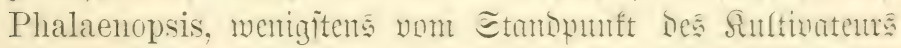

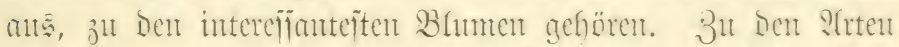

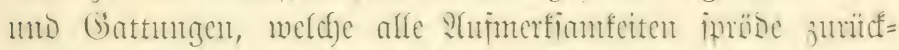
weijen uno Deren es vicle giebt, getören bie Phalaenopsis nicht, uno ich finde, baj fie bei mir mit bem tuthigen

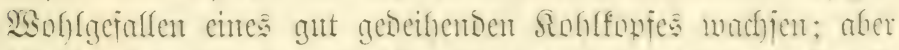

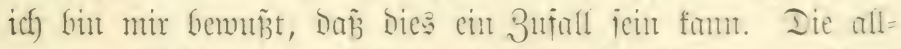

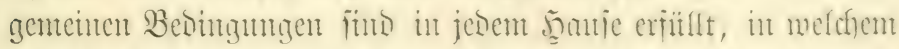
inbijche Srchineen gut geneifhen, aber von Beit 3 ul Beit fommen 


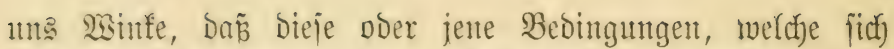
nidf)t überall vorfintoen, für bas Bébeifen Der Phalaenopsis

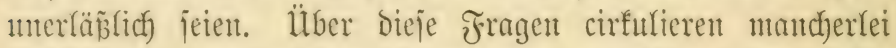

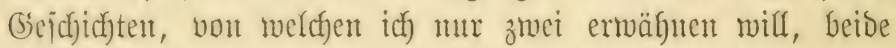

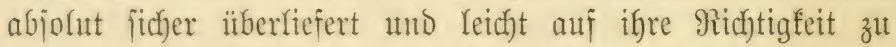
priifen. Die Sorjtände Der Sew=Ssärten bejhloffen, ein

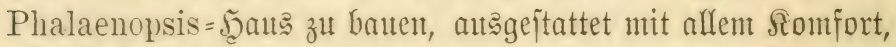

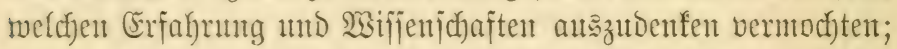
aber als es vor 6 ober 8 Jahren erïffintet wutroe, gebieh

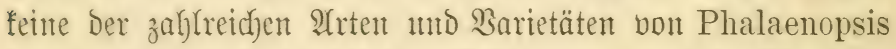

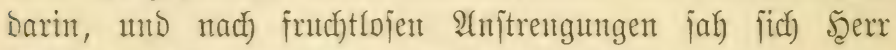

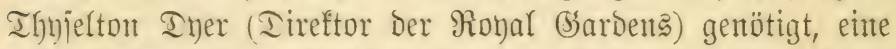
andere Bernendung fïr das Şauts zu finten, und es bient

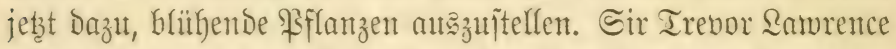

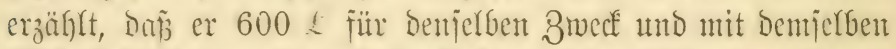
Mišerfolg fortwarf; uno bod) faun jeber ficher anmefymen, Dañ eben bieje Phalaenopsis in 9 von 10 gut eingeridjteten

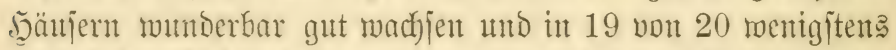

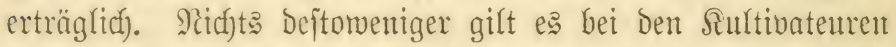

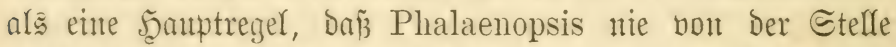
entfernt werden follten, wo fie gut gebeifen, ifre Şafen fün gefeiligt mie ber, an weldyen Solraz jeine Rever auffing. Uno man fam bies nidy)t eimmal fïr jo unvernitnftig erflären,

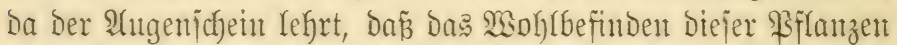

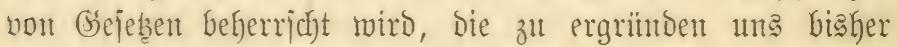
nicht geglïcft ift.

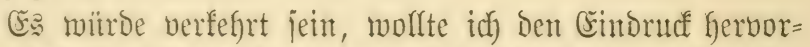

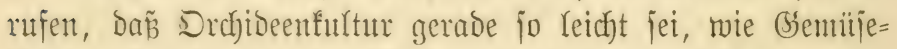

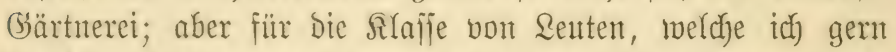
für bieje Sulturen begeiftem mödfte, Gabent bie Saunen, melche Phalaenopsis und einige andere Ârten zeigen, gentau io menig zu bedenten, wie die Ed)recturifie der Tiefe für cintr

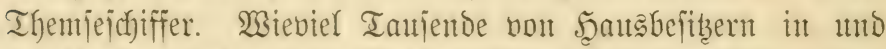
um Innon haben eit "Bischen (Ślas" für Die Suttur von 


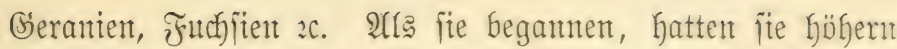
Égrgeiz, aber Mišerfolge haben jie im Laufe Der Beit be= jheibener gemacht, went nicht gar berzmeifeln lafien, umo mun begnïgt jitf) Der arme Mann mit irgend etroas, was

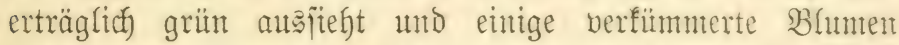

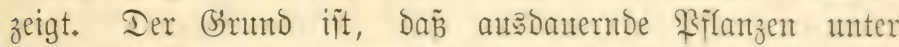
Ğlas jefr jorgfältig befandoelt fein wollen, und da alfe um=

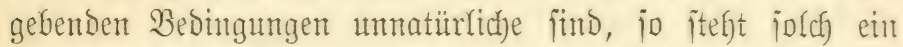
unghïcfficher Siefbaber zwijchen Injeftenplage auf Der cinen Seite und Meflthan auf ber anderen, rwie zmiichen dent Teufel und Der tiefar See. Inter jolchen Ulmïtünden fönnent felbit Yeidyt zu ziefende RfFlanzen ifjre Saunent befommen und ifrr Unbefagen itber bie Befandonng in itberraichender Form zeigen, zumal da ifr Reben von Gejejent beferricht wirb,

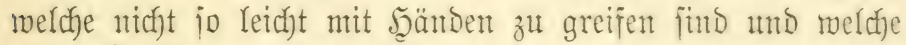
feine Q̈̈nderung zulafijen. Iie Drchibeen jebocf, welche zu fultivieren ein unbemittelter Mann unternefmen fann, ipielen

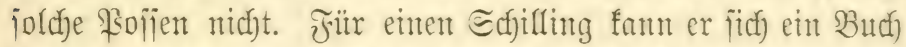

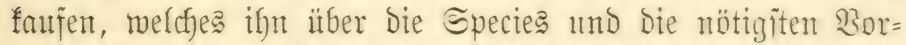
fenntnifife bełehrt; eine weitere 2 atsgabe von 5 E wird ifm für

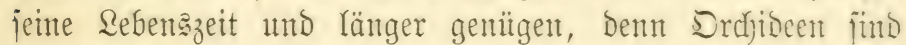

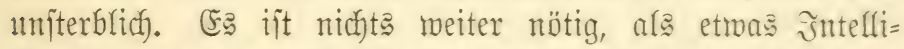
genz. - scidyt einmal şärme, memn er "falte Drchibeen" für jeine Eammlung gemählt fat; es geniingt bei biejen,

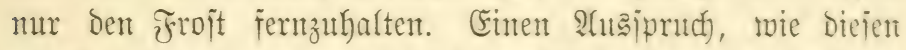

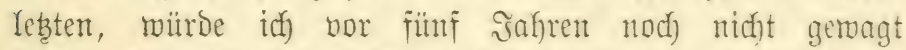
haben, bis idf St. M(tbans bejucht hatte, aber in Dem Salthauje biejes Błhmempalajtes, mit meldhent berr $\widetilde{J}$. Ean= Der ben alten Burgflecfen berjob̈nert hat, waren alfe (be= fitelle gefülllt, bevor bie becizung ganz fertig mar, mo nit fiel bas Thermometer auf Den Gefrierpuntt. Cin meientlicher

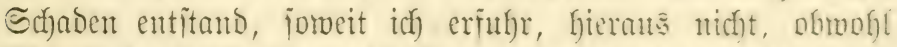

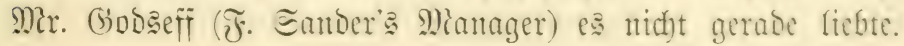




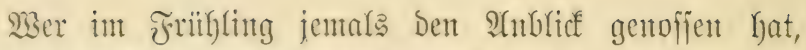
went bieje Ferber von Odontoglossum z̆ blithen beginten,

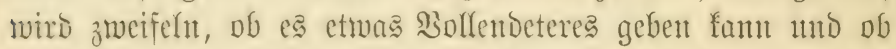

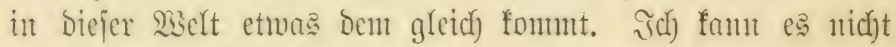

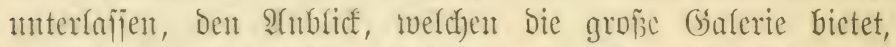
menigitens anmähernd zu bejd)reiben. Mlan ftelle fidd einen 400 Fñ langen แnd 6 Fun breiten forribor vor, in welchem

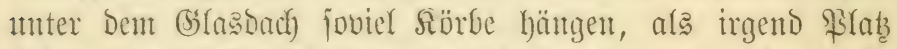
Gaben; man ftelle fid Dan vor, Dañ von jebent bicjer förbe

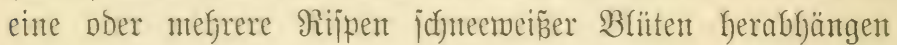
(mie viele es jein mögen, ift nicht eimmal amuähernt zut

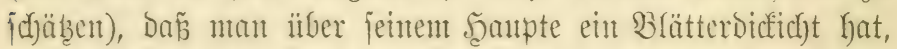

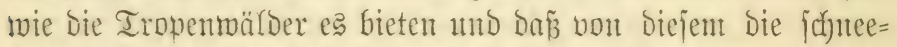

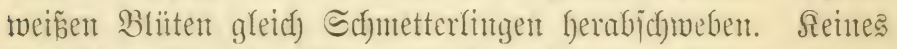

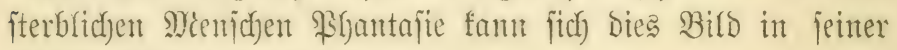
ganzen B́lorie ausmalen.

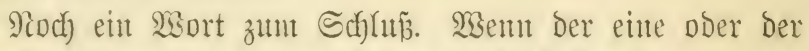

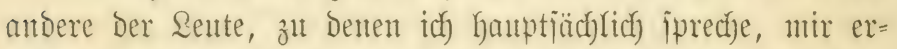

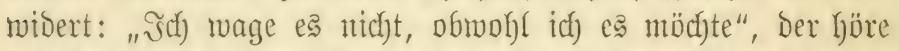
Die Erfafrung eines Entfuftaften, wie Seerr Caftle fie in feiner flemen Edjrift "über Drdjibeen" erzählt. Der betr. Şerr hatte einen Bilašfaften an Fenfter feines Wohnzinmmers,

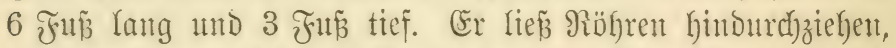

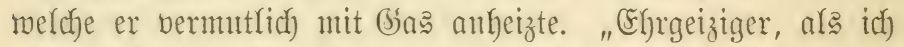
es war," fagt Mar. (Eaftle, "unternahm es Der Beffizer, in

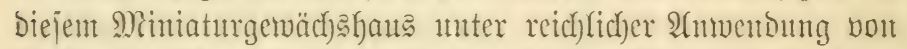

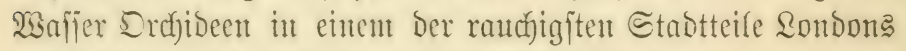

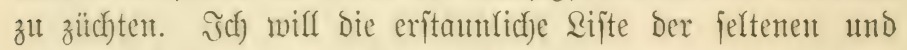

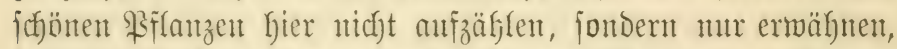

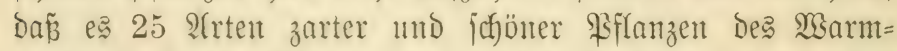

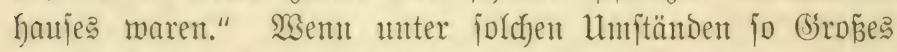
erreidjt werben founte, mit meldjem Redjt fant mant Dam noch) von Sdjwierigfeiten bei ber Drchidoenfultur reben! 


\section{Sedjftes fandtel.}

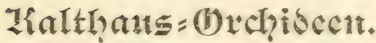

Siejes Thenta mitrde jeden gebildetent Sejer, ja, jedent Bejëzer einer Bifla interejiteren, went man ifn zu ber Üther=

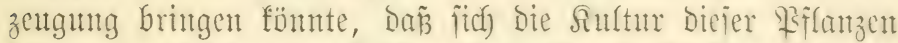
jegr gut mit jeinen praftijad)en Intereî̄en in Einflang bringent

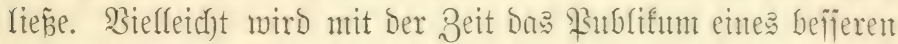

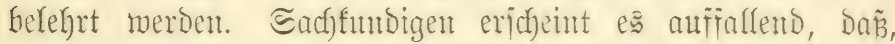
trobsom Erchibeen idjon io befant find und io viel vout

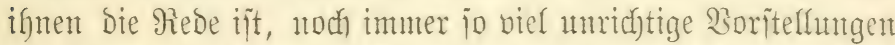

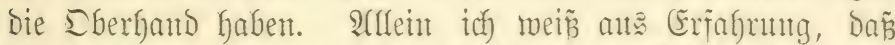

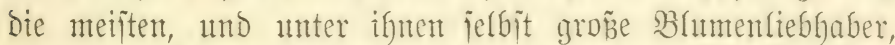

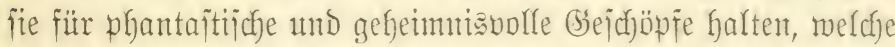

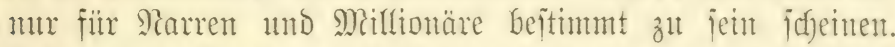

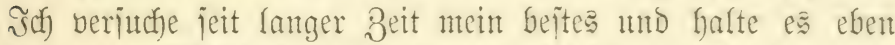

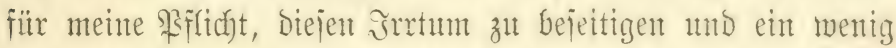
mefre Sidft in bieje Afngelegentyeit zu bringent. Neeiner Meinung nad) Gaben bie Facfmänner icfuld an ioldhen Mis $=$

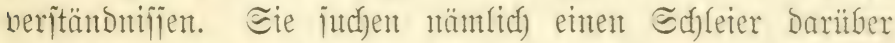

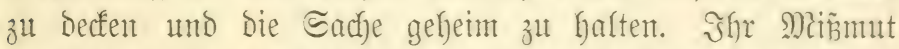

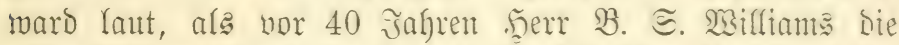

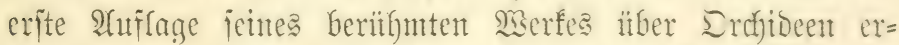

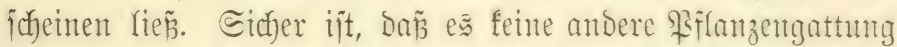

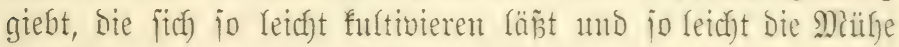
bezahlt madyt, wie Die Salthaus=Drchideen.

Fajt alle Grattungen biejer genaltigen Familie weiten

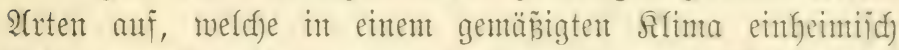

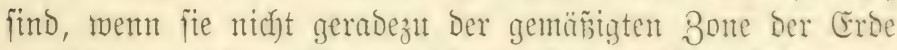

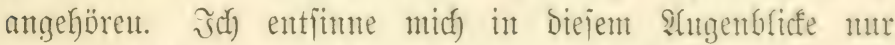

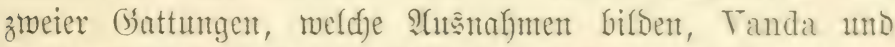

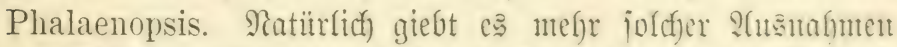
- jelbjt beim ভdjreiben biejer Beilen fielen mir cin Inţento 


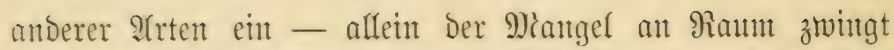

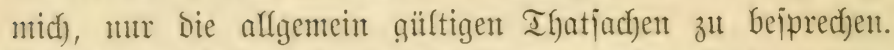
(5s giebt mindeftens hundert Sbattungen und mef)r benn zwei= taujento Srten, bie iiberall gebeigen, jolange fie froftfrei ge= halten werben. AUtlein ein vernienftiger Mlentid) wird ficf (d)on mit bent gropen Siattungen Odontoglossum, Oncidium, Cypripedium uno Lycaste begnitgen, mo an biejen und if)ren unzäffigen Bartetäten faum er viel Freube erfeben. Sie

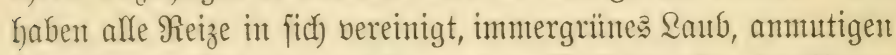

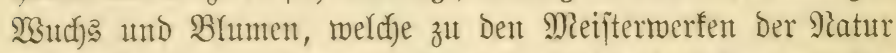
zu rectunen fint. Der arme Mam, welcher biefelben mit Er:=

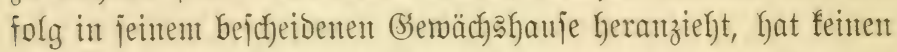

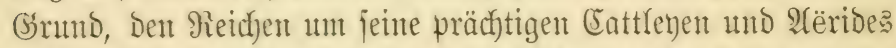

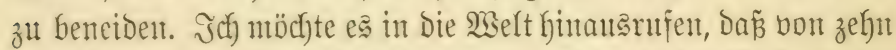

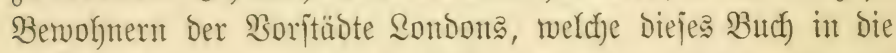

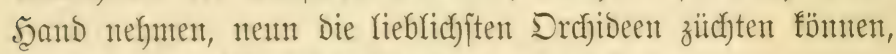
twent fie mur Den Mut hätten, es zu verjud den.

Die EDontogloijen nefymen natïrlidy Den eriten Rang ein, umb es fällt ichwer, alle ifre Sorzüge aufzuzählent.

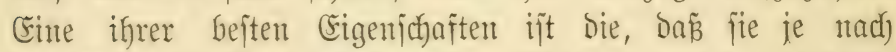

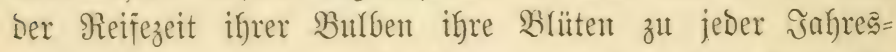
zeit entfalten. Unto gerabe Gierin liegt Der grofe wert für Den Siebfaber. Ein (Särtner, Der ifnen Die richtige \$flege

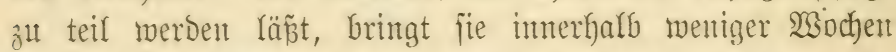
allejant zur Blitte. So gemäfren groß̃e Sammtungen it

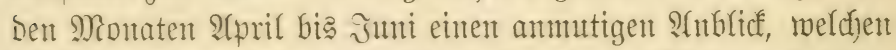

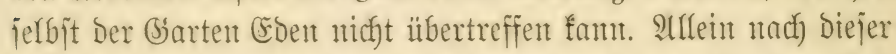

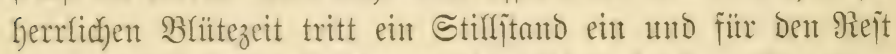

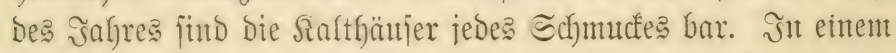

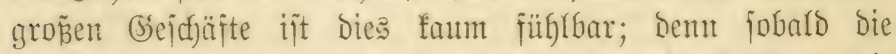
Sonntoglofien abgebliifgt haben, fonment andere ?rten mit Den mannigfaltigiten Farben zur Blitte. Umiontebr aber madjt fich Dieje \$seriobe bem Siebhaber bemerffor, Der mur eine bejchränfte $\mathfrak{A}$ nzagh von Töpfen hat, Denu ex hat feine 


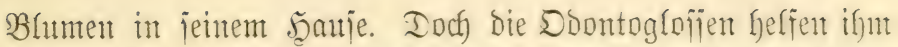

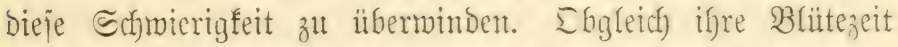
in bas Früfjafyr füllt, jo halten fie nidyt inmer ftreng an

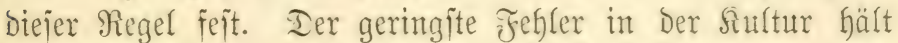

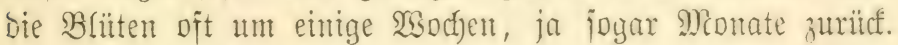

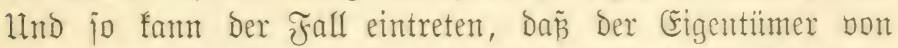

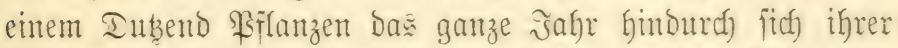
Blitten erfrent, mand)mal gar zwwei ober megrere Biijpen zu

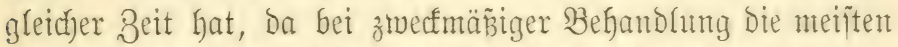

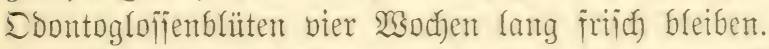

Ein anderer Borzug, Den fie mit einigen 2 rtent der

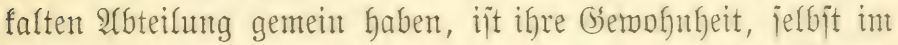

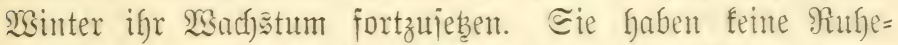
zeit; Das ganze Jahr findurch find fie mit ber Bildoung neuter Bulben, friichen Saubes und junger $\mathfrak{S}$ urzeln beifjäftigt, bis Die Entitefung Des BBhntentriebes jie zrwingt, ifre ganze firaitt

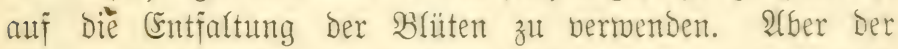
Winter ift bie widitigite Beit, und idf glaube, eint jeder wird

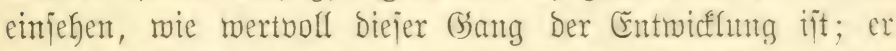
bringt $\mathfrak{A}($ brwech) šlung in bie langen, öben Tage, wo das Sebent anderer Biflanzen itillzuitefen icheint. כijt es io gering an=

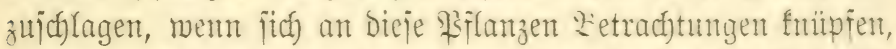
wie man fie an einem Sonntagmorgen bein Iurdfwantern

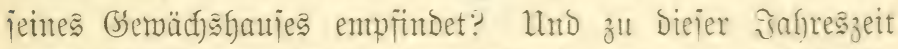

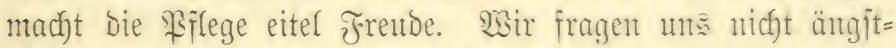

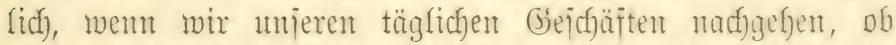

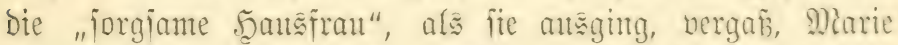

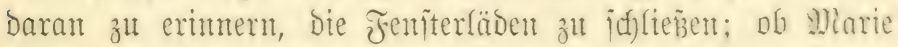
Diejent Befenle, falls er gegeben wurde, gefordfte; ober of bie verbañten gatentluffflappen nidyt mieber anfyejprungen

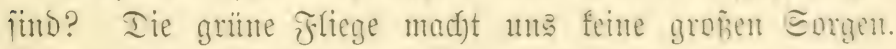

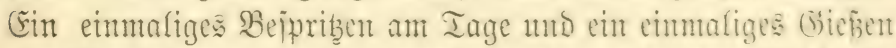

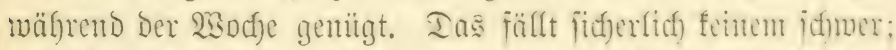

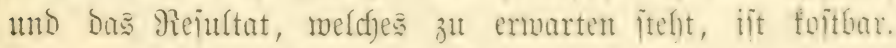


Eefre wentige von bentu, bie Odontoglossum furtivieren,

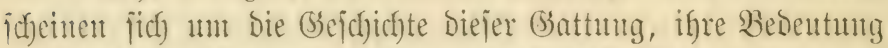
in Santol gefiummert zul habent, und bod) ift es ein cigenes Ding mit ifnen.

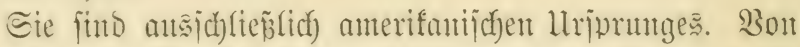
Der Porogrenze Mlexifos bis zur Sütogrenze von \$eru - mit

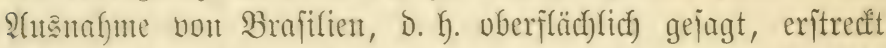

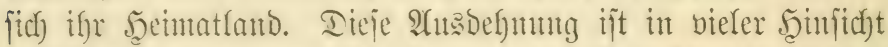
anff̈allend z̆t nemnen. Die Temperatur allein fam nidjt bieje (Sirenze bejtinmen, meil cinerjeits bie an jefre falte Standorte gewöfytten Sophronitis uno einige Der fälteiten (Eattfer)ent ebenfalla von Brafitien fonmen. Sndorerfeits findet man Odontogl. Roezlii, eine felyr heine Şarietät, und Odontogl. vexillariuum, ebenfalls eine warme Species, bis in bieies (Sebiet fintein berbreitet. Sarnm Dieje nidjt einent S(f)ritt

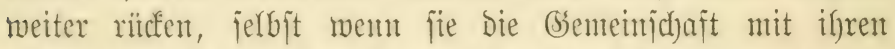
Sdjwejtern ofent in Den Bergen, Den Sophronitis, autd) ver= weigen jollten, if̂t unto bleibt uns ein Rätjel. SAndersimo triffft man fie im überfluñ an.

Die Sammler fömnen fajt mit Sidferbeit beredyuten, wann alfe jene Odontoglossum-Diftrifte, Die jie Durdhjtreift

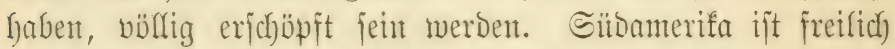

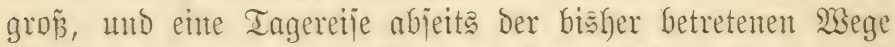

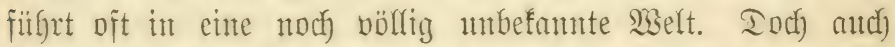
Dem wird bald ein Entoe geiebzt fein. Die Engländer allein Gaben ganze Provinzen iffrer foitbaren Echäbe beraubt, und Die ganze cinilifierte selt ftïrzt fitch hentzutage auf joldje Innternefymungent. Man giebt uns bie traurige Berficheruntg,

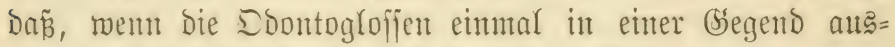

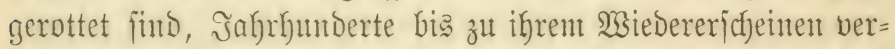
gehen werben. Dic meijten anderen Drdjibeen vermegren fid

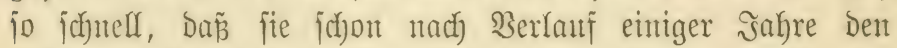

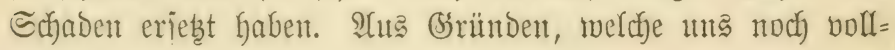


ftänbig unbefannt funt, machen jebod) Die EDontogfoijen cine

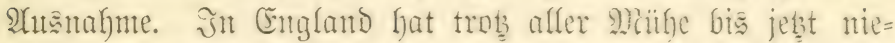
mand mit Griolg Riflanzent ans Eamen berangezogen; nidft einntal ieine beiten Bïdfter, Die Şerrent (Eonfion und Beitd)

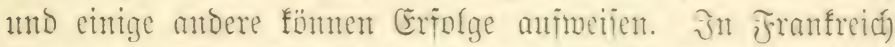

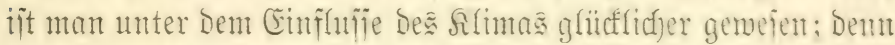

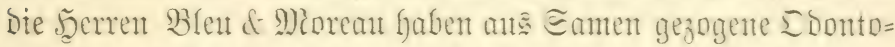

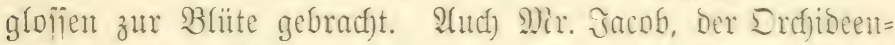

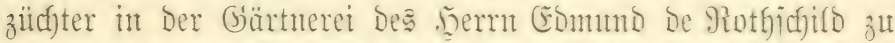
Armainvilliers, hat eine beträd)tliffe Bafl junger sämlinge.

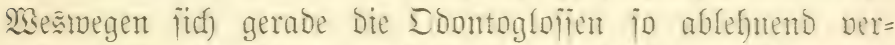

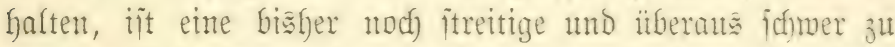
beantwortende Frage; Dem audf an importierten uno wifben Eremplaren jün Eamentapiefn ungemein ielten, eine Errid)einung, meldye jonft mur nod) bei Cypripedium beobadjtet ijt. Die

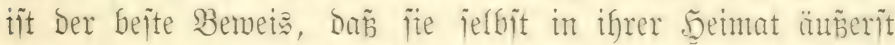

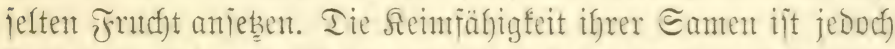
jehr bebentend, uno jie find wiberitandäfähiger mie bie anderen Srcfibeen. Iod) geniigt bies leiser nidft, bie Berfuite rajaf

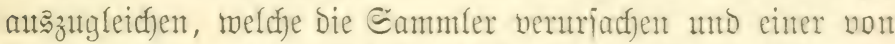

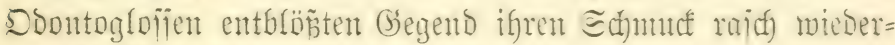
zuverichaffen.

Эd) wifl fier eine Benfadftunty, meld)e bon foern

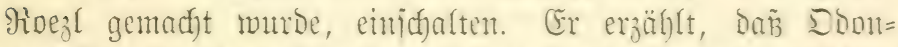

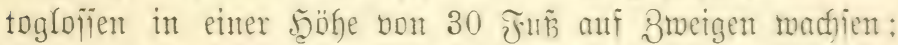

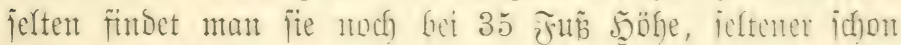
Gei 25; höher ober tiefer itchigen fie nie. Şier fiegt ofme

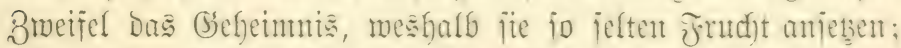
allein, idf) will hier feine meiteren Erffärnngen afgehen, benn je tiefer man in biejes Thema einzubringen indfit, Deito methr Gäufen fidf) bie E(f)wierigfeiten. Bet un in (Enropa idfeint

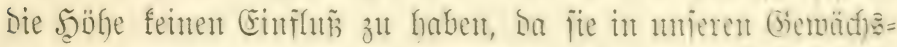
Gäujern mit jedem Etanovorte vorlieb nefmen, uno blühen, jo 
Yange Die J̧auptbebingungen für ihr Gebeifen vorhanbent fint. Stllein in Der Şeimat feint Der Same nur eben in biejer

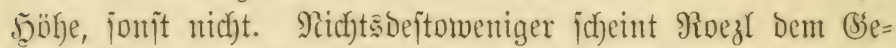
r)einntis auf bie Spur gefonment zu jein, und bie Bufunft wirb vieffectht mefre Ridft in bie Frage bringen.

Der Royal Horticultural Society von Englano gebuifgrt

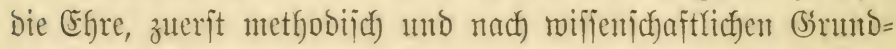

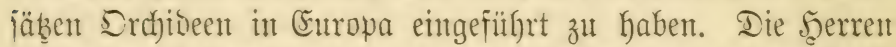
Weir und Fortune waren ifre erjten Reijenden; ein Dritter, Theodor 5̧artweg, entoectte in safre 1842 Odontoglossum Alexandrae, alfein er jantote mur troffene Eremplare Gerüber.

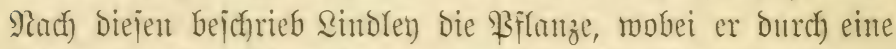

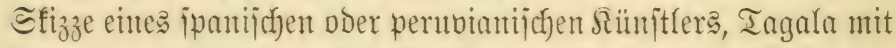
Pamen, unterftitżt murbe. Şierbei geriet er in einige Jrrtïmer, won benen uns jebod) Der wiffeniffaftlidfe weniger angeft. (5r

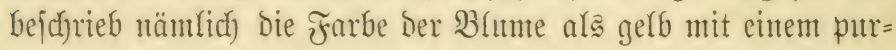
purnen Centrum. So war aud bie Beidumung Tagala's, weld)e noc) heute exiiftiert, foloriert. Das ijt un fo merfwirrdiger,

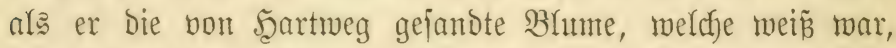
nor fidf) Gatte. Affrein gelbe Odontogl. Alexandrae fint jeitoem gefunden worben, und wir verbanfen ber Royal Hort. Soc. Die Entbectung und (Fintiübrung aud) biejer munber= vollen Prflanze. Tohn SBeir, Der Reijende Der Bsejellid)aft, fante int כahre 1862 lebende Eremplare herïber, uno man

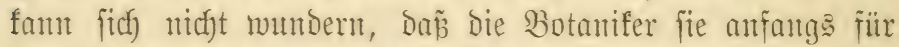
cine Reulfeit Gielten. Alls joldye benante Bateman bie

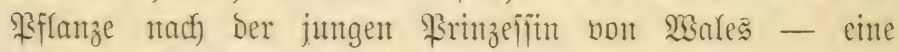

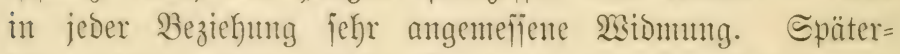
fin nafyment einige reidje 2(mateure, wie Der Jeerzog von

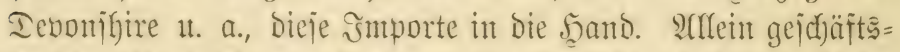

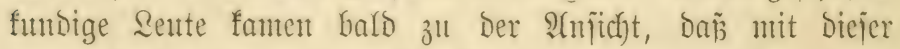
nenen Einfüfrung ein grofser Berdienft berbunden swar, un

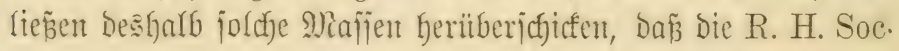




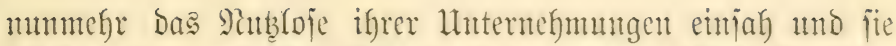
infolgebefien aufgab. Bejonders zu nennen find fier bie 5eerren Rolfiffon in Tooting, Beitd) \& Eofn in Egerjea und Sor \& Co. in Clapton, bie fidh ani bieje Ilnternefmungen

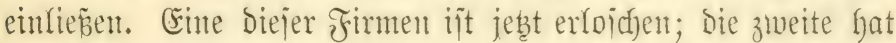
fich bejonbers auf bas Büchten bon Szybriben von Erchibcen gelegt, wäfrento bie leb̧te ifre llnternefmungen nod) fortiebzt.

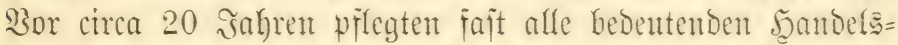
gärtnereien Sondons Sammler ansanjenden; bod) bie meiften Iafien hentzutage Durdi) Rorrefpondenten bon Beit zu Beit Sendungen heritberifjiffen. Ient bie Altagaben für einent

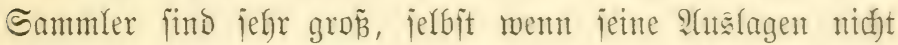
Die fïr ifn beitimmte Sunme überifyreiten - gejchneige Dent,

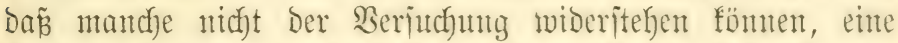
fingierte Redfuntug aufjufteflent. Ferner fint auf ber Yangen Beije grobe Berlufte an \$fflanzen zu befürdyten, was bei jïb= amerifanijajen intportationen bejonders häufitg Der Falf ift.

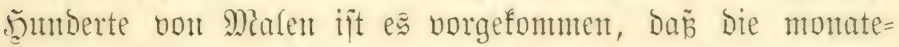
Iangen Mithen, Gejafren und Reiben Der Eanmter uno

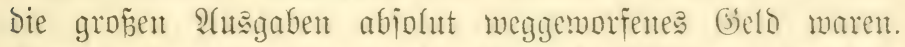
Zwanzig = ober breipigtanjend gejanmelte Siflanzen, bie hod) von ben Bergen ober ats undurdjoringlicfun sisüloen gefolt, jorgfältig in Riften gepacft und verladen twaren, Deren Fracht allein fich anf 300 bis 800 \& belief - idf) habe periönlidy Fälfe mit erfebt, wo biejerbe 500 \& itherichritt - maren bei ifrer Qandung in Englano ofme jede Epur von Reben! Eelbit

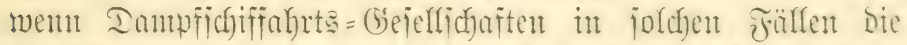

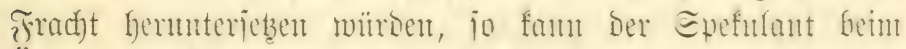

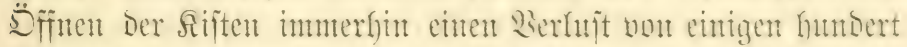

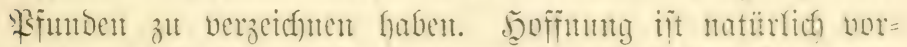

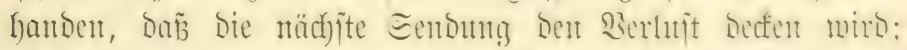

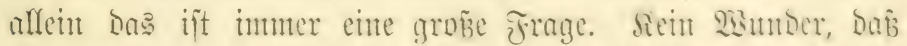

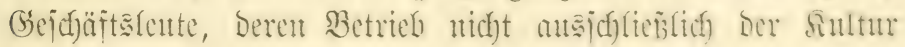




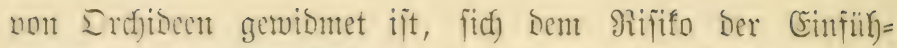

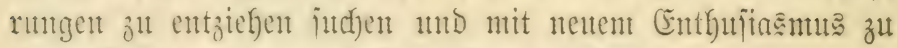

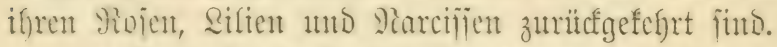

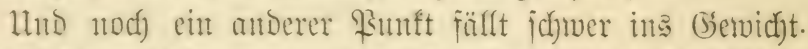

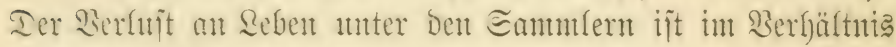
gröber gemejen, als bei irgento cinem anderen Huternefmen.

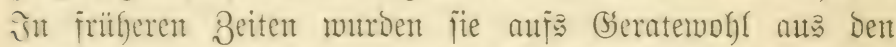

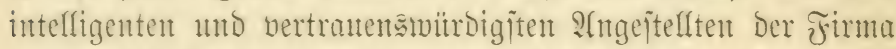
anšgemühtt. Bejonder bie Buberläjïgfeit fiel idjwer ins Gervidgt. So Yenfte irgeno ein anjtändiger junger Mann,

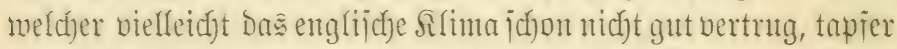
jeine Edf)ritte in bie ungejundejtent Gegenden, wo bie nötigen

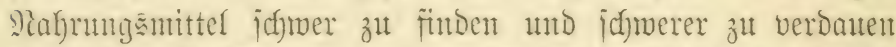
finto. Iag fïr Tag maren jeine đleiber vollitündig Durch)näpêt, uno bas Fiefher in jeinen verjojiebenen Biejtalten fam jo regefmäB̈ig, wie bie Tage auf einander folgen. Mronatelang

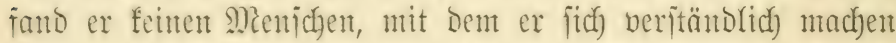

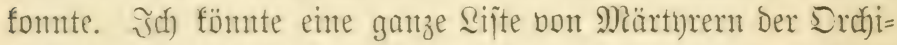

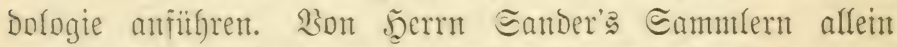
ging Jalfenberg in \$anama, Slaboch in Merrifo, Enores an

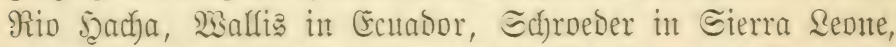
Innold auf Dem Srinoco, Tigance in Brafitien unl B̧rant in

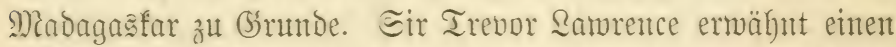
Fall, mo cin eiffriger Forjdjer 14 Tage lang bis an ben Baudd) im Mrorajt herumbatete, un cine jeltene \$iflanze aujzufinton.

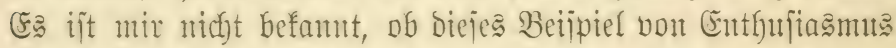
auf Thatiachen herntht; allein wir wijijen, daís es Eelten=

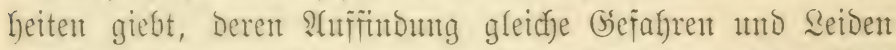

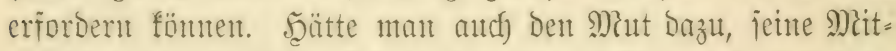
menjajen anzujponten, fidf) int Derartige Seibent und (b́efalgrent

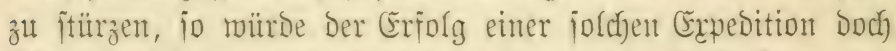

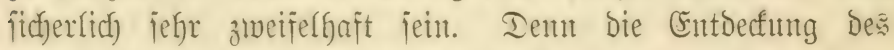
Stmobrtes einer nenten ober wertbollen Erdjibee ift mur Der 


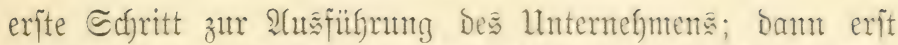
beginnen bie eigentlidjen Edymierigfeiten. Der Piflanze bab= faf̣t zut werdent, jie von iffrem Etandorte jidjer in bewohnte Bsegenden zu bringen, dort zu verpacten und jie Durd) bas von

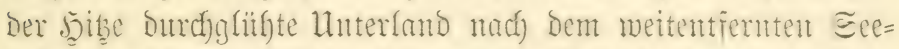

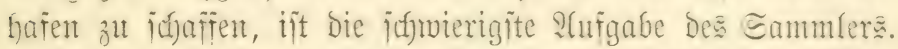

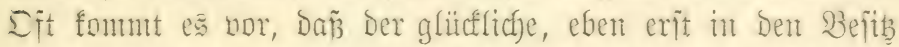
iemes Edjaties gelangte Entbecter erfianft mo nidjt bie nötige Sorgralt ali jeine Fiflanzen verwenten fant, woburdf

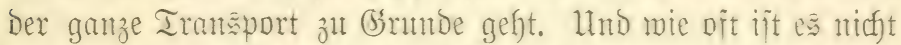

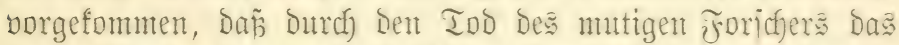

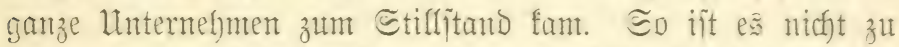

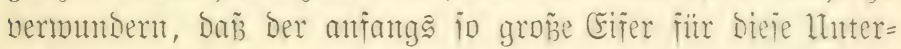
neffmungen mefre und mefre er(ojich und eine Firma nadf Der anderen Davont 2 fbjtand nafm.

Mie idfon oben bemerft, ijt bie Seemat Der SDonto= glojïen Sildomerifa, und zmar trifït mant fie in Dent ges birgigen Gregenden bieies Croteils fajt überall an. W3enngleich) e马 iibereilt jein mitrde, zu jagen, melches bie ichönite Der Ercfibeen iït, jo miro niemano beitreitent, Da Odontoglossum crispum Alexandrae als bie fönigin biejer Gattung zu

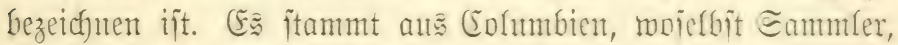
bie jidf) auf bie Euche Dantach begebent, in Bogota ifhr bautpt= quartier aufalidjlagen pflegen. Bon fier aus madjen fie ifre Etreifzüge; entweder begehen fic fid) zefn Tagereiien meit nad) Dem Eithen, um Dort ifgre Eperationen zur Stuffindung guter Barietäten mit breiten Bhumenblättern zu begimnen,

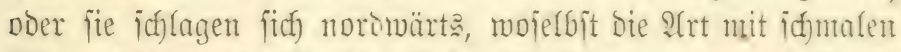

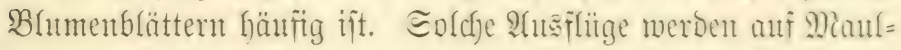
tieren gemacht. Ifyre erifte Eorge beim (Eintreffen anf ifrem

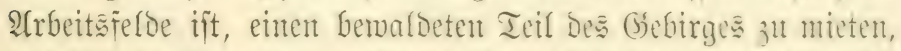

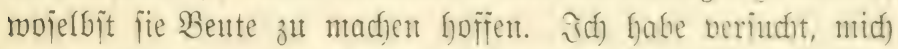

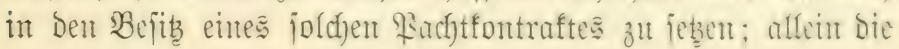


Sache miro gefeim gefralten. Siadjoent Der fiontraft $a b=$ geich)Lifien iīt, nefmen fie 20, 50 oder 100 Eingeforne, je nad) Unitänden, an, weld)e jofort mit bem Fälfen Der Bäume begimnent miijien. Snzmijd)en bleibt Der Sammler zuriicf un errichtet ats 5ुolz eine fintreid)end geräumige Baracfe, tweld)e

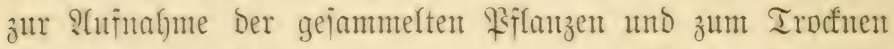

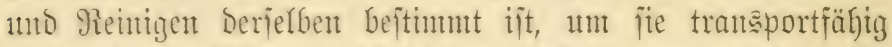

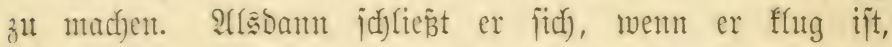
jelbjt jeinen Senten an, unt Durde) jeine (Gegentwart iffren

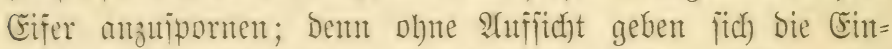

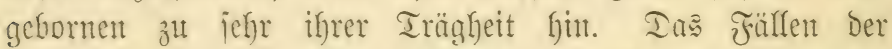

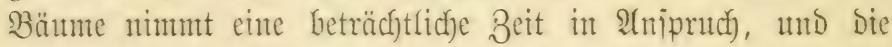
?rrbeit ift oft mit mur jefre mentig Erfolg gefrönt. MRant barf

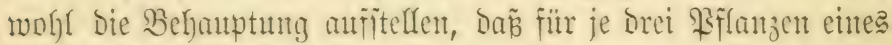
Odontoglossum, meldje jetzit in Eurropa fultiviert merien, ein Baum ber Art jum Epjer fiel. Ins will viel jagen, menn man bebenft, Daj jeit vielen Jahyen Taujente und aber

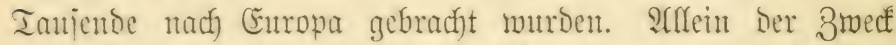

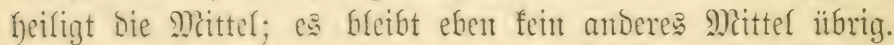

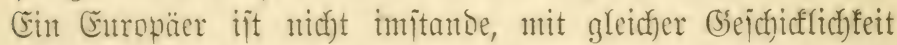

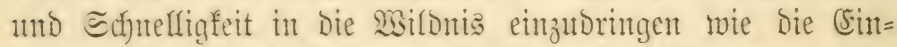
gebornen, unb Dieje jint z̆um Grffettern Der Bäume zu träge,

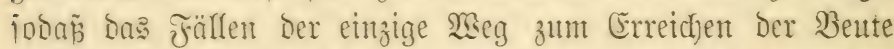

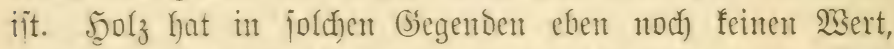

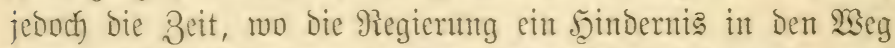
Yegen mirb, riicf́t immer näher. Iurdjidnnittlich) werben auf jebent Baume nicfyt mefyr als fünf Odontoglossum crispum

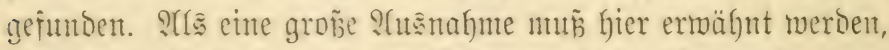

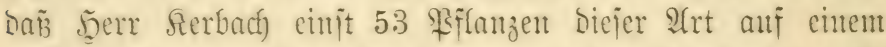
cinzigen Baum uorfanto. Iañ Dagegen andere $\mathfrak{A}$ rtent, wie z. B3. Od. gloriosum, eine minder mertvolle $\mathfrak{Q}(\mathrm{rt}$, in 50 ober 60 Cremt= pharen angetroffen werden, ijt feine Eeltenheit. Iie Cin= 


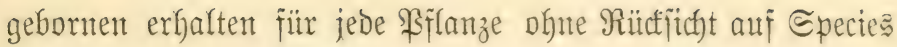
und Dualität 50 Sfennig.

Jit Der fontraft abgefaufen, jo fefret Der Sammler nadf iemem Speidjer zuriaf́, mojelbjt er bie ganze Bente einer

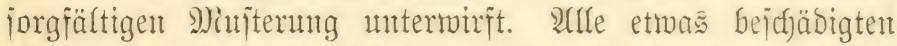
Bffanzen merben ausgelejen, um ein Faulmerben auj ber bevoritegenden langen Reije zu verhüten. Nadjoem alle ge= reinigt und getrodfuet find, werben jie mit fupferbraft an Stäbe bejejtigt, weldje in Silïten feîtgentagelt merben. Die

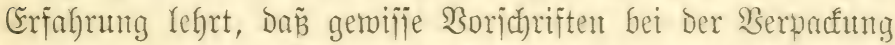
ftrentg zu beadten jind. Tie Etöcte baben gemölynlid) einen

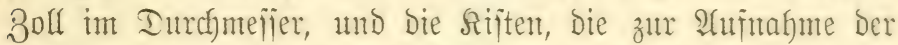

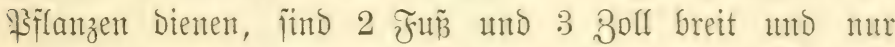

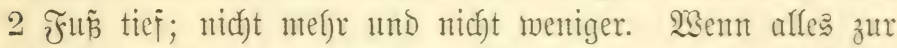
2(breije geriijtet iijt, werben bie Maultiere mit Den Siijten be= pacft, und Der Butg jebzt fich) nad) Bogota in Bemegung, weldhes bielleicht nad) einem Marjoje bon zehn Iagen er= reidjt wiro. Şier wirb nodymals und zum lebeten Male eine iorgfältige llnterjudfung Der Rif̈ten vorgenonmen unD

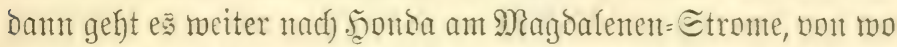

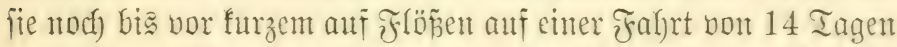

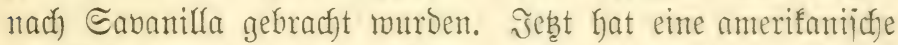

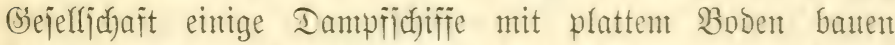
lafien, welche jeb̧t biejelbe Strecfe in 7 Iagen zutrüfflegen, und jo Die Befafren der Reije un bie Sä̈lfte vermintorn. Trob alledem fund biejelfen idjrecffid) genutg. Said)t ber ge= ringite Suftzug fühlt bie idjwille 2 tmojphäre in Der Beit,

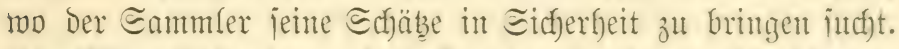
Die Rijten jind oben auf Deck untergefrad)t, Da bie idumere,

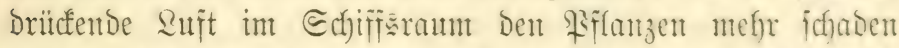
mürbe, als obent bie bremtente Sonne. Seinmandoeffen jïn

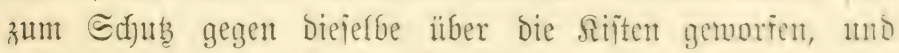
Darïber ijt eine Iecfe von Palmblättern gebreitet, bie fort $=$ 


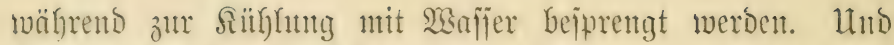

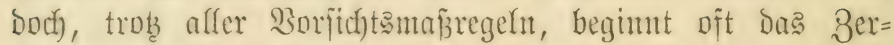
ftörungatwerf in Den Siften.

Jebod) nod) cine andere Sorge bebriact Dent Eanmuler. Bitelfeidf)t ift bas Dampfidjiff, anf tweld)em er jeine Schätze zul verlaben hofft, bei jeiner SAffunft in Sabanilla bereits ab= gefafyren, itt welchem Falle er tagelang anf Den nächjten

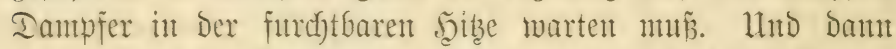

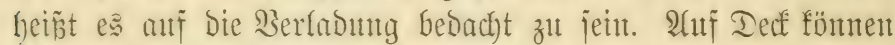
bie Bflanzen von Seewaffer bejuäbigt werben, int Sdjiffes= raum ijt bie Quft zut Dritcend und in Der MRitte Des Sdjiffes

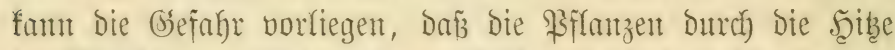

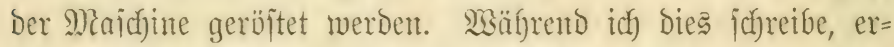

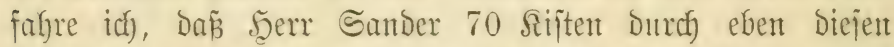
ütbelitano, wie man vermutet, verloren hat. So gänzlich)

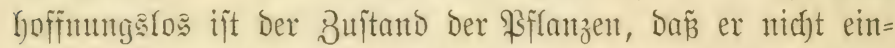
ntal eine Unteriutduntg Derjelben in Ânbetracht Der fojten mnternefymen will. Sie riegen in Southantpton, wojelbjt fie für jebermant offen fteben, und jeder ber Beteifigten wird Demjenigen Ianf wiffen, weldjer fie fortholt. Der Rejer fanm

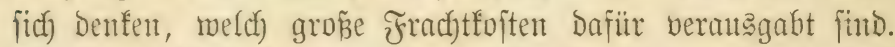
Die Royal Mail Company nimmt fïr Fradyt von Mlanzanifla

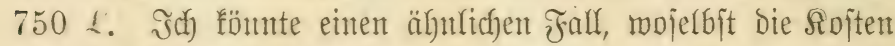
noch höher waren, bon einer Sentung mit Phalaenopsis

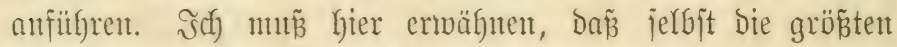

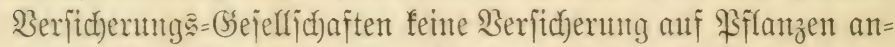
nefmen; Der Figentitmer hat Die ganze Bjefahr ferbjt zut tragen.

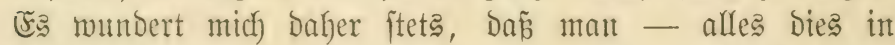
Betrad)t gezogen - Die \$fflanzen jo billig verfaufen fant. Biele hoffent natitrlich auf ein meiteres Fallent ber Preije und bas wirb ficferlicf) bei einigen 2 (rten eintreten. Affrein bie Bsejchäf̆łlente ermarten Das Ssegenteil.

Od. Harryanum erimnert midy immer - jo bizarr ber S3ergleidf jein mag - an cin Bjemitter. Der Sontrajt ifrer 


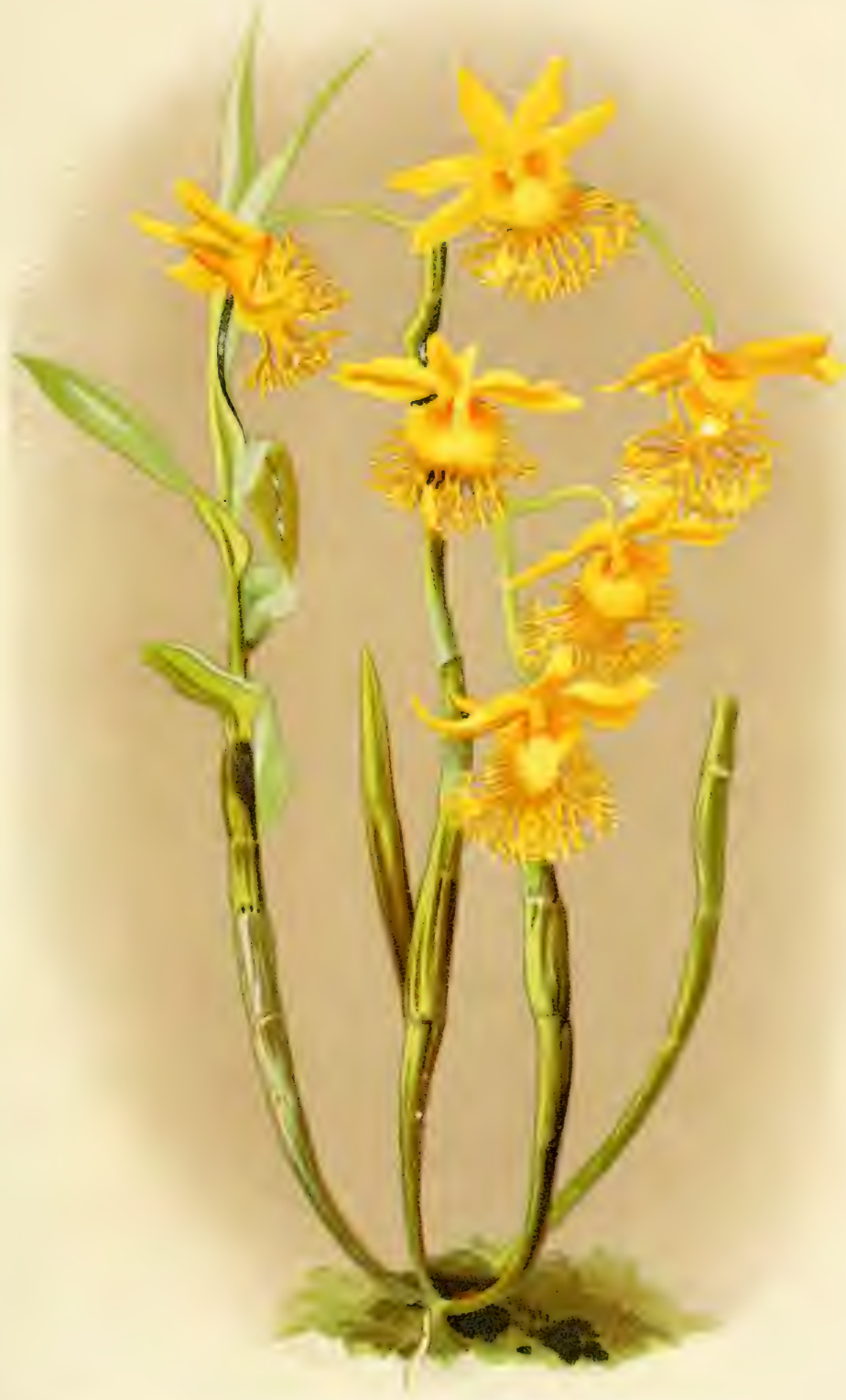



bicfen, Graumen Flecten mit Dem fimmelblauten Sdylumbe und Der breiten ichneeigen Lippe machen ftets auf midy biejen (Fin= brucf. Der Bergleich flingt vermunftgmiorig, alfein ,on est

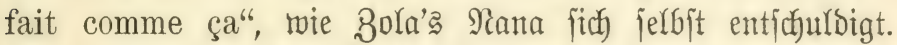
Dieje auffallende \$fflanze "Harryanum" zu nennen, ift höchjt feltjam. Dả Publifum intereffitert fitc) mentig für bie $\mathfrak{u n}=$ ¡tände, welche bie Benenmung einer Błflanze veranlajijen. Afllein, wenu es irgent eine giebt, die einen bejonderen Namen be= anjprudft, rejp. verbient hätte, fo ift es meiner Mieinung nach

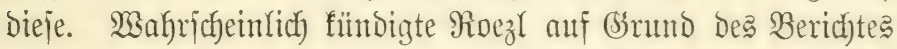
irgent eines jeiner Jndianer - was jedod) fpäter jeinem (be= bäd)tnis entfiel — bie Enttocḱng eines neuen Odontoglossum in Derjerben (biegent an, wo nad) jeinem Tode O. Harryanum gefunden wurbe.

Dieje Prophezeiung ift jpäter als ein Beijpiel jentes Initinfte马 Gingeftellt worben, welcher biejen berilfunten @anmler zu Yeiten pflegte. Die erften $\mathfrak{B f l a n z e n ~ w u r b e n ~ i m ~ J a h r e ~} 1885$ in einer flemen Sifte von Senor Pantod)a in Columbien an

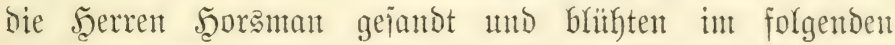

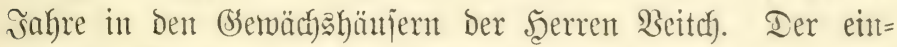
fältigite Menjidy, weldyer diejes 23 under, Das bon einem nod) unbefannten Bsebiete fam, fieft, fann fich Die AGufregung voritellent, welche bie Entfaltung biejer Blumten Gerborrief.

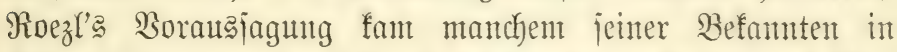
Srinnenung; aber Seerr Sander jeb̧te ein nod) gröñeres Ber= trauten auf jeines alten Freundes Erfarfinum. Er fanote auf Der Stelle einen Sanmler nach Dem Drte, Dent Roezl angegeben, aber ielbit niemale bejudft hatte, uno biejer war io gliteffidy, Dort jenen S(f)ats in Mafien anzutreffen.

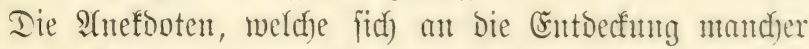
Drchibeen fnitpfen, werben vieffeicht eine Tage ge fammelt und veröffentlicht werben, unt id glaube feit,

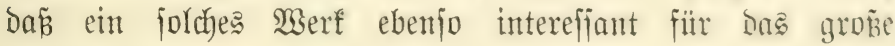


Pabrifun jein mird, als für bie eigentficfen fenter biejer Bflanzen.

Bis jeşt habe ich mur vou Columbijchen Sonontoglofien

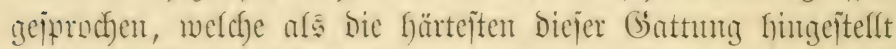
merDen. In serjelben Iemperatur wie fie, wadhien aud

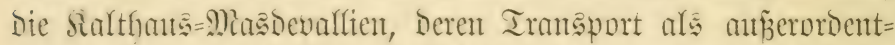

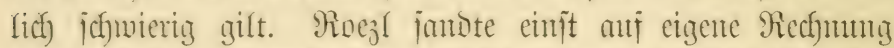

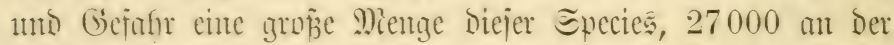
Bahl, Die zu jenter Zeit cin fleines Berntögen repräjentierten,

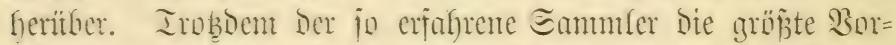
ficf)t beim Berpacfen hatte walten Gajjen, waren beim S̈ffinen

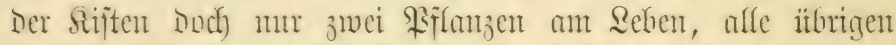

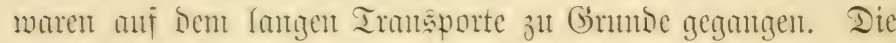

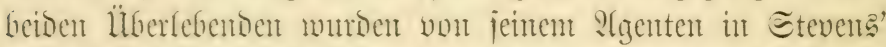

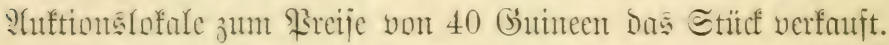

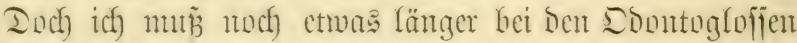

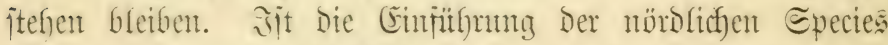

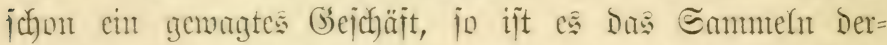

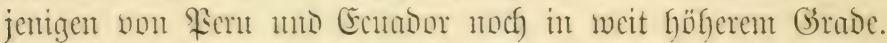

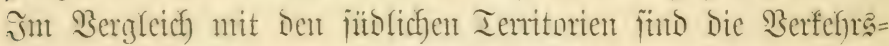
mege in Columbien, woiclbit Die Bevölfenmig civilifierter ijt,

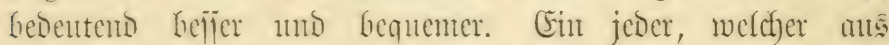
Diejen ïislicfen Provinzen cine Eenoung von Odontoglossum naevium in guter Beichaffentheit auf Den Minft bringen fömute, mitroe mit einem Ecflage cin reicfer Mian jein. Ier Etmonort Diejes Odontoglossum iĩt wohl hefannt, mo

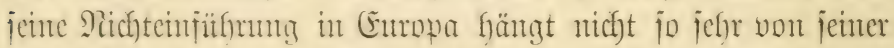

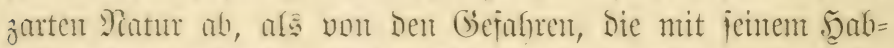
Gaftwernen verbunden finto. Sein nod) fo fuifher Sanmer

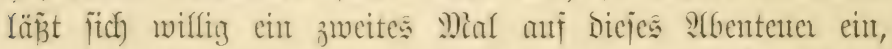

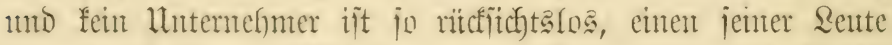

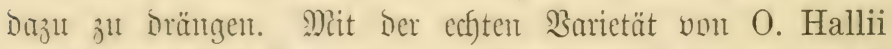

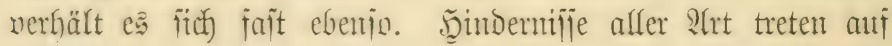




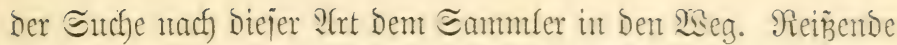
Ströme hat er zu paifieren mo iteile ?abänge zu erflettern, von Denen ein Fehltritt ifm Den fidferen Iod fringen mitrde. UnD Dann Geint, es Denjelben 2 seg mit ben belabenen Maul=

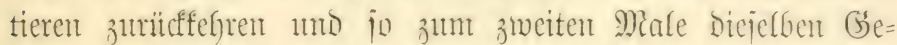
fafren zu itferminton. Die Rorama=Berge gelten hentjutage

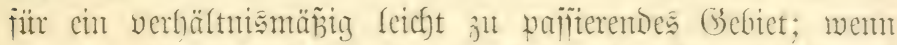

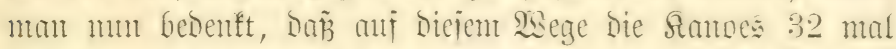
cutfaben and weder befaben werien ntifijen, io fam man fitch (eicht cinte Buritellung Davon machen, wa cin "ichmieriger

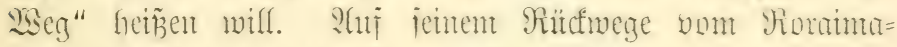

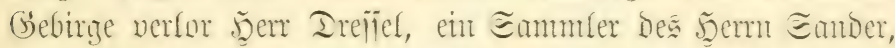

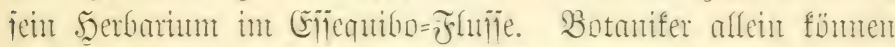
Das Iroitloic eines jufchen Berfultes beurteilen, mo man

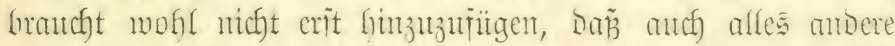
in Den Fluten veriant.

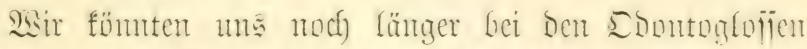

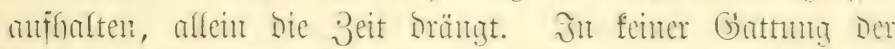

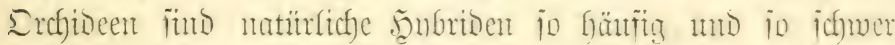

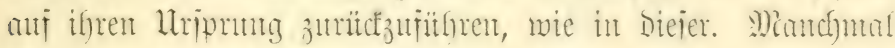

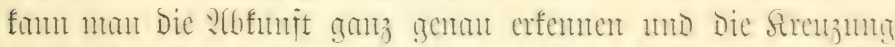

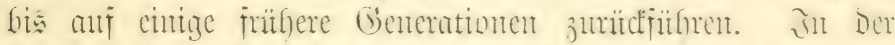

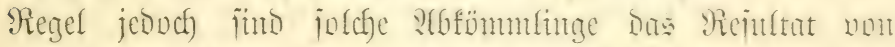

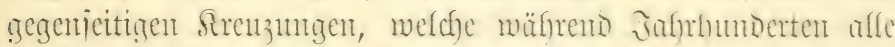
mögfichen ïbertaichenden Berbindungen emgegangen f̈nts.

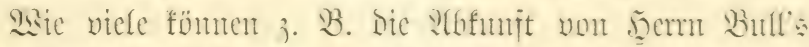
Odontoglossum delectabile verfolgen - cinter D̃ubioe mit

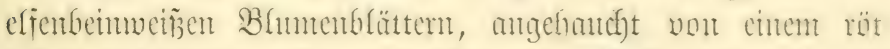

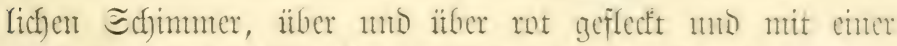

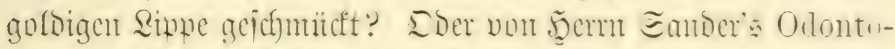
glossum Alberti Edwardi, Defïen më̈d)tige Pictalen wn éntent

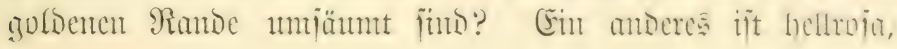




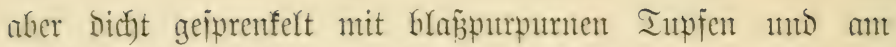
Rambe mit gleichfarbigen Flectent verjehen, bic po bicht zut=

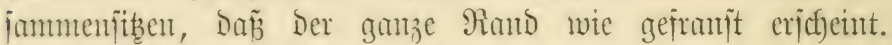
Solche Rlcindoien finden fich gan n merwartet unter Den Sinfithnnngen. Sicin Beichen verrät ifyre Serrlichfeit, als bis

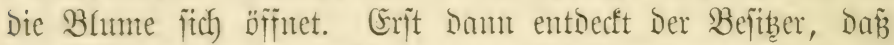

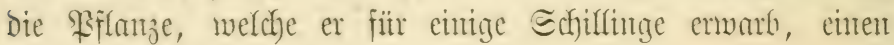
Bert vou mebreren Bunteen hat.

Lycaste ijt eme andere Gattung, bie ebenfalls in

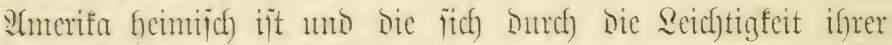

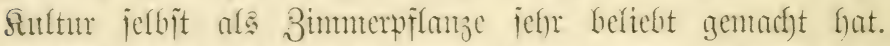

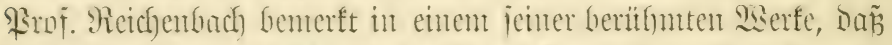
viele Rente, Denen Erchibeen jonjt mbefmut f̈ns, bieje Epecies

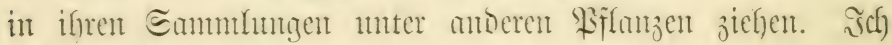
rebe von Diejer Gattung ofne jenes Boruteir; Dent in meinen

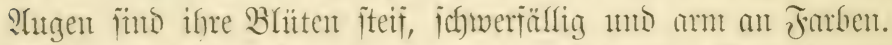

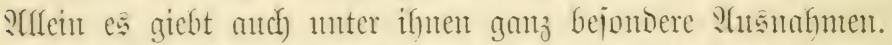
Bor allen Dingen jottet Lycaste Skinneri alba, bie idntec=

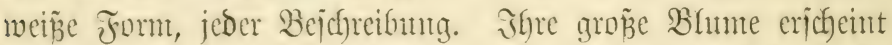
wie ans Marmor genteizelt, und bies miforingliche rohe

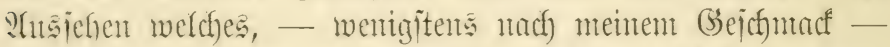
Der gefürbten Stammart eigen ifit, ifit hier zut fajt jumgfräı=

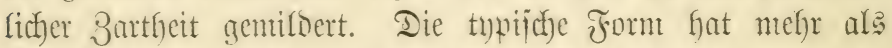
100 Farbenvarietöten anjumeijen. Alle Schattienungen von

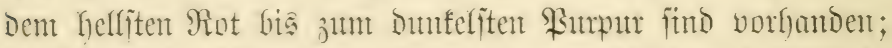
io ijt oft bie Sipwe fo tief pumpumot gefärobt und babei io

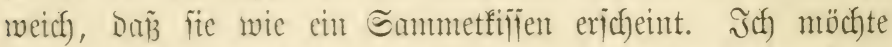

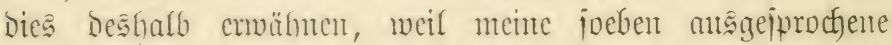
Rlbneignng gegen dieje Riflanze itch eigentlich mut anf bie

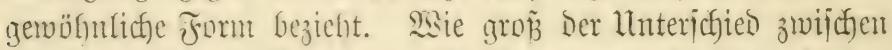
Den verichiedenen Barictüten iit, fann man leicht nach Den

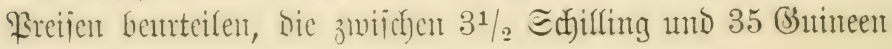
id̆ronuten. 


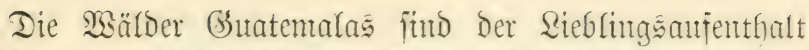
Diejer Sucaiten, und ich Gabe nie gehört, Dañ bas Eanmeln Der= jelben mit jo vielen 2tbentenern verfnitpit ift, wie bei Den SDon= toglofijen. Im grozen Banzen find fic afle ofme bejondere Echwierigfeiten zu erfalten, mo iffr leidfter Transport uno

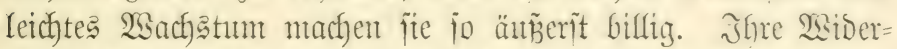

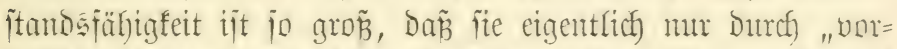

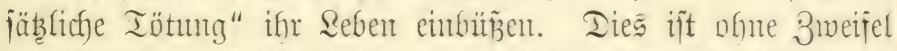

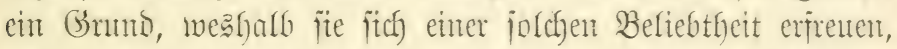
allein es giebt nod) mef)r an ifnen 3 ut loben. Mitten im

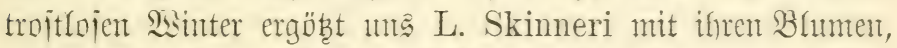
und alfe andoeren S(rten entfalten mefre ober weniger iffere

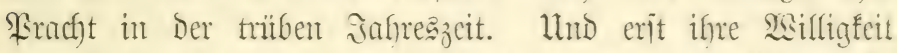

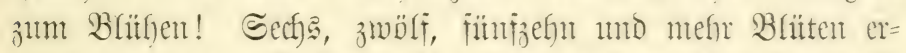
zentgt ente einzige Buthe, welche fint eine lange Beit ifre volle

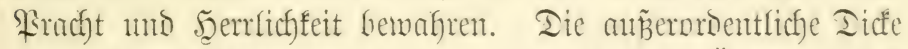
ifrer Bhumenblätter macht ite gegen plïbliche S̈hnernng Der

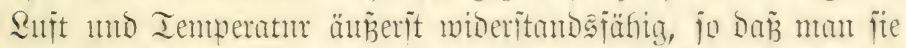

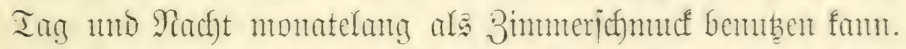

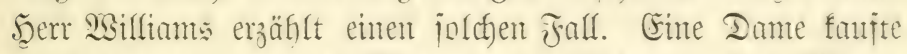
L. Skinneri in volfer Blitte am 2. Fefmar, Die Riflanze

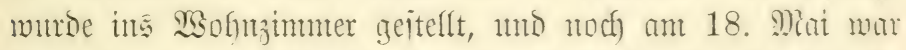

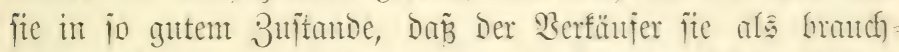

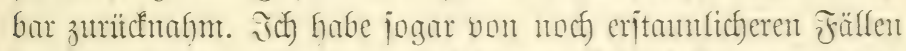
berichten förent. Eine etwas jeltentere : Irt ijt L. aromatica,

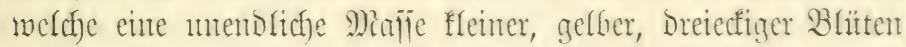
mit herrfichem Infte zum Borichein bringt. Jdf) feme feinte

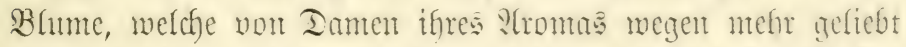

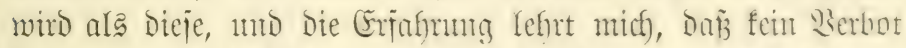

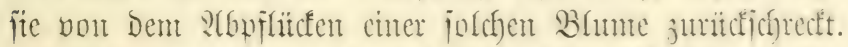

L. cruenta verdient fait benfellon Ruf, mo fitr bic

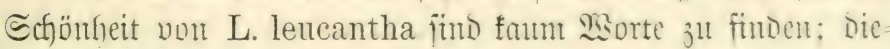
fethe Darf jefre woht der L. Skimneri alba an Die Exte 


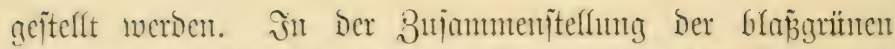

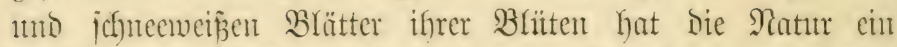

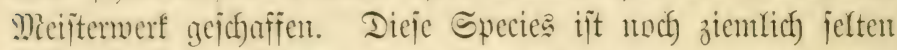

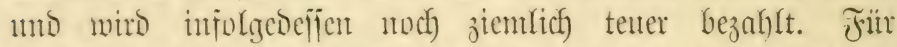
Semer und Bürchter haben bie Sucajten noch einen anderen

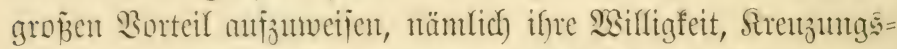

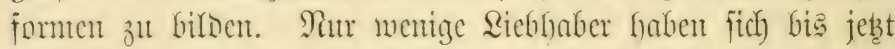
anf biejes Grperiment cingelafjen, unb bie Gejhäftêlente haben mentiger 3eit nober Neignnty Dafitt. Sie nefmen lieber Rrent= żungen vor, Seren Erfolg mit Sicherfeit zu berechnen iłt. Dafer fins erjt wenige Berjuche Damit gemtacht worden unt,

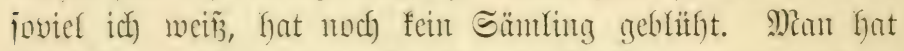
jedoch Davon ichon viele nicht allein Direfte Serrenzungen, jontoen anch) joldbe mit äfurfichen Gattungen, wie Zygopetalum. Anguloa und Maxillaria erzielt.

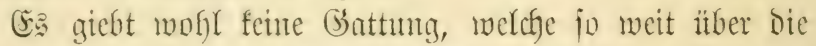
Groe verfreitet iit, wie Cypripedium, and Bantoffelblume gentant, wenigitens miñte ich feine anbere jut nemen. Bon

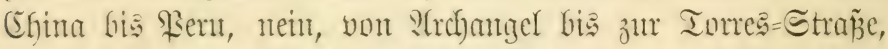

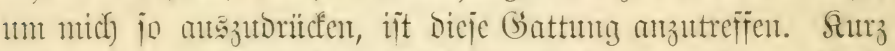

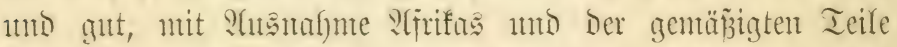

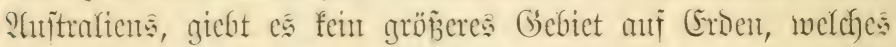
nicht (Eupripesien erzentgt. Lno as giebt Botanifer, melche

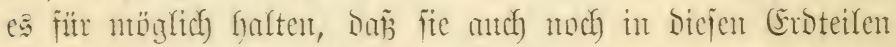

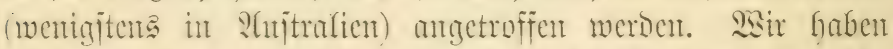

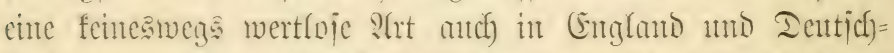

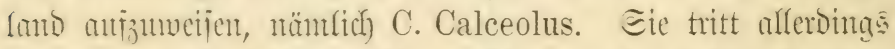

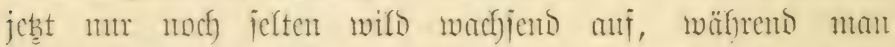

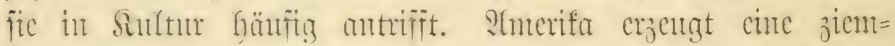

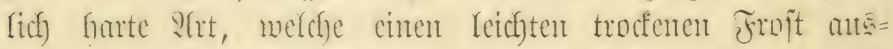
hält. Hujeren majjen ssintern bagegen ijt fie nicht gemadjent.

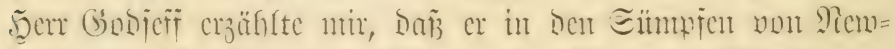
Jerien, weldbe fïl einige Mlonate int 2sinter volfitänoig ju= 
frieren, Cypripedium spectabile in größ̄ter Ïppigfeit fahe machjen jefen. Şier in England mitrde biejelbe : Yrt jelbit an ge= ichirben Etellen unfehlbar zu Grumbe gefien. ${ }^{2}$ ) Jene herr=

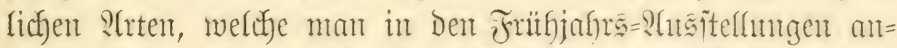
trifft, find alle in rufhentem Buftande eingejitfurt mordent. Bon Den $\mathfrak{B} e r e i n i g t e n$ Etaaten $\mathfrak{N o r d}=\mathfrak{2}(\mathrm{m} e r i f a s$ fommen Cypripedium candidum, C. parviflorum, C. pubescens uto

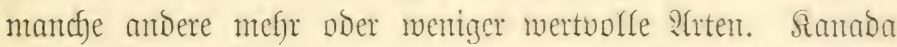
1nto Sibirien erzengen C. guttatum, C. macranthum uno

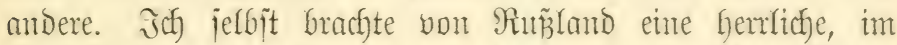
Dूandel unbefannte Epecies mit einer grożen goloigen Blume nach Englani; allein alle funlten nach und nach. Ieshalb find jene ichönen, Garten Barietäten, Deren Sulttur io leicht

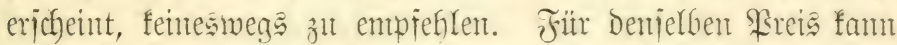
man andere erwerben, Die von heizeren Begenden fommen und ntefr an unjere Sulturbedingungen gemöhnt find.

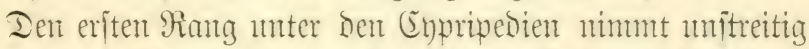

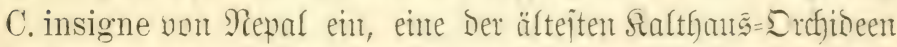

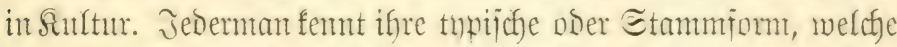

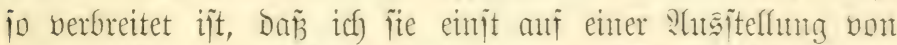
Bimmerpflanzen in Meitminjter vorfand. Mit Recht barf man

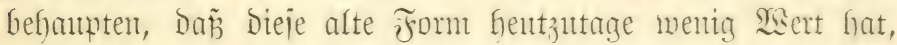

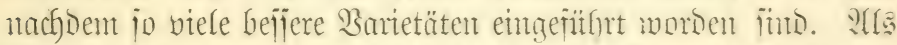
Beweis Dajür mag angejüfrt werden, baß̉ vor nicht fanger 3eit cine fleine Siflunze yon C. insigne, matitrlich eine

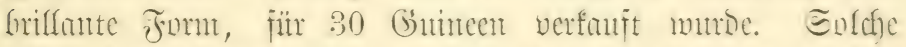
Fäffe fonment vour 3eit zit 3eit vor unto gehören jul Den

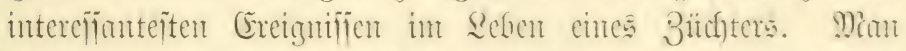

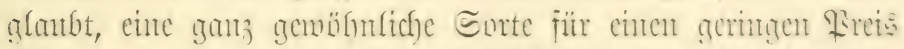

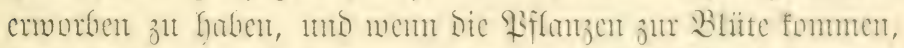

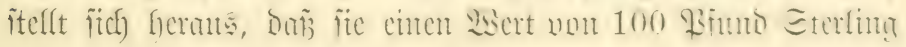

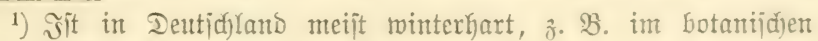
Barten zu BerYin. 
voer mefyr repräjentieren. Seder erfahrene Sanmter faum viele jolcher Beijpiele anjitfyren, uno ich mill hier Den Rejem die (Bejejchichte von C. Spicerianum als jeflagenden Betweis vor 2litgent fitfyren.

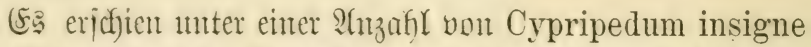
in Der Sammlung ber Fran Epicer in Iwicfenthant. Erftant utber bie ungewöfntliche nente (Erid)einung unter Den altgewofnten

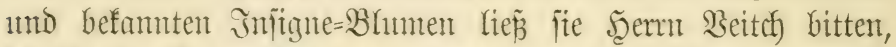
biejelbe in stugenjicfein zu nefmen, und mit Frente erwarb

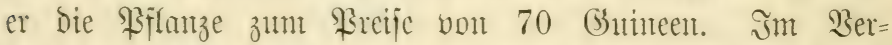

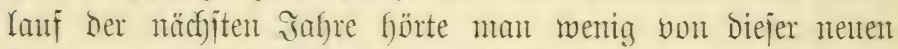

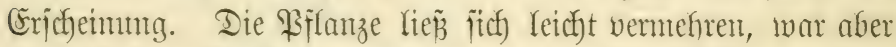
ifres Gohen Fireijes wegen mur etwas fitr Mitlionäre ober

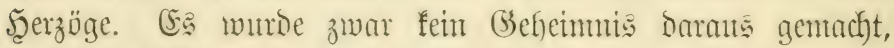

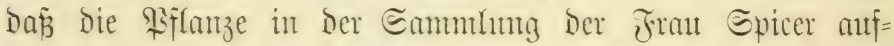
getatcht war; aber eine frembe Dame um Shifflünnt itber Den Hriprung biejer Reufeit zut bitten, galt in Den Rugen Der Gejchäftăleute als eine zul gewagte Berlebzung Des gejellichaft=

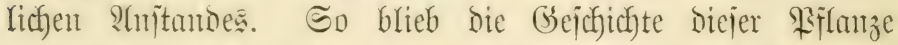
noch) im Dumfeln. Die Szerren Epicer waren mo fund nod) hente weitfin hefannte $\mathfrak{B}$ apierfabrifanten; aber zwijhen der Fabrifation des अapieres mo indijchen srchideen beitehen boch) weber innere noch äuñere Bejiefungen. Mian erfufr

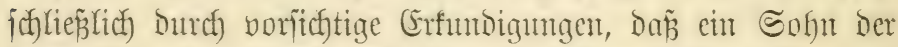
Frant Spicer eine Ihee=\$lantage in Iffjam hätte. Das ge=

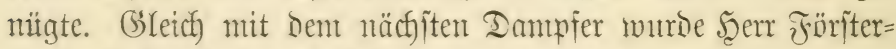
mann nach ?fifant anf bie Enthe antggejand, wojerbjt er nach

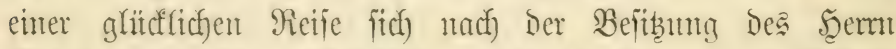
Spicer aufmachte. Dajelbjt angelangt, bat er um ?trbeit,

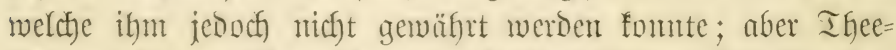

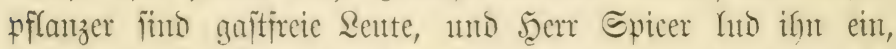
für 1 ober 2 Inge bei ifm zlt bleiben. (Dod) zeigte jich) im Saufe Der Unterfaltuntg feine Belegentheit, Das Gejpräch 


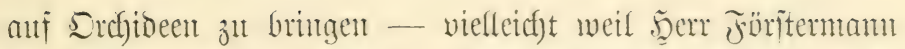
bie Sache zut fein anfing. Simes Inges jedoch hlu ber Sheranfifeher Der Iheefarm ifn zur Teifnatme an einer Jago eint, welcher Crinlabumig er mit Frenton folgte. (B̧elegentlich) bemerfte Diejer: „Min werben an ber Etelle vorbeifonmen, wo

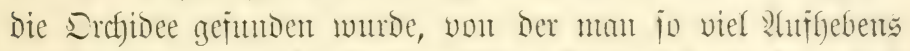
in Englanis gentacht hat". Mian fum fïch Denten, da

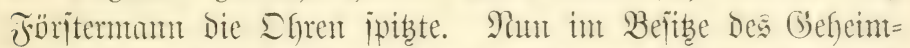
mifijes, verabicfiencte er fidf) vour jeinem (Bajtgeber umb machte fidf) an bie Strbeit, weldye ifm megen ber un= gefenten Menge ber Biflurzen wentig Schmierigfeiten bereitete.

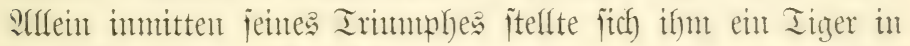

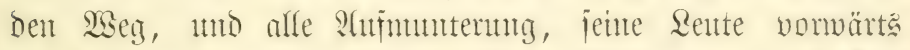
zat bringen, war vergebens. Şerr Föritermum war fein

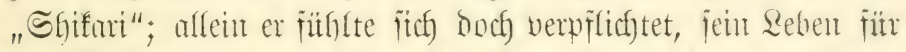

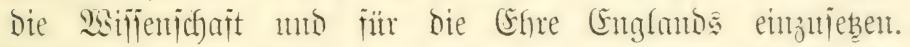

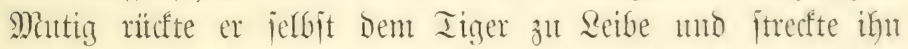

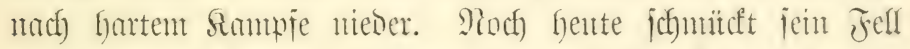

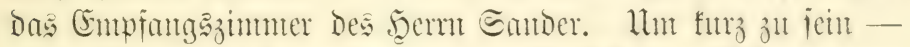

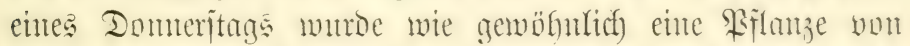
Cypripedium Spicerianum in Eteven: Stuftion fofofule füt

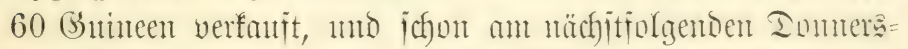

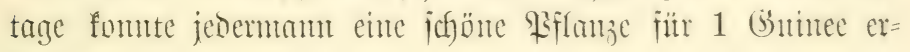
werben.

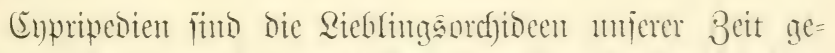

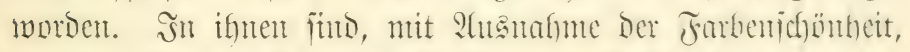

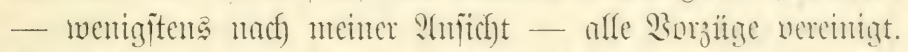
Denur fein cirbiges hat cine flare, beitinunte Farbe, jelbit Das herrfiche Cypripedium nireum ift nicht rein meij.

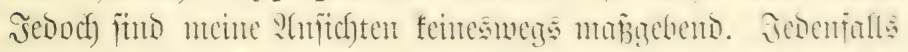

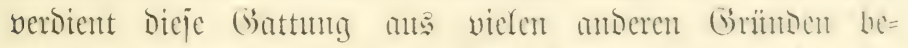

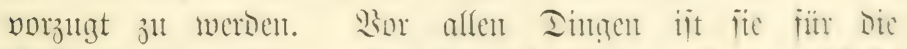

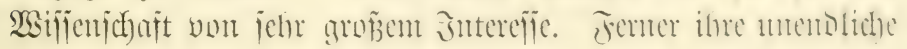




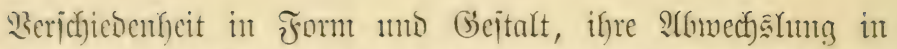
Den Farbentönen, ifrere leichte Sultur umo ifye Willigffeit zur Strenzbefrutchtuln, ${ }^{1}$ ) von ber man anf einten ficheren (Erfolg

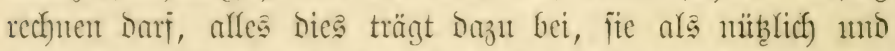

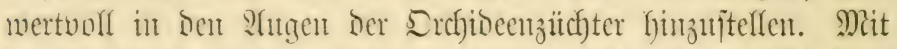
Der Sultur Derjenigen Epecies, Die aus jefn heip̃en \&änben fommen, fund natitrlich હ(f)wierigfeiten verfunton; bie Salthaus= arten jeboch) gebeiffen itberall, jo lange man ifynen gentigento

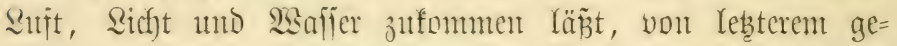

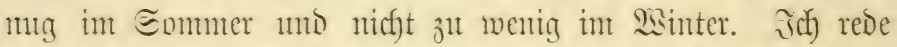
Gier nicht von ben amerifaniijchen und fitbirifchen S(rten, mit Deren Sultur für sen Riebfaber mur ein żmeifelfajter Errfolg

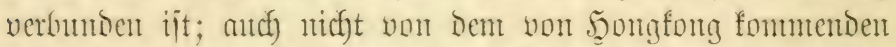
Cypr. purpuratum. Tieje machen jelbit Dent gemiegtejtent Bitchter Garte Afrbeit.

Hnter Den Miärturem ber Srchionologie nimunt Şerr Fearce einen erïten Plabe ein. Jhm verbanfent wir n. a.

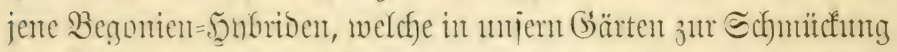
Der Beete 2c. jo heinifich gemorden fint. Er war ber Ent= Decfer ber brei Erigintalanten, welche bie Etmmuteltern aller

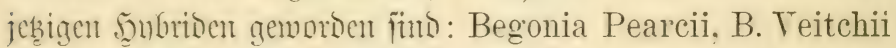

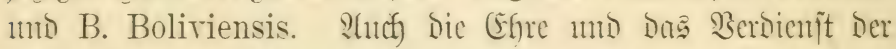

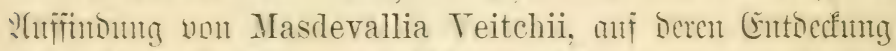

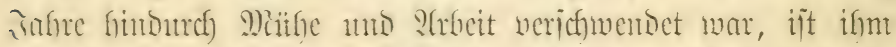

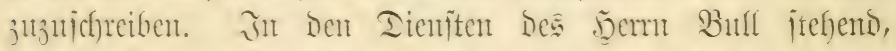
iegelte or jum jumeitemmal nadh Pent, um nochunals eine

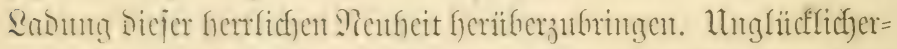

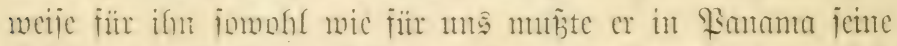

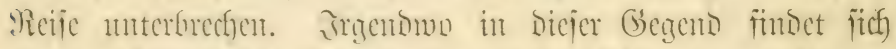
cine mräd)tinge Iftrt, Cypripedium planifolium, vout Der $j e=$

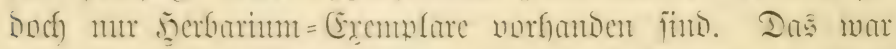

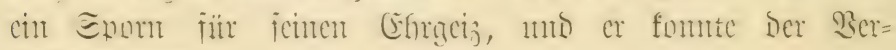

1) Siehe Srapiter itber şnbridifiterung. 
inchung, antch Diejer Siflanze habhait zu werben, nicht mider= itehen. Irob aller Mabynutgen Der Dortigen Eimgebonnen,

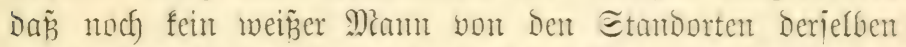

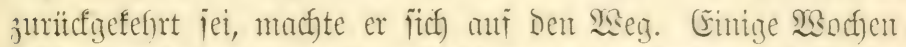
ipäter murbe ex bon Den Jimbintern in elendent Buitumbe

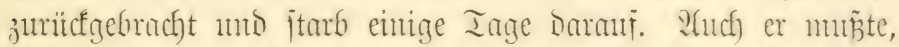

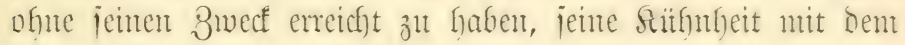
Iode Gü̈ent.

Iie Battung Oncidium ït ebentalls mo ansichlicểlich

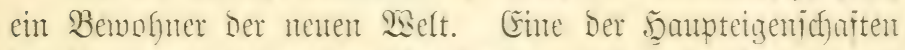
Der Encibien ijt ifure herrliche Farbe. Jch habe Seute ingen

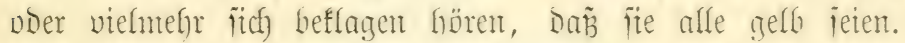

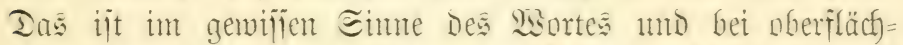
licher Betrachtung wahr; Dem wohl Der gröbite Ieil weitt

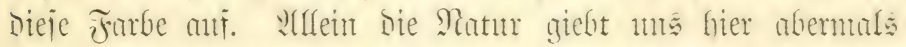

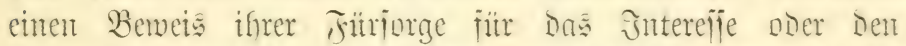

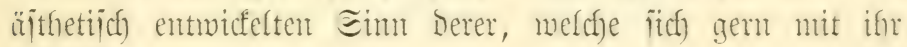
bejchäftigent.

Б口ätten wir feine Encióten in mneren falthänjen, io

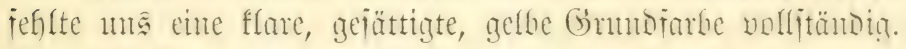

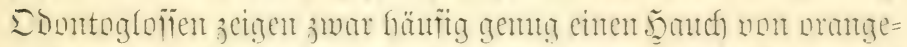
gelber oder rötlich gelber Farbe; allein im grop̃en Gamzen iोt

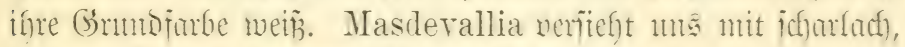

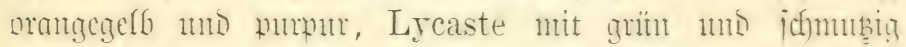
gel6, Sophronitis ntit formeïu mo Mesospinirlium nte

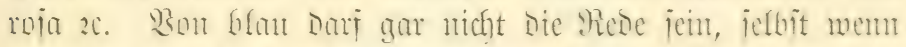
man, wie es io hänfị imtümlicf) geidficht, Utricularia ju

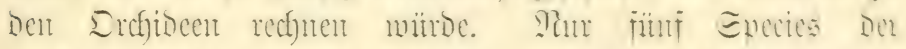
aro)

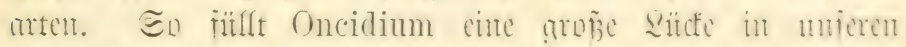

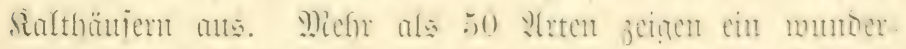

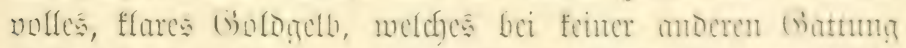
icinesgleidfen findet. Eo Oncidium macranthun! Whit Yetht 
iit es als ein Meijtermerf Der Natur a betrachten und ifum

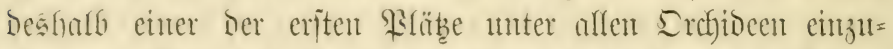

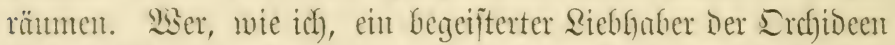

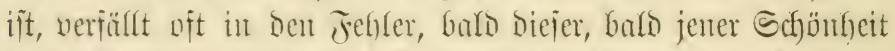

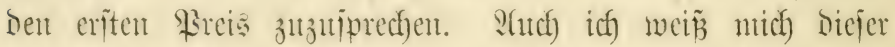
Simbe ichulbig. Darm, meine Rejer, fucht jelbitt eine Gelegenfeit, Dicjes Oncidium macranthum in jeiner Bracht

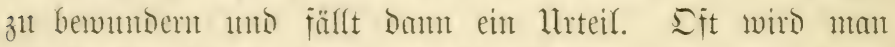
jeboch fïr lange Beit anf bie Folter geipant, bevor es jeine mumbervollen Blitten jur Entfaltung bringt. Dr. Wallace berichtet von einem Falle, wo 18 lange Monate

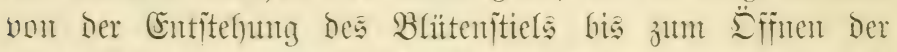
eriten Blïte vergingen. Die lange Taner ifner Echönlfeit

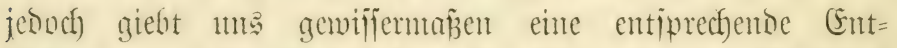

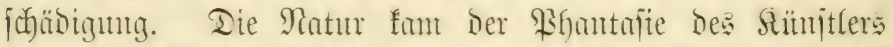
zuvor, als ite biejes Oncidium macranthum jchuf. So unt

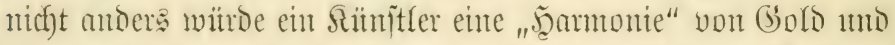

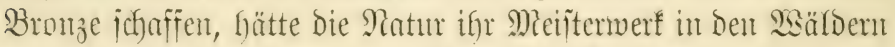
wou Ecuador nerborgen gefalten. Fajt ebenjo jübu und ferrlicf) futh Oncidium serratum, $O$. superbiens ınd O. sculptum zu nemen, beren Rippe allerbings benenteni)

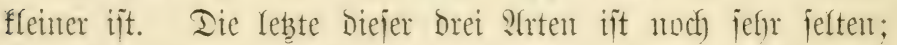

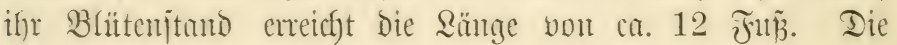

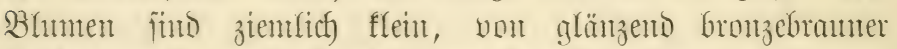
Farbe und jo fein gefräujelt, wie fein Sräujeleijen es hervor= bringen fömte, mit einent goldigen Rande vou unenticher

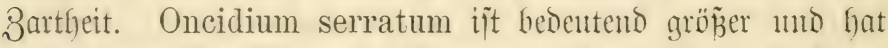

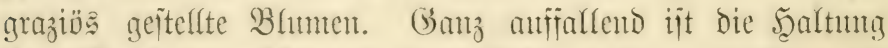

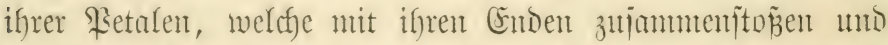
jo einen Sreis von Granngorbiger Jarbe um bie Sänle

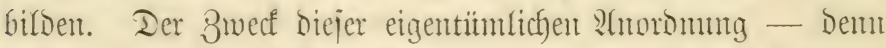

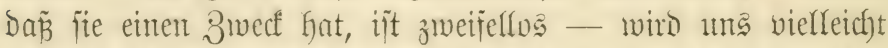
mit ber Beit offenbart werden. Der S(ntalogie nach) zu nuteilen, 


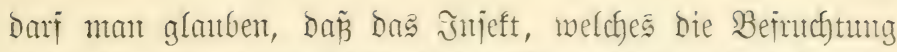

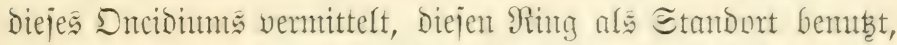

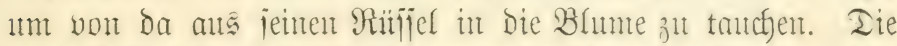
vierte Diejer Epecie., Oncidium superbiens, verdient zu Den Schönheiten eriten Range gerechnet zu werDen. Die braun=

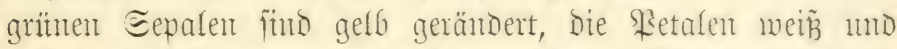
mit einer blañ-purpumen farbe gezeid)net. Sie Sippe iit iefor flein, wurpum und mit einer gologelben Grhöhung veriehen.

(s)anz abnorm uno jonnerbar ijt Oncidium fuscatum. Bon Der Rücfieite betrachtet, tritt uts bie Form eines ge=

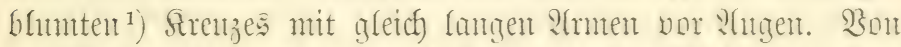
Der Bornerjeite geiefen, wird Das unterite GSfied von einter

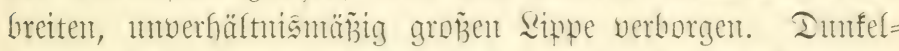

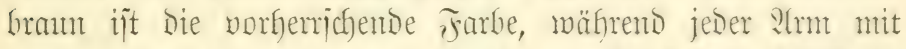

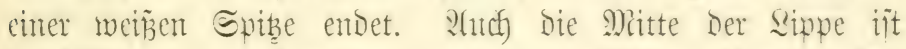
Dunfelfrann, untgehen von einer etwas Gelleren 3one, melche

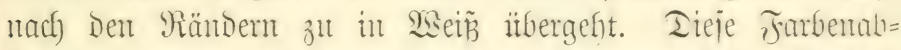

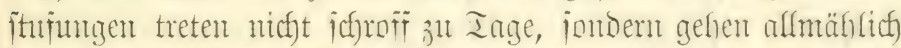
in cinander itber. Botanifer mitroen nit grožen intercile cine jolche Bhume zerlegen; aber bie Gélegentheit Dazn bietet

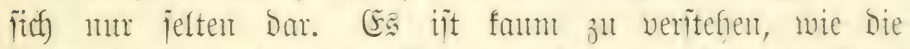

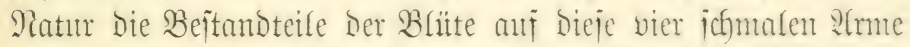
uno eine Sippe hat bejefränfen fömnen. Mrancfimal jeigt bicje

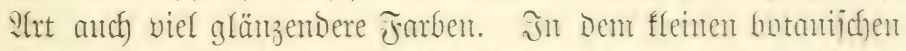

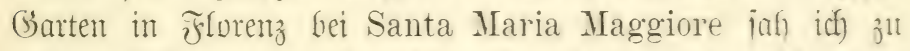
meinem (Exitanten ein Oncidium fuscatum mit einer icharfach)roten Rippe unD vou ciner itherans glänzenton Fürbung.

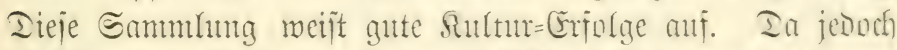
Erchideen in Stafien noch wentig befannt finto, fo afute man nicht, was für einten bedententoen repräjentierte. Mian Dari woht fagen, Doñ gerabe bei Encidient

1) ఏ̨eraldifide Bezeidnnung. 


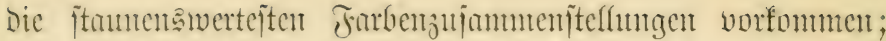

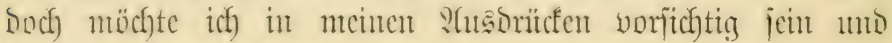
feine unuötigen Beinorte gebranthen.

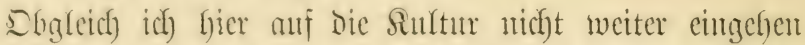

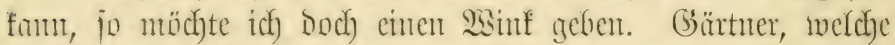

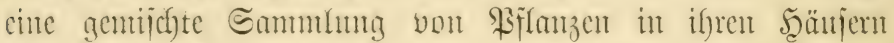

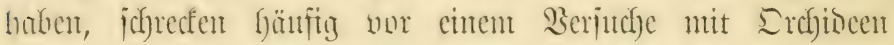
jutritcef wegen Des llngezicfers, wovon Ieß̨tere häufig befallen meroen, mo weil fie Das Rüuchen mit Iabaf nidf)t vertragen

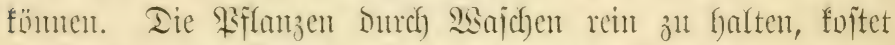

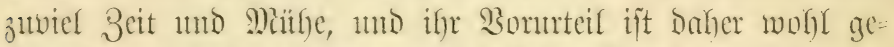

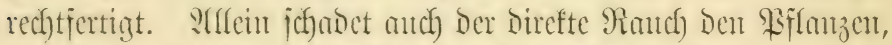

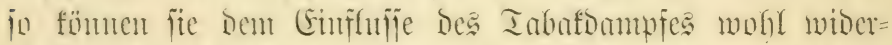

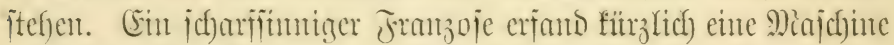

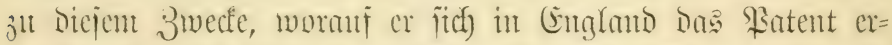

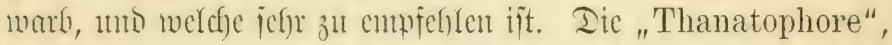
mie Dicjelbe genamut woird, tötet jebes injeft in Bereiche ifyes

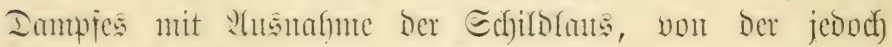

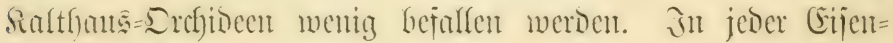

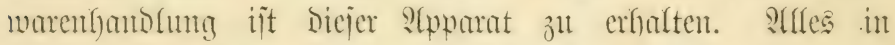
allen genummen: fïr bie unembliche frente, bie nus

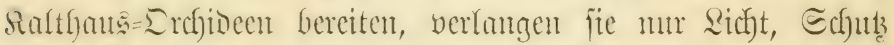
nor Der heî̉en Sommerionte mo vor Froft in winter,

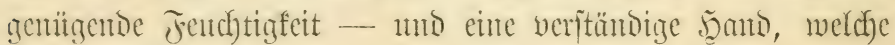
fiir ite jorgt.

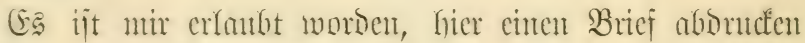

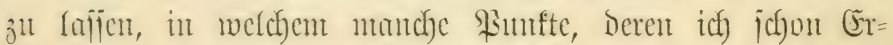
wäfmung getfou, noch Dentficher flargelegt werden. Dent Entfufüriten who Diejer Brief wenig Frente bereiten, or wird

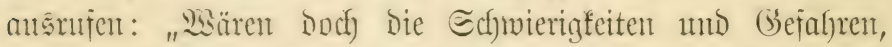

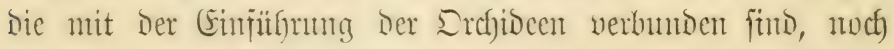

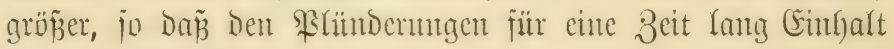
getfan wäroe!" 
19. Jamuar 1893.

Sefyr geefrter Şerr!

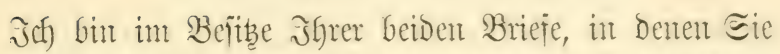
Nachfirage nach Cattleya Lawrenceana, Pancratium Guianense um Catasetum pileatum falten, mo bitte 3u entichuldigen, Dä̃ ich) Diejelben erït heute beantworte.

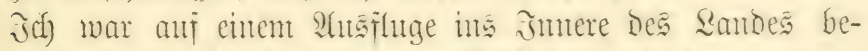

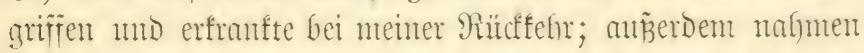

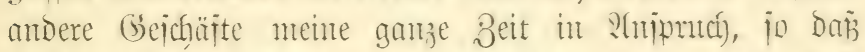

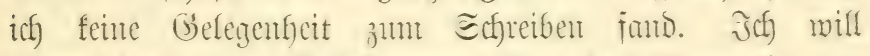

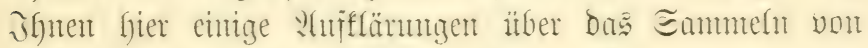
Erchibeen in Diejer Colonite getent. 23or etwa 6 ober

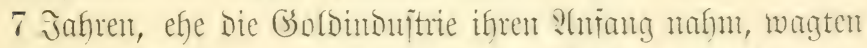
es mur wentige, in bas ômere des Sandes einzubringent.

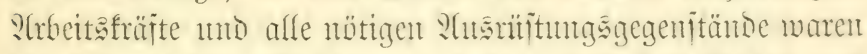

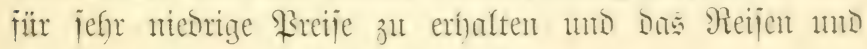
Der Iaujabandel mit Berwim verbunden.

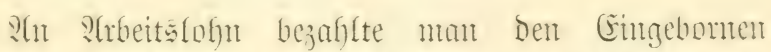
1 Echilling pro Ian, währeno Echiffer, welche Die Böte Dutrd)

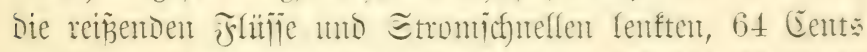
erfielten. Dentzutuge werden eritere mit 64 bi 80 Cents

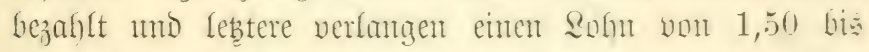

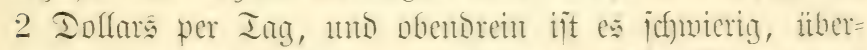

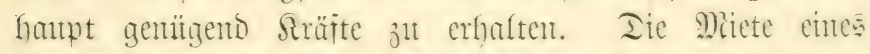

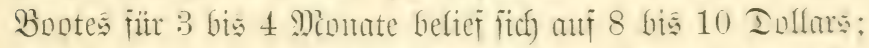
jeb̧t hat man fait Diejelbe Eumme pro Iang zul bezahlent,

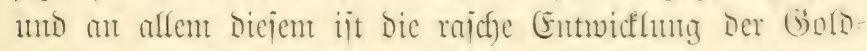
induitrie idulo. Redfut man eine Reije non 25 Ingen

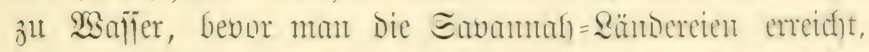

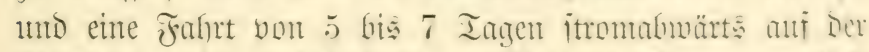

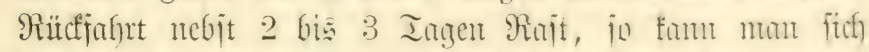

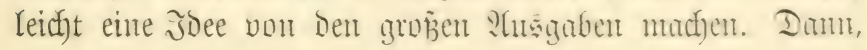


mach Berlanf von ca. 3 Mionaten, menn ntan mit bent

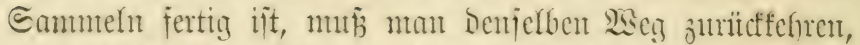

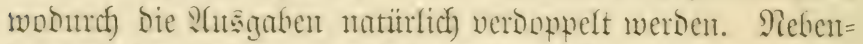
bei fund bie Gefanten zul hedenfen, weldbe mit einer joldgen Bontiant Durch Die Etromidnulfen verbumben fints. Sit genug fonmut es vor, bari in Den Etrontidnellen bie Böte mitiant ber foitharen Sabung verloren gehen; ja, jumeilen

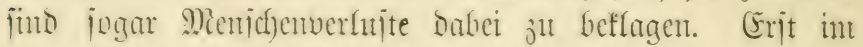

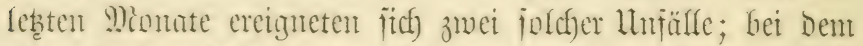
enten fanden 7 Rerjonen, bei dent mberen 12 ifren Iob

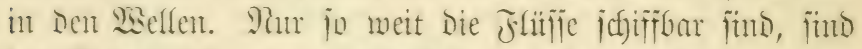

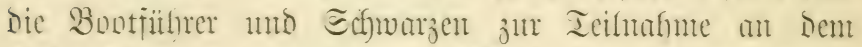

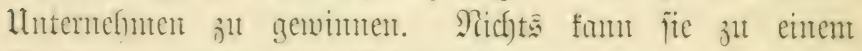
Borbringen miter Die Jnbiancriatänme benegen, ba fie

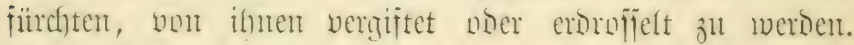

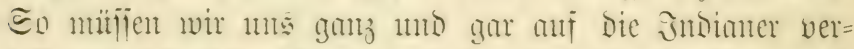

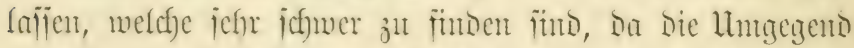
wn Rojario mu idfwadh hevolfert ift. Bor ca. 4 Safren Grachen unter ben Bemofnem Die Blattem nus Diajem an,

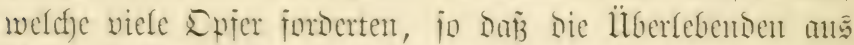
Dicjen Gebiet flitchteten mo ganje Iijtrifte jeither anbemol)nt geblieben füno.

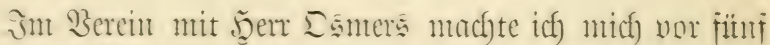

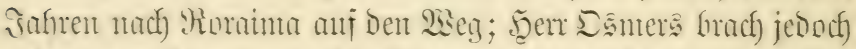

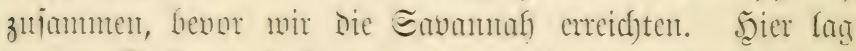

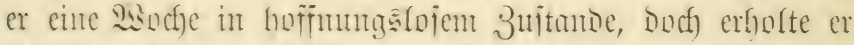

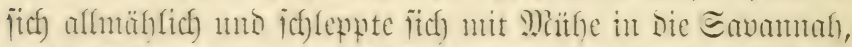

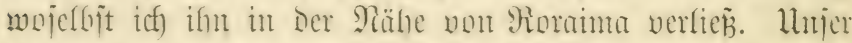
Unternehmen mar nit Erfolg gefrïnt, indent wir ca. 3000)

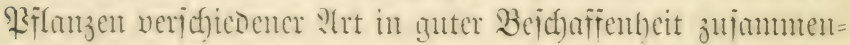

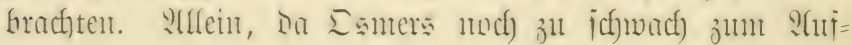

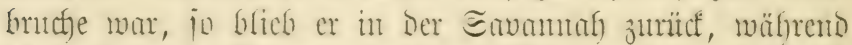

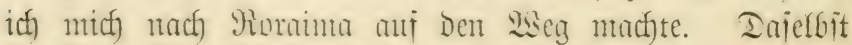




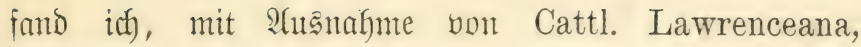
welches von meinen Borgäntgem völlig aus̄gerottet war, alles. Bei meiner Rüuffehr fand id Sonters mefr tot als lebendig vor, ba er einen menten SUnfall jeiner Siranffeit befommen hatte. Dazu hatten ifn fait alle mijere Sente verlafien ans Frurcht vor ien Indiantem, welche Drei ifrer

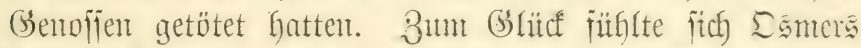

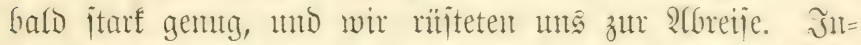

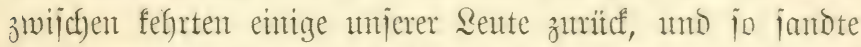
icf DEmers mit eintem Ieil der Bente vorats, währento

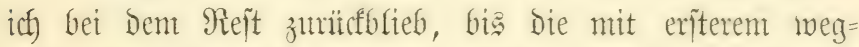
gejanden Sente mich abfolten. Şätten wir eine gentitgento S(nzah)l Siäfte gehabt, jo wäre alles glatt abgelaufent. Dies war nodf, Gevor bie groß̈e Eterbfichfeit ntnter Den Indianem auftrat.

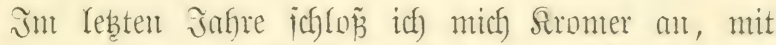
Dem ich) anf meinem Ritctiwege zuäanmentraf. Strom=

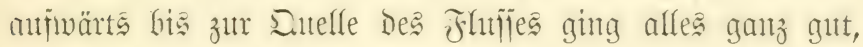
aber Dam hatten wir nit manthen Echmierigfeiten zut fämtefen. WSir fomten mu 8 Intianer, welche in Den Golominen gearbeitet hatten, zur Teifluahme an unjerem Iluternefunen gewinnen, da bie ganze Gegend verlafjen war, uno hatten einem jeden pro Ing einen halken Iollar

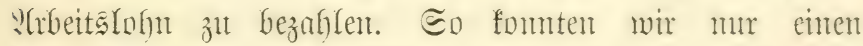
Teil ber gejammelten Siflanzen in bie Eabamath fort= idfaffen unb muñten unjere Reute verichiebene Male zut= ritcfenten, um Den Rejt mijerer Bente jut holen. Mist er= reichten bie Eavamulg farb verhungert, ba wir mutr menia Nafrumgsmittel befonmen fomuten. Cattl. Lawrenceana

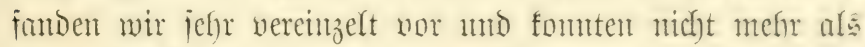

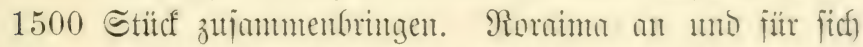

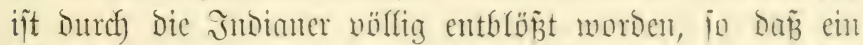
P(bjutchen diejer Gegend verlorene Mäiffe mar. In Der 
Untgegento von Roraima hielten wir un ca. 14 Iage auf uno janmelten cine Mienge Utricularia Campbelliana, U. Humboldtii uno U. montana. Yutc) Zygopetalum, Cypr. Lindleyanum, Sobralia, Liliastrum, Cypr. Schomburgkianum, Zygopetalum Burkei ı. a. fanten wir gentïgeno vor, währento mir von Onc. nigratum ıut 50 Eremplare mntrajen. $30 n$ Cattl. Lawrenceana bagegen war feine Spur vorthomben. Sbgheidf) mijere Solleftion nicht jefr. grop war, jo hatten wir boch ants Plangel an genitgenten

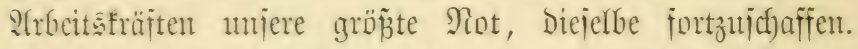

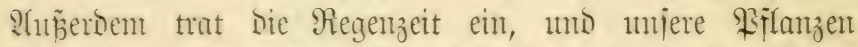

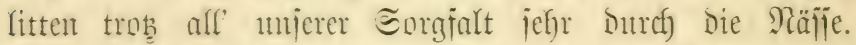

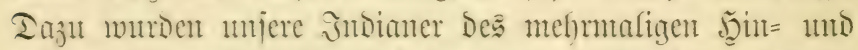

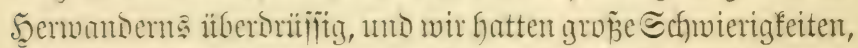
Das Sumbinggebirge วu erreichen. Jn biejer Beit maren mir itets nafe baran z̧u verfungern; żwar fanden wir einige Nafhungsinnittel auf Dent Auntbinggebirge, Doch gingen

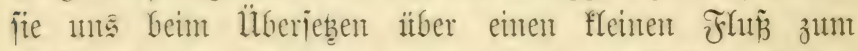

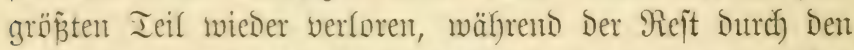

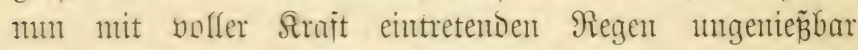

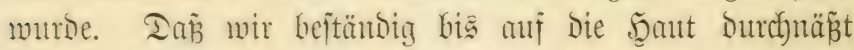

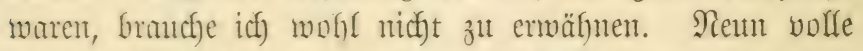
Inge gebrinteftent wir, um unjere \$iflanzen ïber dą Gobirge sut jofaffen, mojelbit um miner Bont erwartete, um

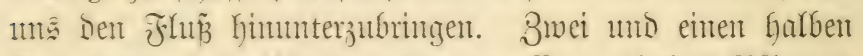

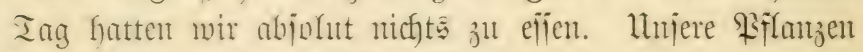

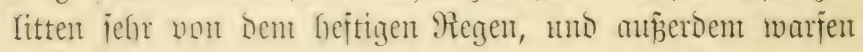

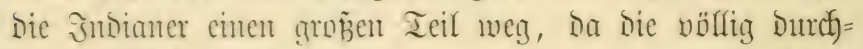

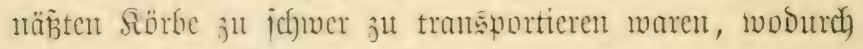
wir bie bejten \$flanzen vertoren.

S.ı unjerem Biel angelangt, nuñten wir 8 Tage auf miler Boot warten, Da infolge Des Regens bie Frlitfle angeidfwoffen waren uno baburdh bie Fafrt erifhmerten. 


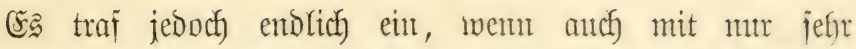
wentig Rebensmitteln. Sobald wir Dic erĩten Gorbntinen emeichten, berjah ums einer meiner frembe mit frijchen Rebensmitteln, und Dam jeb̧ten wir ueu gejtärft unjere Reije fort. Bei Den Sapurifällen jedoch erwartete un

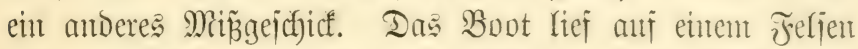
fejt und jauff, woburch unjere Biflanzen unter sisajer ge=

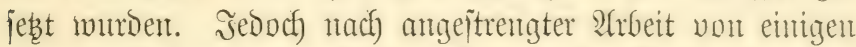
Stumben gelang cs uns, das Boot wieber flott ju machen

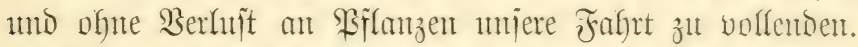
Beim Shtspadfen Der Echäbe jeigte es ftch, Dañ wir im gauzen mur 900 Cattl. Lawrenceana nach Samje gefrucht hatter, wowon unr ber britte Ieil gut jul nemter war, mäfreno zmei Drittel ihfecht oder zul flein waren. Die

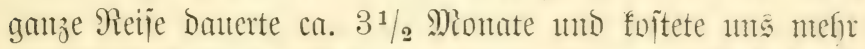

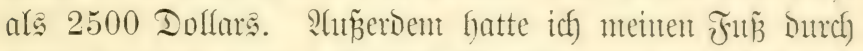
einen Splitter eines verfaultem Bammitammes vergiftet nul lag 4 Mionate lantg mit Den feitigiten Echmerzen Danneder.

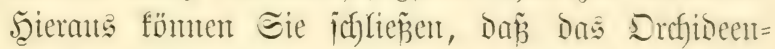
Sammelu fein Bergnitgen iitt, und ich möchte Eie Darmif

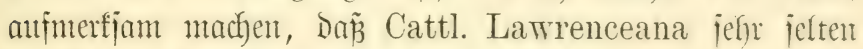
im Jnnem bes Rambes geworven ît.

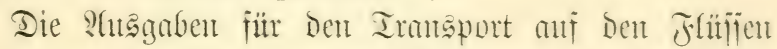
find infolge der Gordombuttrie ungemöhulich) hod), ju,

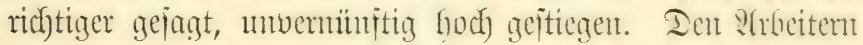
nuñ man nefen der Berpflegung 64 Cent bis 1 Dullar

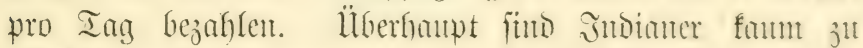

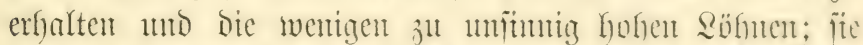

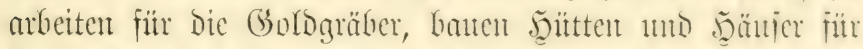
fie, roben bas lluterfor ans uns jagen fïr fiec. Eelbit wem betr Sromer io glitcflich gewejen märe, 3000 bi 4000 Cattl. Lawrenceana ju finten, jo hötten wir ma Mianget an Irä̈gern Diejelben nich)t fortichaffen fönnen. 


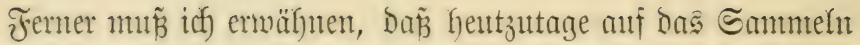
you Errfyideen eine Gébithr von 100 Dollar zut entrichten ift, weldye Mix. Sromer ebenfalls zu bezahlen hatte, und

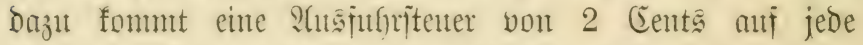

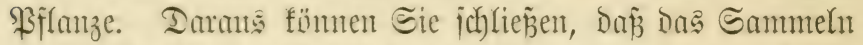

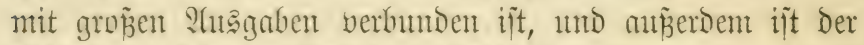
Griolg ein jelns zweifelfafter, jelbit Damn, wem man mit ถer Gegend, Den Eitten und (B)ebrändfen Der Gingeborenen nolfitänoig vertrant ift. Unjere reste Erpebition fojtete

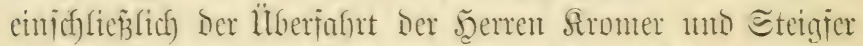
2500 bi 2900 Dollar. Liem Eic abjolut Cattl. Lawrenceana huben undfen, jo nuts ich Shnen raten, ielfit ferither zll icficfen; wh Eie aber Erfolg haben werden, ijt cine

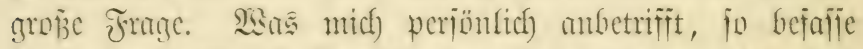

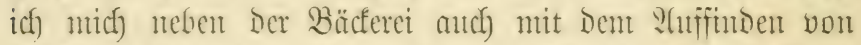
Góld uns werbe mich in cintigen Mionaten ebenfalls in Die Sabmunal hegehen. In Falle Sie cine Expedition untemefmen witriden, ftelle idf) ifnen die beiten (Fm=

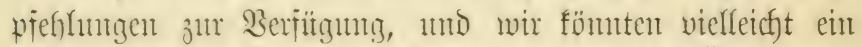
Qhfommen treffen. Jebenfalls witmen Eie Die Überfahrts=

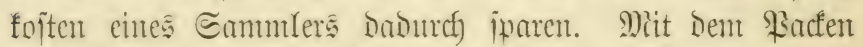

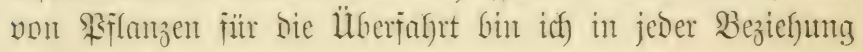
vollitündig vertrant.

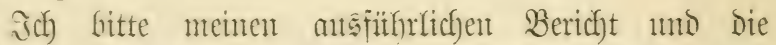

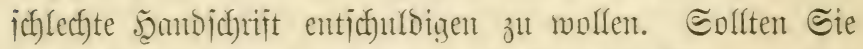
zu cinter Expenton gencigt jein, jo bitte ich Sie, mir eine Rijte Der gemiunjhten Riflanzen einjenten zu wollen, Damit ich) Jnnen Den Etmoort Dex Rfflanzen mitteilen famm; C. superba 3. B. wäd)it nidjt in Demielben Diftrift wie C. Lawrenceana, jondern viel jitolidjer.

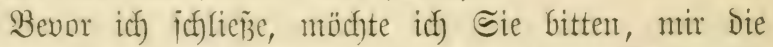
Preije von 25 Der icfönten und bejten jitbamerifanijøen Srchideen mitzuteilen, Dic id) fïr meine eigene Eanmlung 
Gahen möchte, als Cattleya Medellii, Cattl. Trianae, Odontogl. crispum, Miltonia vexillaria, Cattleya labiata 2 .

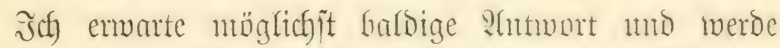

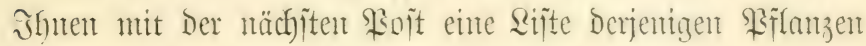
jenton, bie in biejer Solonie anzuttreffen funt.

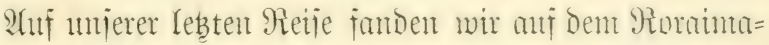
gebirge, wojelbit mir îthentachteten, ein nentes Oncidium mit gewaltigen Butben, viclfeicht mang ca auch) ein Catasetum

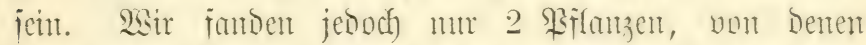

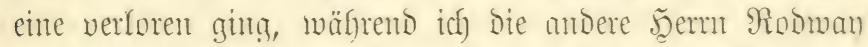
anvertrante. Sie hatte jeboch jut jefr mif Der Reije ge= litten uno itarb ab, ofme jemales geblitht jut haben, io

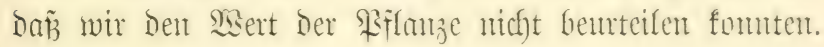

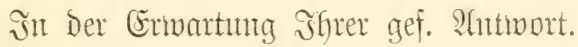

Jitr ergebenter

Seyler.

P. S. Eollten Eie cinen Eammler nach) fier ienten

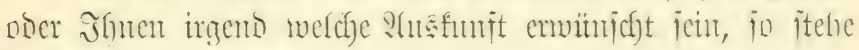
ich gent zu Dienjten.

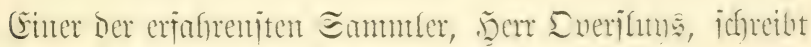

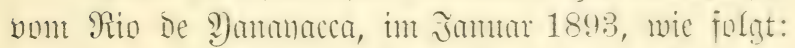

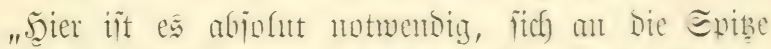

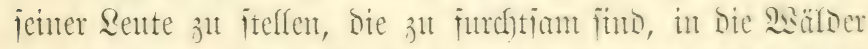

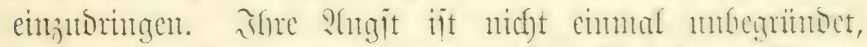
Dem bie meitten weroen franf mo Das (Findringen it un=

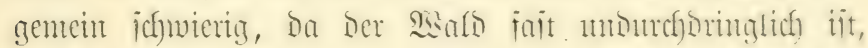

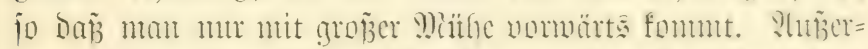

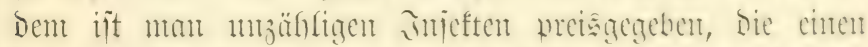

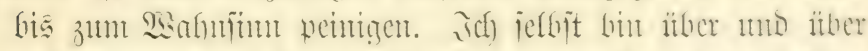

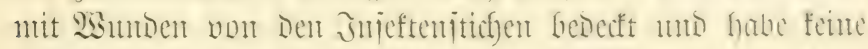

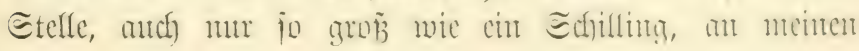




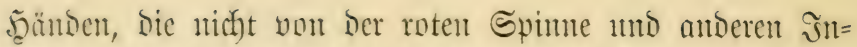

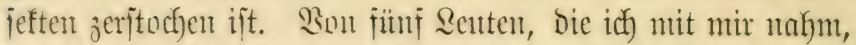

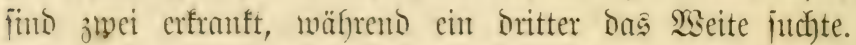

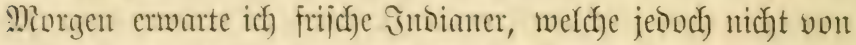

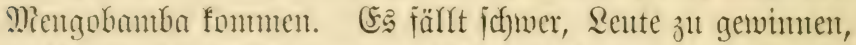

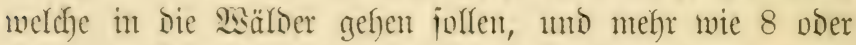
10 fanm ich nicht anjellen, ba ich beftänoig lyinter ifnnen

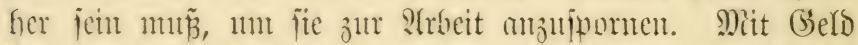
fann man hier nichts emeichen; man mun eben BSlitef flaben mo jemte Rente gut zu befmbehn wifjen. Sie ver=

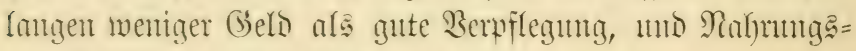
mittel futo hier jebr jofwer zu erhalten.

Die Srefiocen fonmen jefre vereingelt vor, mur ein cinjiger Banm trutg orei Exentulare. Tie höchiften nut

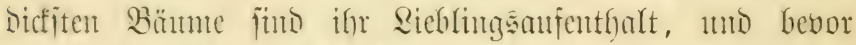

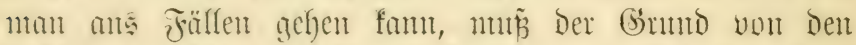

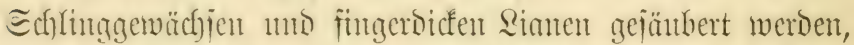

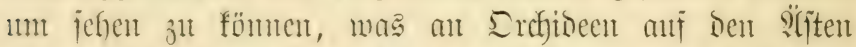

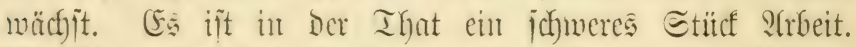
Die Ratur hat bicje Eattlena ants beite gejchitht. Siemem

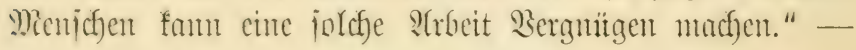
Ђeier bricht ex plöblich jeinen Brief ab:

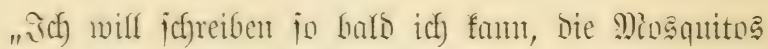
lajjen mich feinen :Gugenblict in Rube." 


\section{Siebentes finpitel.}

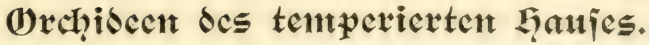

Drchibeen Des temperierten Şaujes funt joldje, melche

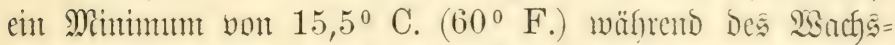

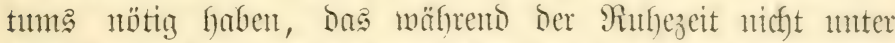

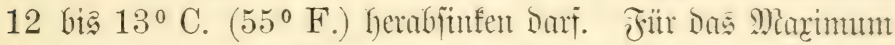

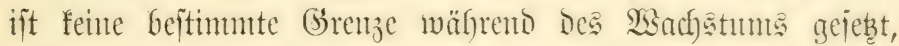
mäfyrent Der Ruthezeit bagegen mun Eorge getragen werben, Da erfolge vorfommen. SGuf Gronto biejer Besinguntgen mitifen

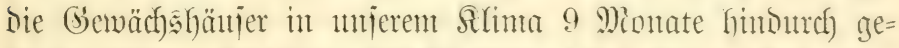
Geizt werien. Srchibeen des Marmbanjes vertantgen bas ganze

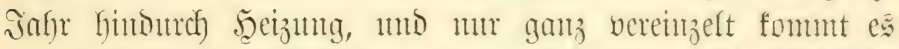

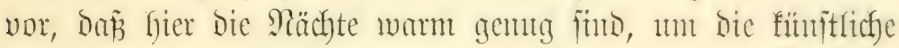
Erroärmumg einftellen zu fömuen.

3n Dicjem troffench Thenta ither Ientperatumen will ich

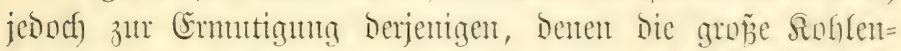

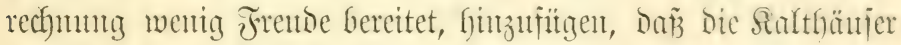

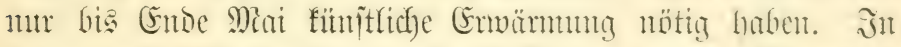

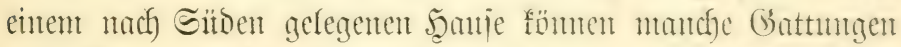
und S(rten, weld)e fïr gewöhntich ar "temperiert" bezeidynet

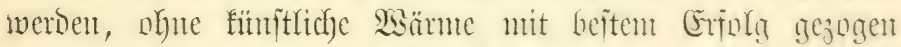

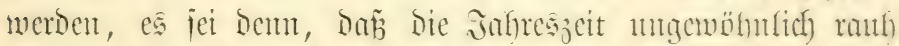

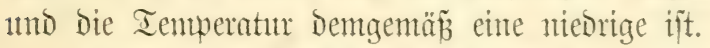

Temperierte Srefiocen fonmen non einer inthtropifichen

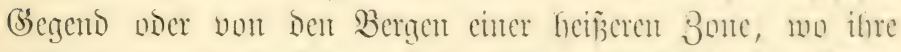

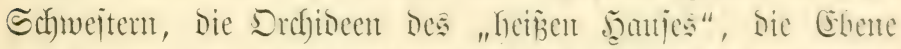

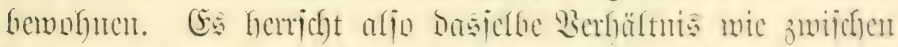

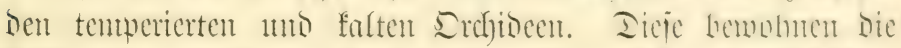

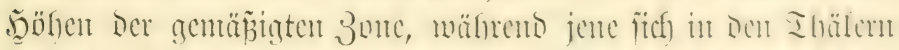

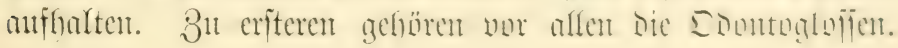




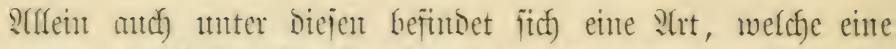
temperierte Sultur heanjputht, Odontoglossum vexillarium, von manch)en Botanifern med Miltonia vexillaria genumut.

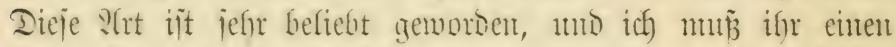

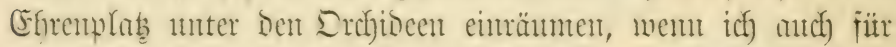

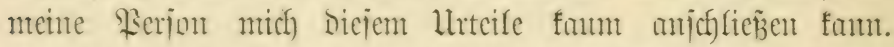

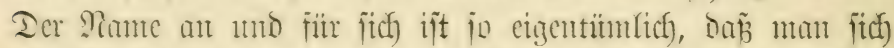

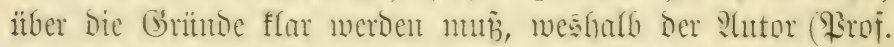

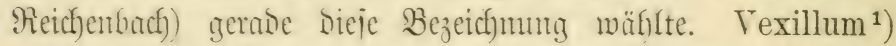
- Fafne - iit cin genitgeno befanntes $2 \mathrm{sunt}$ - vexilla-

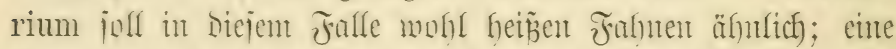

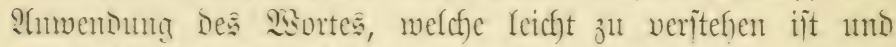
welche Denjenigen, melche mit Den alten rönijchen Regintents=

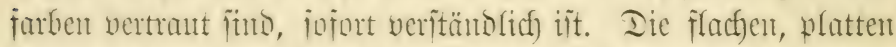

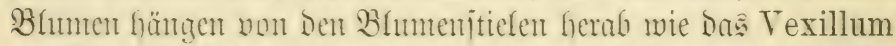

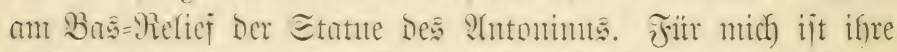

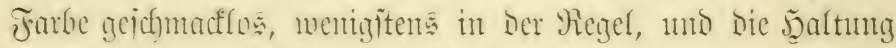
int ganzen iteif; allein sie Miobe nimme, wie in munchen

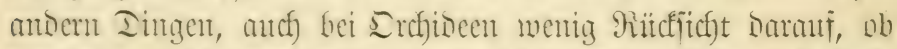

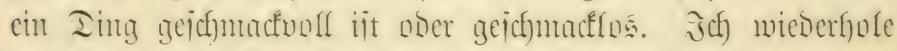

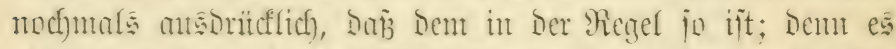
giefot manche mijchäb̧baren Exemplare, Deren Fomben briffant

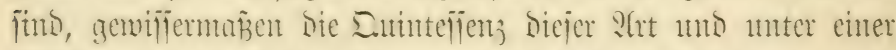

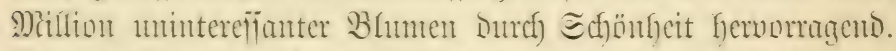
Iie flemiten von Diejen veroienen ofne Bueifel ieflit von

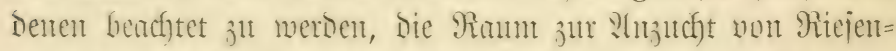
cremplaren foufen. Eie machjen rajch zu groñen Piflanzen

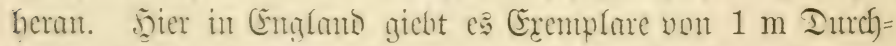
melïer, Die fumberte von Blumen zun jelben Beit hervorbringen

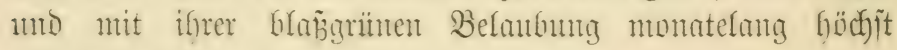

1) Die römiichen Bieiter hatten fleine bannerähnliche Vexilla

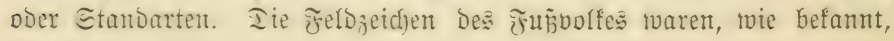
bie Reginnabler. 


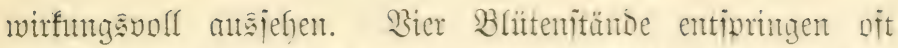

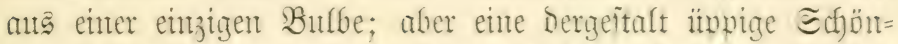
heit jollte im ganzen genoffen und feuter fritichen :Hafnie unterzogen merisen.

Eingefitfut untroe Diejes Odontoglossum von Cofumbient.

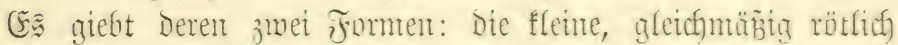

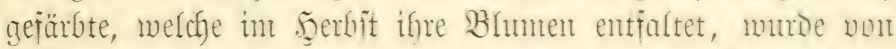

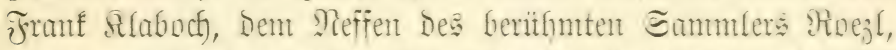

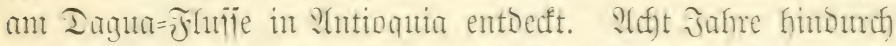
verjuchte er, flente Eendungen biejer Piflanze lebend nadh Ĕmopa zu bringen; alfein jeine Piühe iafien vergebens, jämt=

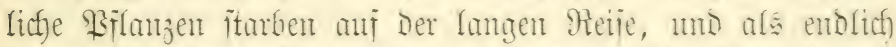

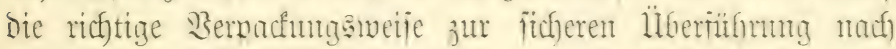

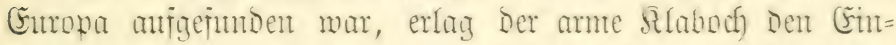

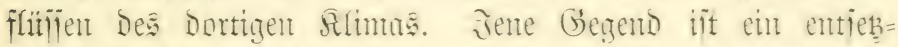

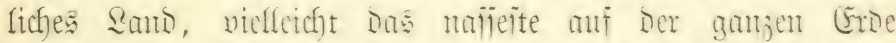

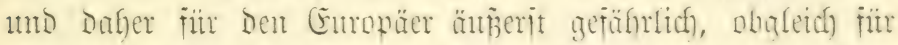
Eammler jefne ergiebig, Da nefen Diejent Odontoglossum nuch verichiedene wertvolle Cattlenen Dort vorfonment. Atber

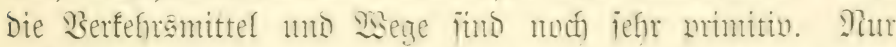
mittelit Ranoes und mit Diffe Der indianer fönten Die Eammler ifre Bente forticfafien. Disem Günter withten, wic

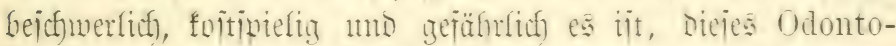

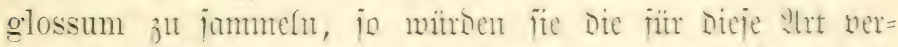

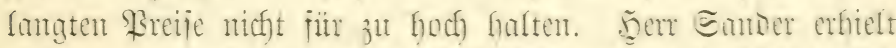

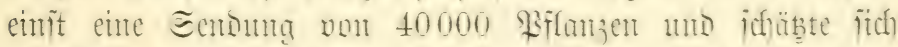

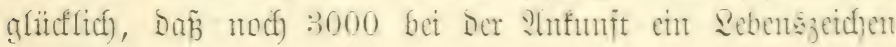
in fich Gattert.

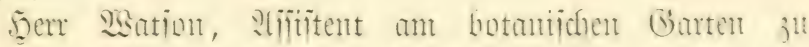

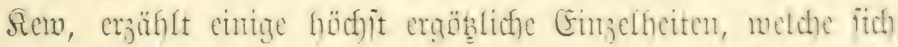

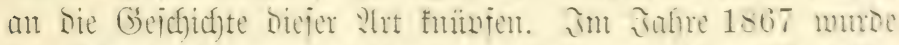
Das Odontoglessum vexillarium jum eritemul non trofefion

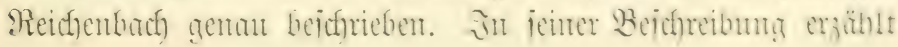




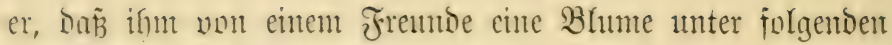
Bebingungen geliebent worben jei:

1. Fie feinem andern zul zeigen,

2. nidft viel Davon zut reden,

3. feine Beidjum bavon zut machen,

4. fie nicht photographieren zn Yaffen, IIIID entolich)

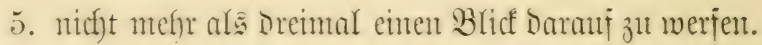

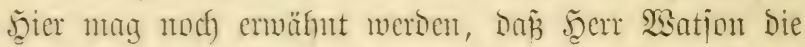

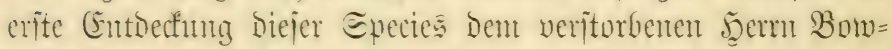

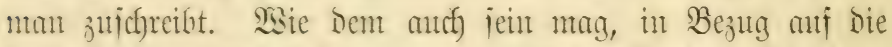

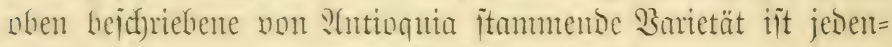
falls Der won mir gegebene Beridyt ber richtige.

Tie mbere Barietät itummt an Der Gegend vou Frontino, ca. 250 englijche Meilen nördficf) von ber oben beichrichenen, uns ijt - Botanifer witroen jagen jelbit=

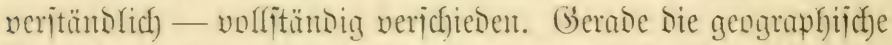

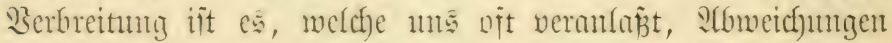

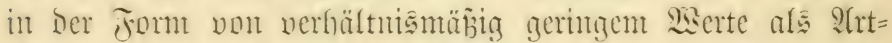

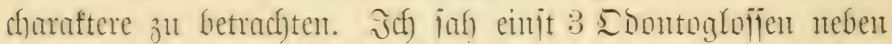
cinmor gejtellt, welche jelbjt cin Semer fïr Barietäten Der= jelben Siflanze gehalten hahen witroe, wäre er nicht ganz genan mit ifmen nertrant. (5) maren Odontoglossum Williamsi, Od. grande uno Od. Schlieperianum. Od. grande

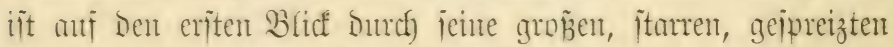
Bhtumen von gelber, fram geiprentelter Farbe zoll erfemten.

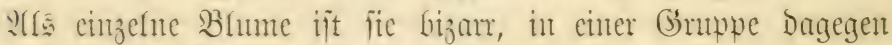

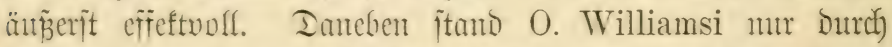

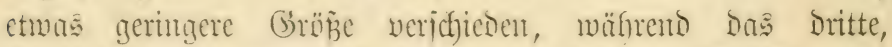

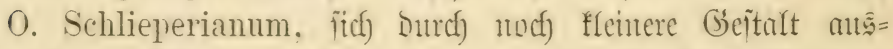

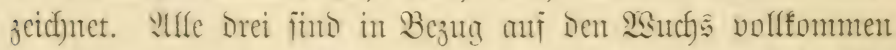

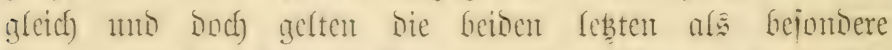

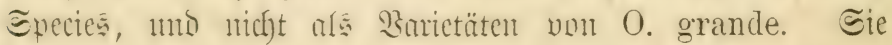


finto alle beide burch ca. 10 Rängengrabe 1 mo 10 Breiten= grabe von 0 . grande entfernt, umb man fam mit Sicherheit jagen, Dã feine Bwijhyenforment in Den Daz̧wijchen liegenton Gebicten vorfonment. 10 Rängengrabe bebenten aber Dort

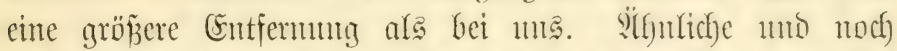

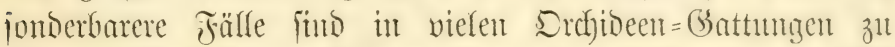
fintoen. Ias Odontogl. vexillarium vou frruntino wäd)it fiuffler uno hat bedentento gröpere Bhunen, die vou den reinjten bis zu Den Dumferfiten Farben variieren. Ecine Błlitte=

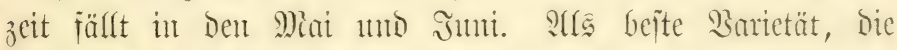
nicht ifres gleichen findet, ift Odontoglossum vexillarium

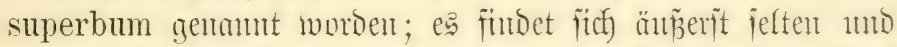

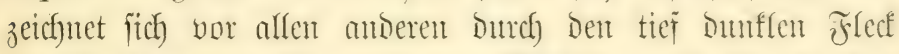

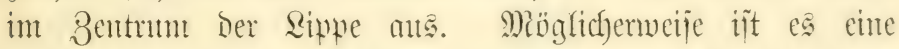

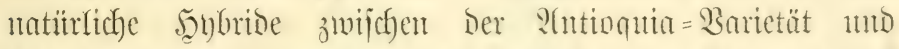
Odontoglossum Roezlii. Die :(tûfitht, ein ffentes Stüct

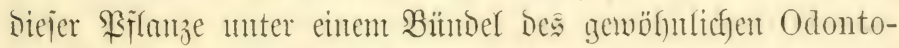

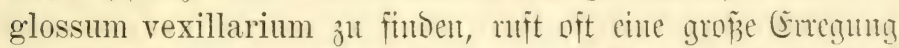

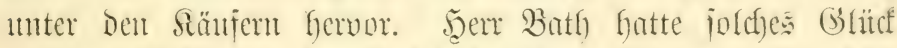

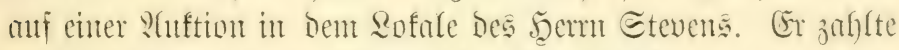

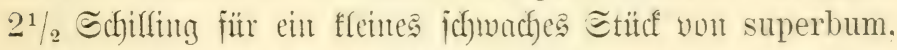
welches er, banf ber jurgfältigen \$iflege, bie er ifm mo

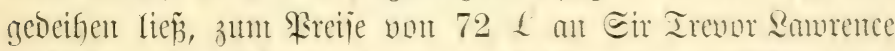
nerfante, Der fich frente, eine joldhe cinjige Riflanze in

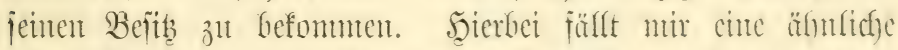
fleine (bejchich)te ein. Unter ciner : (uzaf) von C'spripedium

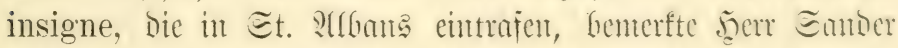

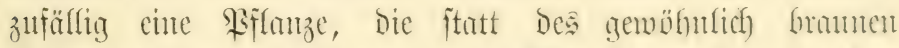

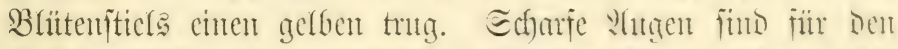

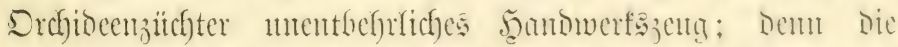

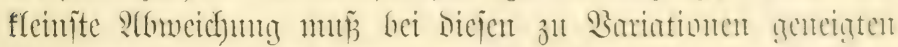

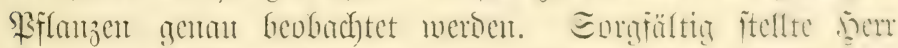

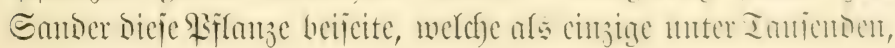




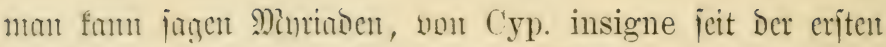

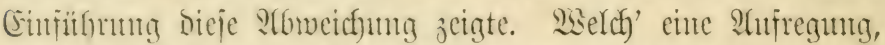
als fich) Die Blume iffrnete, Die vollftündig golbgelf war! Die

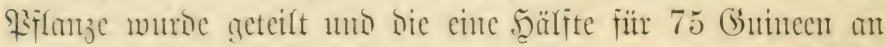

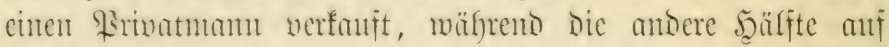

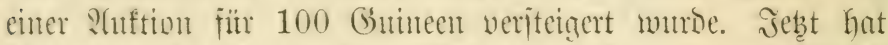

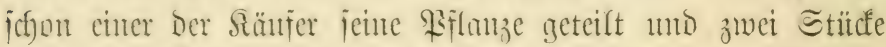

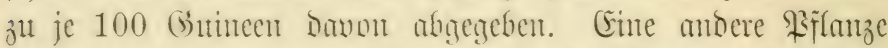

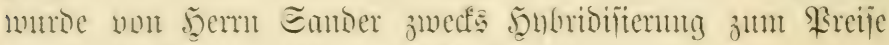

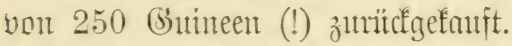

Bein शieberichreiben Diejer Zeilen fällt mir cin ähnliches

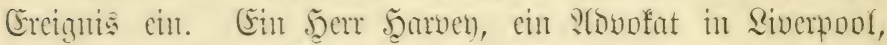

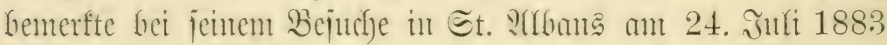
cine PBflanze von Laelia anceps, an Deren Butben bie Ringe

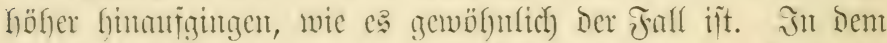

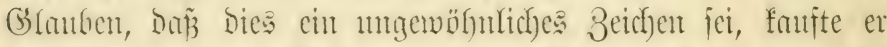

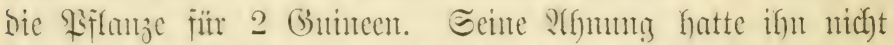
Getrogen; an 1. Dezentber 1888 verfante er bicjelbe \$if́lanze wiederum an D̃em Ember zum \$reije vou $200 \%$ Sie

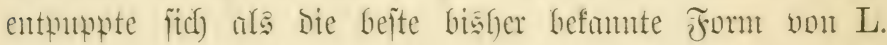
anceps, wou rötficfucifier Farbe, welche zu Csfren Des be=

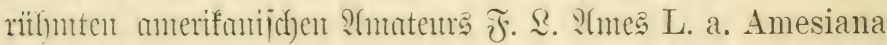

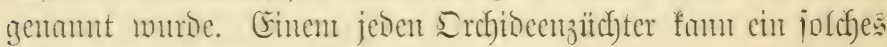
(G)ïnd Gevoriteben.

Die Gattung Der Cattleyen (Laelia ntit inbegriffen) ijt

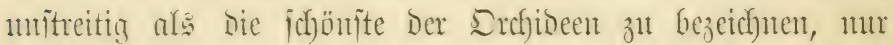

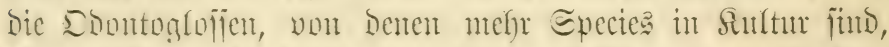
fönten ifnen chenbitrtig jur Ecite gejtellt werden. હectjig verichiedene Barictäten und Epecies vou Cattleya merben

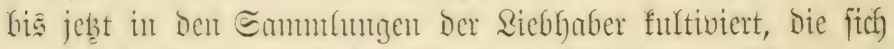
ganz bejonsers nit sicjer Grmppe bejchäftigen. S(ber and inter Den verjoficdenen jogentunten :Trten fund viele reid) an Barietüten, itber Deren botanijchen sert jeboch bie

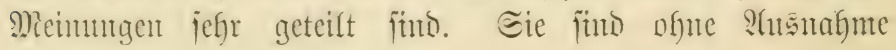




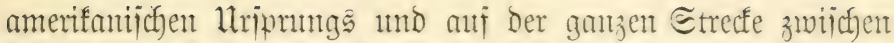

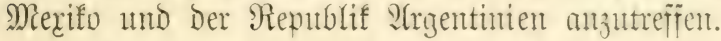

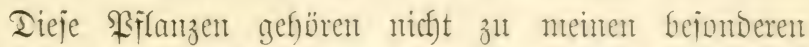
Rieblingen, an: Denjelben Grinton, ans weldjen meine 2 b neigung gegen Odontoglossum rexillarium entipringt. Die Sattlenen find io antoringlich ichön, fie haben io grox̉e

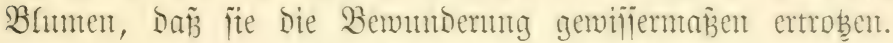
Und bocf ijt in grożen Ganzen ifjre (Erichemung cine recht fintiche zu nemten! Eie fonmen mir vor wie ein antige=

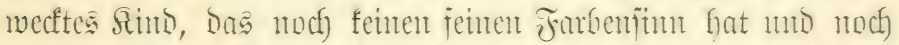

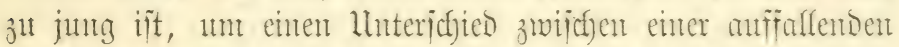
min cincer reizenden Form zu finden. Iocf, icf Darf nicf)t zul meit gefren.

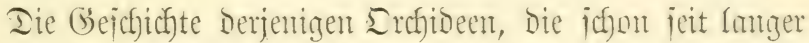
Beit in mierer fultur furb, fan man nicht gentu veriolgen. Die erfte Cattlena, welche mentes asijem nach Emona ent= gefitfret mutroe, war C. violacea Loddigesii. Wite Der ?amte jofon andentet, war fie von Der Gefamten Fimn Sobotiges,

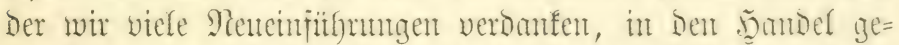
bracht worden. Bwei Jafle ipäter erichien C. labiata, ani Die wir noch genanter juthifffonmen werben. Inu fom C. Mossiae von Earacas unt endidf C. Trianae von Iolima

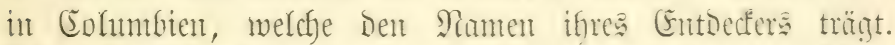
Sberit Trian Darf nicht in Bergeffenfeit genten, Da er ats Diejer abgeidgrofjenen Begend ftunmt nub ein Botanifer iit. Sism

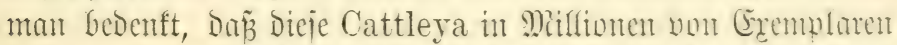

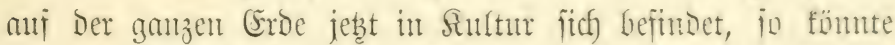

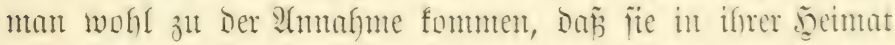

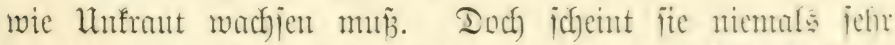
fäuffig angetroffen morben zu jein, ja, angentrfeflich iit fie io

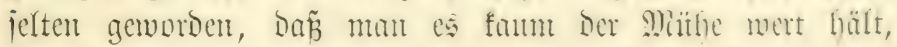
Sammler banach atizulienten. 


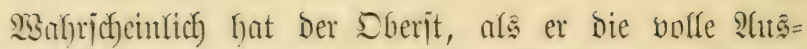
rottung bicjer Epecies fonmen jaf, Dem übel vorgebentgt uns it einem verborgenen Winfel Durd) Shupflanzung einiger Eremplare Dieje Cattlena vom ficheren Untergange gerettet. Mit Cattleya Mossiae verfält $c^{3}$ fich fajt ebenjo, wem nidat nod) forlimmer.

Dieje Thatjachen entharten cine \$sannug. Smuerfarb

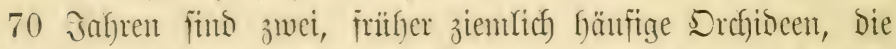

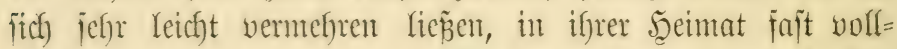

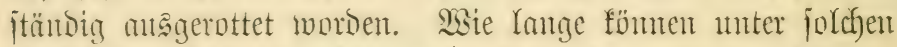
Umịtänถen jelten vorfonmentoe Erremplare erfalten bleiben,

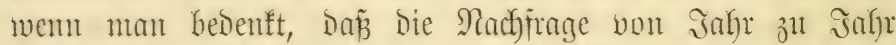

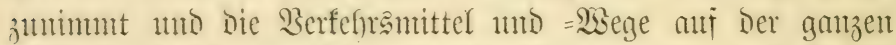
Welt io leid)t gemtadht werben? S(rten, weldhe auf Jnfelu

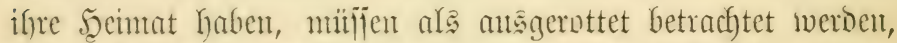
wem ite nidft, wic Laelia elegans, ifyre Buffucht in umer= reichbaren Slippen Kaben.

F. it: Dies mutr eine Frage Der Beit; aber wir wolfen Goffen, Dar bie Regienungen Dem Einthalt thun werDen, Gevor es 3ll juät icin wiro. Şerr Burbioge, Surator Des Gotan. Gartens

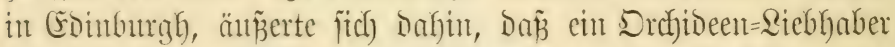
Gier und ba cine \$Fflanzung in Der Şeimat ber Drchiocen an= Yegen, uno fich) jorgfälting anf Die Sirenzıng Derjetben legen

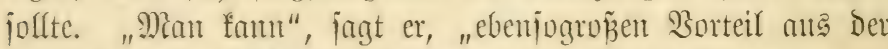

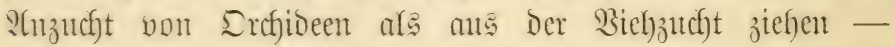

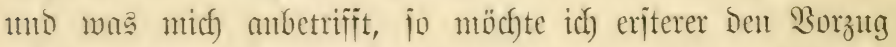

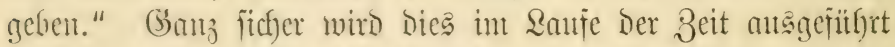
werben, wenn autd) nicht jo jefyr wegen Der Büthtung von รู) Darf zit liefern.

Diejenigen, weldye glantben - mol es giebt deren

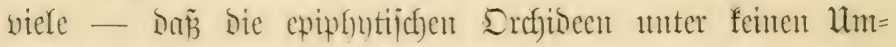

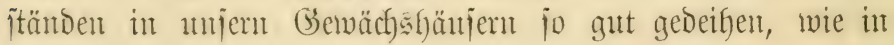


ifrer Şeimat, büriten mit ifrer Befauptung jegr im Ĵrtum jein. Sweifellos ijt es möglich), jagen jite, Diejelben mit Crojolg Geranzuziefen und zum Blïfen zu bringen umb bei jorgäältiger

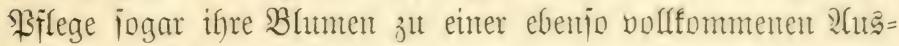

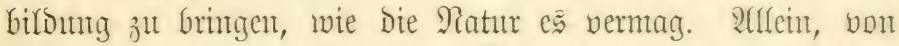

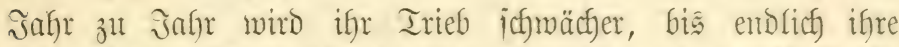

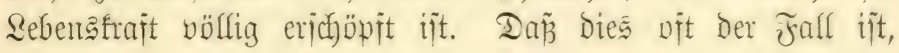
fann man żwar nicht leugnen; aber wern man Siflanzen fieft, bie jeit mefre Dem 20 ober 30 Jufren von ifren $B e=$ fithern gefegt uno gepflegt find und von Jahn zut Jafh an

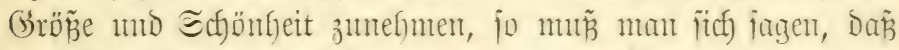

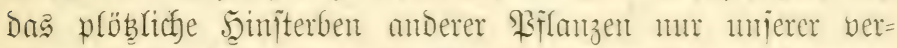

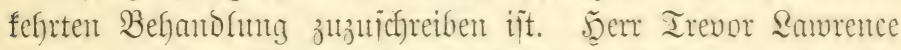

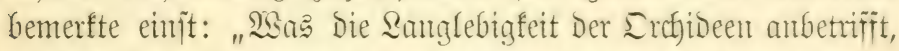
jo beithe ich ein (Exemplar, welches, wie ich heitimmt weiñ,

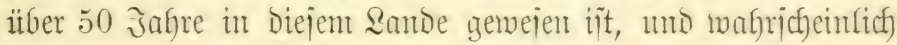

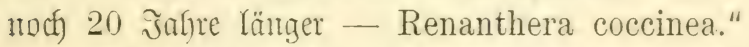

Die ichönten Eremplare von Eattlenen in Der Sulleftion Des Şerm Etebenjon Clarfe fint von fleinen, importierten Stïcfen herangezogen worden. Söäbe es noch mefn હamum=

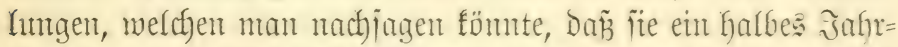
Gumbert unter Derjelben jorgiamen Szand geitanden Gätten, io

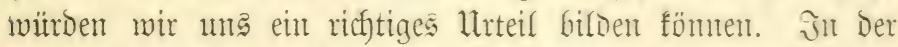
Regel aber find Die Daten Des Crinfauf nicht genum notiert worben; erjt in Den leģten Jafhren verwendet mun mefr Eorg= falt Darauf.

Şier muñ eine (Eattleya erwägnt werben, welche vor mindeptens 70 Jahren bis zu ifrer sisebereinfüflung im

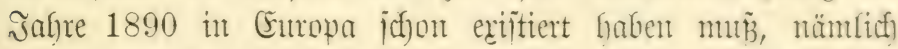
Cattleya labiata autumnalis. 怤em wir cine mefro ben

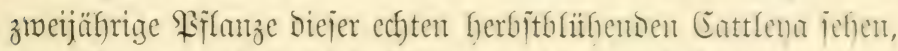

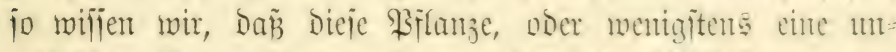

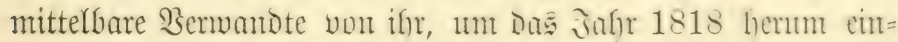


gefithrt moroen jein mun; Dem fo weit wie befannt, ift nie eine Prflanze an Eamen herangezogen worben. ${ }^{1}$ )

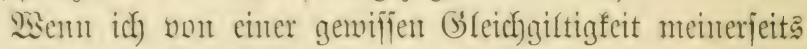

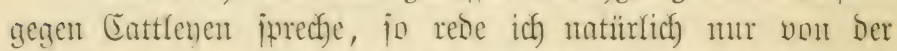

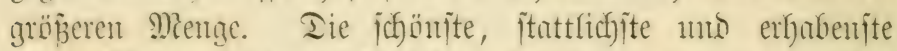
alfer Bhumen ijt unbejtreitbar Cattleya Dowiana noer C. aurea, melche mur eine geographijche Barietät Der erịteren

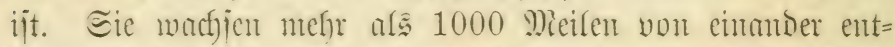
Fernt, bic eine in Colnmbien, bie andere in Evita Rica.

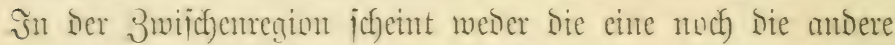

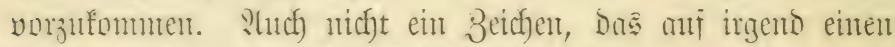
Bujanmenthang Der beinen Bartetäten hinmeijen fönnte, iit

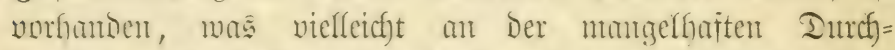

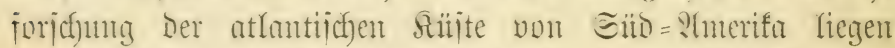

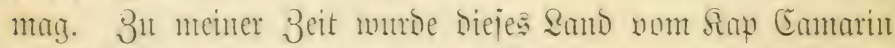

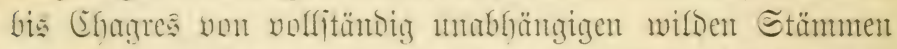

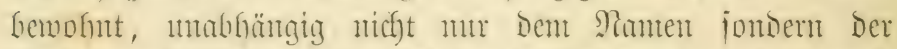
Eache ntacf). Tem bie Piowquito= Jubimer werben audf) rechtlich) als mtahfängig angejefen; cintge fumbert Dutadrat=

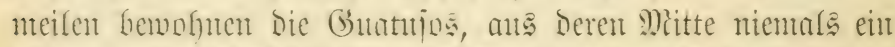

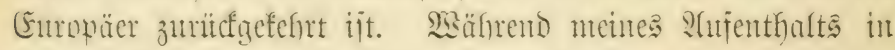
Diejent Gschiete maren mur bie Inlamantas als mentiger

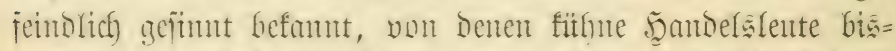

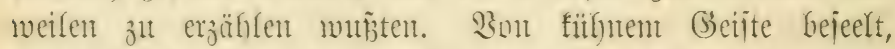
mande icf) Den Berjutch, eine (Expesition zu Diejen Talamancas

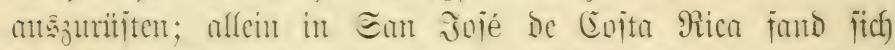

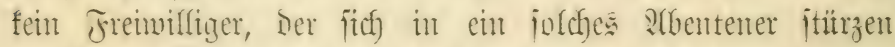

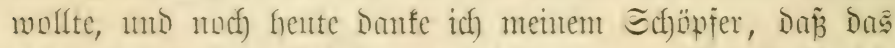

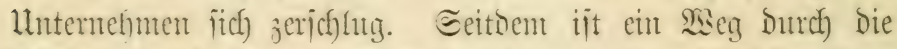

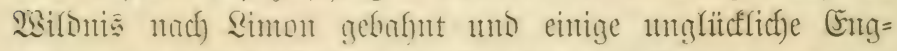

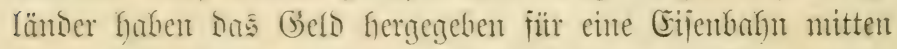
Durch Das Gsebiet Dicjer wilsen Etämme. Bon cinem

1) Siebe Das Rapitel: "Eine verichollene Srabibee" 


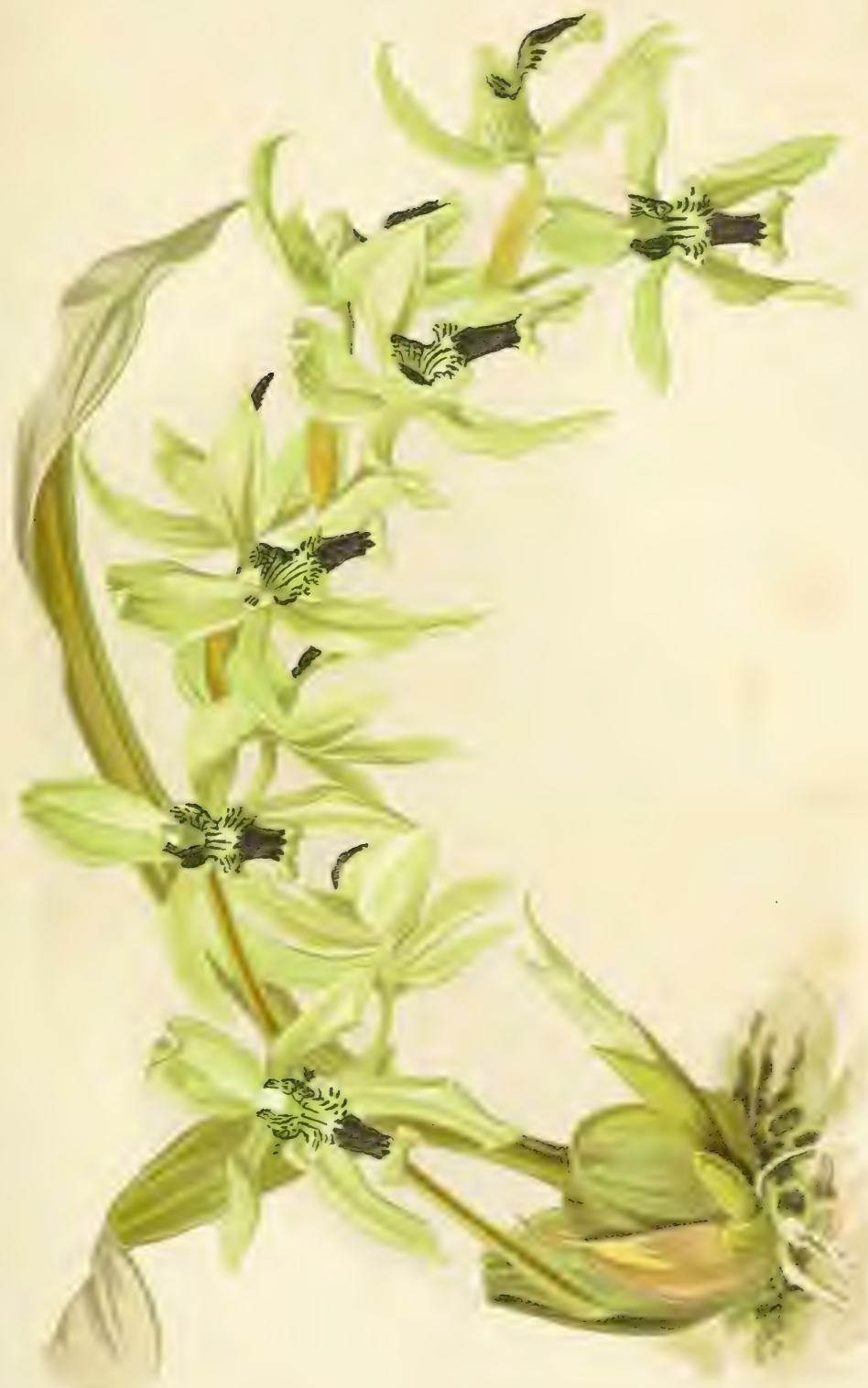





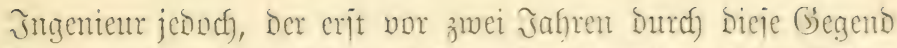

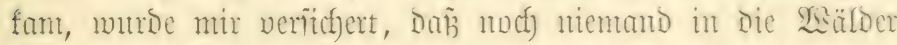

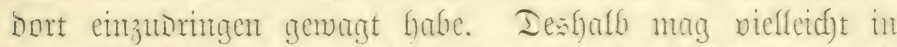
ifnen ein Berbundmisuglied zmijchen Cattleya Dowiana und aurea verforgen jein, mas jenoch chenjogut bezweifelt merden mag.

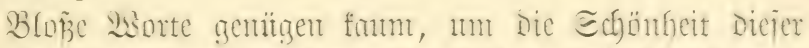

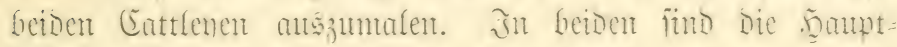
farfen gell und famefintot worberricheno, aber mit jehr widftigen Mindiffutinnen. In Cattleya amrea find dic

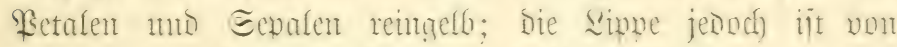

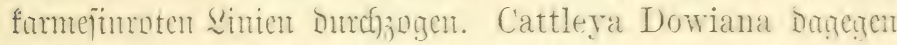

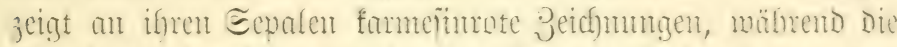

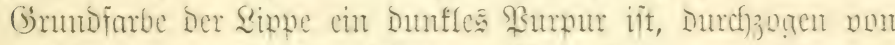

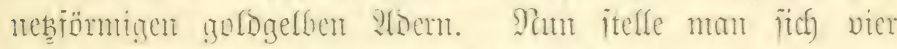
jolcher Bhunen, jede einen flathen Fun freit, an eirent Blitteniticle vor! 9tber Worte reichen da nicht aus.

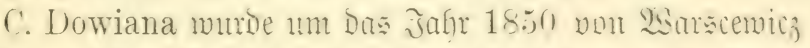

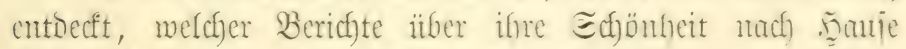
iande, die fanm glanblich ichienen. Ecme ?hajagen muden von manthent fïfl Denfenden (Engländer als gamj numb̈glich) bezeichnet, und ba leider jente wentigen nad) (Eurupa gejanten Prfanzen nutermegs itarben, jo mar bic Eache vorlüurita zu Ende.

Şier mag cin anderer Umitand neueren Datums er=

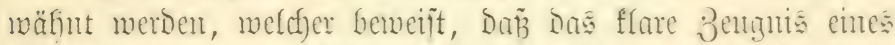

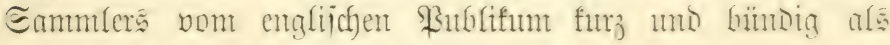
unmöglich rejp. erlogen bezeichnet murbe.

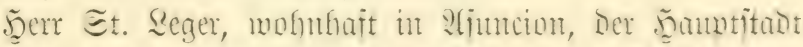
Raraghats, teilte cinem Freunde brieflidf cine vielleidft etmas iehr warm entpintone Bejchreibung einer in jener begento vorfommenten Erchisee mit. Iicje Echildenma erreate in

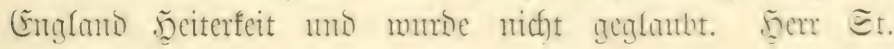




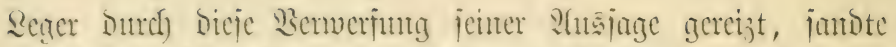
cinige getrodincte Bfumen als Beweis herieffer, um bic lln=

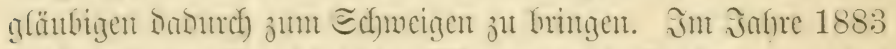

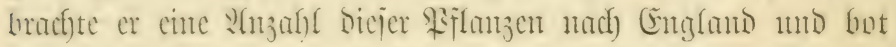

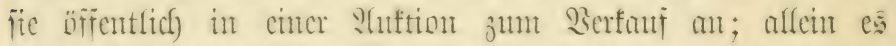

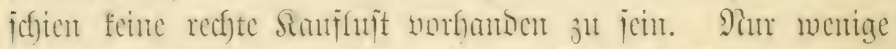

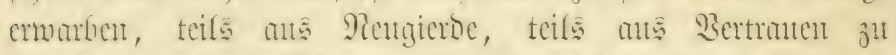
Et. Seger, cintuge Piflmigen für eine jefre geringe Emmme.

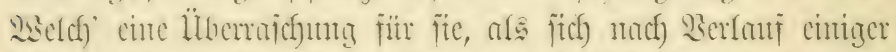

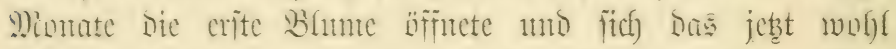

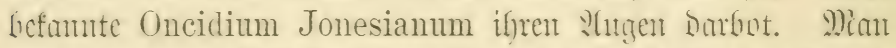

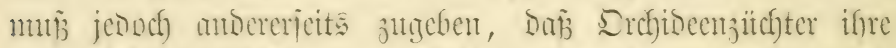

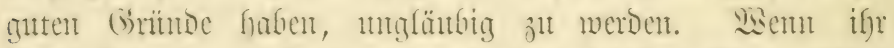

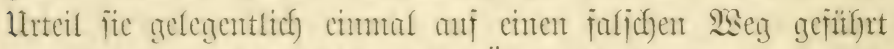

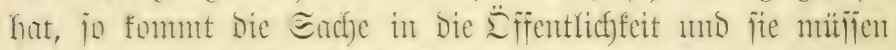

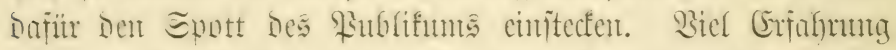

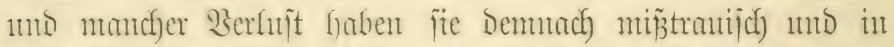

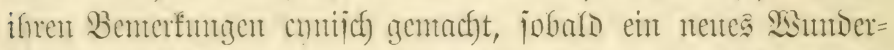
Dinty von Drejisee angertiejen miro. Gerende in Diejent Falle

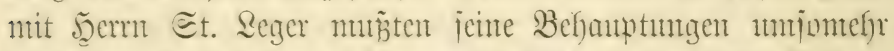

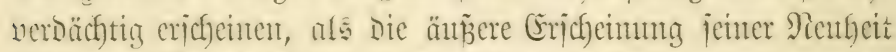
ielir viel sifnulidbfeit mit Oncidium Cebolleta, einer jaĩt

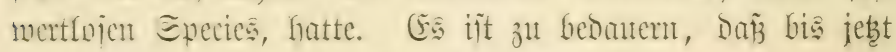
Dieje Schöngeit jefre fifwer zu fultivieren ijt.

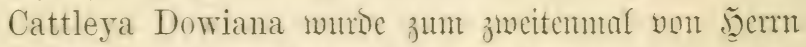

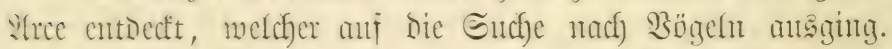

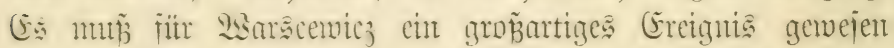

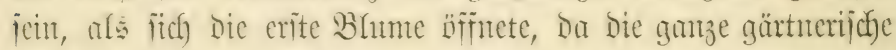

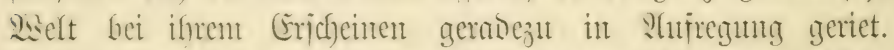

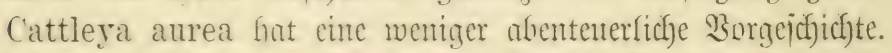

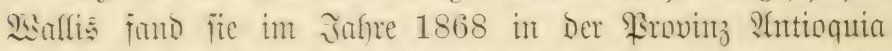

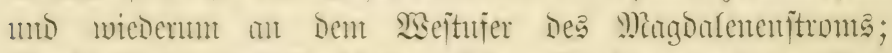
fie ijt jeooch) änjerit je(tent. Dieje Cattfena wiro in ifrer 


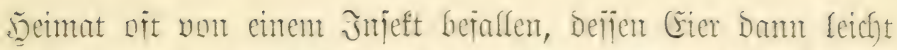

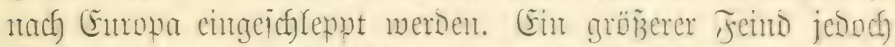
iit Die Strege, welche fich oft an Cattl. Mendellii zeigt, megen beren Riebfaber und Büchter ein Bonurteil gezen Dieje

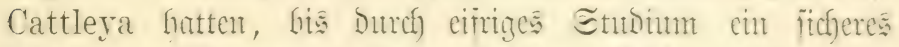

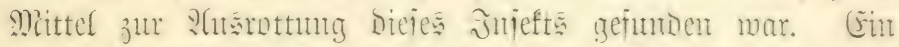

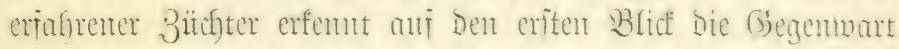

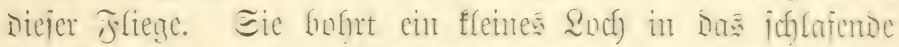

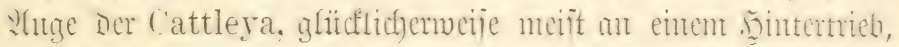

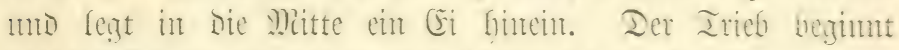

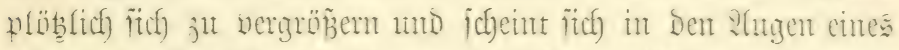

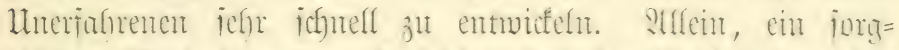

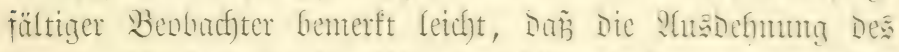
jumgen Irtebs in Die Sänge nicht nit ber jumefmenten

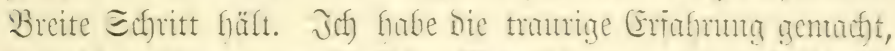

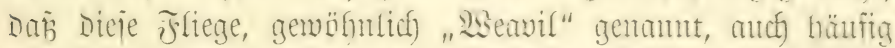
an Laelia purpurata vorfonmt und ïferfant alfe (Eattfenen

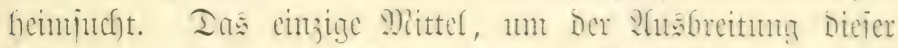
Fflege vorzubengen, bejteft Darin, alle Gejallenen Iete afjuichneiden mo zu verbremen. N2an fam alio Cattleya Mendellii efenjo gejahrlos mie andere Cattlenen intportieren, wem ite nicht ju ciner nuginutigen Beit geionmelt iit.

Unter Die Gerrficfjiten, ieltenijten uno wertuoliten Cattenen iit ('attleya Hardyana ju red)nen, wafhicheinlich) cine nation= fiche 5ngutioe juijchen Cattleya aurea mo Cattleya gigas

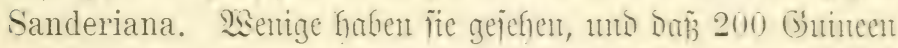
fïr eine Siflange gezahlt werben, ijt abjolut feine Eeltenlheit.

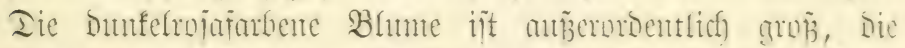
Sippe magentafarben, gofogelb genoert. Qfn C'attleya San-

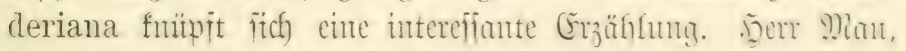

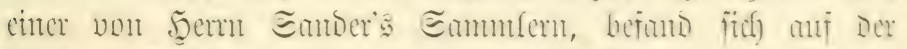
Euche nadf) Odontoglossum crispum in BBognta. Stuf ienten

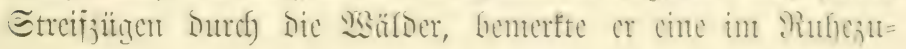




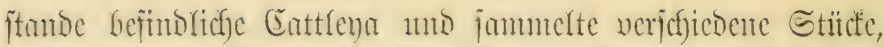

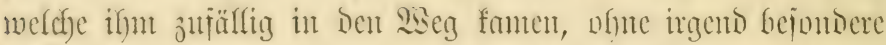

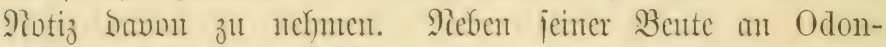

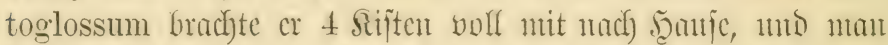

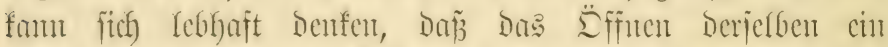

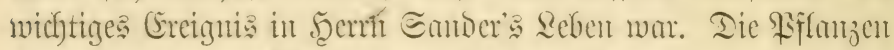

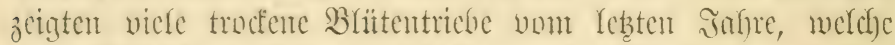

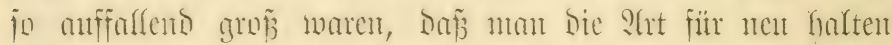

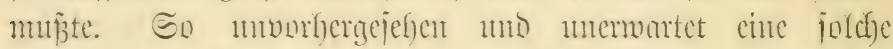

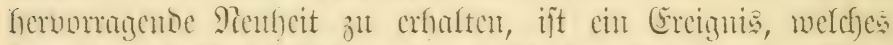

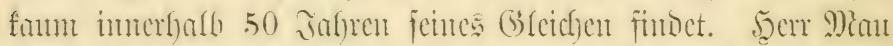

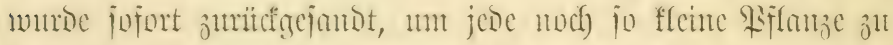

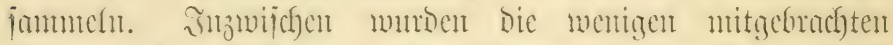

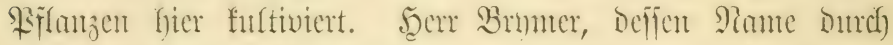
Das Dendrobium Brymerianum uniterblich) gentacht ijt, er= warb cin Etïd, welches muter jorgfältiger Sinltum f̈rd Galo

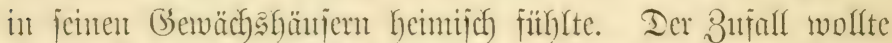

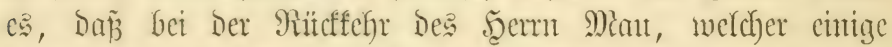

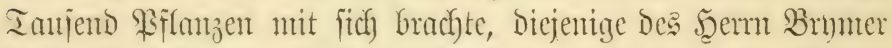
ifre erite Blume entfaltete. Das war ein zrueites widhtiges

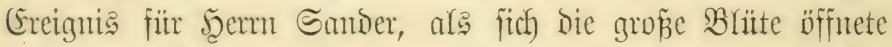
mo inte rofigen Sepalen und \$etalen entfaltete, mo babei

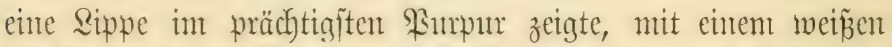

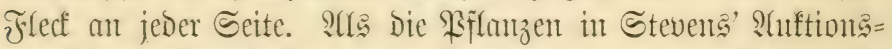
rofale zum Berfanf fonmen follten, war Sem Brumter jo

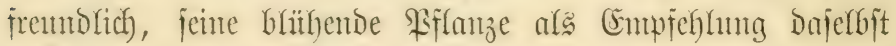

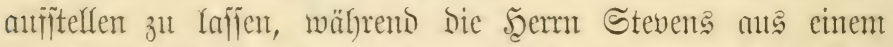

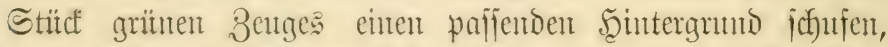

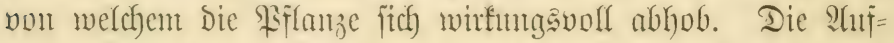
regung, weldye an Diejen Iage anf Der Shlftion herrichte, ift fanm ju bejdbreiben. Ier Grtrag joll bie Eumme vou 2000 \& nod) ïberjafritten Gaben.

Unter Den Gefantejten (Enttlenen, als Mossiae, Trianae,

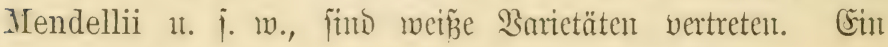




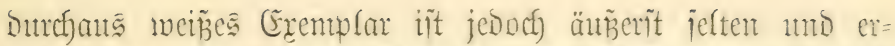
jielt itets enten hohen Preis. Die ichöntite von allen iit Cattleya Skinneri alba. Eeit vielen Generationen fummeln Die Bemohner von Coita Rica jedes fleme Etïcé, Defïen ïe habhait mersen fönten, nm ïe anj Den Däcfern ifrer an Erde gebanten Rirchen anzunflanzen. Roejl mo jeme Dor gänger fautjen ofne viele Überrebung von Den Fiteitem Dieje

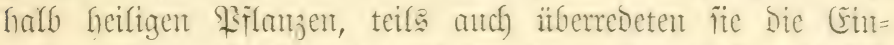

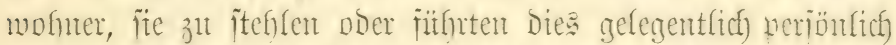

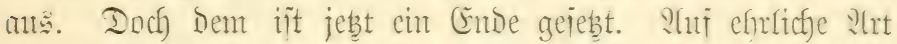

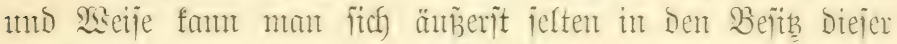

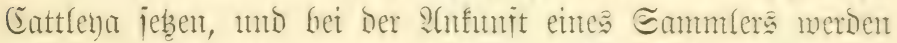

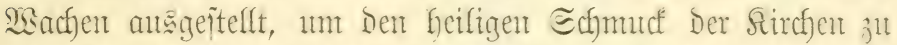

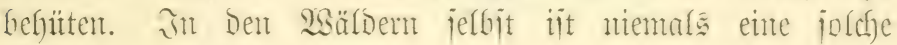
Bflanze angetroffen worden.

Dafielbe gilt von Laelia anceps alba. Iie Gattumy Laelia untericheidet fitc) von Cattleya Dutch Das Borfindon=

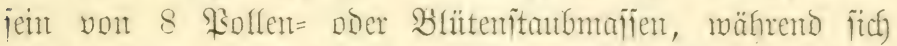
hei Cattleya Deren 4 finden. In meinen ?fugen iit bieic Gattung in ganzen nuch reizender. Saten fömen faum fafien,

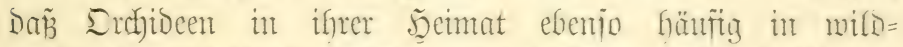
unchjentem 3uitande vorfommen wie Fingerfut und Söwent=

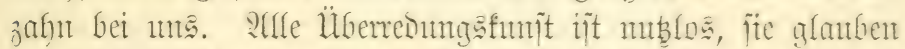

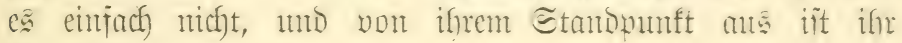
Unglaube zut verîtelyen.

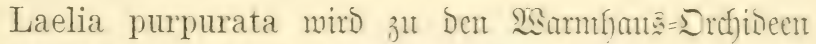
gerechnet, Laelia anceps bayegen erforbert nicht jo arofie Särme. Dhandfe flthtivieren fite im Salthanie, went fie fie Der vollen Eome ansietsen. Eelbit bic jeflechtejte from iit noch ichön ju nenten. Sch juth einit in Der Eammlung bes

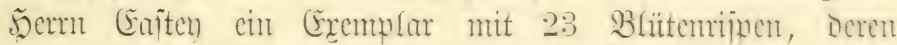

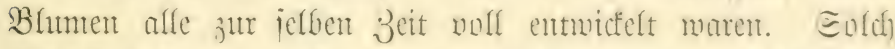

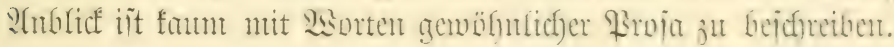




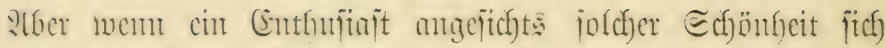

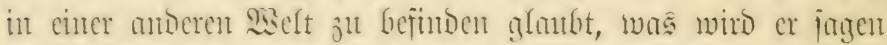
ober cmpfinnen, wenn er bie ichneeneipe 2sarietät, Laelia anceps alba, fieht!

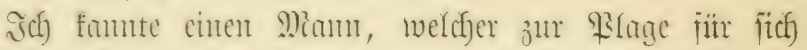
umb annore bei jeber Ǵelegenfeit eine vulgäre Bemerfinty jutr

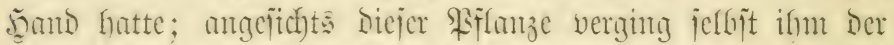

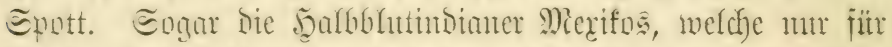

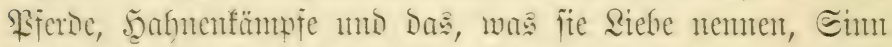

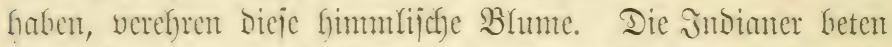
fie arabezut an. Sisie ifre Stanmengenoffen in Sitben Die Cattleya Skinneri alba von Gseneration jut (Śeneration anf iffre firchen pflanzen uns gleichjam als fycilig veref)ren, jo janmeln fie Die Laelia anceps alba uno ffllanzent fie in Der Nähe ifrer bुitten an. Eo jorgïültig jammelı fie jenes fleme Eremplar, Dañ man weder Das eine noch Das moere jemale

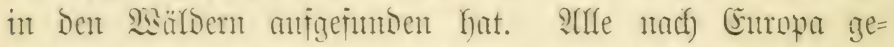

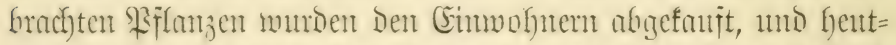
zuttage ift e zll meroen. Lie crite Laelia anceps alba fam vor ca.

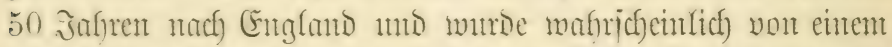

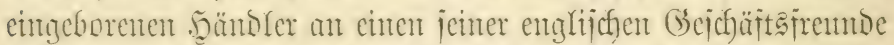

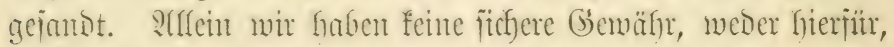

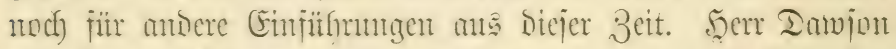

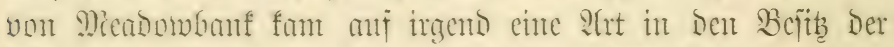

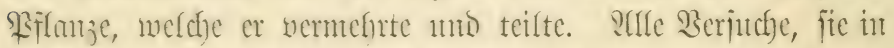

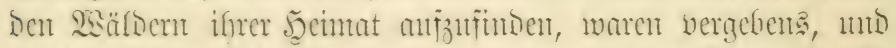

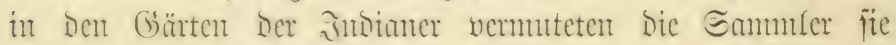

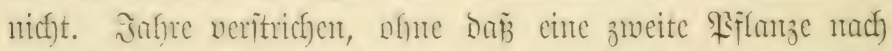

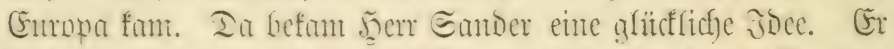

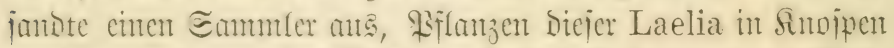

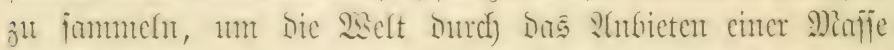

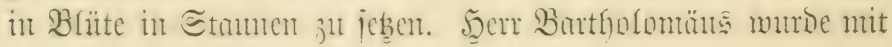


bizier Dififiton beaniftragt, weldhe er infofem löite, als er ca. 40 SFflanzen mit Blittentrieben emwarf. Eorgiältig he=

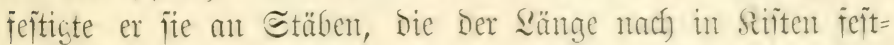

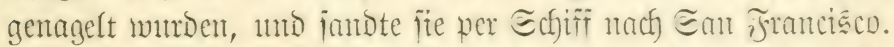
Yon hier Durcheilten ite mit Echnellyngägeidhmindigfeit bie

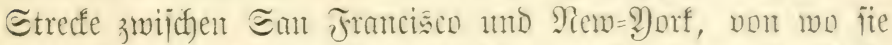
ofne Berzug anf Der LImbria, Die Damals ifne erite Seije machte, nach Siverpool geichiff̈t mutroen. QYlles ging jomeit ganz gut, und mit Bertranten jah) Şerr Eanter Der Galdigen

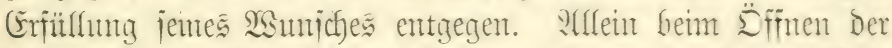

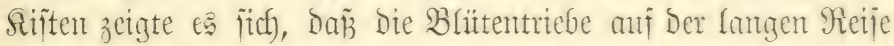
aub Dantel ait frijcher Sufft allejamt vertrochnet waren. Noch cimmal iit Derjelbe Berjuch wieberfolt morden, jedod mit gleichen $\Re$ ejultate. Die Blïtenffnojpen von L. anceps fönmen Die Ecefuft nicht vertragent. - Die Catasetum-2(rten gehören 3twar nicht zu Den Echönheiten Der Framilie; mit ?tuŝnahme von Catasetum pileatum, gemöfnlicf) C. Bungerothi ge= namit, mo C. barbatum gieft es woht feine, welche einer bejonderen Beactutung wert ijt. C. fimbriatum iit zmar jefre Gïbjch ju nemmen, aber nutr jelten bringen wir jie

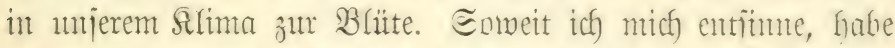
ich) mur cinmal eine Prflanze geichen, weld)e in Begriff mar,

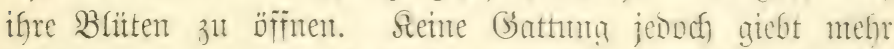
Material zum wijenjogatticfen Etudium mie gernoe bie

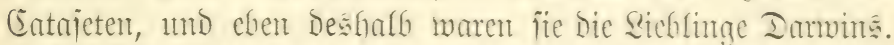
Eelfit Pich)=Botanifer, weldhe aumerfiom bas Gedeihen iffer

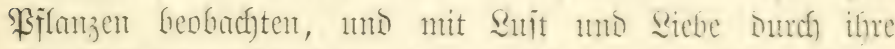

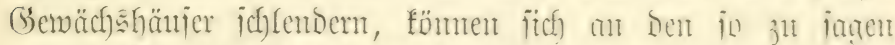
afrohatiichen Borjteflumigen Der Eatajeten ergöbent. Die

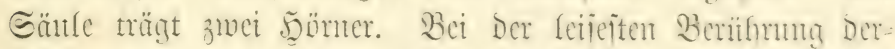

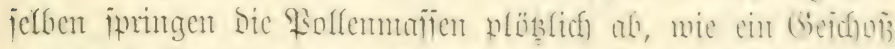

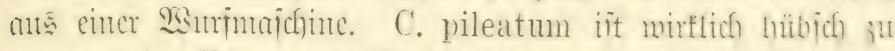

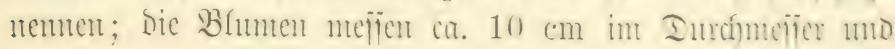




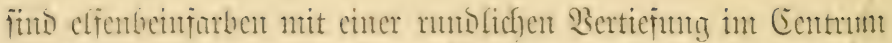

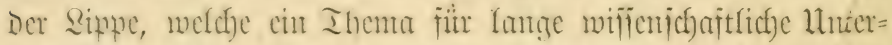

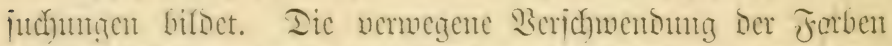

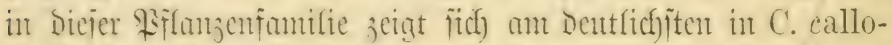
sum, einer Pietheit, melde non Earacas itanunt. Die

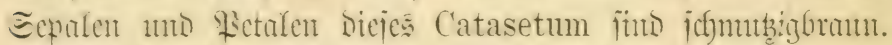

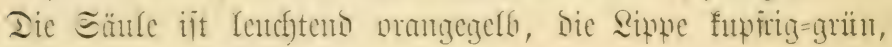
an Der Epike prangegelf.

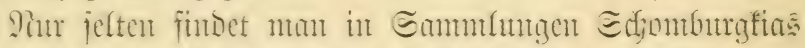

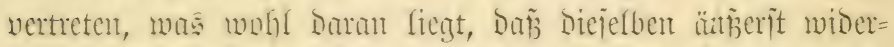

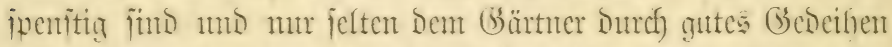

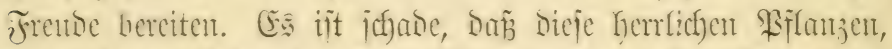
unter Benen Schomburgkia tibicinis Den eritten Rang cin=

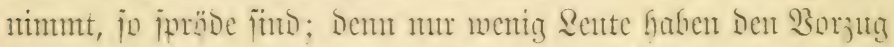

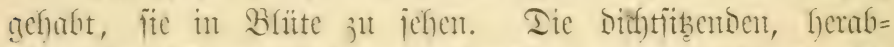
Gängentoen Bhtment Gohent cine Dunfle, murmutrote Farbe, Die

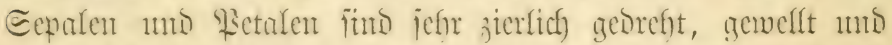

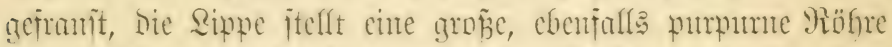

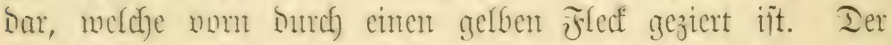

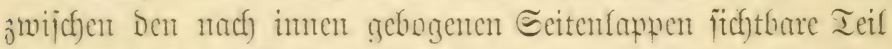
iit vou itarf hervortretenden farmoifinuroten Reitten burchzogen.

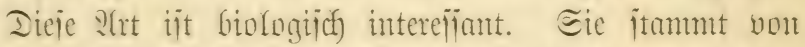

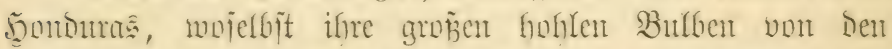

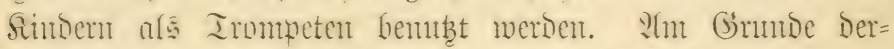

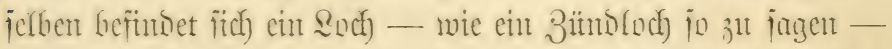
Defijen 3med von Den Botanifern nuch nicht ieitgejtellt iit. ${ }^{1}$ )

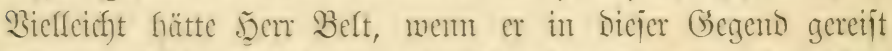

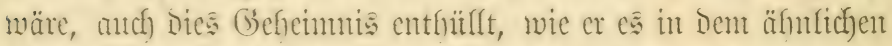

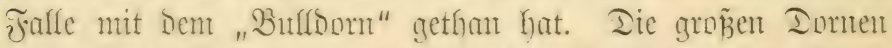

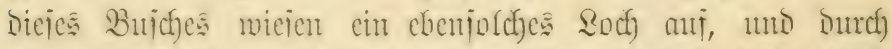

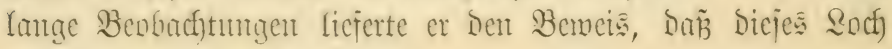

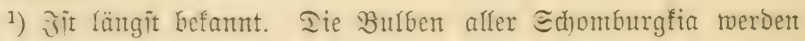
bon Stmeijen betoognt. 


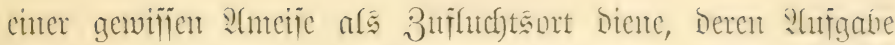

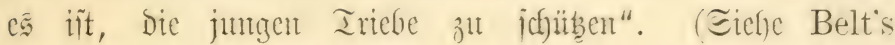
..Naturalist in Nicaragua", こ. 218.) Jntporteme femten nutr zul gentau bas Jnjeft, meldjes Schomburgkia tibicinis

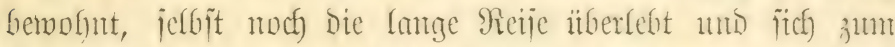
Stantpfe itellt, wem die Siiften geöfintet werden.

Sic meijten Ientrobien finto zut Den temperierten Erchi=

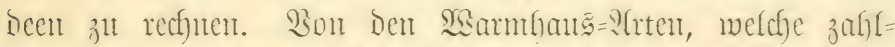

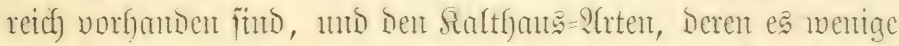

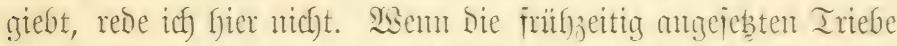
am 1. Juni icfon gut entwicfelt fint, wem bas

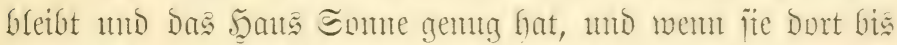

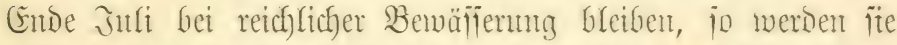

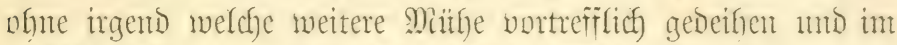

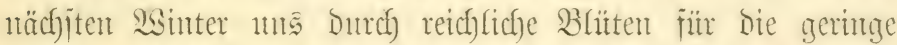

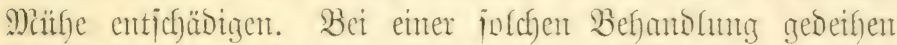
D. Wardiantm, Falconeri, crassinode, Pierardii. crystallinum, unter Umitänton auch Devonianum uns vor aflent

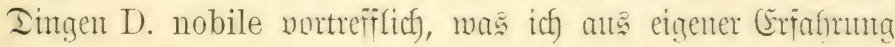
Gebaupten famm.

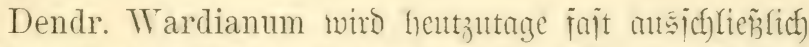

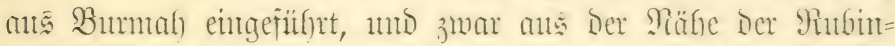

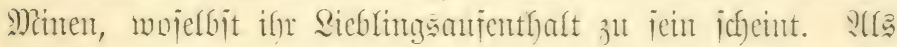

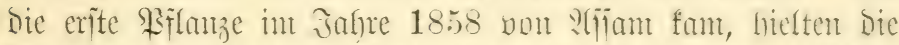
Botanifer fie fïr cine Barietät von D. Falconeri. Dicier

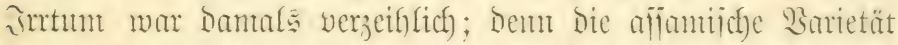
fat mentiger fräftige unto Dabei herabfänngente Butlfen mie mijere hentigen Exemplare. Bor Der : Ymerion von Bumath

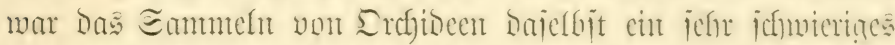

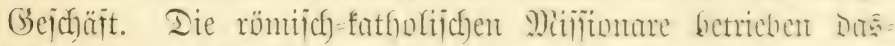

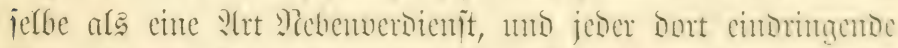

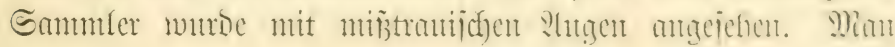


verfot ifm, sie ?hanem Der Etant Bhamo jut verfajien, mo=

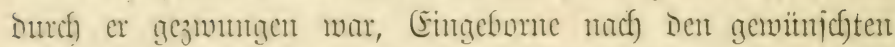

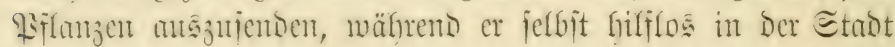
liegen bleihen muß̈te. Eeine Mivalen, Die (Beijtfichen, melde befier mit Den Eitten mo Der Eprache bes Randes vertrant

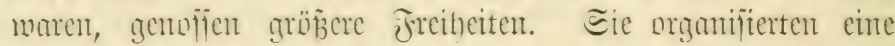

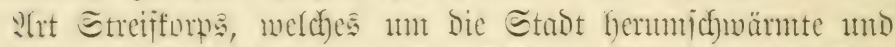

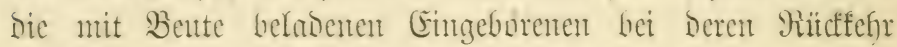
itherfiel. Ilnjweifelhajt crfielt anch irgend jentand ben

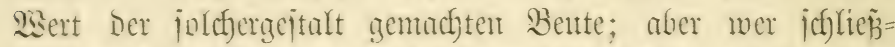

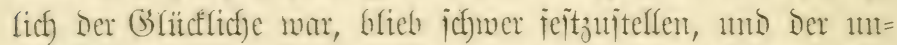

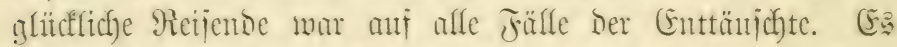

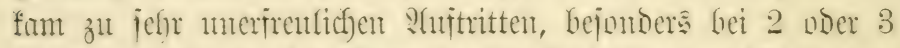

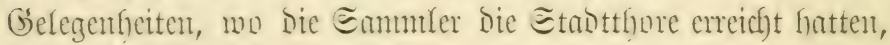

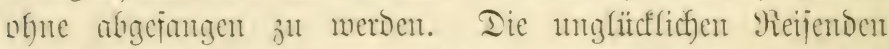

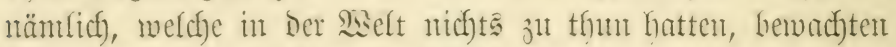
furtwäfyrento sie 4 Ifore Der Etant, rannten von einent 3 !un

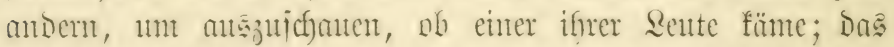

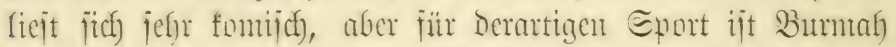

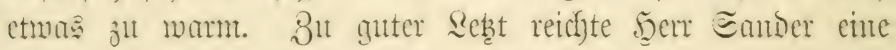

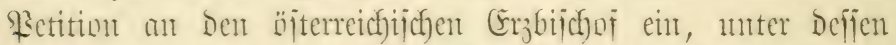

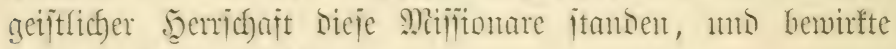
baburd) einige (Erleidyterung.

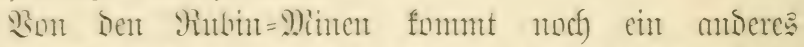

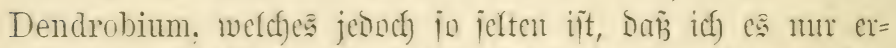

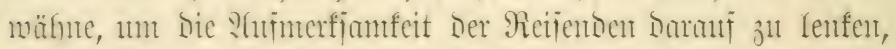

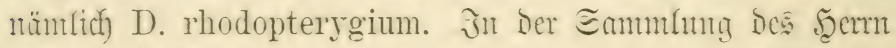
Irenor sampence befimbet noer hefand fich cin Exemplar,

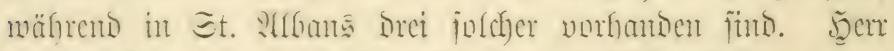

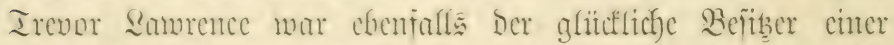
iffarladfroten Epecies non Błurntah; allem bie Pitlanje ging cint, benur ite cincu ?anten exhelt, nus fein jueites Exentular ift

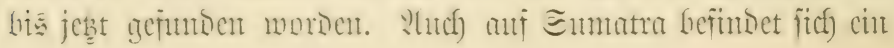


ioldes icharlachrotes Dendrobium. D. Forstermanni, weldes

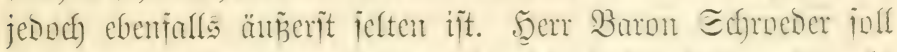

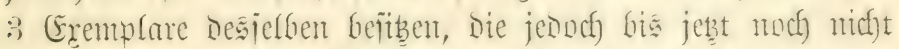
geblitht haben. Ein anderes iefrr intereflante Dendrobium nou Burmaf) iĩt D. Brymerianum, bei Den mir jeiner CEnt=

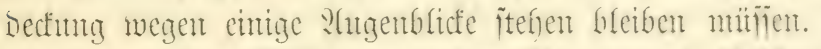

(5)

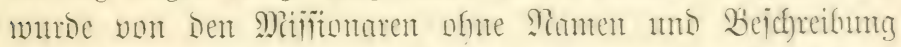

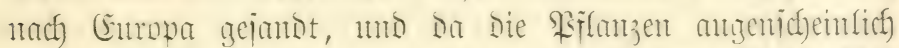

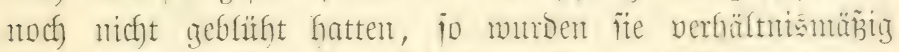

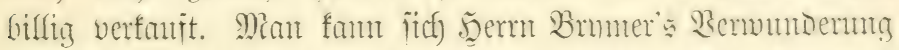

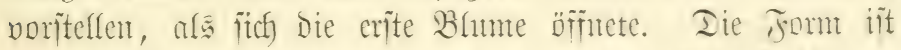

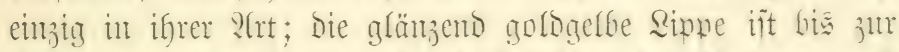

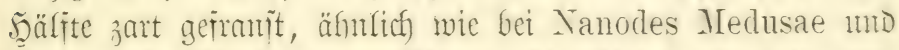
Brassavola Digbyana. 然arum bie Natur es mit einer jold anfifalfenden sippe veriefen hat, ift cine Frange, weldge fitf), wie vicle andere, jedem, anch Dem gedanfentojeiten Srchibeenziuchter, von jelcit anforängt.

Dendrobium nobile ijt io gut befaunt, bah e faum ciner Ermäfnumg bedari. Bielfeicht fonme Die Beit,

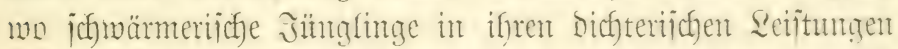

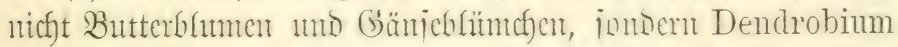
nobile anfütgen; mtr mit einent guten Reint wirto es ieine Ectimierigfeit haben.

Picht io gewöhutlich iit Dendrobium nobile rar.

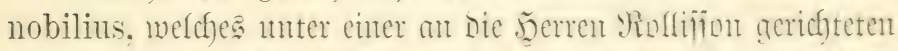

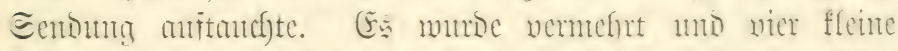

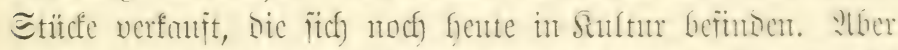

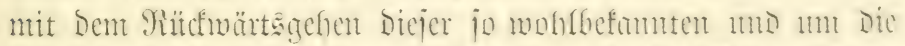

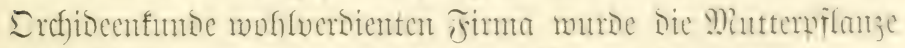

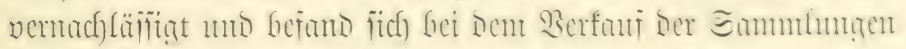

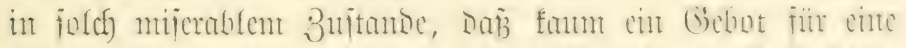

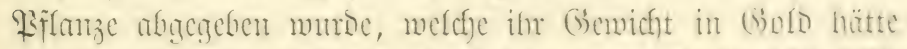

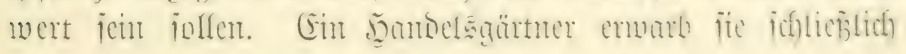




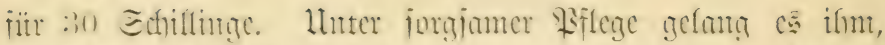

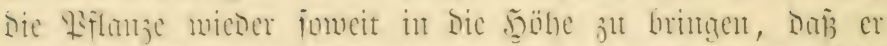

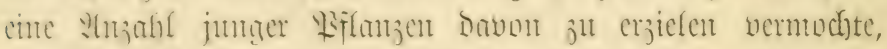
muranf er bic Minterpflanze für 40 L verfanfte. Plfer von

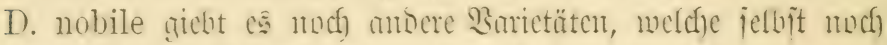
wertuoller als $\mathrm{D}$. nobile nobilius finto.

D. nobile Sanderianum hat vicf sïfnulichfect mit

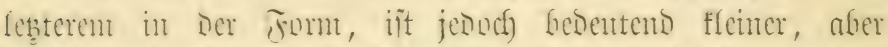

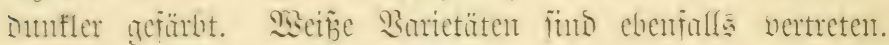

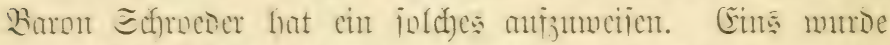

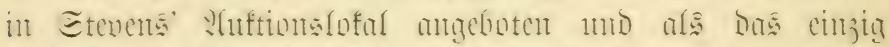

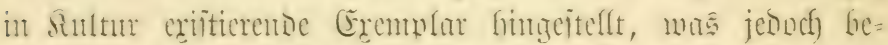

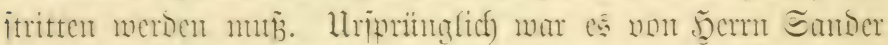
mit D. nob. Sanderianum impurtiert morner. 43 ! murnen

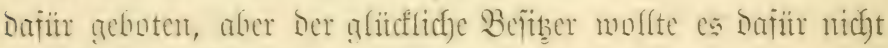
hingeben, Da sllginos bei Dembrobien iefnr ieltent finto.

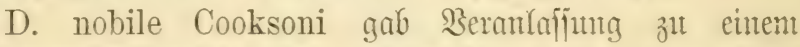
untiebianten פ)

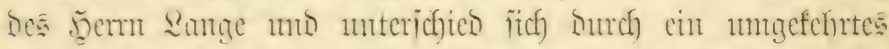

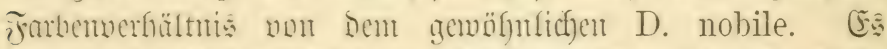

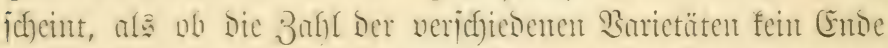
hat. 13 sem allgentein befant wäre, wie viel intereffantes

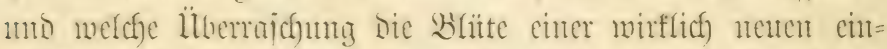

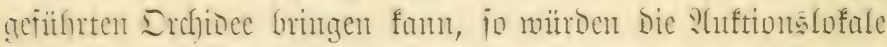

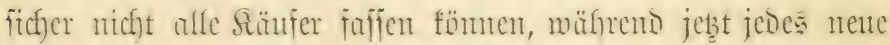

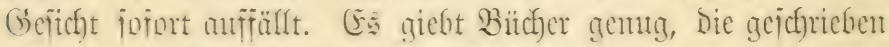
finto, um mehr Sich)t itber Dicje Fiflanzenfantile jut verbreiter.

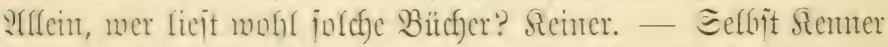

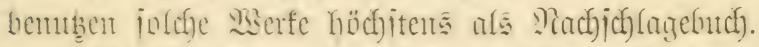

Die Eepalen ant Petalen Des D. n. Cooksoni fino an

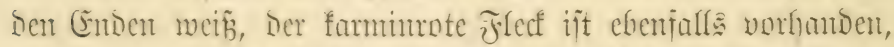

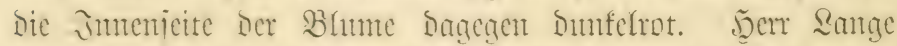

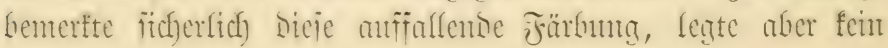




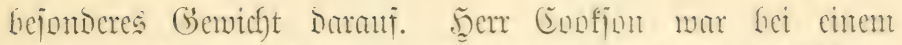

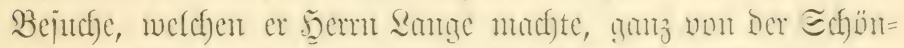

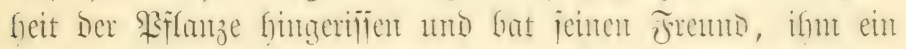

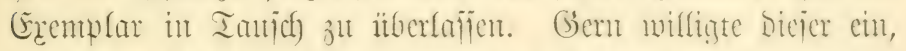

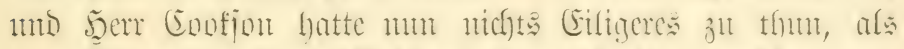

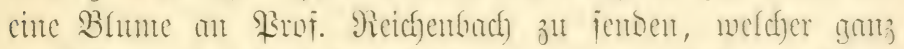

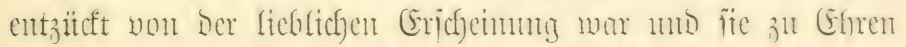
Des Eenoer: D. n. Cooksoni numnte. Ingegen mroteitiente

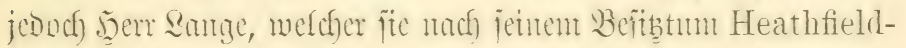
sayeanum bentunt mifien woflte. Reid)enfach) jesod) meigerte

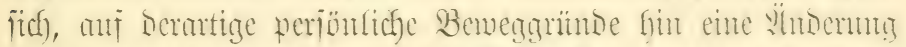

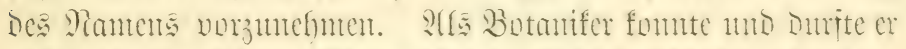
nicht andor verfafhen: :

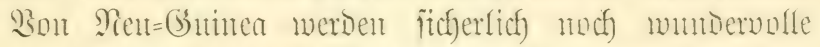

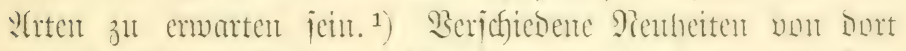

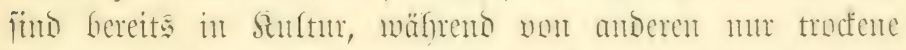

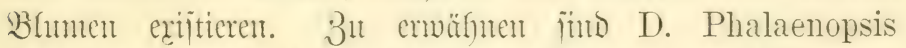
Schroederianum, D. Goldiei, cinc Bintetät von D. superbiens, aher nuch gröżer als Dieje. In tisii, fenter I). Broomfieldianum, wefches an Laelia anceps

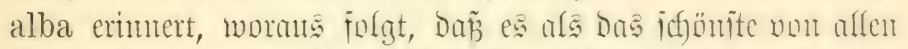
Dentrobien zut bezeichnen ijt. Sie Ectjönfeit Dicier Enectes

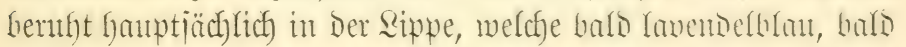
rot angehautcht eridheint. Sine anoere s(rt ijt jefhe mahe mit D. bigibbum verwanot, jeond) größ̌er in Fon nut mit ppizeren Sepalen; fie ijt D. Statterianum getnuft moroen; Die

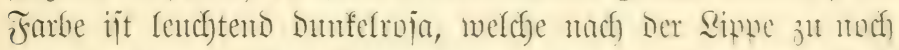

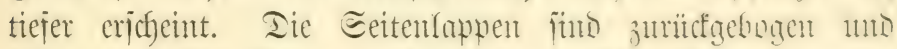

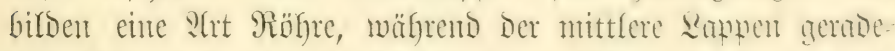

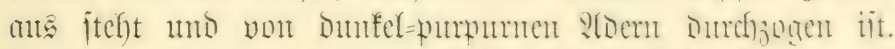

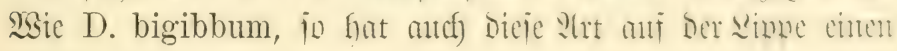

1) Eind inzmiid)en eingetrofien; Dendrobium Victuriate Augustae

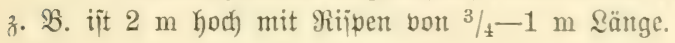




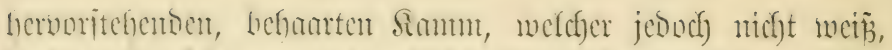

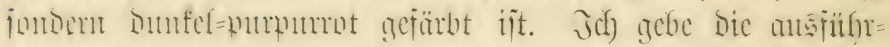

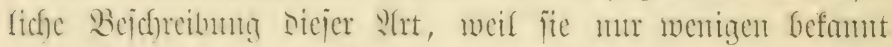

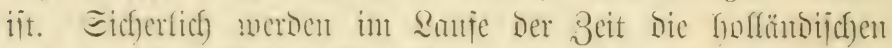

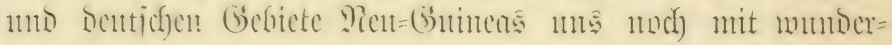
Garen Renteiten verfehen. ${ }^{1}$ )

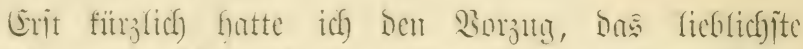
Dendrobium. meldes ich je gejefien flabe, ju fremundern: D. atro-violacemn. Dic prëchtigiten Błhnten fiängen wic Iranben fommter, ca. 10-12 an einen Iriche. Die Eepraten

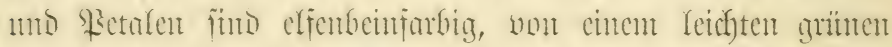

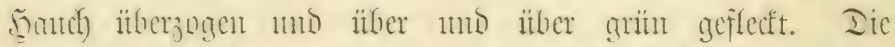

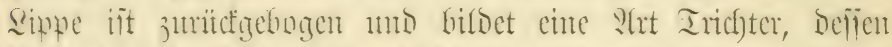

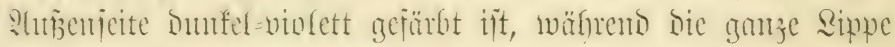

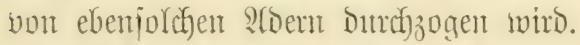

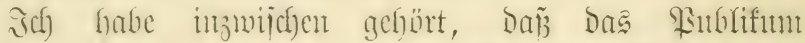

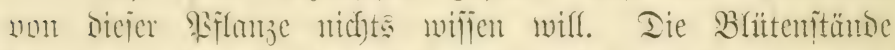

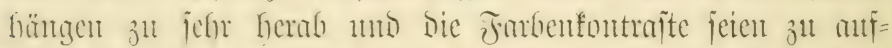

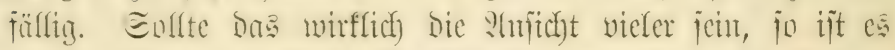

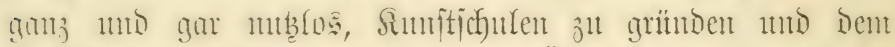

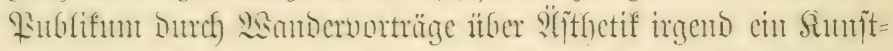

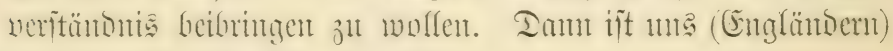
ciu Siun für Sumit verjagt. ${ }^{2}$ )

1) Sef)r ridhtig! Saga das Deutiche (bebiet betrifit, jo jitto jedod)

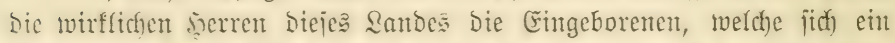
(Findoringen in ifre bebiet ebenjo energijd) wie erfolgreid) verbeten haben.

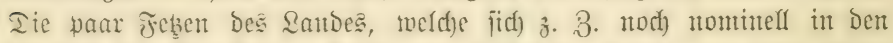

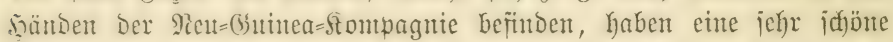

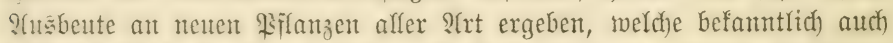
wijienjchaitlich bearbeitet ïnt. Das Borbringen anderer Sammler haben trow Der von Berlin aus erlafienten Weijungen Die Dortigen S(utoritäten nach) Mëgliçfeit gefindert.

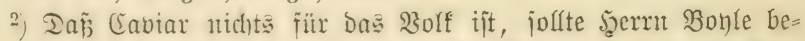
fant iein, wentgitens mujte es vor mu 300 Jahren bereits ein Eng= Yänber, mag iein Plame Shafeipeare ober Baco gelautet faben. 


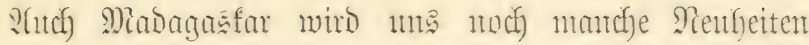
Iiejerr. (5. iiberrajcht. Ia ñ ein jordes

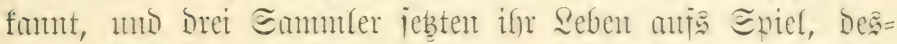

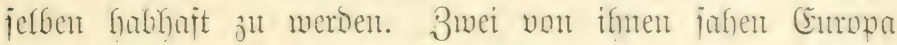

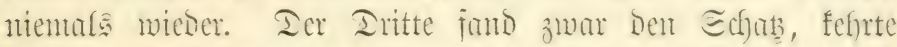

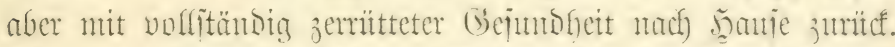

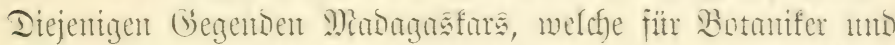
Eanmuler am meiften interefie haben, mitifen in Der That

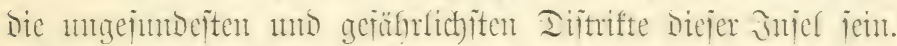

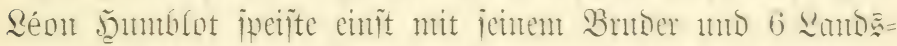
lenten in Tamatave, won wo ite ifle Erforichungarecie zut

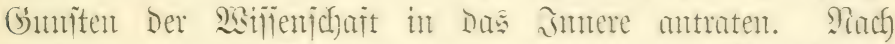
3erfanf von 12 Dionaten war er ber einjige lïherfebento.

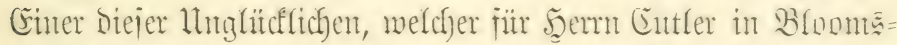
(ntri) Etrect, Eomonon, Echmetterfinge mo Bö̈gel jommelte,

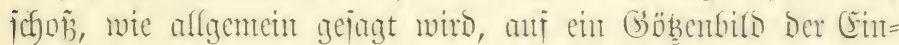
geborenen. Q.u

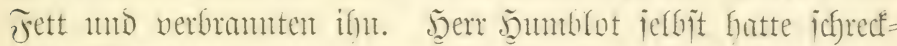

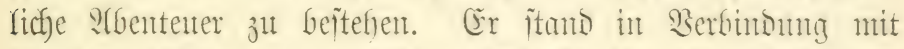

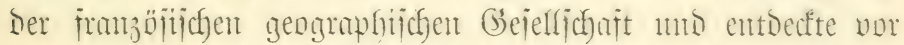
ca. 10 Jafren Phajus Humblotii uno Ph. tuberenlosus in

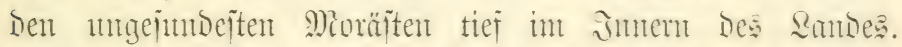

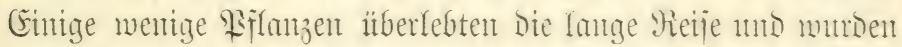

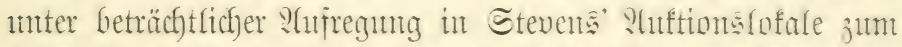

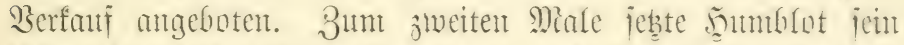

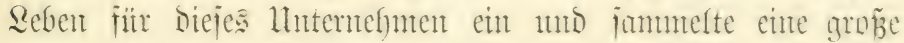
Mienge fït bुerrn Eander, aber um welchen Rreis! 3mölf

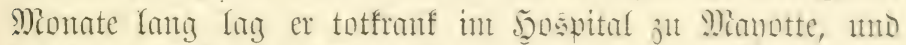

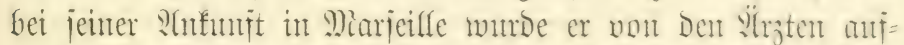
gegeben. Ph. Humblotii ift in Der That ein simoner nou

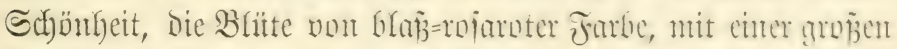
Dunfelroten Ripne uno ciner hellgrïnen Eünte verjechen. 
Ein jener, meldfer jeinen "Iormun" genitgento femut,

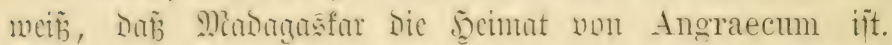

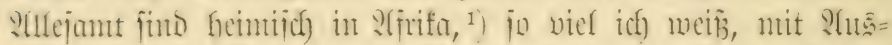
ntafme Des A. falcatum, melches jontorforemeije von Japan itanmut. Dian mun in Der That vernuten, Dan bicje Epecies

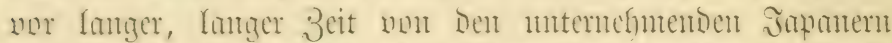
nach) Dorthin gebradht uno afflimatifiert mure. Egenjo fomiich) ît, Dañ Dic einzige Aërides mo cin Dendrobium,

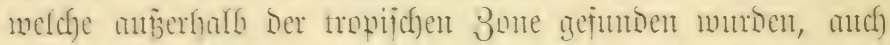

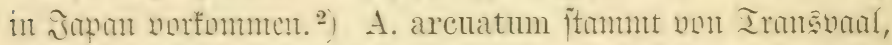

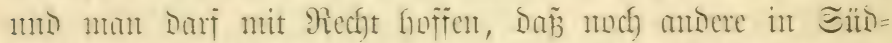

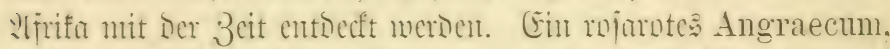

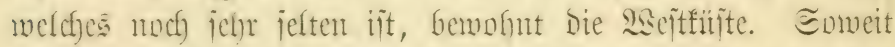

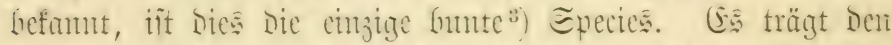

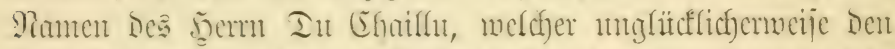
Finnourt vergejen hat. selle B̧erjuche, Den Etantont in jein

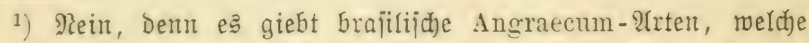

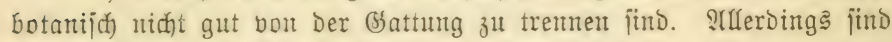
eร jäntlich) fleinfrütige 2trten, „botanical Orchids" im ichlimmiten Sinne bes Wortes.

2) Dieje Methode, pflanzentgeographifde હctwierigleiten zu

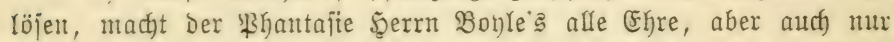
Diejer. Mestwegen Die Japaner, falls fie je Madagaß̌ar betraten, må abjolut unermiejen ijt, gerabe eine direft unjob̈ne Bflanze mit nach Şauje nahmen, Deren fiultur nod) Dazu gar nidft leidft iît,

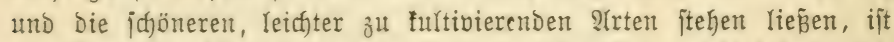
eine frrage, weldje jeíbit unjer phantajievoller $\mathfrak{A}$ utor nidjt fofort miro

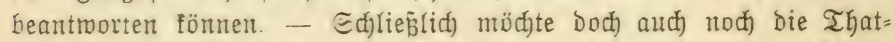

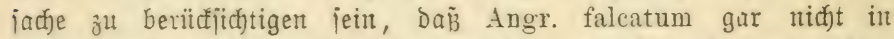
Madoagasfar twädjt, aljo nid)t von bort irgent rofit eingejübrt werden founte.

3) Angraecum Chailluanum ifit nicht bunt, es ift tief crênte= farben und hat etma bie Jarbe von etwas angealtertem Elfenbein.

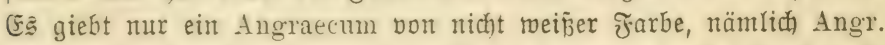
citratum, bei roldjem biejer gelb=meiñe farbenton nod) ctroas mefr poncé i ijt. 


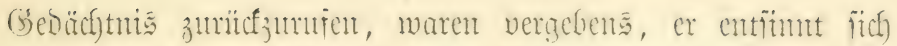

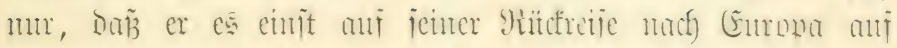

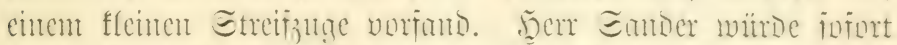

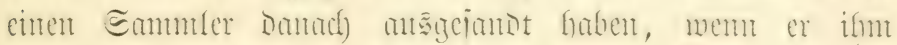

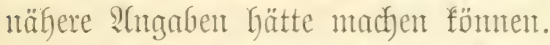

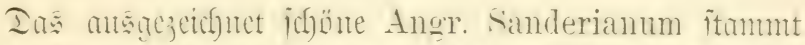

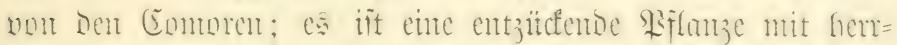

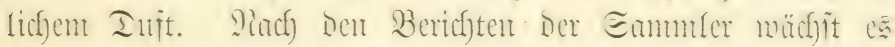

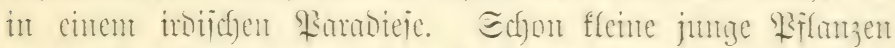

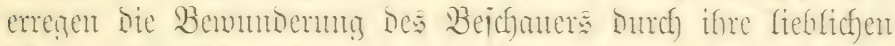

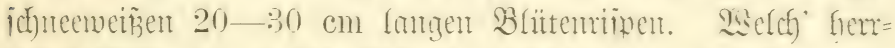
liches Bild ein in Et. N(than gejüchtetes aroize Exemplar mit meterlangen Blittenripen bot, ift jefruer zlt be ichreifen. A. Scottianum itumunt von Barzibar mo mune

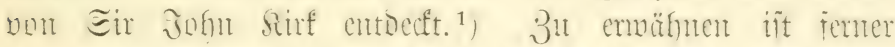
A. cautatum nou Eiema Seone, meldes Durch iente Enome, bie $15-20 \mathrm{~cm}$ lang fint, an A. sesquipedale erimert. But biejer (somple für noch A. Leonis no Kotschyi zh recfunt Deren Epontre Die Ränge von ca. $10 \mathrm{~cm}$ er= reichen, währento Diejenigen von A. Scentianum uno Ellisii

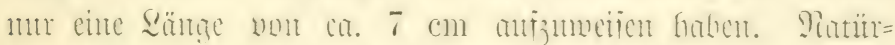

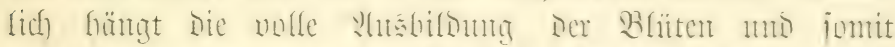

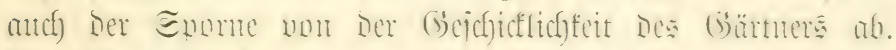

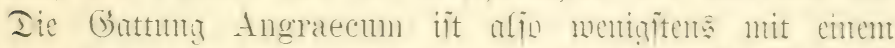

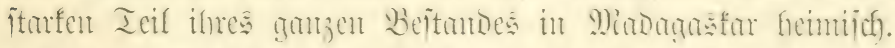

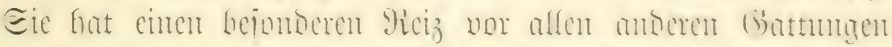

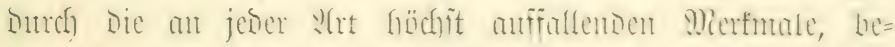
jonters in ber Etruftur ber "Ë̈ute", aljo gerribe in Dem Engane, weldyent alle Botanifer Die hördite Beachtuna

1) Rein. Der Entrecter Dieier jänen Pflanze ijt ein Deutider

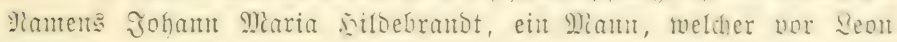
Sumblot uno befier als Elis unjere Sientutis der flora von Mabagą̇tar geförbert hat. 


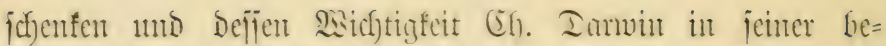

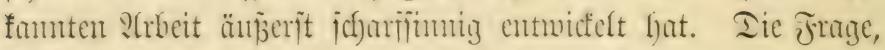
wh bie Pflumze Angraecum oder Aeranthus sesquipedalis

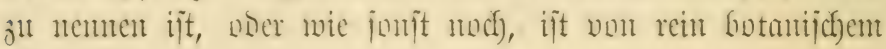
जntereffe.

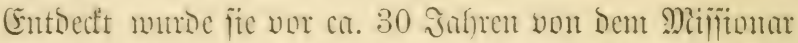

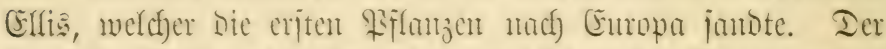

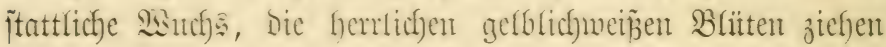
Die Bemunterming aller mij jitch), und Der Eporn bietet ein

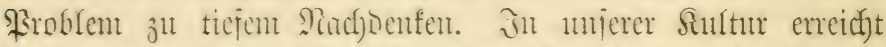

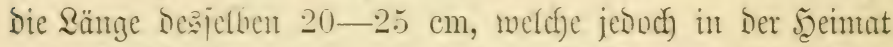

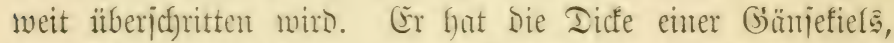

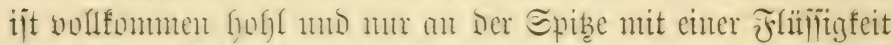

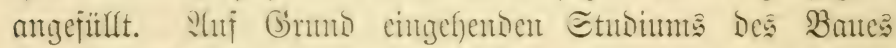

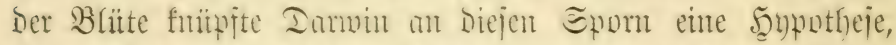

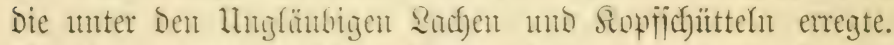
Bei ber mugemöbulidyen \&änge des Epontes madyte er

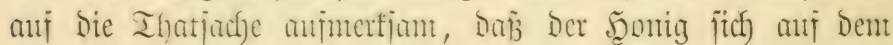

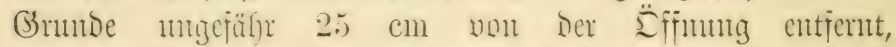

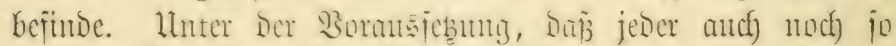

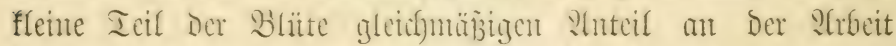

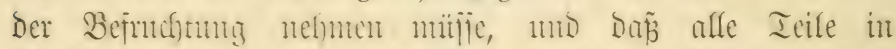

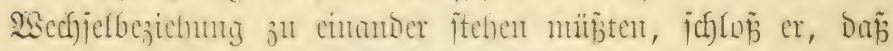

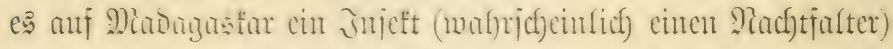

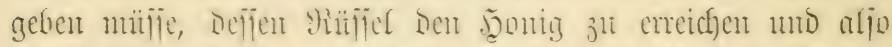
Die Boffenmajien menjutfolen imjtande jei. Sä̈re Der Neftar

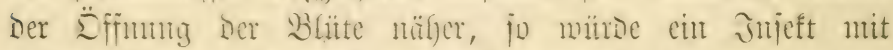

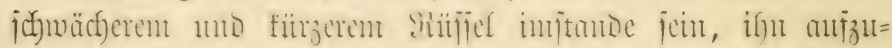

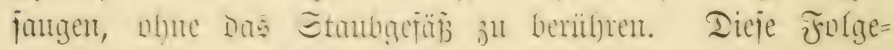
rung zeigt bie intenjue geijtige simift Dombin in iffrer ganzen

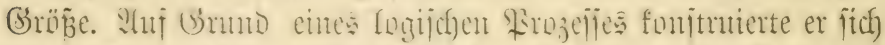

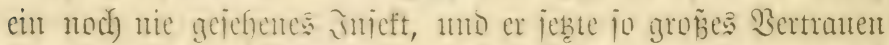

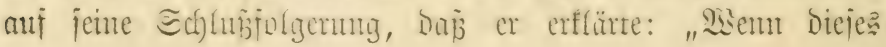




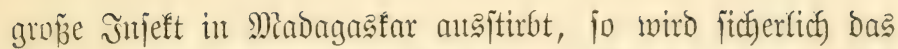
Angraecum sesquipedale ebenfalls zu Gruttoe gehen." $\$ \mathfrak{b}$ Dorming Befantutung Durch bie Afuffindung eines folchen

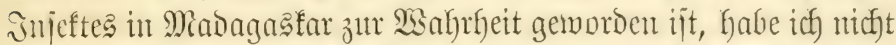
feitjtellen föment. 'Stber man lacht nicht ftraf́los ïtber Darmint. Range vor jeinem Tobe mutrbe in Sitd=Braftien ein Nacht=

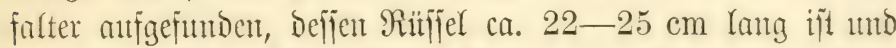
Desfall zur Befruthtumg Diejes Angraecums lang gentug wäre. Bei Dem heutigen Stant Der gauzen Frage herrifht jedoch

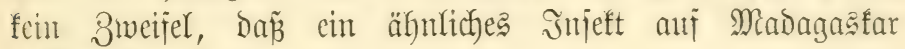

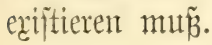

\section{Addtes fanpitel.}

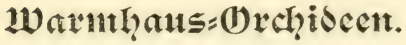

In Den vorfergeffemben Sapiteln habe id mein Bejte

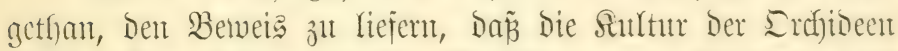
abjofut fein Geheimuis ift. Die Naturgejebe füno flam umb

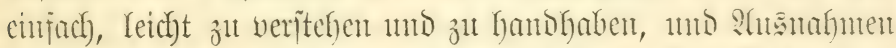
gient es mur in einigen wenigen Fällen. Dic Sultur ber

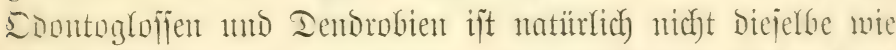
Dic Der Rojen; aber eine einigermañen intelfigente Ferjon lent

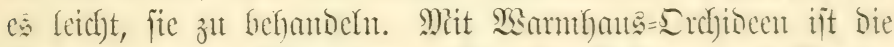

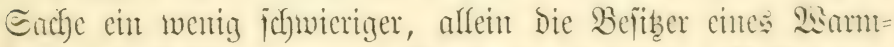

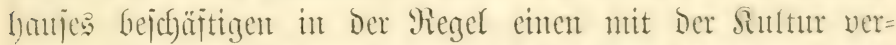

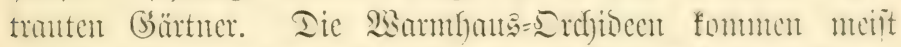

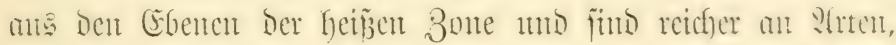

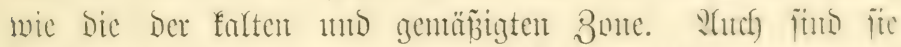

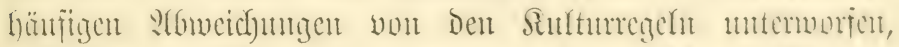
mo eben beshalb nicht jedcrmtann jut emtefeflen. Eie ner= 


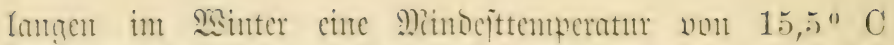

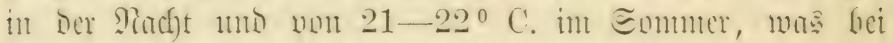

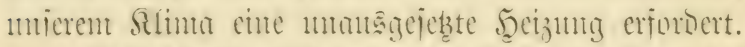

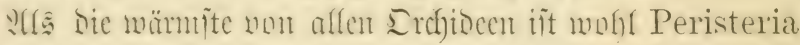

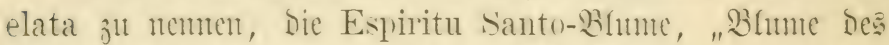

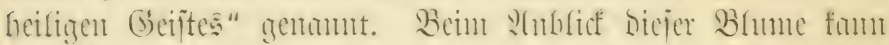
man bie Echmämerei ber Esminer weritchen, als ifnen bie=

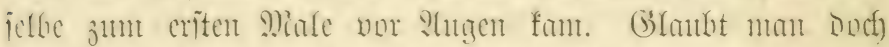

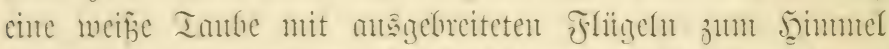

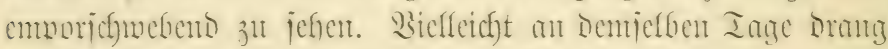

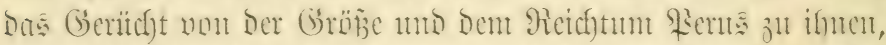

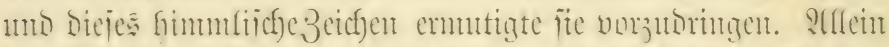

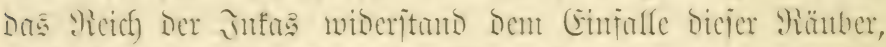

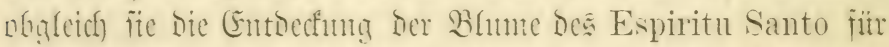
cin guintitige 3eichen bielten. Peristeria elata it cine jo

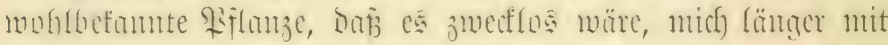

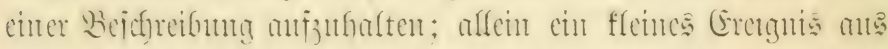

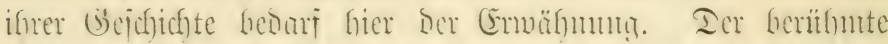

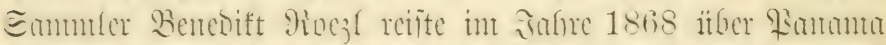

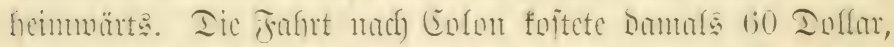

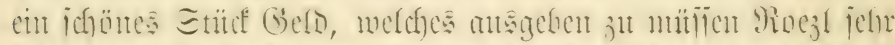

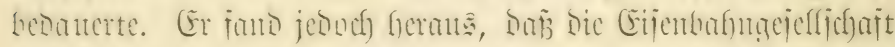

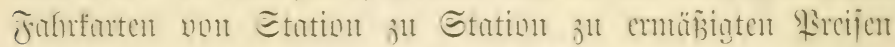

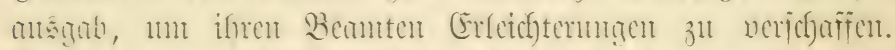

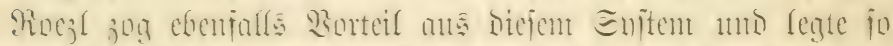

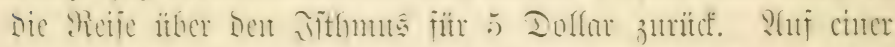

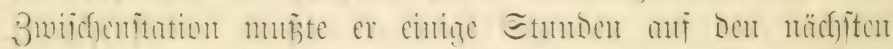

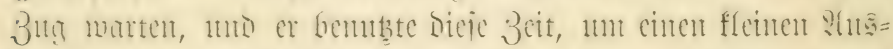

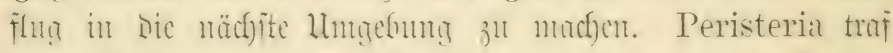

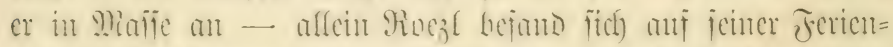

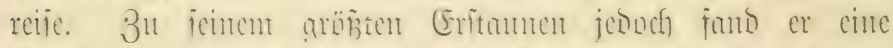

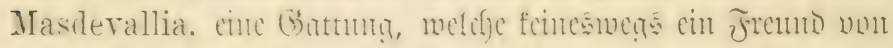
alfuniel Eome it, im brisciten Eommenichente, Ecite an 
Seite Der Peristeria. (5r foumte Dem Irange nicht mider=

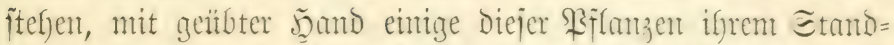

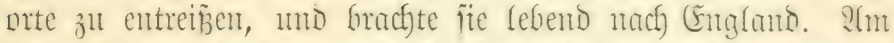
felfon Inge, als fie zum Berfauf angefoten murden, fam Die

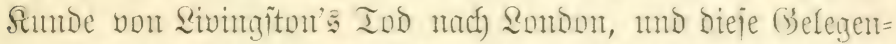
feit bembend tante er jeine Rranze M. Livingstoniana. Purr wentige feruten ben Etandort biejer ielteniten alfer Mcas = Devallien, mo fein (Exremplar ijt ieitsem mieder nach (Emona

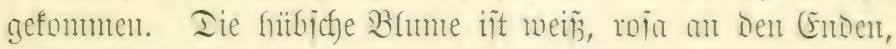
mit getben Echmeifen an ben 3 Eepaten. Eie gebieh in Der nä̌chiten Untgebung ber Etation Enfebras an Der Ranama=

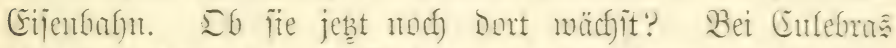

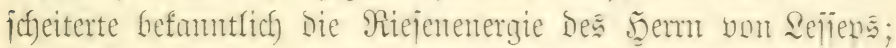
Die begento fatte menigiten voribbergefento ein ganz anderes

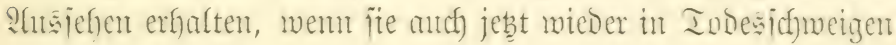

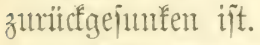

Die BanDen gefören zweifellos zu Den "würmiten" Gattungen, und nimmt Vanda Sanderiana Den eriten Fluts

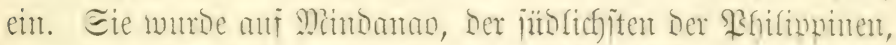

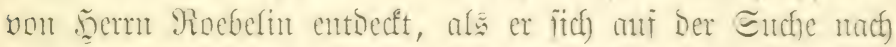

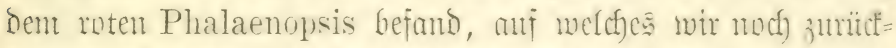
fommen merden. Vanda Sanderiana iit bie immojunteite

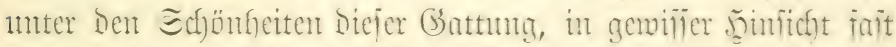

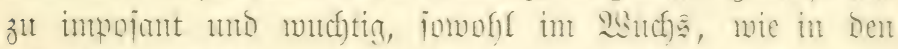
gewaltigen Bhäten. Dicic hahen cinen Durchmefier non

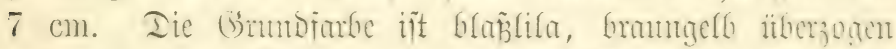

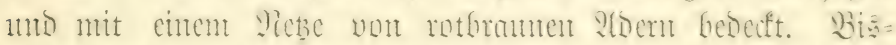
meilen fitzen mehr al: 12 B Bliten an einem Blittenitanto,

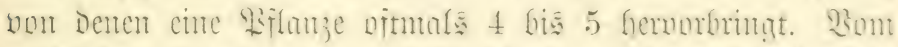

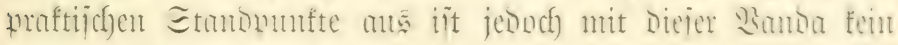
gutes Gejechäft verbumbent.

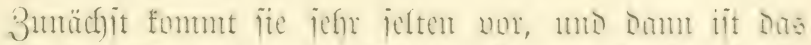

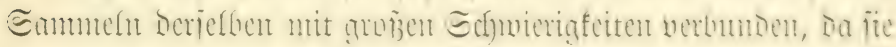


hodf Dben auf Bäımen wächit, welche zu biejem Bmecfe ge=

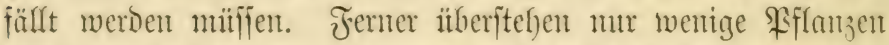

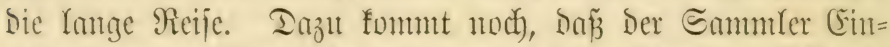
geborene zum Eammeln anftellen mun, weldye pro Bfflanje,

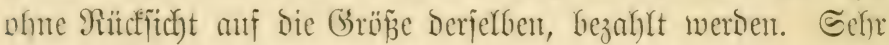

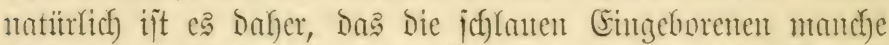

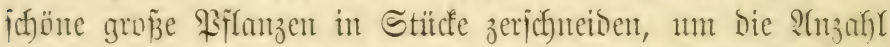

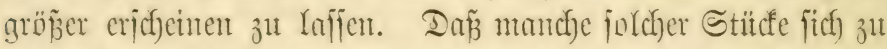

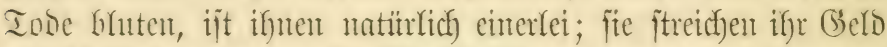

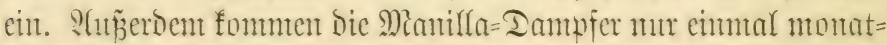

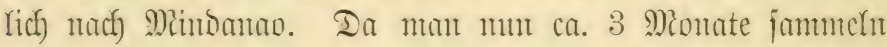

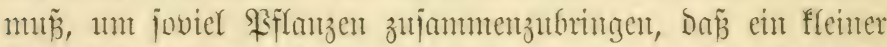
Borteil Dabei heranfonmt, jo haben am Gnde biejer Beit alle Fiflanzen, welche in Den erijten Iagen gejanmelt murben, iffere Rehengfraft eingebuint; Dem Vanda hat feine Butllen und

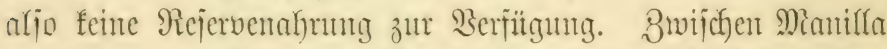
und Singapore ijt unt alfe vierzefn Tage Dampfichifffahtots= Ber=

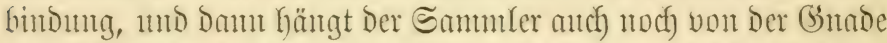
voer Untynade des Sanpitäns ab, ob bicjer eine jolche Radumi an Borb refmen will. Jnt Gejahenden Falle errichtet ber

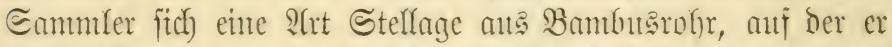

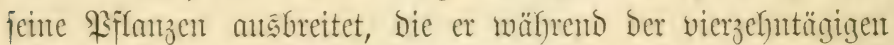

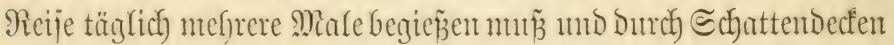

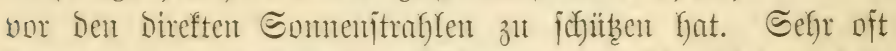

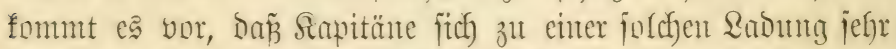
jchwer ober gar nicht veriftehen wollen. In Singapore an=

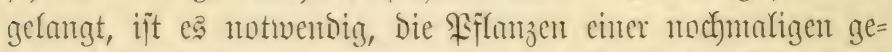

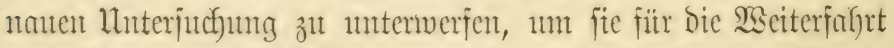
nach Şatje verjandfälig zu machen. S(tuch) Dam mirb oft

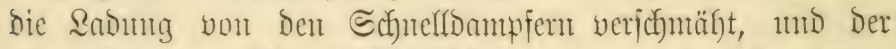

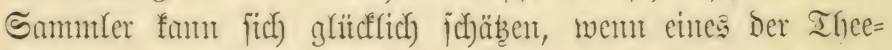
fobiffe in Singapore fich jeiner erfornt mo ifn fant feiner foitharen Bente in 35 Ingen nach) (Englanto Gringt. 
Bieft man alle bicie Niühen und Befahren in $\mathfrak{B} e=$

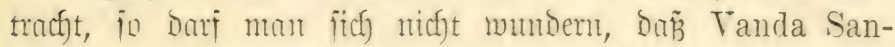

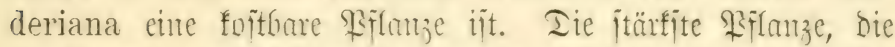
je Europa crreichte, mumbe von Sir Irevor Samtence fïr 80 Ginineen crmorben. Eie hatte 8 Triebe, von Denen bie

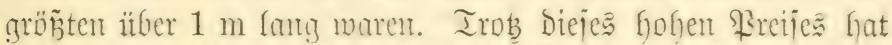

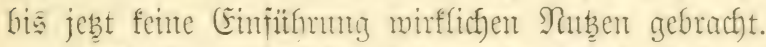

Die hauntiächliche beimat ber Bamba=?lrten ijt כava. im vollen Echmucfe iffer sichten, Dumfelgrïnen Belauthng

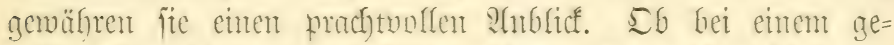
wififen ?(lter bie unteren Blätter abfallent, fann idf) nich)t jagen.

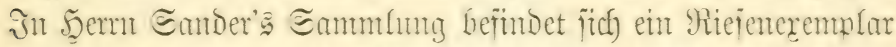
von Vanda suavis mit eff Trichen, welches im Jahre 1847 ein= gefiüf)rt mutroc. Ier längite Irieb min̈t ca. $5 \mathrm{~m}$ und ijt von ohen Gis unten im Befithe aller Blätter. Bei idflect)ter Bultur

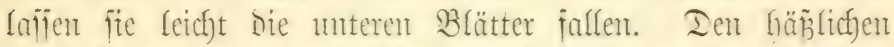

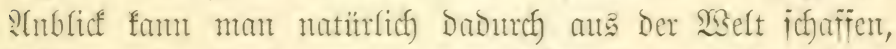
Dan man Den Trieb vom Etmmme abjanteioet mo Den oberen Ieil besielfen friich cimpflanzt. Das icjönjte Exemmlar, von Dem

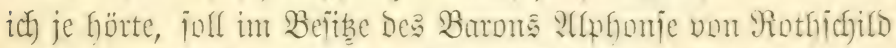

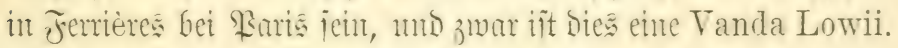

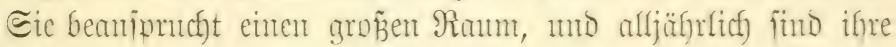
12 Etümme nit cinter $\mathfrak{U}$ nzand von $3-4 \mathrm{~m}$ fangen Blitten=

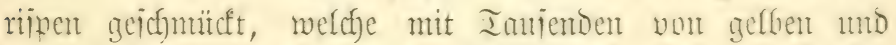

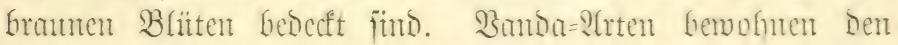

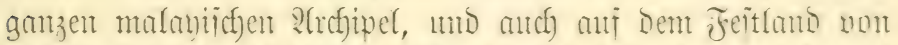
Jubien finto fie nidyt ielten. Die herrlidge Vanda teres fommt

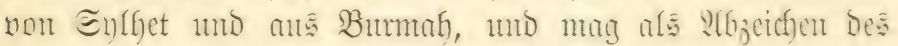

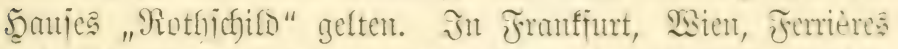

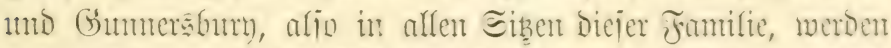

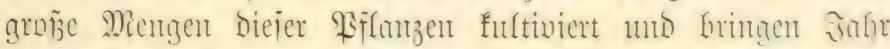

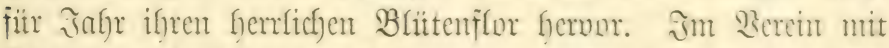
Falmen mo Farnen bieten jee ein Bils, an melchem jitf) 


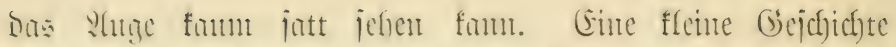

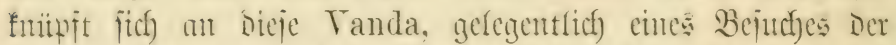

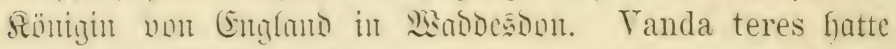

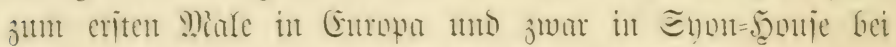

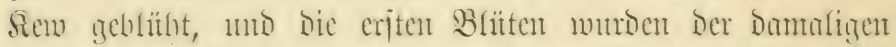

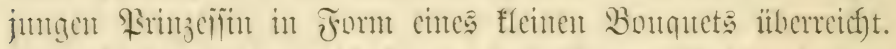

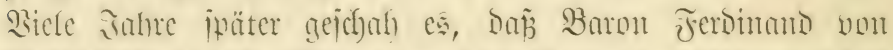

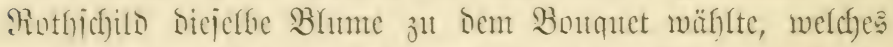

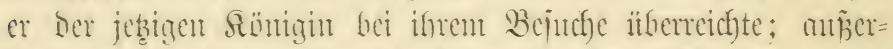

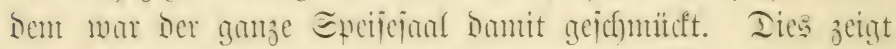

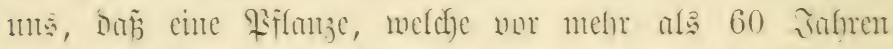

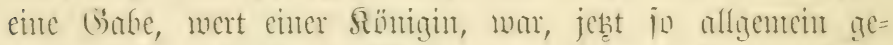

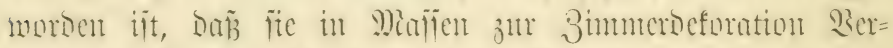

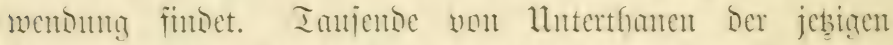

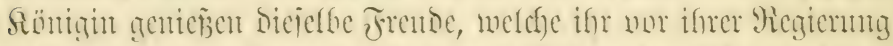

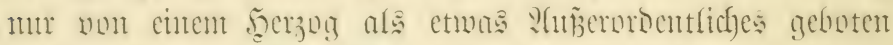

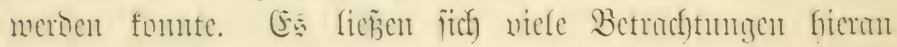

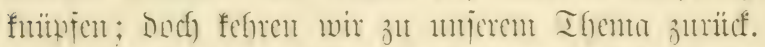

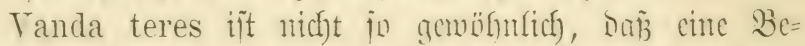

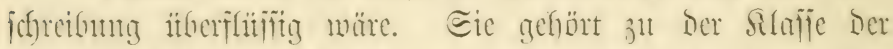

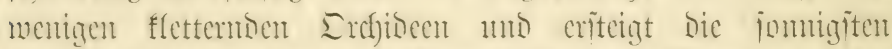

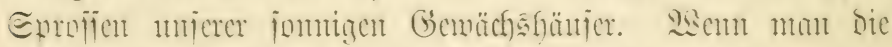

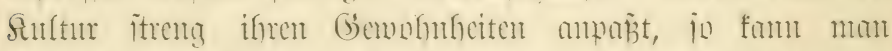

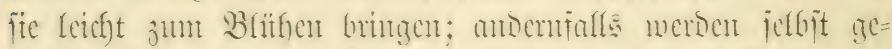

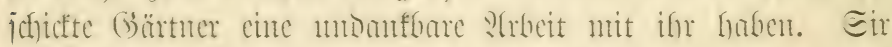

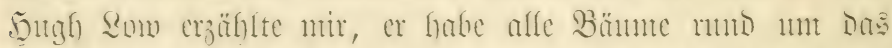
Gounementent iffer nüchiten Wermanoten, Yanda Hookeri. Geffecinet, weiche

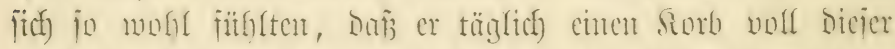

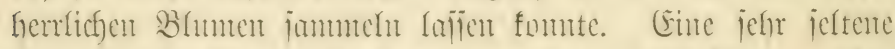
Barietüt iit Tanda limbata von ber innel Simnor; ifne

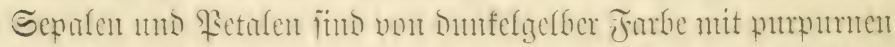

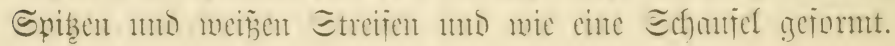




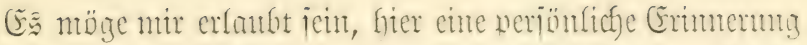
cinzuichalten in Der Dofofmung, Daj einer meiner Leier vielfeid)t

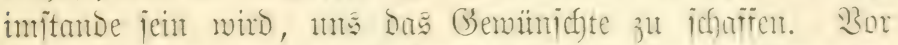
langen Jahren, weldbe mir jest wie cinte moere Exijtenz wor meiner jesigen erjobeinen, anj einer Reije in Bomed, beinchte

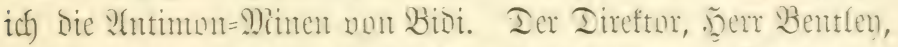

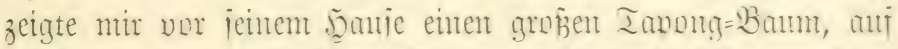

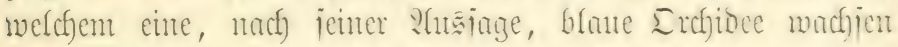
pollte. Jn Bezul anf Die Namen Dex Sreffideen waren mil

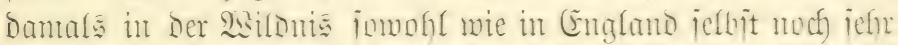

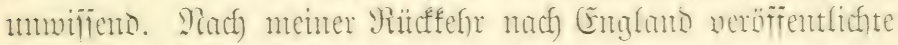

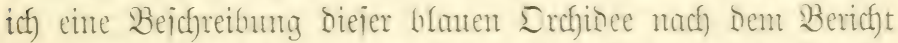

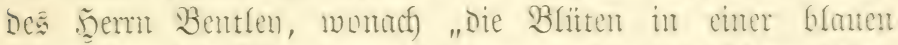

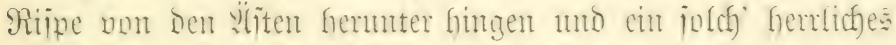

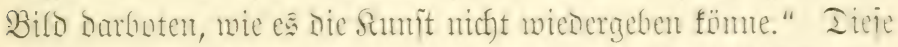

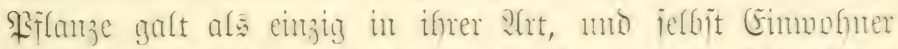

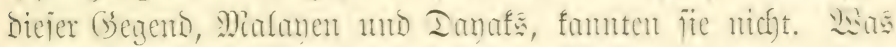

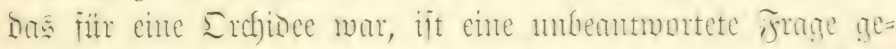

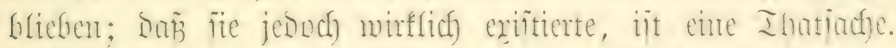
Den Benten iande Die Riflange an Den Sireftur Der Dituen=

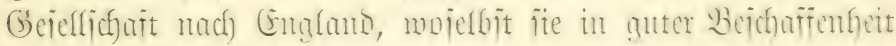

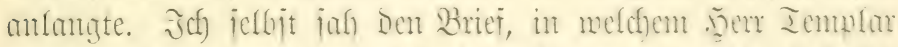
Den Emprang beitütigte mo emen Efred von 100 \& Dapür

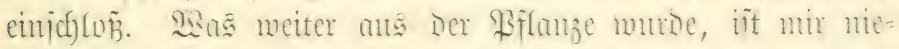

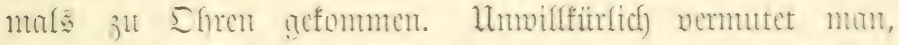

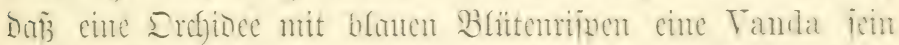
muis. Sie Bejefreibum may fïr Vanda cofrulea pajieno

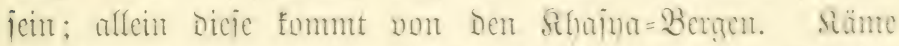
Vanda cuerulescens von jener Gegento, jo häten wir sic Wntwort anf Die Jrage gefumon; allew fie ftanme ans Bumulh mo iit mic Vanda coerulea nicht mi Bümen

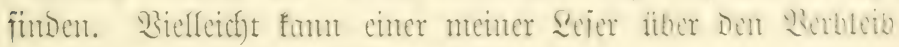
Der Bffanze Des Serrn Ientplar SUafuntt geben. 
Iie Renanthera-?trten verlangen in allgemeinent grofe

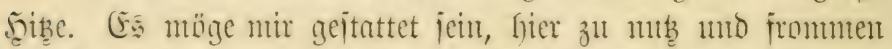

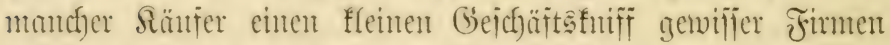
feitjunagefn, hei meldyem es fich zumeift um R. coccinea

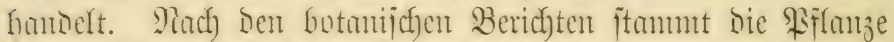

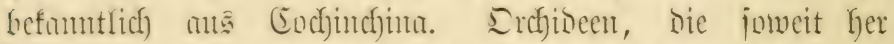

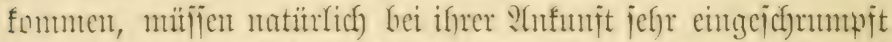

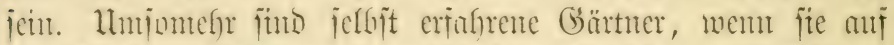

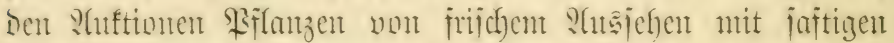
Blättern erbficfen, ütherzengt, Da $\tilde{B}$ Diejelben in bejter $\mathfrak{B} e=$ ichaffentheit uno volfer Qebensfraft finto. Dent Stricheine

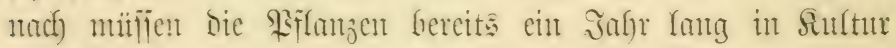
gemejen jein, und man fanft im volfen Bertrmen auf cin fenteres gutes Gebeigen. Nan jul wit jebod) tritt

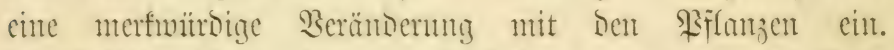

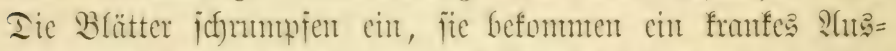
ieflen nto nach Berfanf ciniger

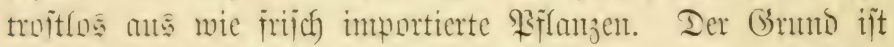

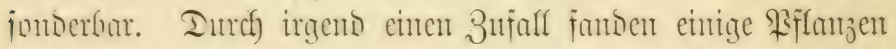
Diejer Renanthera coccinea ifrer $\mathrm{secg}$ nach Rio, wo fie

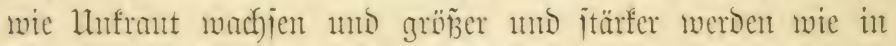

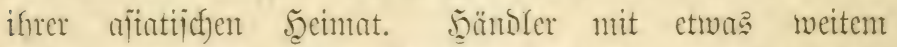

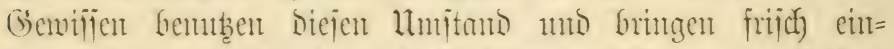

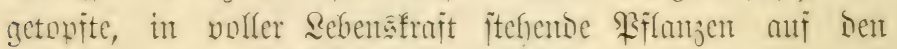
entgliichen Minfft. Bei Der geringen Entfermung fonmen bie Piflanjen in frijchem, jaftigen 3uitande an nub werben jum

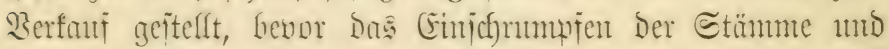

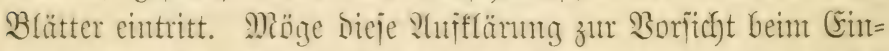
fanfe mahnen! (5s ijt bie alte Gejobichte: fanft gut etablierte

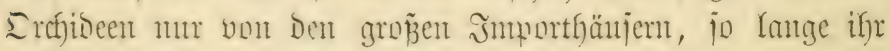
micft vorzieft, eure Siflanzen jefler heranjuzief)en.

Renanthera coccinea ijt eine zweite flettembe Epecies mo verlangt noch mefir wie $V$. teres einen Pratab unter Dem 


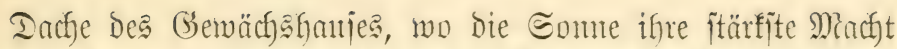

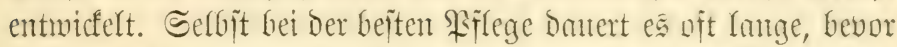
fie ifre enelgeformten, Dunffelroten und orangegelben Blïten ent= faltet. S(nocreriets wächjt fie itanf mo raich) uno ijt an uno fïr fich) vou Deforativem Siserte. Der Serzog von Devon=

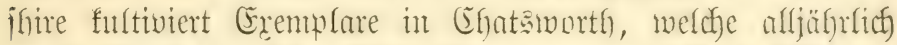

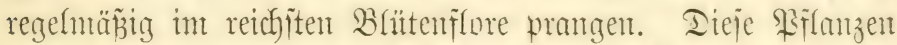

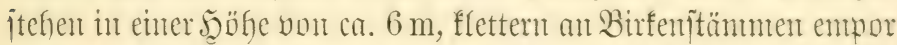
mon nefment iffren jeţigen Etandort jeit ca. 50 Jafrent ein. But

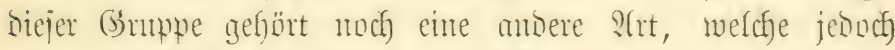
unter Dem Namen Tanda Lowii befamter irt, als unter Dem Namen Renanthera, uno meldye man jull Den botanijajen

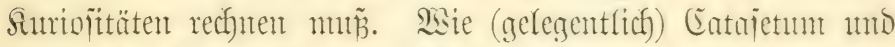

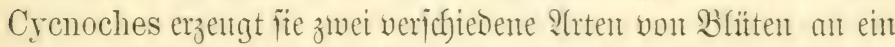

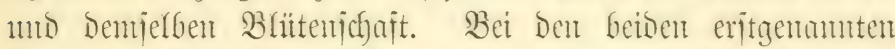

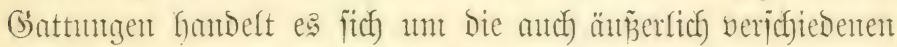
mämılichent ınเ weiblichen Blüten, bei R. Lowii bugegen

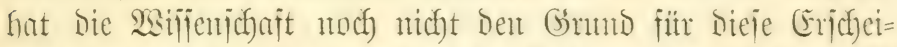
mung entoectt. Sie gemöfulliche Färbung Der Bhiiten ijt griinlicfgelb, mit brantren Flecten; ïe mejpen ca. $8 \mathrm{~cm}$ im Durchmejer mb befleiben einen Blïtentrieb vou ca.

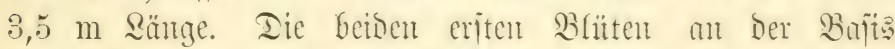
aher bifben einen itrengen Sontrajt jut Den itfrigent. Eie find bedeutent fleiner, werichieden in iffer Gejtalt, ichwarjyelf

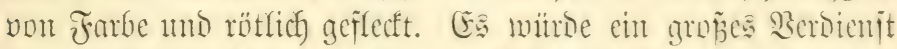
fein, Richt in bieje jut Beit noch Dunfle Frnge jut bringen.

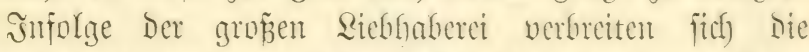
Srchiocen hentanutage mit Echnelligfeit ither sie ganje Eroe,

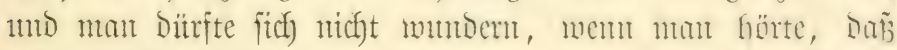
gelegentlich) irgent eine Sart, in ein anderes prifientes

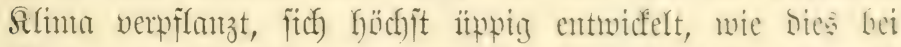
R. coccinea beobachtet morden ift. Bis jestit fam ich nuch

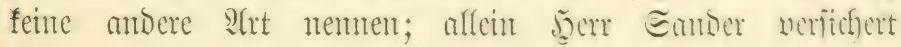




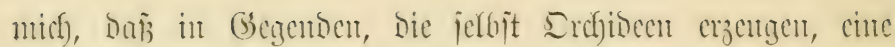

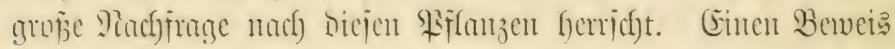

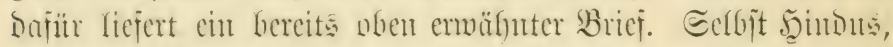
Efincjen, Sapancjen mo Eimejen von hofent Shume jählt

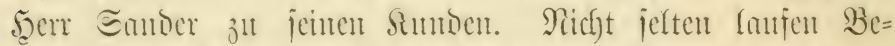

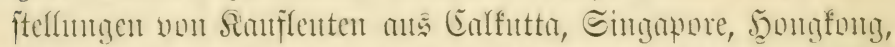

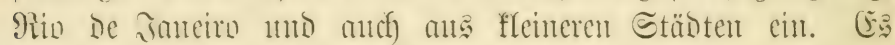

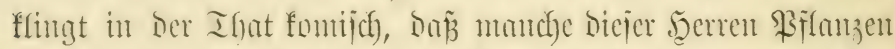

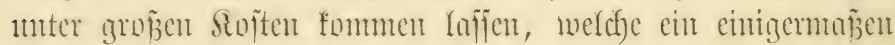
intellianter Eingeborener in beliefiger Pienge nus mit ge=

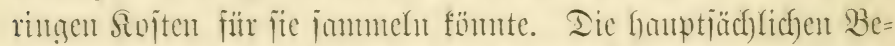

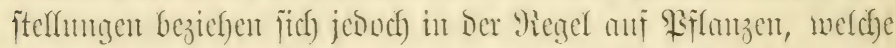

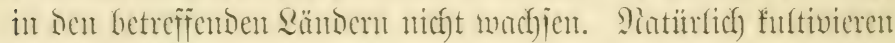

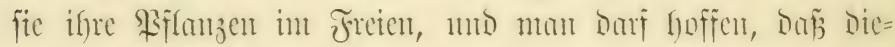

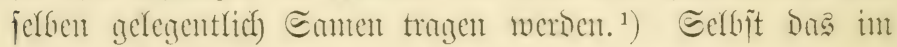
vorigen Sarpitel crmähnte Angr. sesquipedale wimro in

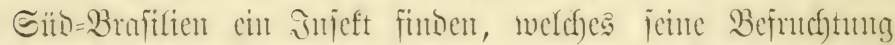

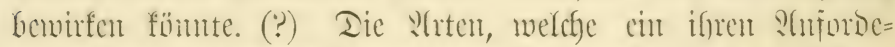

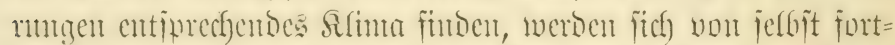

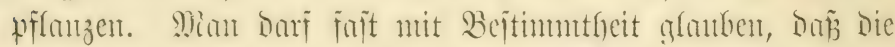

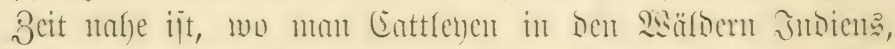
Denoruben an ?(nnzonentrum mo Phalaenopsis in Den

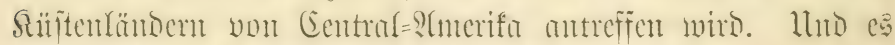
gieht viele, bie nugenuldig biejer Zeit entgegenjehen.

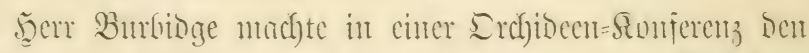

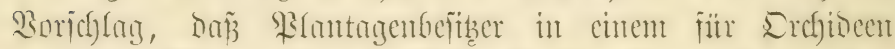

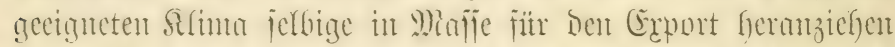
jullten. Sic sobee ijt ansigezeidyet, mis ment infolge Der

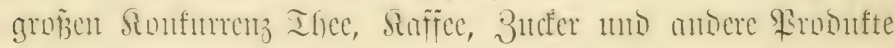

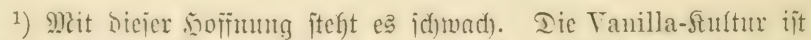

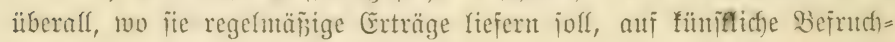
tung angewiejen. 


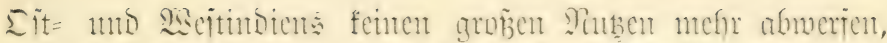

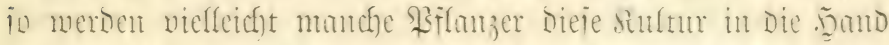

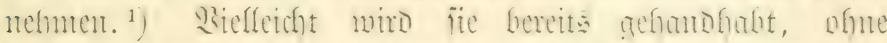

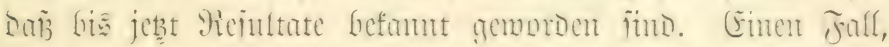

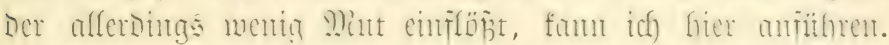

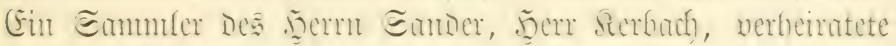

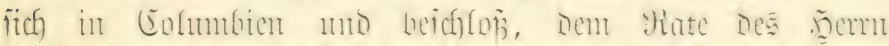

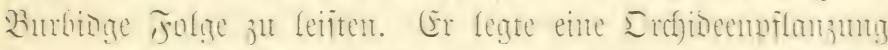

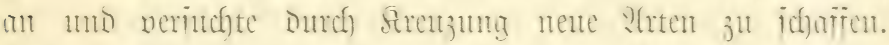

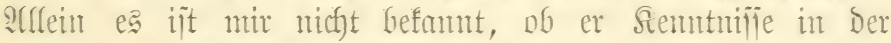

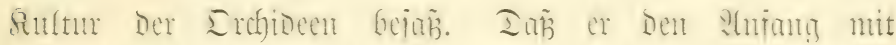

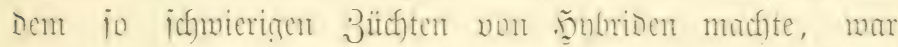

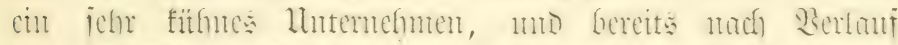

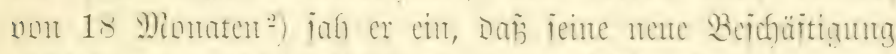

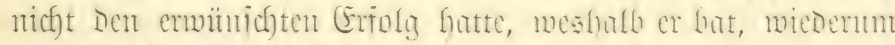

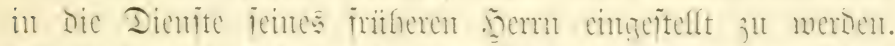

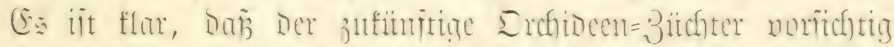

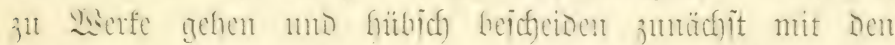

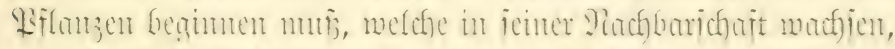

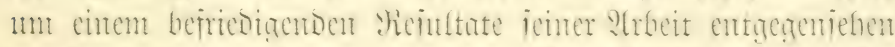

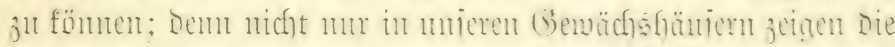

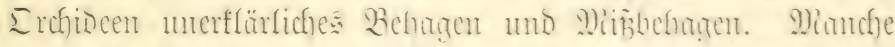

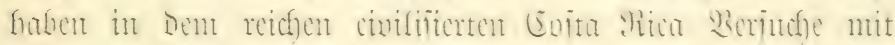

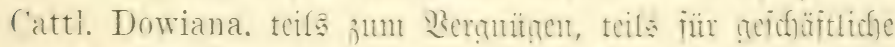

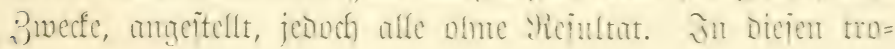

1) Sit rängit geichefen. Эd) eximere an beern v. Türfheim,

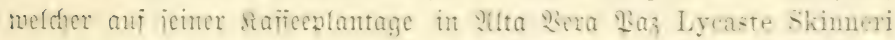
alba für Den Erport züd)tet. Sb Englänoer es biäher gethan haben,

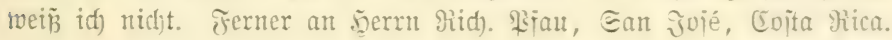

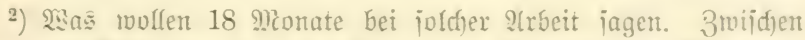

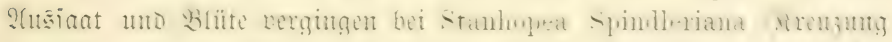
bon St. oculata und tigrina) \pm Jafre, bei (Cattlenen fömen 10 jafre Darüber Gingefen. (ङiefe baz 厄dhlūffapitel. 


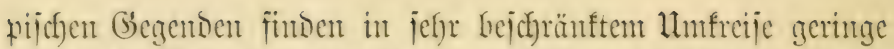
flimatijache Bertünorungen jtatt, weldhe mi Siflanjen, Deren

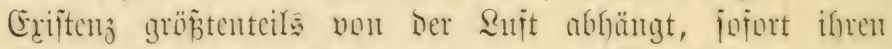

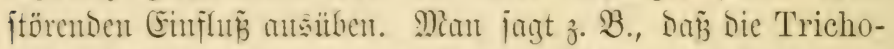

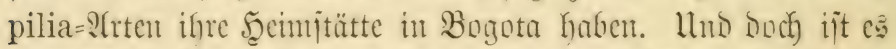
cine Thatjache, Dan fie in munttelfarer Päbe bicjer Etadt

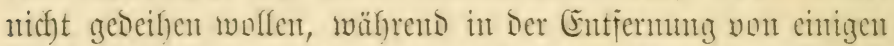

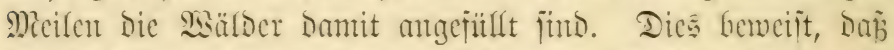

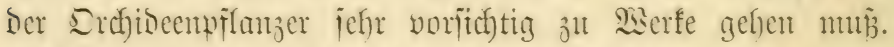
Piflanzen, wetche in serielthen Gsegento wachjen, mun er ver= mefren und nicht ithereilig jeine Eammlung anf ben Diantt

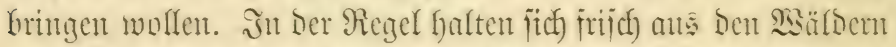

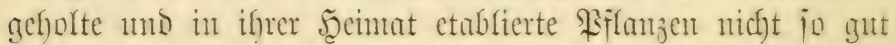

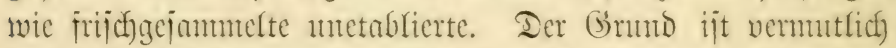

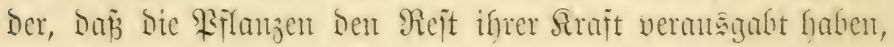

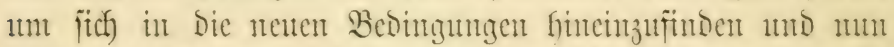

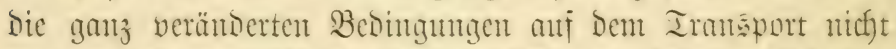

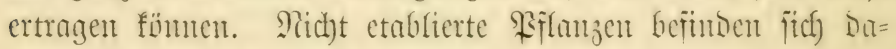

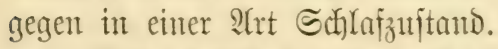

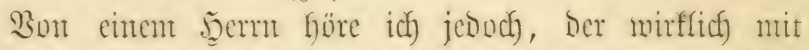

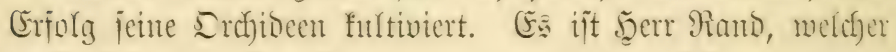
in Brafilten an Rio Negro eine nen angelegte Piflanjung non Hevea Brasiliensis, cinem Dort einfheimiichen fiantichufbanme

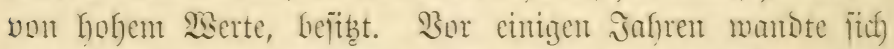

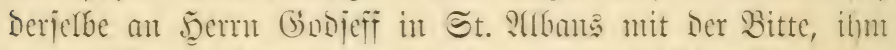
einige Vanda Sanderiana uno anoere ififtiche ?frten $3^{11}$ jenden. In Ianjh jujicte er ein neues Epidendrum,

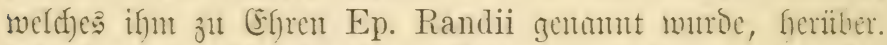

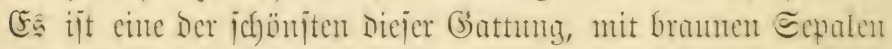
mo Retalen, einer farmefintoten sippe and jwei groijen weiñen Ecitenflitgeln. Er fultiviert ant Rio Picanro bicies Epidendrum twie noch manche andere einfeimijoche :(rt anf

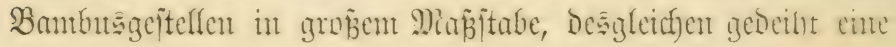


weiß̄e Cattleya superba, welche Şerr Rand perjöulich) ent= Decfte, vorzilglich) unter jeiner 和fege. Nach jeinen lebten Berichten icheinen fich) bie orientalijchen ?(rten jenjeits des

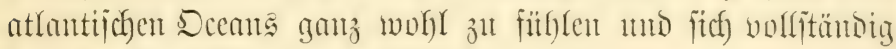

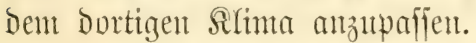

Stlle Tanda-STrten jollten in ber Seeimat Der Cattl. superba gut gebeifen, itberbaupt jene Brfanze, welche eine

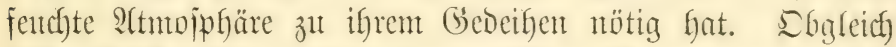
fajt alfe Cattleyen mit einer mittleren Iemperatur vorfieb nelfmen, jo verfantgen boch einige menige dos Bon zimeien, Cattl. Dowiana umb Cattl. aurea, hathe idf

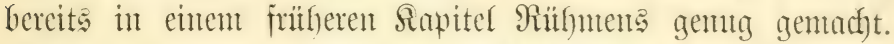
In Sa. Catfharina in Brafitien wächjit Cattl. guttata Leopoldi im $\mathfrak{B} e r e i n$ mit Laelia elegans mo L. purpurata. $\mathfrak{B} o r$

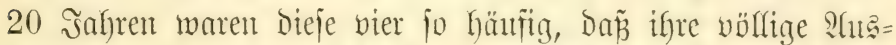
rottung ganz unmöglich jehien. Doch fein Sanmuler bejucht

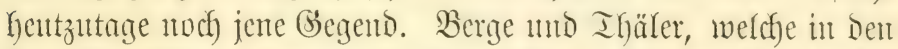
buntepten Farben prangten, liegen obe und jeden Echmucfes

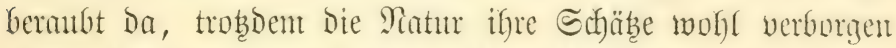
Gieft. Laelia elegans mo Cattl. guttata Leopoldi ivachjen

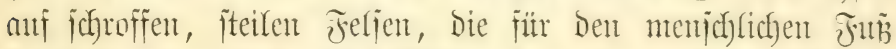

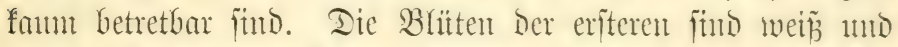

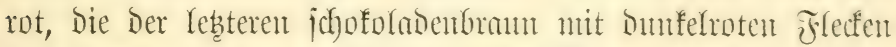

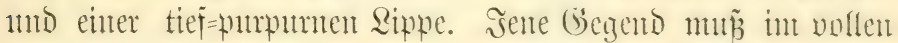

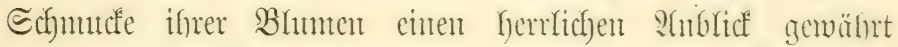

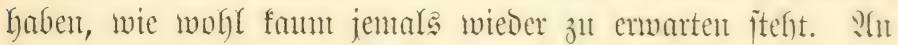

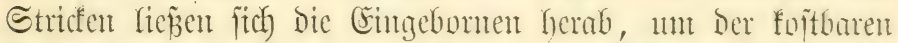

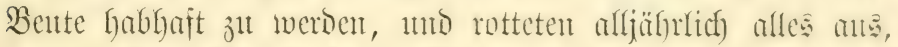
was in ifren Bereich famt. Plut wenige Exemtrlare, Die mif

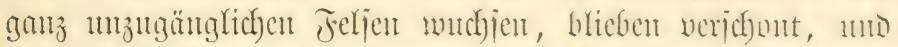
cin jeber Befitzer einer L. elegans jollte jith) jeines (Eigentum

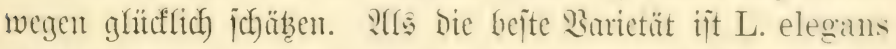
Statteriana ju nemten. Ter fumminnte Flect an EnDe 


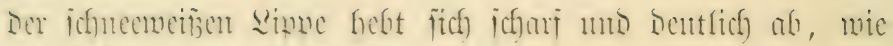
you Räurtlerfand gcichaffen.

Cattl. guttata Leopoldi ftammt ans einer anderen

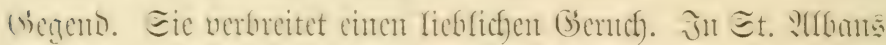

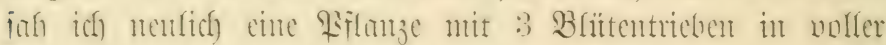

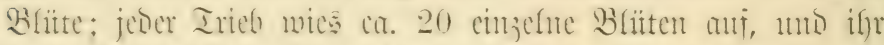

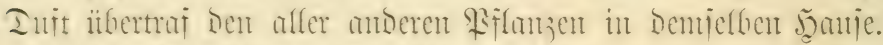

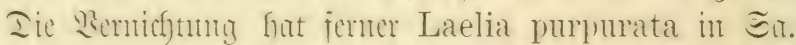
Gatharina ereilt, non wo bie beiten in Sinltur befundichen

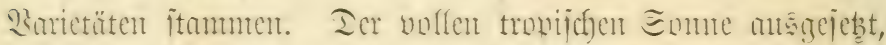

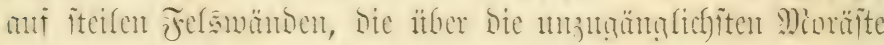

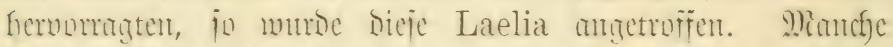

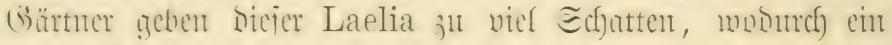
gutes Rejultat ımmöglich miro.

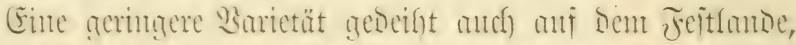

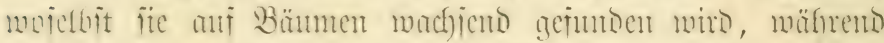

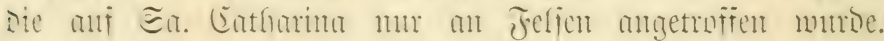

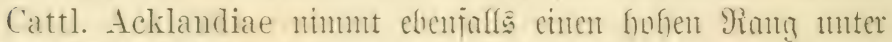

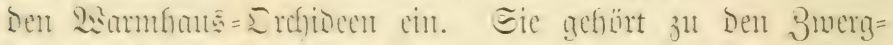

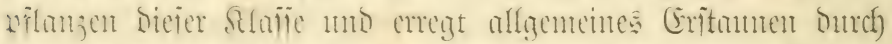
Die anfallende Gribize ifner Blitten, Deren fie zmei an jenent

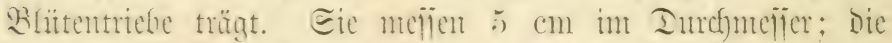

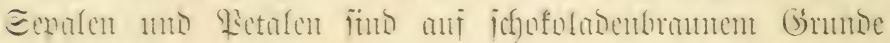

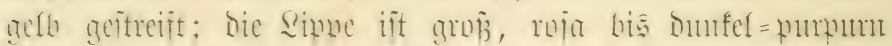
nefïrot. Eic itammen nou Bahia, mojelbit fie Ecite an Eeite mit Cattl. amethrstoglossa mädfit, cincr reizemben ?hten,

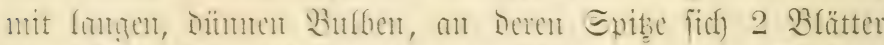

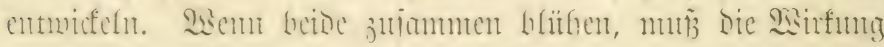

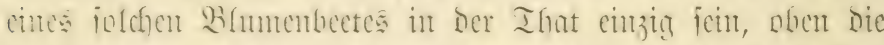

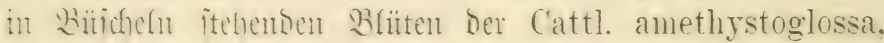

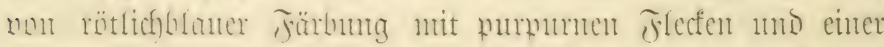

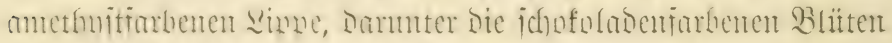
Dor Cattl. Acklandiae mit ifmen mofortenen Sipwen. 


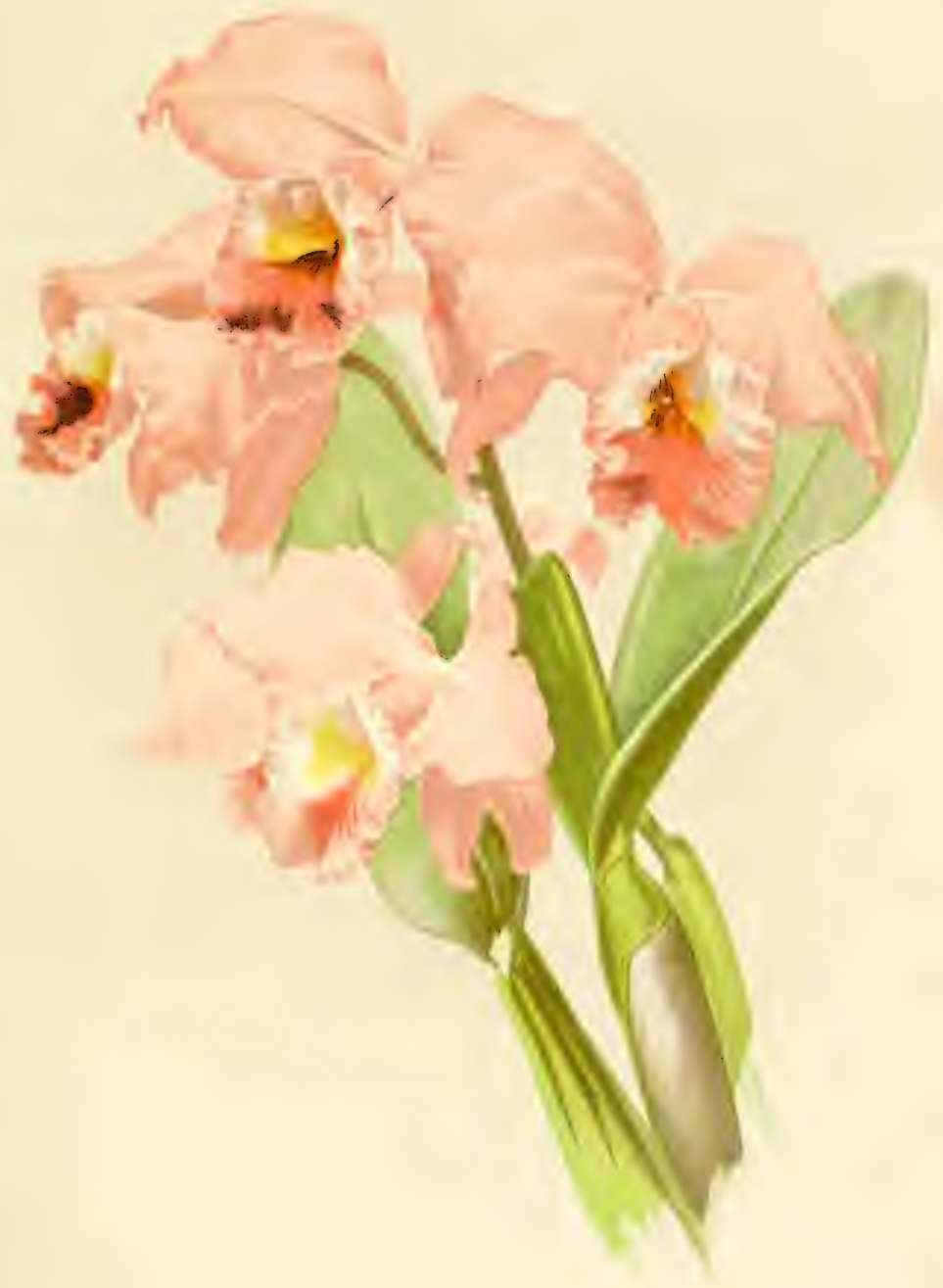



Cattleya superba fonmut, wic icjon bemerft muroc,

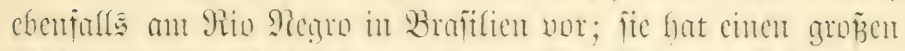

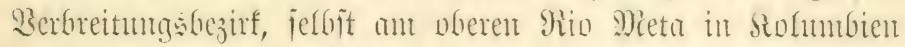
iit fie anzuttreffen. Görturer halten wegen ifgrer idfwierigen

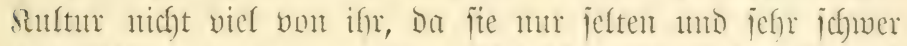

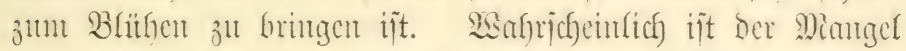
an genitgento ftarfem Eomnenichent Der Grums. Baron

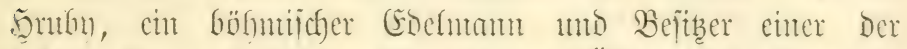
hefannteiten mo bejten Eommunutgen Siterreichs, Dazut ein

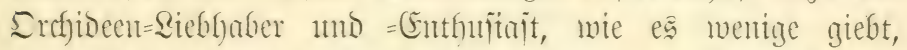

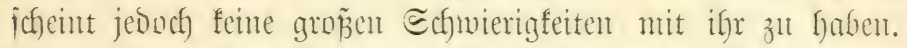

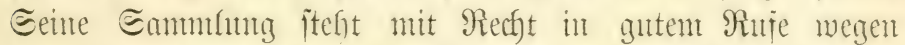

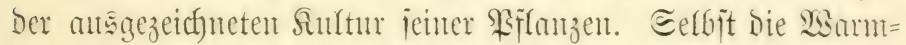

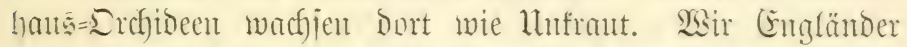

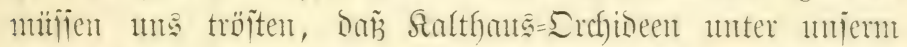

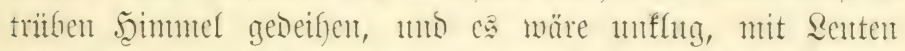
wetteifern zu wollen, benen ein helfes trochnes Rlima gemilfe Sulturen erlecichtert. Der Surrator des botanifichen (s)artens in Sicw verfichert mis jedoch) in jeiner flemen, aber

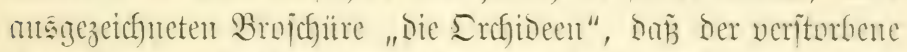
Derr Enuer Cattleya superba mit Erjolg fultivierte, mo

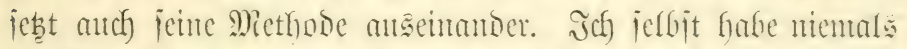
Die Bhume gejeben. Šerr

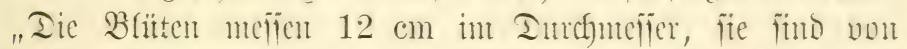

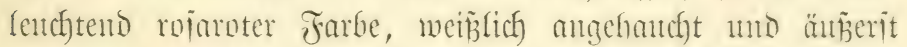
wohlriechents. Sie sippe ijt nierenfömig, Dunfel=purputrn mit

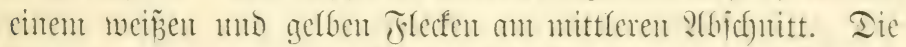

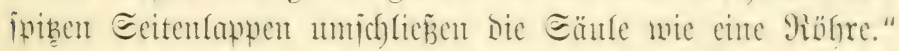
int Derierben Gegeno wie Cattleya superba mächit ınter gantz ungewöhulicf) lluitünton Galeandra Devoniana.

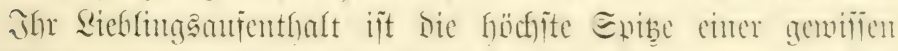
Falme in Eitmpien, bic iellit Dic Eingeborenten menen Der

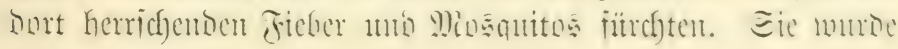




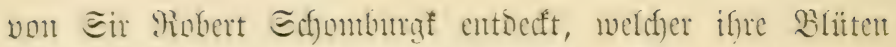
mit mijem Fingerfun (Digitalis) verglich). Tie röfrenföm

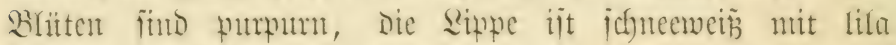
Etreifen int Siblutude.

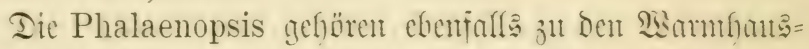
Erefibeen mo nefmen als eine ber älteren Gattmugen

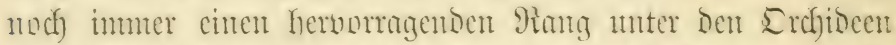

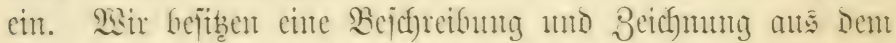
inthre 1750 , wäfrent erit in infure $18: 38$ eine lebente

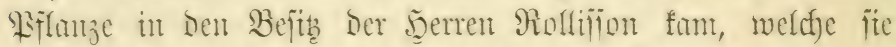

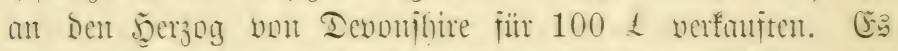

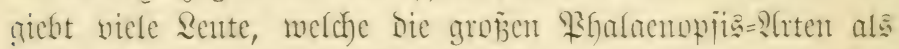

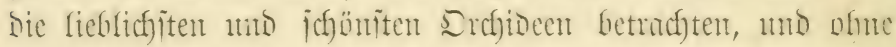
3meifel mis ifnen ein huher sert jugeitmonen werben. Dic

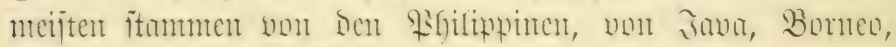

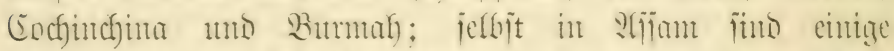

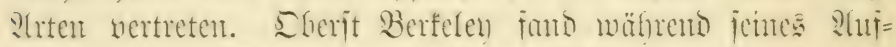

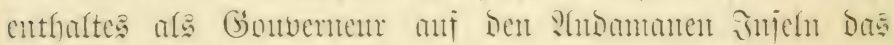
inneemeize Phal. tetraspis mo Das pumume Ph. speciosa,

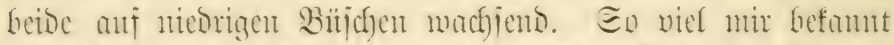

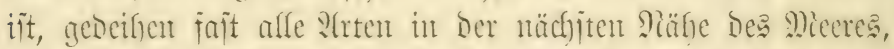
wo Die ?(tmoipläre nit Ealz getränft iit. Dies iit bet ber

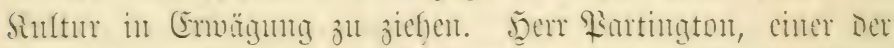

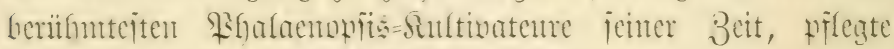

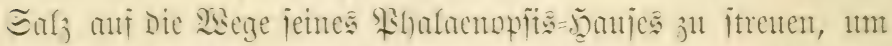

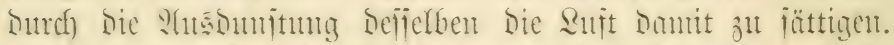

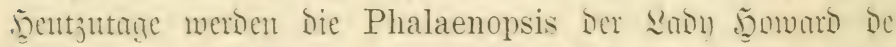

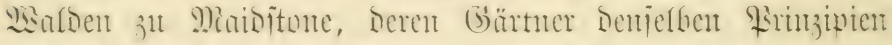
flulbigt, als bie bejten angeiefon. Iicie Wiflangen itehen unter

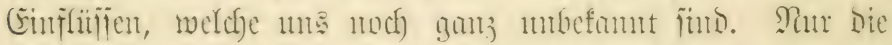

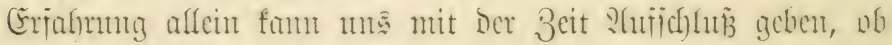

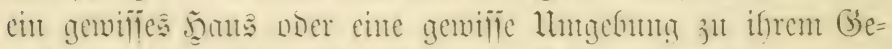

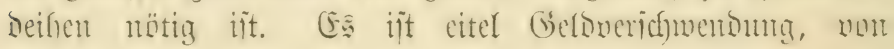


Beit zul Beit Silnoerungen in Der Sultutr vorzunefment.

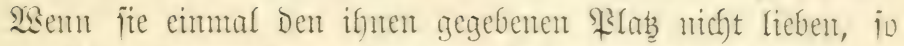

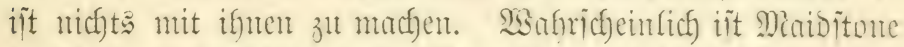

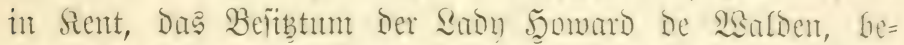
jonders fitr ifre Beditfunffe gecignet; jebenfulls aber veritent

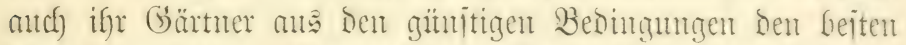

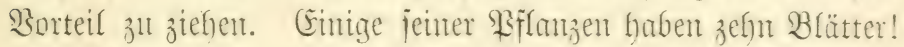
Iem Raien mag of ieltian flingen, wem eine jolche That= jache einer (Ermäf)meng wert erachtet miro, alfein eine ein=

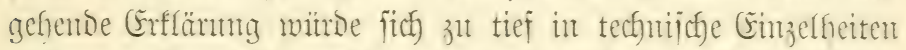
verfieren. Bemerft mag fier noch meroen, Don̈ anch) Die heriffunte

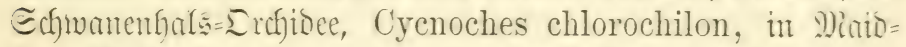
itnue befier gedeigt, als ingendino jonjt in Conglanto.

Die eriten, welche Phalaenopsis in Gnglano einiutfrten, warent bie Şerren Rollififon in Tonting, Deren Beichäft ichon jeit Joffren nicht mefy beiteft, bas aber in Den Stmuafen

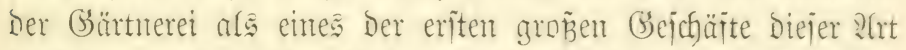
fortleben wiro. In Jafne 1836 jüfluten fie ein lebendes Erentplar von Phalaenopsis amabilis ein, welche ?lut bereits 80 Jufre frither bejchriehen uno abgebildet mar. Ëinige

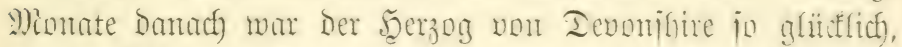
Ph. Schilleriana lebend nach) Emona jut idfaffen. Der ner=

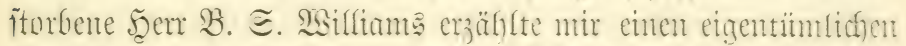

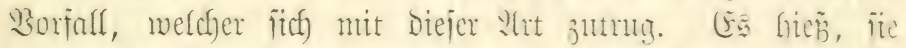

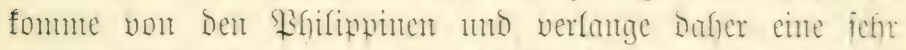
marme, fentchte Iemperatur. Bufüullig jebonf) geriet ein flemes Etüd in eins jeiner Sulthä̈lier in Doflonwan, meldges Dort

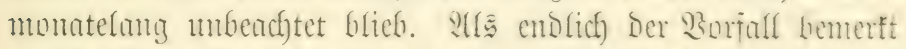

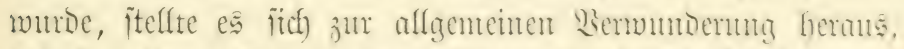

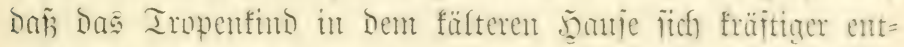
widelt hatte als ba, wo ihm, mie man amahm, bie ridftige

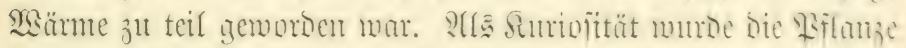

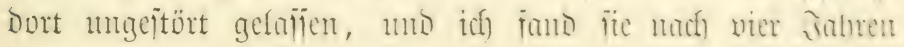




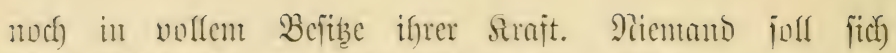

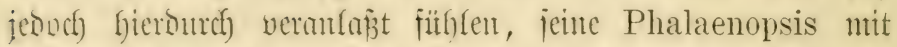

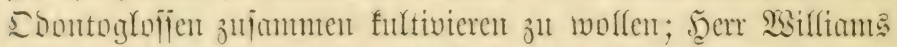

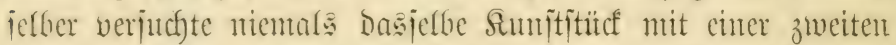
Riflanze. Iiejes Borfunmmis ijt ente jenter İtherrajchnngen, weldye ein Srdyibeenjitchter von Beit jut Beit erlebt.

(5) giebt viele jeltene Saten biejer Gattung, weldhe man in Ratalogen fam verzeichnet funbet. Fin Riebraber, Denen eine Rentheit noer Ecltenheit jtete willfummen ijt, will ich fier cintige ?amen antïfren. Ph. Manni, Genannt zu

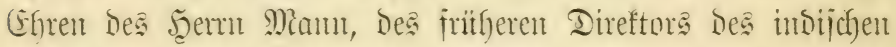
Foritoepartements, ît gelf unto rot; Ph. cornucervi gelb

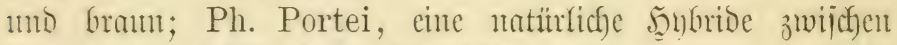

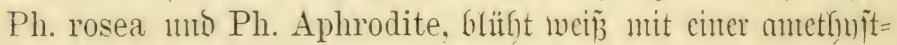

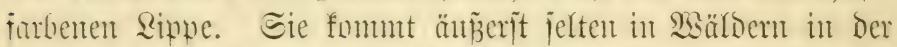

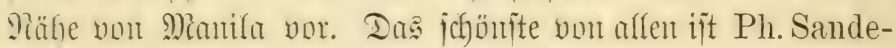
riana, an meldes fïh wieberum cine fleme Srzällung fuitpft.

Sobald es den Gingebonten der Pfilippunen verjtändich)

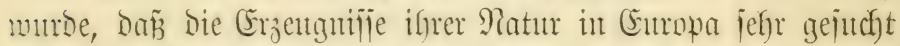
maren, erzälften fie von einer jobrladfroten Barietät cines Phalaenopsis, metche das Jृerz Der Sammler vor Frende

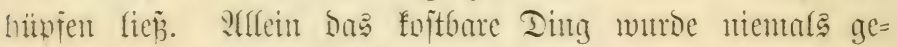

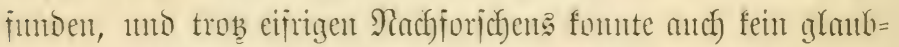
mutroiger Benge anfgejumben wersen, weldyer bie Piflanze mit

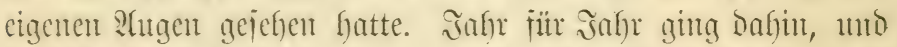
Sas idfarfadrute Phalaenopsis wurde Gegemitamb Des Sputtes.

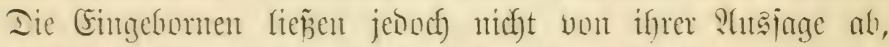

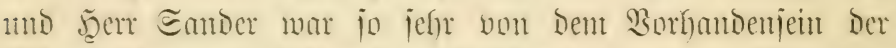
Riflanje ütherzengt, Daz er beim Eröffnen eincr neuen Damufer=

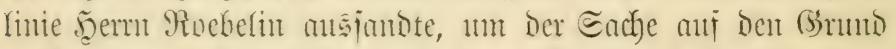

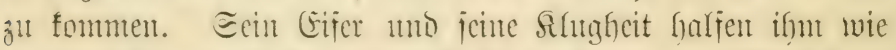

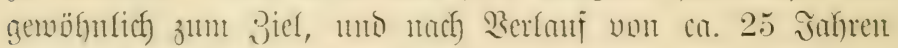

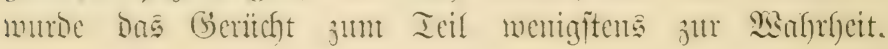


Ph. Sanderiana iit freilich nicht icharfachrot, aber wenigitem Dunfetroja und jebenfalls eine herrliche Prilanze.

Iemielben Sammler veromfen wir Die Einfüf)rung cinte Der itolzeiten Aerides, A. Lawrenciae, mit mach

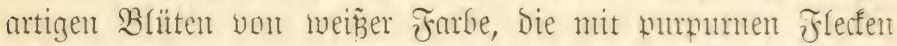
mo einer tieiputwumen Sippe gejiert find. Neben ber feert=

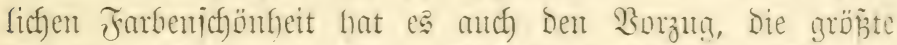

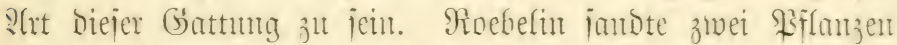
ans Dem ferniten Siten nach Somie, von Denen er weder eine Blitte gejefen noch) cinte Beichreibung erffalten hatte. Irei

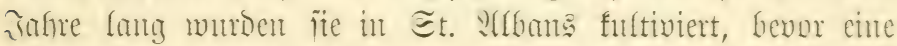
Derjelben zut Blitte fam. Bu einer Pluftion nach Sonton

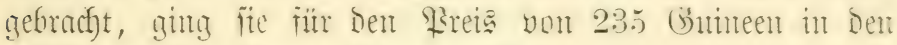
Befith von Sir Trevor Ramrence itber.

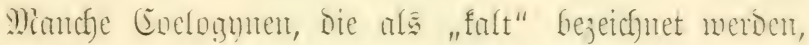

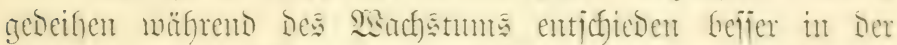
Temperatur ethe sismbanice. Coelogyne cristata itoumt von Mepal, wo fie ïch in heipeiten Eomenichein an mogliten fühlt. Iodh idf mill mur einige wenige mrüffren, bie cine warme Temperaturt nötig Gaben.

3u Den anffallenditen uno jugleidf) idjöniten ber Battung gefört Coel. pandurata an Bonen. Iie Rijue iit mehs

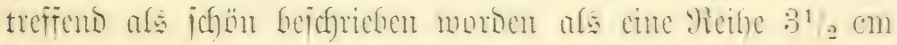
Uneeiter, grianer Fröjche mit icfwarzen Bungen. Die gange

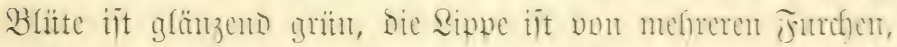

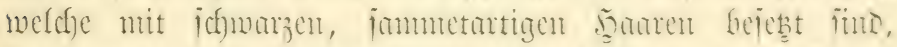

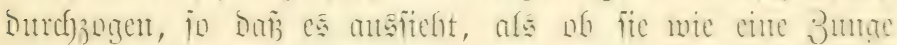

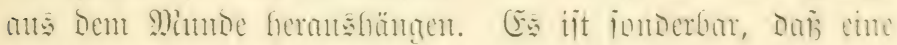

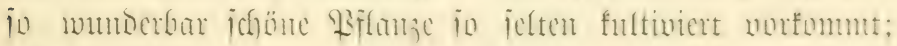

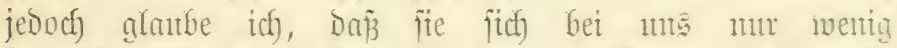

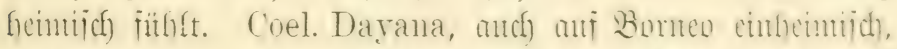

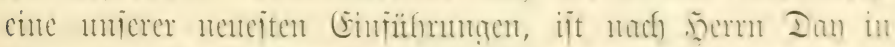

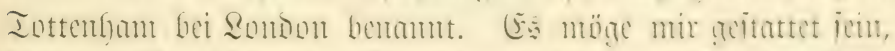

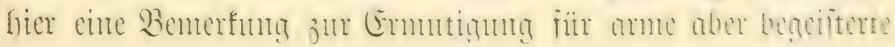




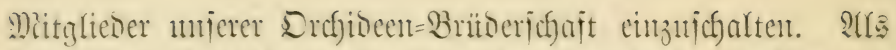

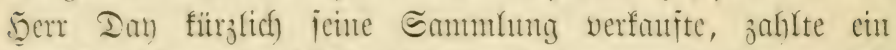

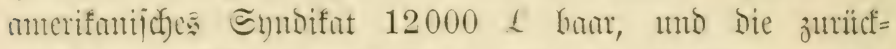
gebliebenten Siflanzen brad)ten anf ber Stuftion weitere 12000 \&; jo wenigiten lantet ber bisher nicht wider= mifene Bericht. ${ }^{1}$ ) Coel. Dayana ijt jelten no sentzu=

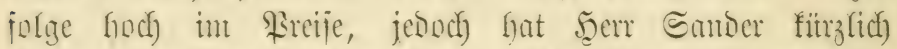

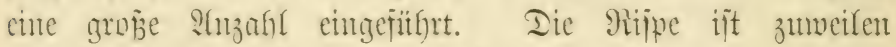

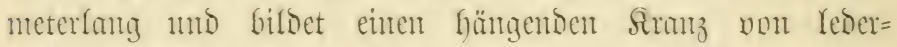

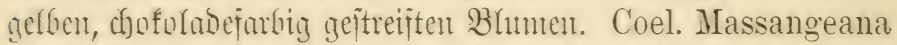
non stijan gleicht ber vorfergefenton, jesuch ift bie Rippe

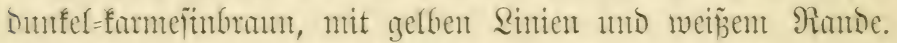
Die nenteite und bei meiten ichönte ber ganzen Gattung ijt

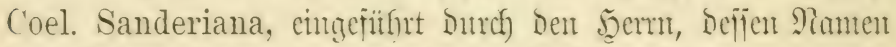

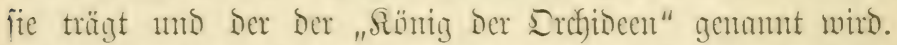

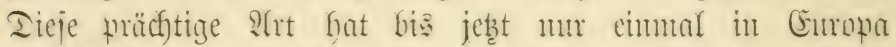

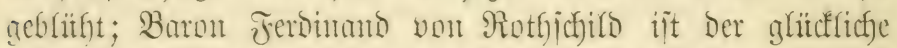

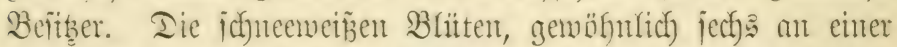

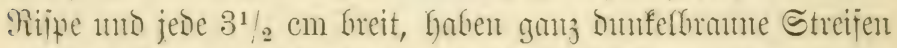

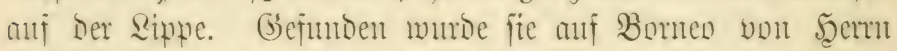
Föritermann, semielfen Sommler, Der das prachtoflfe,

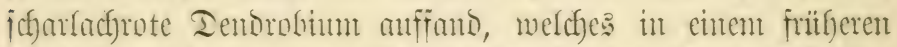

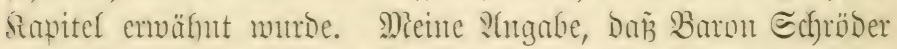

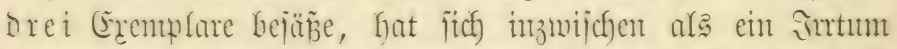

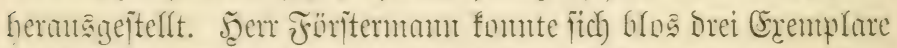
verichaffen, von melchen zmei anf Der Reije eingingen; Şent

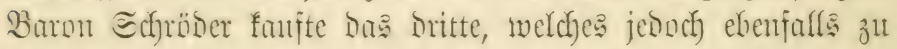

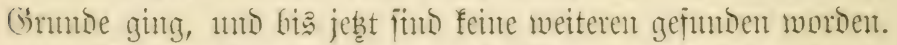

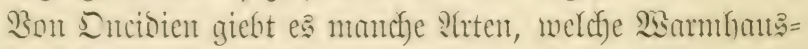
Sulturr verlangent. Bu ifnen gehört $\mathrm{O}$. splendidum, Deffen

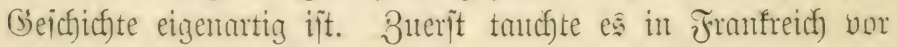

1) 2iobei nidgt geiagt iịt, was ñerm Ian bie Eammlung ge= foitet fat. 


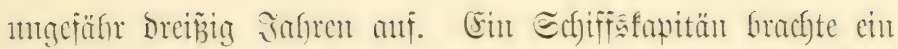

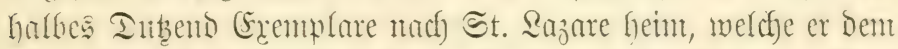

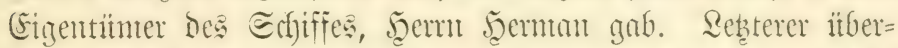

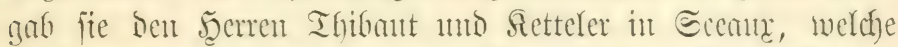
Die Eremplare teilten, bie bann in verichiedene. Shänoe fanten.

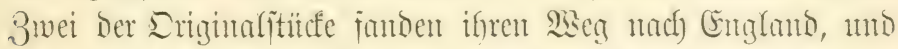

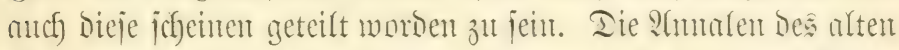
Pluftion Den Preis fïr ein winjiges Etiuf mit eintem Blatt bis auf

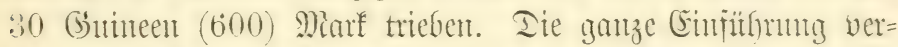

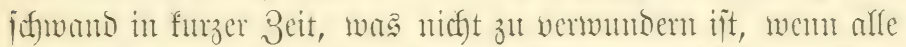

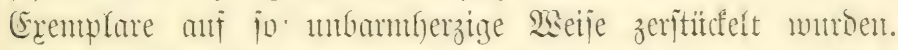

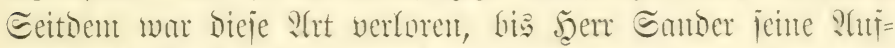
merfiamfeit anf fie Yenfte. Sein Beridft war vorfanden, wo= her fie gefonmen war. Ier Pame des Ectjiffes oder jelbyt Der

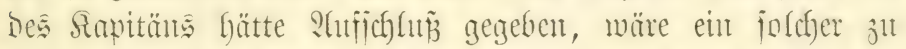
finden gemejen; Dem ntan hätte Dan ans Dem Echiffistuge= Gutch erjegen fömten, weldye bäfen Gejutcht worden maren.

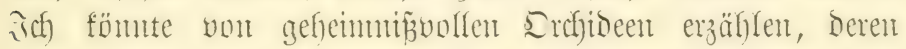

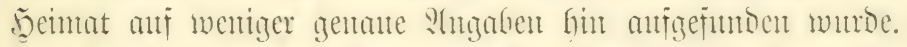

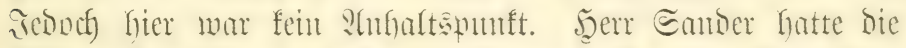
Piflanze jorgiältig beobachtet, wälnento noch einige Exemplare

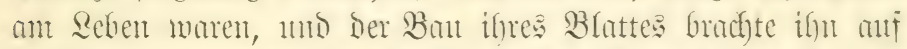
Die Bermutung, Dañ fie Der Ffora von Central=?lmerifa an=

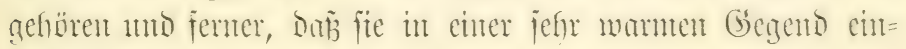
Geimifich jein mitife.

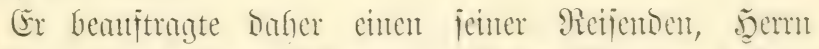

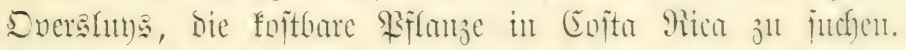

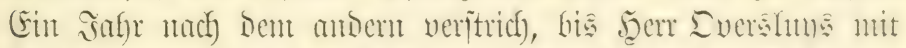
Entichiebentheit erflärte, Dã 0 . splendidum viefleich)t int

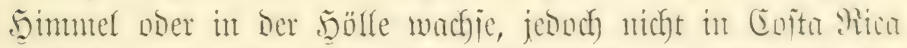

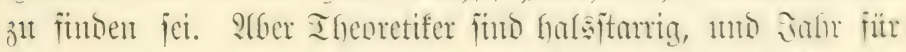

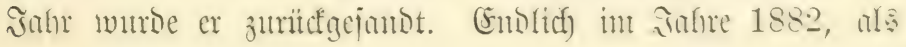




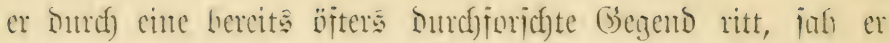

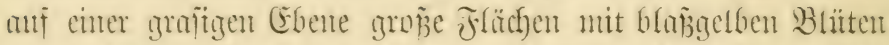

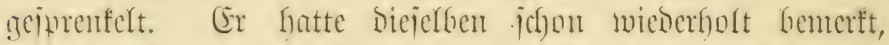

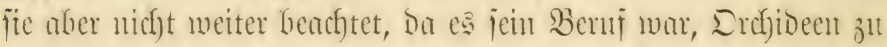

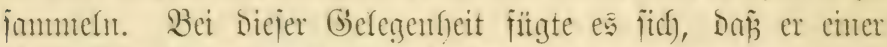
Siejer Diafjen mafhe fan mo mu bas gejuchte Oncidium

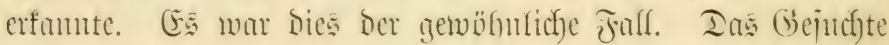

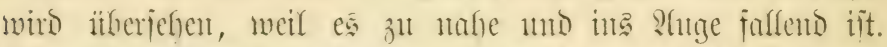

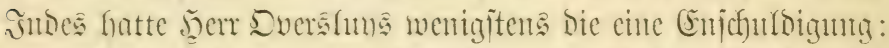
wer founte afuten, ein Oncidium in foufen Graje, Dex vollen Sumenthibe anggejeht, zu funden?

Oncidium Lanceanum ift walnricheinlich bie "märntite"

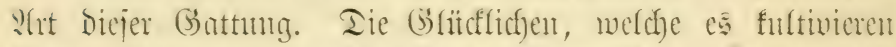
fömen, befanten, es biete fei!ne Echmierigfeit; aber mentu es

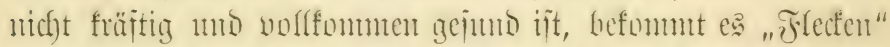

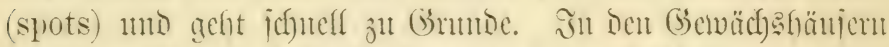
Der ... Yew Plant \& Bulb Company" zut Cordefter (mun er=

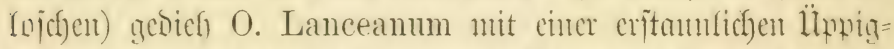

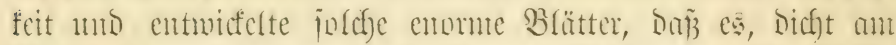
(j)laje bängens, mitten int Enmmer Bejchattung itforfitititg

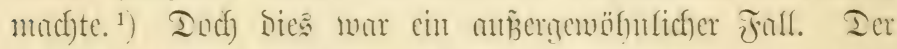

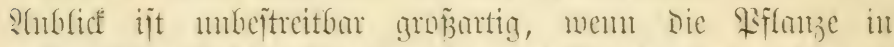

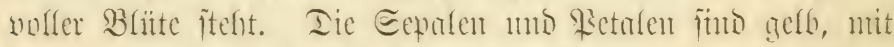

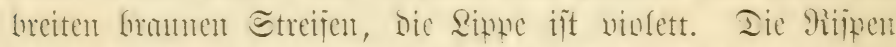

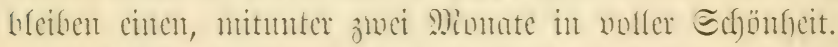

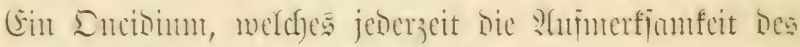

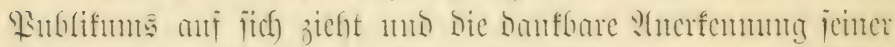

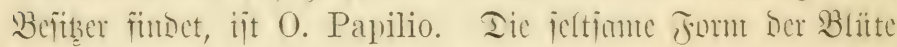

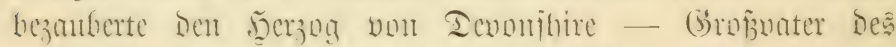

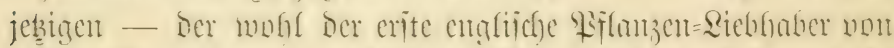

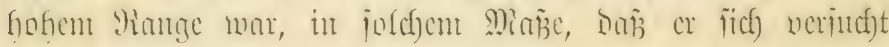

1) Eorlte bie Eeeluft Gierbei mitgeminft haben? 


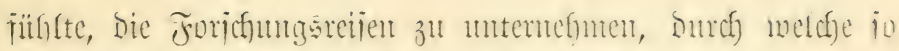

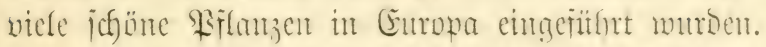

Die "Echmetterfing

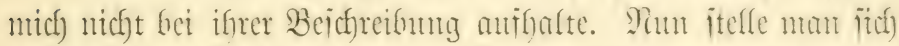

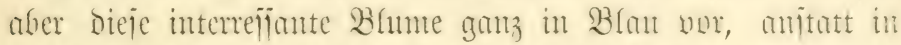

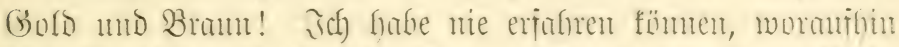

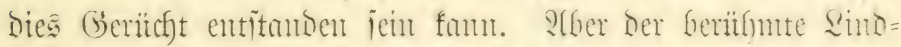
(el) jtarf mit Dent unerichütterficfen Bertranen, Don cun

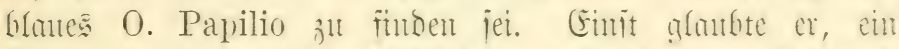
Eremplar jut haben, jesodg biafhte es, and mit ientem Trimmb

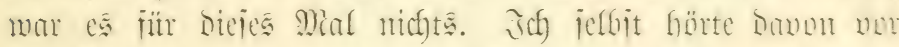

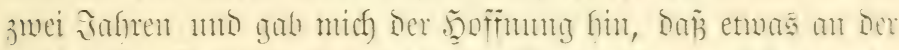

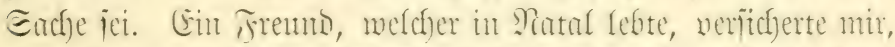

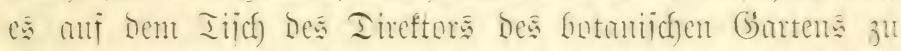

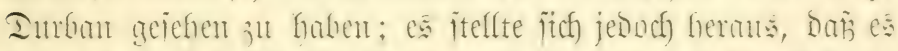

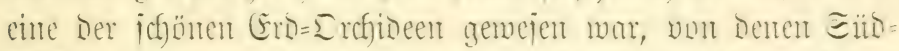

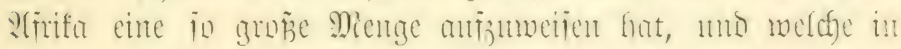

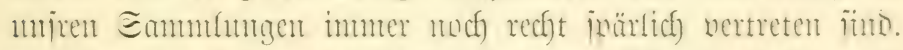

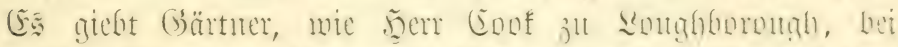
melchen Disa grandiflora wie llufrunt mächit. ñom

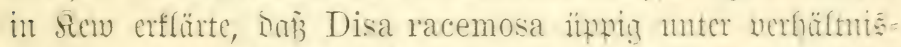

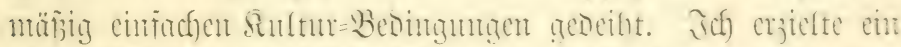

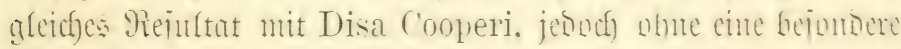

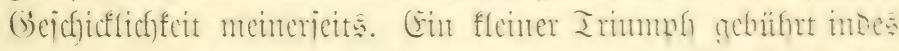

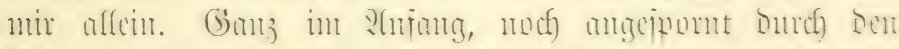

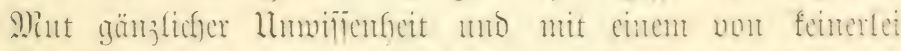

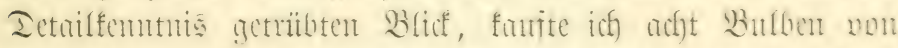

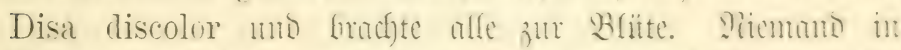

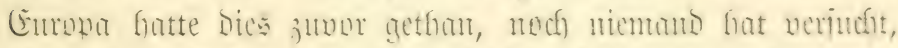

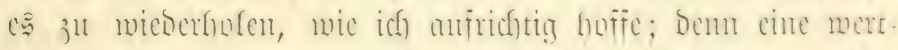
loiere Bhume giebt es nicht. Indf hier hien es - Eog

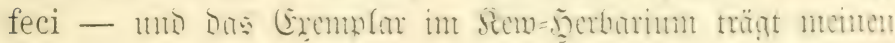
Namen. 


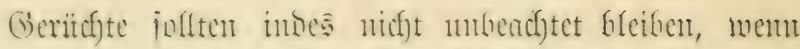

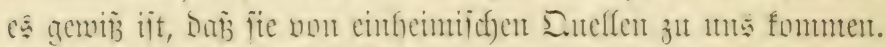

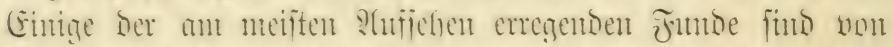
je fer mifnerfimen Eingebunen ju nerbanfen genejen. Die (Bejoficfte nou Phalaenopsis Sanderiana habe icf) bereit: er= jühlt. Ein Sull mar es ferner, meldyer ben Eammler anf Die Exur Der nenten gefben Calla bradfe. Tie blane Utri-

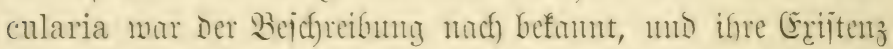

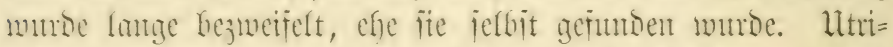

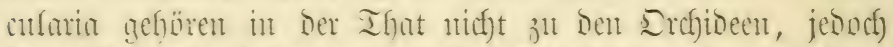

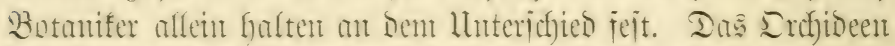

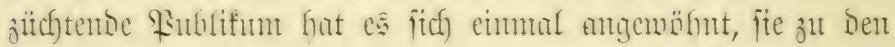

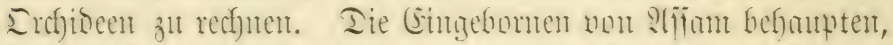

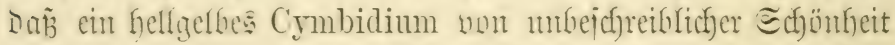

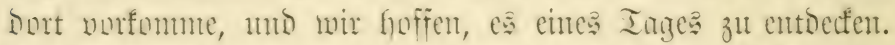

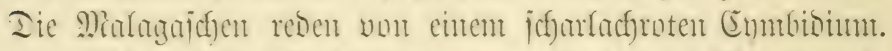
Die meiten Entiontoren vertragen fo viel siäme, als

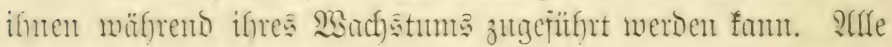

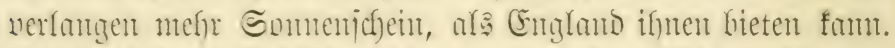

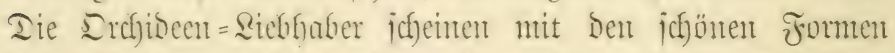
Dieier Gattung nicht io befoment zu iein, als fie es jolltent.

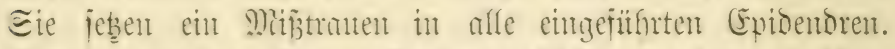
Mandfe mertloje strten alferbing gleichen in ifrent Dabitus

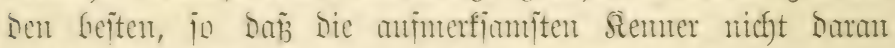
Denfen, anf einer SUfftion zu funfen, wenn fie fein Bertnaten

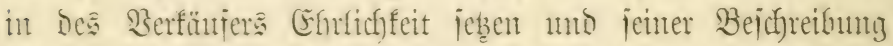

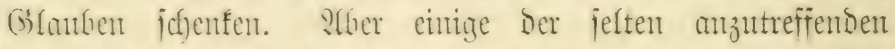

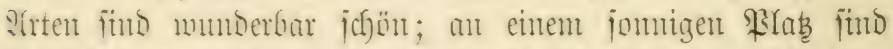
fie leicht ju jiefen mo gar nitfl fojtipielig. Epidendrum rhizophorum ijt in E. radicans ${ }^{1}$ ) ungetmuit

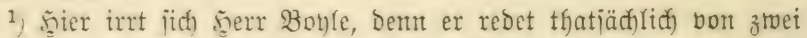
ganz veriditedenen \$iflanzen. Iie Beborzutgung De? slamens radicans ijt mijienj(haftlid) gered)tiertigt. Ep. radicans wächit nur in Nerrico unb Gruatemala, nidyt in Brajilien. 
morDen, ein Name, weldher muj bie mexifaniiche Barietät zu

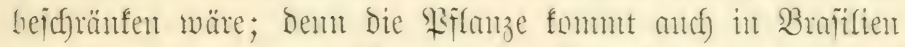
nor, jeboch mit einent huterichies. Iie erĩtere wächit ani

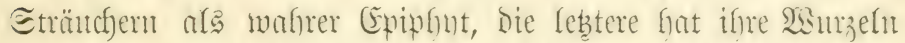

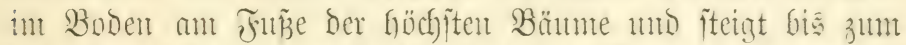

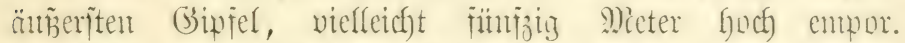
Die Blitten zeigen ehenfalls Lhterichiede; aher in allgemeinen

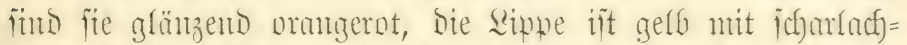

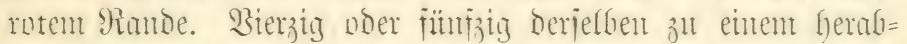
hängenten Biticher vereinigt, gewäf)ren einent prächtigen Shrbficf.

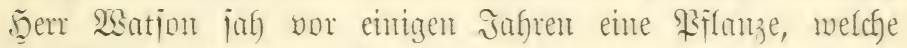
86 Blïtenföpfic trutg. Die Blütezeit Danert Drei Mionate.

Epid. prismatocarpum ijt efenfalls recht fithifh, mit

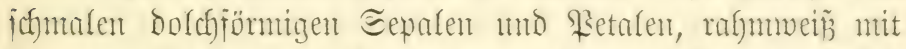

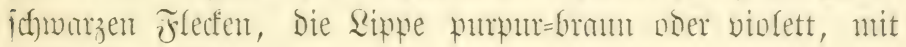
blǟgelbem $\Re$ ande.

Bon den vielen Dendrobien fïr Warmbant

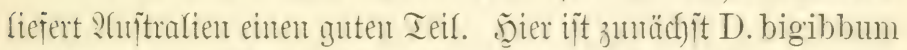

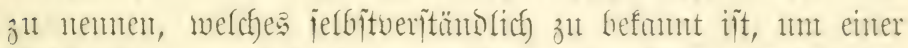

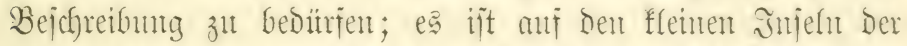

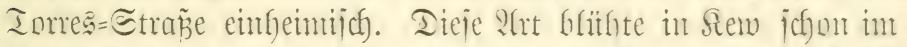

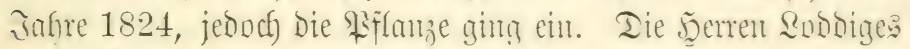

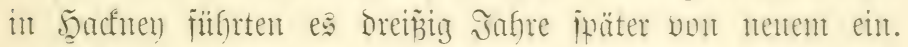

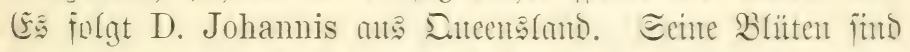

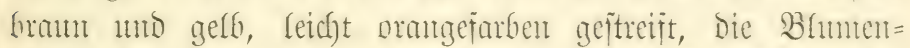
blätter find jeltjam georeft. D. superbiens, efrenfalls von oer

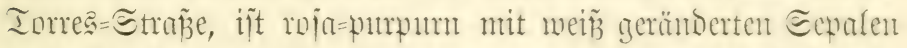

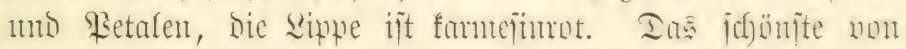
allen ift D. Phalaenupsis. (s) entuvifelt unmittelfor unter

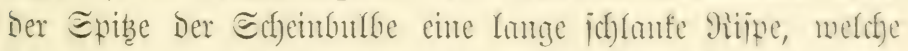

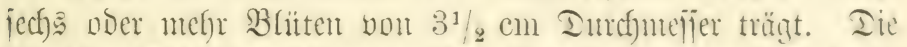

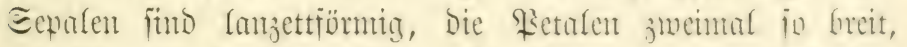

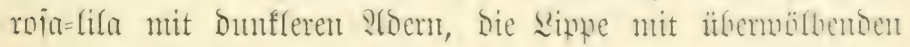




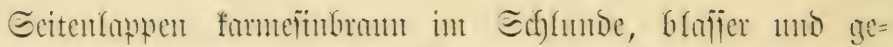

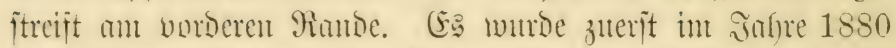

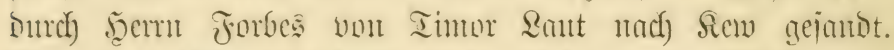

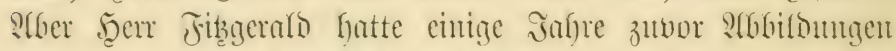

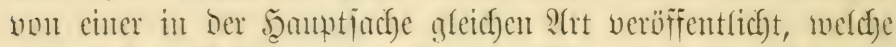

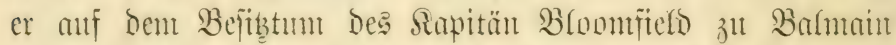

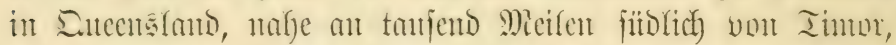

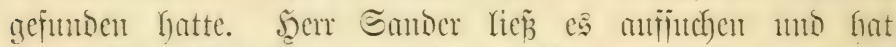

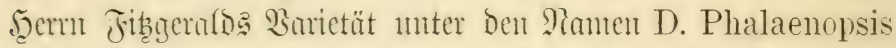

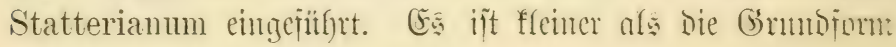
und farmefinfarben aujtatt lifa.

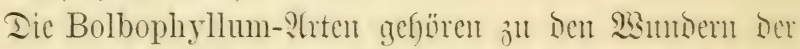

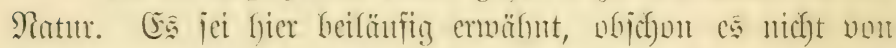

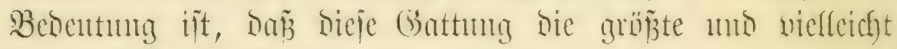
bie flemite aller Drrbioeen enthält.

B. Beccarii fyat Błätter, weldye $1 / 2 \mathrm{~m}$ lantg 1 min 20

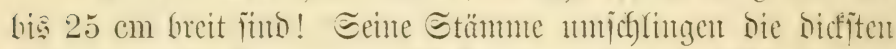

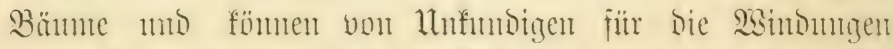

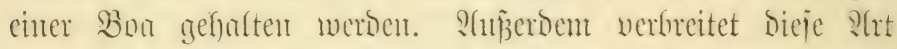

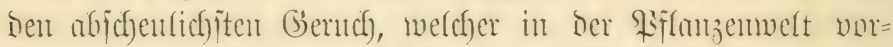

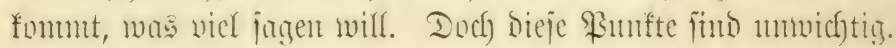

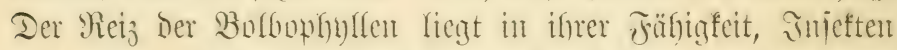

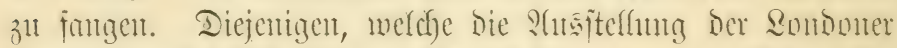
Gortenbant=bejeffichaft int "Iemple" int vorigen iafure be=

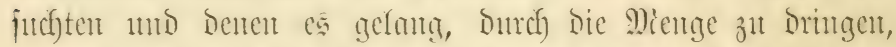

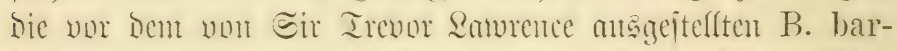
bigerum verianmelt mar, fomuten etwas bavon jechen. Dicic

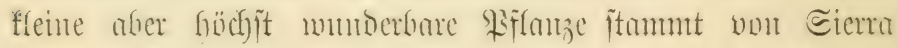
2enne. Die lange gello Rippe ijt mit ber Sönle mur gant;

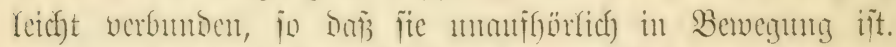

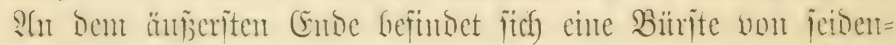
artigen banten, wetche fich) bor= 


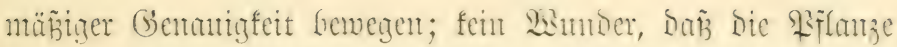

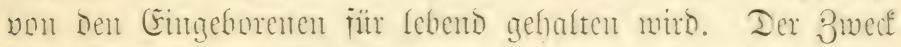

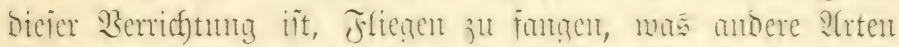

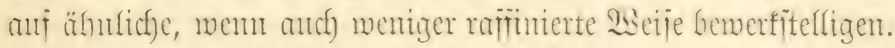

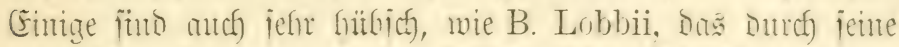
reine, flare Erangenfarbe Den Blicf iefielt. Die Simpe Galan=

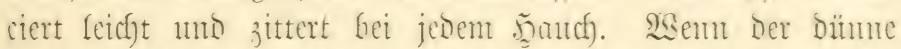

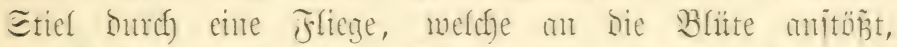

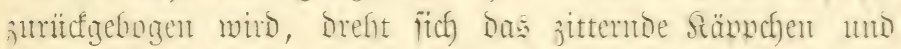
hängt hervoriteheno; cin mberes leid)tes Echütteh, mem

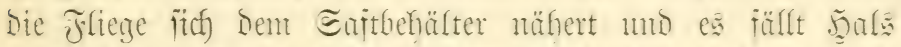

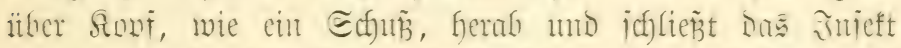

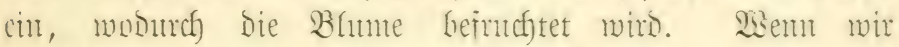
mitnichten, in einem flutgen Stinde Jnterefie zur Botanif

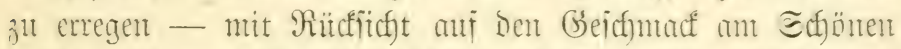
- io wimben wir ifn B. Lobbii verichaffen mijifen. B. Dearei iit auch iefn niedsich), golobraum mit rot geflects, mit emem ureiten nberen Eenalum, mo ichr ichmalen flattemben Retalen; Die mnteren Eevalen hahen breite rote Etreifen, Die Sinpe it getb unb natitrlich wie inmer eingelenft anf einer S.rt von

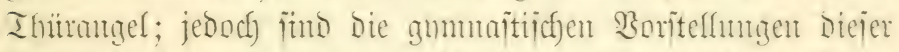

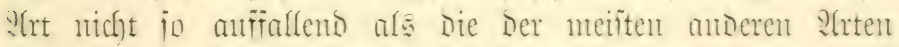
Diejer Gattung.

Grin neues Bolboyhyllum. B. Godseffianum. umurne

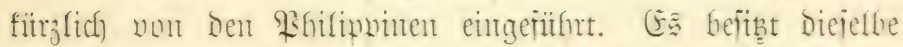

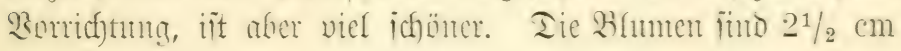
Greit und Gaben bie Farbe von "altem Gold" mit famefinmten Etreifen an Den Fetalen. Das obere Eepalun

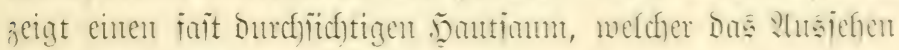
von Silberiticferei hervomut.

Bis jur Cinfithmung Des Bolbophyllum Reccarii im Jahne 1867, mumen bie Grammatophntlen als sie theien

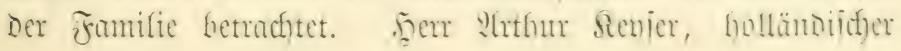


Refitsent zu Selangenr, erzäflt von einem Eremplar, meldyes

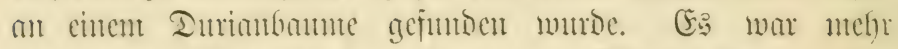
als zmei Meter hoch, itfer vier Mieter freit, truț

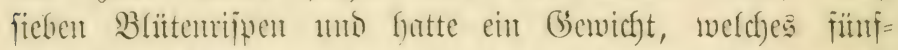
zef) Mian faum zut tragen imjtande waren.

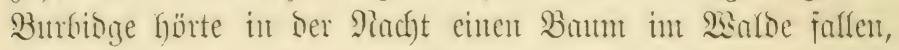

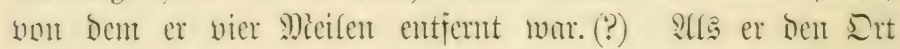
aufindete, fours or anf Dem Etanme cin Grammatophyllum,

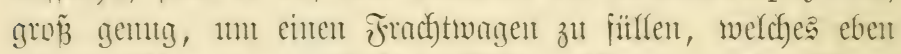

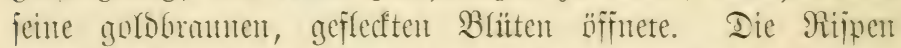

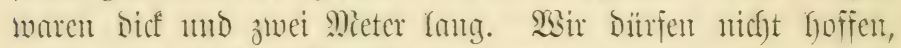
Dañ wir je foldhe Sulofie in Guropa zu jefen befonment.

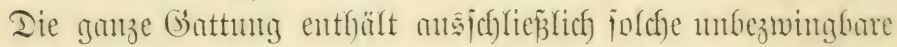
(Geitalten. G. speciosum erreich)t in ber Sulttur zuvei Nieter

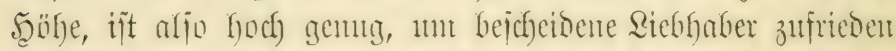

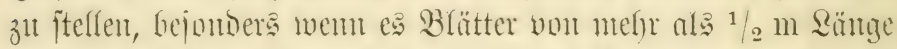

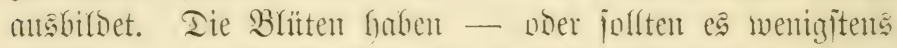
- $8 \mathrm{~cm}$ in Dutchmelfer; fie fins tiefgelb ntmo pumpurot

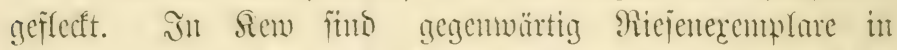

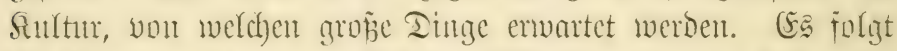
G. Measureseanum, nach) Şerm Dienjures, cinem Gefanten

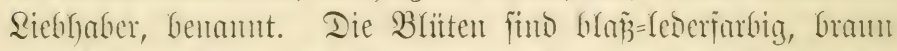

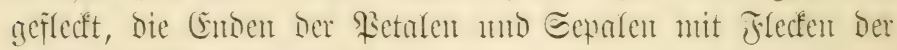
gleichent Nientree getitpfelt.

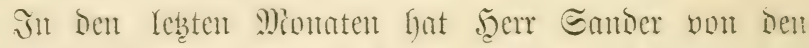
Prgilippinen G. multiflorum erfalten, welches nicht mur bas

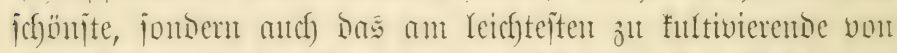

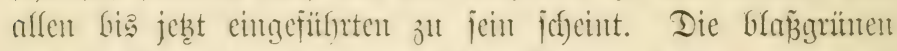

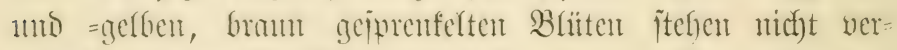
cinzelt wie gewöfnulich, jonbern fanm einen (Eentimeter vou

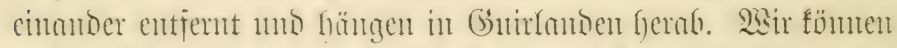

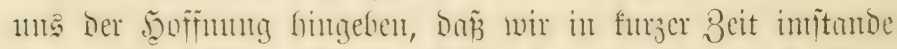

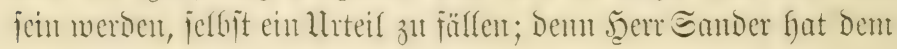




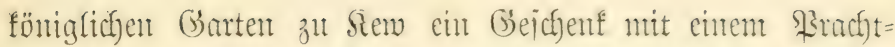
exemplar gemadat, welches mitreitig Die gewaltigite Erchioee iit, bie jema(s nach Europa ge(nach)t murbe. Eie it im

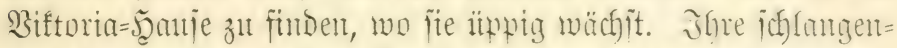
ähnlichen Etämme fint $3 \mathrm{~m}$ hoch, und bie alten Blitten= jtänbe ragten bis zu $6 \mathrm{~m}$ ફ̧öhe empor.

\section{Ilantes \&apitel.}

\section{Eine veriftyollente (I)}

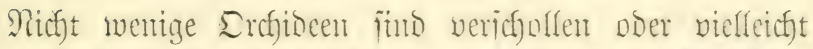

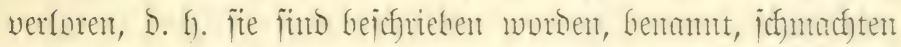

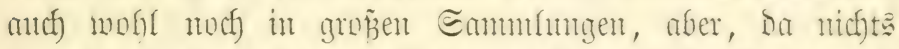

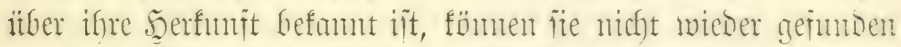
merien. Dies ijt Der Fall mit Cattleya Jongheana. Cymbidium Hookerianum mo Cypripedium Fairianum. Jenod) eine, anf welche Der Iitel nuch vor einigen Ingen gentan ge= pañt hätte, ijt inzwijchen miebergeimben: ('attleya labiata rera. Eie mar bie erite, welche Den Plamen Énttenn trutg, obichon micht bie erite an Diejer Gattung, melde cut= Decft wntroe; Dem bies war C. Loddigesii. Dieje ijt eminge Jafbre länger befannt, murne jeboch bantals Epidendrum

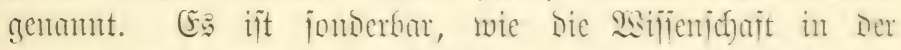

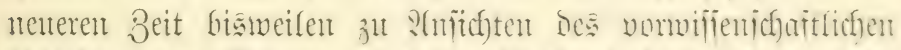

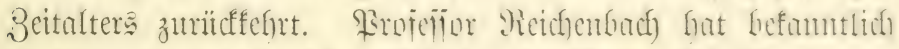

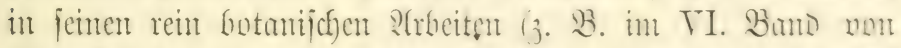

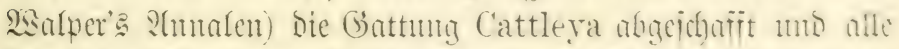
S.rten Deriefben unter Epidendrum gefurad)t. Cattl. labiata

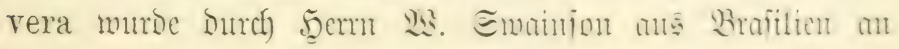




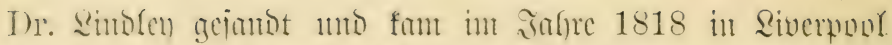

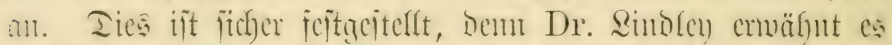

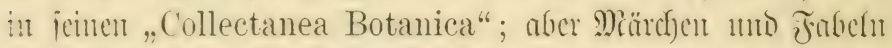

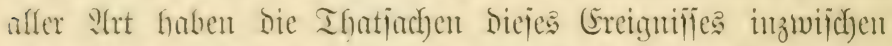

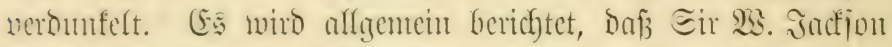

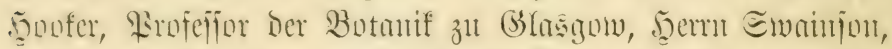

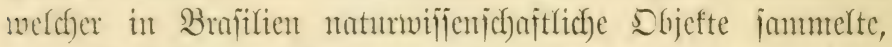

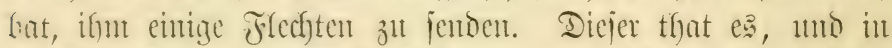

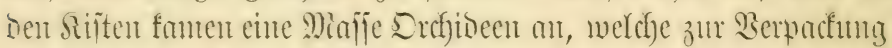

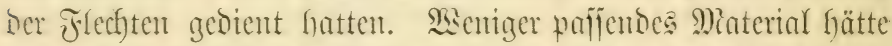

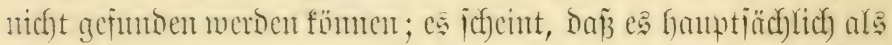

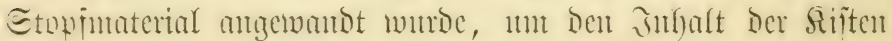

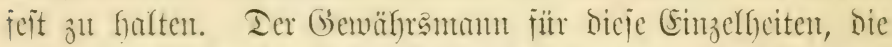

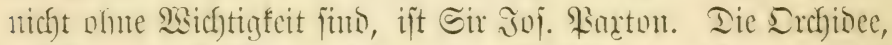

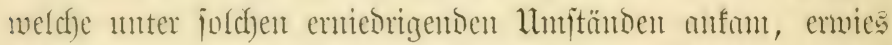
fich) als net, und Rind(en gah ifrr Den Namen Cattl. labiata;

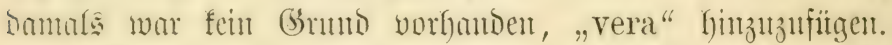
(Ex itellte eine nene Gattung anf mo rettete jo für alle

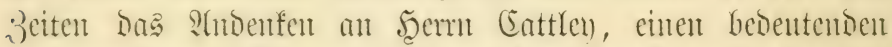

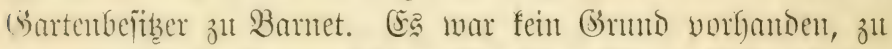

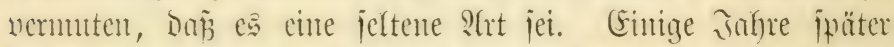

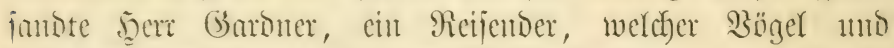

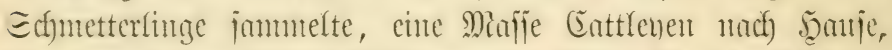

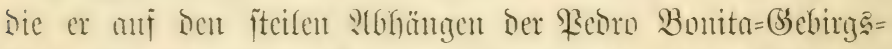

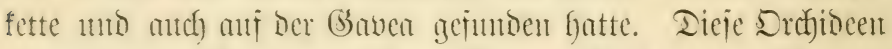
gingen einige Beit unter bem ?amen (C. labiata. Parton aratulierte domale fich mo ber Welt in feinem "Flower

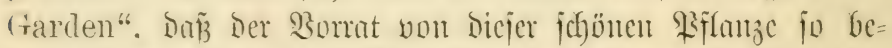

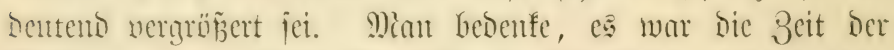
Fontmagen, wo Botanifer nicht fo vick Belegenheit fatten,

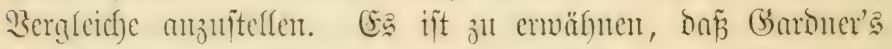
Eattlena an nächiten vermanot nit Der yon Emainjon emgejührten mar; fie iot hentzutnge fiefount als r. labiata 
Warneri. Dic echte Form fout jeboch unverfenture Dierfmale.

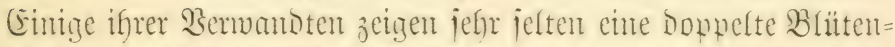
icheioe, welche aber bei C. labiata vera nie fehlt, uno es iit eine interefiante Frage, meshalb bieje allein ifme Blitten jo jorgfältig bejchitbzt. Wian fömute — mit Dent nütigen Worbefalt - vermuten, baj ifr Etmonort noch fenchter jei, als ber von anderen Bartetäten. Eobam haben einige Fiflanzen Blätter, weldhe rot, andere joldye, weldhe grün ani

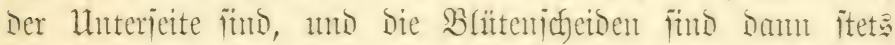

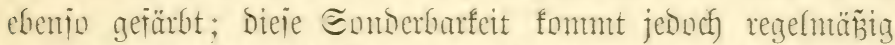
unr bei C. labiata Warneri vor. Dritten - uno bies itt Der

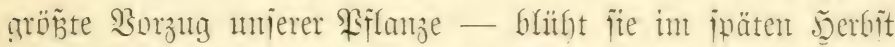
mo fiutllt jomit eine Ritcfe in Der Blittezeit mierer Srchioeen

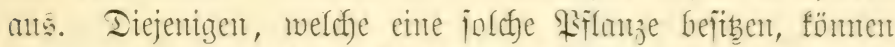
Das ganze Jufne Gindurch Eattlencu in Blitte frahent; aber untr

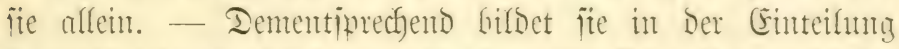
Der „Reichenbachia“ eine Seftion fitt ïtch allem, als bie

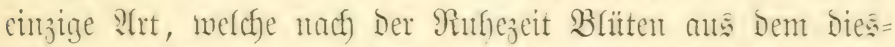
jährigen Iriefre bringt. Eeftion II enthält Die :(rtent, meldhe noch vor Der Ruthejeit aus Dem Jafurestrieb Glïhen, Eeftion III Diejenigen, welche ats bent vorjährigen Triebe nach ber $\Re u$ he=

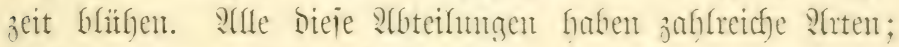
C. labiata vera fteht jedoch allein.

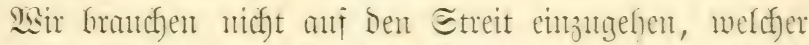

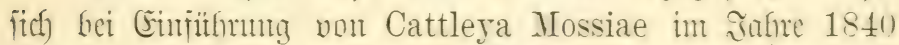

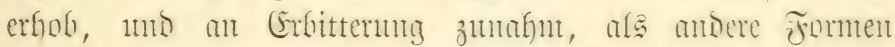

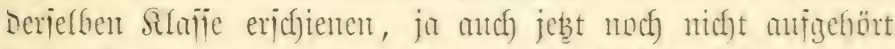

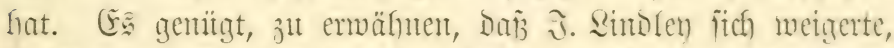

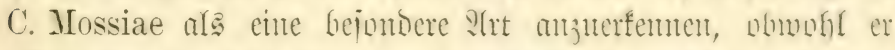

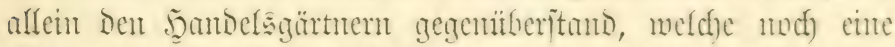
Echar von begeifterten Sieffiabern finter fitch hattem. Ier aroß̃e Botanifer erflärte, er föme in Der pröchtigen mentr

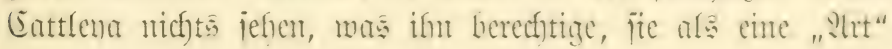


nou Der Gereits befumten C. labiata ju unterjcheiben, an:

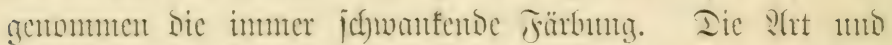

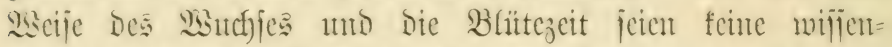

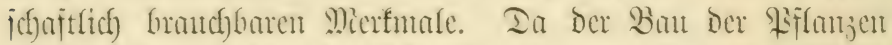

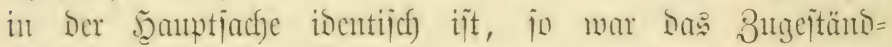

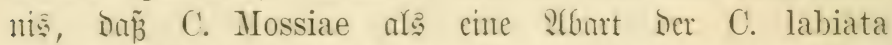

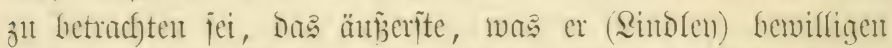

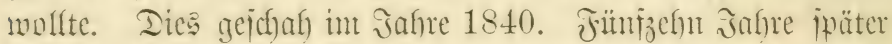
fan C. Warscewiczi, jeß̧t gigas genamt, in ben Şantel, ein Jafn inäter C. Trianae, C. Dowiana in Jahre $1866^{\circ}$ und C. Mendellii im safre 1870 , alle, gentut gentommen, nutr Formen von Cattl. labiata. Bei jener Einfüfnntug

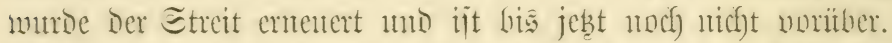

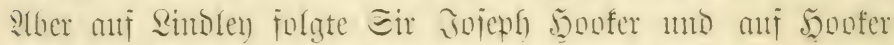

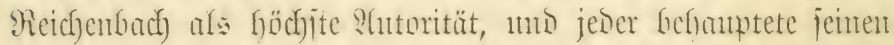
Etmonunft. Eelbitueriänolich find viele Cattlenen als jo=

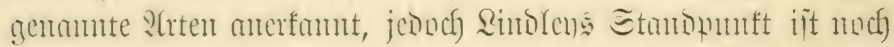

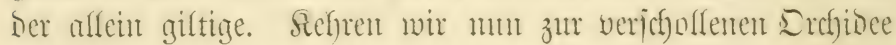
zแnüct.

Wit Der Beit mutro Der Sient wou C. labiata rera

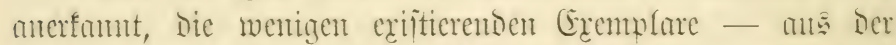

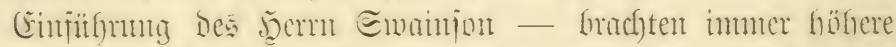

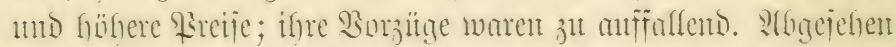

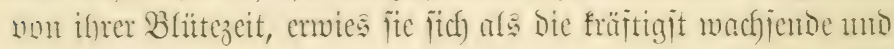
ant Iciduteiten ju fultivierente Cattleya. ज्ञne Form max mentigiten io jefön als bie ingend einer moteren, mo fite jeigte

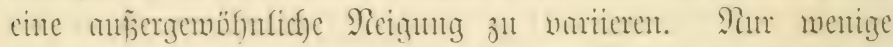
Hiflangen waren, wie gejagt, in Sultur, aber bieje enthielten

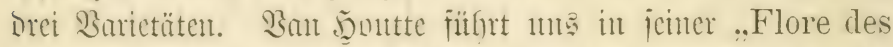

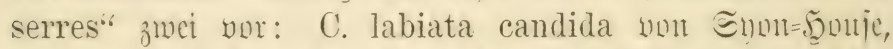

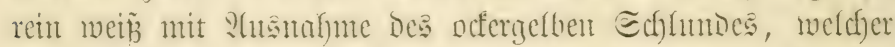
Lei allen Cremplaren vorfonmt, und C. labiata picta, sumfel=

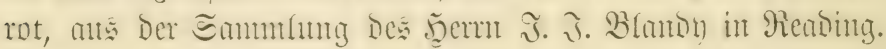


Iie Dritte, C. labiata Pescatorei, wein mit Dunfelrotem Fleef anf Der Sippe, war erit CFigentum Der bुerren Fontget=Chau=

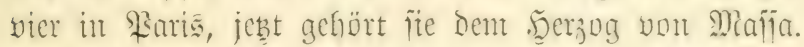

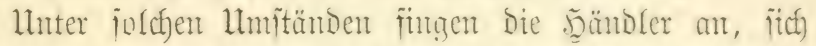

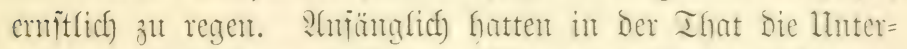
nefmung

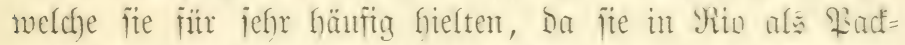

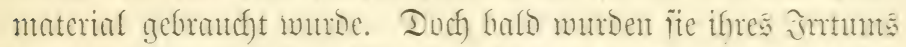

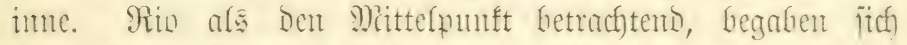

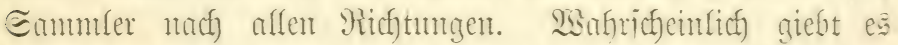
fein bedentendes ammortgeidäajt weder in (Fngland noch anf Dent Feitlanoe, melche nicht Geld - und bisweifen recht hedentento Eummen - aufgewendet hat, um C. labiata vera

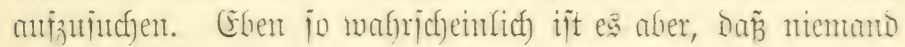
hei Dicier Evefulation verforen hat; Dem ofmoht man von Der acjuchten Fĩlanje feine Exur fand, ja nicht eimmal ein

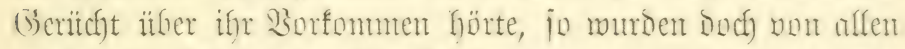

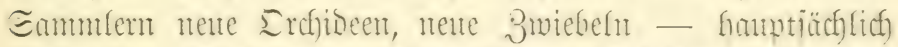

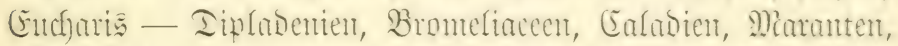

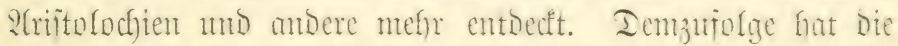

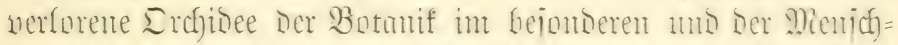

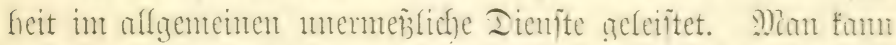

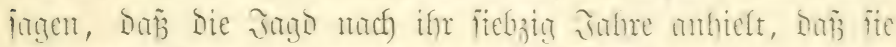

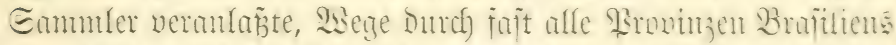
cinzuichlagen; fait alle - Dem es giebt nodh hentzutane gan?

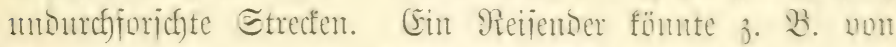

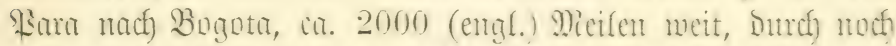

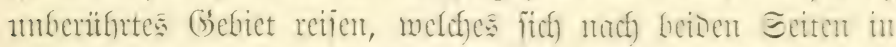

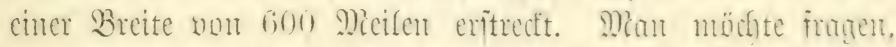

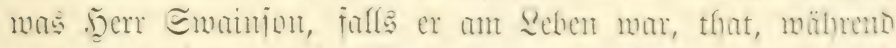
ieine Entoctimng io bie siselt anfreate. Er lebte men, uno

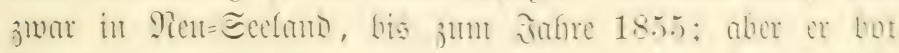

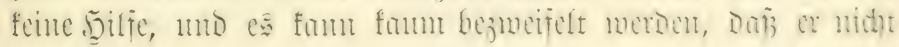


imitante war, jolche ju leiften. Die Srchibeen waren wofle

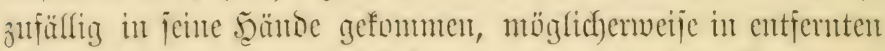

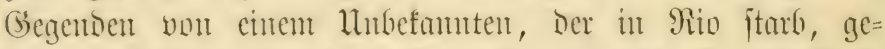

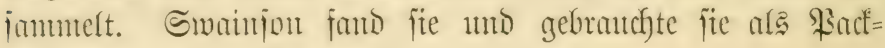
mtaterial für jeine Flechten.

97icht mentiger jontoerbar ijt es in bicjer merfwitroigen

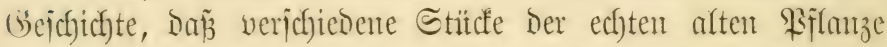

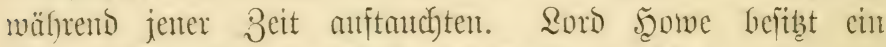

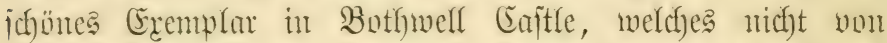

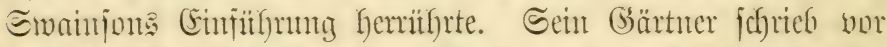

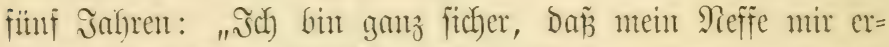
jühlte, bas fleme Stitcf, weldyes ich von ifm vor vierzig

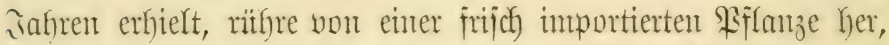
meldye mit einem Schiff ber Şerren 5ुorafall angefonment war."

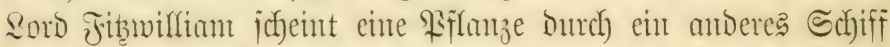
erfalten zut habent. Seboch Der itannenterregendite Fall ereignete

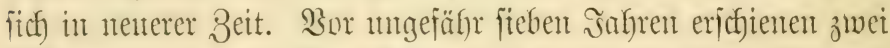

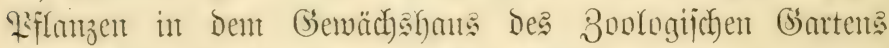

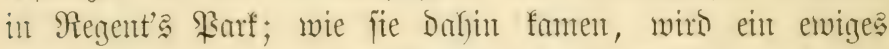
Geheimmis bleiben. Socr Bartlett, Der Direftor Des Boolo= giichen Grartens, verfante fie fïr einen hohen Preis; soch fonnte er eine gleiche Summe, bie ifm gefoten muntio für

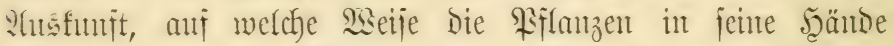
gefonmen waren, nidht versienten; Denn er war nicht im= itande, eine foldhe jut erteilen. Shyte Bmeifel foment fie in Gejellichaft ciniger S(ffen an; aber wan mo ats weldher

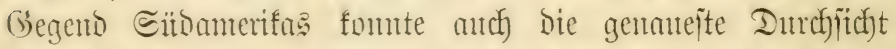

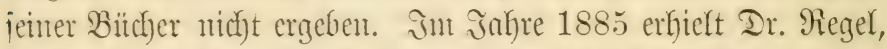
Eireftur Des Gotanijchar Gartens zu St. Fetershutg, einige SFflanzen.

Uno mut berichten bie Garten=Beitungen, bap̃ bie ver=

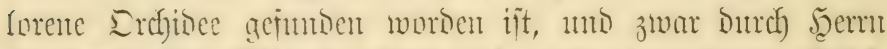

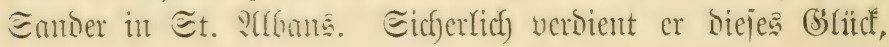


wem ein zwanzigjäfriges Suchen jo genannt werben famt.

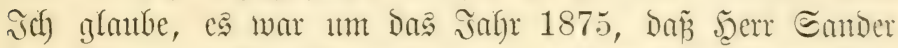
ben Sammler atrutb amsande, welcher fïnf Jahre hin=

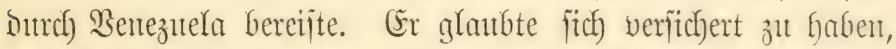
Daj bie Rflanze nicht in Brafflien jut jutchen jei. Nach

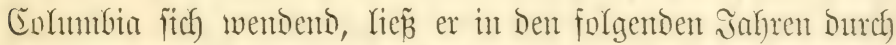

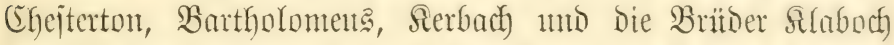

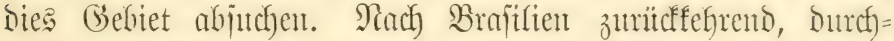

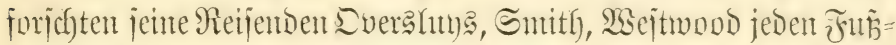
Greit Ranbes, weldyen Swainjon, jeinen Bitchent nadh, betreten

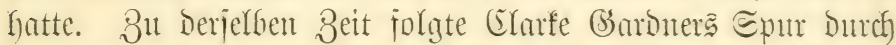

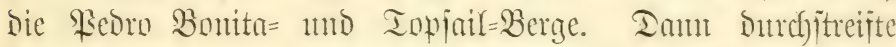
Somers bie ganze Rifitenliute Brafitiens von Norden nady Sitoen, fitr welche Reife er fïnf Jahre gebratchte. Entolid) muternafym Digance bie Suche, itarb aber noch in Demijerben

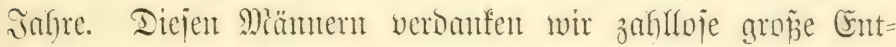
Secfungen. Hun blos die bedentenditen zut ermähnen: jo fanto Strutolo Cattleya Percivalliana. 3on Cofumbien mutroe ge= fracht: Odont. vex. rubellum, Bollea coelestis, Pescatorea Klabochorum; Entith jantste Cattleya O'Brieniana, Élarfe bie Bucrgcattfeyen pumila mo praestans; Rawrencejon fant Cattleya Schroederae, Cheítertor Cattleya Sanderiana, Digance Cattleya Diganceana, weldye an 8. Eeptember

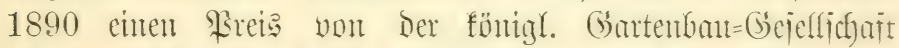

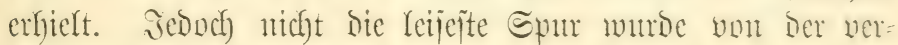
Iorenten Drthibee entidect't.

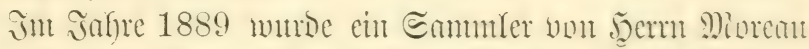

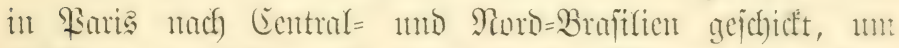

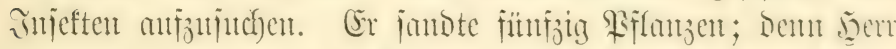

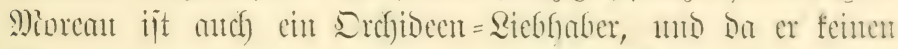

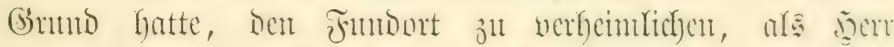
Somber bei einem Bejuche ben jo lange gejutchten Echats or

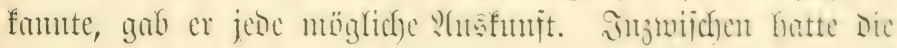




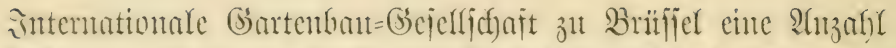

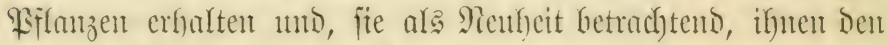
Pamen C. Waroequeana gegeben, in weld)ent Irrtum fie

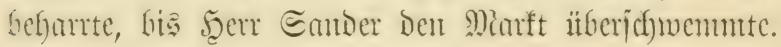

\section{Beluntes faptitel.}

\section{Einte (I)}

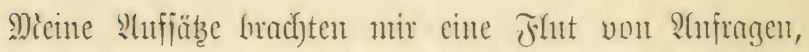

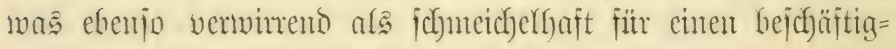

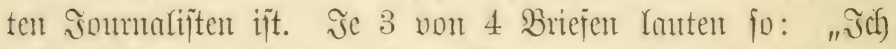

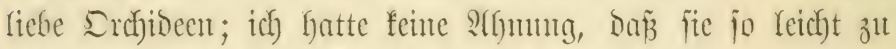
fultivieren unto jo billig finto. Id w will bannit anfangent.

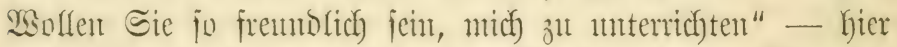

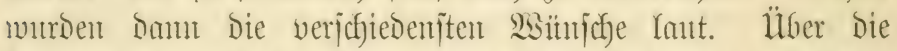
Temperatur 1 mo bie Bhumentöpfe, die S(11

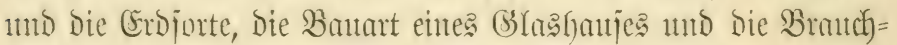

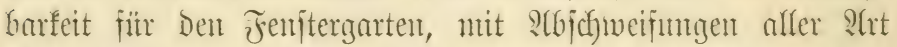

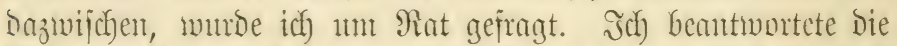

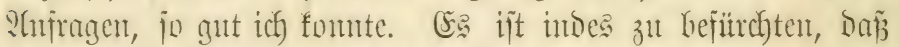
Die gentauejten Fragen und Die weitgehendĩten Bcantwortungen

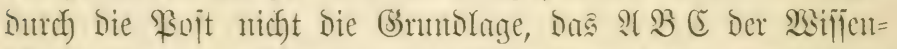

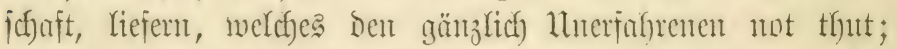

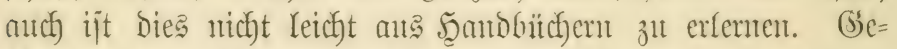

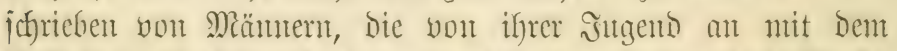

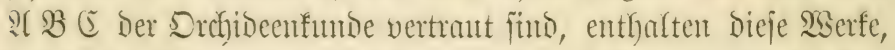

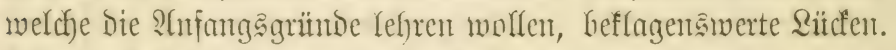
(rs ift mentig, mas idf) in ber Eache thum faun; doch im 


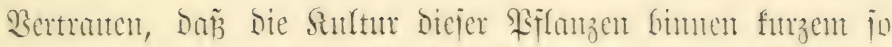

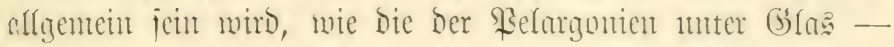

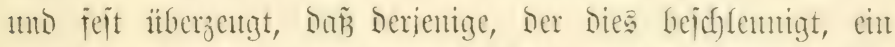
wiff(icher ssoflthäter in jeinem Fache ijt - Gin ich) Gereit, alles

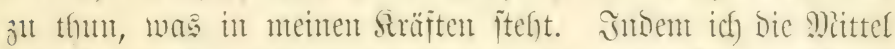

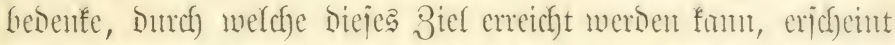

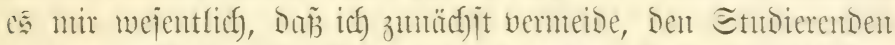

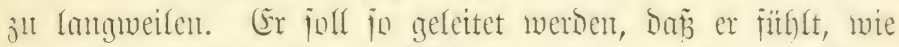

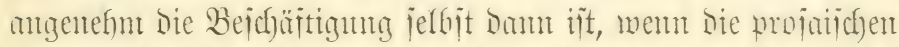

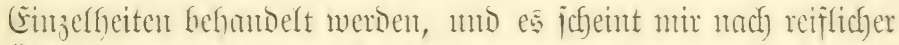

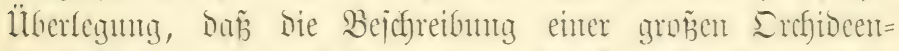

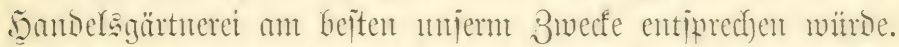

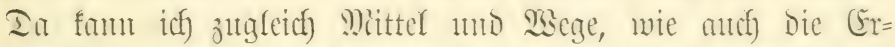

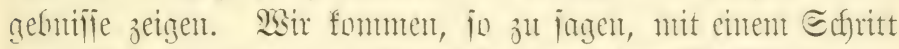

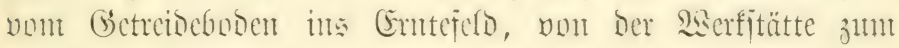
vollenteten Grzengnits.

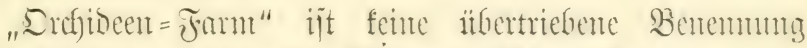

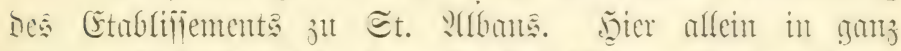

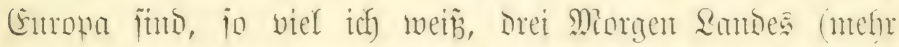

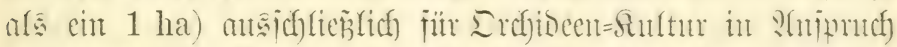

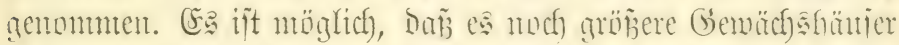

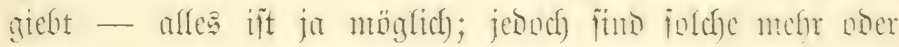

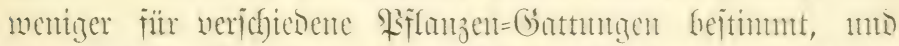

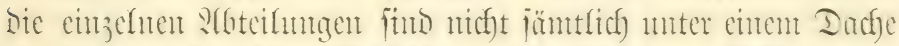

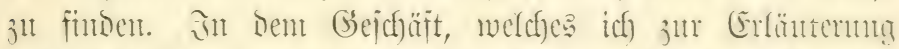

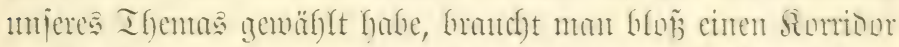

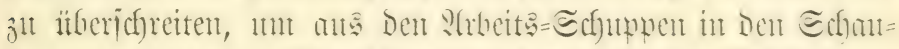

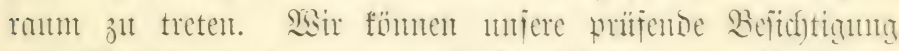

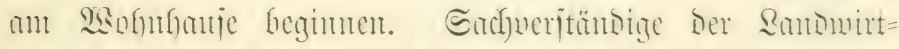

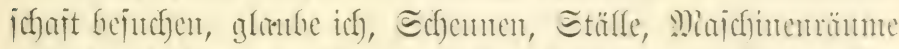

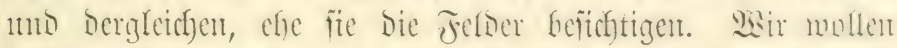

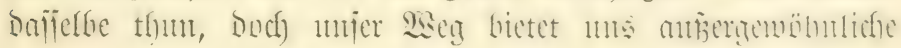




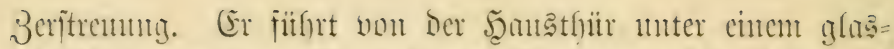

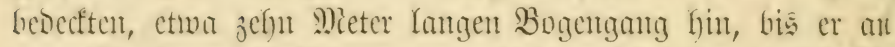

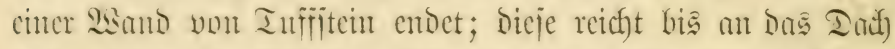

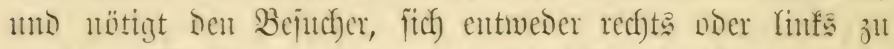
wenton. SEafjer rimut herunter uno füllt plätjcherno in ein

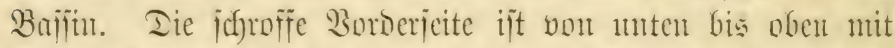

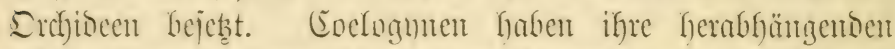
Blittenripen verloren, Die bis vor furrjer Beit Den Felfen wie

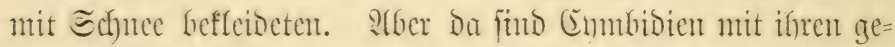

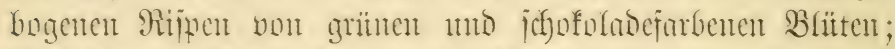

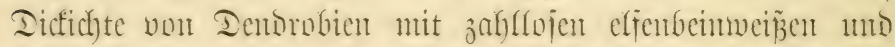

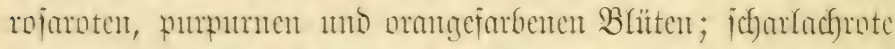

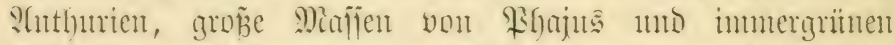
Ealonthen, nit Duscenden vou Blitteniftengeln ats Den freiten

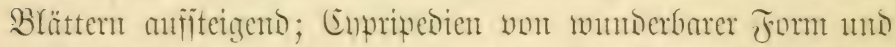

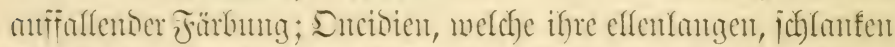

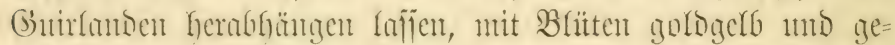

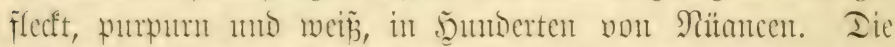

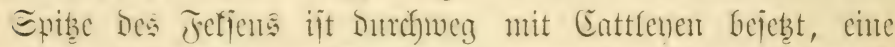

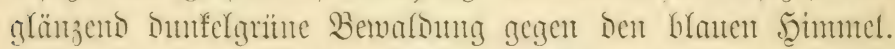
Die Trianaes fimo fajt voritber, buch fommt fier und da cine

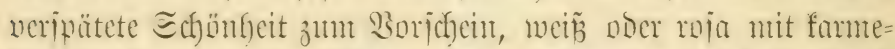

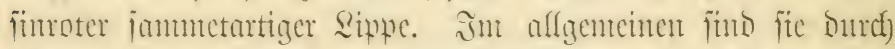

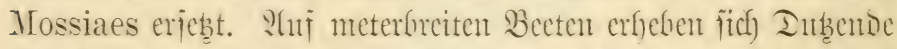

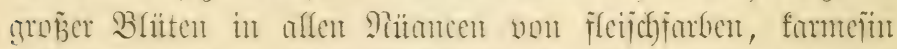

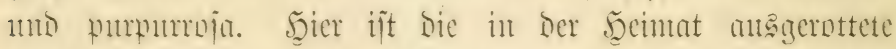
Laelia elegans von jo itümnntigent mo ïppigem sistchje,

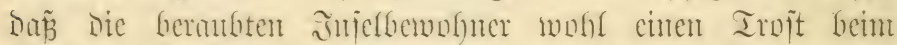

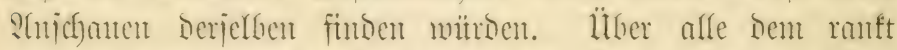

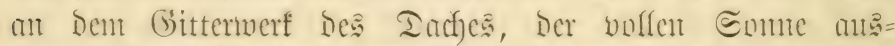
gejeb̧t, Tanda teres cmpor, in Blättem un Etamm num

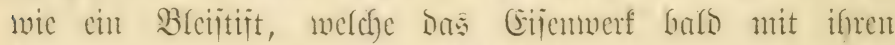

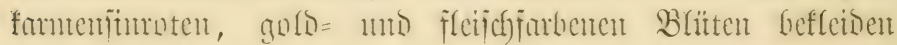




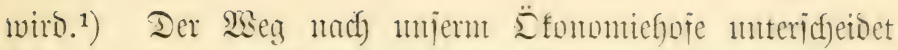

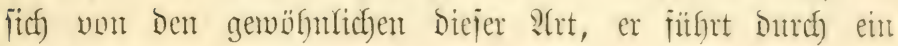
3auberlanto.

Wir fünon eine Ifyitr Dutrch) eine Felientartie maśftert,

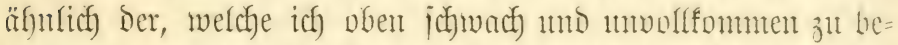

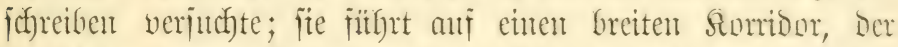
in jeiner ganzen Ränge von ca. $130 \mathrm{~m}$ mit mexricanijachen

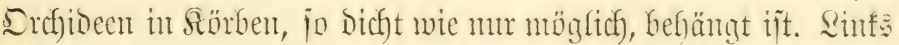

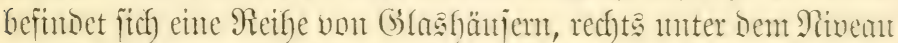

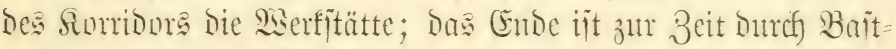
matten veriperrt. Doch Deutet bieje Bwijhenmand Daranf Gin,

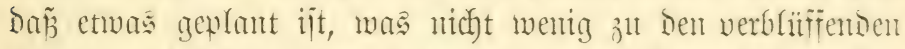

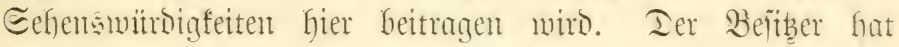

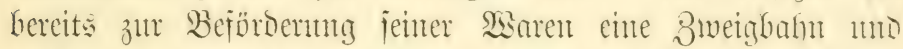

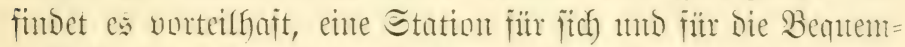
lichfect jeiner Sumben jut haben. Şinter Der Bmijchemmanto

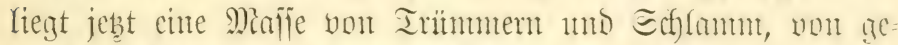

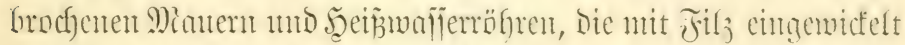

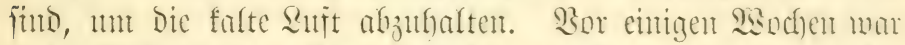

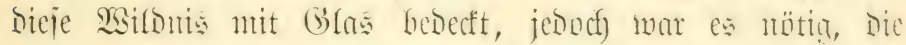

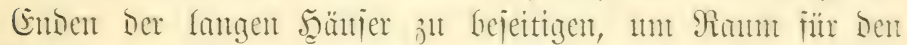

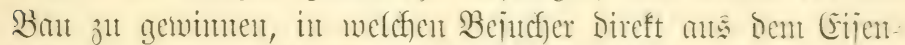

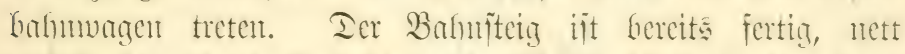

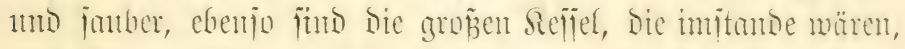

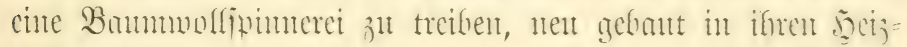
jtellent.

Eminige Regionfeit Gietet jidf) mijeren B̊ficten, menn mir

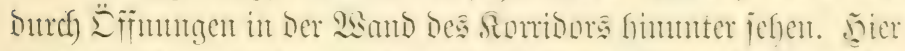

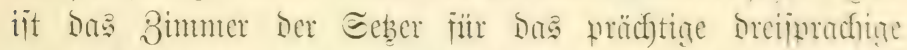

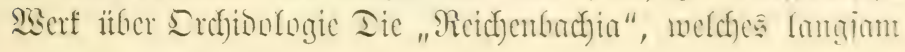

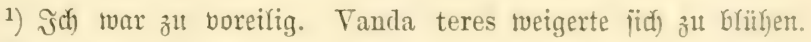
(2(mmerfung Des : Berfaijers.) 


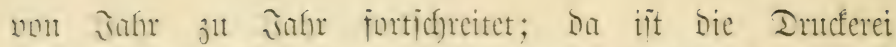

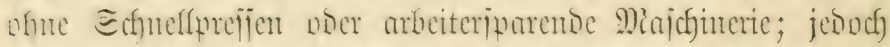

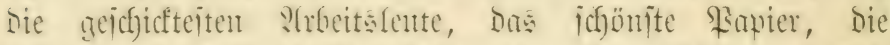

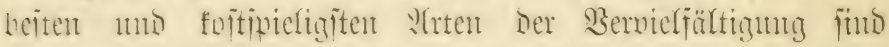

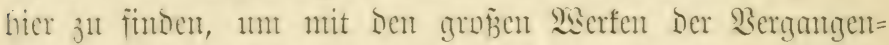

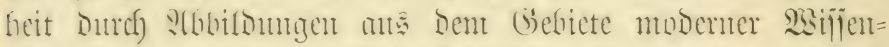

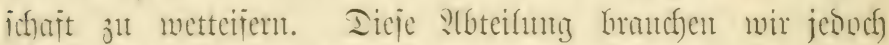
nicht zut bejuchen, andf) nicht Dic Reäume weiter muten, wo meechanijche ?(rbeiten berrichtet werben.

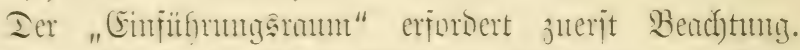

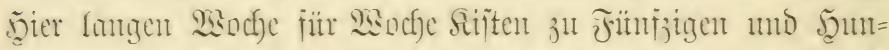

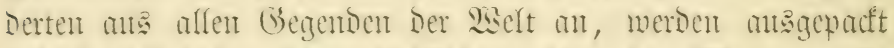

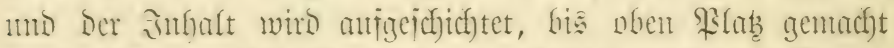
mernen fum. Es i ĩt cin lantger, freiter, nieoriger Raum,

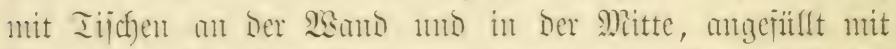

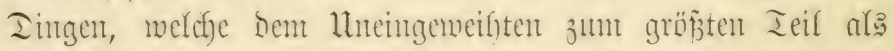

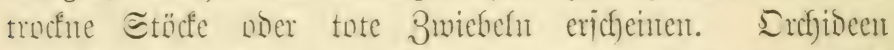

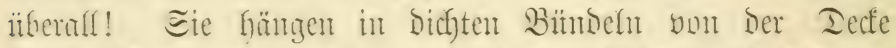

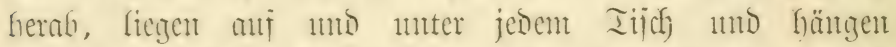

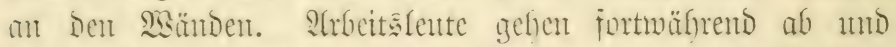

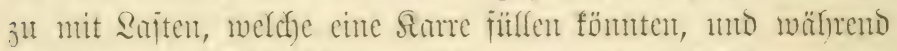

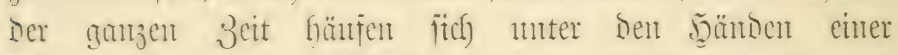

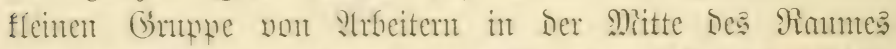

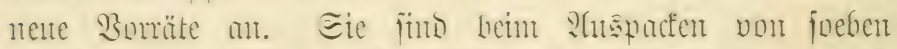
angefonmenen Siiten, welche, 80 an ber Bahl, geitem abento

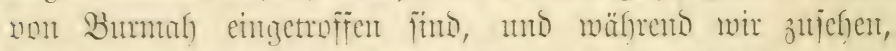
Gringt ein Buriche ein Ielegramm, 50 weitere Sijten vout

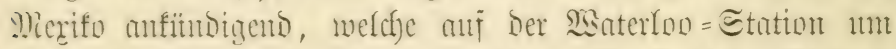

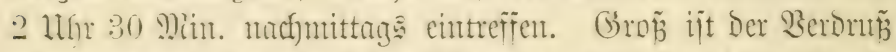

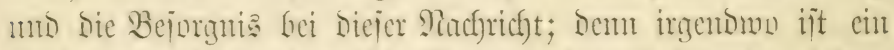

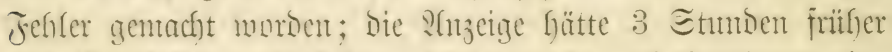
cintreffen joffen. Ercfibeen Ditrfen nidf)t auf Etationen ein=

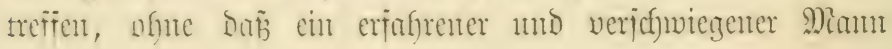




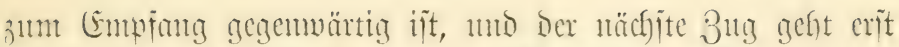

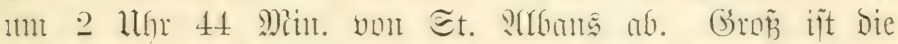

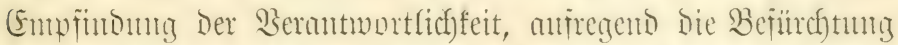
cincer Sibernuärtigfeit, welche Duref Diejes Berjehen hervor= gentfen mird.

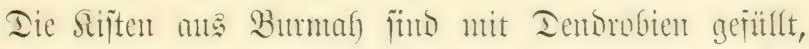
crassinode mo Trardianum in Echichten jo Dicht wie möglich

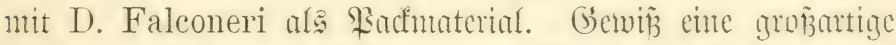

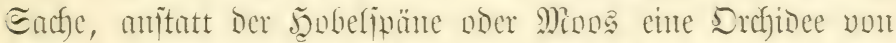
gert jut verwenden, Doch ebenjo pafjend mie cinträglich;

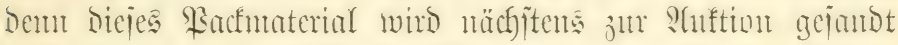

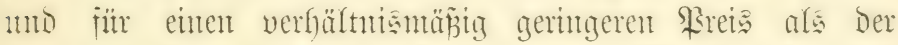

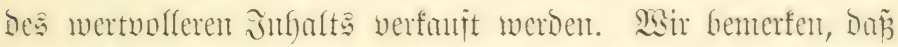

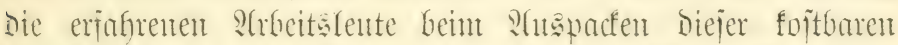

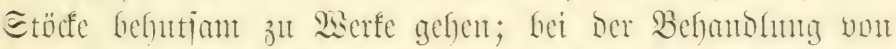

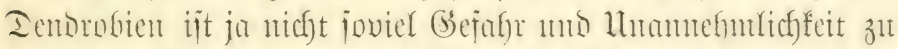

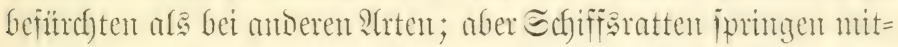
unter herans mo verjeben innen abjohentiche Bifie. Eforpunte

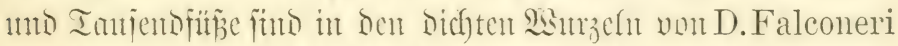

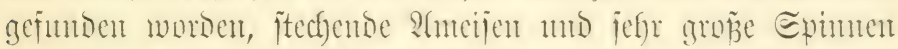

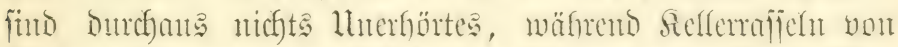

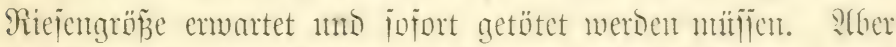

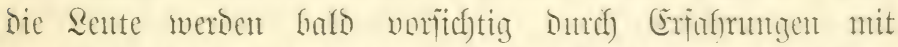
weit gefäfreficheren Rnonngen. ज̂n Den Diafien von Arundina

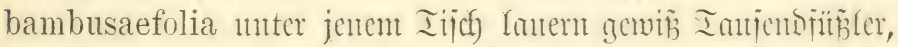

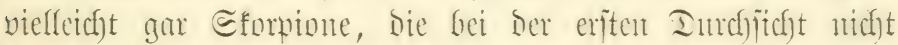

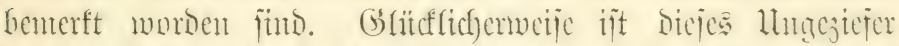

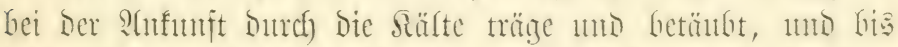
jebst ift feiuer vou Den Senten - vorfidutig wie fite f̈nts -

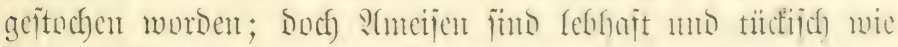
Daheint. Fine Ecmonnig von Epidendrum bicornutum verlanąt

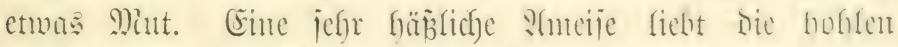

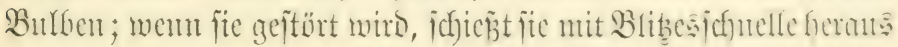




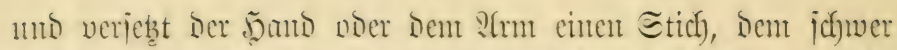
ju entgehen ijt. S(m fenwierigiten fint bie Sijten zut hanto= bahen, meldhe Schomburgkia tibicinis entfialten. Dieje

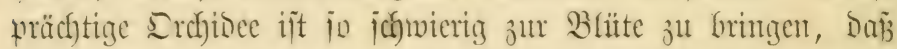
jefre wentige es verjutchen. Sich hathe bie Blitten mur zmeimal

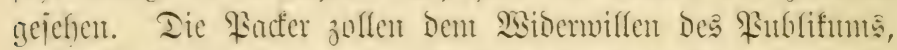

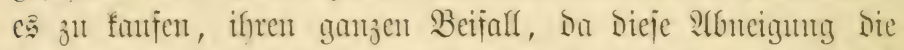

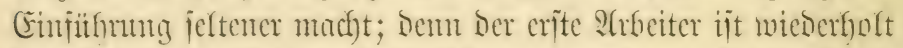

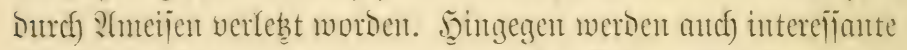

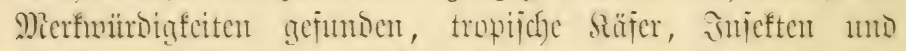

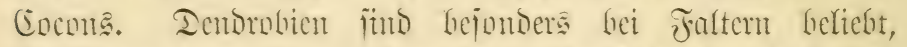
D. Wardianum ijt mit ifren Geipinften bescect, jeboch) finto bicie gewöfunfich leer. Bisfer fraben Die Rente fente

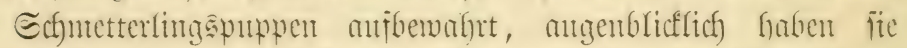

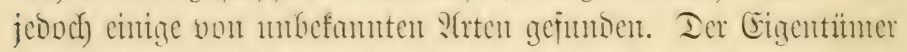

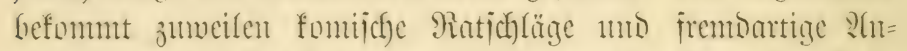

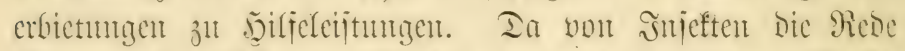

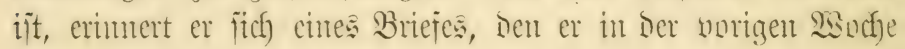
erfielt. Or lautet aljo:

\section{Meine Serren!}

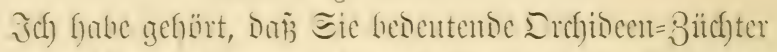

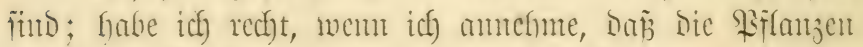

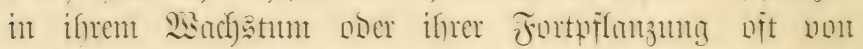

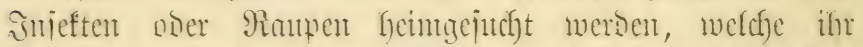

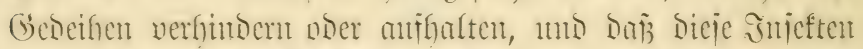
Doer Ranpen Durch fleine Echlangen vernichtet merben

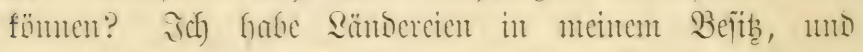

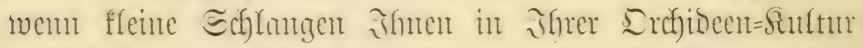

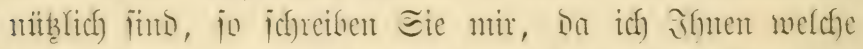

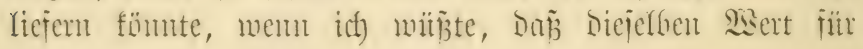
Sic haben.

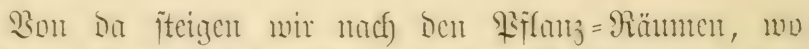

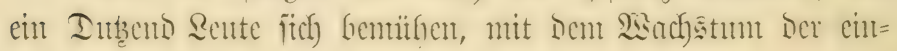




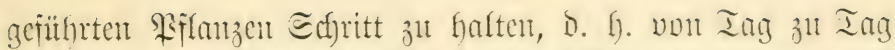

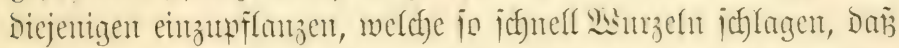
Berzögerung nachteilig jein witrode. Die breiten Sijche in ber

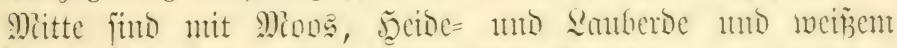
Eande bebectt, an jeber Ecite find J̧anblanger mit Eiehen

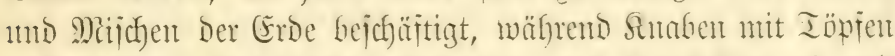

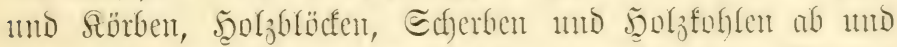

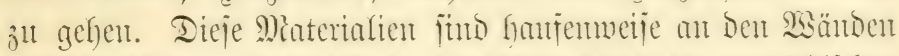

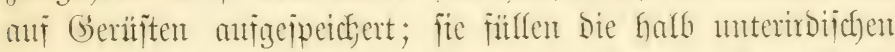

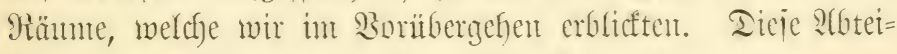
luntg unjerer Farm gleicht einer Fabrif́.

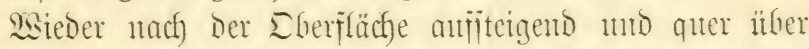

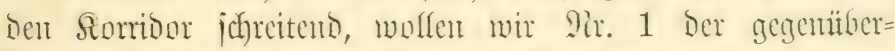

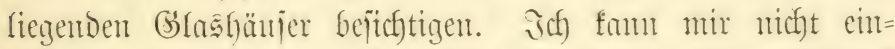

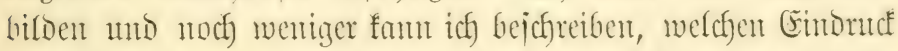

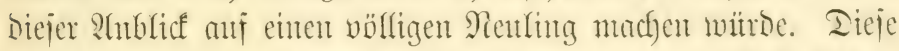
bänjer - es fint Deren zwölf nebenemander - finto $60 \mathrm{~m}$

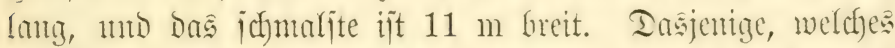
mir betreten, ift mitr fïr Odontoglossum crispum 1 mil einige Massovallien bejtinmt. Şier waren cinte Iage 22000 Töpfe; mefrere Ianjend fino verfant, andere Tampane herein= gefracht worden, und bie Shazahl, welche en gegenmärtig ent=

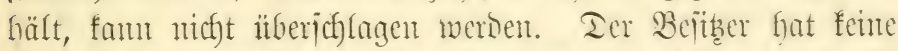
3eit, fith in Dergleidfen Berechnungen einzulaffen, er treitut en gros-5ูantol. Mian telegraphiere cune Bejtelfung anf tmijeno

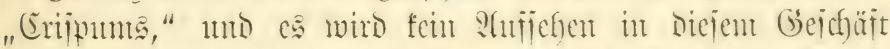

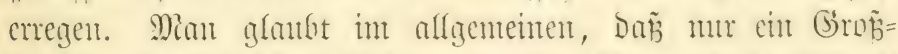
fämoler einen joldhen ?aftrag erteifen füme, Doch bies ijt

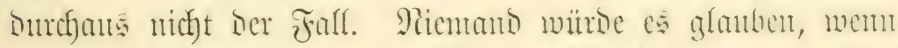

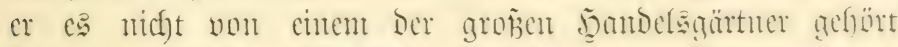

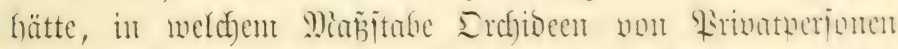

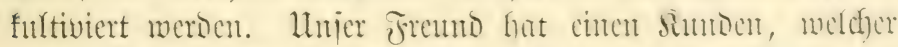
feinen Borrat von $O$. crispum allein anf ber siobe non 


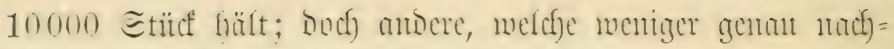
zäf)len, mögen viefleidgt noch) mehr haben.

Ier Ifiur gegenther iit cum hohes Geriint mit Etufen,

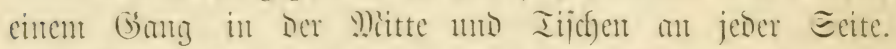
Iieje fun nulf won 0 . crispum, jenes mit ciner noer juet

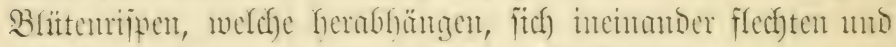
mieser mipuärts iteigen. Sitcht alle f̈uts gegenuärtig in Bhlite. Dicjen iefenomerten ?hulict merden wir erit im

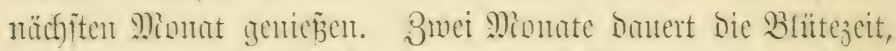

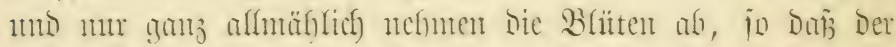

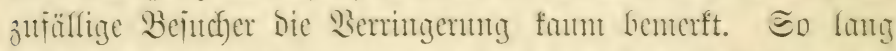

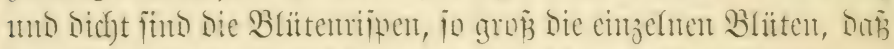

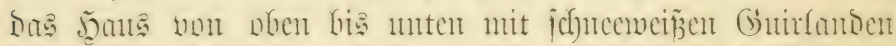

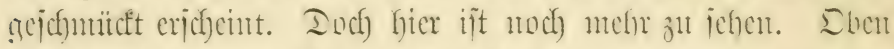
befunon fidf gange Reifen wou Sibrben mit herabhängenden

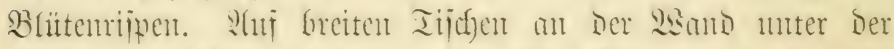

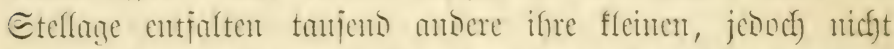

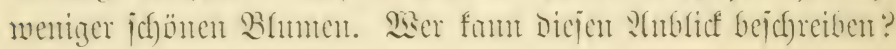
sich bin es nidft imitande!

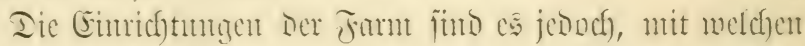

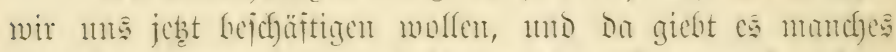

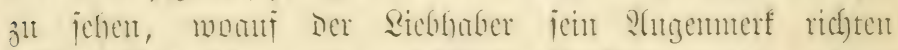

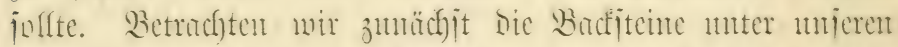

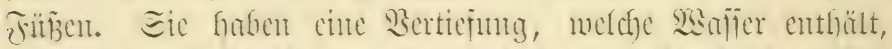

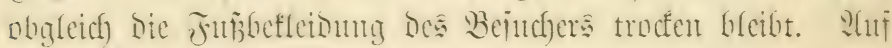

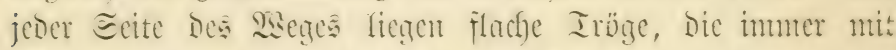

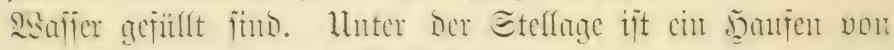

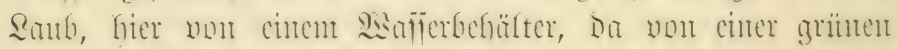

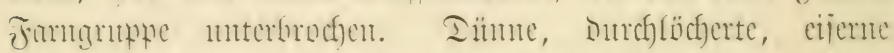

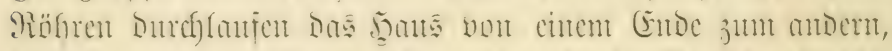

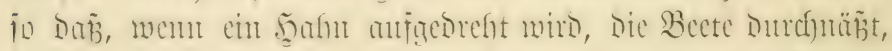

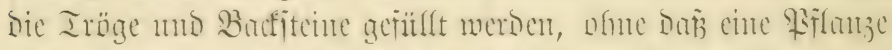

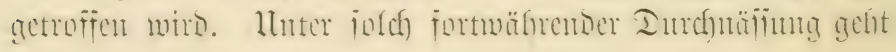


Das Santb unter Den Iijchen in Berwejung ïber und cntwicfelt

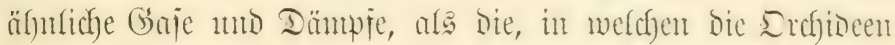
in iffrer beimat fitch jo wohl bejumben haben. Pach Diejem

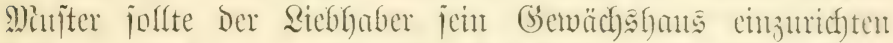

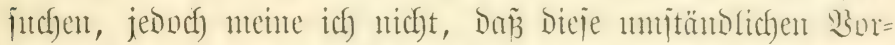
ridftungen alfe wejentlid) fints. Natr wem, wie fier, mit Der Patur gewetteifert wirs, joldhe Bhymen und Sundlen Gervorzutbringen, wie cis nur unter jo jeltenten Besingungen

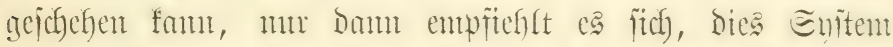

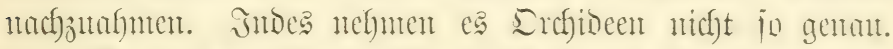

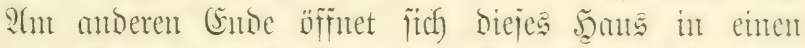

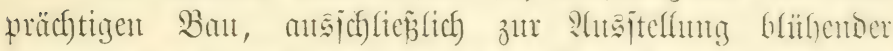

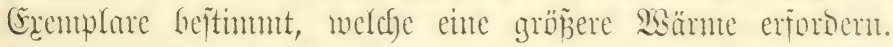
Er ijt $100 \mathrm{~m}$ fang, $9 \mathrm{~m}$ breit 4 mo $6 \mathrm{~m}$ hoct. Die Darin befindicfen Röfren witroen antentandor gelegt nahezu

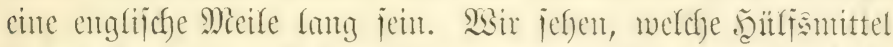

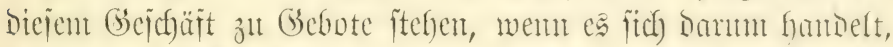

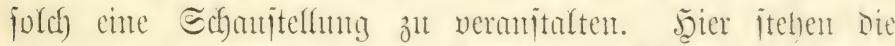

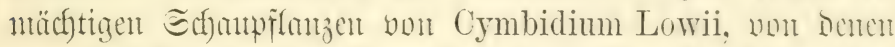

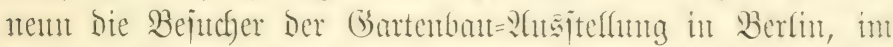

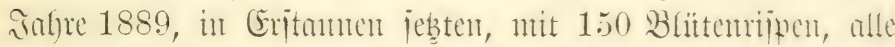
zu gleicher Beit geöffutet. 2sir benterfen hier cine Mentuge

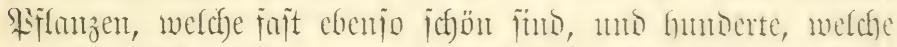

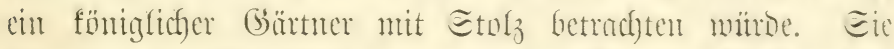

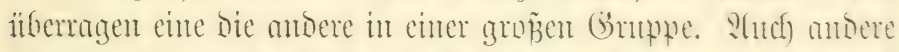
(Emmbisien fün hier, aber nicht bas ichüne C. eburneum. Ecint

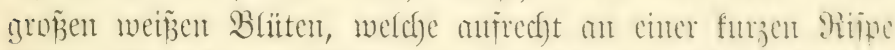

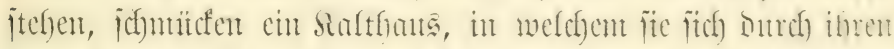

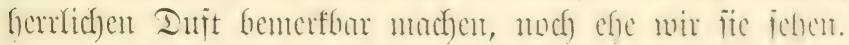

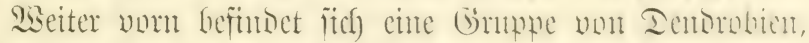

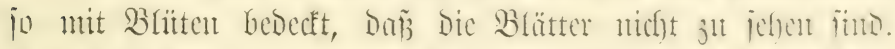

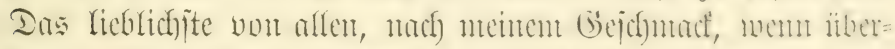

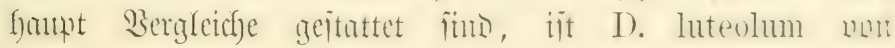




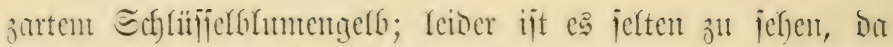

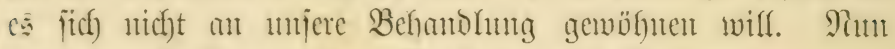

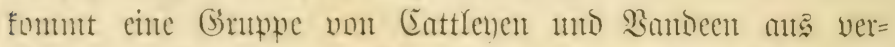

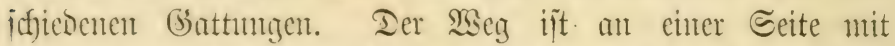

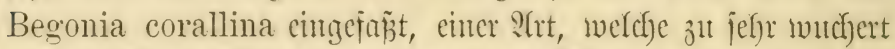

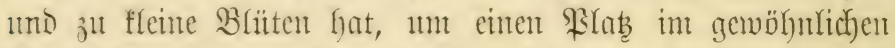

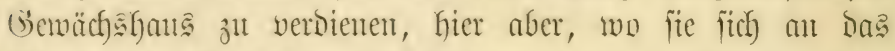

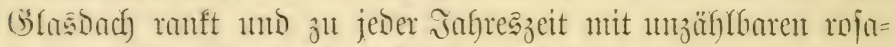

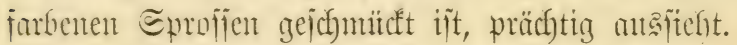

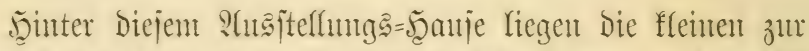

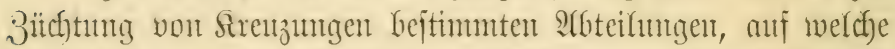

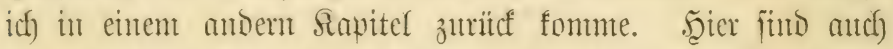
Die PGalaennpfis, Die 3anneen, Die Bollea, Pescatorea, Anoectochilus uno ambere zierliche aber eigenfinnige Echön= heiten untergebracht.

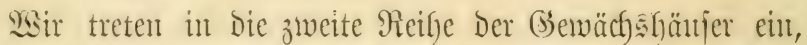

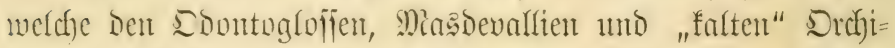
Deen vorbefalten ijt. Sie fint ebenfo gejüllt uvie die vorfer=

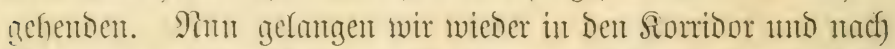
Shrmmer Drei, weldes vou (Eattlenen mo Dergleichen in $\mathfrak{B} C=$

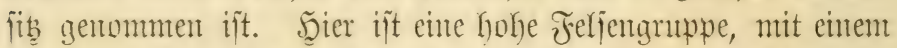

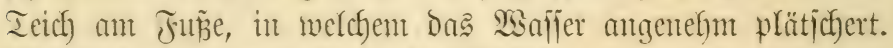

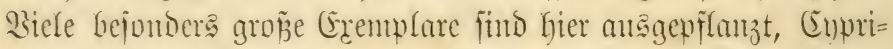
Hesien, Eattlenen, Eofralien, Sigajus, Raelien, Bngopetalum mo fumbert andre, afles Firnchteremplare, dos will jagen mit

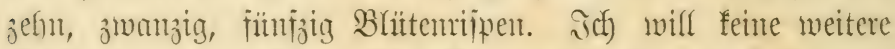
Beichreifung verjuchen. Saser Remer ift, Dem wirs bie ein=

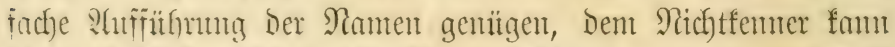

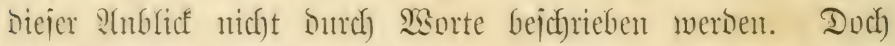

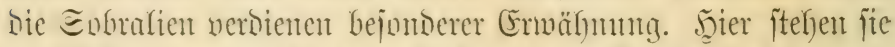

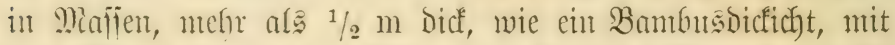
ciner Nienge iffer gronen Blitten ant Enoe Der Etengel, int all= 


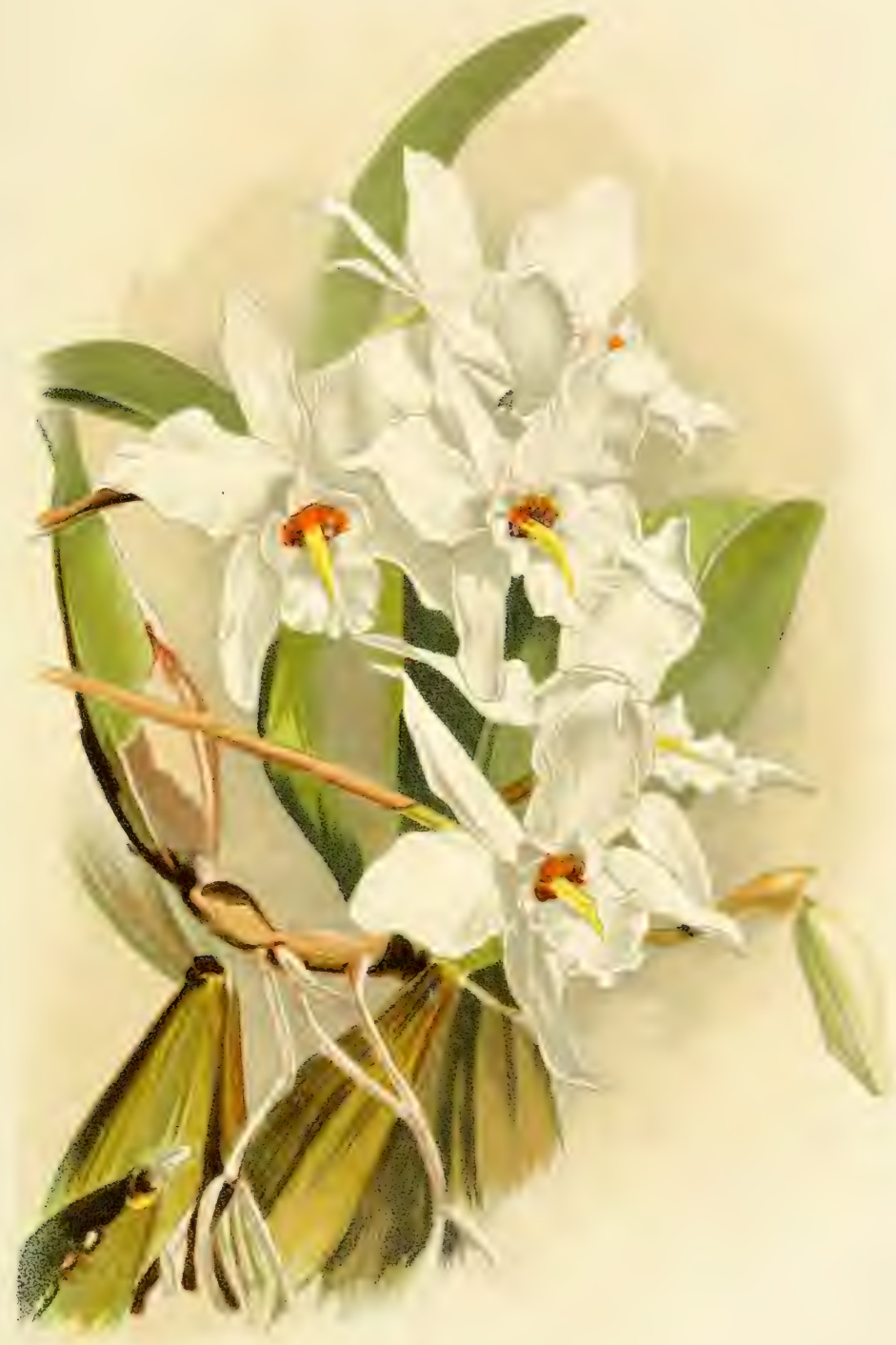





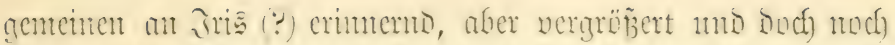

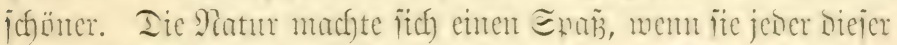

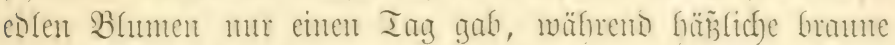

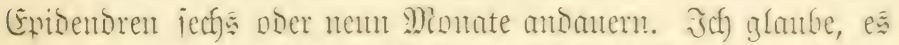

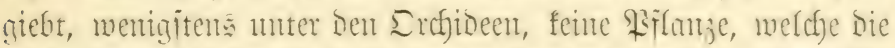

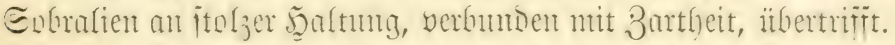

îch bari mich nicht zu lange bei hem 23 merfaren vor

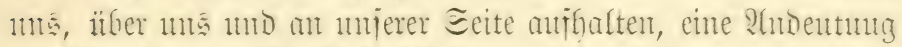
mun gentrgen. Ia fint Sörhe mit Laelia anceps, $1 \mathrm{~m}$ in Intrantefer, ganz io wie fie von Banme abgenonmen find

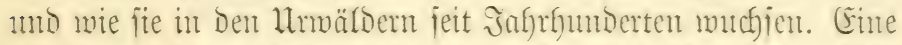
von ifmen, bie weiñe Barietät - weldje äitetfijch gebildete lln= glänfige anbeten fönten, and wenn fie jonit an ?ichts glanben

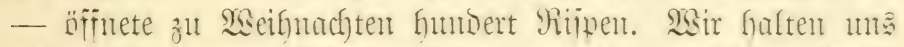

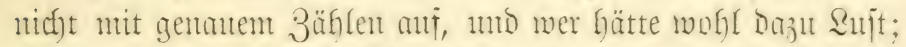
aber cin Gegeifterter Piflifter zählte eines Iages bie Bhument ner ungehentren Miafie jener Laelia albida, mo jtelfte feit, Dan fie fith anf zweifnumert und elf beliejen, wenn er nicht, wie jemano vermutete, Blïten uno Blittentiänose vermechielt hat, in weld)en Falle Die Bahl nit zmei ober Drei multipliziert

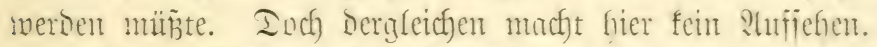

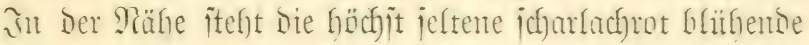
Utricularia, meld\}e in Den Bhattwinfeln enter Triesea mädjit, wie in einer immer gefüllten Edyle; bis jebst ijt jeonch iffe Blitte in Enropa blo mit geijtigen Sangen geiehen moroen.

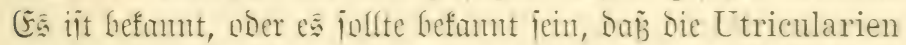
nicht ju ben Srcfibeen gefören, ja gamidgt cimmal in bie ent=

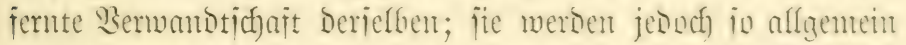
mit ifuen fultiviert mo verlangen cime io äfnliche Bebanto=

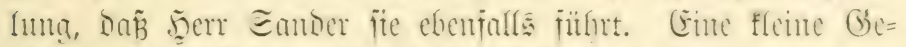
idficf)te fnithit fitch an bic joföne $U$. Camplelli.

(5. mar jur Beit Der allgentemen iaģ nach Cattleya

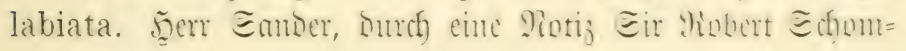




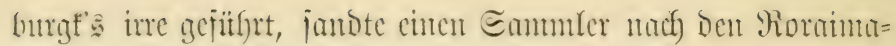
Bergen un Gutanta, mit Den gentejenen Bejehl, mur añ Dieje

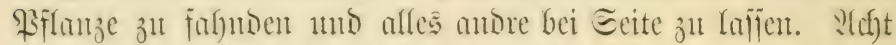
Q)ionate lang manberte bicjer nuter Den Jndianern anf mo

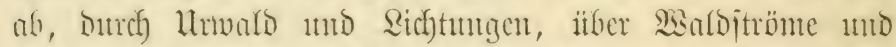
Feljen, fand aber weber C. labiata noch bie jeltjante von ミir

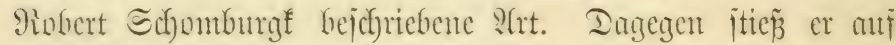
Die joüne Utricularia Campbelli, no trok bes erfjaltenten

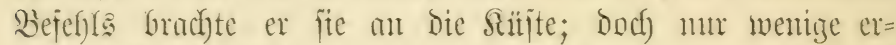

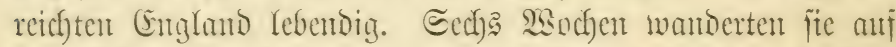

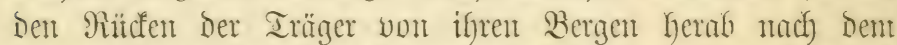

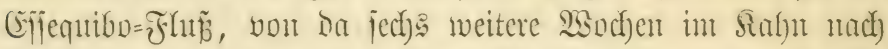
Georgetom mit zwanjigntaligen llmlaben, Dam wursen fie

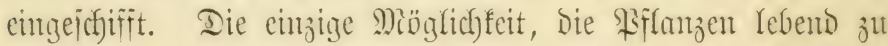
erfalten, ijt, fie nugeitört in Den Mionghallen zut lafjen, in melchen fie wachjen, ebenjo wie mbere s(rten in ben Blatt= winfelı Der Vriesea.

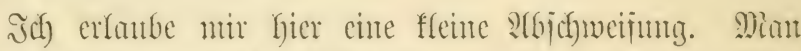

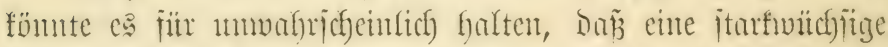

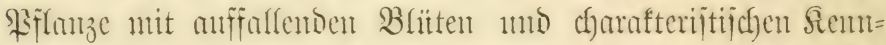

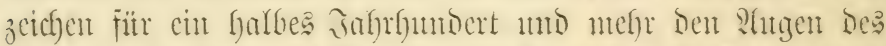
Eammlers entgeben jollte, Der Dodf mit Der Sache vertrant ijt uno crmmutert wirs, feine Sinjten ju iparen, went bie

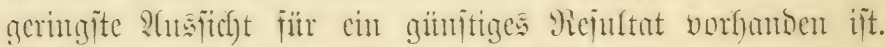
Doch wen bie näferen llmptünde in Betracht gejogen werden,

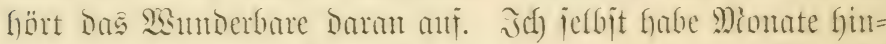

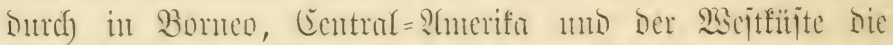

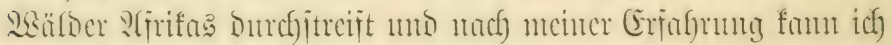

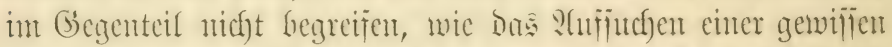

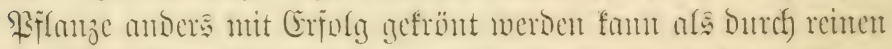

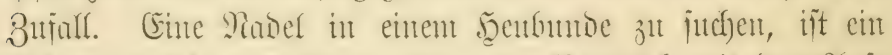

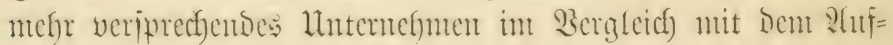

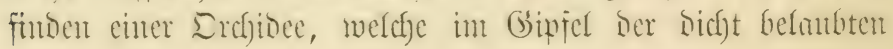




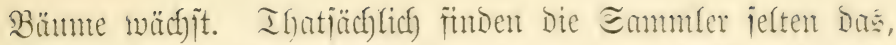

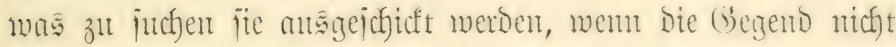

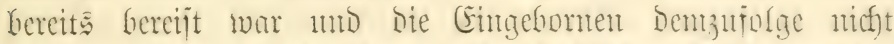

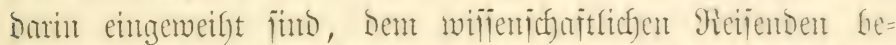

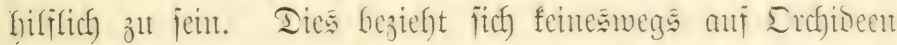

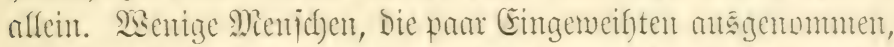

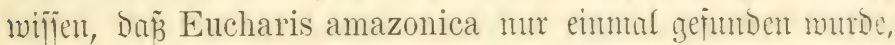

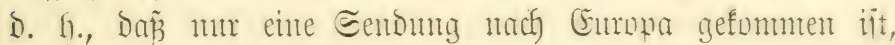

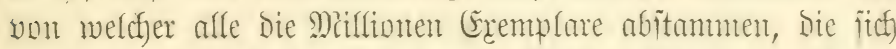

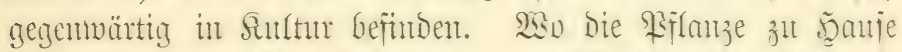
iit, wifien wir nicht. Fajt ein ganzes Dienjchenalter fintourch

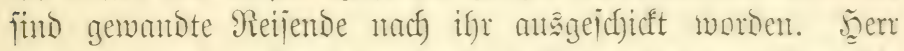
Eamber nafm auch an Der Enche teil, und hat wentigiteñ bie

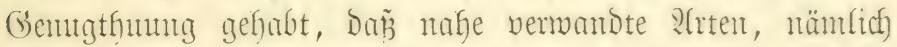
Eucharis Mastersii und Eucharis Sanderiana, Durch jeine

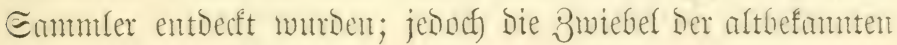
S(rt ift noch) zut jutchent.

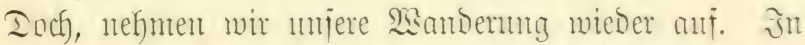

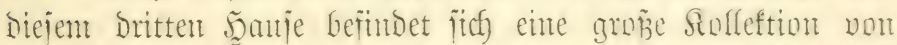
Cattl. Trianae, melche jo joüt in vorigen Juffre artam, Daß̉ Sie B(ütenicheionen fitch ju gleicher Beit mit Denten won Cattl.

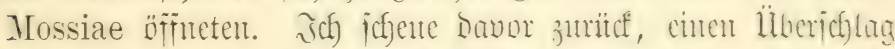
3̆l machen, wie viel tanjeno Blitten gegenuärtig offen funto. Eo wie bie Ebontoglofien ifre Siidge mit icfnecmeiben Guntranden, io bebecfen bieje Cattlenen bie ifrigent mit ifnen

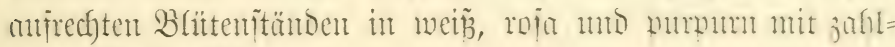
Iojen Farbent= Nitancen.

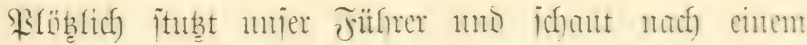

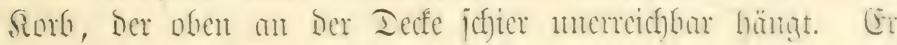
cuthält cinten glatt ansichenden Gécgenitano, icfre artint und

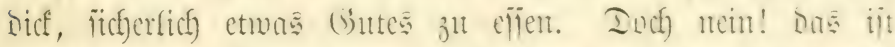

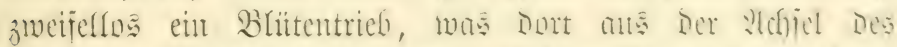

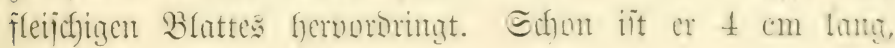


Dicf wie cin Bleititift, mit einer Dicfen Smojpe an ber Epiţe. Iergleichen angenthme Ïberraichungen hat jumeifen ber

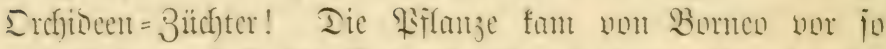

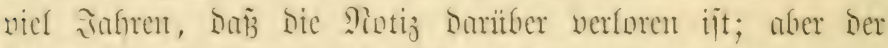

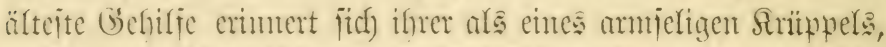
meldjer, jwiichen Q

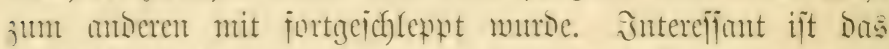

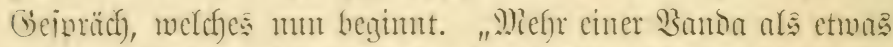

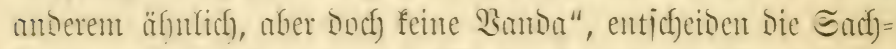
Ennoigen bis mif meiteres. Eie ber hejonderen Sorgfalt der nerantwortlicf)en நünto anemtyeffent, gehen wir meiter.

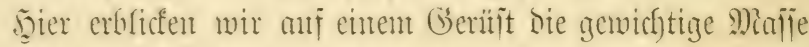
Des gröb̆ten Catasetum, meld)es jemals gejunten, ja vou

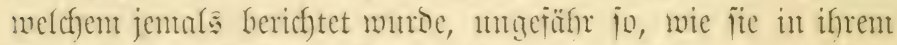
heimatlichen sasto in Ginatemala gelegen haben mag. Die

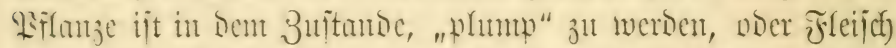

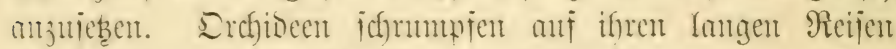

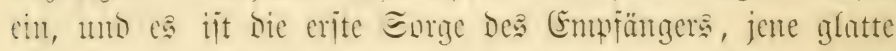

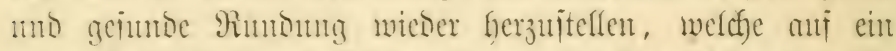

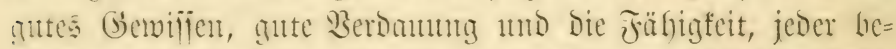

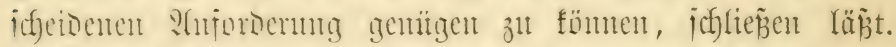
Llnter Den Etellagen fün Imijente verborter Etöcfe, żl=

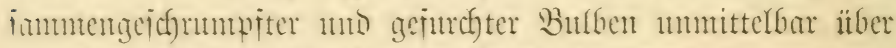

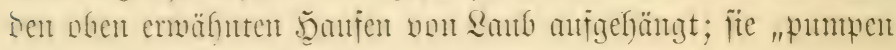

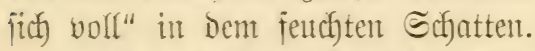

In gröbere Eremplor von Catasetum - es finto

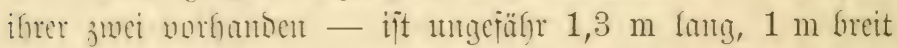

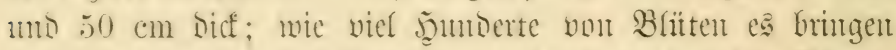
mirs, entjicht fidf) Der Berechmung. Jdf mad)te bic Bemer=

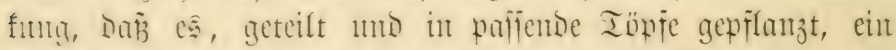

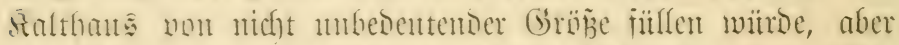
the Ieifung Desielfen idfeint nicht benbüthtigt jut iein. Dor

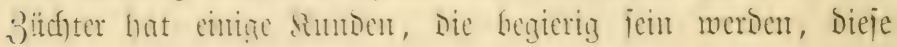




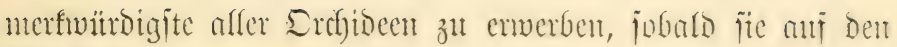
Marft fommt.

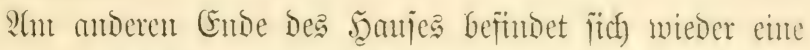

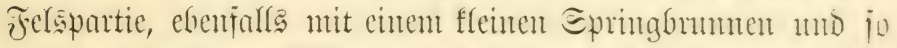

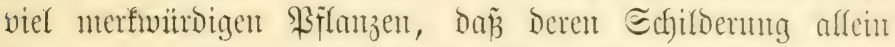

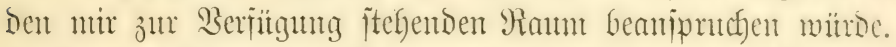
Doch glitclichermeije iteht obentan cine Cattleya Mossiae,

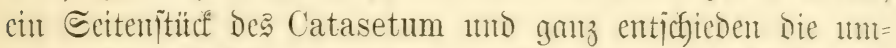

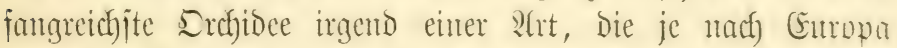

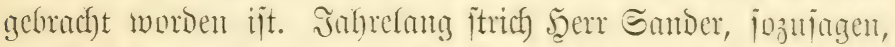

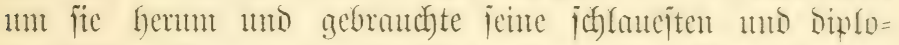

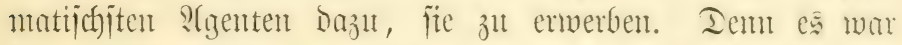

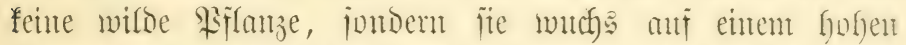

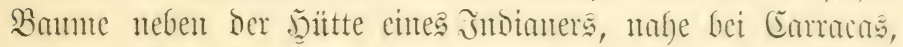
mo wor jein Cigentum jo gut wie bie Frühte jeiner Siflun=

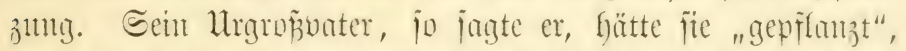

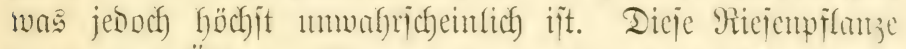
Gatte zmei Silte Des Baumes ammant und bedecte fie io

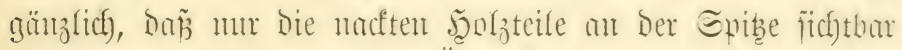

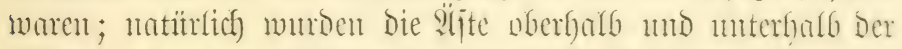

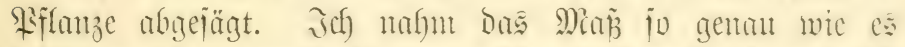

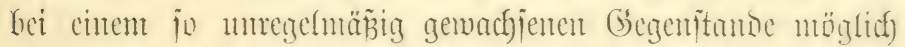

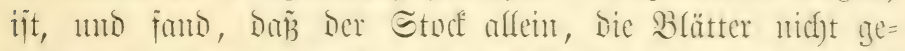

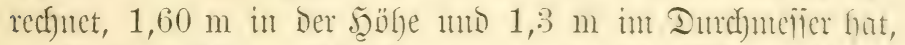

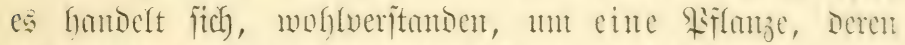

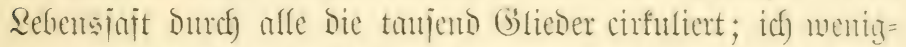

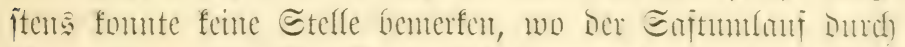
Bermunbung oder Siranffecit gejtört mar, woer wo anjerhalb

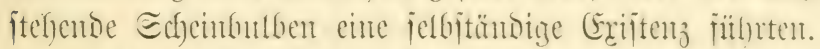

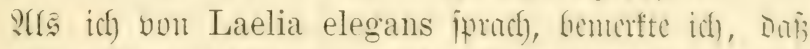

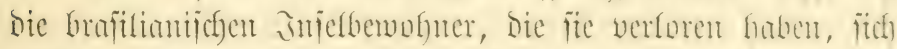

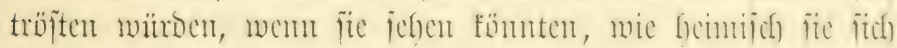
in Der Berganmmig füflt. Der fremolicfe Eejer hält Dies 
vieffeidft füt cine l̈thertreifunty, Dodf bem ijt nicht jo. Die

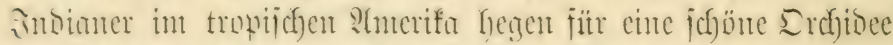

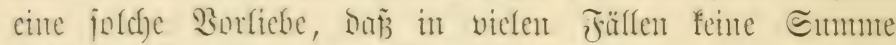

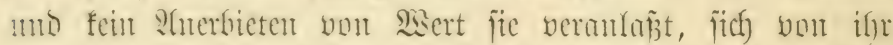

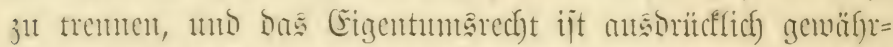

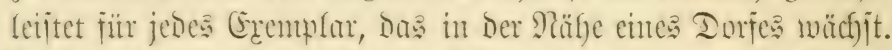

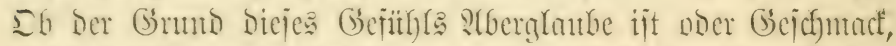

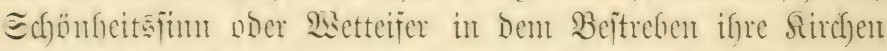

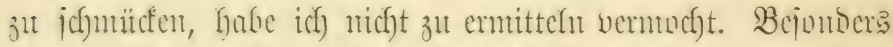
jtarf iit ca in Coita Rica entwidelt mo dort menigitens

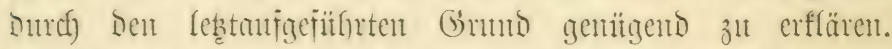

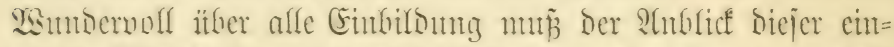
jomen

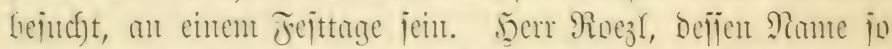
hefannt muter Den Botanifern ijt, Ginterliể cine Bejchreigung

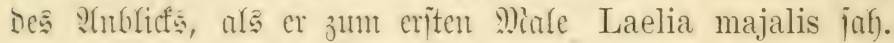
Er jugt: "Die Sirche war beftünzt mit ifren Gintirfanden, mo

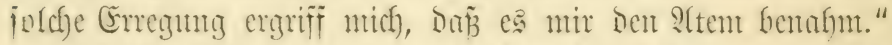

Iiejer Bericht ijt gan glantwuitndig; biejenigen, welche

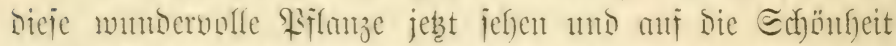

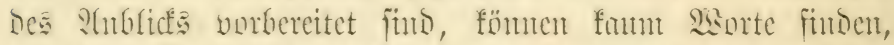

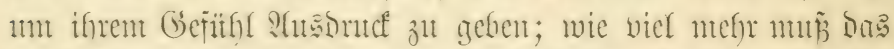
Der Foll gemejen jein bei cincm Enthufifajten, Der unworbereitet

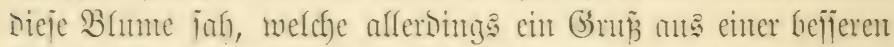

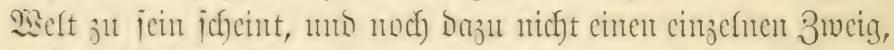

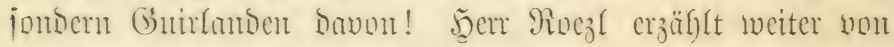

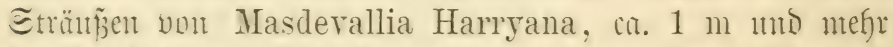

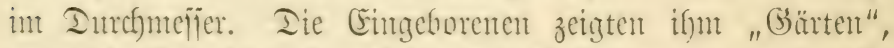

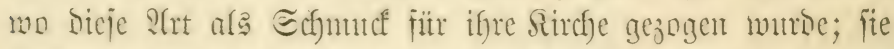
waren zwar nicht fittiviert, aber ficherlich) angepflanzt und eritrecten fitch itber viele Mlorgent.

Der Intimer, Dent bie ohen hejofriebente Cattleya Mossiae gehörte, weigerte fith) jafrelang, fith) vont iffr zut 


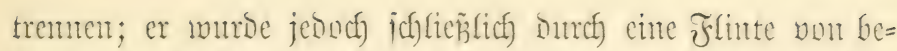

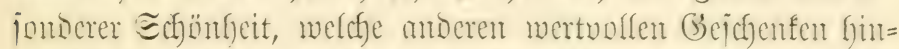
zugeititgt munde, umgeitimunt. Fine Laterna magica itfut

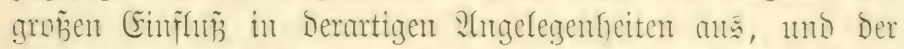

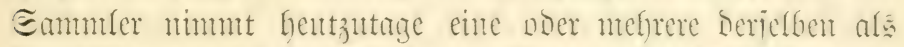

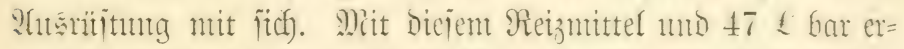
marb jeer Ember jeine erite C. Mossiae alba; jedoch itt bies in

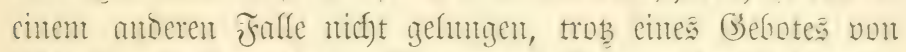

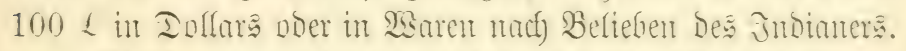

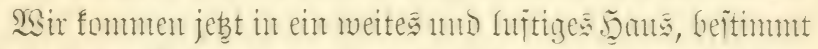

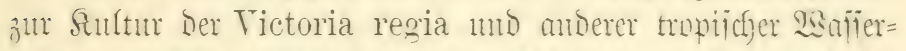

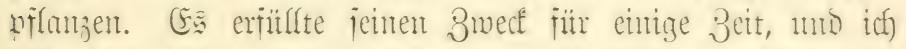
hathe bieje Siflanzen nie wieber unter Imitünoen gejegen, Die io gecignet waren, ifgre Reize zur Entfaltung ju bringen. In

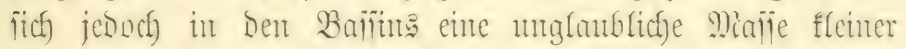

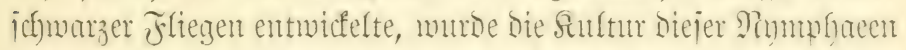
anfgegeben. EFinige menige mutroen beibchalten, gernoe gentra,

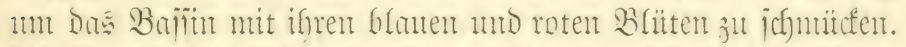

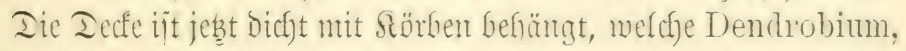
Coelogyne, Oncidium, Spathoglottis und iolche Prten cut=

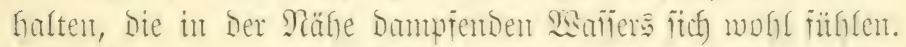

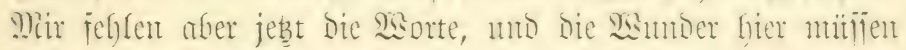
ntrbejchrieben bleiben.

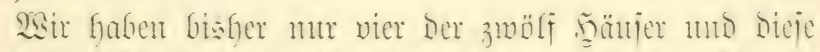

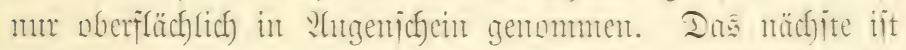
miederm ein temperiertes, voll von Gattlenen, Encioten, Die

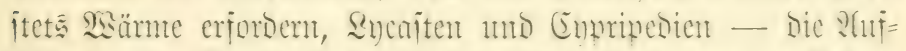

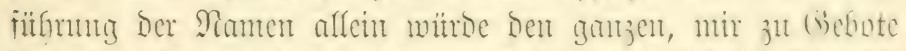

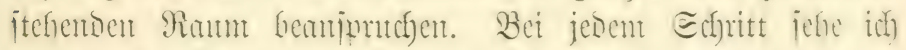

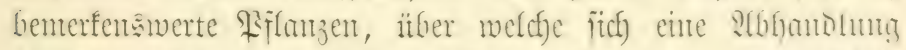

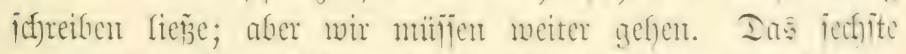
bam ift wieser falt und für Odontoglossum und Der= gleichen beĩtimmt. Das fiehente enthält Denbrobien. Beim 
Eintritt jehen wir aher vor mo cine Lycaste skimneri, welde

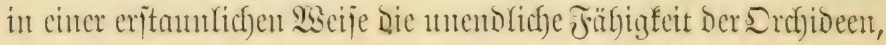

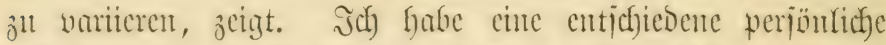
P(bneigung gegen bicje Strt mit ifrer miforinglichen mo an=

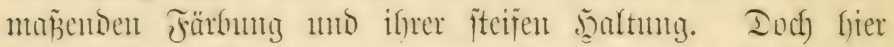
jefhen wir ein Eremplar vou ganz hervorragender Echüuneit.

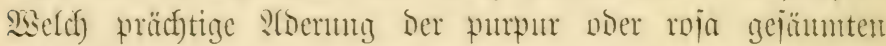
Blätter! Sieldy ein Gínng Der jummetnen Sippe, bie von farmefin in tiefes Domfelutubinrot itbergeht!

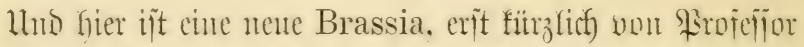

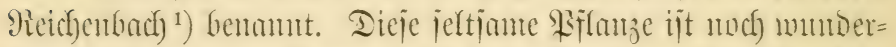
Garer, als bie ganze 3erwantichaft Der Sncisien. Jifre Eepalum iit $8 \mathrm{~cm}$ lantg, Der "Echywarzteil" $13 \mathrm{~cm}$, mit cinter riefitgen

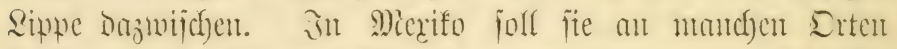

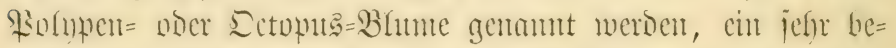

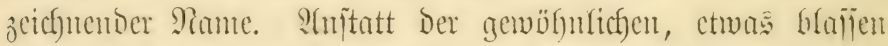

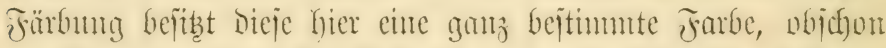

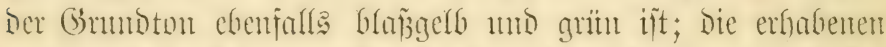

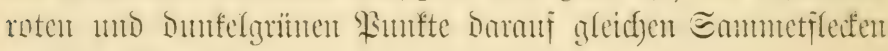
auf Monjelin.

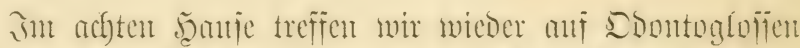

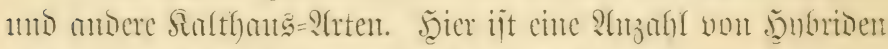

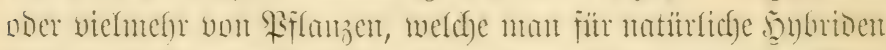

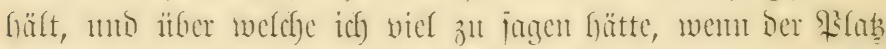

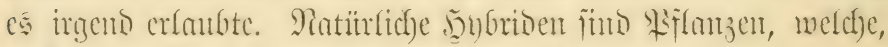

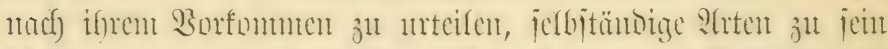

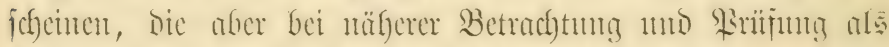

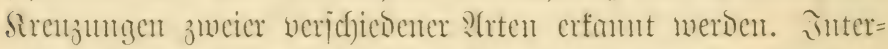

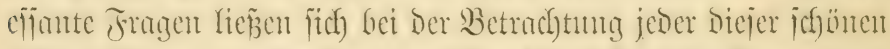

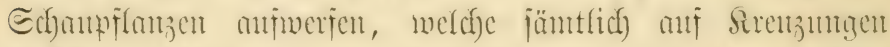
jmijcfen Odontoglossum Lindleyanum mo O. crispum

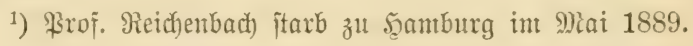




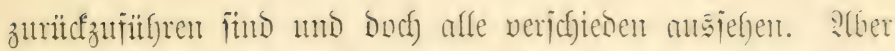
mir müfifen weiter żm neunten Şanje, von melchem Das zefinte fich abzineigt.

Şier iit es marm, und Dämmerung herricht ïffer bem Ieile, wo Die S(ntgeförigen verichiedener fochtropiicher Gaattungen Fich von Den Etrapazen Der Reije erfolen, wieder mit Eajt füflen und $\mathfrak{W}$ urzeln johlagen.

Dieje zarten Simber Der Tropen mögen wofgl ïtber eine

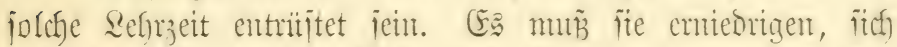

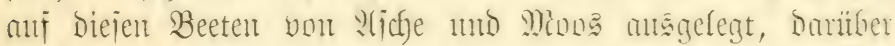
verfefort antigehängt, ober wohl gar in bie slidge eingerfirlangt

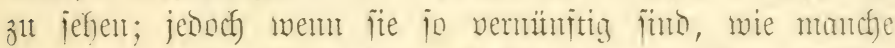
glauthen, werDen fie einjeflen, da

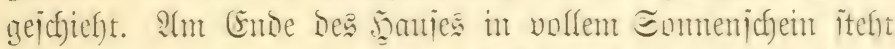
cin flemer Son woun Tanda teres, jo bicht als iffre iteifen

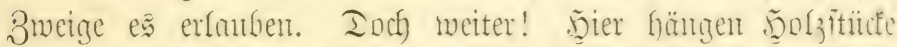
jo verfault, Dan fie faum zuilammenfalten; Doch winzige gritme

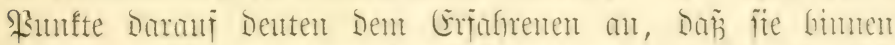

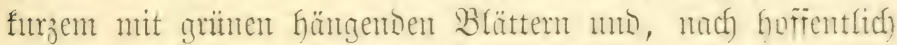
abermals furzer Beit, mit Glanen, meiñen and icfartad)roten Blitten von Utricularia geidfnutate jein merden. Bon Diejem

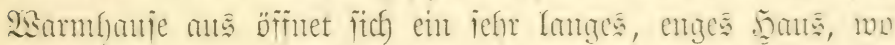
Salthan mieber nit Eajt fïllen. Biele banon zeigen bereit it tarfen

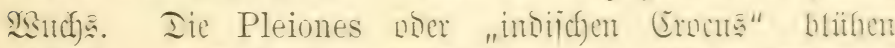

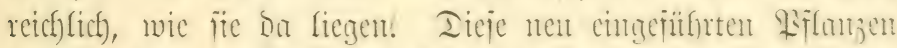

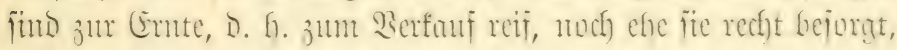

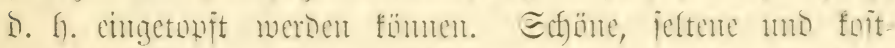

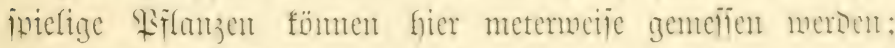

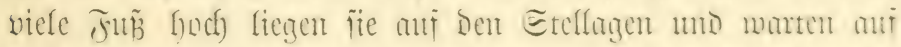

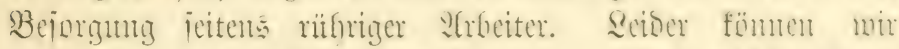

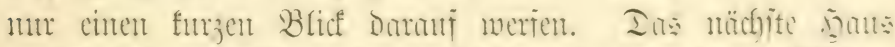

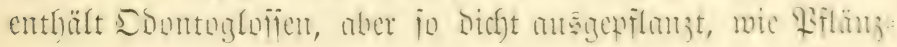




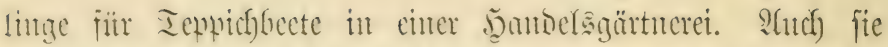
marten, his fie an bie Reife fommten, am in Töpfe gejebt zut

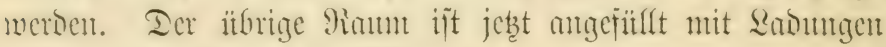
vout nett angefommenen $\mathfrak{B f} f(a n z e n$.

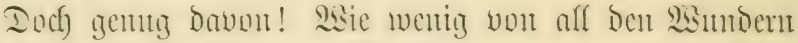

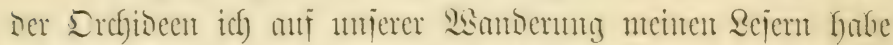

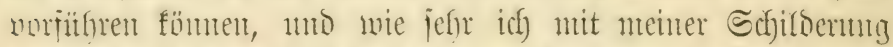

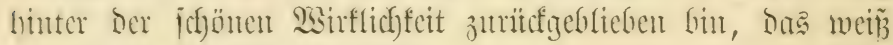
niemand beffer als idf jelbit.

\section{Elftrs Eampitel.}

\section{ïbev ฮีïchุtu1ta vout (I) fortitert.}

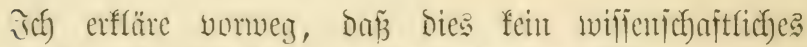

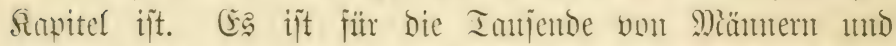

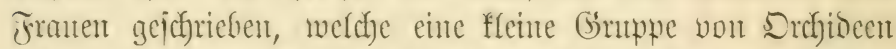
mit Riefe pffegen und bas Simbernerf ifres Banes mit eben

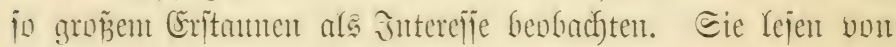
jolchen Büdytuntgen, je jefen bie Rejultate in mertuolfen

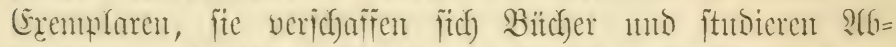

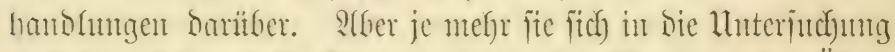
wertiefen, Dejto mefyr foument fie in ber Regel ju ber llber=

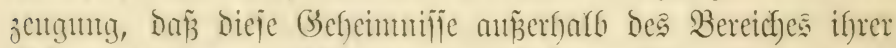

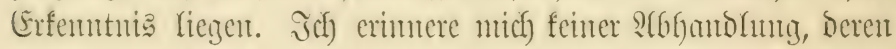
cmitfafter Bwed es ift, bie Hutingemeiften zu belefrent.

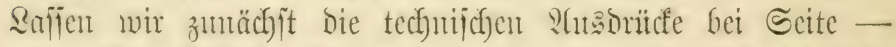

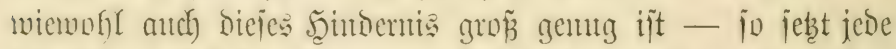

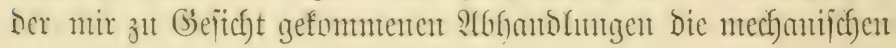




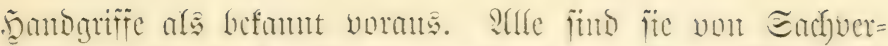

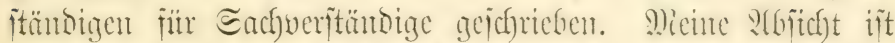

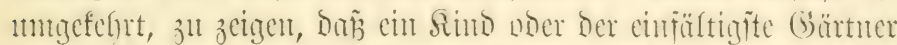

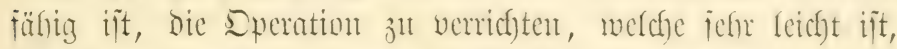
jobalo man weiñ, wie man zu Werfe zut gefen bat.

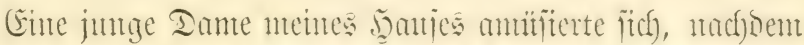
fie chen etros mit Cypripedium befant gemoroen war,

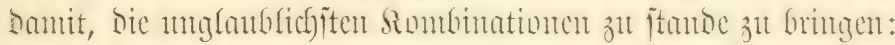
Dendrobium mit Odontoglossum. Epidendrum mit Oncidium, Oncidium mit Odontoglossum mo Dergleichen mefr. (s.5 i i t

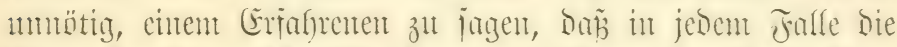

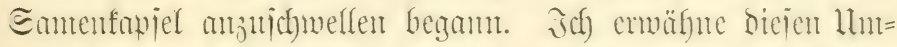
jtonto unt zum Beweis, wie einfach bieje Enerationen fund, jo= farb eimmal cin Sisuf gegchen mo verîtanden iit.

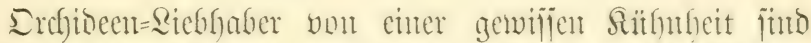

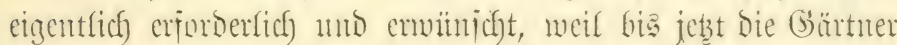

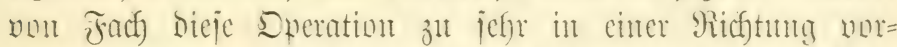

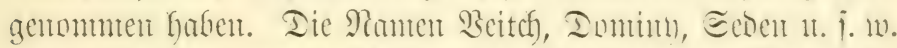

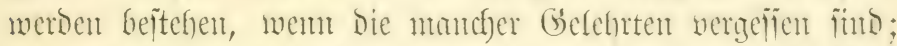

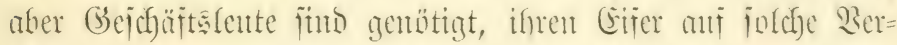

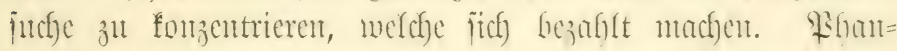

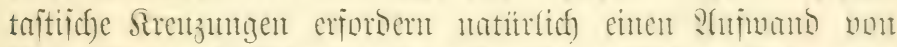

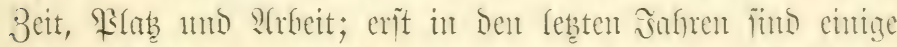

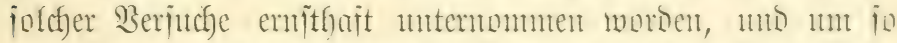

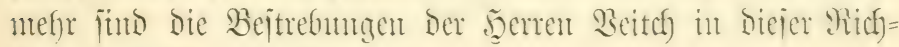

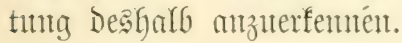

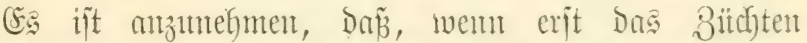

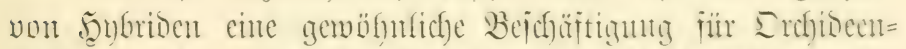

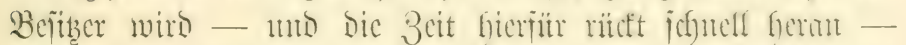

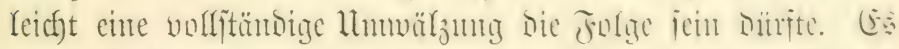

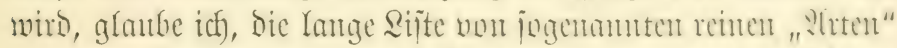

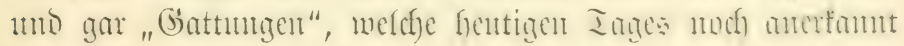




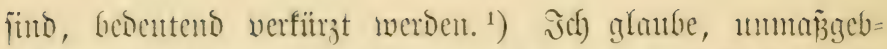

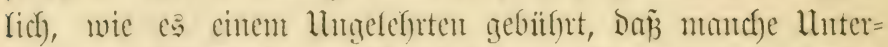
fofeibung

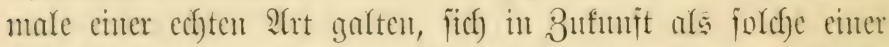
frubribe antweijen werben, als bas Pejultat einer vor Jaffe= fumberten ftattgefabten Sirenzung. Beweijen läp̃t fïch) io ctmas meijt nich)t, Da Das menichfiche Seben jo fun ijt; aber

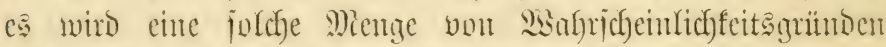

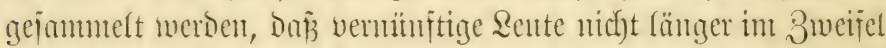

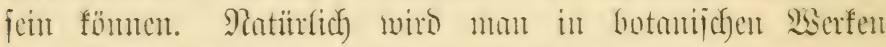

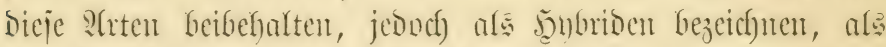

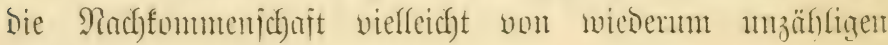
5ૃy)brioent. $\left.{ }^{2}\right)$

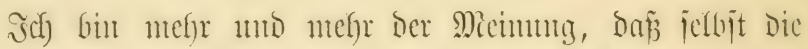

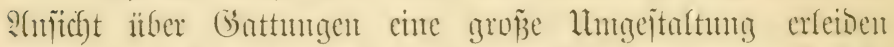

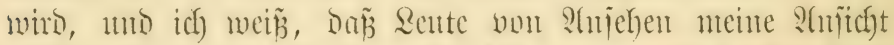

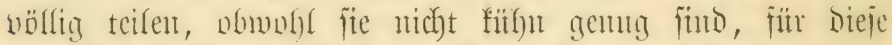

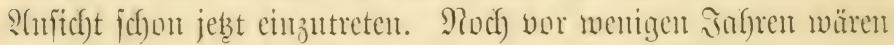

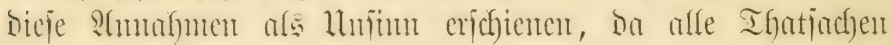

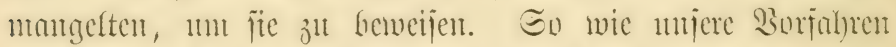

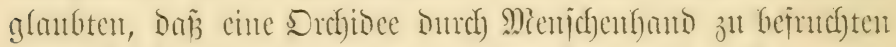

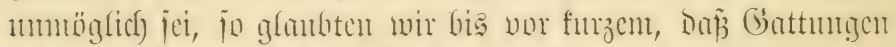

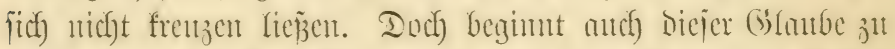

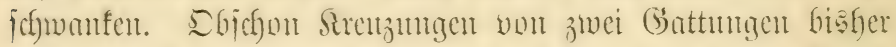

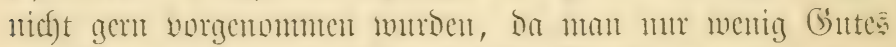

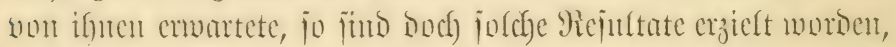

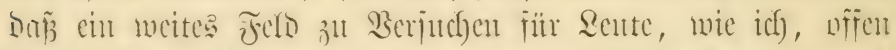
fiegt. 㐘m Cattleya mit Sophronitis, Sophronitis mit

1) $\mathfrak{D a s}$ glautbe idd audc). $\Re$.

2) 2(ud) Diejer Faff ijt inzwiid)en cingetreten. C'ypripedium ('ur-

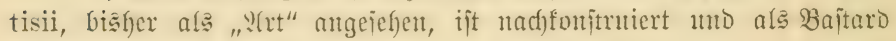
nachgetwiejen worden. 
Epidendrum. Odontoglossum mit Zygopetalum. Coelogyne mit Calanthe gefrengt imn, io borf man alles crmarten.

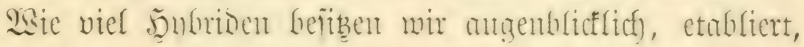

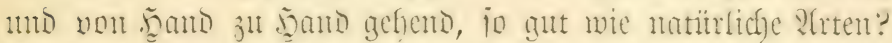

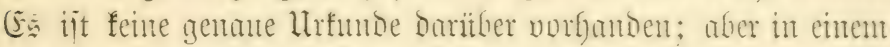

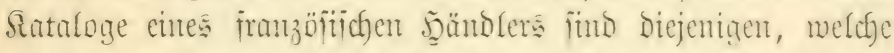

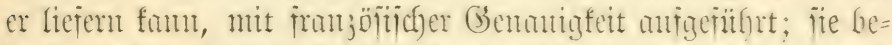

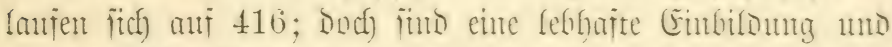
fanimänniicher Ahternefomungägeijt Den Franzoien nicht meniger (b)arafterijtiich als Gsenantigeteit.

in bem wertwollen „Manual" Der Şerren Beitch), in

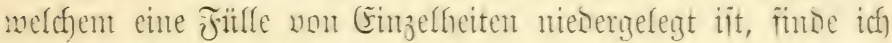
10 5ufritom nou Calanthe, 13 nou Cattleya uno 15 vou

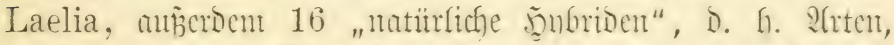

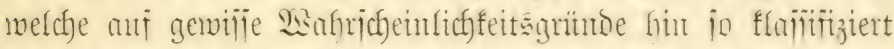
Finn, jobann die munderfare Sophro-Cattleya, Den Bujtard

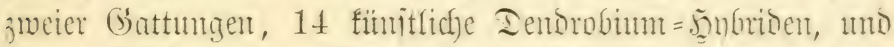
cine, möglichermeije natitrliche, jomie 87 fimitliche (Enpripesimm=

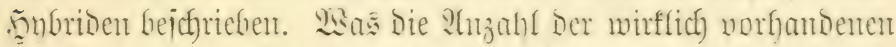

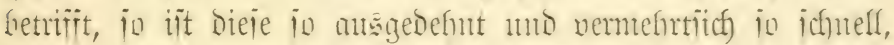

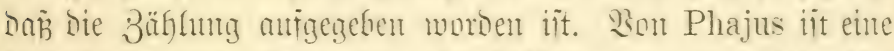

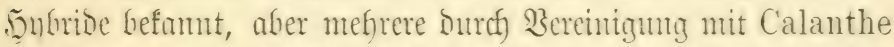
entitundene; von Chysis zmei, von Epidendrum eine, von Miltonia eine mo zwei iogentunte "natitrliche"; von Masde-

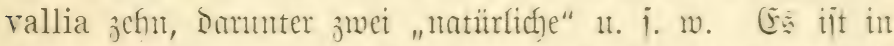

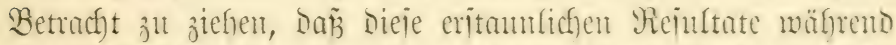

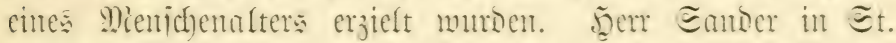

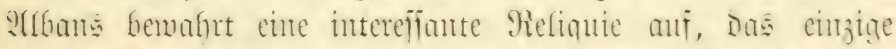

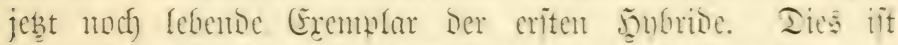
('attl. hylbrida. Die erite biejer Gattung, meldbe berr Iominu,

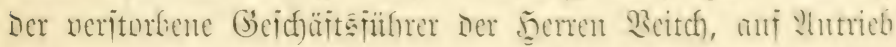

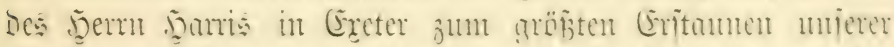

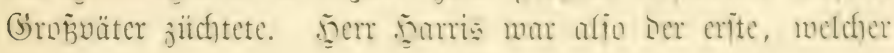




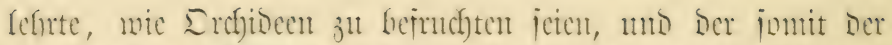

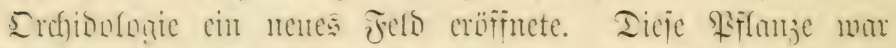

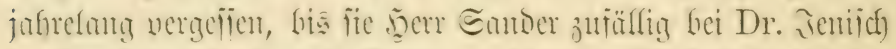

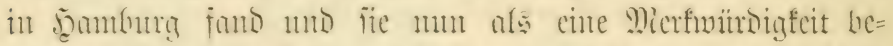

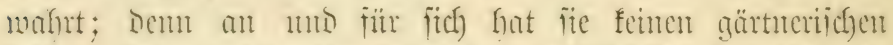

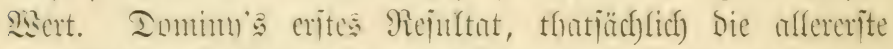
3 tur B(üte gebrachte Garten=5ु)bride, war Calanthe Dominyi,

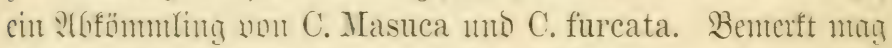

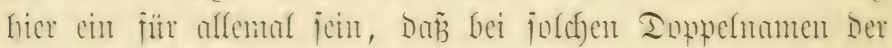

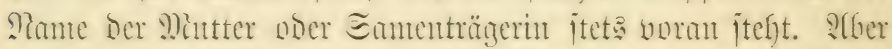
noch ein meiteres Jnterejīe iit mit C. Dominyi verfnitpft. Betoe EEltem gefören zu Der Teratrifolia-Gruppe der Ealanthen,

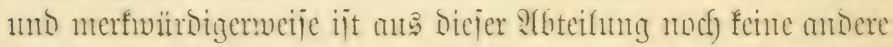

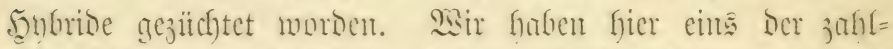

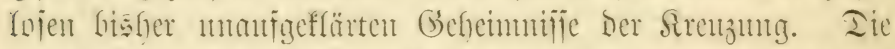

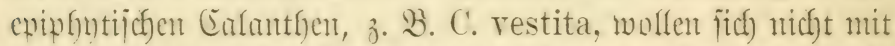
inen terreptrijchen, wie C. veratrifolia, frenzen foijen, uno cbenio

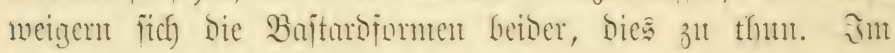
iaffre 1859 blithte C. Veitchii, mis C. rosea (welche in Der Regel noch Limatodes rosea genamut wird) uno C. restita entitanoen. Sicine Erchioce ijt jo allgemein befount, mo feine

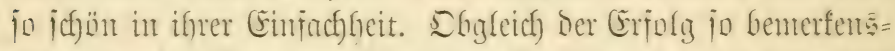
mert anto Der sieg Dozut ichenthar fo leicht mar, jo vergingen

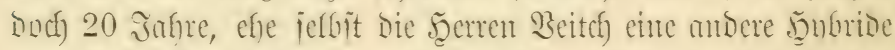
won Ealanthe ergielten. Im Janfre 1878 brachte Eeden jour Enlanthe Sedeni an C. Teitchi mo C. restita jum

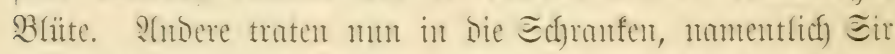

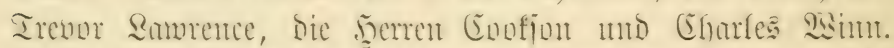

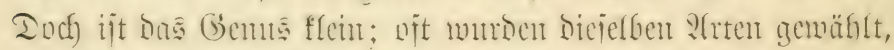

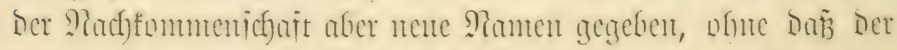

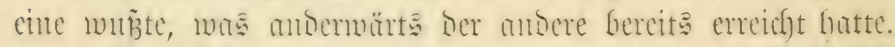

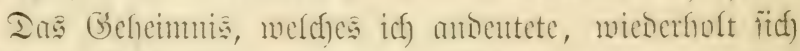

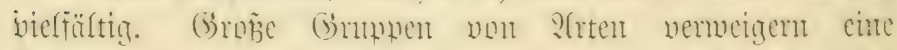

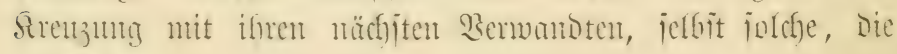




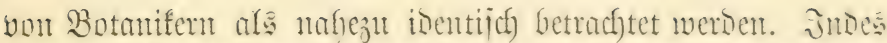

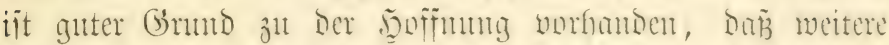

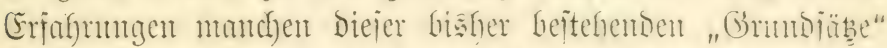
bejeitigen werisen. $\Xi_{0}$ ift wienerfoult mo nuch in Den letsten

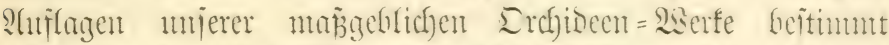

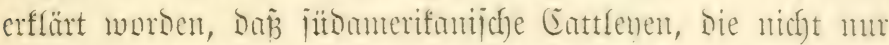

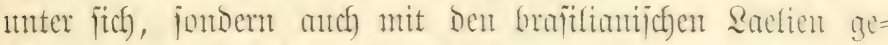

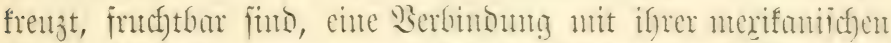

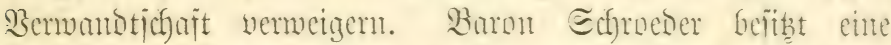

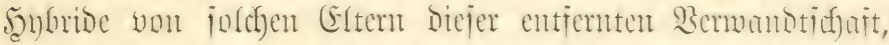
nämlich) von Cattl. citrina (nterifmiicher) no Cattl. inter-

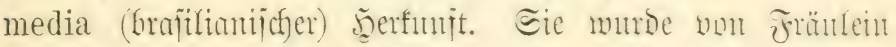
Damis zu Samberfurt in Rent gezïthtet, aher leiser mu in

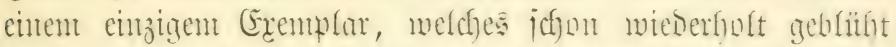
hat. Şer Ember fat Cattl. guttata Leopoldii an Bräilien mit Cattl. Dowiana an Eofta Prica gefienjt, mas Cattl. Chamberliana ergab; Laelia crispa mb Brafilien mit Der= jelben Roflempflanze, murats Laelio-Cattl. Pallas cutituno: Laelia flava ans Brofflien mit Cattl. Shimneri ma Goitu Rica, mormts Laelio-Cattl. Marriottiana entituns: Laelia

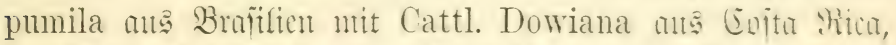
Das Frobuft heibt Laelio-Cattl. Normami; Laelia Dio-

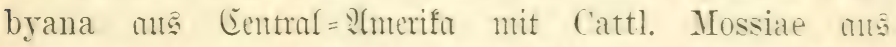
Benezuela, Laelio-Cattl. Digbrana-Mossiae gefuenis; Cattl. Mossiae mo Bentejucla mit Laelia cimabarina ms

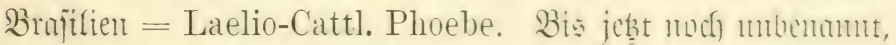

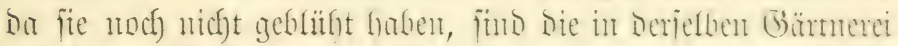
gezüd)teten Baptarie wu Cattl. citrina ma Mixico mo Laelia purpurata ats Bronilien; Cattl. Harrisoniate nus Brafilien mit Cattl. citrina; Latelia anceps ats lererico mit

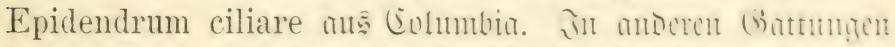

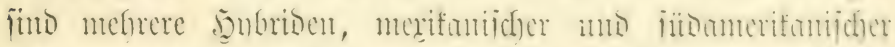

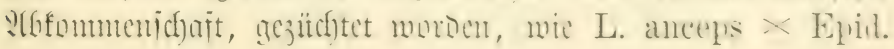


(iliare, Sophronitis grandiflora $\times$ Epid. radicans; Epid. xanthinum $\times$ Epid. radicans.

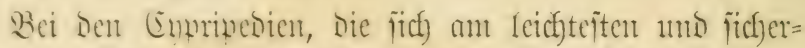

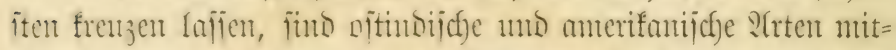

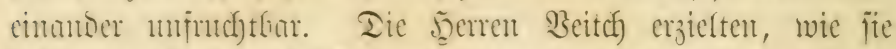
bejtimm glauten, ford) cine firenzung in einem Falle. Eech)=

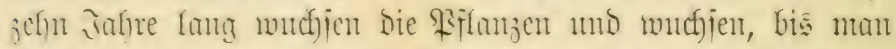
alanthe, fie mitroen wie gemöhnlich) z̆ brithen verweigent.

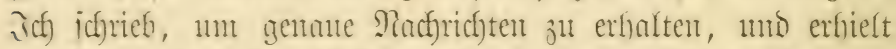

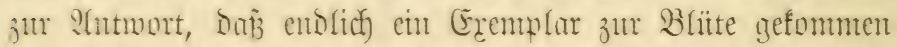

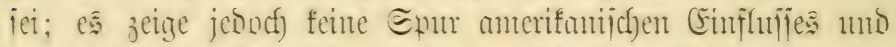

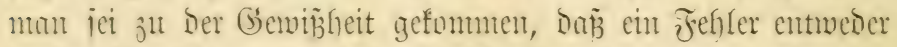

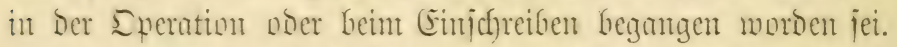

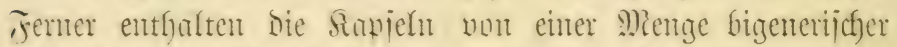

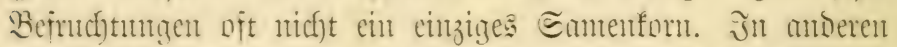
Tällen ichien Der Same ausigezeidfutet zu jein, aher er weigerte fich) jut feinten, mo femer zeigten gemifife Sümlinge von 2 Gat=

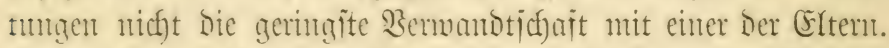

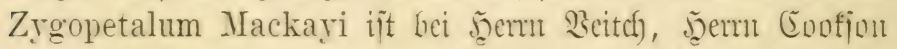

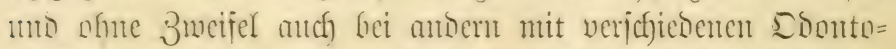

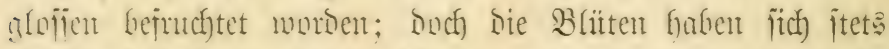
uls Die Do Zygopetalum Mackayi ermiejen, was, je mefn man Dorither nuthoenft, Deito merf(ärlicher ericheint.

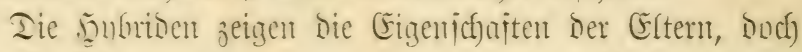
neigen fie fïh) im allgemeinen, wic in ben erwäfnten ertrem=

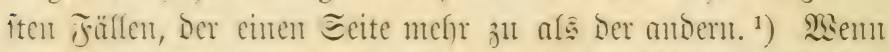
cine Cattleya noer eine Laelia von ber einblättrigen Eeftion mit einer ber zmeiflättrigen befruchtet worsen iit, jo bringen cinige Piachfonment ans Derieflen Sapiel zw mur eins, nut anocre mieder tragen an verichienenen Trieben

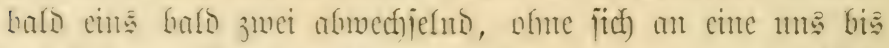

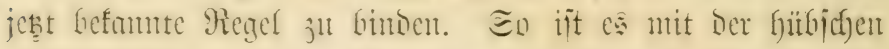

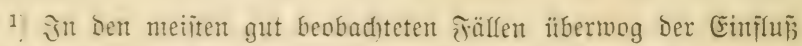
Dew 23atera, aljo Der Biflanze, tweldje Den Rollen lieferte. - K. 


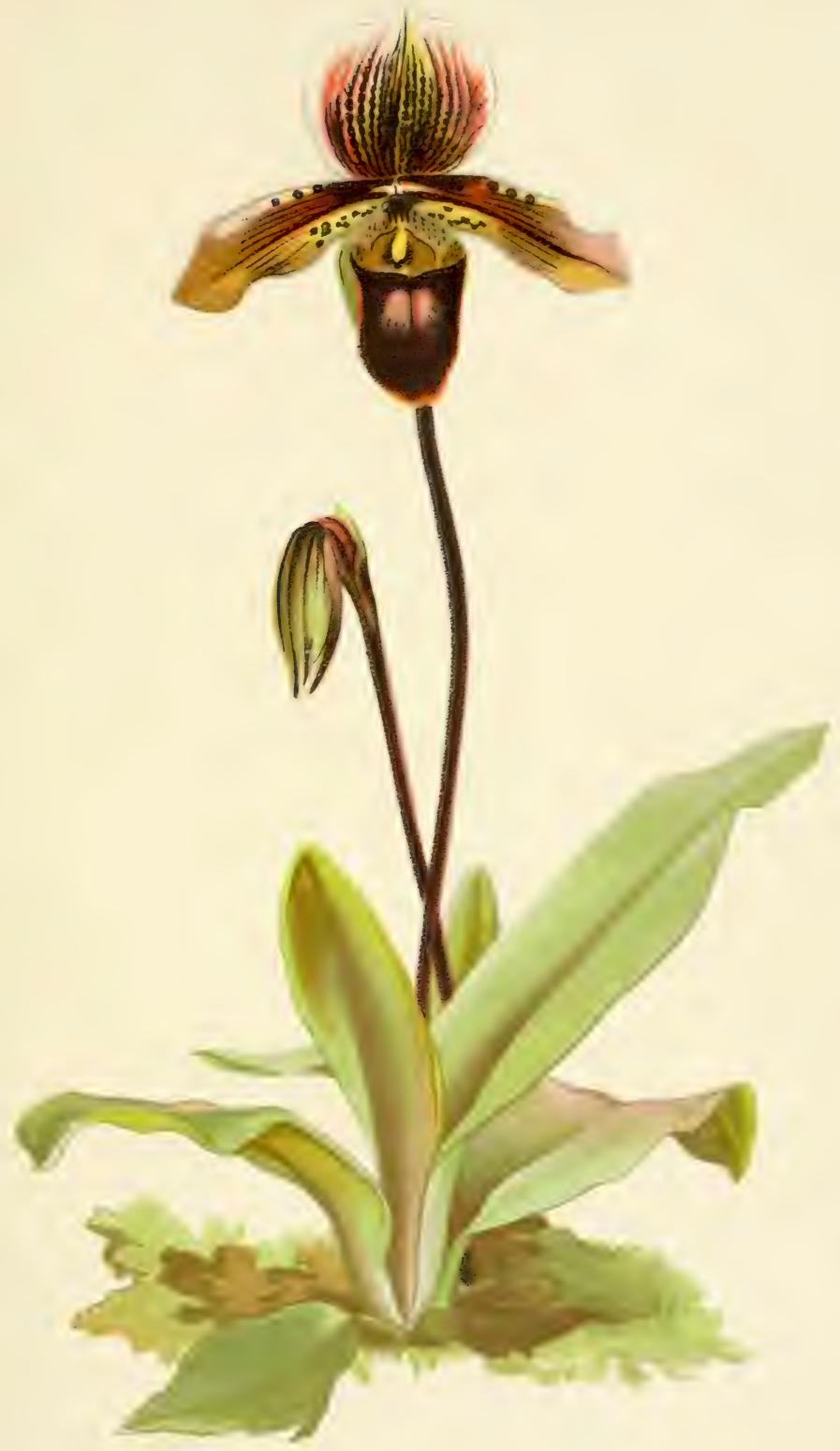



Laelia Maynardii an L. Dayana uno Cattleya dolosa.

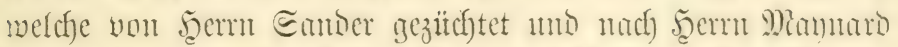

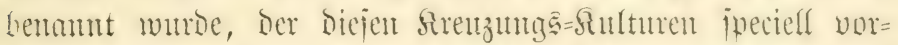
ftefyt. Catt. dolosa hat żwei Blätter, L. Dayana fat ein

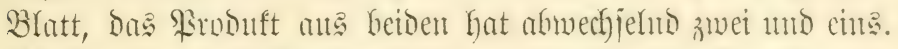
Die Eepalen mo Fetalen fino vou gleidjer roja-famefintoter

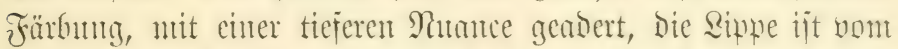

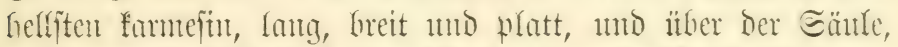

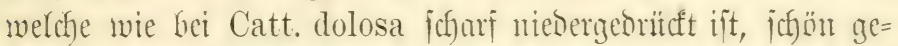

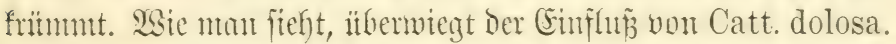

Die erfte Gattung $=$ Sreuzumg erfordert fordon iffer eigenten

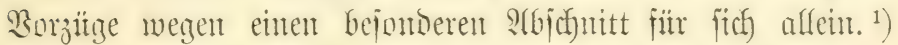
Sophro-Cattleya Batemaniana wutro bei .̧erm 3eitch vou Sophronitis grandiflora mol Catt. intermedia erziclt; fie blithte im Shugitit 1886. Die Retalen mo Eepalen fino

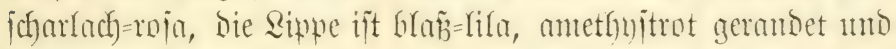
rofaputruturn betropft.

Unter Denorobien iit mur eine natïrliche songrioe feft= geftellt, ofue 3meifel cin stbënmmling won D. crassinode

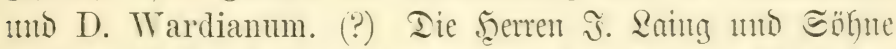

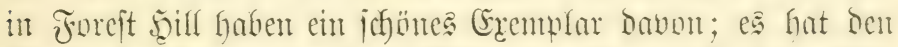

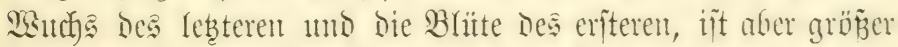

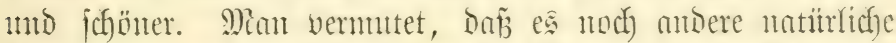

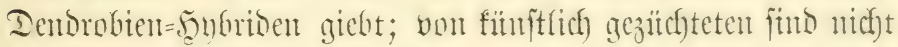
wentiger als fïllfzig vorfanden.

Phaius - oft Phajus geidfriegen - ift io nuthe mit

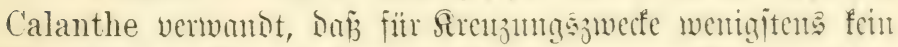
Unterichico in Betracht fonmut. Dontin) geman Ph. irroratus non $\mathrm{Ph}$. grandifolius mo Calanthe restita; Eenen mudyte Dicielfe Arcuzung, getrandf)te aher bie Barietät C. r. rubro-

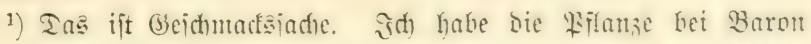
Edröber in the Irell bei Staines blithend geiehen. Eie it entidniedent mehr interefiant als hjübid). 
oculata mo crficlt Ph. purpureus. Dicjer Evifolg ijt um jo

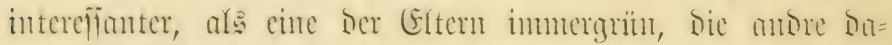

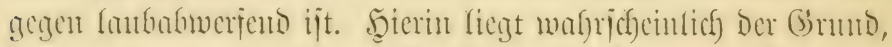

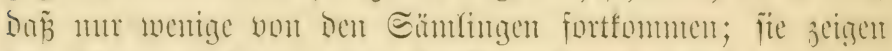

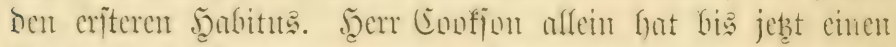

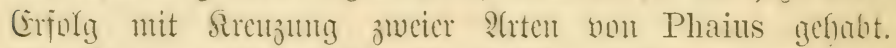
Ph. Conksoni ijt aut Plh. Wallichii uno Ph. tuberculosus

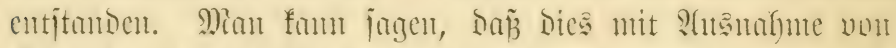

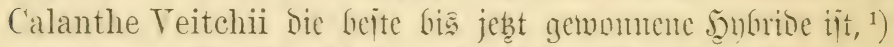

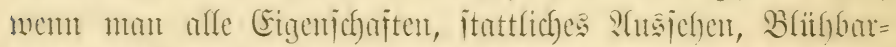

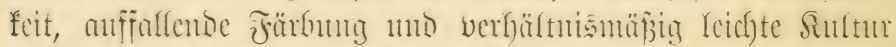
in Betracht jicht. Ginc Butlle bringt hio zut vier Blitten=

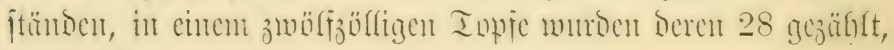
jeder mit untgefäfr 30 Bhlïtent.

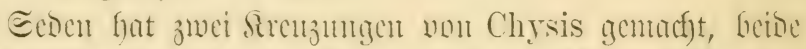

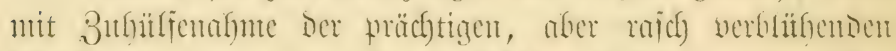

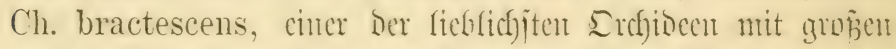

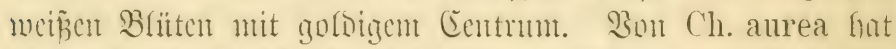

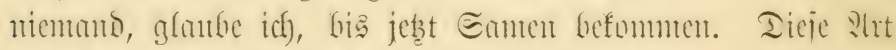

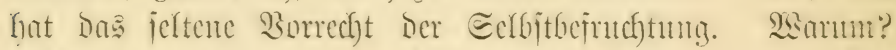

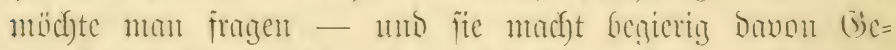

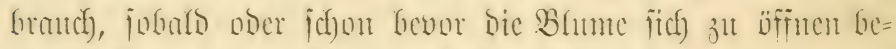

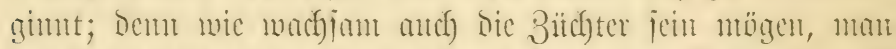

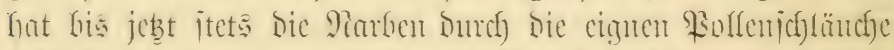

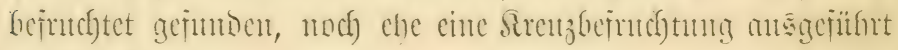
werion fomte.

(Fin fonfrios Epidendrum iit erjiclt mornen, nünticf) Epid. O'Brienianum non Epid. evectum uno Epid. radicans,

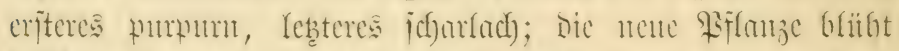

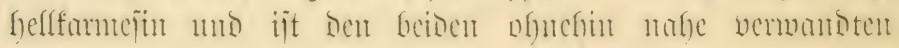

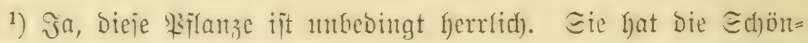

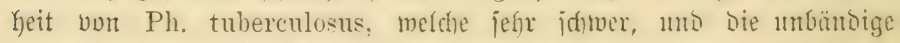
Sebensfraft von $\mathrm{Ph}$. Wallichii, welde Yeid)t zu fultivieren ijt. 


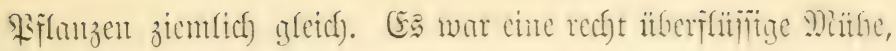
Dieje Sirenzutrg vorzunelyment.

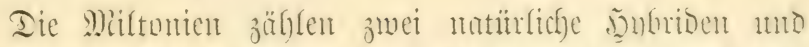
cine fiumftliche, Mil. Blenana von Mil. vexillaria mo Mil.

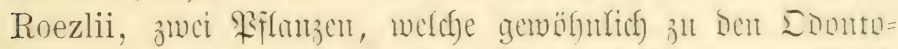

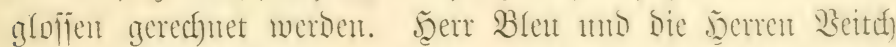

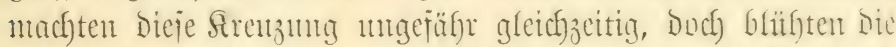
Eänllutge be erjeren in Jafre 1889, Die Der lez̧teren erit 1891. Şier Gaben wir cinten Beweis fitr Den B̧orteil, welchen

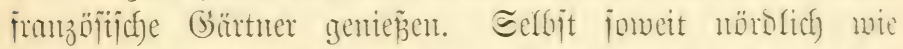
Faris madhten cin heiterer bुinmel und reichlicher Eomten=

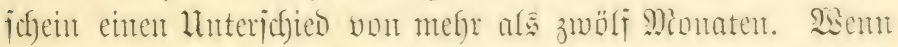

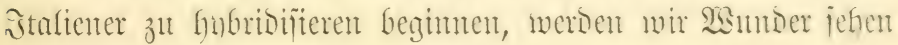
- unio erift Briechent und Eggpter!

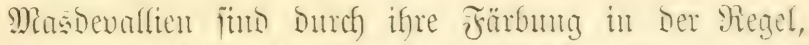

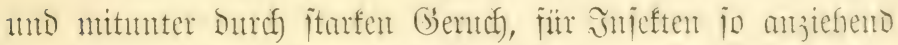

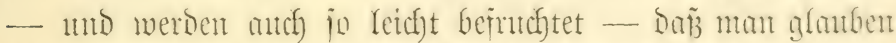

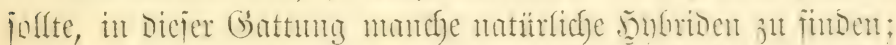

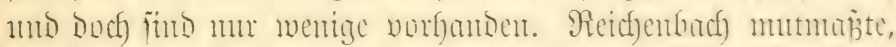

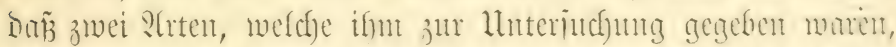

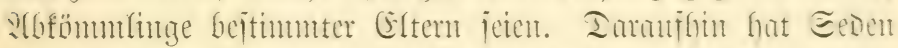

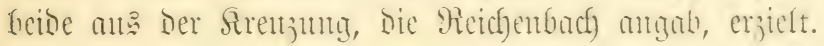

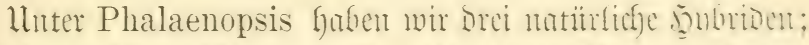
Ph. intermedia erichien jucrit unter cincm Immant nou

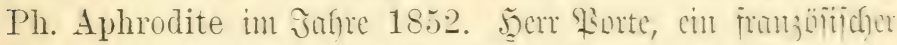

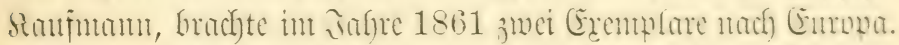

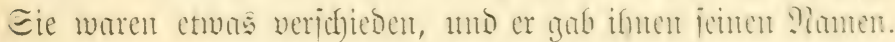

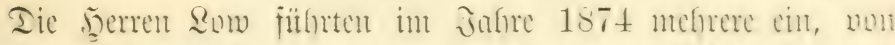

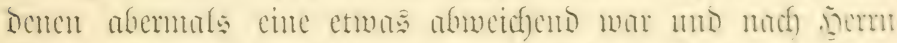

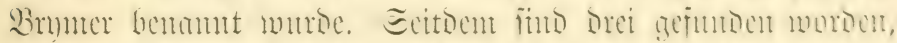

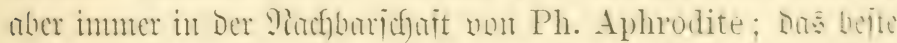

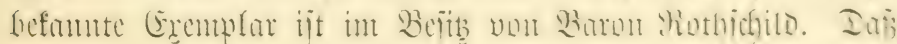




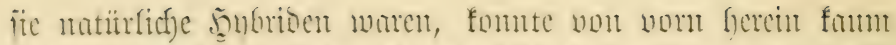
bejtritten merien; Ecoen frenzte Ph. Aphrodite nad)trüglich) ntit Ph. rosea mo licferte Damit Den erperimentelfen ?ach)weis. Inice Gartenthubrion fun Ph. F. L. Ames, cin Ergenuis von Ph. amabilis m 11, Pll. amabilis mo Ph. violacea gejitchtet nto nad) Der

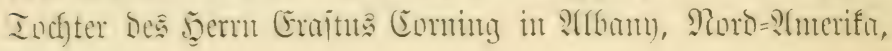
benamnt.

llnter Den Sncisien gient es bis jebt nut zuci natür=

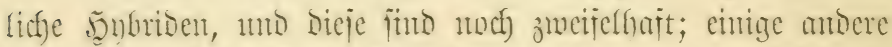

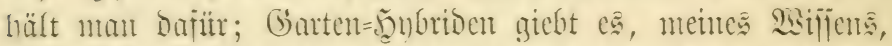

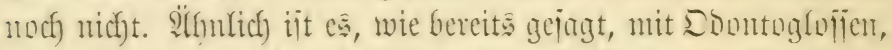
jeond befruchten bieje fich gegenjeitig jo (eicht in matïrlichen

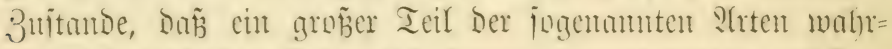

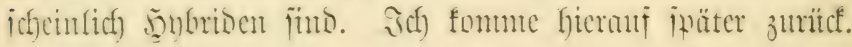

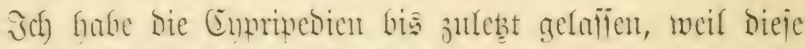
antheroriontlich interrefiante Gattung etmas mefre verlangt, als

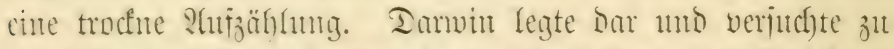

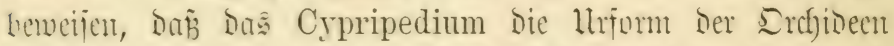

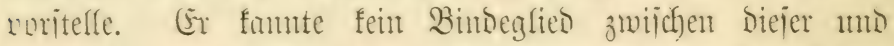

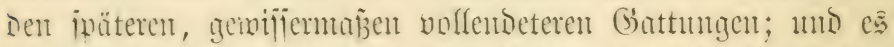

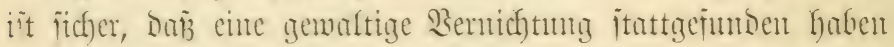

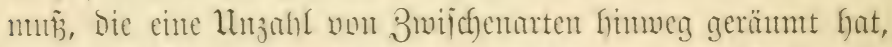
mäfremo sieje cinjige Gattnng, meldye an einc frithere und

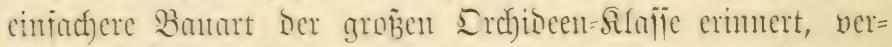

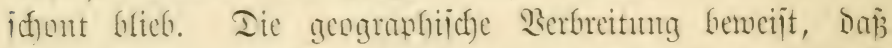

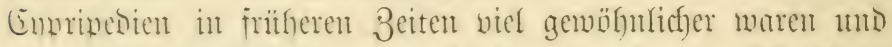

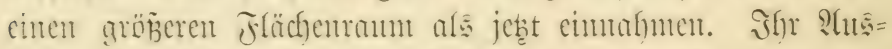

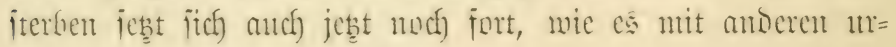
iprïnglichen Formen ber Fall ift.

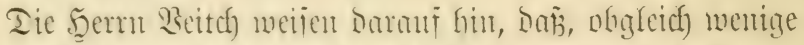

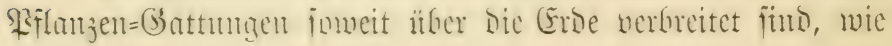




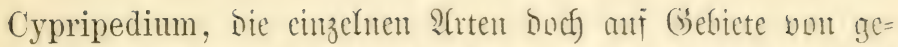

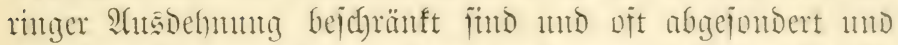

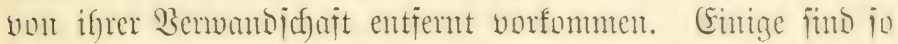

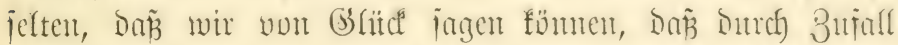

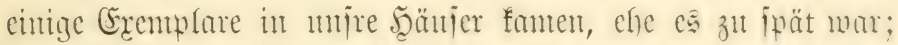

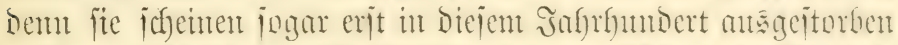
3n fein. Tie 5eerren Beitch füfren cintige ichlagente Beipiele an. Stlle von Cyp. Fairieanum exiftierenton Gremplare find

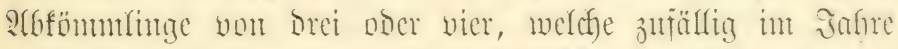
1856 eingefüfnet mutron. Zwei હtückchen von Cyp. superbiens fanden fich) in einer Ectronng vour ('yp. barbatum,

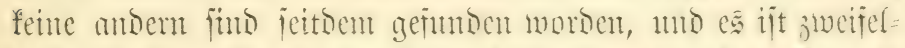

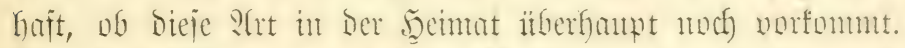
Nutr brei Prflanjen yon Cyp. Mastersianum munom cut= Dectt. Seerr Bulf erfielt fie in einer Rifite mit ver=

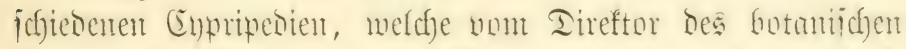

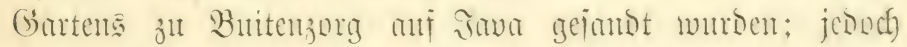
founte weder biejer frem, nod fein Plachfolger cin moeres

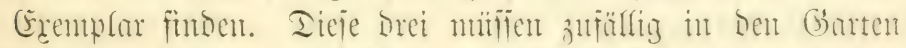
gefunment jein, vielfeich)t als (Bejejenf cincs reijenton 5ูolländers.

Cyp. purpuratum iit in gुongfong fojt ansigcitorfon mo

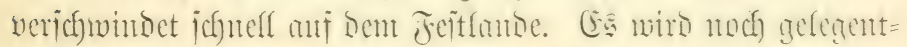

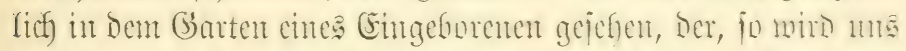

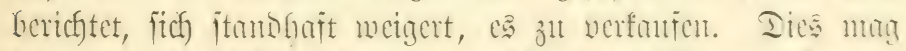
Denen unglantolich ericheinen, welche Den Efincien femen,

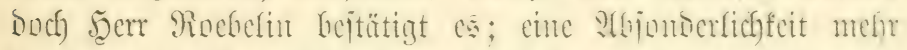

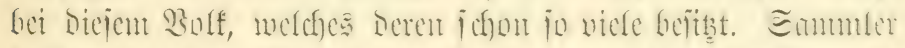
hoffen cine nene nemat vou ('yp. purpuratum in Fomoia

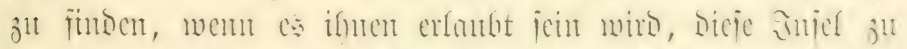

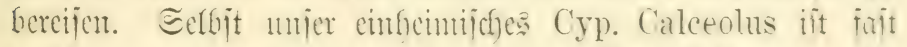

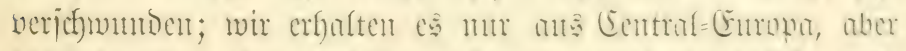

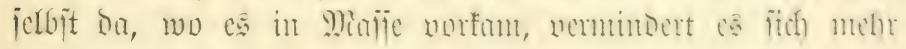




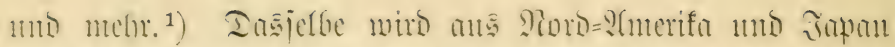
berichtet. int Direften Gegenjats fierzu fteht bie Thatjadhe, Son (Eupripedien fich) mit merfwitroiger Reichtigfeit vernefhen,

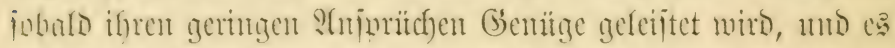

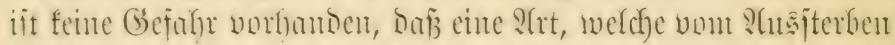
gerettet wurde, unter ber Riflege bes Mienichen umfonument wirs.

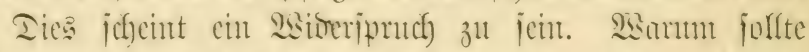

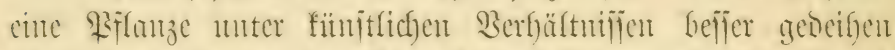

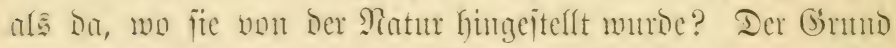
liegt in bent altertimtlichen Bau Der Enpripesien, weldyer

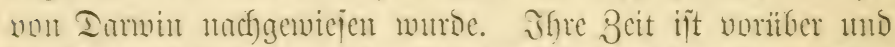

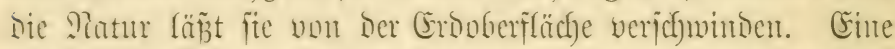

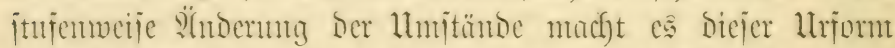

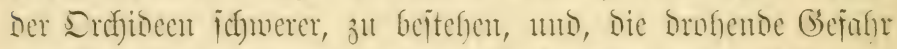

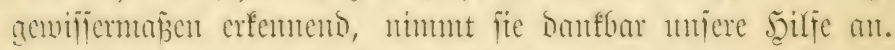

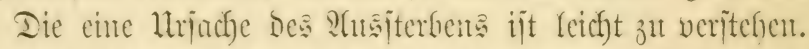

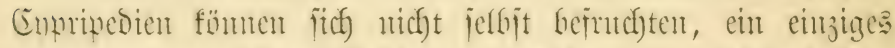

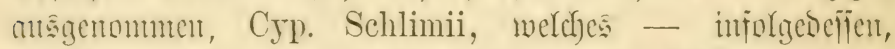

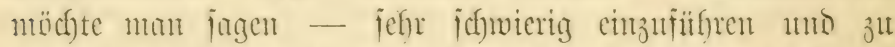

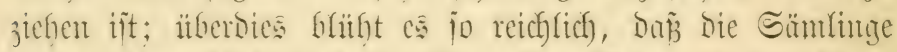

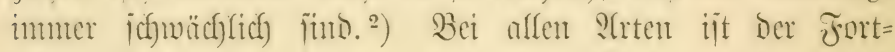

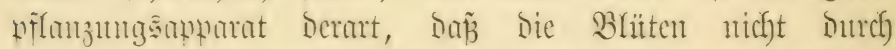
Bufall hejmeftet werben fömen, uno mu wenige Snjeften

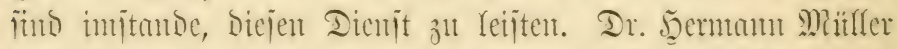
heofuadetete fefjr emitg Crp. Calceolus. Er bemerfte jedoch

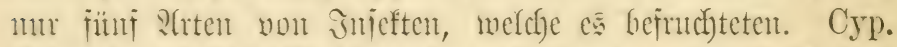
Calceolus beïtht

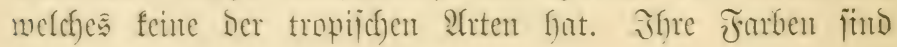

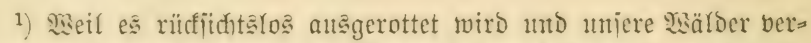

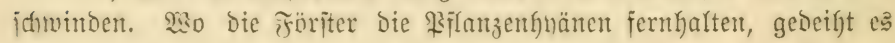
twunberidjön.

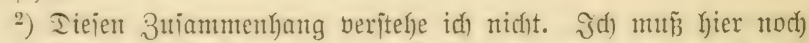

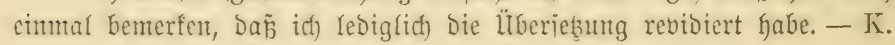




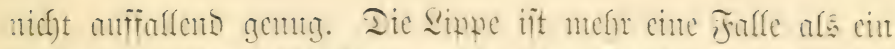

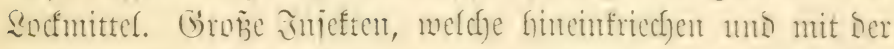

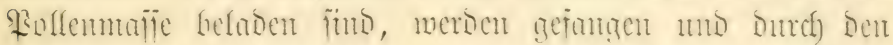

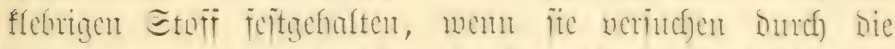

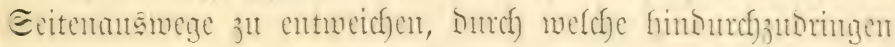
flemere Injeften zut folwach finto.

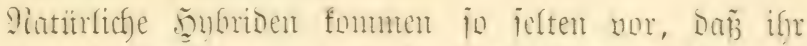

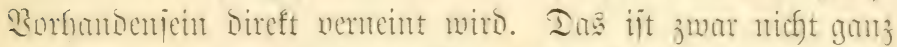
ridftig; ment wir jebodf) Den Ban Diejer (Gattung in Betratht

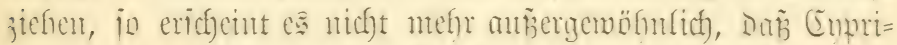
pesien fitch jo jelten untitrlich bejutchten.

Eattlenen, EDontogfoijen mo ähnfiche Sirten leben

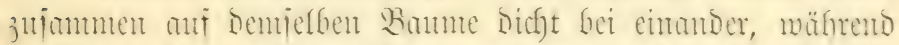

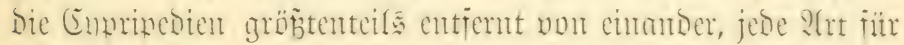

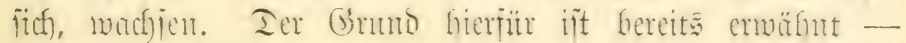

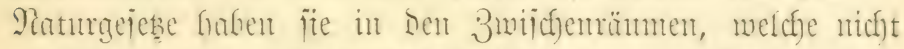
gecignet waren, eine verurteilte battung zu erfalten, ats. jterbent lafijent.

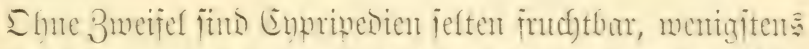

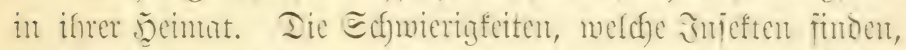

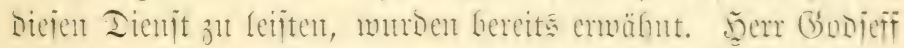

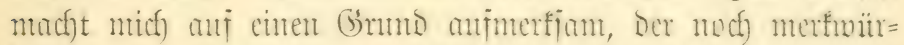

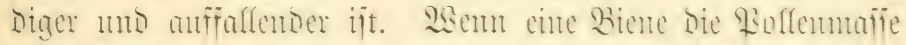
ciner Cattleya 3. B. Forträgt, fä̈ngt biejelbe am Shopf oner ber Bruit mittelft cine flebrigen Etoffes, Der ficf) an Der Pollen= maje Gefundet, jo bari, wem fie jut cinter anderen Blunte fliegt,

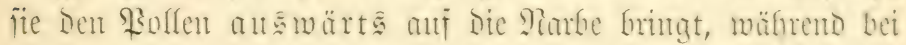
Den (Enpripedien fein jofcher Etofif norfanden ift; Dic flebrige

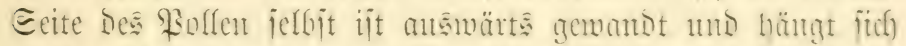

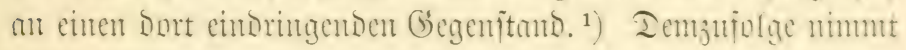

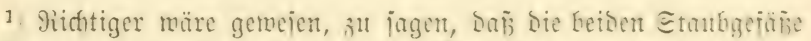

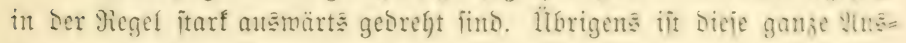
füfrung jtarf anfedtbar. - K. 


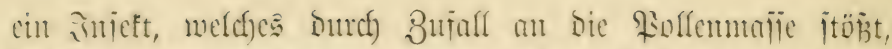

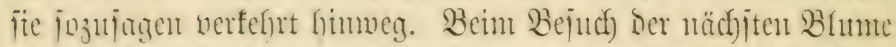

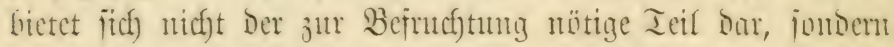

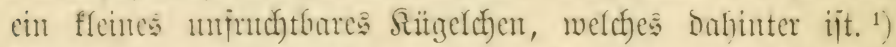

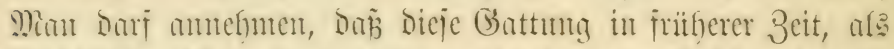

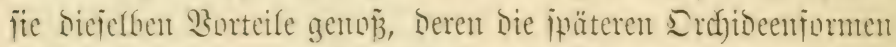

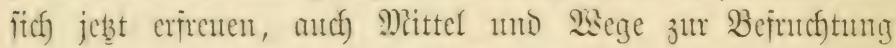
Gatte, weldhe gegenwärtig verichumon finto.

llnter joldgen unginitigen llmitünden ijt es nicht zut crmarten, ojt Eamenfupieln an eingejithrten Enpripedien zu

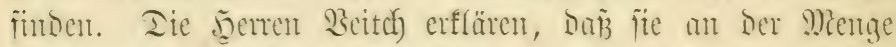

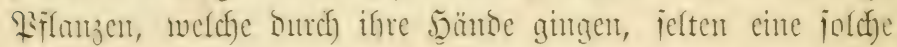

1) Ias märe richtig, menn die Jnjeften das ganze @tanfgeiäi

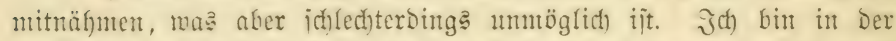

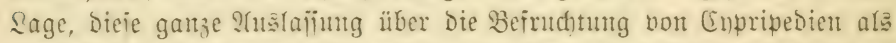

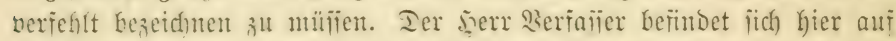

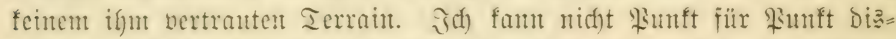
futieren, midnte aber mur anf zueierlei aumerfiam madhen. Tie Cinpri= peoten find in unieren Sanm mo ieben reidflich Sament an, iobant fimo ite leidht auf vegetativem

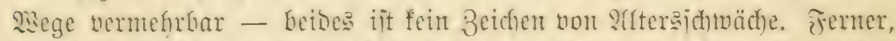
Sa bie Befrudhtung nur burdh intieften benirft werben fann, io mürben wir eher ein ungefentes sterhen unter gemijien J̀njeftentabteifungen antefmen müijen, Dur(d) melches bie sur Bejructung umungängfid nutigen Tiere vermindert oner wernichtet murben. Eine majlenhaite

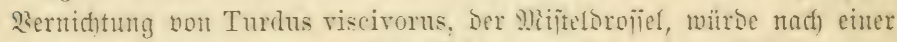

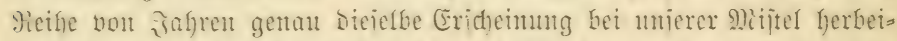

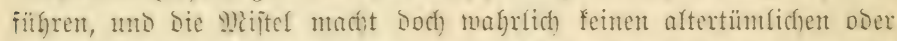

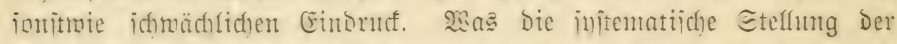
(invripedien angeft, io glaube id) allersings, bañ zmichen ifnen uno Den ïbrigen Erdfibeen ief)r viele Bindeglieber fehlent; ich gefhe indelien

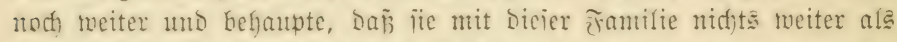
Den aflgemeinen Tupu Der Mionocotnlen gentinjam haben, im ïbrigen aber einer ganz anderen formenteife angehören. Eic nefmen jïh mter

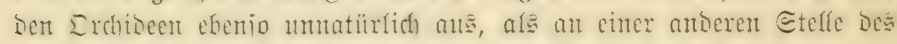

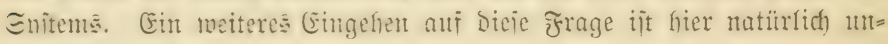
möglich). $-\mathrm{K}$. 


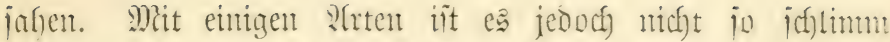

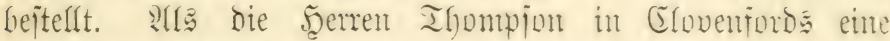
: (nzaf) von Cyp. Spicerianum einfitf)rten, als es zum eriten mal anf ben Darft fam, fanden ite cintige Sanieh, an melchen fie eintige buntoert Sämlinge erzogent. Sapięn, vol! von reifen Eamen, funben ̈̈̈h) ferner oft an importierten Cyp. insigne.

In Den anfigefitfrten Füflen finten wir bie Grflümung fïr cine anjergewöbntiche Thatiache. 5ुybrioen ober au

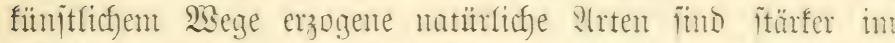

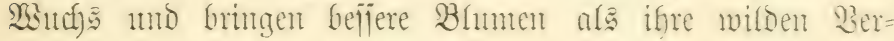

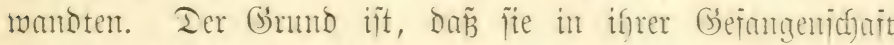

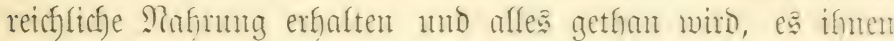

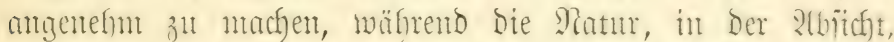

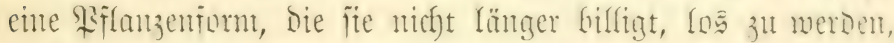
fie vernachläjifigt and verfuntgem läp̃ t. ${ }^{1}$ )

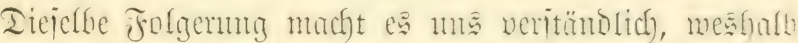

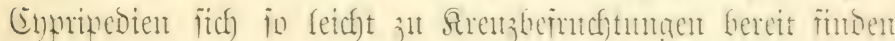

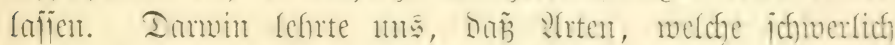

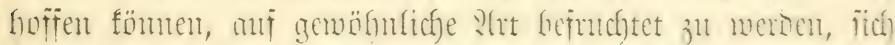

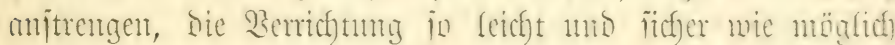

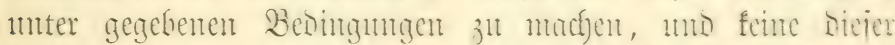

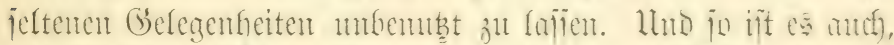

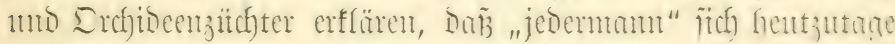
mit Dem Strenzen von Cupripedien beichäftigt.

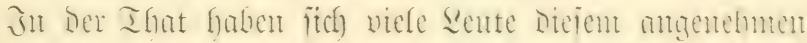

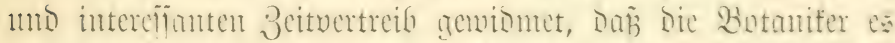

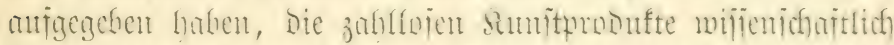

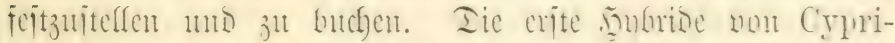
pedium wurbe non Iominu in infle $186 ! 9$ acjitchtet unD nad) Dr. Darris bentannt, Der, wie beretis gejont, ihn jur

1) W3ie brutal! Gilaubt Sherr Bonle an bie 9tatur als eine bes wuşt Gandelnoe $\mathfrak{B}$ sotenz? ? $\mathrm{K}$. 


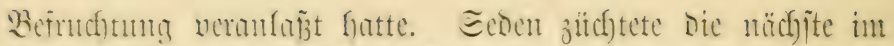
iothe 1874, mümlich Crp. Sedeni von Cyp. Schlimii uno Crp. longiflorum, benterfenwert ab der cinzige bis jebt be=

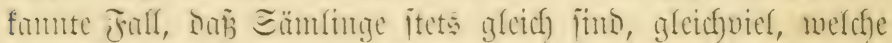

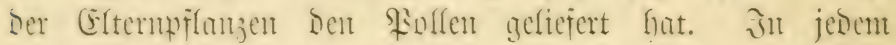
motoren Falfe f̈mb fie verichienen, je nachoen bic Junftiunen Der Eytern wechjelut.

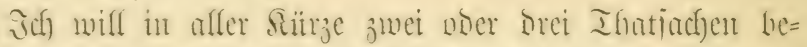

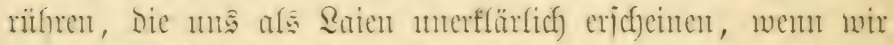

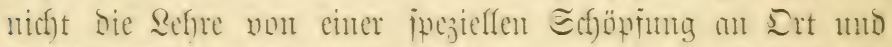
Etelfe jufajien mulfer. Oncidium cucullatum mächjt ïppig

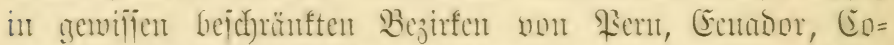

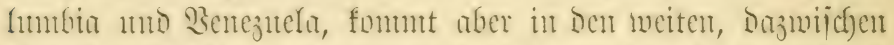

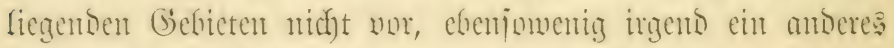

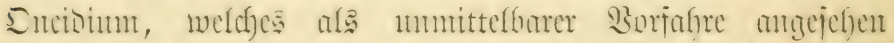

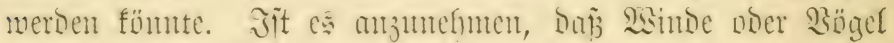

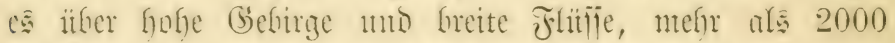
Dieifen meit, nach vier verj(f)ienenen Ridftungen tmigen, un

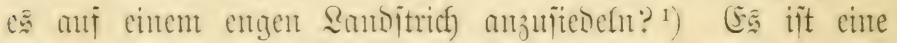
icfunicrige Jrage; ich fitr meinen Ieil mtöchte eher Denfen,

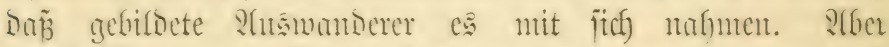

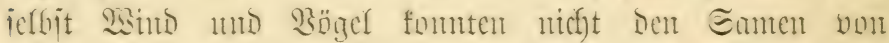

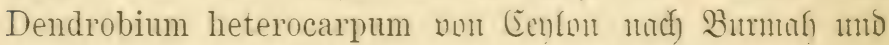

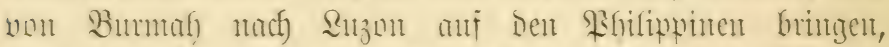

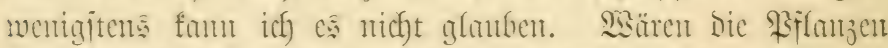

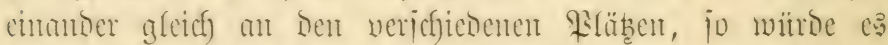

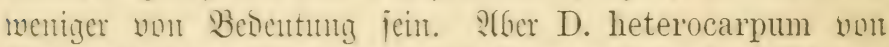

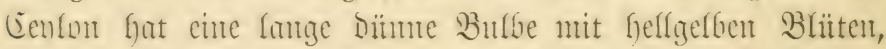

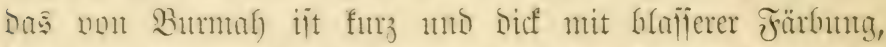

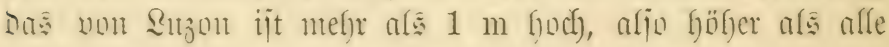

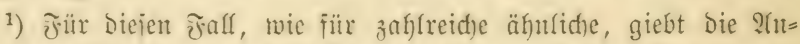
nahme cinter (Eişzeit eine gute Erflärung. O. cuctullatum ijt eine aus $=$ geiprod)ene Berg=srdibee. 


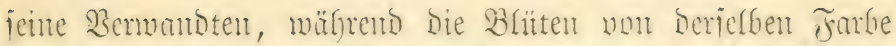

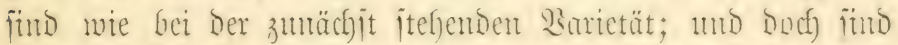

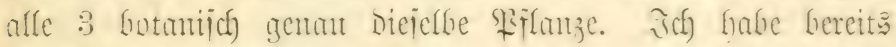

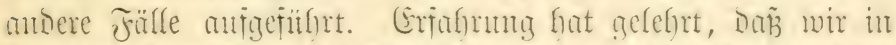

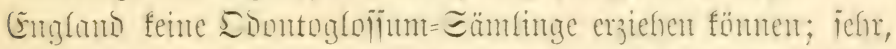
jefrr menige finto überfont bis jeşt gemonnen worden. Ber=

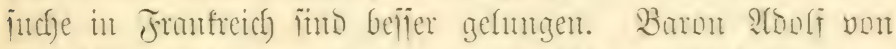

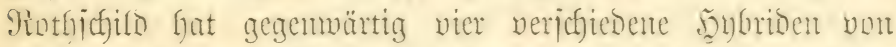

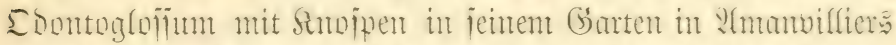

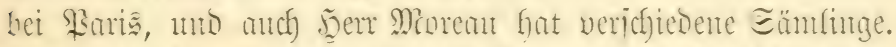

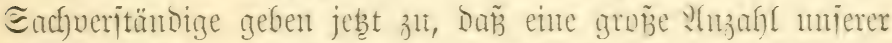

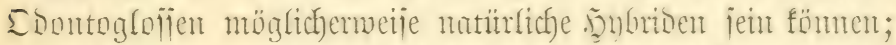

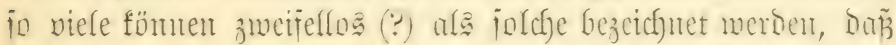
Das Epefulationa felo fait feine Grrenzen fat. ${ }^{1}$ ) 0 . excellens iit jitcher (?) ein : Plof́nmmling von O. Pescatorii mo O. triumphans, O. elegans von 0 . cirrhosum uno 0 . Hallii, 0. Wattianum von 0 . Harryanum un 0 . hystrix. (⿺辶一 mun

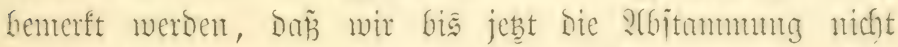

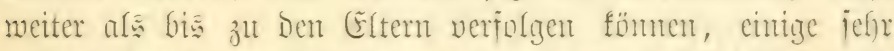

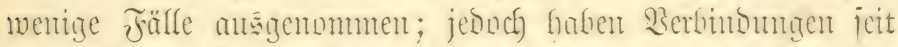
umbenffichen Beiten itattgeimben. Shne Bweifel fün Die Erchibeen Die jüngiten Simber Der Fflora, aher zugleich) ifnee

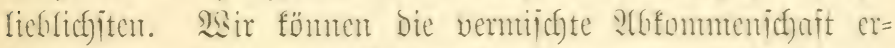
femten von 0 . crispum Alexandrae, gepant mit 0 . gloriosum, O. luteo-purpureum uno O. Lindleyanum. Dicje

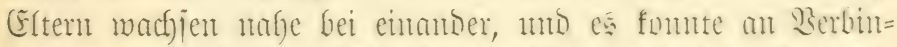

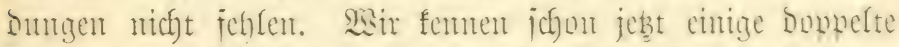

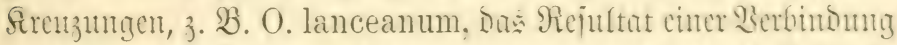
3miidfen 0 . crispum Alexandrae mo 0 . Rückerianum.

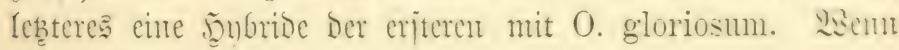

1) Bis jebst iit, wie ñerr Bonle jelbit an anoeren Erten sugegebon Gat, nod) fein EDontogloïum fünitlid) nachgefifbet worsen; von imifellos ijt aljo feine giebe. 


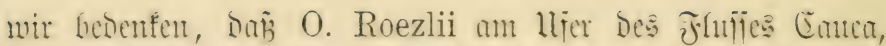
O. vexillarium höher finnuf, Dajegen 0 . vexillarium superbum zmijefen beisen mächit, jo fümen mir oreiit bie Somperfurfeit cines Greiten Dunflen Ffledens anf Der Sippe

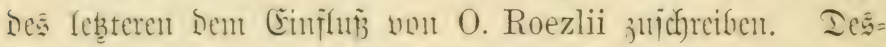

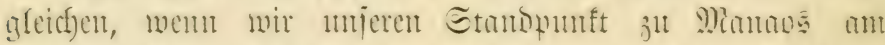
S(mugontenftrom nefmen, fo funden wir im Elten Cattleya superba, im siejten C. Eldorado mo in Der Mitte C. Bry-

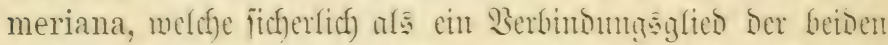

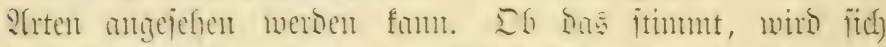

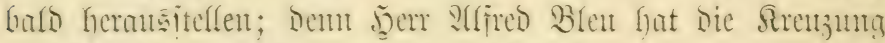
zmichen C. superba mo C. Eldorado nurgenummen und

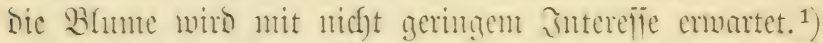

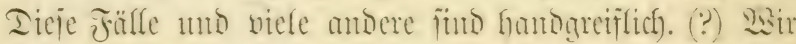

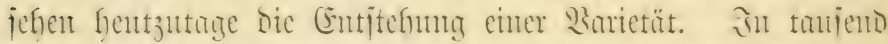

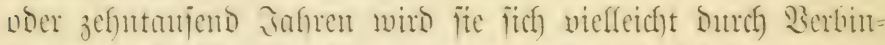

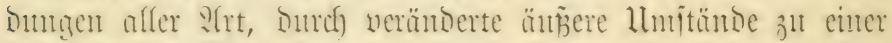

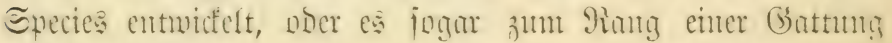
gebractit Gaber.

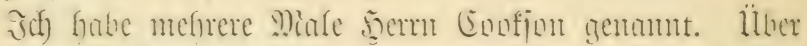

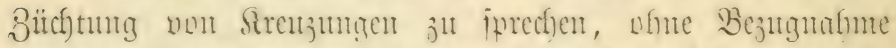
anf jeine erjtannlichen Reijtungen, mitrde in ber That un=

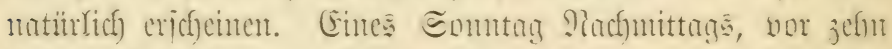

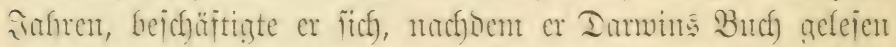
foutte, mit ber Erforichung ber Etruftur einiger Enpripedien

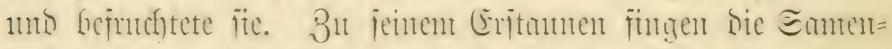
fapjefu an ju ichmelfen, mo jult gleicher Beit jitieg ñem

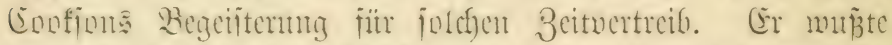

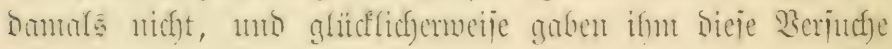

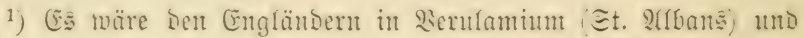

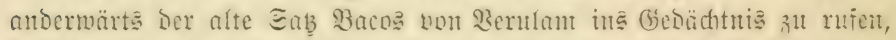

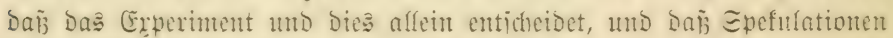
itid) Yeidft ins Blaue berlieren. - K. 


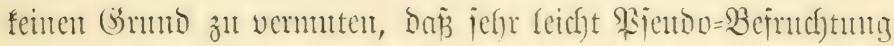
Durch ingent ctwas bemirft wernen fanm. Eo untgemein

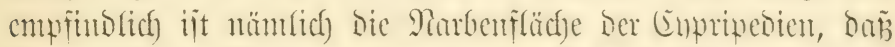

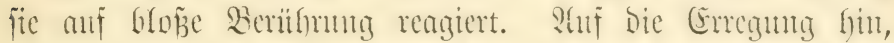

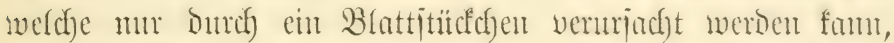
wiro fie alfe äuñerfich fitchtbaren Stabien ber Befund tunt

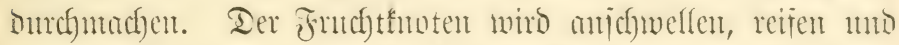

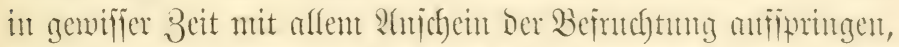

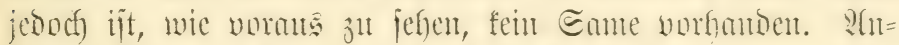

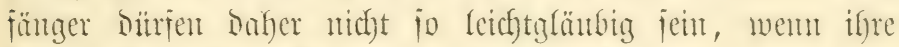
fiit)nen Berjuche and viefverjprechens erjdenent.

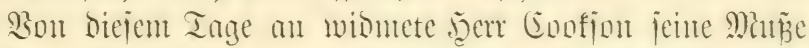

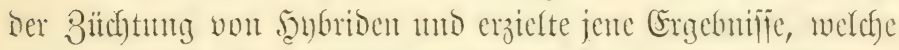

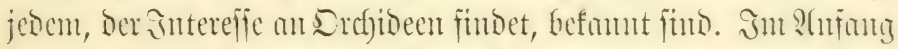

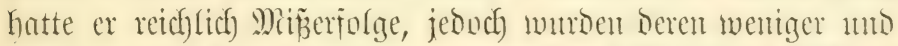
mentiger, jo ba

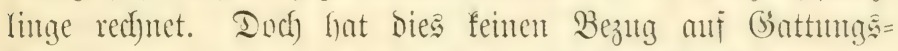
sirenzungen, weldyen er bis jebt jeine Shujuerfjumfeit wentiger geichenft hat. Mit Supripebien anfangento, hat er jeţt 94

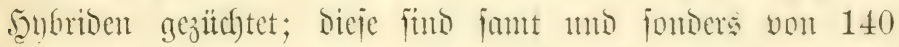
Eamentapjeh gewounen. Şou Ealanthen erjiclte er 16

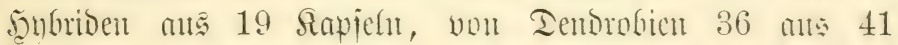

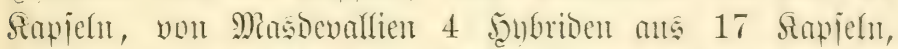

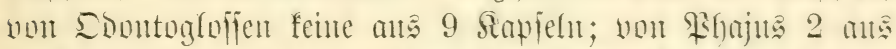

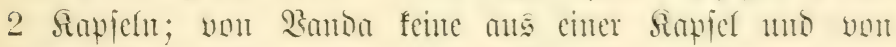

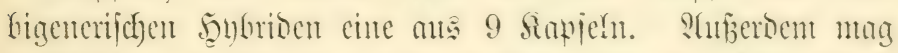

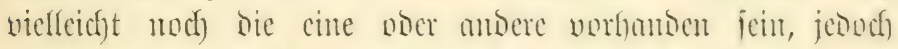

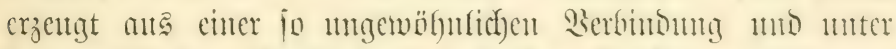

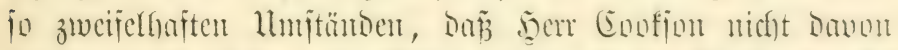

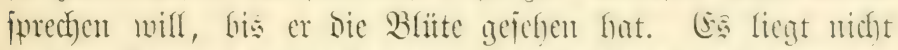

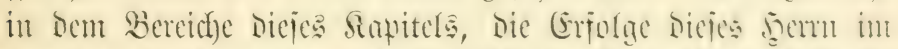

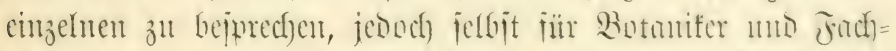

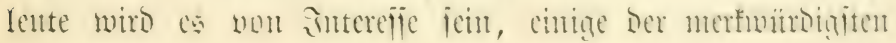


Sirenjungen femmen zut lemen; Demu fie finto bisher nich)t ver= offentficht. Эch fübre auf gut (3)litcl folgende an:

$\begin{array}{cc}\text { Phajus } & \text { Wallichii } \\ \text { Laelia } & \text { praestans } \\ " & \text { purpurata } \\ " & " \\ " & n \\ \text { Cattleya } & \text { Mrginata } \\ " & \text { Trianae } \\ " & \text { Percivalliana } \\ " & \text { Lawrenceana } \\ " & \text { gigas } \\ " & \text { crispa } \\ " & \text { Dowiana } \\ " & \text { Schofieldiana } \\ " & \text { Leopoldii }\end{array}$

Cypripedium Stonei

$\begin{array}{ll}" & \quad \text { Sanderianum } \\ " & \text { Spicerianum } \\ . & \text { Jo }\end{array}$

$\times$ Phajus tuberculosus

$x$ Cattleya Dowiana

$\times \quad "$

$\times$ Laelia grandis tenebrosa

$\times$ Cattleya Mendellii

$x$ Laelia elegans Cooksoni

$\times$ purpurata

$\times$ " harpophylla

$\times$,

$\times$ Cattleya Mossiae

$\times \quad, \quad$ Gaskelliana

$\times \quad$,

$\times \quad " \quad$,

$\times \quad$ "gigas imperialis

$\times \quad$ Dowiana

$\times$ Cypripedium Godefroyae

$\times \quad$ Spicerianum

$\times \quad$ Veitchii

$\times \quad, \quad$ Sanderianum

$\times \quad$ vexillarium

Dendrobium nobile nobilins $\times$ Dendrobium Falconeri

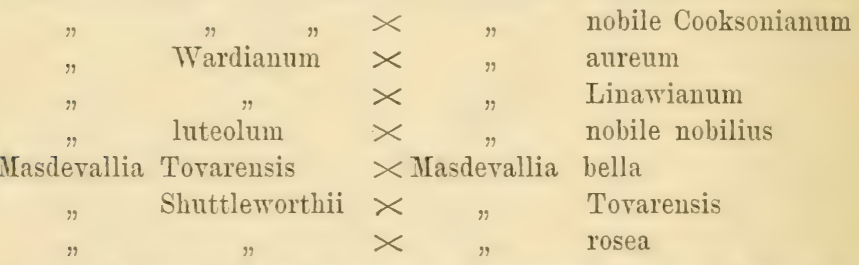

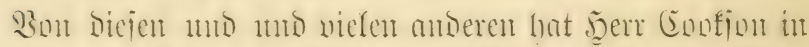
Diejem : Angenblid 15000 Piflamzen. Da mein Endzued Der

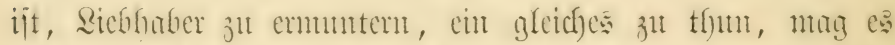
mir vergönnt jein, etmas ju Gerïfren, wa nuter anderen llnt=

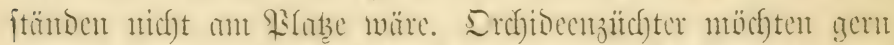

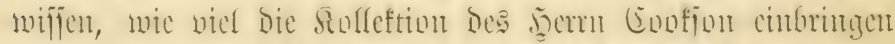

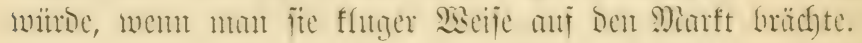




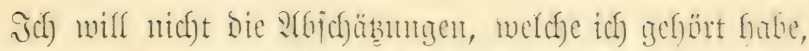

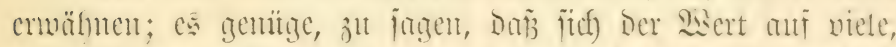

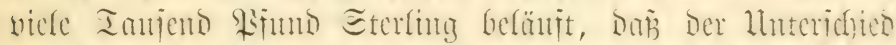

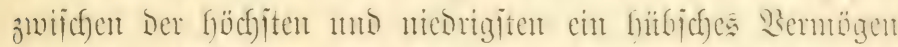

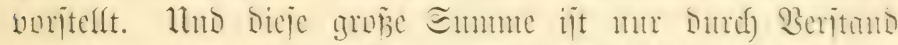

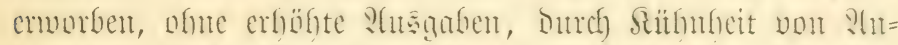

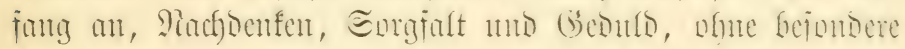

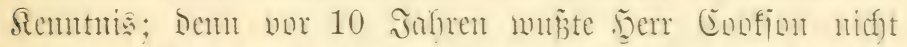

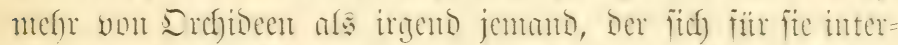

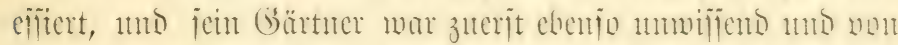

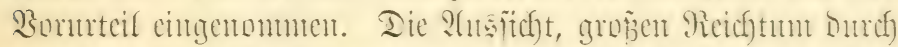

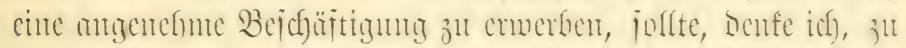

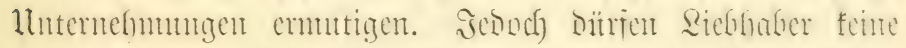

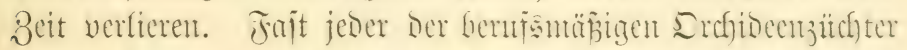

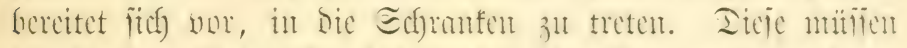

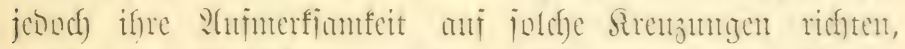

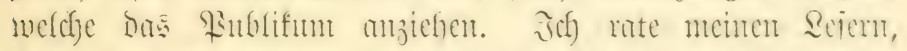

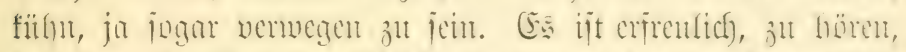

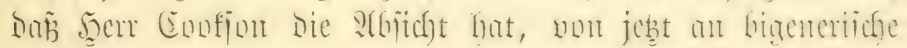
Beprutchtungen anfmerfiam zu beobad)ten. ${ }^{1}$ )

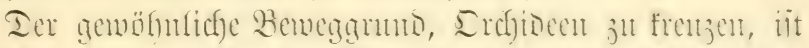

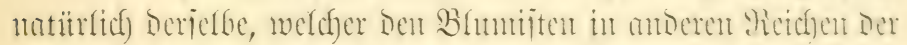

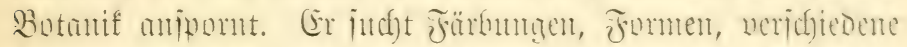

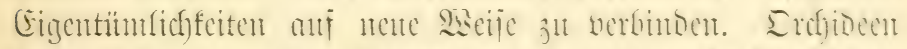

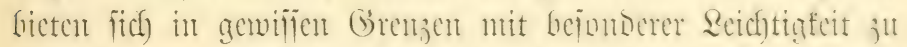

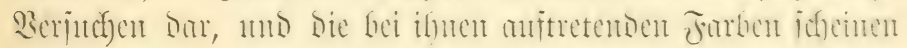

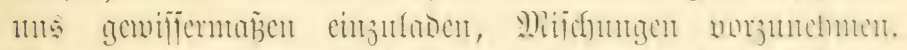

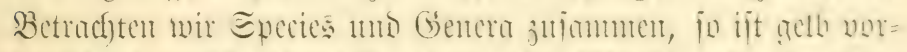

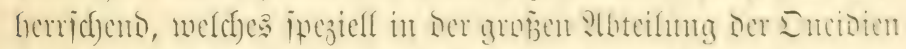

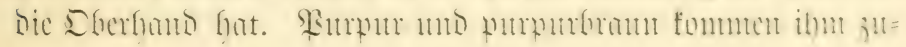

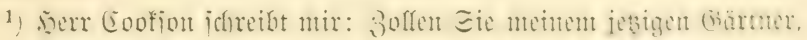

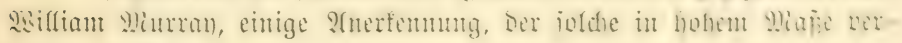
Dient. - Sutor. 


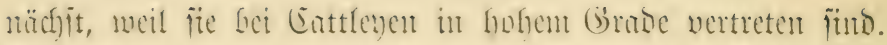

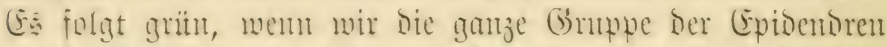

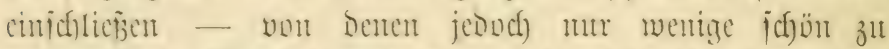

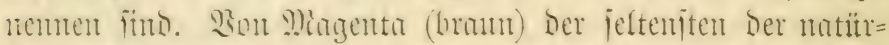
lichen Förbungen, foben wir nur wenige; fantepin in tanjens

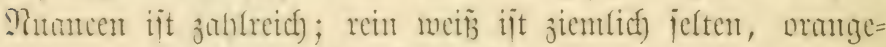

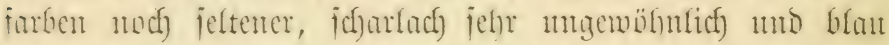

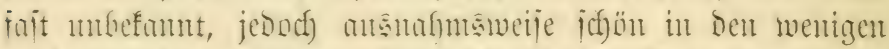

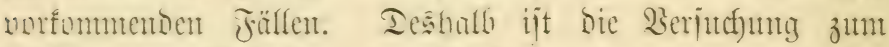

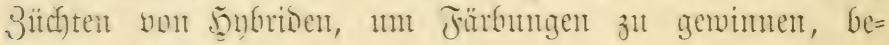
ionbers ftart.

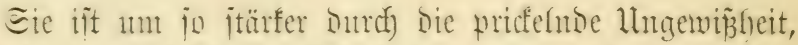

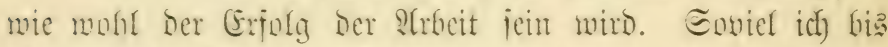
jest gefount noer gelejen habe, ijt nemand in jtande, Begefn aufjujtelfen, melche anf bas Rejultat Der Berbindungen

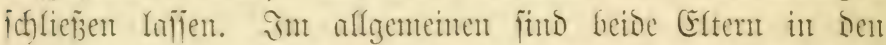

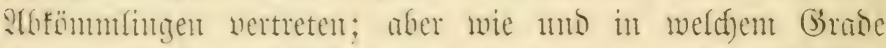

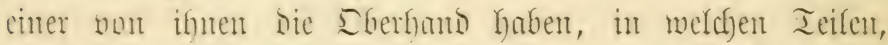

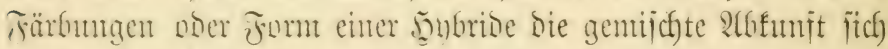

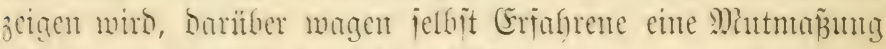

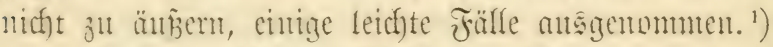

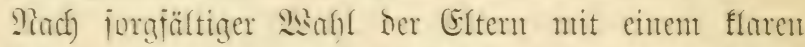

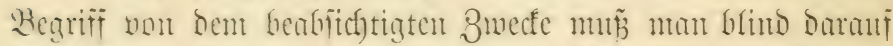
low gehen. Eefre nit wiro bas gemitndate Biel in geföriger 3eit erreicf)t, iefre bif fonmt etwas ganj Ilnermartetes zum

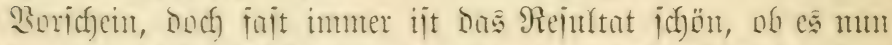

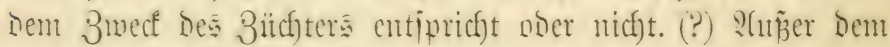

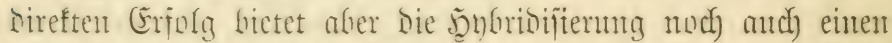

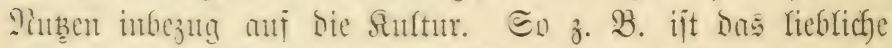
Cypripedium Fairieanum in idfuierig zu fultivieren, ba

1) Эิก allen non mir unteriudten Füllen, tvo bie (Eltern jidjer

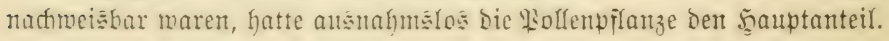

$\mathrm{K}$. 


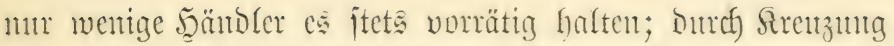
mit Cyp. barbatum von Miount Sprir, einer wetterfarten falten $\mathfrak{A}$ rt, erfalten wir Cyp. vexillarium, welches bic fejte

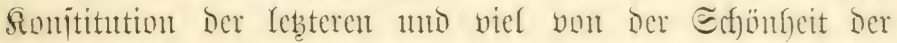
eriteren hat. Cyp. Sanderianum ans bem malanifonen

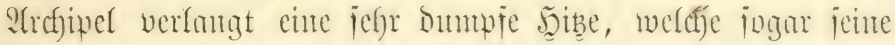
Berwanden jelten Yichen. (Fs ijt jedoch mit Cyp. insigne gefreagt worben, welches itberall geneigt, unt obgleich bie Eämlinge bis jest nicht geblüht haben, jo ijt boch nicht zut

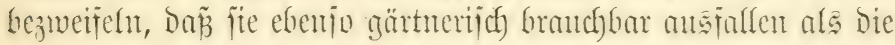
vorher genamuten. Tie icföniten Barietäten von Cyp. insigne fins in groper Menge zu forden Buecten verwentet morden. Sir haben ferner das anfaflente Cyp. hirsutissimum; es hat Eepalen von cinent nubeitinmten Gerfogrïn,

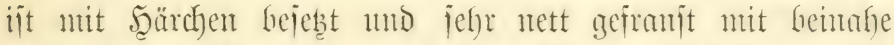
verblitfenden funtrait vou hellen Pimpur. Fitr's erite ijt

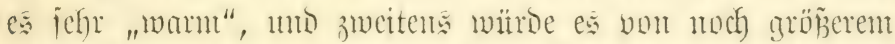

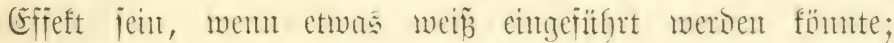
es ijt mit Cyp. niveum gefrenst morden, mo jumeritcht $=$

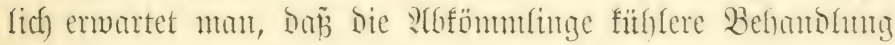

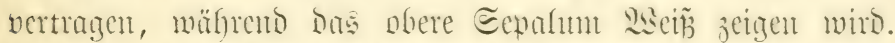
Ebenjo ijt bie reizento Masdevallia Tovarensis mam, wei⿸̈ mo niebrig; gefteugt mit Masd. bella minme fie beren Figen=

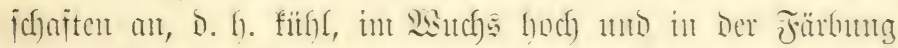

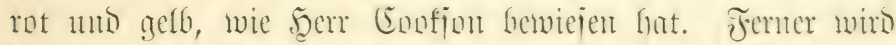

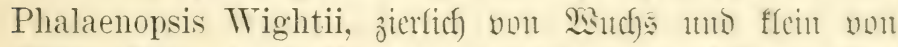
Blïte, fräftig Durch Sirenłumy mit Phal. grandiflora, vlnte jeonch igren zarten Farbenton zn verfieren.

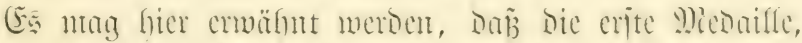
melche vou ber Royal Hortieult. Society fïr cinten Eämling

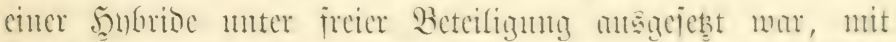
Laelia Arnoldiana in infure 1891 gemonnen mme. Iie=

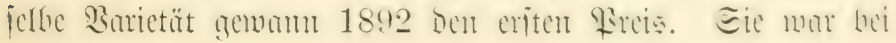


5ुerm Sander vou L. purpurata mo Cattl. labiata ges 3ongen, im Jaljre 1881 angejät mo Grïfte 1891.

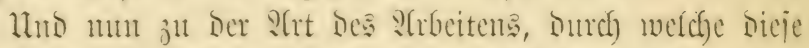

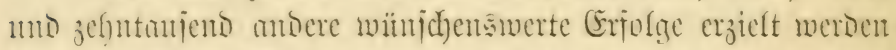

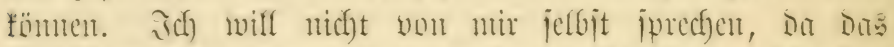

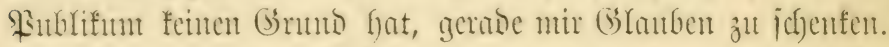

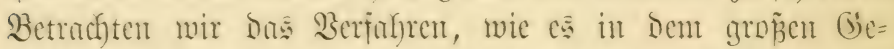

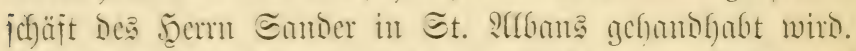

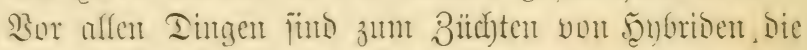

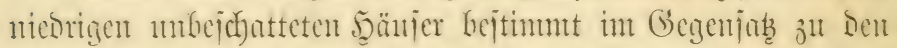

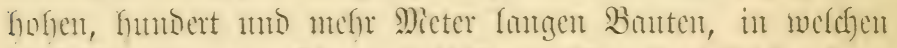

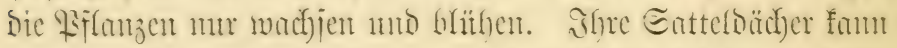

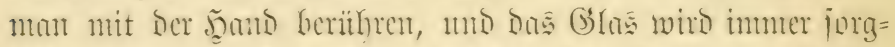

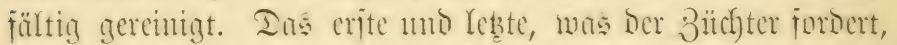

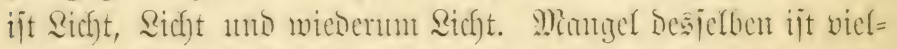

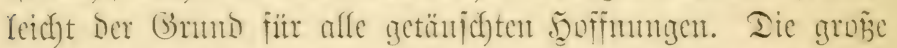

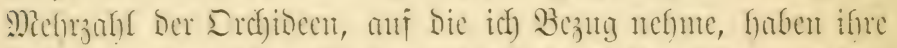

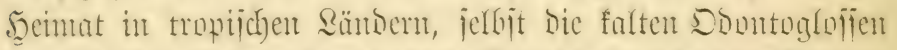

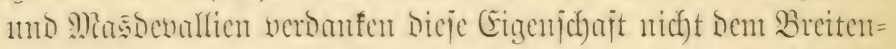

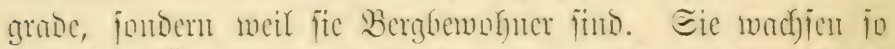

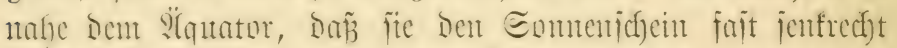

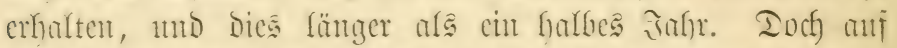

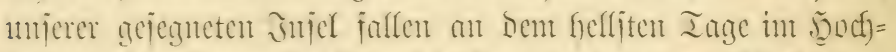

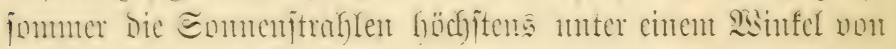

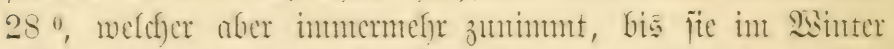

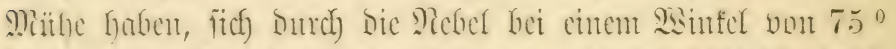

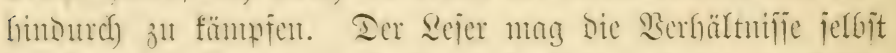

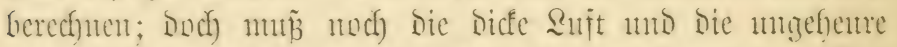
Bafle ber nebligen Iage in Betracht gejogen merden. Sisir

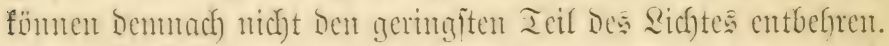
Ier reifente Eame mun Dicht unter bem Gifaje ftehen, und

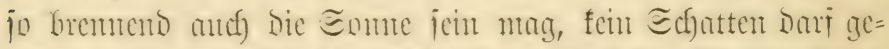

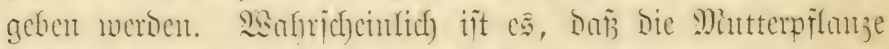

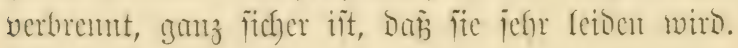




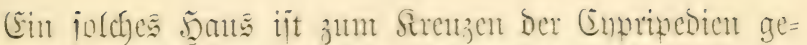

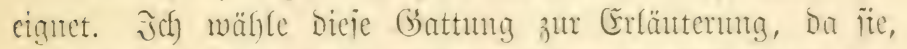

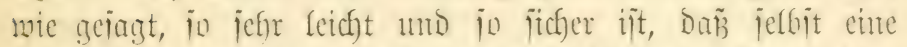

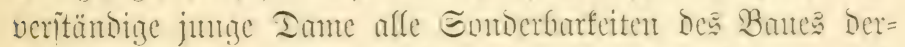

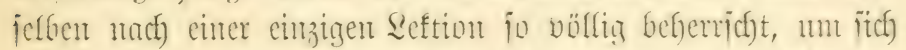
mit ifne ehenjo gut mie mit Denorobien, Dntioien, Donutu=

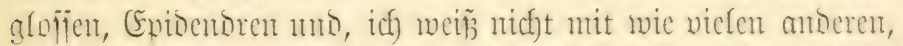

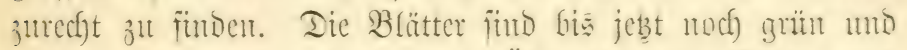
glatt, mit mantfen jonderbaren Überrejten bon Blitten und

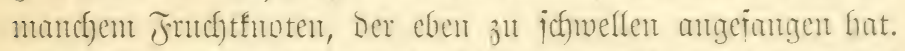

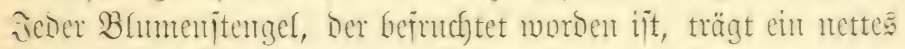
(Étifett, welches Den Namen Des 23ater und Das Datum Der Streuzung angiebt.

Die Patur verfiert feine Beit, man fam fajt ingen, Die Echeibe heginnt jofort manuchwelfen. Der Ieil, welchen

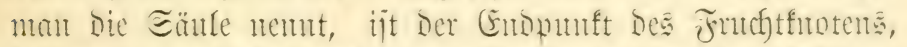
Ser Drei, ober jects, and neur (Eentimeter von bem Blitten= itengel finter ber Blïte cimninme. Edfon nach mentigen

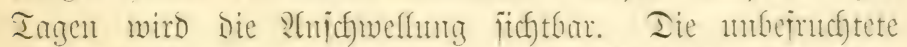
Bhlume füllt zull bejtimmten Beit ab, jenodg Die bejutchtete

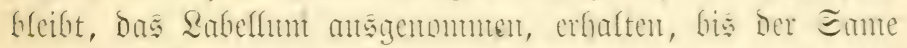
reif iit, was vieffeicht nad) cinem frafhen Jofnre eintritt; ielbit=

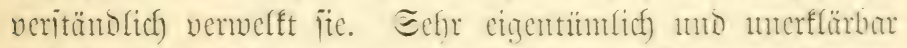

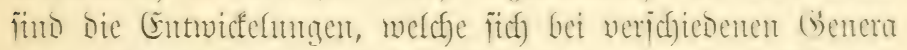
noer jelgit Epecies nuch Der Bepruchtung zeigen. Bei Den

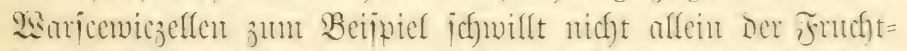
fnotent, jontom bie ganze Säıte ant. Phalaenopsis Luddemanniana ijt Gejonoer merfwitrotg. Die ichönen Etreifen und Frlecfen in roja, Grant and pumpur nefinten jofort cinte gritul=

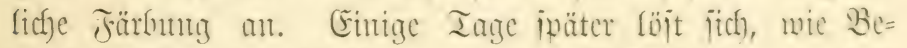

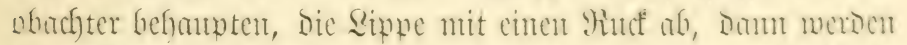

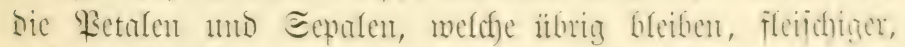
bicfer uns bicfer, mähreno bie ?tütancen verblaffen uno Das 
(G)

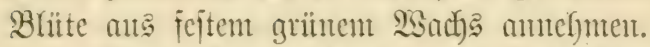

Unjer Cypripedium miro Den Eamen in mugejähr 12 Mionaten, vieffeicht auch etmow mefre oner weniger Beit reifen. Iann flabst bie Sapjel, meldye $21 / 2 \mathrm{~cm}$ lantg it mo $1 \mathrm{~cm}$ im Iutchmefier hat. Fुerr Miannard, verantwort=

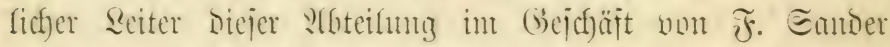

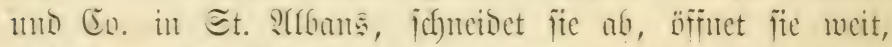
und ftreut bie Ianjente vou Eamen, viefleteft 150000 ,

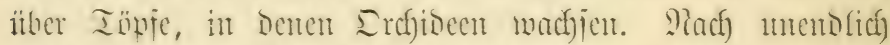

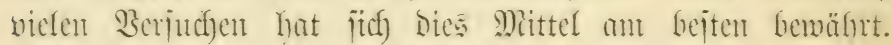
Ier Eame, feiner als ein Etanbfom, beginnt fojort anzits=

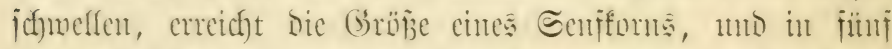

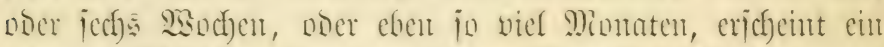
mingige Blättchen, Dann eine fleme Blatt, und in vier bis jünf Jafnen fümten wir bie Blitte

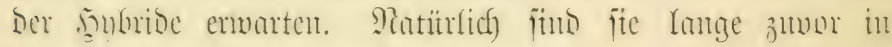
ifre bejondern Töpfe gepflangt wordent.

Eeltiante Ereignilife fonment ojt bei Derartigen Berjuchen

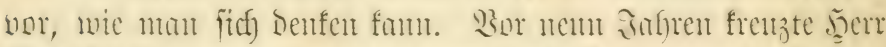
Gunjcfi Catasetum macrocarpum unit Catasetum callosum.

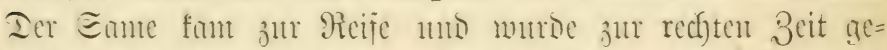
fäet, jenuch feimte nichtô an Der betreffenten Etelle. Santge

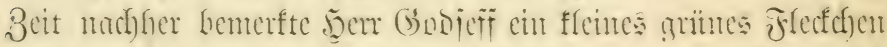

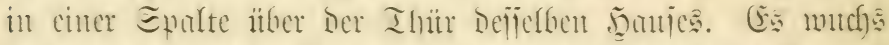

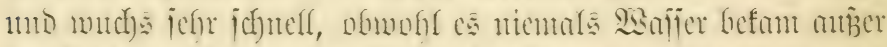
Durth chutu jeltenen Bufall, his Die Semter es jür ein junges

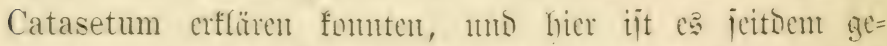

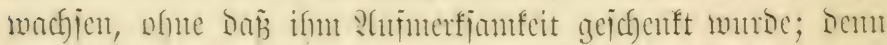

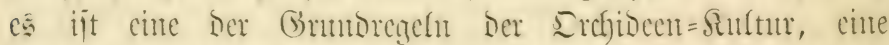

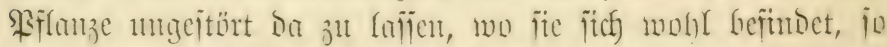
frembartig anch) bie ltmittüno ericheinen mögen. Diejeg

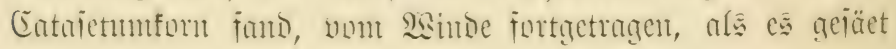




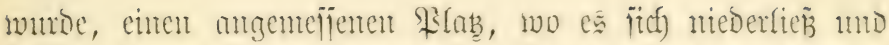
Ichendig murbe, mäfrend alle jeine (sefüforten, fitr weldfe be=

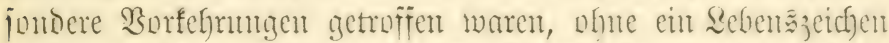

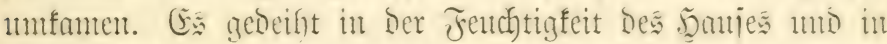

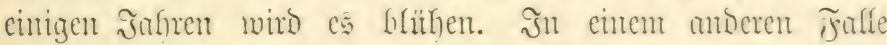

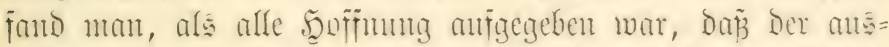
geiäcte Eante anjgehen würDe, unter Dem föbzenen (s)itter=

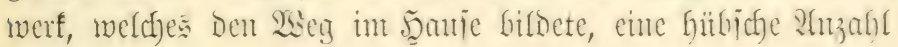
von ๔ämlingent.

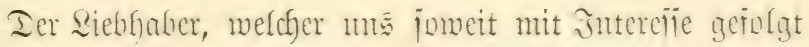
iit, wirb fragen, wie lange es banem fanm, bis wir ein Rejultat unjeres Beriaforens ermarten fömuen? Zuerit mun

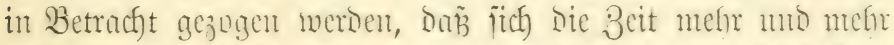
verfitrzt, je nach Den Erfahmungen, weldhe wir gentad)t haben.

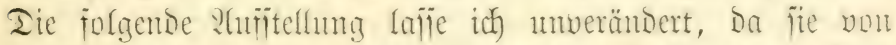

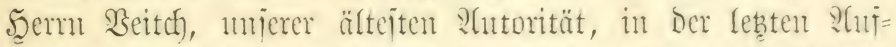

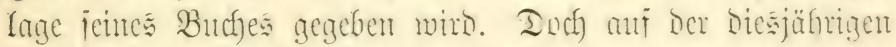

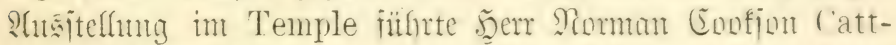
leya William Murray, cinen vfbënmuling non Catt. Mendellii $\times$ Catt. Lawrenceana. nor, eine foerfiche Biflanze, unelofe

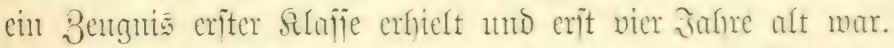

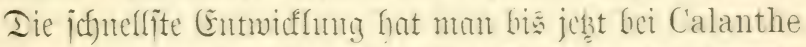
Alexandri geimnen, nit welcfer Deerr Enofinu cin Benguis

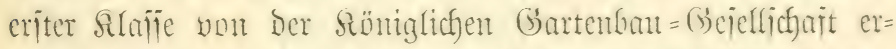

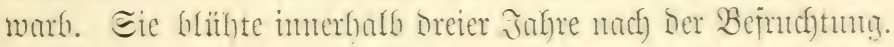

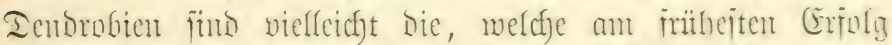

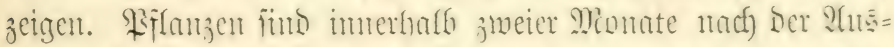
jaat pifiert worben nuth haben im vierten Jafne geblitht.

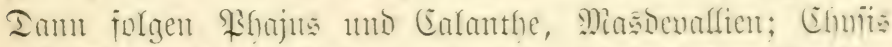

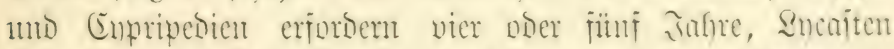
fieben bis acht, Sachen und Cattlenen zehn bis jubli Jolure.

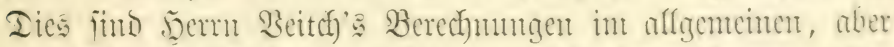

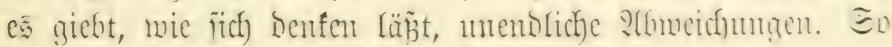




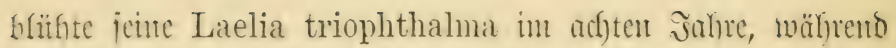
ieine Laelia caloglossa biš zum neunjchnten zögerte. B̧e= junners eigentimuliche Etreiche madht dic Gattung Zygopetalum. Z. maxillare gefrenjt mit Z. Mackayi verlangt

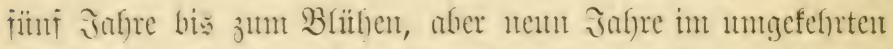

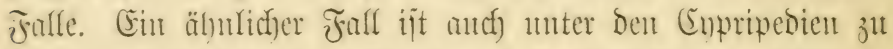
finoert. C. Schlimii, gefrengt mit C. longifolium, blitht in

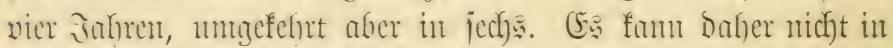

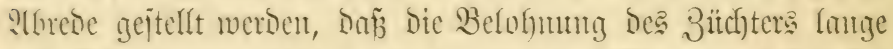

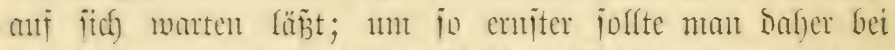
Den Stenzungen Daranf Gedacht jein, Rejultate anzultreben, welde Des wartens wert finto. 


\section{Stein's \\ Orchideenbuch.}

\section{Beschreibung, Abbildung und Kulturanweisung}

der empfehlenswertesten Arten.

Mit 184 Textabbildungen. Gebunden, Preis 20 M.

Die Orchideenkultur hat in Deutschland während des letzten Jahrzehnts ausserordentlich an Ausdehnung gewonnen, und in den weitesten Kreisen anerkennt man jetzt das Berlürnis, Orchideen nicht mehr als einzelne Prunkblumen, sondern auch in grossen Mengen in den Handelsgärtnereien zu ziehen. Für Orchideenliebhaber und Kultivateure machte sich daher auch ron Jahr zu Jahr mehr der Mangel eines guten Handbuches fühlbar, welches eine korrekte Schilderung der Arten und Varietäten mit einer Kulturanweisung verbindet und die empfehlenswertesten Formen in guten Abbildungen bringt. Deswegen glaubt der Terfasser mit der vorliegenden Zusammenstellung Gärtnern und Liebhabern in gleicher Weise zu dienen, da in ihr alle kulturwerten Arten berücksichtigt sind.

\section{Reichenbachia.}

Chromolithographische Abbildung, Beschreibung und Kulturanweisung der

\section{schönsten Orchideen.}

Unter Mitwirkung wissenschaftlicher Autoritäten herausgegeben von

F. Sander in St. Albans, England.

4 Bünde mit je 48 Tafeln nebst Text. Preis 580 M.

Auf Tafeln im Format von 40 zu 54 Centimeter sind die Orchideen in natiurlicher Grïsse und Farbenpracht chromolithograpi-ch so vollendet abgrebildet, wie es bei Blumen bisher rielleicht niemals gelungen, und der in deutscher, englischer und französischer surache abgefasste Text fusst auf den Erfahrungen jahrelanger Kultur-Praxis. 
Verlagsbuchhandlung Paul Parey in Berlin SW., Hedemannstrasse 10.

\section{Vilmorin's Blumengärtnerei.}

\section{Beschreibung, Kultur und Verwendung}

des gesamten Pflanzenmaterials für deutsche Gärten. Dritte, neubearbeitete Auflage,

Unter Mitwirkung von

\section{A. Siebert,}

Direktor des Palmengarteus zu Frankfurt a.M. herausgegeben von

A. Yoss

in Berlin, früher Institutsgäirtner in Göttingen.

Hit 12:-Textabbildungen u. 400 buuten Blumenbildern auf 100 Farbendrucktafeln. Zwei starke Bände in Gross-Lexikonformat.

In Halbleder gebunden, Preis 56 M.

Ein Buch, wie diese dritte, neubearbeitete Auflage ron Tilmorin's Blumengärtnerei gab es bisher nicht; es war noch nie die Follstïndigkeit, unbedingte Zurerlässigkeit und praktiselıe Branchbarkeit erreicht, welche von Gärtnern und Liebhabern sehnlichst gewïnscht war.

Der erste, über 1200 Seiten in Gross-Lexikonformat umfassende Teil ist eine mit 1272 schwarzen und 400 farbigen Abbildungen geschmiickte, nach Familien und Gattungen geordnete, vollständige

\section{Deutsche Gartenflora mit 100 Farbendrucktafeln}

(Freilandpflanzen, Kalt- und Warmhauspflanzen).

An der Spitze befindet sich ein alphabetisehes lerzeichnis aller im Werke rorkommenden Pflanzennamen, sowohl der botanischen (einschliesslich der Synonyme) wie der deutschen, und durch den Druck ist kenntlich gemacht, welches die gültige, korrekte Bezeichnung ist.

Im zweiten Band, dem angewandten Teil des Werkes, finden sich in übersichtlicher Weise die Grundziige der Gartenkultur behandelt, und dann folgen Aufstellungen und Register der rerschiedensten $\Lambda$ rt. Es sind nämlich die Pflanzen gruppiert, je nachdem sie sich zur Einfassung und Beflanzung von Rabatten, zur Einzel- oder Gruppenserwendung, zu Teppichbeeten oder Trupps eignen; die Schattenpflanzen, die wohlriechenden Pflanzen, die Pflanzen mit farbigem Laub, die Schlingpflanzen, die Pflanzen mit Zierfrüichten etc. sind zusammengestellt. Desgleichen sind die Blumen nach ihrer Farbe geordnet und in einem Blïtekalender nach der Zeit ihrer Blüte. Des weiteren enthält dieser Teil eine ausführliche, durch zahlreiche Pläne erläuterte Anleitung zur Anlage einheitlicher Gartenszenerien, farbenreicher Blumenteppiche, Rabattenbepflanzungen $\mathrm{u}$. s. w.

Trotz des grussen Lmfanges hat das Werk im Tertranen auf einen aussergewöhnlich grossen tbsatz einen in Aubetracht des Gebotenen ïberraschend niedrigen Preis.

Was ein Konversations-Lexikon für das allgemeine Wissen bedeutet, das leistet der neue Vilmorin für die Ziergärtnerei und ist deshalb unentbehrlich für Jedermann, der nach Beruf oder Neigung sich mit einem Garten beschäftigt. 


New York Botanical Garden Library
QL $66 . A 1$ B 6551896 gen

Boyle Frederick/Uber Orchideen. Deutsch

(

|| |||||||

35185000580025 
